\title{
Overview of Groundwater Quality in the Piceance Basin, Western Colorado, 1946-2009
}

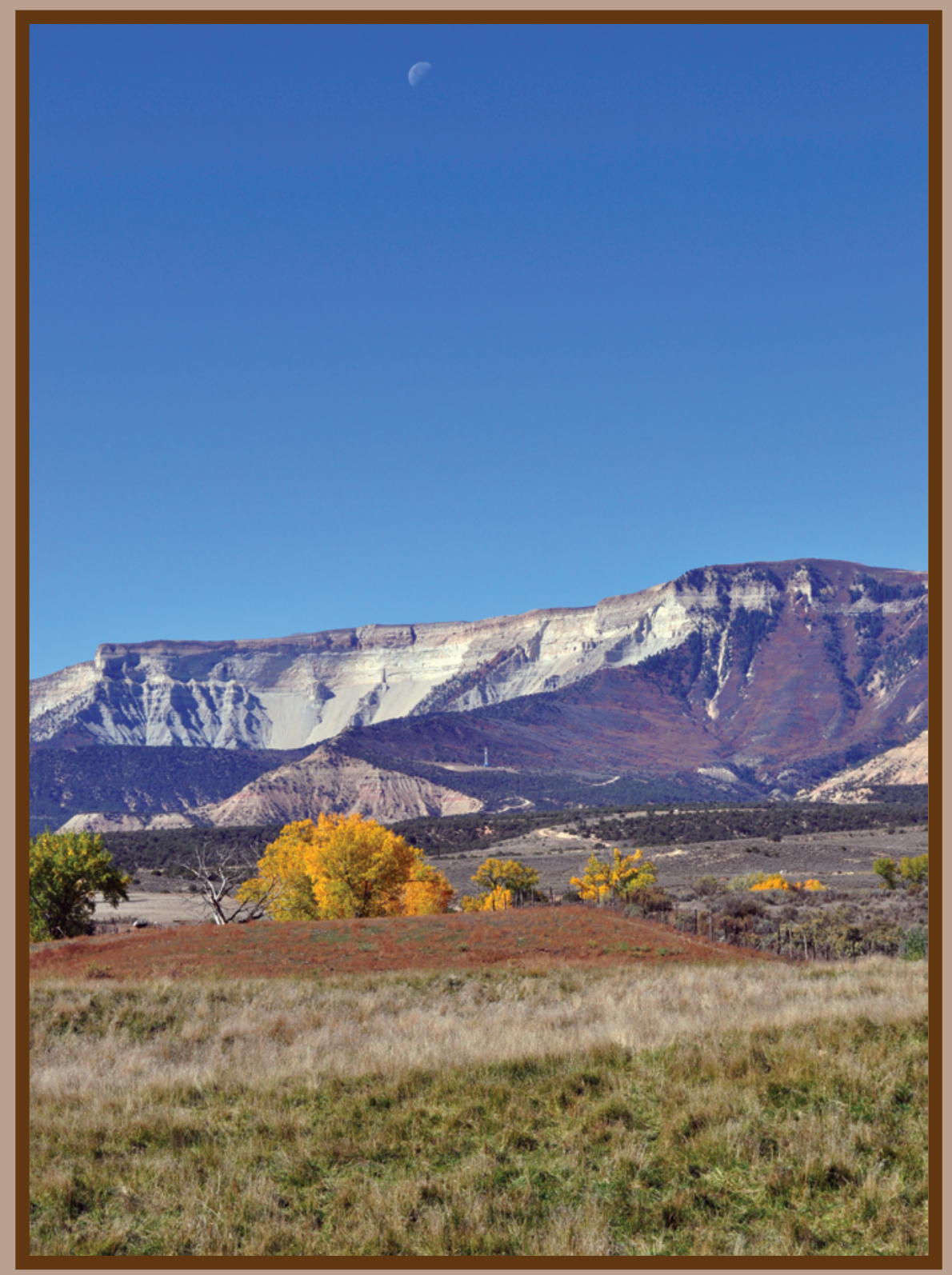

Scientific Investigations Report 2012-5198

U.S. Department of the Interior

U.S. Geological Survey 
COVER. Drill rig at base of Roan Cliffs near Rifle, Colorado (photo taken by Judith Thomas, U.S. Geological Survey). 


\section{Overview of Groundwater Quality in the Piceance Basin, Western Colorado, 1946-2009}

By J.C. Thomas and P.B. McMahon

Prepared in cooperation with (in alphabetical order): Antero Resources, Bureau of Land Management, Bureau of Reclamation, Chevron Corporation, Cities of Grand Junction and Rifle, Colo., Colorado Department of Agriculture, Colorado Department of Natural Resources, Colorado Department of Public Health and Environment, Colorado Division of Wild life-River Watch, Colorado Oil and Gas Conservation Commission, Colorado River Water Conservation District, Delta County, Colo., EnCana Oil \& Gas (USA) Inc., Garfield County, Colo., Gunnison Energy Corp., National Park Service, Natural Soda, Inc., North Fork River Improvement Association, Oxy Petroleum Corporation, Petroleum Development Corp., Rio Blanco County, Shell Oil Company, Solvay Chemicals, Towns of Carbondale, De Beque, Palisade, Parachute, Rangely, and Silt, Colo., U.S. Forest Service, West Divide Water Conservancy District, and Williams Companies, Inc.

Scientific Investigations Report 2012-5198

\section{U.S. Department of the Interior U.S. Geological Survey}




\section{U.S. Department of the Interior \\ KEN SALAZAR, Secretary}

\section{U.S. Geological Survey \\ Suzette M. Kimball, Acting Director}

U.S. Geological Survey, Reston, Virginia: 2013

For more information on the USGS - the Federal source for science about the Earth, its natural and living resources, natural hazards, and the environment, visit http://www.usgs.gov or call 1-888-ASK-USGS.

For an overview of USGS information products, including maps, imagery, and publications, visit http://www.usgs.gov/pubprod

To order this and other USGS information products, visit http://store.usgs.gov

Any use of trade, firm, or product names is for descriptive purposes only and does not imply endorsement by the U.S. Government.

Although this information product, for the most part, is in the public domain, it also may contain copyrighted materials as noted in the text. Permission to reproduce copyrighted items must be secured from the copyright owner.

Suggested citation:

Thomas, J.C., and McMahon, P.B., 2013, Overview of groundwater quality in the Piceance Basin, western Colorado, 1946-2009: U.S. Geological Survey Scientific Investigations Report 2012-5198, 204 p. 


\section{Contents}

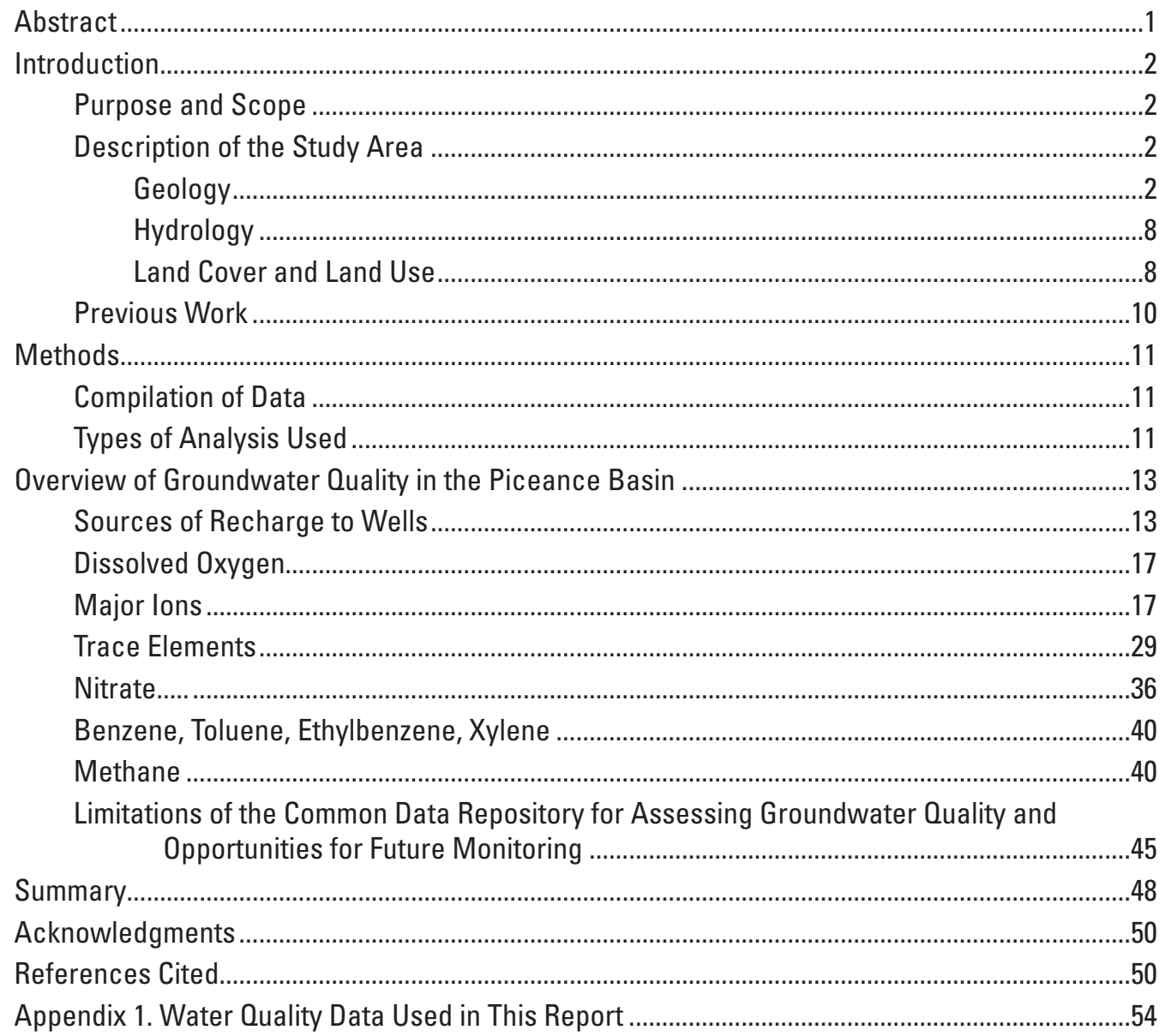

\section{Figures}

1. Map showing Piceance Basin study area, western Colorado............................................

2. Map showing generalized geologic structures, Piceance Basin, western Colorado.......4

3. Map showing generalized geologic units in which water wells were completed, Piceance Basin, western Colorado

4. Map showing distribution of sites by geologic unit for wells in the Piceance Basin,

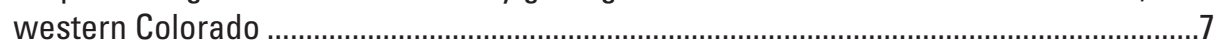

5. Map showing land cover and land use, Piceance Basin, western Colorado....................9

6. Diagram of stable isotopic composition of water from water wells compared with stable isotopic composition of snow from Grand Mesa, Colo., and produced waters from the Mesaverde Group, Piceance Basin, western Colorado.

7. Map showing concentrations of dissolved oxygen in groundwater from wells in the Piceance Basin, western Colorado

8. Map showing concentrations of dissolved solids in groundwater from wells in the Piceance Basin, western Colorado 
9. Trilinear diagram of major-ion chemistry of groundwater by dissolved solids and geologic unit of aquifer well in which is screened, Piceance Basin, western

Colorado

10. Trilinear diagram of major-ion chemistry of produced waters from Mesaverd Group and Wasatch Formation, Piceance Basin, western Colorado.

11. Map showing values of $\mathrm{pH}$ in groundwater from wells in the Piceance Basin, western Colorado

12. Map showing chloride concentrations in groundwater from wells in the Piceance Basin, western Colorado

13. Map showing sulfate concentrations in groundwater from wells in the Piceance Basin, western Colorado

14. Map showing fluoride concentrations in groundwater from wells in the Piceance Basin, western Colorado

15. Map showing arsenic concentrations in groundwater from wells in the Piceance Basin, western Colorado.

16. Map showing barium concentrations in groundwater from wells in the Piceance Basin, western Colorado

17. Map showing manganese concentrations in groundwater from wells in the Piceance Basin, western Colorado

18. Map showing iron concentrations in groundwater from wells in the Piceance Basin, western Colorado

19. Map showing selenium concentrations in groundwater from wells in the Piceance

Basin, western Colorado

20. Map showing nitrate concentrations in groundwater from wells in the Piceance Basin, western Colorado.

21. Map showing benzene concentrations in groundwater from wells in the Piceance Basin, western Colorado

22. Map showing toluene detections in groundwater from wells in the Piceance Basin, western Colorado

23. Map showing methane concentrations in groundwater from wells in the Piceance Basin, western Colorado

24. Diagram of stable hydrogen and carbon isotope compositions of methane swater wells in the Piceance Basin, for water samples with at least $1 \mathrm{mg} / \mathrm{L}$ of methane

25. Map showing groundwater-quality sites in the Piceance Basin common data repository and oil and gas wells permitted by Colorado Oil and Gas Conservation Commission and shown on permit layer

26. Map showing groundwater-quality sites in the Piceance Basin common data repository and domestic and municipal wells permitted by Colorado Division of Water Resources

\section{Tables}

1. Geologic units and U.S. Geological Survey National Water Information System descriptions of aquifers represented in the Piceance Basin common data repository

2. Data sources that contribute to the Piceance Basin common data repository of groundwater data, 1946-2009

3. Number of sites, samples, and water-quality results in the Piceance Basin common data repository and in the dataset analyzed in this report. 
4. Categories of groundwater-quality data used in the overview analysis by source in the Piceance Basin common data repository, 1946-2009..

5. Number and percentage of water-quality samples discussed in this report with associated well-depth, depth-to-water, and well-type information.

6. Number of water-quality samples by county, geologic unit, and aquifer, Piceance

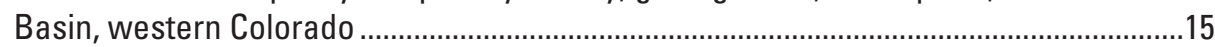

7. Summary of analyses of data in the Piceance Basin common data repository for constituents discussed in this report.

8. Concentrations of selected water-quality constituents discussed in this report by county, geologic unit, and aquifer

9. Concentrations of selected water-quality constituents relative to three dissolvedoxygen concentration thresholds 


\section{Conversion Factors}

Inch/Pound to SI

\begin{tabular}{lcl}
\hline \multicolumn{1}{c}{ Multiply } & By & \multicolumn{1}{c}{ To obtain } \\
\hline & Length & \\
\hline foot $(\mathrm{ft})$ & 0.3048 & meter $(\mathrm{m})$ \\
mile $(\mathrm{mi})$ & 1.609 & kilometer $(\mathrm{km})$ \\
\hline & Area & \\
\hline square mile $\left(\mathrm{mi}^{2}\right)$ & 2.590 & square kilometer $\left(\mathrm{km}^{2}\right)$ \\
\hline & Flow & \\
\hline cubic foot per second $\left(\mathrm{ft}^{3} / \mathrm{s}\right)$ & 28.32 & cubic decimeter per second $\left(\mathrm{dm}^{3} / \mathrm{s}\right)$ \\
\hline
\end{tabular}

Temperature in degrees Celsius $\left({ }^{\circ} \mathrm{C}\right)$ may be converted to degrees Fahrenheit $\left({ }^{\circ} \mathrm{F}\right)$ as follows:

${ }^{\circ} \mathrm{F}=\left(1.8 x^{\circ} \mathrm{C}\right)+32$

Temperature in degrees Fahrenheit $\left({ }^{\circ} \mathrm{F}\right)$ may be converted to degrees Celsius $\left({ }^{\circ} \mathrm{C}\right)$ as follows:

${ }^{\circ} \mathrm{C}=\left({ }^{\circ} \mathrm{F}-32\right) / 1.8$

Vertical coordinate information is referenced to the North American Vertical Datum of 1988 (NAVD 88).

Horizontal coordinate information is referenced to the North American Datum of 1983 (NAD 83).

Elevation, as used in this report, refers to distance above the vertical datum.

Specific conductance is given in microsiemens per centimeter at 25 degrees Celsius $(\mu \mathrm{S} / \mathrm{cm}$ at $\left.25^{\circ} \mathrm{C}\right)$.

Concentrations of chemical constituents in water are given either in milligrams per liter (mg/L) or micrograms per liter $(\mu \mathrm{g} / \mathrm{L})$.

\section{Abbreviations Used in This Report}

$<\quad$ less than

$\geq \quad$ greater than or equal to

$\mu \mathrm{g} / \mathrm{L} \quad$ microgram per liter

$\mathrm{ft} \quad$ foot

$\mathrm{ft}^{3} / \mathrm{s} \quad$ cubic foot per second

$\mathrm{mg} / \mathrm{L} \quad$ milligram per liter

$\mathrm{mi}^{2} \quad$ square mile

BTEX benzene, toluene, ethylbenzene, xylene

DO dissolved oxygen 


\title{
Overview of Groundwater Quality in the Piceance Basin, Western Colorado, 1946-2009
}

\author{
By J.C. Thomas and P.B. McMahon
}

\section{Abstract}

Groundwater-quality data from public and private sources for the period 1946 to 2009 were compiled and put into a common data repository for the Piceance Basin. The data repository is available on the web at http://rmgsc.cr.usgs.gov/ cwqdr/Piceance/index.shtml. A subset of groundwater-quality data from the repository was compiled, reviewed, and checked for quality assurance for this report. The resulting dataset consists of the most recently collected sample from 1,545 wells, 1,007 (65 percent) of which were domestic wells. From those samples, the following constituents were selected for presentation in this report: dissolved oxygen, dissolved solids, $\mathrm{pH}$, major ions (chloride, sulfate, fluoride), trace elements (arsenic, barium, iron, manganese, selenium), nitrate, benzene, toluene, ethylbenzene, xylene, methane, and the stable isotopic compositions of water and methane.

Some portion of recharge to most of the wells for which data were available was derived from precipitation (most

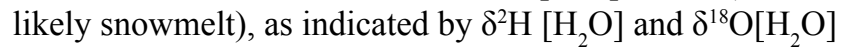
values that plot along the Global Meteoric Water Line and near the values for snow samples collected in the study area. Ninety-three percent of the samples were oxic, on the basis of concentrations of dissolved oxygen that were greater than or equal to 0.5 milligrams per liter.

Concentration data were compared with primary and secondary drinking-water standards established by the U.S. Environmental Protection Agency. Constituents that exceeded the primary standards were arsenic (13 percent), selenium (9.2 percent), fluoride ( 8.4 percent), barium (4.1 percent), nitrate (1.6 percent), and benzene ( 0.6 percent). Concentrations of toluene, xylenes, and ethylbenzene did not exceed standards in any samples. Constituents that exceeded the secondary standard were dissolved solids (72 percent), sulfate (37 percent), manganese ( 21 percent), iron (16 percent), and chloride (10 percent). Drinking-water standards have not been established for methane, which was detected in 24 percent of samples. Methane concentrations were greater than or equal to 1 milligram per liter in 8.5 percent of samples. Methane isotopic data for samples collected primarily from domestic wells in Garfield County indicate that methane in samples with relative high methane concentrations were derived from both biogenic and thermogenic sources. Many of the constituents that exceeded standards, such as arsenic, fluoride, iron, and manganese, were derived from rock and sediment in aquifers. Elevated nitrate concentrations were most likely derived from human sources such as fertilizer and human or animal waste.

Information about the geologic unit or aquifer in which a well was completed generally was not provided by data sources. However, limited data indicate that Quaternary deposits in Garfield and Mesa Counties, the Wasatch Formation in Garfield County, and the Green River Formation in Rio Blanco County had some of the highest median concentrations of selected constituents. Variations in concentration with depth could not be evaluated because of the general lack of welldepth and water-level data.

Concentrations of several important constituents, such as arsenic, manganese, methane, and nitrate, were related to concentrations of dissolved oxygen. Concentrations of arsenic, manganese, and methane were significantly higher in groundwater with low dissolved-oxygen concentrations than in groundwater with high dissolved-oxygen concentrations. In contrast, concentrations of nitrate were significantly higher in groundwater with high dissolved-oxygen concentrations than in groundwater with low dissolved-oxygen concentrations. These results indicate that measurements of dissolved oxygen may be a useful indicator of groundwater vulnerability to some human-derived contaminants and enrichment from some natural constituents.

Assessing such a large and diverse dataset as the one available through the repository poses unique challenges for reporting on groundwater quality in the study area. The repository contains data from several studies that differed widely in purpose and scope. In addition to this variability in available data, gaps exist spatially, temporally, and analytically in the repository. For example, groundwater-quality data in the repository were not evenly distributed throughout the study area. Several key water-quality constituents or indicators, such as dissolved oxygen, were underrepresented in the repository. Ancillary information, such as well depth, depth to water, and the geologic unit or aquifer in which a well was completed, was missing for more than 50 percent of samples.

Future monitoring could avoid several limitations of the repository by making relatively minor changes to samplecollection and data-reporting protocols. Field measurements for dissolved oxygen could be added to sampling protocols, 
for example. Information on well construction and the geologic unit or aquifer in which a well was completed should be part of the water-quality dataset. Such changes would increase the comparability of data from different monitoring programs and also add value to each program individually and to that of the regional dataset as a whole. Other changes to monitoring programs could require greater resources, such as sampling for a basic set of constituents that is relevant to major waterquality issues in the regional study area. Creation of such a dataset for the regional study area would help to provide the kinds of information needed to characterize background conditions and the spatial and temporal variability in constituent concentrations associated with those conditions. Without such information, it is difficult to identify departures from background that might be associated with human activities.

\section{Introduction}

Western Colorado is undergoing large-scale energy development especially in the Piceance Basin. As this development proceeds, potential short and long-term changes in groundwater quality will require ongoing monitoring and assessment. A substantial number of water-resource datasets, publications, and other materials have been developed and are potentially available for use in assessing existing groundwater quality. However, these data have been stored in disparate formats among numerous agencies, energy companies, private consulting firms, universities, and stakeholder groups. In order to take advantage of existing data and to integrate them with data from ongoing studies, the U.S. Geological Survey, in cooperation with public and industry partners, developed a web-accessible common data repository to provide energy operators, researchers, consultants, agencies, and interested stakeholders equal access to existing water-quality information. The following is an alphabetical list of cooperators involved in this study: Antero Resources, Bureau of Land Management, Bureau of Reclamation, Chevron Corporation, Cities of Grand Junction and Rifle, Colo., Colorado Department of Agriculture, Colorado Department of Natural Resources, Colorado Department of Public Health and Environment, Colorado Division of Wildlife-River Watch, Colorado Oil and Gas Conservation Commission, Colorado River Water Conservation District, Delta County, Colo., EnCana Oil \& Gas (USA) Inc., Garfield County, Colo., Gunnison Energy Corp., National Park Service, Natural Soda, Inc., North Fork River Improvement Association, Oxy Petroleum Corporation, Petroleum Development Corp., Rio Blanco County, Shell Oil Company, Solvay Chemicals, Towns of Carbondale, De Beque, Palisade, Parachute, Rangely, and Silt, Colo., U.S. Forest Service, West Divide Water Conservancy District, and Williams Companies, Inc.

\section{Purpose and Scope}

This report provides an overview of groundwater-quality data that have been compiled and put into a common data repository for the Piceance Basin from public and industry sources for the period 1946 to 2009. This overview describes the availability of groundwater-quality data in the Piceance Basin in terms of three characteristics: spatial (where sample sites were located), temporal (what is the period of record), and analytical (what constituents were sampled). Spatial variability in constituent concentrations are described and, where possible, examined relative to human and natural factors. This overview provides a basis for developing regional groundwater monitoring strategies to fill data gaps, to improve the understanding of groundwater quality in the basin, and to increase the comparability of data derived from existing monitoring programs.

\section{Description of the Study Area}

In this report, the Piceance Basin refers to an area of approximately 9,500 square miles in western Colorado extending from north of Rangley to south of Delta and from Glenwood Springs to the Colorado-Utah state border (fig. 1). The study area combines structural basins, hydrologic basins, and political boundaries whose selection has been based in part on mineral-resource extraction, water resources, and cooperator interest.

\section{Geology}

The Piceance structural basin is a downwarped region surrounded by uplifted regions including the Axial Basin arch to the north, the Grand Hogback to the east, the Gunnison uplift to the southeast, the Uncompahgre uplift to the southwest, and the Douglas Creek arch to the west (fig. 2). Quaternary alluvial, terrace and valley-fill deposits constitute the unconsolidated materials in the valleys. Tertiary sedimentary bedrock units crop out in the center of the downwarped region, and uplifted regions are underlain primarily by Cretaceous sedimentary rocks (fig. 3).

Quaternary sedimentary deposits and Tertiary and Cretaceous sedimentary rocks were the primary host materials for aquifers represented in the common data repository (table 1). Quaternary deposits included alluvium, terrace deposits, and valley fill deposits (fig. 4). The Green River and Wasatch Formations were the primary Tertiary units in which wells in the study area were screened. The Green River Formation, of early Tertiary age, rests conformably on the Wasatch Formation (older Tertiary rock). The Green River Formation has been divided into four members (from youngest to oldest): Evacuation Creek, Parachute Creek, Garden Gulch, and Douglas Creek (Bradley, 1931). The name "Evacuation Creek Member" was abandoned and its associated rocks are assigned to the lower part of the Uinta Formation in the 


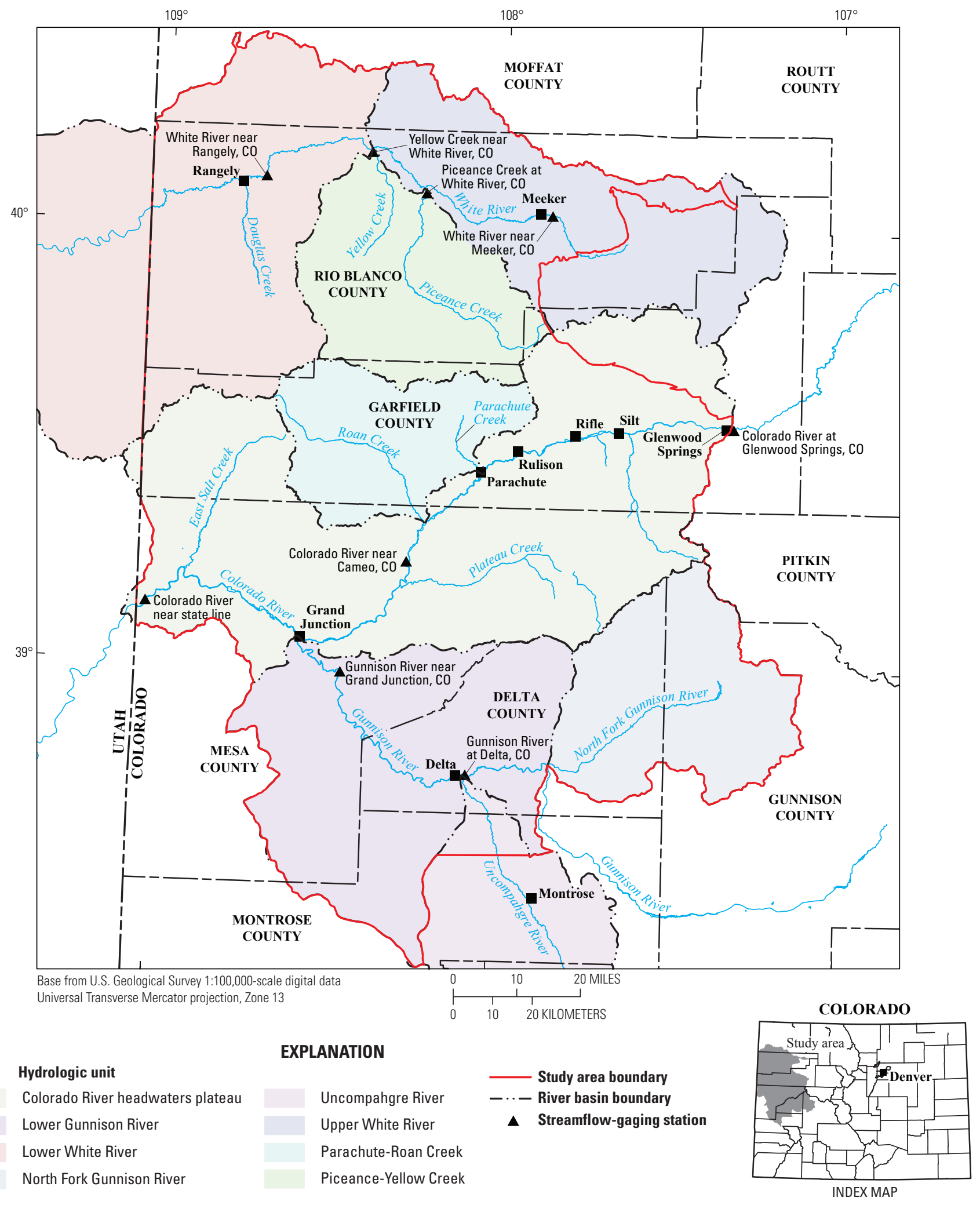

Figure 1. Piceance Basin study area, western Colorado. 


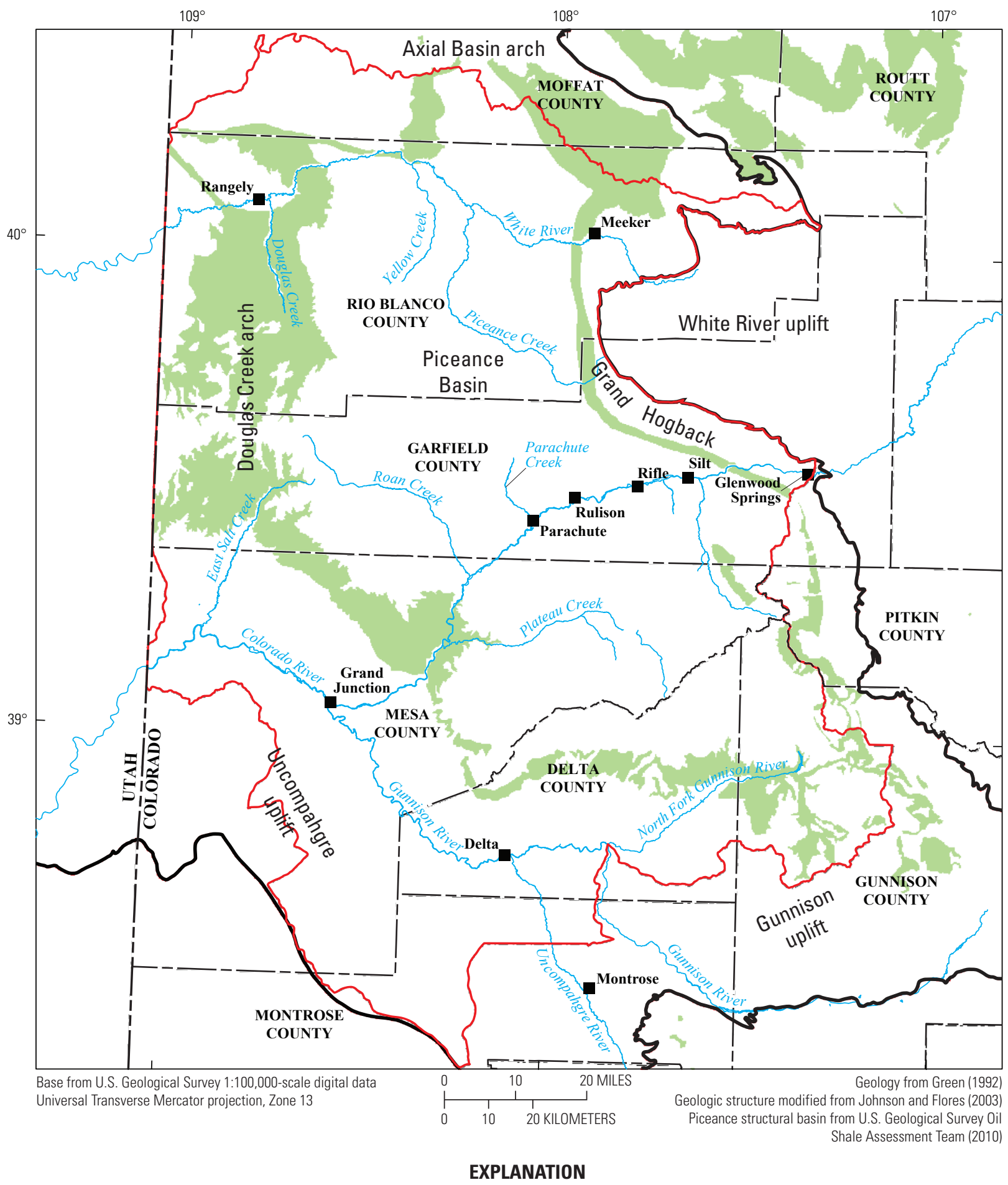

Cretaceous Mesaverde Group, Williams Fork Formation, Iles Formation, Hunter Canyon Formation

Piceance structural basin boundary

Study area boundary

Figure 2. Generalized geologic structures, Piceance Basin, western Colorado. 


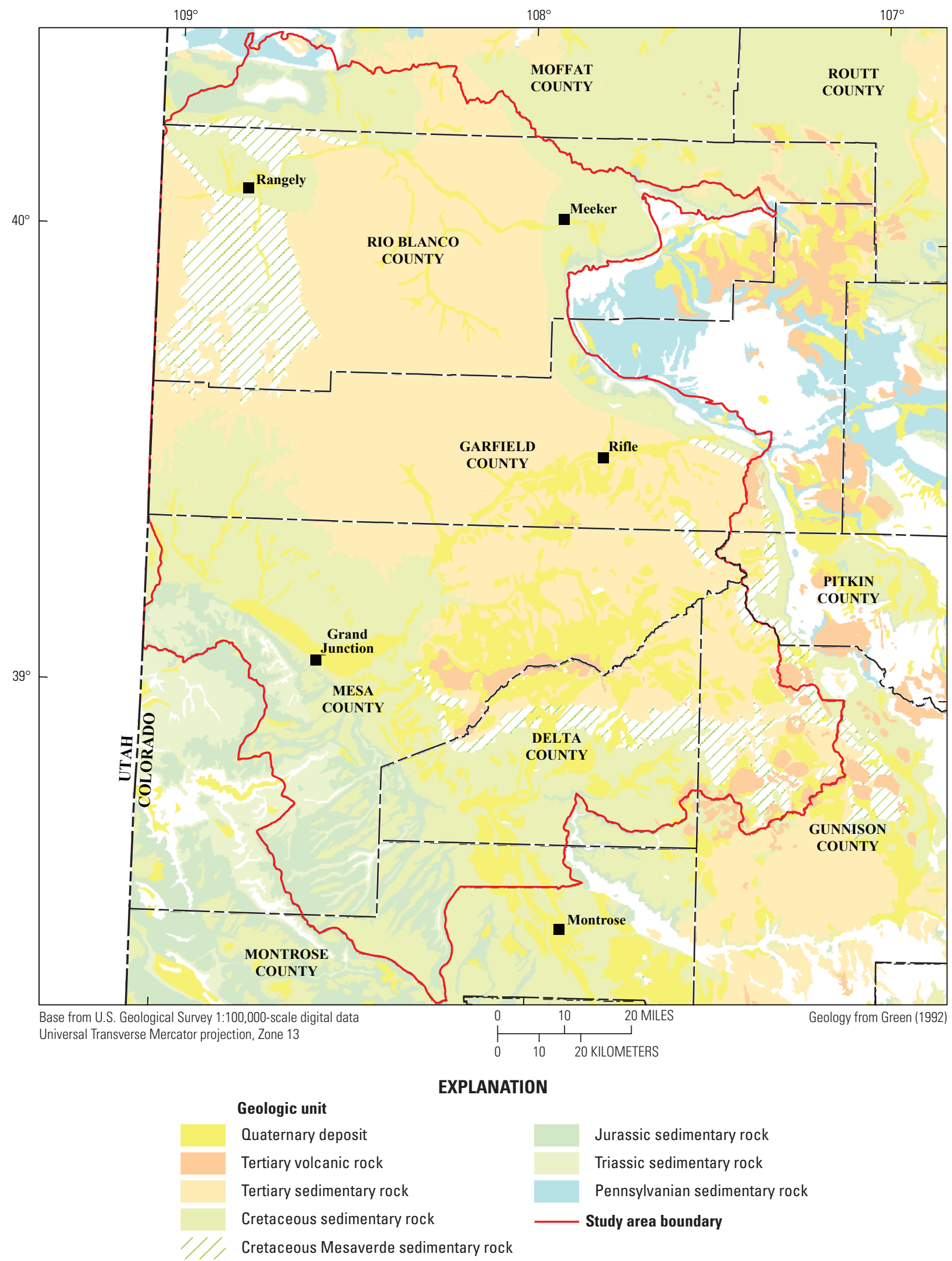

Figure 3. Generalized geologic units in which water wells were completed, Piceance Basin, western Colorado. 
Table 1. Geologic units and U.S. Geological Survey National Water Information System descriptions of aquifers represented in the Piceance Basin common data repository.

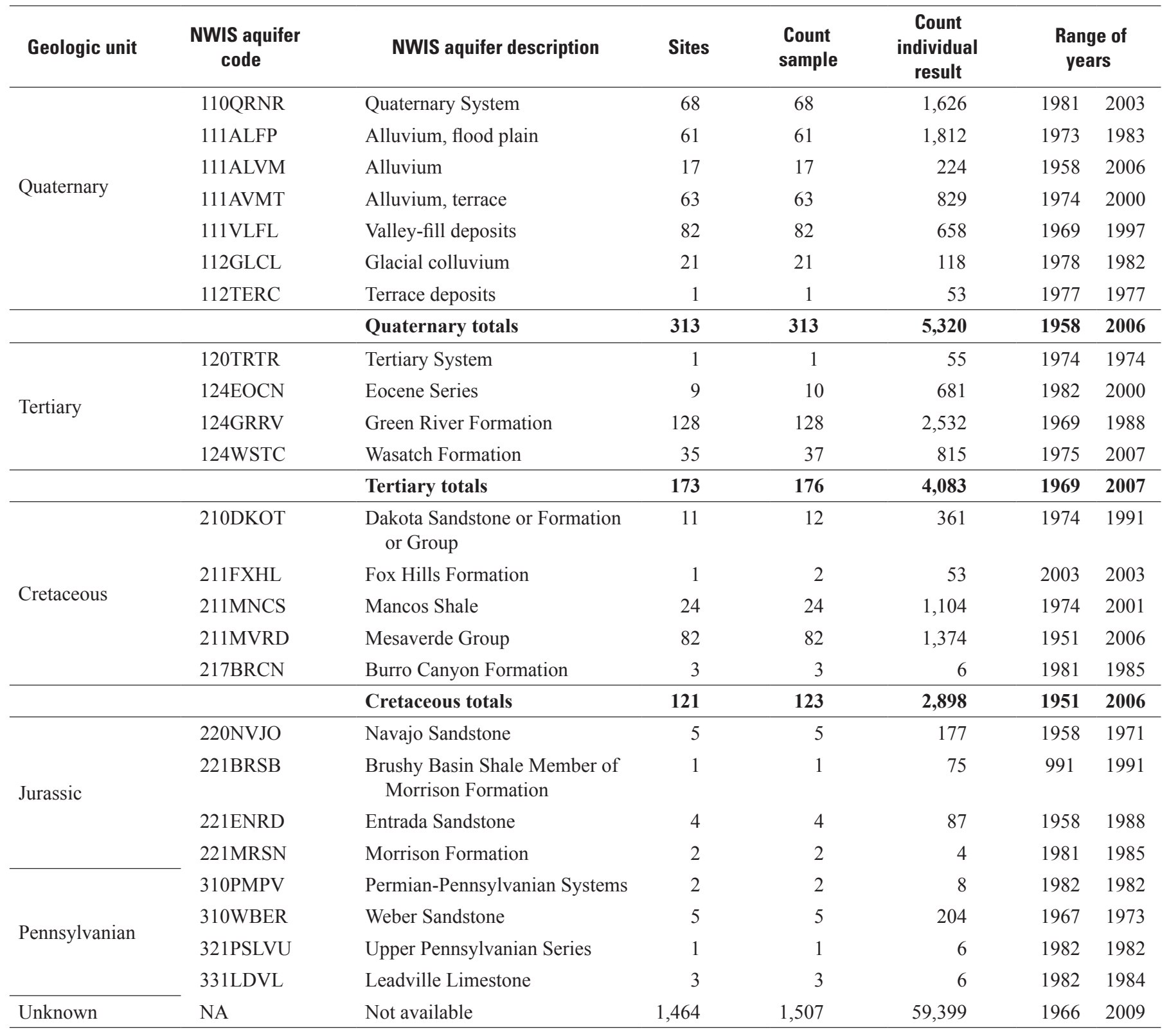



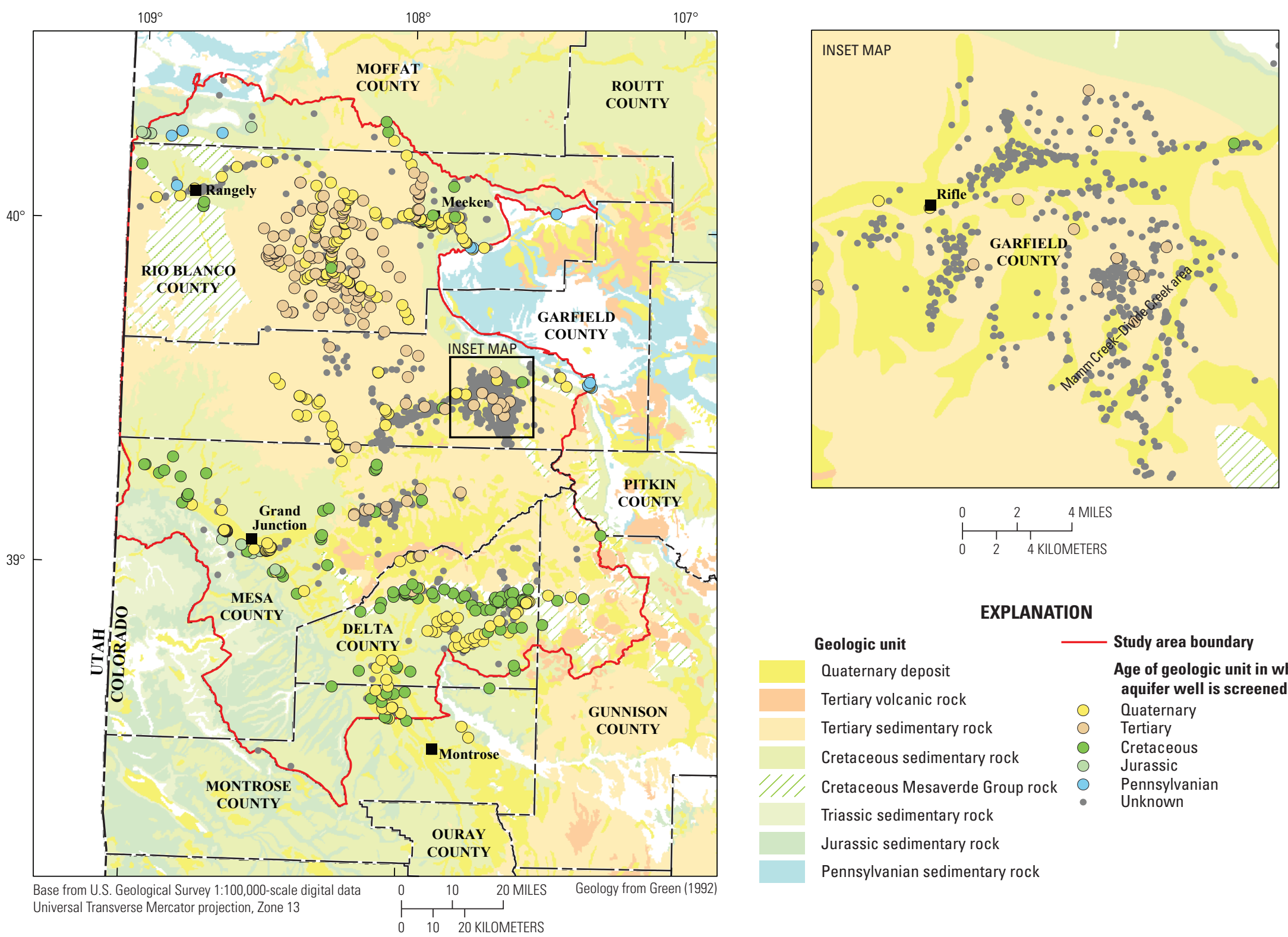

EXPLANATION

\section{Geologic unit}

\section{Quaternary deposit}

Tertiary volcanic rock

Tertiary sedimentary rock

Cretaceous sedimentary rock

Cretaceous Mesaverde Group rock

Triassic sedimentary rock

Jurassic sedimentary rock

Pennsylvanian sedimentary rock

Figure 4. Distribution of sites by geologic unit for wells in the Piceance Basin, western Colorado. Geologic unit designation was based on aquifer descriptions from original data source and was not reclassified for this study. 
Piceance Basin, a revision in nomenclature based on stratigraphy (Cashion and Donnell, 1974). The Parachute Creek Member is a dolomitic marlstone containing soluble minerals (such as halite and nahcolite). The Garden Gulch Member is a shaley, dolomitic marlstone, and the Douglas Creek Member is primarily sandstone. The Green River Formation is well known for rich oil shale deposits (for example, the informally named Mahogany oil shale zone). The Wasatch Formation consists of varicolored mudstone with sandstone lenses (Johnson and Flores, 2003). Some of the sandstone lenses, such as the Wasatch "G" interval of the Wasatch Formation, are notable reservoirs of natural gas near the towns of Parachute and Rulison (Nelson and Santus, 2010) (fig. 1). The Wasatch Formation is a source of potable water and also contains rich natural gas deposits (URS Corporation, 2006).

Cretaceous sedimentary rocks of primary interest in the study area were the Mesaverde Group, the Mancos Shale, and the Dakota Sandstone. The Mesaverde Group consists of the Iles Formation and the Williams Fork Formation. The Williams Fork Formation is an important natural-gas-producing unit within the study area. The Iles Formation contains several members, such as the Corcoran Sandstone, Cozzette Sandstone, and Rollins Sandstone Members (Reinecke and others, 1991). The Mesaverde Group was formed by a series of marine transgressions and regressions followed by periods of fluvial deposition (Johnson, 1989) which produced a sequence of marine shales, siltstones, and sandstones capped by a thick sequence of terrestrial fluvial deposits. The fluvial deposits of the Mesaverde consist almost entirely of lenticular channel sandstones and fine-grained flood-plain deposits. Recovering natural gas from channel sandstones, the primary reservoirs, requires a dense drilling pattern (Nelson and Santus, 2010). The Mancos Shale is composed of massive, fossiliferous marine shale with interbedded sandstone, siltstone, and devitrified volcanic ash layers. The Mancos is wholly or partly the lateral equivalent of the Niobrara Formation, Cody Shale, and lower Pierre Shale in Colorado, Montana, Nebraska, South Dakota, and Wyoming (Green, 1992; Wright and Butler, 1993). The Dakota Sandstone is composed of interbedded well-consolidated sandstone, conglomerates, shale, and coal (Brooks and Ackerman, 1985).

\section{Hydrology}

The Colorado River flows through the middle of the study area from Glenwood Springs to the Colorado-Utah state line and has annual mean streamflows of 3,340 cubic feet per second $\left(\mathrm{ft}^{3} / \mathrm{s}\right)$ at Colorado River below Glenwood Springs, $3,830 \mathrm{ft}^{3} / \mathrm{s}$ at Colorado River near Cameo, and 6,140 $\mathrm{ft}^{3} / \mathrm{s}$ at the state line (fig. 1) (U.S. Geological Survey, 2011). In the northern portion of the study area, Piceance and Yellow Creeks flow into the White River; annual mean streamflows at their mouths are $35 \mathrm{ft}^{3} / \mathrm{s}$ and $2.8 \mathrm{ft}^{3} / \mathrm{s}$, respectively. The White River flows east to west in the northern portion of the study area with average annual streamflows of $620 \mathrm{ft}^{3} / \mathrm{s}$ at White River near Meeker and $727 \mathrm{ft}^{3} / \mathrm{s}$ at White River below Boise Creek near Rangely. In the southern portion of the study area, the Gunnison River has annual mean streamflows of $1,960 \mathrm{ft}^{3} / \mathrm{s}$ at Gunnison River at Delta and 2,560 $\mathrm{ft}^{3} / \mathrm{s}$ at Gunnison River near Grand Junction (fig. 1).

Sources of recharge to aquifers in the study area include snowmelt from upland areas, irrigation in valleys, and upwelling of groundwater from deeper aquifers. Surface-water basin boundaries roughly serve as groundwater basin boundaries, where groundwater elevations in the study area generally follow the topography. Thus, groundwater flows from recharge areas in uplands to discharge areas in valleys. Major surface-water drainages such as the Colorado, White, and Gunnison Rivers were interpreted as regional discharge areas for groundwater. In the Piceance and Yellow Creek drainage area, recharge occurs from higher elevations and groundwater flows toward Piceance Creek and then to the north. Owing to geologic structural controls, groundwater moves upward along fractures from deeper aquifers to shallower aquifers in the north-central part of the drainage area (Weeks and others, 1975; Taylor, 1987). In Mesa County near Grand Junction, artesian aquifers have existed within the Entrada Sandstone, the Wingate Sandstone, the Salt Wash Member of the Morrison Formation, and the Dakota Sandstone (Lohman, 1965). The Williams Fork Formation is a semiconfined aquifer under artesian pressure specifically in the Garfield County area, where upwelling can occur along conduits such as well bores or natural fractures (Tyler and others, 1995).

\section{Land Cover and Land Use}

Land cover in the study area includes forest, rangeland, agricultural land, and urban areas (fig. 5). Of the 9,500 square miles $\left(\mathrm{mi}^{2}\right)$ in the study area, about half the total area $\left(4,700 \mathrm{mi}^{2}\right)$ is forest; about 40 percent $\left(3,400 \mathrm{mi}^{2}\right)$ of the total area is rangeland. Located predominately in valleys and adjacent to rivers, about 6 percent $\left(633 \mathrm{mi}^{2}\right)$ is agricultural land, and less than 1 percent of the total area $\left(69 \mathrm{mi}^{2}\right)$ is urban land. Grand Junction (population 54,694), Delta $(8,708)$, Rifle $(8,675)$, and Meeker $(2,409)$ are the largest population centers in the study area (U.S. Census Bureau, 2011).

Oil shale has historically been an energy resource of great interest in the northern part of the Piceance Basin. Oil shale is a leaseable mineral according to the Mineral Leasing Act of 1920 and Executive Order 5327. In the early 1900s an executive order from the President established the Naval Petroleum and Oil Shale Reserves within which the oil shale resources of Colorado were managed (U.S. Department of Energy, 2011a). In 1974, oil shale prototype leasing was initiated in the Piceance Basin; two leases (Tracts C-a and C-b) were awarded in order to determine methods of extracting oil shale while maintaining the environmental integrity of the area affected (Weeks and others, 1975). The prototype leasing program for Tracts C-a and C-b ended owing to various political and economic reasons and little was done with oil shale on Federal 

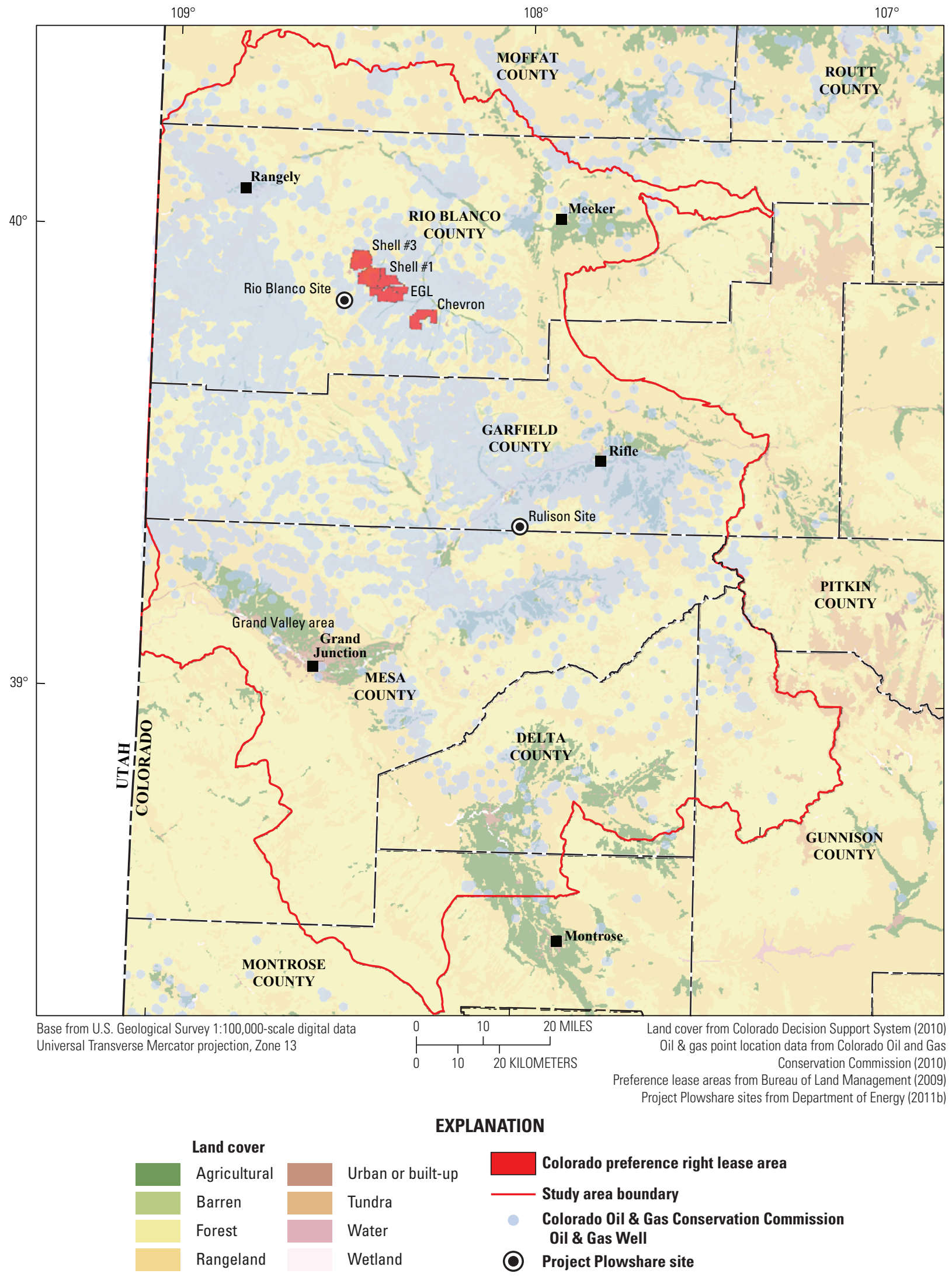

EXPLANATION
Land cover 
lands in the Piceance Basin until 2005 when the Energy Policy Act was enacted. Beginning in 2005, the Bureau of Land Management began nominating entities for research, development, and demonstration (RD\&D) projects targeted at developing oil shale in an economically sustainable and environmentally responsible fashion. In 2007 in Colorado, three RD\&D leases of 160 acres each were awarded - one to Shell Frontier Oil and Gas, Inc., one to E.G.L Resources, Inc., and one to Chevron USA, Inc. (Bureau of Land Management, 2011a). Ten thousand acres were identified as preference right lease areas for commercial development within a 10-year term, and with the potential for 5-year extensions (Bureau of Land Management, 2011a).

Nahcolite is also a leasable mineral and is associated with oil shale in the Green River Formation in the Piceance Basin. As such, it cannot be disposed of during oil shale production, and thus it becomes an integral factor in determining the ease of mining oil shale in the Piceance Basin. The U.S. Bureau of Mines characterized nahcolite resources in the Piceance Basin in the late 1970s by use of samples obtained from a 10-foot-diameter shaft drilled to the nahcolite (saline) zone in the northern part of the basin near Piceance Creek (U.S. Geological Survey Oil Shale Assessment Team, 2010). On the basis of this information, White River Nahcolite Minerals LLC began solution mining nahcolite on a Federal lease tract in the Piceance Basin. In 2003, AmerAlia Inc. (Natural Soda Inc.) purchased the operation and produced about 103,000 tons of sodium bicarbonate in 2007. This facility is the only one currently (2009) producing sodium bicarbonate from nahcolite in the Piceance Basin. The method of mining involves drilling to the target bed, injecting hot water to dissolve the nahcolite, and then pumping the nahcolite-bearing brine to the surface through a production hole adjacent to the injection well. The brine is placed in holding ponds where excess water is removed, which allows the sodium bicarbonate to recrystallize (U.S. Geological Survey Oil Shale Assessment Team, 2010).

Natural gas development has increased in the study area since 2000, in part due to advancements in the application of hydraulic fracturing of tight gas resources (Wasatch Formation and Mesaverde Group) which has allowed successful extraction of natural gas from previously inaccessible sources (Nelson and Santus, 2010). In Garfield County alone between 2004 and 2010, the Colorado Oil and Gas Conservation Commission issued 13,775 application permits to develop (fig. 5) (Colorado Oil and Gas Conservation Commission, 2010). Natural gas development on public lands in Rio Blanco County increased from 58 permits in 2001 to 200 permits in 2006; a total of 2,556 multiple well pads is projected during the next 20 years (Bureau of Land Management, 2011b).

\section{Previous Work}

The Piceance Basin in western Colorado contains energy, mineral, and other natural resources that have been the subject of numerous studies and publications. Selected publications were summarized as they relate to water resources within the study area. In 1874, the Green River Formation was identified in the Piceance Basin, and subsequent studies have focused on the energy resources of this formation (for example, oil shale) (Donnell, 1961). In 1964, the first investigations of water resources were conducted that document the geohydrology of the Piceance Basin (Coffin and others, 1968; Coffin and others, 1971). Several reports discussed simulated effects of oil-shale development and assessed oil shale resources by using information learned during earlier prototype leasing in the 1970s (for example, Weeks and others, 1975; Taylor, 1987). These studies were conducted primarily in Rio Blanco County in the Piceance Creek and Yellow Creek drainages and were focused on oil shale resources. Around the time of oil shale prototype leasing, the U.S. Department of Energy conducted nuclear gas-stimulation experiments under Project Plowshare at several locations including Rio Blanco site and Rulison site, both of which lie within the Piceance Basin (National Nuclear Security Administration, 2010; U.S. Department of Energy, 2011b) (fig. 5). During this time, groundwater-resource studies focused on planned energy development and small-scale prototype investigations.

More recently, URS Corporation completed a hydrogeologic study of the Mamm Creek-Divide Creek area in Garfield County (fig. 4), located 8 miles southeast of Rifle, that described regional hydrology, geology, and groundwater chemistry (URS Corporation, 2006). In a second phase of investigation in 2007, S.S. Papadopulos \& Associates, Inc., resampled domestic wells (identified in the phase I investigation) for water quality, composition of hydrocarbon gas, and stable isotopic composition of methane. It then sampled produced water and collected natural gas samples from gas wells to characterize potential sources of hydrocarbon contamination (S.S. Papadopulos \& Associates, Inc., 2008). These studies provided information on groundwater resources, groundwater quality, and activities related to natural gas production. As a follow up to these studies (URS Corporation, 2006; S.S. Papadopulos \& Associates, Inc., 2008), McMahon and others (2010) examined sources and sinks of nitrate and methane in groundwater from the Wasatch Formation in Garfield County.

Groundwater quality in the Colorado headwaters drainage, including the Grand Valley area (fig. 5), is discussed as part of surface-water investigations focused on salinity and selenium (Butler and others, 1996; Butler and Osmundson, 2000; Hamilton and others, 2003). Other studies of salinity and selenium in surface water and the effects of irrigation practices in the lower Gunnison River Basin (Butler and others, 1996; Butler and Osmundson, 2000; Thomas, 2009) discuss groundwater but not comprehensive groundwater investigations. 


\section{Methods}

Groundwater-quality data were compiled into the common data repository and a subset of these data was used in this report. These data were summarized to provide information about the period of record for each constituent, number of values, number of censored values, and exceedances of drinking-water standards. The data were evaluated spatially within the study area and statistically to compare concentrations of selected constituents at different sites.

\section{Compilation of Data}

A repository of available groundwater-quality data was compiled from local, State, and Federal agencies and private entities (consulting, energy, and mining companies) to assist in the analysis of water resources in the study area (table 2). The data repository is available on the web at http://rmgsc.cr.usgs. gov/cwqdr/Piceance/index.shtml. The goal was for the repository to contain the most comprehensive set of groundwaterquality data in the study area to date. For a variety of reasons, however, not all results from known data sources were loaded into the repository: data sources that did not participate, data not received in electronic format, metadata insufficient, and data submitted late. For these reasons, this report represents the most comprehensive set of groundwater-quality data available for the Piceance Basin as of August 2009.

As of August 2009, the data repository contained 8,635 samples and more than 480 constituents, but only a subset of these samples $(1,545)$ was analyzed in this report (tables 3 and 4). The following constituents were selected for analysis: stable isotopes of water, dissolved oxygen, major ions (dissolved solids, $\mathrm{pH}$, chloride, sulfate, fluoride), trace elements (arsenic, barium, iron, manganese, selenium), nitrate, benzene, toluene, ethylbenzene, and xylene, methane, and isotopic composition of methane. Duplicate results (the same sample submitted to the repository by two or more source agencies) were removed prior to analysis. For sites with more than one sample, only the most recent, comprehensive groundwater sample was used for analysis. Where major-ion data were available, a charge balance was computed as part of the water-quality review, gross errors were flagged in the database, and those results were removed from data analysis. In using these datasets to describe water quality in the Piceance Basin, it is important to remember that the repository as a whole is a collection of multiple studies with multiple objectives. Thus, it is not surprising that the samples were not all analyzed for the same set of chemical constituents. Many samples, however, also lacked important ancillary information that was needed to place them in proper hydrologic context. Such ancillary information included well depth, depth to water, well type, and geologic unit or aquifer. Only 33 percent of the samples had well-depth information, 19 percent had geologic-unit or aquifer designations, and 5 percent had depth-to-water information, but all did have well-type information (tables 5 and 6).

\section{Types of Analysis Used}

Various types of analysis were used to evaluate groundwater-quality data for this report. Concentration data were evaluated spatially by plotting them on a map of the study area with either geology layer or land use layer. Stableisotope results use the standard delta $(\delta)$ notation, in per mil $(\%$, parts per thousand). Stable oxygen-iostopic composition of water samples $\left(\delta^{18} \mathrm{O}\left[\mathrm{H}_{2} \mathrm{O}\right]_{\text {sample }}\right)$ are equal to

$$
\delta^{18} \mathrm{O}\left[\mathrm{H}_{2} \mathrm{O}\right]_{\text {sample }}=\left(\left({ }^{18} \mathrm{O} /{ }^{16} \mathrm{O}\right)_{\text {sample }} /\left({ }^{18} \mathrm{O} /{ }^{16} \mathrm{O}\right)_{\text {ref }}-1\right) * 1000
$$

where

$$
\begin{aligned}
& { }^{18} \mathrm{O} /{ }^{16} \mathrm{O} \quad \begin{array}{l}
\text { is the ratio of the oxygen- } 18 \text { to } \\
\text { oxygen- } 16 \text { isotopes in the sample and } \\
\text { the reference (ref) material. }
\end{array}
\end{aligned}
$$

Values of $\delta^{2} \mathrm{H}\left[\mathrm{H}_{2} \mathrm{O}\right]$ and $\delta^{18} \mathrm{O}\left[\mathrm{H}_{2} \mathrm{O}\right]$ were referenced to the Vienna Mean Ocean Water scale (Coplen, 1988).

Groundwater composition was represented by using trilinear plots of major anions and cations. Of the 1,545 sites, 1,007 were classified as domestic wells on the basis of information provided by the data source (table 4 ). Because more than half of the wells were being used for domestic water supply, data were compared with drinking-water standards even though none of these standards are required or enforced for domestic water supplies. Where applicable, constituents were compared to constituents listed in primary and secondary drinking-water standards established by the U.S. Environmental Protection Agency (USEPA). The USEPA National Primary Drinking Water Regulations (herein referred to as "primary standards") are legally enforceable standards that apply to public water systems. Primary standards are intended to protect public health by limiting the concentrations of contaminants in drinking water and were used in this report to provide a context for evaluating groundwater quality in the Piceance Basin (U.S. Environmental Protection Agency, 2010). Data also were compared with National Secondary Drinking Water Regulations (herein referred to as "secondary standards"), which are nonenforceable guidelines for constituents that may cause cosmetic effects (such as skin or tooth discoloration) or have poor aesthetic qualities (such as poor taste, odor, or color) in drinking water (U.S. Environmental Protection Agency, 2010). The geologic unit and aquifer in which a well was completed were important information for evaluating water-quality data and were used to group concentration data by constituent. Water-quality samples were stratified by county, geologic unit, and aquifer, and results for sample counts greater than or equal to 10 were considered sufficient and retained for further analysis and presentation in this report.

The determination of chemical properties of waters can result in nondetects (zero and negative values, "left-censored data") because of analytical limits of the laboratory analysis. In a dataset of this breadth, nondetect data can reflect several forms of bias, such as antiquated methods for storing nondetects and changes in the meaning of "less than" values. Therefore, 
Table 2. Data sources that contribute to the Piceance Basin common data repository of groundwater data, 1946-2009. Duplicate data and all but the most recent sample and results were removed.

\begin{tabular}{lrrrrr}
\hline \multicolumn{1}{c}{ Agency Name } & Sites & Samples & \multicolumn{2}{c}{ Date range } & Results \\
\hline Antero Resources & 159 & 223 & $7 / 12 / 2005$ & $7 / 16 / 2009$ & 9,749 \\
City of Grand Junction & 3 & 67 & $6 / 22 / 1988$ & $10 / 28 / 2008$ & 813 \\
Colorado Department of Agriculture & 43 & 43 & $3 / 31 / 1998$ & $10 / 26 / 2000$ & 354 \\
Colorado Oil and Gas Conservation & 1,048 & 2,861 & $4 / 2 / 1956$ & $10 / 14 / 2008$ & 63,234 \\
$\quad$ Commission & & & & & \\
EnCana Oil \& Gas (USA) Inc. & 496 & 2,991 & $5 / 4 / 2001$ & $4 / 13 / 2009$ & 119,233 \\
Mine Consultant & 24 & 24 & $8 / 10 / 1995$ & $12 / 6 / 2006$ & 139 \\
Oxbow Mining & 1 & 1 & $8 / 10 / 2006$ & $8 / 10 / 2006$ & 5 \\
U.S. Forest Service & 1 & 3 & $9 / 27 / 2006$ & $6 / 27 / 2007$ & 71 \\
U.S. Geological Survey & 1,228 & 2,391 & $7 / 16 / 1946$ & $4 / 27 / 2009$ & 35,433 \\
Willams & 12 & 16 & $8 / 30 / 2006$ & $10 / 6 / 2008$ & 699 \\
Wright Water Engineeres, Inc, CO & 15 & 15 & $4 / 26 / 2002$ & $7 / 17 / 2002$ & 75 \\
\hline
\end{tabular}

\begin{tabular}{|c|c|c|c|c|c|c|c|c|c|c|c|c|}
\hline \multirow[b]{2}{*}{ Agency Name } & \multicolumn{12}{|c|}{ Constituent catergories ${ }^{1}$} \\
\hline & Information & $\begin{array}{c}\text { Inorganic } \\
\text { com- } \\
\text { pounds, } \\
\text { major ions, } \\
\text { metals }\end{array}$ & $\begin{array}{c}\text { Inorganic } \\
\text { compounds, } \\
\text { major ions, } \\
\text { nonmetals }\end{array}$ & $\begin{array}{c}\text { Inorganic } \\
\text { compounds, } \\
\text { minor ions, } \\
\text { metals }\end{array}$ & $\begin{array}{c}\text { Inorganic } \\
\text { compounds, } \\
\text { minor ions, } \\
\text { nonmetals }\end{array}$ & $\begin{array}{c}\text { Micro- } \\
\text { biological } \\
\text { constitu- } \\
\text { ents }\end{array}$ & Nutrient & $\begin{array}{c}\text { Organic } \\
\text { and other } \\
\text { com- } \\
\text { pounds }\end{array}$ & $\begin{array}{l}\text { Organic } \\
\text { com- } \\
\text { pounds } \\
\text { and } \\
\text { pesticide }\end{array}$ & $\begin{array}{c}\text { Physical } \\
\text { character- } \\
\text { istics }\end{array}$ & $\begin{array}{c}\text { Radio- } \\
\text { chemical } \\
\text { com- } \\
\text { pounds }\end{array}$ & $\begin{array}{l}\text { Stable } \\
\text { Isotopes }\end{array}$ \\
\hline Antero Resources & 892 & 892 & 1,465 & 1,081 & 573 & 442 & 669 & 1,338 & & 1,507 & & \\
\hline City of Grand Junction & & 70 & 73 & 278 & 76 & 73 & 99 & 16 & & 116 & 8 & \\
\hline Colorado Department of Agriculture & & 84 & 82 & & 21 & & 41 & & & 126 & & \\
\hline $\begin{array}{l}\text { Colorado Oil and Gas Conservation } \\
\text { Commission }\end{array}$ & & 6,611 & 14,223 & 3,838 & 1,924 & 2,018 & 3,852 & 12,835 & 101 & 10,014 & 272 & 1,392 \\
\hline EnCana Oil \& Gas (USA) Inc. & 5,807 & 8,401 & 20,101 & 5,396 & 3,174 & 3,945 & 6,857 & 23,415 & & 24,995 & 25 & 2,427 \\
\hline Mine Consultant & & & 20 & & 34 & & 15 & & & 70 & & \\
\hline Oxbow Mining & & & 1 & & & & 1 & & & 3 & & \\
\hline U.S. Forest Service & 27 & & 20 & & & & & & & 21 & & \\
\hline U.S. Geological Survey & 2,459 & 4,024 & 6,740 & 5,261 & 1,541 & 3 & 2,881 & 1,952 & 486 & 9,175 & 384 & 196 \\
\hline Willams & 38 & 64 & 140 & 128 & 48 & 28 & 60 & 81 & & 95 & & \\
\hline Wright Water Engineeres, Inc, CO & & & 15 & & 2 & & 13 & & & 45 & & \\
\hline
\end{tabular}

Wright Water Engineeres, Inc, CO

${ }^{1}$ Examples of constiuents for "Information" would be sampling purpose, methods, analyzing agency. Examples for "Inorganics, Major, Metals" would include calcium, magnesium, and sodium. Examples of constiuents for "Inorganics, Major, Non-metals" would include chloride and sulfate. Examples of constiuents for "Inorganics, Minor, Metals" would be manganese and iron. Examples of constiuents for "Inorganics, Minor, Non-Metals" would be arsenic and selenium. Examples of constiuents for "Microbiological" would be fecal coliform and iron reducing bacteria. Examples of constiuents for "Nutrient" would be nitrate as N and total phosphorus. Examples of constiuents for "Organics, Other" would be benzene, toluene, and methane. Examples of constiuents for "Organics, Pesticide" would be alachlor and chlorpyrifos. Examples of constiuents for "Physical" would be field parameters and depth to water. Examples of constiuents for "Radiochemical" would be uranium and tritium. Examples of constiuents for "Stable Isotopes" would be carbon-13/carbon-12 ratio in methane and deuterium/protium ratio in methane. 
Table 3. Summary of groundwater-quality-data reduction from site, sample and qw_result tables for data analysis for Piceance retrospective water-quality data base. Duplicate data and all but the most recent sample and results were reviewed.

\begin{tabular}{lcc}
\hline Table name & $\begin{array}{c}\text { All groundwater } \\
\text { data }^{1}\end{array}$ & $\begin{array}{c}\text { Groundwater data } \\
\text { summarized }^{2} \\
\text { here }^{2}\end{array}$ \\
\hline Site & 3,058 & 1,587 \\
Sample & 8,635 & 1,587 \\
QW_RESULT & 229,805 & 53,924 \\
\hline
\end{tabular}

'In Piceanc Basin common data repository; see table 3.

${ }^{2}$ See table 4.

these results should be considered with this limitation in mind (Helsel, 2005a). Where multiple censoring levels exist for a single constituent, data were not edited to a common censoring level for summary statistics in this report (Helsel, 2005b). Summary statistics were computed by using Kaplan-Meier methods when no more than 50 percent of the observations were censored (Helsel, 2005b). Maximum likelihood estimations were computed for greater than 50 observations and where 50 to 80 percent of the observations were censored. The data range, censoring levels, and maximum observation were reported where more than 80 percent of the observations were censored (Helsel, 2005b).

Dissolved-oxygen concentrations and methane concentrations were also used to stratify concentration data for each constituent. Dissolved-oxygen data were used to determine if a sample was oxic (dissolved-oxygen concentrations greater than or equal to 0.5 milligrams per liter $(\mathrm{mg} / \mathrm{L})$ ) or anoxic (dissolved-oxygen concentrations less than $0.5 \mathrm{mg} / \mathrm{L}$ ) (McMahon and Chapelle, 2008). Methane concentrations greater than or equal to $1 \mathrm{mg} / \mathrm{L}$ were considered high; methane concentrations less than $1 \mathrm{mg} / \mathrm{L}$ were considered low (McMahon and others, 2010). The nonparametric Wilcoxon rank sum test was used to identify statistically significant differences $(p<0.05)$ in average constituent concentrations belonging to different groups (for example anoxic as compared to oxic samples) (Helsel and Hirsch, 2002).

\section{Overview of Groundwater Quality in the Piceance Basin}

This report provides an overview of constituents, which include stable isotopes of water, dissolved oxygen, major ions (dissolved solids, $\mathrm{pH}$, chloride, sulfate, fluoride), trace elements (arsenic, barium, iron, manganese, selenium), nitrate, benzene, toluene, ethylbenzene, and xylene, methane, and isotopic composition of methane. These were analyzed because they accounted for most of the exceedances of water-quality standards in the data repository. Other data, such as dissolved-oxygen concentrations and water and methane isotopic values, provide understanding with respect to the water sources and fate and transport of the selected constituents.

\section{Sources of Recharge to Wells}

Recharge to most wells in the study area was derived from precipitation, as indicated by the ratio of deuterium $\left({ }^{2} \mathrm{H}\right)$ and protium $\left({ }^{1} \mathrm{H}\right)\left(\delta^{2} \mathrm{H}\left[\mathrm{H}_{2} \mathrm{O}\right]\right)$ and the ratio of ${ }^{18} \mathrm{O}$ and ${ }^{16} \mathrm{O}$ in water $\left(\delta^{18} \mathrm{O}\left[\mathrm{H}_{2} \mathrm{O}\right]\right)$ values which plot along or near the Global Meteoric Water Line (GMWL) (Craig, 1961).

Data for Grand Mesa snow and for Mesaverde Group produced water were plotted to provide additional context for these stable-isotope values in the study area. Grand Mesa snow samples were intended to generally represent precipitation from higher elevations and colder temperatures, whereas produced waters from the Mesaverde Group were intended to generally represent produced water and/or deep groundwater.

Extensive faulting and fracturing in the study area and drilling for natural gas provide potential conduits through which deep groundwater can move upward into freshwater aquifers. Chemical and isotopic characterization of deep groundwater allows its presence in the shallow aquifers to be recognized and the ability to know from which deep geologic unit it originated. These comparisons were somewhat qualitative because the isotopic composition of various types of produced waters in the study area were not well characterized, nor was the composition of water from below the sampled aquifers but above the gas-producing intervals. Most of the groundwater samples plotted near the snow samples, indicating that they were probably recharged at higher elevations or colder temperatures (or both), similar to the conditions on the Grand Mesa.

Methane concentrations were greater than $1 \mathrm{mg} / \mathrm{L}$ (high methane concentrations) in 75 samples, but only 4 had associated $\delta^{2} \mathrm{H}\left[\mathrm{H}_{2} \mathrm{O}\right]$ and $\delta^{18} \mathrm{O}\left[\mathrm{H}_{2} \mathrm{O}\right]$ concentrations to provide an understanding of sources of recharge for such groundwater samples. Hydrogen and oxygen isotope concentrations of samples with low methane concentrations (less than $1 \mathrm{mg} / \mathrm{L}$ ) plot relatively close to those of snow samples. Hydrogen and oxygen isotope concentrations of samples from two sites (report numbers 164 and 807 in appendix 1) had high methane concentrations and plot along the GMWL but away from Grand Mesa snow and report number 807 plots closer to produced water from the Mesaverde Group (fig. 6). Hydrogen and oxygen isotope concentrations of samples for report numbers 226 and 465 (appendix 1) had high methane concentrations and plotted to the right of the meteoric water line, similar to hydrogen and oxygen isotope concentrations of samples of produced water from the Mesaverde Group. These data indicate that samples from those two sites may represent much older and deeper formation water than samples from other sites. Report number 226, a domestic well in Garfield County, is likely completed in the Tertiary Wasatch Formation (completion interval not provided by the data source) (URS 
Table 4. Categories of groundwater-quality data used in this overview analysis by source in the Piceance Basin common data repository, $1946-2009$.

\begin{tabular}{|c|c|c|c|c|c|c|c|c|c|}
\hline \multirow{2}{*}{$\begin{array}{l}\text { Agency Name } \\
\text { Antero Resources }\end{array}$} & \multirow{2}{*}{$\begin{array}{r}\begin{array}{r}\text { Total } \\
\text { sites }\end{array} \\
159\end{array}$} & \multirow{2}{*}{$\begin{array}{c}\begin{array}{c}\text { Domestic } \\
\text { wells }\end{array} \\
159\end{array}$} & \multirow{2}{*}{$\begin{array}{c}\text { Agri- } \\
\text { cultural } \\
\text { wells }\end{array}$} & \multirow[t]{2}{*}{$\begin{array}{l}\text { Observation } \\
\text { wells }\end{array}$} & \multirow[t]{2}{*}{ Other } & \multirow{2}{*}{$\begin{array}{c}\begin{array}{c}\text { Sam- } \\
\text { ples }\end{array} \\
159\end{array}$} & \multicolumn{2}{|c|}{ Date range } & \multirow{2}{*}{$\begin{array}{r}\text { Results } \\
6,526\end{array}$} \\
\hline & & & & & & & $8 / 25 / 2005$ & $7 / 16 / 2009$ & \\
\hline City of Grand Junction & 3 & & & 3 & & 3 & $5 / 7 / 2002$ & $10 / 28 / 2008$ & 74 \\
\hline Colorado Department of Agriculture & 43 & 33 & & 10 & & 43 & $3 / 31 / 1998$ & $10 / 26 / 2000$ & 354 \\
\hline $\begin{array}{l}\text { Colorado Oil and Gas Conservation } \\
\text { Commission }\end{array}$ & 506 & 449 & 1 & 10 & 46 & 508 & 9/12/1977 & $10 / 14 / 2008$ & 11,810 \\
\hline EnCana Oil \& Gas (USA) Inc. & 354 & 293 & 12 & 49 & & 354 & $8 / 8 / 2001$ & $4 / 13 / 2009$ & 13,213 \\
\hline Mine Consultant & 24 & & & 24 & & 24 & $8 / 10 / 1995$ & $12 / 6 / 2006$ & 139 \\
\hline Oxbow Mining & 1 & & & 1 & & 1 & $8 / 10 / 2006$ & $8 / 10 / 2006$ & 5 \\
\hline U.S. Forest Service & 1 & & & 1 & & 1 & $6 / 27 / 2007$ & $6 / 27 / 2007$ & 23 \\
\hline U.S. Geological Survey & 468 & 100 & 25 & 331 & 12 & 468 & $3 / 29 / 1951$ & $4 / 27 / 2009$ & 13,139 \\
\hline Williams & 12 & 11 & 1 & & & 12 & $8 / 30 / 2006$ & $10 / 6 / 2008$ & 593 \\
\hline Wright Water Engineeres, Inc, CO & 15 & & & 15 & & 15 & $4 / 26 / 2002$ & $7 / 17 / 2002$ & 75 \\
\hline
\end{tabular}

\begin{tabular}{|c|c|c|c|c|c|c|c|c|c|c|c|c|c|}
\hline \multirow[b]{2}{*}{ Agency name } & \multicolumn{11}{|c|}{ Constituent catergories $^{1}$} & \multirow[b]{2}{*}{ Sediment } & \multirow[b]{2}{*}{$\begin{array}{c}\text { Stable } \\
\text { Iso- } \\
\text { topes }\end{array}$} \\
\hline & $\begin{array}{l}\text { Informa- } \\
\text { tion }\end{array}$ & $\begin{array}{l}\text { Inorganic } \\
\text { compounds, } \\
\text { major ions, } \\
\text { metals }\end{array}$ & $\begin{array}{c}\text { Inorganic } \\
\text { compounds, } \\
\text { major ions, } \\
\text { non-metals }\end{array}$ & $\begin{array}{c}\text { Inorganic } \\
\text { compounds, } \\
\text { minor ions, } \\
\text { metals }\end{array}$ & $\begin{array}{l}\text { Inorganic } \\
\text { compounds, } \\
\text { minor ions, } \\
\text { non-metals }\end{array}$ & $\begin{array}{l}\text { Micro- } \\
\text { biological } \\
\text { constitu- } \\
\text { ents }\end{array}$ & Nutrient & $\begin{array}{l}\text { Organic } \\
\text { and other } \\
\text { com- } \\
\text { pounds }\end{array}$ & $\begin{array}{l}\text { Organic } \\
\text { com- } \\
\text { pounds } \\
\text { and } \\
\text { pesticide }\end{array}$ & $\begin{array}{l}\text { Physical } \\
\text { charac- } \\
\text { teristics }\end{array}$ & $\begin{array}{l}\text { Radio- } \\
\text { chemical } \\
\text { com- } \\
\text { pounds }\end{array}$ & & \\
\hline Antero Resources & 636 & 636 & 1,072 & 908 & 436 & 314 & 477 & 954 & & 1,093 & & & \\
\hline City of Grand Junction & & 12 & & 42 & 11 & 4 & 3 & & & & 2 & & \\
\hline Colorado Department of Agriculture & & 84 & 82 & & 21 & & 41 & & & 126 & & & \\
\hline $\begin{array}{l}\text { Colorado Oil and Gas Conservation } \\
\text { Commission }\end{array}$ & & 1,683 & 2,959 & 1,181 & 464 & 199 & 957 & 2,609 & 24 & 1,634 & 46 & 1 & 53 \\
\hline EnCana Oil \& Gas (USA) Inc. & 689 & 1,255 & 2,661 & 1,046 & 492 & 457 & 978 & 2,606 & & 2,859 & 20 & & 150 \\
\hline Mine Consultant & & & 20 & & 34 & & 15 & & & 70 & & & \\
\hline Oxbow Mining & & & 1 & & & & 1 & & & 3 & & & \\
\hline U.S. Forest Service & 9 & & 7 & & & & & & & 7 & & & \\
\hline U.S. Geological Survey & 715 & 1,656 & 2,547 & 2,252 & 589 & 3 & 1,243 & 256 & 126 & 3,385 & 233 & & 134 \\
\hline Williams & 33 & 56 & 122 & 112 & 42 & 24 & 52 & 71 & & 81 & & & \\
\hline Wright Water Engineeres, Inc, CO & & & 15 & & 2 & & 13 & & & 45 & & & \\
\hline
\end{tabular}

${ }^{1}$ Examples of constiuents for "Information" would be sampling purpose, methods, analyzing agency. Examples for "Inorganics, Major, Metals" would include calcium, magnesium, and sodium. Examples of constiuents for "Inorganics, Major, Non-metals" would include chloride and sulfate. Examples of constiuents for "Inorganics, Minor, Metals" would be manganese and iron. Examples of constiuents for "Inorganics, Minor, Non-Metals" would be arsenic and selenium. Examples of constiuents for "Microbiological" would be fecal coliform and iron reducing bacteria. Examples of constiuents for "Nutrient" would be nitrate as $\mathrm{N}$ and total phosphorus. Examples of constiuents for "Organics, Other" would be benzene, toluene, and methane. Examples of constiuents for "Organics, Pesticide" would be alachlor and chlorpyrifos. Examples of constiuents for "Physical" would be field parameters and depth to water. Examples of constiuents for "Radiochemical" would be uranium and tritium. Examples of constiuents for "Stable Isotopes" would be carbon-13/carbon-12 ratio in methane and deuterium/protium ratio in methane. 
Table 5. Number and percentage of water-quality samples discussed in this report with associated well-depth, depth-towater, and well-type information.

\begin{tabular}{ccccccc}
\hline \multicolumn{2}{c}{ Number (percentage) of samples } & & \multicolumn{3}{c}{ Number (percentage) of samples by well type } \\
\cline { 1 - 2 } \cline { 5 - 6 } Well-depth data & Depth-to-water data & & Domestic & Observation & Irrigation & Other \\
\hline $529(33$ percent $)$ & $75(5$ percent $)$ & & $1,045(66$ percent $)$ & $444(28$ percent $)$ & $39(2$ percent $)$ & $58(4$ percent $)$ \\
\hline
\end{tabular}

Table 6. Number of water-quality samples by county, geologic unit, and aquifer, Piceance Basin, western Colorado. [Most samples discussed in this report lacked information about geologic unit or aquifer in which well was completed]

[--, Not present, not sampled, or unknown]

\begin{tabular}{|c|c|c|c|c|c|c|c|}
\hline County & Geologic unit & Aquifer & Sample count & County & Geologic unit & Aquifer & Sample count \\
\hline \multirow[t]{7}{*}{ Delta } & Quaternary & -- & 25 & Mesa & & Mancos & 13 \\
\hline & Tertiary & Undifferentiated & 1 & & Other & -- & 3 \\
\hline & Cretaceous & Mesaverde & 20 & & Unknown & -- & 95 \\
\hline & & Mancos & 4 & Moffat & Quaternary & -- & 1 \\
\hline & & Dakota & 1 & & Tertiary & -- & 0 \\
\hline & Other & -- & 0 & & Cretaceous & Mesaverde & 2 \\
\hline & Unknown & -- & 72 & & Other & -- & 10 \\
\hline \multirow[t]{6}{*}{ Garfield } & Quaternary & -- & 14 & & Unknown & -- & 9 \\
\hline & Tertiary & Green River & 4 & Montrose & Quaternary & -- & 4 \\
\hline & & Wasatch & 31 & & Tertiary & -- & 0 \\
\hline & Cretaceous & Mesaverde & 1 & & Cretaceous & Mancos & 1 \\
\hline & Other & -- & 0 & & & Dakota & 6 \\
\hline & Unknown & -- & 979 & & Other & -- & 0 \\
\hline \multirow[t]{5}{*}{ Gunnison } & Quaternary & -- & 1 & & Unknown & -- & 6 \\
\hline & Tertiary & -- & 0 & Rio Blanco & Quaternary & -- & 41 \\
\hline & Cretaceous & Mesaverde & 1 & & Tertiary & Green River & 31 \\
\hline & Other & -- & 0 & & & Wasatch & 4 \\
\hline & Unknown & -- & 1 & & & Undifferentiated & 9 \\
\hline \multirow[t]{3}{*}{ Mesa } & Quaternary & -- & 58 & & Cretaceous & Mesaverde & 6 \\
\hline & Tertiary & Wasatch & 8 & & Other & -- & 3 \\
\hline & Cretaceous & Fox Hills & 2 & & Unknown & -- & 139 \\
\hline
\end{tabular}




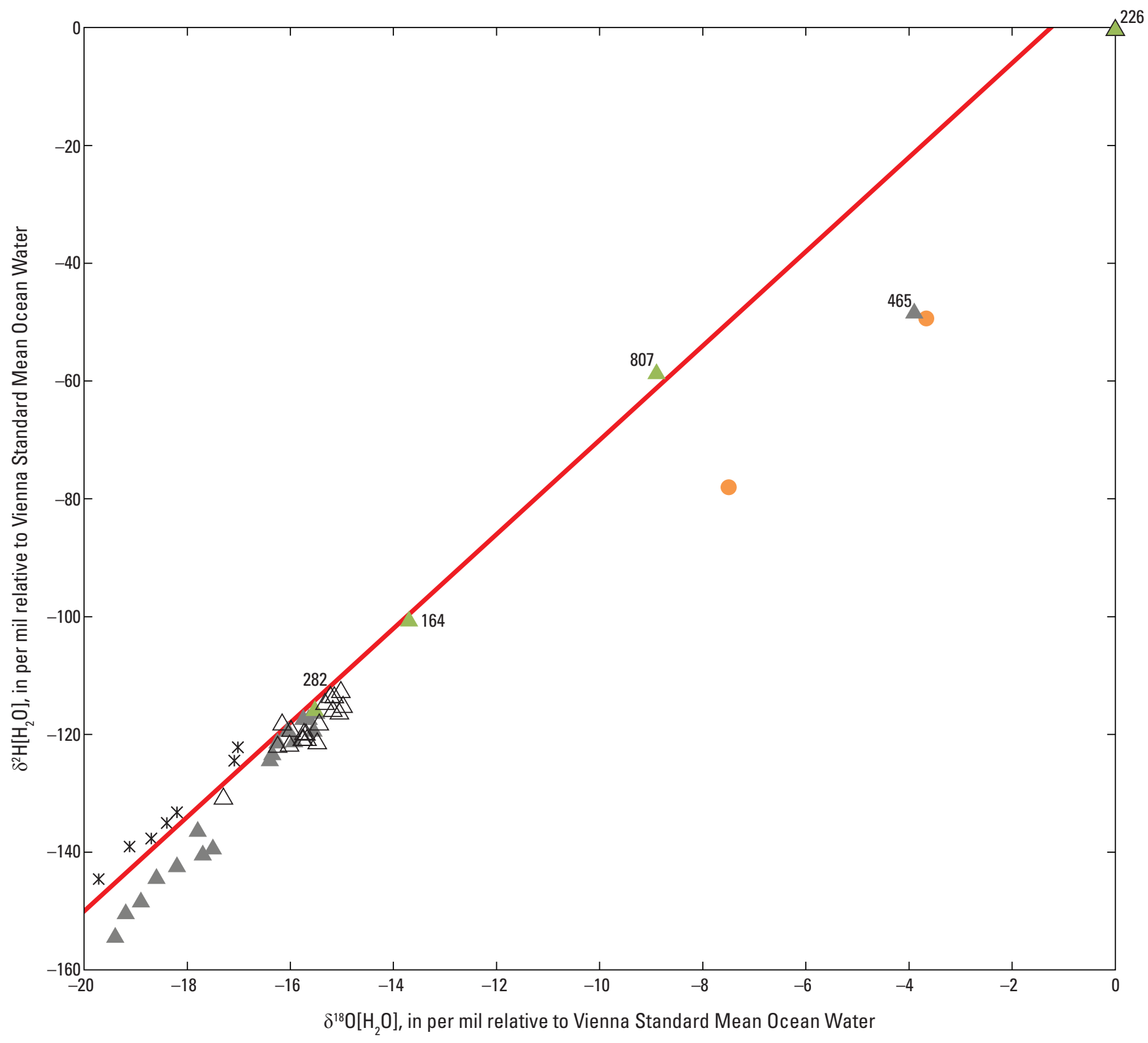

\section{EXPLANATION}

Global Meteoric Water Llne of Craig, 1961

Methane concentration

$\triangle \quad$ Less than 1 milligram per liter

$\triangle \quad$ Greater than or equal to 1 milligram per liter

- Unknown

Mesaverde Group produced water, Garfield County, Colorado

* Snow from Grand Mesa, Colorado

164 Report number

Figure 6. Stable isotopic composition of water from water wells compared with stable isotopic composition of snow from Grand Mesa, Colo., and produced waters from the Mesaverde Group, Piceance Basin, western Colorado. Report No., report number as provided in appendix 1. Grand Mesa snow data are unpublished U.S. Geological Survey data (George Ingersoll, U.S. Geological Survey, written commun., 2006). 
Corporation, 2006). Report number 465 , an observation well in Garfield County, is completed in the Cretaceous Mesaverde Group.

\section{Dissolved Oxygen}

Reduction-oxidation (redox) processes affect the chemical quality of groundwater in all aquifer systems. Redox processes can mobilize or immobilize potentially toxic metals associated with naturally occurring aquifer materials, contribute to the degradation or preservation of anthropogenic contaminants, and generate undesirable byproducts such as dissolved ferrous iron, hydrogen sulfide, and methane. Dissolved-oxygen (DO) concentrations can be used as an indicator of redox processes within groundwater systems (McMahon and Chapelle, 2008). For example, McMahon and others (2010) mapped a low-DO zone in the Wasatch Formation south of the town of Silt in Garfield County, where both denitrification (the primary sink for nitrate in groundwater) and biogenic methanogenesis were observed. In general, such maps of DO concentration can be used to assess an aquifer's vulnerability to contamination by a variety of redox-sensitive chemicals. DO concentrations greater than or equal to $0.5 \mathrm{mg} / \mathrm{L}$ generally indicate oxic conditions and DO concentrations less than $0.5 \mathrm{mg} / \mathrm{L}$ indicate anoxic conditions (McMahon and Chapelle, 2008). In oxic groundwater, dissolved chemicals such as nitrate, uranium, and selenium tend to persist, but constituents such as methane and BTEX (benzene, toluene, ethylbenzene, and xylene) tend to degrade. In anoxic groundwater, conditions favor mobilization of arsenic from aquifer solids, production of biogenic methane, and degradation of dissolved nitrate (Chapelle and others, 1995; McMahon and Chapelle, 2008).

DO concentrations, measured between 1983 and 2009, were available for 733 wells (representing 47 percent of the wells in appendix 1). Concentrations ranged from less than 0.1 to $14 \mathrm{mg} / \mathrm{L}$, with a median of $3.2 \mathrm{mg} / \mathrm{L}$ (table 7). Ninety-three percent of the samples had DO concentrations indicative of oxic conditions (table 7.). DO samples were collected primarily in Garfield County near Rifle and many of the anoxic wells were located in the Mamm Creek-Divide Creek area (fig. 7). Few of the sample data included information on the completion intervals, but at least $10 \mathrm{DO}$ samples were assigned to the Wasatch Formation in Garfield County and to Quaternary deposits in Mesa County. Of those, 70 percent and 68 percent of those samples, respectively, were characterized as oxic (table 8).

Because DO in groundwater is obtained from the atmosphere, DO concentrations in groundwater typically decrease with depth below water as it is consumed by redox processes. Understanding the relations between DO concentration and depth in aquifers is helpful for placement of water-well screens in relation to the distribution of certain redox-sensitive chemicals. Because of the lack of well-depth and water-level information, this study did not examine the relation between DO concentration and depth at the regional scale (table 5).
An alternative method of analysis uses DO data stratified by geologic unit or aquifer, but those data also were generally lacking (table 8).

\section{Major lons}

Dissolved solids (DS) are widely used in evaluating water quality because they can be correlated with certain major ions and with specific conductance to determine water-quality characteristics such as salinity (Hem, 1989). This information can suggest water sources; deeper sources tend to have high DS concentrations and are also high in certain constituents such as chloride. DS concentrations measured between 1958 and 2009 were available for 684 wells, and 497 concentrations (72 percent) exceeded the secondary drinking-water standard of $500 \mathrm{mg} / \mathrm{L}$ (table 7). DS exceedances were relatively widespread in the study area (fig. 8). DS concentrations ranged from 139 to $66,600 \mathrm{mg} / \mathrm{L}$, with a median of $840 \mathrm{mg} / \mathrm{L}$ (table 7). The highest concentration was in a sample from an observation well that was screened in rocks of Tertiary age in Rio Blanco County (appendix 1). Of samples for which the geologic unit or aquifer was designated, only the Wasatch Formation in Garfield County had 10 or more DS values. The median DS concentration in that unit was $1,135 \mathrm{mg} / \mathrm{L}$ (table 8), which exceeds the secondary standard. The National Academy of Sciences recommends that water with DS concentrations greater than $7,000 \mathrm{mg} / \mathrm{L}$ should not be used for watering livestock (ALL Consulting, 2003). Where the geologic unit was known, DS concentrations greater than $7,000 \mathrm{mg} / \mathrm{L}$ in the study area were found in association with rocks of Tertiary and Cretaceous age (fig. 8 and appendix 1).

Major ion chemistry was used to evaluate the composition of dissolved solids within the study area (fig. 9A). Major ion chemistry of DS concentrations greater than $1,000 \mathrm{mg} / \mathrm{L}$ were mostly sodium-sulfate or sodium-bicarbonate-sulfate waters, whereas major ion chemistry of DS concentrations below $1,000 \mathrm{mg} / \mathrm{L}$ were mostly mixed cation bicarbonate waters (fig. 9A). Major ion chemistry was also plotted by geologic unit where this information was available. Waterquality samples from wells screened in Quaternary units were mixed cation-sulfate or mixed cation-bicarbonate waters (fig. 9B). Water samples from wells screened in Cretaceous units were mostly mixed cation - sulfate waters. Waterquality samples from wells screened in aquifers with unknown geologic units plot into three water types; type 1, calcium magnesium-bicarbonate water; type 2, sodium-bicarbonate water; and type 3, sodium-sulfate water. Samples from wells screened in aquifers with unknown geologic units that plot with water type 1 may be most similar to samples from wells screened in aquifers from Tertiary geologic units. Type 2 and type 3 waters have cation compositions similar to those of produced waters from Mesaverde and Wasatch wells (fig. 10), but they typically have lower chloride and DS concentrations than the produced waters. 
Table 7. Summary analyses in the Piceance Basin common data repository of constituents discussed in this report.

$[<$, less than; $\geq$, greater than or equal to; $>$, greater than; N/A, not applicable; --, unknown]

\begin{tabular}{|c|c|c|c|c|c|c|c|c|}
\hline \multirow[b]{2}{*}{ Constituent } & \multirow[b]{2}{*}{$\begin{array}{l}\text { Observa- } \\
\text { tions }\end{array}$} & \multicolumn{3}{|c|}{ Value } & \multicolumn{4}{|c|}{ Detections } \\
\hline & & Minimum & Median & Maximum & Detections & $\begin{array}{c}\text { Percentage } \\
\text { of total } \\
\text { observations }\end{array}$ & Date range of & observations \\
\hline $\begin{array}{l}\text { Dissolved oxygen, water, unfiltered, } \\
\text { milligrams per liter }\end{array}$ & 733 & 0.1 & 3.2 & 14.0 & N/A & N/A & $8 / 3 / 1983$ & $7 / 16 / 2009$ \\
\hline $\begin{array}{l}\text { Total dissolved solids, milligrams } \\
\text { per liter }\end{array}$ & 686 & 139 & 840 & 66,630 & N/A & N/A & $7 / 14 / 1958$ & 7/16/2009 \\
\hline $\mathrm{pH}$, standard units & 1,444 & 3.47 & 7.63 & 12.8 & N/A & N/A & $7 / 14 / 1958$ & $7 / 16 / 2009$ \\
\hline $\begin{array}{l}\text { Sulfate, water, filtered, milligrams } \\
\text { per liter }\end{array}$ & 1,229 & $\begin{array}{c}<5,<2.5,<0.31 \\
<0.30\end{array}$ & 155 & 18,000 & 1,211 & $98.5 \%$ & $3 / 29 / 1951$ & $7 / 16 / 2009$ \\
\hline $\begin{array}{l}\text { Chloride, water, filtered, milligrams } \\
\text { per liter }\end{array}$ & 1,203 & $<1,<0.1$ & 22.2 & 18,000 & 1,173 & $97.5 \%$ & $3 / 29 / 1951$ & $7 / 16 / 2009$ \\
\hline $\begin{array}{l}\text { Fluoride, water, filtered, milligrams } \\
\text { per liter }\end{array}$ & 1,138 & $\begin{array}{c}<2.5,<2.0,<1.0 \\
<0.5,<0.4 \\
<0.1\end{array}$ & 0.64 & 95 & 1,045 & $91.8 \%$ & $7 / 14 / 1958$ & 7/16/2009 \\
\hline $\begin{array}{l}\text { Iron, water, filtered, micrograms } \\
\text { per liter }\end{array}$ & 589 & $\begin{array}{c}<100,<70,<60 \\
<50,<40 \\
<30,<18 \\
<10,<9,<8\end{array}$ & 40 & 110,000 & 321 & $54.5 \%$ & $4 / 5 / 1968$ & 4/27/2009 \\
\hline $\begin{array}{l}\text { Arsenic, water, filtered, micrograms } \\
\text { per liter }\end{array}$ & 256 & $\begin{array}{l}<1,<15,<2 \\
<20,<3,<5\end{array}$ & 1 & 1,100 & 165 & $64.5 \%$ & $12 / 19 / 1971$ & $10 / 28 / 2008$ \\
\hline $\begin{array}{l}\text { Barium, water, filtered, micrograms } \\
\text { per liter }\end{array}$ & 193 & $<100,<200$ & 100 & 5,300 & 162 & $83.9 \%$ & 4/5/1968 & 4/13/2009 \\
\hline $\begin{array}{l}\text { Manganese, water, filtered, micro- } \\
\text { grams per liter }\end{array}$ & 681 & $\begin{array}{c}<20,<15,<10 \\
<5,<3,<1 \\
<0.2\end{array}$ & 6 & 8,900 & 368 & $54.0 \%$ & $4 / 5 / 1968$ & $4 / 26 / 2009$ \\
\hline $\begin{array}{l}\text { Selenium, water, filtered, micro- } \\
\text { grams per liter }\end{array}$ & 551 & $\begin{array}{c}<20,<15,<5 \\
<4.8,<2.4 \\
<2.0,<1.0 \\
<0.7\end{array}$ & 5 & 1,640 & 298 & $54.1 \%$ & $5 / 30 / 1972$ & $10 / 6 / 2008$ \\
\hline $\begin{array}{l}\text { Nitrate as nitrogen, water, filtered, } \\
\text { millgrams per liter }\end{array}$ & 791 & $\begin{array}{c}<0.4,<0.32 \\
<0.29,<0.24 \\
<0.16,<0.09 \\
<0.08,<0.07, \\
<0.06,<0.05 \\
<0.04\end{array}$ & 0.206 & 179 & 601 & $76.0 \%$ & $7 / 14 / 1958$ & $7 / 16 / 2009$ \\
\hline $\begin{array}{l}\text { Benzene, water, unfiltered, recover- } \\
\text { able, micrograms per liter }\end{array}$ & 808 & $\begin{array}{c}<5,<1,<0.5 \\
<0.4,<0.3 \\
<0.2,<0.001 \\
\quad<0.0005\end{array}$ & NA & 160 & 11 & $1.4 \%$ & 9/13/1989 & $\begin{array}{l}10 / 1 / 2008 \\
\text { (all data } \\
7 / 16 / 2009)\end{array}$ \\
\hline $\begin{array}{l}\text { Toluene, water, unfiltered, recover- } \\
\text { able, micrograms per liter }\end{array}$ & 808 & $\begin{array}{l}<5,<3,<2,<1 \\
\quad<0.5,<0.4 \\
\quad<0.2,<0.005\end{array}$ & NA & 14 & 38 & $4.7 \%$ & $\begin{array}{c}6 / 2 / 1997 \\
(9 / 13 / 1989 \\
\text { all data) }\end{array}$ & $\begin{array}{r}9 / 30 / 2008 \\
(7 / 16 / 2009 \\
\text { all data })\end{array}$ \\
\hline $\begin{array}{l}\text { Ethylbenzene, water, unfiltered, } \\
\text { recoverable, micrograms per liter }\end{array}$ & 806 & $\begin{array}{l}<5,<3,<2 \\
<0.5,<0.001 \\
\quad<0.0005\end{array}$ & NA & 4.8 & 6 & $0.7 \%$ & $\begin{array}{l}9 / 13 / 1989 \\
\text { (all data) }\end{array}$ & $\begin{array}{l}7 / 16 / 2009 \\
\text { (all data) }\end{array}$ \\
\hline $\begin{array}{l}\text { Xylene (all isomers), water, unfil- } \\
\text { tered, recoverable, micrograms } \\
\text { per liter }\end{array}$ & 526 & $\begin{array}{c}<3,<1.5,<0.003 \\
<0.0015\end{array}$ & NA & 3.6 & 7 & $1.3 \%$ & $\begin{array}{l}9 / 13 / 1989 \\
\text { (all data) }\end{array}$ & $\begin{array}{l}7 / 16 / 2009 \\
\text { (all data) }\end{array}$ \\
\hline $\begin{array}{l}\text { Methane, water, unfiltered, recover- } \\
\text { able, milligrams per liter }\end{array}$ & 874 & $\begin{array}{c}<0.01,<0.001 \\
<0.0008\end{array}$ & 0.001 & 36.7 & 207 & $23.6 \%$ & 6/2/1997 & $7 / 16 / 2009$ \\
\hline
\end{tabular}


Table 7. Summary of analyses of data in the Piceance Basin common data repository for constituents discussed in this report.-Continued

$[<$, less than; $\geq$, greater than or equal to; $>$, greater than; N/A, not applicable; --, unknown $]$

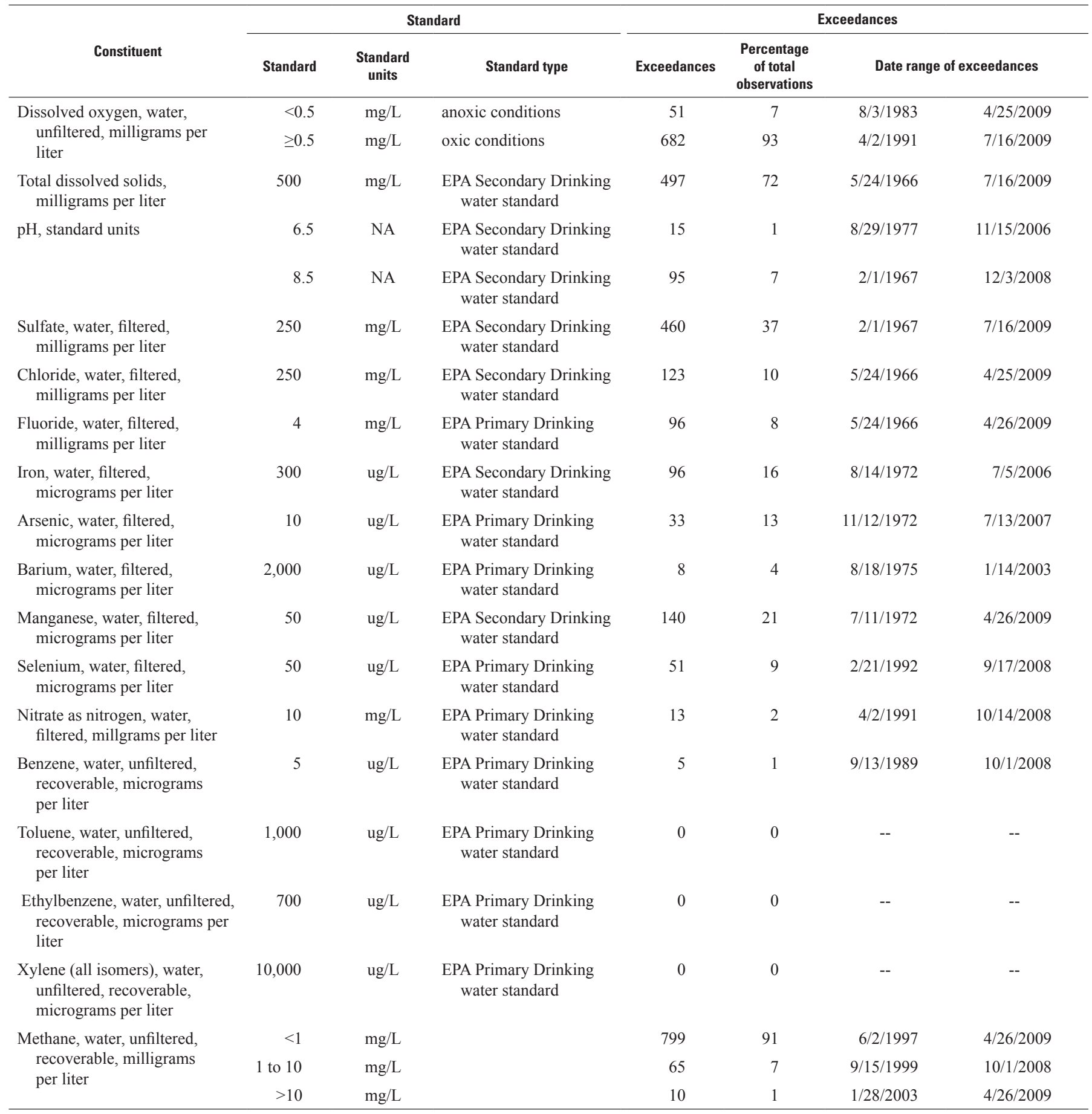



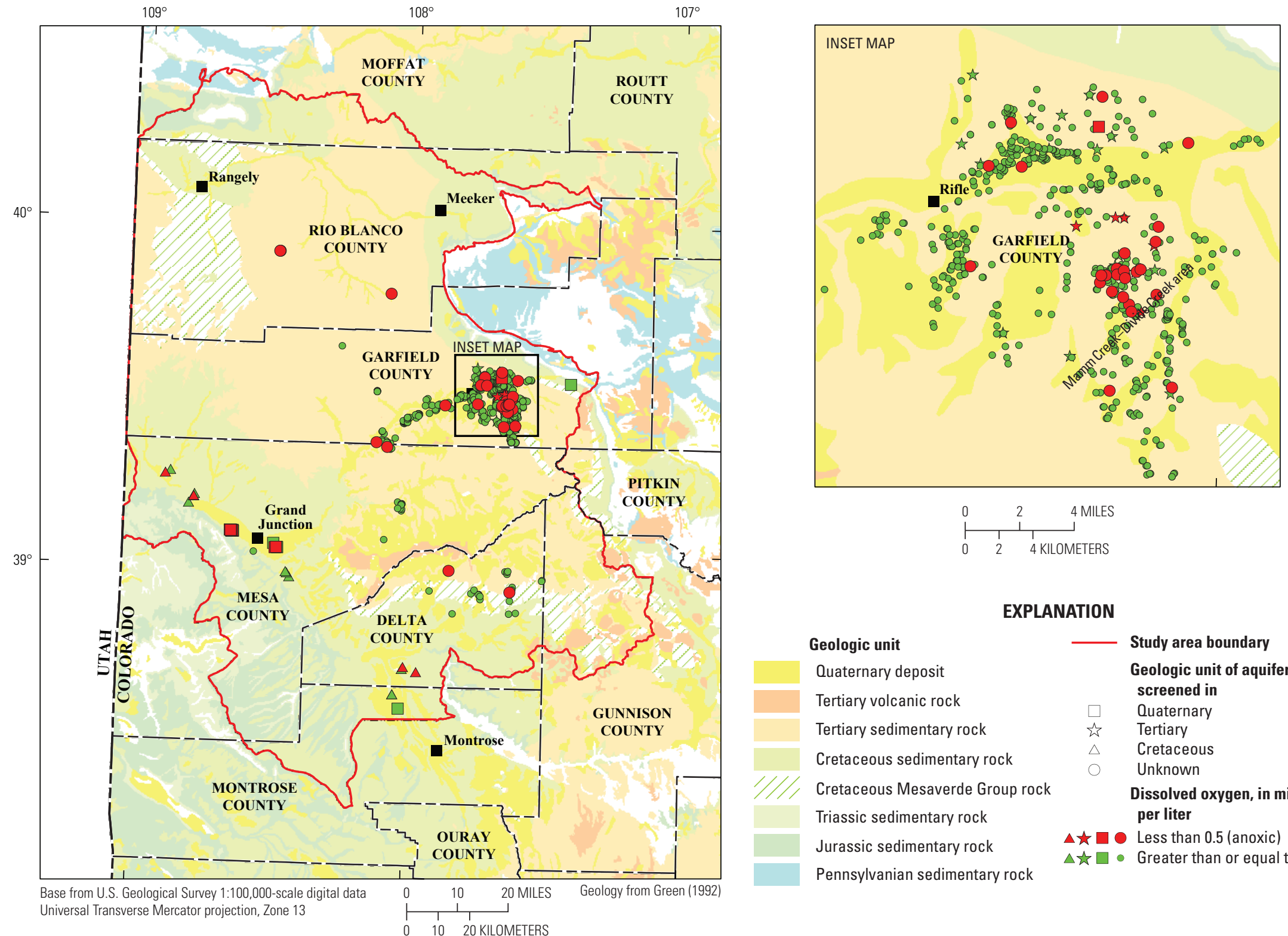

\section{EXPLANATION}

\section{Geologic unit}

Quaternary deposit

Tertiary volcanic rock

Tertiary sedimentary rock

Cretaceous sedimentary rock

Cretaceous Mesaverde Group rock

Triassic sedimentary rock

Jurassic sedimentary rock

Pennsylvanian sedimentary rock

Figure 7. Concentrations of dissolved oxygen in groundwater from wells in the Piceance Basin, western Colorado. $[<$, less than; $\geq$, greater than or equal to $]$ 
Table 8. Concentrations of selected water-quality constituents, by county, geologic unit, and aquifer. The majority of samples discussed in this report lacked information for geologic unit or aquifer.

[mg/L, milligrams per liter; $\mu \mathrm{g} / \mathrm{L}$, micrograms per liter; MCL, maximum containment level; SMCL, secondary maximum containment level; --, not applicable; $\geq$ less than or equal to; $<$, less than]

\begin{tabular}{|c|c|c|c|c|c|c|c|c|c|c|c|}
\hline \multirow[b]{2}{*}{ County } & \multirow[b]{2}{*}{$\begin{array}{l}\text { Geologic } \\
\text { unit }\end{array}$} & \multirow[b]{2}{*}{ Aquifer } & \multicolumn{3}{|c|}{ Dissolved oxygen } & \multicolumn{3}{|c|}{ Dissolved solids } & \multicolumn{3}{|c|}{ Sulfate } \\
\hline & & & $\begin{array}{c}\text { Median } \\
\text { concentration } \\
\text { (range), } \mathrm{mg} / \mathrm{L}\end{array}$ & n & $\begin{array}{c}\text { Percentage } \\
\text { of oxic } \\
\text { (02 } \geq 0.5 \mathrm{mg} / \mathrm{L}) \\
\text { samples }\end{array}$ & $\begin{array}{c}\text { Median } \\
\text { concentration } \\
\text { (range), } \mathrm{mg} / \mathrm{L}\end{array}$ & $\mathbf{n}$ & $\begin{array}{c}\text { Percent } \\
\text { exceedances } \\
\text { (SMCL=500 mg/L) }\end{array}$ & $\begin{array}{c}\text { Median } \\
\text { concentration (range), } \\
\mathrm{mg} / \mathrm{L}\end{array}$ & $\mathbf{n}$ & $\begin{array}{c}\text { Percent } \\
\text { exceedances } \\
\text { (SMCL }=250 \mathrm{mg} / \mathrm{L} \text { ) }\end{array}$ \\
\hline \multirow[t]{2}{*}{ Delta } & Quaternary & -- & -- (--) & 0 & -- & $--(-)$ & 0 & -- & $761(2.1-3,243)$ & 17 & 71 \\
\hline & Cretaceous & Mesaverde & -- (--) & 0 & -- & $--(--)$ & 0 & -- & $18(1.0-510)$ & 17 & 6 \\
\hline \multirow[t]{2}{*}{ Garfield } & Quaternary & -- & $0.9(0.1-1.7)$ & 2 & 50 & $4,773(2,819-6,726)$ & 2 & 100 & $490(170-3,729)$ & 6 & 67 \\
\hline & Tertiary & Wasatch & $1.5(0.1-8.6)$ & 23 & 70 & $1,135(558-5,281)$ & 20 & 100 & $357(15.7-2,882)$ & 29 & 62 \\
\hline \multirow[t]{2}{*}{ Mesa } & Quaternary & -- & $0.9(0-2.3)$ & 28 & 68 & $6,460(--)$ & 1 & 100 & $2,907(548-5,582)$ & 19 & 100 \\
\hline & Cretaceous & Mancos & $0.9(0.1-3.5)$ & 9 & 68 & $4,460(3,820-16,100)$ & 6 & 100 & $2,800(1,224-8,400)$ & 12 & 100 \\
\hline \multirow[t]{2}{*}{ Rio Blanco } & Quaternary & -- & $--(--)$ & 0 & -- & $1,908(214-3,664)$ & 3 & 67 & $340(13.0-1,741)$ & 29 & 59 \\
\hline & Tertiary & Green River & -- (--) & 0 & -- & 926 (909-944) & 2 & 100 & $140(4.2-850)$ & 31 & 26 \\
\hline
\end{tabular}

\begin{tabular}{|c|c|c|c|c|c|c|c|c|c|c|c|}
\hline \multirow[b]{2}{*}{ County } & \multirow[b]{2}{*}{$\begin{array}{c}\text { Geologic } \\
\text { unit }\end{array}$} & \multirow[b]{2}{*}{ Aquifer } & \multicolumn{3}{|c|}{ Chloride } & \multicolumn{3}{|c|}{ Fluoride } & \multicolumn{3}{|c|}{ Nitrite+nitrate } \\
\hline & & & $\begin{array}{c}\text { Median } \\
\text { concentration } \\
\text { (range), } \mathrm{mg} / \mathrm{L}\end{array}$ & n & $\begin{array}{c}\text { Percent } \\
\text { exceedances } \\
\text { (SMCL=250 } \\
\text { mg/L) }\end{array}$ & $\begin{array}{l}\text { Median concentration } \\
\text { (range), mg/L }\end{array}$ & n & $\begin{array}{c}\text { Percent exceed- } \\
\text { ances (MCL=4 } \\
\mathrm{mg} / \mathrm{L})\end{array}$ & $\begin{array}{c}\text { Median } \\
\text { concentration (range), } \\
\mathrm{mg} / \mathrm{L} \text { as } \mathrm{N}\end{array}$ & $\mathbf{n}$ & $\begin{array}{c}\text { Percent exceed- } \\
\text { ances (MCL=10 } \\
\text { mg/L as } N \text { ) }\end{array}$ \\
\hline \multirow[t]{2}{*}{ Delta } & Quaternary & -- & $8.76(6.40-11.0)$ & 4 & 0 & $0.68(0.50-1.50)$ & 4 & 0 & $--(--)$ & 0 & -- \\
\hline & Cretaceous & Mesaverde & $14.0(2.70-180)$ & 11 & 0 & $0.80(0.20-5.40)$ & 11 & 9 & $--(--)$ & 0 & -- \\
\hline \multirow[t]{2}{*}{ Garfield } & Quaternary & -- & $20.5(12.0-281)$ & 6 & 17 & $1.10(0.48-2.44)$ & 6 & 0 & $7.52(1.37-13.7)$ & 2 & 50 \\
\hline & Tertiary & Wasatch & $43.3(<1.0-1,116)$ & 29 & 7 & $0.97(0.33-5.10)$ & 29 & 7 & $0.40(<0.038-6.73)$ & 27 & 0 \\
\hline \multirow[t]{2}{*}{ Mesa } & Quaternary & -- & $367(20.0-1,201)$ & 20 & 70 & $0.54(0.11-1.21)$ & 19 & 0 & $5.78(--)$ & 1 & 0 \\
\hline & Cretaceous & Mancos & $215(34.5-4,880)$ & 10 & 30 & $0.71(0.30-5.52)$ & 10 & 10 & $2.64(<0.04-7.58)$ & 7 & 0 \\
\hline \multirow[t]{2}{*}{ Rio Blanco } & Quaternary & -- & $19.0(4.00-270)$ & 29 & 3 & $0.80(0.10-33.0)$ & 29 & 21 & $0.30(<0.08-0.54)$ & 4 & 0 \\
\hline & Tertiary & Green River & $13.0(0.1-1,700)$ & 31 & 10 & $5.00(<0.10-47.0)$ & 31 & 52 & $<0.08(<0.08-<0.08)$ & 4 & 0 \\
\hline
\end{tabular}


Table 8. Concentrations of selected water-quality constituents, by county, geologic unit, and aquifer. The majority of samples discussed in this report lacked information for geologic unit or aquifer-Continued

[mg/L, milligrams per liter; $\mu \mathrm{g} / \mathrm{L}$, micrograms per liter; MCL, maximum containment level; SMCL, secondary maximum containment level; --, not applicable; $\geq$ less than or equal to; <, less than]

\begin{tabular}{|c|c|c|c|c|c|c|c|c|c|c|c|}
\hline \multirow[b]{2}{*}{ County } & \multirow[b]{2}{*}{$\begin{array}{c}\text { Geologic } \\
\text { unit }\end{array}$} & \multirow[b]{2}{*}{ Aquifer } & \multicolumn{3}{|c|}{ Iron } & \multicolumn{3}{|c|}{ Manganese } & \multicolumn{3}{|c|}{ Arsenic } \\
\hline & & & $\begin{array}{l}\text { Median } \\
\text { concentration } \\
\text { range, } \mu \mathrm{g} / \mathrm{L}\end{array}$ & $\mathrm{n}$ & $\begin{array}{c}\text { Percent } \\
\text { exceedances } \\
\text { (SMCL=300 } \\
\mu \mathrm{g} / \mathrm{L})\end{array}$ & $\begin{array}{l}\text { Median concentration } \\
\text { (range), } \mu \mathrm{g} / \mathrm{L}\end{array}$ & $\mathbf{n}$ & $\begin{array}{c}\text { Percent exceed- } \\
\text { ances (SMCL=50 } \\
\mu \mathrm{g} / \mathrm{L})\end{array}$ & $\begin{array}{l}\text { Median concentration } \\
\text { (range), } \mu \mathrm{g} / \mathrm{L}\end{array}$ & $\mathbf{n}$ & $\begin{array}{c}\text { Percent exceed- } \\
\text { ances (MCL=10 } \\
\mu \mathrm{g} / \mathrm{L})\end{array}$ \\
\hline \multirow[t]{2}{*}{ Delta } & Quaternary & -- & $40(<10-60)$ & 3 & 0 & $<10(<10-20)$ & 4 & 0 & $1.38(<1-5.00)$ & 3 & 0 \\
\hline & Cretaceous & Mesaverde & $150(10-580)$ & 11 & 27 & $20(2-120)$ & 11 & 9 & $<1(<1-6.00)$ & 11 & 0 \\
\hline \multirow[t]{2}{*}{ Garfield } & Quaternary & -- & $<14(<14-2,400)$ & 6 & 17 & $<10(<10-876)$ & 6 & 33 & $1.61(<1-3.00)$ & 6 & 0 \\
\hline & Tertiary & Wasatch & $9.2(3.0-92)$ & 20 & 0 & $2.1(<0.2-220)$ & 21 & 10 & -- (--) & 0 & -- \\
\hline \multirow[t]{2}{*}{ Mesa } & Quaternary & -- & $<60(<60-7,206)$ & 13 & 23 & $1,410(<10-7,243)$ & 12 & 92 & $1.50(<1-8.00)$ & 4 & 0 \\
\hline & Cretaceous & Mancos & $<10(<10-780)$ & 8 & 25 & $195(30-860)$ & 8 & 75 & $6.43(5.63-7.23)$ & 2 & 0 \\
\hline \multirow[t]{2}{*}{ Rio Blanco } & Quaternary & -- & $70(<10-49,000)$ & 28 & 7 & $45(8-530)$ & 28 & 39 & $5.00(<1-66)$ & 28 & 25 \\
\hline & Tertiary & Green River & $80(9-2,400)$ & 29 & 14 & $<10(<10-100)$ & 31 & 6 & $4.00(<1-150)$ & 27 & 15 \\
\hline
\end{tabular}

\begin{tabular}{|c|c|c|c|c|c|c|c|c|c|c|c|}
\hline \multirow[b]{2}{*}{ County } & \multirow[b]{2}{*}{$\begin{array}{l}\text { Geologic } \\
\text { unit }\end{array}$} & \multirow[b]{2}{*}{ Aquifer } & \multicolumn{3}{|c|}{ Barium } & \multicolumn{3}{|c|}{ Selenium } & \multicolumn{3}{|c|}{ Methane } \\
\hline & & & $\begin{array}{c}\text { Median } \\
\text { concentration } \\
\text { (range), } \mu \mathrm{g} / \mathrm{L}\end{array}$ & n & $\begin{array}{c}\text { Percent } \\
\text { exceedances } \\
\text { (MCL=2,000 } \\
\mu \mathrm{g} / \mathrm{L} \text { ) }\end{array}$ & $\begin{array}{l}\text { Median concentration } \\
\text { (range), } \mu \mathrm{g} / \mathrm{L}\end{array}$ & $\mathbf{n}$ & $\begin{array}{c}\text { Percent exceed- } \\
\text { ances (MCL=50 } \\
\mu \mathrm{g} / \mathrm{L})\end{array}$ & $\begin{array}{l}\text { Median concentration } \\
\text { (range), mg/L }\end{array}$ & $\mathbf{n}$ & $\begin{array}{c}\text { Percentage of } \\
\text { samples }>1 \mathrm{mg} / \mathrm{L}\end{array}$ \\
\hline \multirow[t]{2}{*}{ Delta } & Quaternary & -- & $36(--)$ & 1 & 0 & $7.50(1.30-32.0)$ & 14 & 0 & $--(--)$ & 0 & -- \\
\hline & Cretaceous & Mesaverde & $90(50-2,100)$ & 11 & 9 & $18.0(<1-238)$ & 16 & 19 & -- (--) & 0 & -- \\
\hline \multirow[t]{2}{*}{ Garfield } & Quaternary & -- & $<100(<100)$ & 6 & 0 & $2.00(<1-160)$ & 6 & 17 & $--(--)$ & 0 & -- \\
\hline & Tertiary & Wasatch & -- (--) & 0 & -- & $13.6(5.50-21.6)$ & 2 & 0 & $<0.0005(<0.000-32.5)$ & 27 & 11 \\
\hline \multirow[t]{2}{*}{ Mesa } & Quaternary & -- & $<100(<100)$ & 4 & 0 & $2.72(<1-280)$ & 31 & 10 & -- (--) & 0 & -- \\
\hline & Cretaceous & Mancos & -- (--) & 0 & -- & $9.00(<5-1,000)$ & 11 & 27 & -- (--) & 0 & -- \\
\hline \multirow[t]{2}{*}{ Rio Blanco } & Quaternary & -- & $36(15-56)$ & 3 & 0 & $4.00(<1-9.00)$ & 27 & 0 & -- (--) & 0 & -- \\
\hline & Tertiary & Green River & $<200(<200-800)$ & 10 & 0 & $<1(<1-7.00)$ & 20 & 0 & $--(--)$ & 0 & -- \\
\hline
\end{tabular}

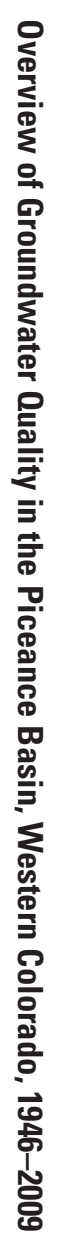


Table 8. Concentrations of selected water-quality constituents, by county, geologic unit, and aquifer. The majority of samples discussed in this report lacked information for geologic unit or aquifer.-Continued

[mg/L, milligrams per liter; $\mu \mathrm{g} / \mathrm{L}$, micrograms per liter; MCL, maximum containment level; SMCL, secondary maximum containment level; --, not applicable; $\geq$ less than or equal to; $<$ less than]

\begin{tabular}{|c|c|c|c|c|c|c|c|c|}
\hline \multirow[b]{2}{*}{ County } & \multirow[b]{2}{*}{$\begin{array}{c}\text { Geologic } \\
\text { unit }\end{array}$} & \multirow[b]{2}{*}{ Aquifer } & \multicolumn{3}{|c|}{ Benzene } & \multicolumn{3}{|c|}{ Toluene } \\
\hline & & & $\begin{array}{l}\text { Median concentra- } \\
\text { tion (range), } \mu \mathrm{g} / \mathrm{L}\end{array}$ & n & $\begin{array}{c}\text { Percent } \\
\text { exceedances } \\
\text { (MCL=5 } \mu \mathrm{g} / \mathrm{L} \text { ) }\end{array}$ & $\begin{array}{l}\text { Median concentration } \\
\text { (range), } \mu \mathrm{g} / \mathrm{L}\end{array}$ & n & $\begin{array}{c}\text { Percent } \\
\text { exceedances } \\
\text { (MCL=1,000 } \mu \mathrm{g} / \mathrm{L} \text { ) }\end{array}$ \\
\hline \multirow[t]{2}{*}{ Delta } & Quaternary & -- & $--(--)$ & 0 & -- & $--(--)$ & 0 & -- \\
\hline & Cretaceous & Mesaverde & -- (--) & 0 & -- & -- (--) & 0 & -- \\
\hline \multirow[t]{2}{*}{ Garfield } & Quaternary & -- & -- (--) & 0 & -- & -- (--) & 0 & -- \\
\hline & Tertiary & Wasatch & $<1(<1)$ & 8 & 0 & $<5(<5)$ & 8 & 0 \\
\hline \multirow[t]{2}{*}{ Mesa } & Quaternary & -- & -- (--) & 0 & -- & -- (--) & 0 & -- \\
\hline & Cretaceous & Mancos & -- (--) & 0 & -- & -- (--) & 0 & -- \\
\hline \multirow[t]{2}{*}{ Rio Blanco } & Quaternary & -- & -- (--) & 0 & -- & -- (--) & 0 & -- \\
\hline & Tertiary & Green River & -- (--) & 0 & -- & -- (--) & 0 & -- \\
\hline
\end{tabular}



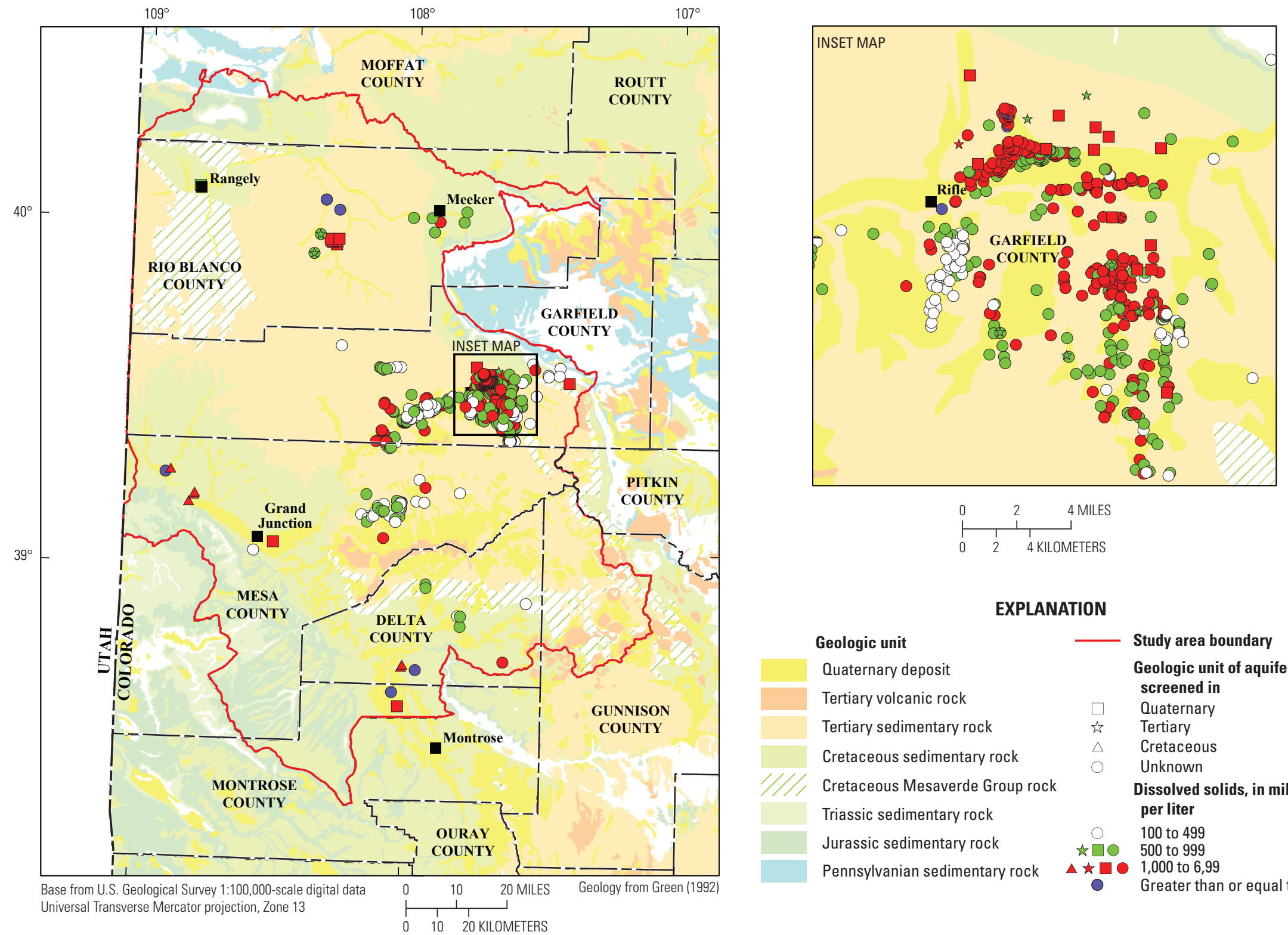

EXPLANATION

Geologic unit

Quaternary deposit

Tertiary volcanic rock

Tertiary sedimentary rock

Cretaceous sedimentary rock

Cretaceous Mesaverde Group rock

Triassic sedimentary rock

Jurassic sedimentary rock

Pennsylvanian sedimentary rock

Figure 8. Concentrations of dissolved solids in groundwater from wells in the Piceance Basin, western Colorado. [ $\geq$, greater than or equal to] 

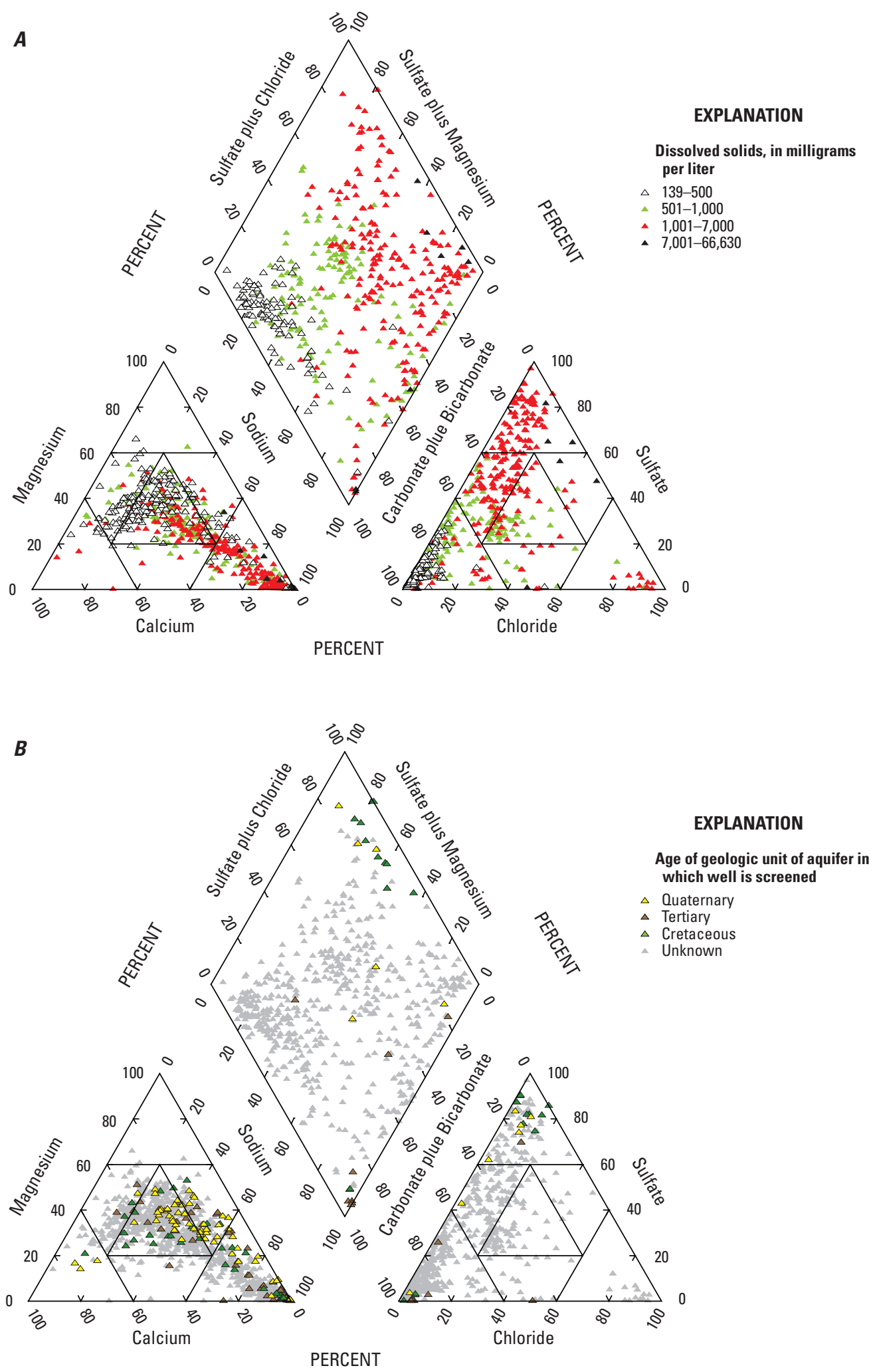

Figure 9. Trilinear diagram of major-ion chemistry of groundwater by $(A)$ dissolved solids, and $(B)$ geologic unit of aquifer in which well is screened, Piceance Basin, western Colorado. 
Figure 10. Trilinear diagram of majorion chemistry of produced waters from Mesaverde Group and Wasatch Formation, Piceance Basin, western Colorado. Produced-water-quality samples submitted to the Piceance Basin common data repository by Colorado Oil and Gas Conservation Commission, 2010.

The property $\mathrm{pH}$ is a measure of the hydrogen ion activity in water and, like water temperature, is often collected as part of a water-quality-sampling program because it provides the most basic understanding of water quality. Values of $\mathrm{pH}$ less than 7 are generally referred to as acidic; those greater than 7 are generally referred to as basic. The normal range of $\mathrm{pH}$ in natural waters is between 6.5 and 8.5 (Hem, 1989). Values of $\mathrm{pH}$ less than 6.5 or greater than 8.5 exceed the secondary standard. Of $\mathrm{pH}$ values collected between 1958 and 2009 for 1,441 wells, only 15 values ( 1 percent) were less than 6.5 (acidic) and 95 values (6.6 percent) were greater than 8.5 (basic) (table 7). The median value was 7.6 standard units. Several of the $\mathrm{pH}$ values greater than 8.5 were measured in samples from wells completed in Quaternary deposits in Rio Blanco County, but the majority of the samples with $\mathrm{pH}$ greater than 8.5 were from unknown geologic units (fig. 11).

Chloride is a conservative constituent - it is not likely to be participate in geochemical processes or be precipitated once mobilized (Hem, 1989). Chloride concentrations, measured between 1951 and 2009, were available for 1,203 wells, and 123 concentrations (10 percent) exceeded the secondary standard of $250 \mathrm{mg} / \mathrm{L}$ (table 7). Like DS exceedances, chloride exceedances were relatively widespread in the study area (fig. 12). Concentrations ranged from less than detection to $18,000 \mathrm{mg} / \mathrm{L}$, with a median concentration of $22.2 \mathrm{mg} / \mathrm{L}$ (table 7). The highest chloride concentration was found in a sample from an observation well screened in the Pennsylvanian and Permian Weber Sandstone in Rio Blanco County (appendix 1). Several of the samples had geologic unit or aquifer designations. Of those samples, only samples from Quaternary units in Mesa County had a median chloride concentration greater than the secondary standard (table 8). Overall, 85 chloride concentrations ( 7 percent) were greater than $355 \mathrm{mg} / \mathrm{L}$, a concentration above which agricultural use should be severely restricted, specifically with respect to irrigation (Ayers and Westcot, 1985). These samples were collected in Garfield, Rio Blanco, and Mesa Counties (fig. 12). Eight chloride concentrations (less than 1 percent) were greater than 4,000 mg/L, similar to some brines (Hem, 1989), 

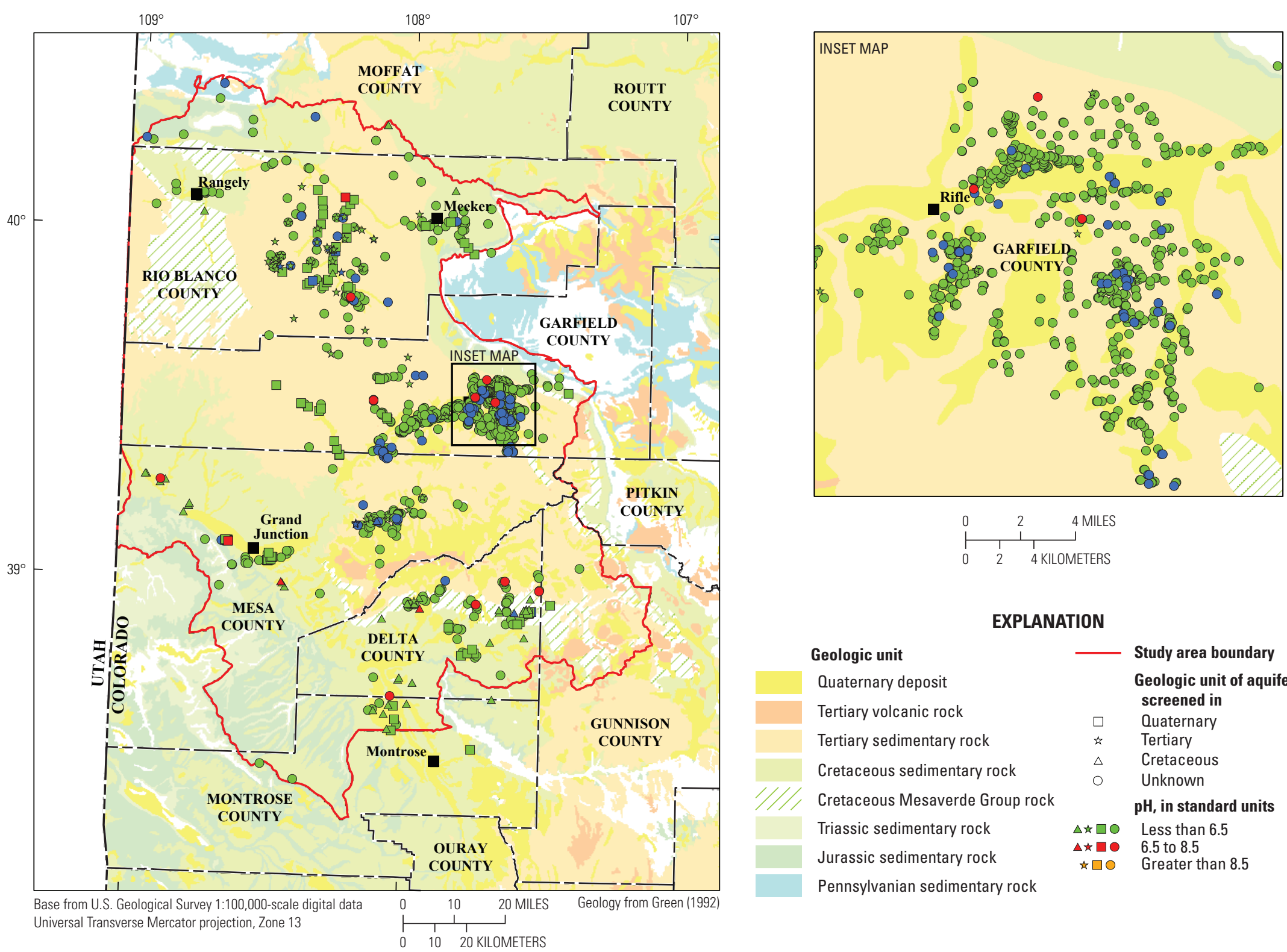

\section{EXPLANATION}

\section{Geologic unit}

Quaternary deposit

Tertiary volcanic rock

Tertiary sedimentary rock

Cretaceous sedimentary rock

Cretaceous Mesaverde Group rock

Triassic sedimentary rock

Jurassic sedimentary rock

Pennsylvanian sedimentary rock 

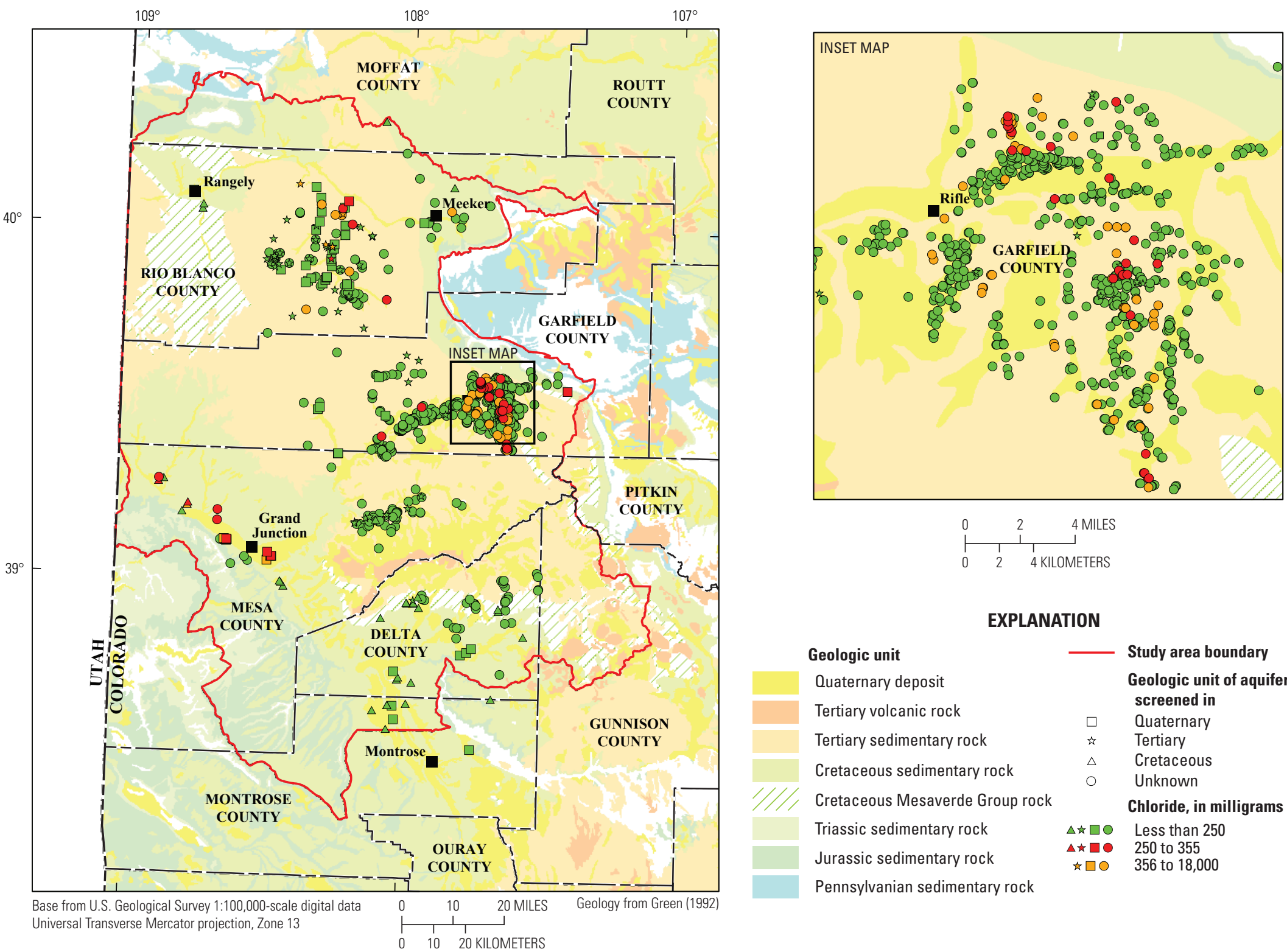

\section{EXPLANATION}

Geologic unit

Quaternary deposit

Tertiary volcanic rock

Tertiary sedimentary rock

Cretaceous sedimentary rock

Cretaceous Mesaverde Group rock

Triassic sedimentary rock

Jurassic sedimentary rock

Pennsylvanian sedimentary rock

Figure 12. Concentrations of chloride in groundwater from wells in the Piceance Basin, western Colorado. [<, less than] 
and these samples were primarily collected in Rio Blanco County (appendix 1).

Sulfate concentrations measured between 1951 and 2009 were available for 1,226 wells and 460 concentrations (37 percent) exceeded the secondary standard of $250 \mathrm{mg} / \mathrm{L}$ (table 7). Like DS and chloride exceedances, sulfate exceedances were relatively widespread in the study area (fig. 13). Concentrations ranged from below detection (commonly less than $5 \mathrm{mg} / \mathrm{L}$ ) to $18,000 \mathrm{mg} / \mathrm{L}$, with a median concentration of $155 \mathrm{mg} / \mathrm{L}$ (table 7). The highest sulfate concentration was found in a sample from an observation well in Garfield County (geologic unit in which it was screened was not reported) (appendix 1). Of the several samples that did have geologic unit or aquifer designations, most had median sulfate concentrations higher than the standard (table 8). These data indicated that subsurface sulfate sources were relatively common in the study area. In the absence of additional information, such as stable sulfur-isotope data, it is difficult to identify those sources. Likely sources include minerals such as gypsum and pyrite (following oxidation). Produced waters are less likely to contribute sulfate to the freshwater aquifers because sulfate concentrations in those waters typically are low (URS Corporation, 2006). Produced waters from the Mesaverde Group in Garfield County, for example, generally contained less than $10 \mathrm{mg} / \mathrm{L}$ sulfate (S.S. Papadopulos \& Associates, Inc., 2008). On the basis of the nonparametric Wilcoxon rank-sum test, sulfate concentrations were significantly higher $(p<0.001)$ in anoxic groundwater than oxic groundwater (table 9) (Helsel and Hirsch, 2002). This result is opposite the result expected on the basis of the redox chemistry of sulfate (McMahon and Chapelle, 2008). The reason for this outcome is unclear, but it could indicate a relative abundance of sulfate sources in the aquifers compared with electron donors, such as organic carbon, to support sulfate reduction under anoxic conditions.

Fluoride has a low solubility and, in natural waters, is typically found at concentrations less than $1 \mathrm{mg} / \mathrm{L}$ (Hem, 1989). Fluoride concentrations, measured from 1958 to 2009 , were available for 1,138 wells, and 96 concentrations ( 8.4 percent) exceeded the primary standard of $4 \mathrm{mg} / \mathrm{L}$ (table 7). Most of these exceedances were in samples from Rio Blanco County (fig. 14). Additionally, fluoride concentrations commonly exceeded the primary standard in the Mamm Creek-Divide Creek area of Garfield County (URS Corporation, 2006). Fluoride concentrations ranged from less than detection (commonly less than $0.5 \mathrm{mg} / \mathrm{L}$ ) to $95 \mathrm{mg} / \mathrm{L}$, with median fluoride concentration of $0.64 \mathrm{mg} / \mathrm{L}$. A sample from an observation well in Rio Blanco County had the highest fluoride concentration (geologic unit in which it was screened not reported) (appendix 1). Of samples for which the geologic unit or aquifer was designated, only the Green River Formation in Rio Blanco County had 10 or more fluoride values and a median fluoride concentration greater than the primary standard (table 8).

\section{Trace Elements}

Trace element data for arsenic, barium, manganese, iron, and selenium in groundwater were included in this report. These trace elements are typically derived from minerals in the aquifer rocks and sediment (Hem, 1989). Arsenic concentrations, measured between 1971 and 2008, were available for 256 wells and 33 concentrations ( 13 percent) exceeded the primary standard of 10 micrograms per liter $(\mu \mathrm{g} / \mathrm{L})$ (table 7$)$. Most of these exceedances were in samples from Rio Blanco County (fig. 15). Arsenic concentrations ranged from less than detection (most commonly less than $1 \mu \mathrm{g} / \mathrm{L}$ ) to $1,100 \mu \mathrm{g} / \mathrm{L}$, with a median concentration of $1 \mu \mathrm{g} / \mathrm{L}$ (table 7). A sample from an observation well in Garfield County had the highest arsenic concentration (geologic unit in which it was screened was not reported) (appendix 1). Quaternary and Tertiary units in Rio Blanco County had the highest median arsenic concentrations ( 5 and $4 \mu \mathrm{g} / \mathrm{L}$, respectively) of those units with at least 10 assigned samples (table 8 ). Arsenic concentrations were significantly $(p=0.008)$ larger in anoxic groundwater than in oxic groundwater (table 9), indicating that reductive dissolution of manganese and/or iron oxide minerals in the aquifer rock and sediment probably was an important mechanism for producing elevated arsenic concentrations in groundwater (Smedley and Kinniburgh, 2002). In fact, iron concentrations were significantly higher $(p=0.001)$ in samples with arsenic concentrations above the primary standard of $10 \mu \mathrm{g} / \mathrm{L}$ than in samples with lower arsenic concentrations. There was no significant difference in manganese concentrations between samples with arsenic concentrations higher and lower than the standard. The arsenic-DO relation also implies that DO measurements indicating anoxic conditions could be a useful guide to groundwater vulnerability to arsenic enrichment.

Barium concentrations measured between 1968 and 2009 were available for 193 wells, and 8 concentrations (4.1 percent) exceeded the primary standard of 2,000 $\mu \mathrm{g} / \mathrm{L}$ (table 7). Samples with barium exceedances were collected in Delta, Garfield, and Rio Blanco Counties (fig. 16). Concentrations ranged from less than detection to $5,300 \mu \mathrm{g} / \mathrm{L}$, with a median concentration of $90 \mu \mathrm{g} / \mathrm{L}$. A sample from an observation well in Rio Blanco County had the highest barium concentration (geologic unit in which it was screened was not reported) (appendix 1). The Mesaverde Group in Delta County had the highest quantifiable median barium concentrations $(90 \mu \mathrm{g} / \mathrm{L})$ of those units with at least 10 assigned samples (table 8).

Manganese concentrations measured between 1968 and 2009 were available for 681 wells, and 140 concentrations 

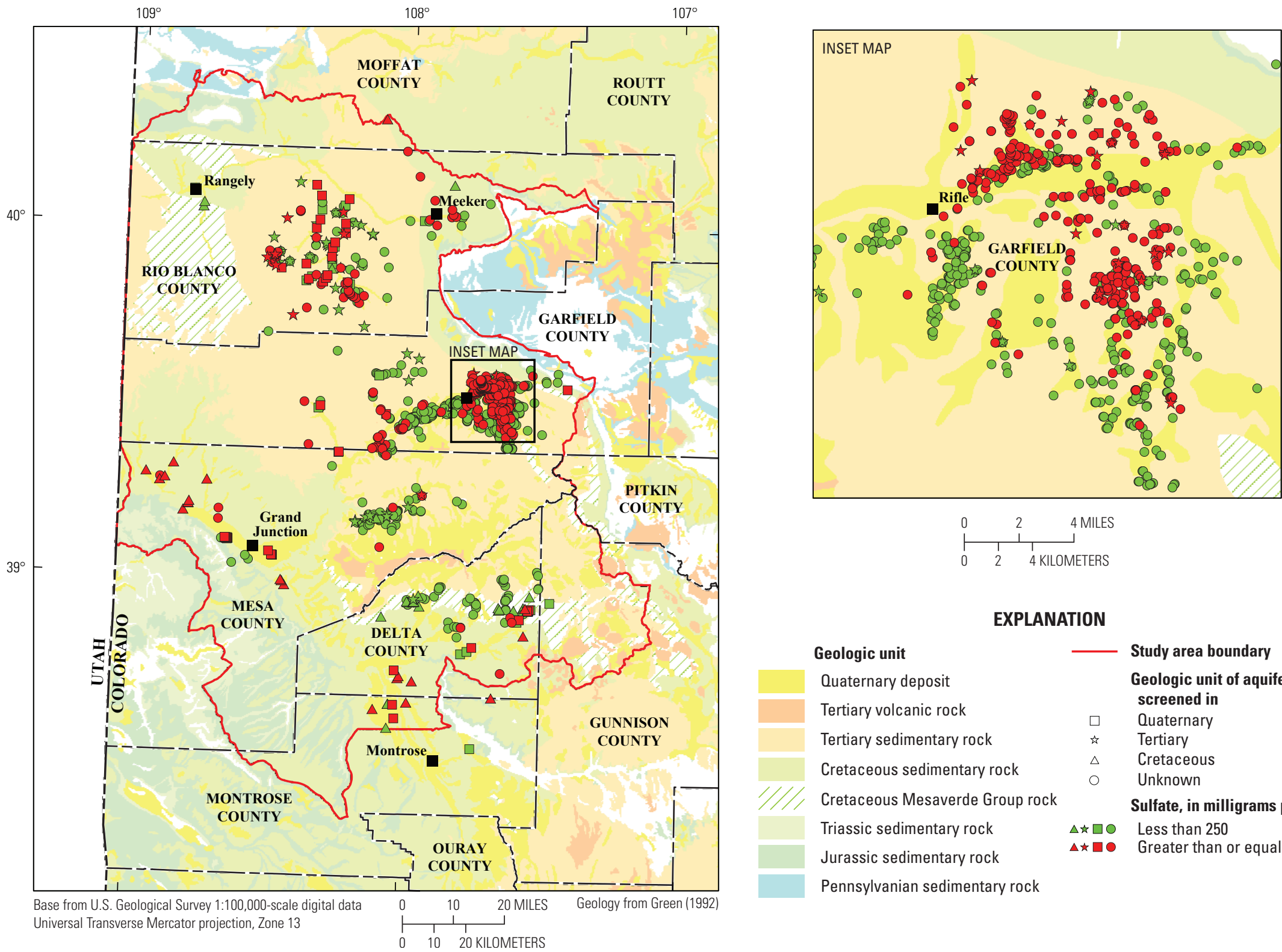

\section{EXPLANATION}

Geologic unit

Quaternary deposit

Tertiary volcanic rock

Tertiary sedimentary rock

Cretaceous sedimentary rock

Cretaceous Mesaverde Group rock

Triassic sedimentary rock

Jurassic sedimentary rock

Pennsylvanian sedimentary rock

Figure 13. Concentrations of sulfate in groundwater from wells in the Piceance Basin, western Colorado. [<, less than; $\geq$, greater than or equal to $]$ 
Table 9. Concentrations of selected water-quality constituents relative to three dissolved-oxygen concentration thresholds. [Reported concentrations below the indicated common assessment levels set to zero. P-values determined from the nonparametric Wilcoxon rank-sum test (Helsel and Hirsch, 2002]

[mg/L, milligrams per liter; $\mu \mathrm{g} / \mathrm{L}$, micrograms per liter; $<$, less than; $\geq$, greater than or equal to;]

\begin{tabular}{|c|c|c|c|c|c|c|c|c|c|c|c|}
\hline \multirow{3}{*}{ Constituent } & \multirow{3}{*}{$\begin{array}{l}\text { Concentration } \\
\text { units }\end{array}$} & \multirow{3}{*}{$\begin{array}{c}\text { Common } \\
\text { assessment } \\
\text { concentration }\end{array}$} & \multicolumn{9}{|c|}{ Average concentration (number of samples) } \\
\hline & & & \multicolumn{3}{|c|}{$\mathrm{O}_{2}$ concentration $(\mathrm{mg} / \mathrm{L})$} & \multicolumn{3}{|c|}{$\mathrm{O}_{2}$ concentration $(\mathrm{mg} / \mathrm{L})$} & \multicolumn{3}{|c|}{$\mathrm{O}_{2}$ concentration $(\mathrm{mg} / \mathrm{L})$} \\
\hline & & & $<0.5$ & $\geq 0.5$ & p-value & $<1.0$ & $\geq 1.0$ & p-value & $<2.0$ & $\geq \mathbf{2 . 0}$ & p-value \\
\hline Nitrate & $\mathrm{mg} / \mathrm{L}$ as $\mathrm{N}$ & 0.29 & $0.87(35)$ & $0.93(547)$ & 0.459 & $0.84(85)$ & $0.95(497)$ & 0.183 & $0.91(183)$ & $0.94(399)$ & 0.015 \\
\hline Sulfate & $\mathrm{mg} / \mathrm{L}$ & 2.5 & $1,320(43)$ & $551(623)$ & $<0.001$ & $1,080(102)$ & $514(564)$ & $<0.001$ & $821(211)$ & $499(455)$ & $<0.001$ \\
\hline Manganese & $\mu \mathrm{g} / \mathrm{L}$ & 5 & $580(31)$ & $151(267)$ & 0.001 & $366(66)$ & $148(232)$ & $<0.001$ & $300(100)$ & $143(198)$ & $<0.001$ \\
\hline Iron & $\mu \mathrm{g} / \mathrm{L}$ & 70 & $315(31)$ & 749 (291) & 0.486 & $354(68)$ & $801(254)$ & 0.856 & $348(108)$ & $888(214)$ & 0.973 \\
\hline Methane & $\mathrm{mg} / \mathrm{L}$ & 0.01 & $1.86(31)$ & $0.54(605)$ & 0.004 & $1.78(80)$ & $0.43(556)$ & $<0.001$ & $1.47(181)$ & $0.26(455)$ & $<0.001$ \\
\hline Arsenic & $\mu \mathrm{g} / \mathrm{L}$ & 3 & $4.57(7)$ & $0.40(45)$ & 0.008 & $4.00(7)$ & $0.41(45)$ & 0.018 & 2.12 (17) & $0.40(35)$ & 0.118 \\
\hline Selenium & $\mu \mathrm{g} / \mathrm{L}$ & 20 & $80(25)$ & $35(271)$ & 0.043 & $51(60)$ & $35(236)$ & 0.120 & $59(99)$ & 28 (197) & 0.289 \\
\hline
\end{tabular}



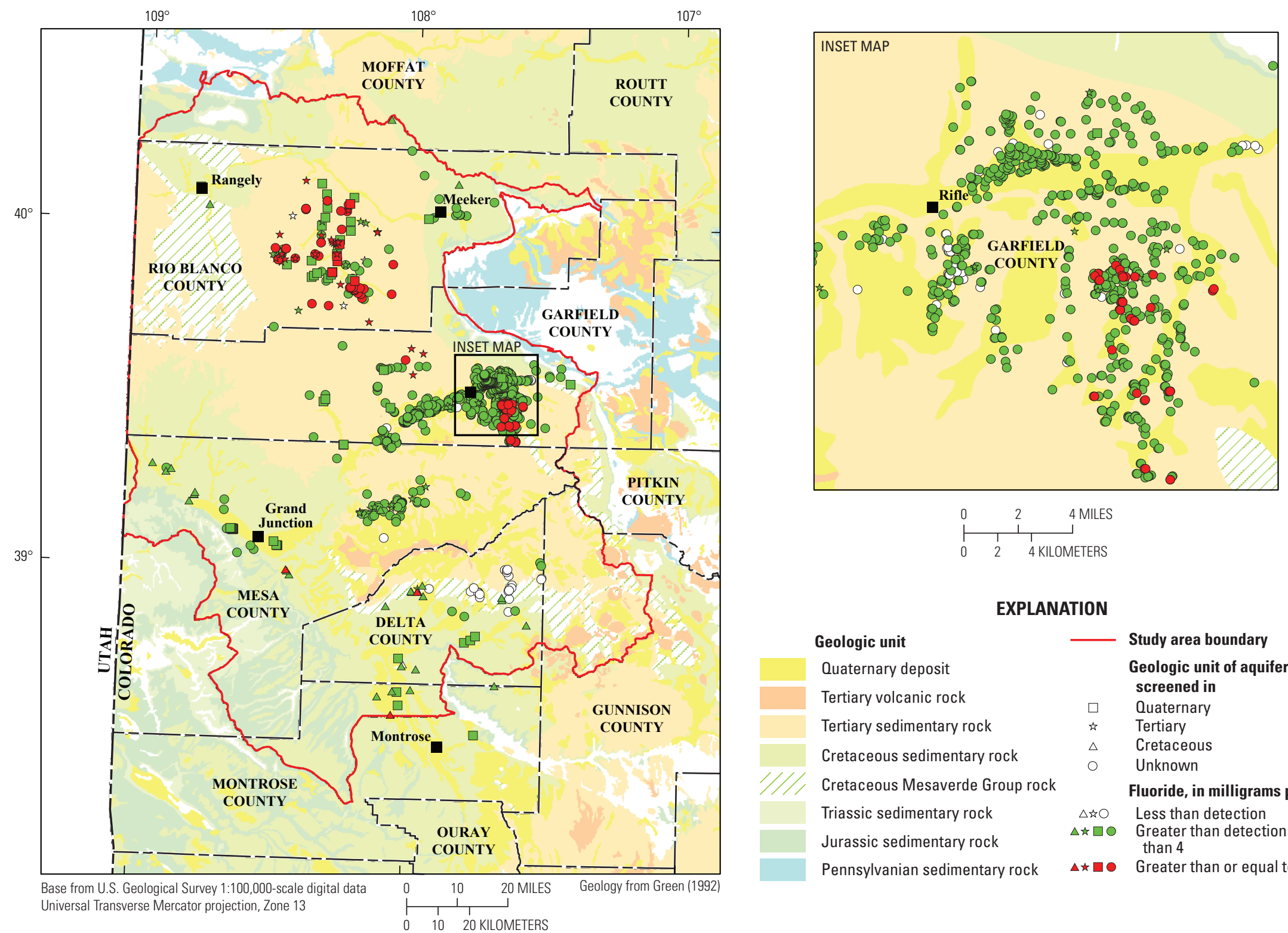

\section{EXPLANATION}

\section{Geologic unit}

Quaternary deposit

Tertiary volcanic rock

Tertiary sedimentary rock

Cretaceous sedimentary rock

Cretaceous Mesaverde Group rock

Triassic sedimentary rock

Jurassic sedimentary rock

Pennsylvanian sedimentary rock

\begin{tabular}{|c|c|}
\hline & Study area boundary \\
\hline & $\begin{array}{l}\text { Geologic unit of aquifer well is } \\
\text { screened in }\end{array}$ \\
\hline$\square$ & Quaternary \\
\hline 动 & Tertiary \\
\hline$\Delta$ & Cretaceous \\
\hline O & Unknown \\
\hline & Fluoride, in milligrams per liter \\
\hline$\Delta \vec{\varkappa} \mathrm{O}$ & Less than detection \\
\hline$\Delta \forall \square 0$ & $\begin{array}{l}\text { Greater than detection and less } \\
\text { than } 4\end{array}$ \\
\hline$\Delta \star \square 0$ & Greater than or equal to 4 \\
\hline
\end{tabular}

Figure 14. Concentrations of fluoride in groundwater from wells in the Piceance Basin, western Colorado. $[<$, less than; $\geq$, greater than or equal to] 

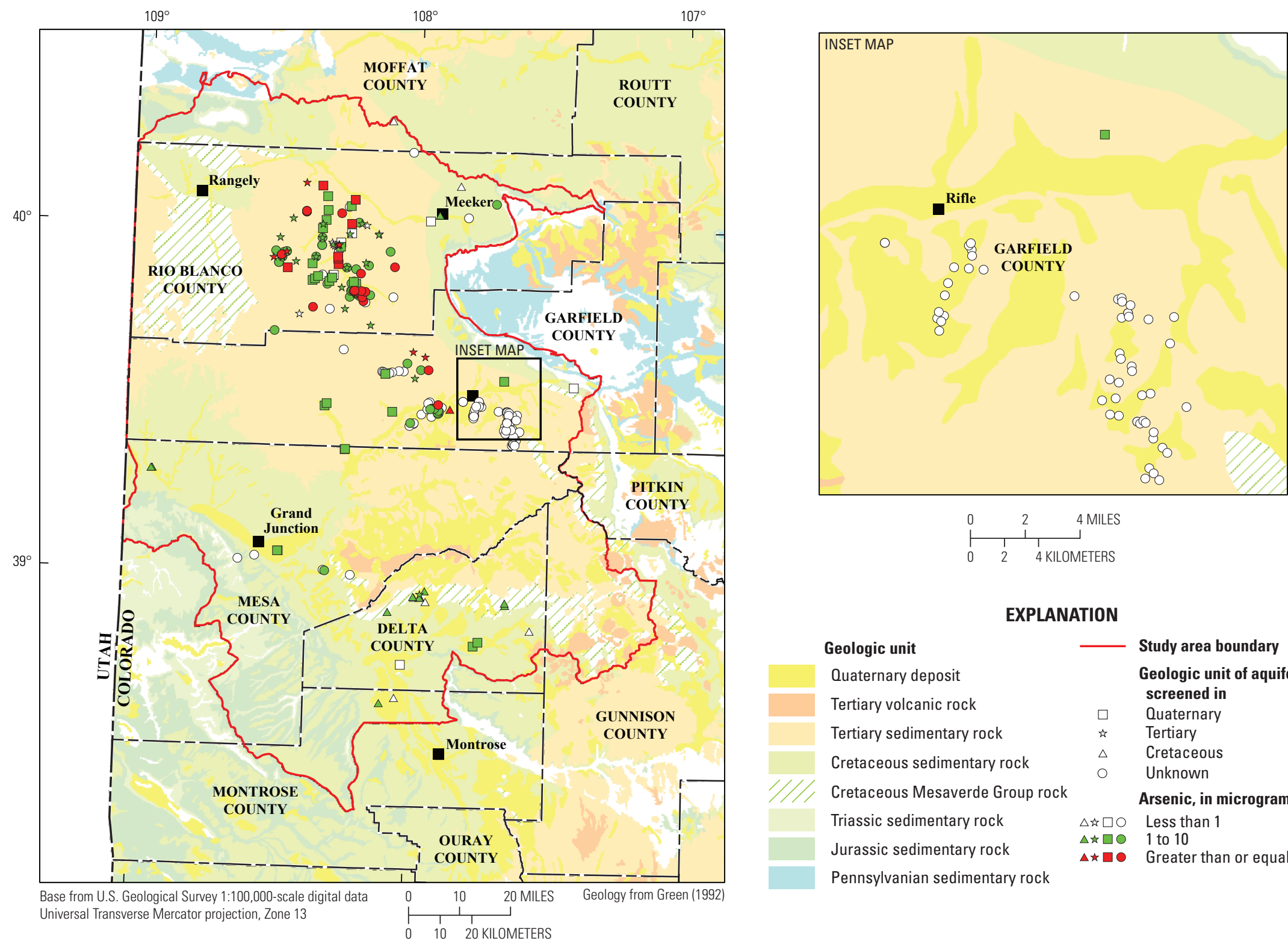

EXPLANATION

Geologic unit

Quaternary deposit

Tertiary volcanic rock

Tertiary sedimentary rock

Cretaceous sedimentary rock

Cretaceous Mesaverde Group rock

Triassic sedimentary rock

Jurassic sedimentary rock

Pennsylvanian sedimentary rock

Figure 15. Concentrations of arsenic in groundwater from wells in the Piceance Basin, western Colorado. [<, less than; $\geq$, greater than or equal to] 

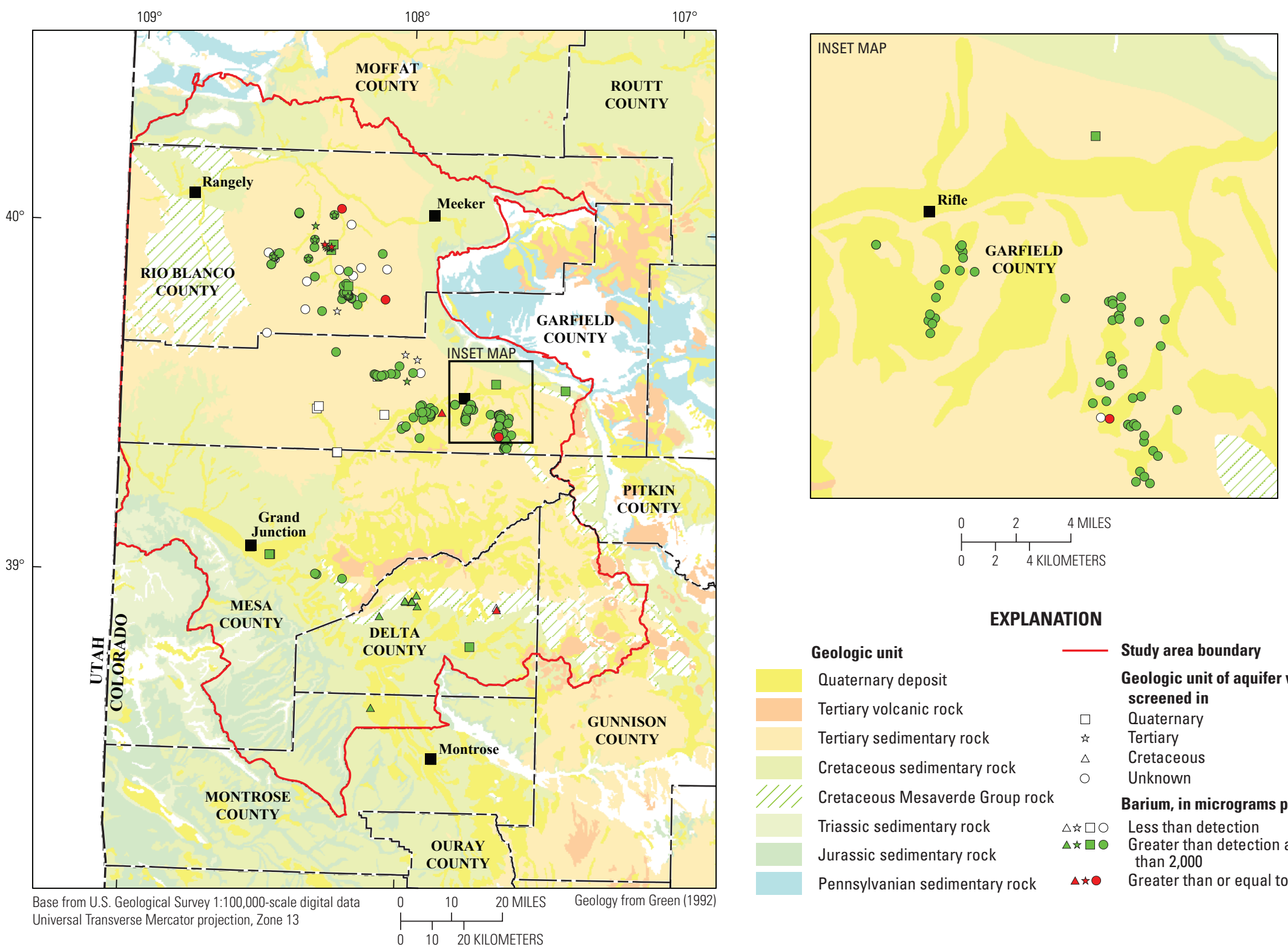

\section{EXPLANATION}

\section{Geologic unit}

Quaternary deposit

Tertiary volcanic rock

Tertiary sedimentary rock

Cretaceous sedimentary rock

Cretaceous Mesaverde Group rock

Triassic sedimentary rock

Jurassic sedimentary rock

Pennsylvanian sedimentary rock

Figure 16. Concentrations of barium in groundwater from wells in the Piceance Basin, western Colorado. $[<$, less than; $\geq$, greater than or equal to] 

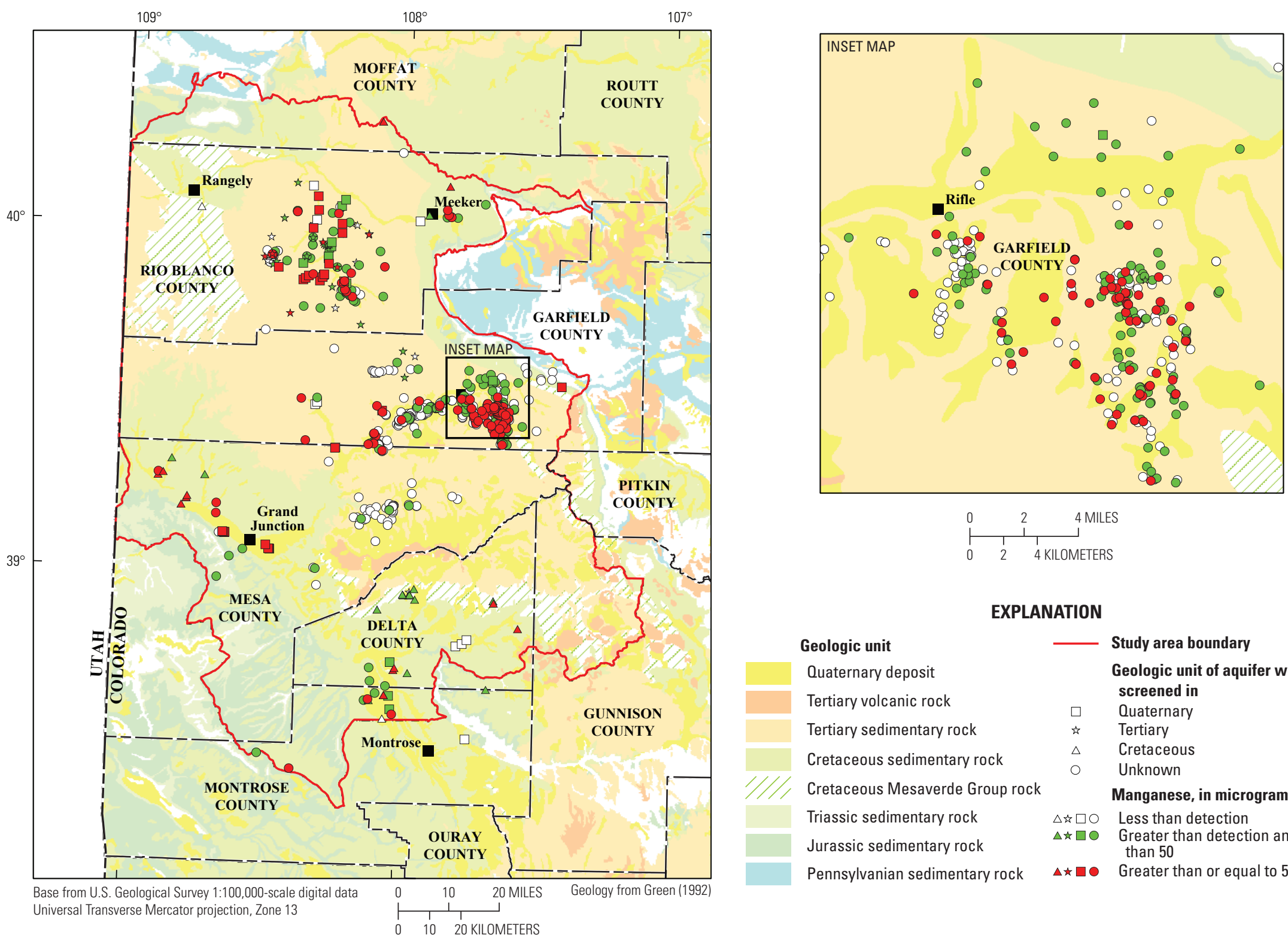

\section{EXPLANATION}

Geologic unit

Quaternary deposit

Tertiary volcanic rock

Tertiary sedimentary rock

Cretaceous sedimentary rock

Cretaceous Mesaverde Group rock

Triassic sedimentary rock

Jurassic sedimentary rock

Pennsylvanian sedimentary rock

\begin{tabular}{|c|c|}
\hline \multirow[t]{2}{*}{ 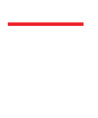 } & Study area boundary \\
\hline & $\begin{array}{l}\text { Geologic unit of aquifer well is } \\
\text { screened in }\end{array}$ \\
\hline$\square$ & Quaternary \\
\hline is & Tertiary \\
\hline$\Delta$ & Cretaceous \\
\hline \multirow[t]{2}{*}{ ○ } & Unknown \\
\hline & Manganese, in micrograms per liter \\
\hline$\Delta$ 蛞 $\square$ & Less than detection \\
\hline$\Delta \leftrightarrow \square 0$ & $\begin{array}{l}\text { Greater than detection and less } \\
\text { than } 50\end{array}$ \\
\hline$\Delta \star \square 0$ & Greater than or equal to 50 \\
\hline
\end{tabular}

Figure 17. Concentrations of manganese in groundwater from wells in the Piceance Basin, western Colorado. $[<$, less than; $\geq$, greater than or equal to] 
(21 percent) exceeded the secondary standard of $50 \mu \mathrm{g} / \mathrm{L}$ (table 7). Exceedances of the secondary standard were relatively widespread in the study area (fig. 17). Manganese concentrations ranged from below detection (most commonly $5 \mu \mathrm{g} / \mathrm{L}$ ) to $8,900 \mu \mathrm{g} / \mathrm{L}$, with a median of $6 \mu \mathrm{g} / \mathrm{L}$ (table 7 ). A sample from a domestic well in Garfield County had the highest manganese concentration (geologic unit in which well was screened was not reported) (appendix 1). Quaternary deposits in Mesa County had the highest median manganese concentration $(1,410 \mu \mathrm{g} / \mathrm{L})$ of those units with at least 10 assigned samples (table 8). Like arsenic, manganese concentrations were significantly higher $(p=0.001)$ in anoxic groundwater than in oxic groundwater (table 9), which indicates that DO measurements also could be a useful indicator of groundwater vulnerability to manganese enrichment.

Iron is the second most abundant metallic element in the earth's crust; however, concentrations in water are generally small (Hem, 1989). Iron concentrations measured between 1968 and 2009 were available for 589 wells, and 96 concentrations (16 percent) exceeded the secondary standard of $300 \mu \mathrm{g} / \mathrm{L}$ (table 7). Most of the concentrations that exceeded the standard were from Garfield and Rio Blanco Counties (fig. 18). Iron concentrations ranged from less than detection (most commonly $70 \mu \mathrm{g} / \mathrm{L}$ ) to $110,000 \mu \mathrm{g} / \mathrm{L}$, with a median of $40 \mu \mathrm{g} / \mathrm{L}$ (table 7). A sample from a domestic well in Garfield County had the highest iron concentration (geologic unit in which well was screened was not reported) (appendix 1). The Mesaverde Group in Delta County had the highest median iron concentration $(150 \mu \mathrm{g} / \mathrm{L})$ of those units with at least 10 assigned samples (table 8). Unlike arsenic and manganese, iron concentrations were not significantly different between oxic and anoxic water samples, nor were they different at DO-concentration thresholds of 1 and $2 \mathrm{mg} / \mathrm{L}$ (table 9). Iron concentrations typically would be expected to be higher in anoxic groundwater than oxic groundwater based on its redox chemistry (McMahon and Chapelle, 2008). The reason for this apparent discordant finding is unclear, but it could be related to sample collection and preservation techniques used. If the sample had been preserved with acid in the field at the time it was collected but filtered later in the laboratory, it is possible that the acid could have dissolved particulate iron in the sample. Ideally, trace element samples would be first filtered and then acidified in the field immediately after sample collection. For example, in a set of 6,906 groundwater samples collected from around the country by the U.S. Geological Survey National Water-Quality Assessment Program, median iron concentrations were significantly $(p<0.001)$ higher in anoxic groundwater than in oxic groundwater $(220 \mu \mathrm{g} / \mathrm{L}$ and less than $10 \mu \mathrm{g} / \mathrm{L}$, respectively) (P.B. McMahon, U.S. Geological Survey, written commun., 2011). Those samples were filtered and acidified in the field immediately after collection.

Selenium is a trace element that occurs naturally in the environment. The processes that control selenium mobilization are strongly controlled by the geochemical environment. Selenium concentrations measured between 1972 and 2008 were available for 551 wells, and 51 concentrations ( 9 percent) exceeded the primary standard of $50 \mu \mathrm{g} / \mathrm{L}$ (table 7). Most of the concentrations that exceeded the selenium standard were from Garfield County (fig. 19). Selenium concentrations ranged from less than detection (most commonly $20 \mu \mathrm{g} / \mathrm{L}$ ) to $1,640 \mu \mathrm{g} / \mathrm{L}$, with a median of $5 \mu \mathrm{g} / \mathrm{L}$ (table 7). A sample from a domestic well in Garfield County had the highest selenium concentration (geologic unit in which the well was screened was not reported) (appendix 1). The Mesaverde Group in Delta County had the highest median selenium concentration $(18 \mu \mathrm{g} / \mathrm{L})$ of those units with at least 10 assigned samples (table 8).

Natural sources of selenium in the study area include authigenic pyrite in Mancos Shale, particularly in Mesa and Delta Counties, and other selenium-bearing solid phases that have been deposited in the aquifer or redistributed by geochemical processes within the groundwater environment (Wright and Butler, 1993). Percolation of irrigation water through selenium-bearing geologic units such as the Mancos Shale has the potential to oxidize and mobilize selenium. This study found that selenium concentrations were significantly higher $(p<0.001)$ in samples containing nitrate at a concentration of at least $1 \mathrm{mg} / \mathrm{L}$ (as nitrogen) than in samples with lower nitrate concentrations. Mean selenium concentrations in the high- and low-nitrate samples were 85 and $7 \mu \mathrm{g} / \mathrm{L}$, respectively (most common censoring level for nitrate and selenium of $0.29 \mathrm{mg} / \mathrm{L}$ and $20 \mu \mathrm{g} / \mathrm{L}$, respectively). The nitrate concentration of $1 \mathrm{mg} / \mathrm{L}$ (as nitrogen) is a proposed national baseline concentration in groundwater (Dubrovsky and others, 2010), and nitrate concentrations above that level could indicate inputs from human sources such as irrigated agriculture or septic leachate. Stratified by DO concentrations, selenium concentrations were significantly higher $(p=0.043)$ in anoxic groundwater than in oxic groundwater (table 9). This result is opposite of the result expected based on the redox chemistry of selenium and may reflect the diversity of geologic units from which samples were collected, however, further sampling would need to be conducted. Analyses of DO-selenium relations in more uniform geologic settings such as the Central Valley of California have shown that selenium concentrations typically are highest in oxic groundwater (Dubrovsky and others, 1993).

\section{Nitrate}

Excessive nitrate in drinking water supplies is a human health concern especially for small children because it may cause methemoglobinemia ("blue baby" syndrome). Elevated sources of nitrate in groundwater are likely to be associated with septic systems, animal manure, or fertilizers applied to lawns and crops (Hem, 1989). Concentrations of nitrite in most of the samples were less than the reporting level; therefore, concentrations of nitrite plus nitrate consisted almost entirely of nitrate and are referred to as such in this report (appendix 1). Nitrate concentrations measured between 1958 

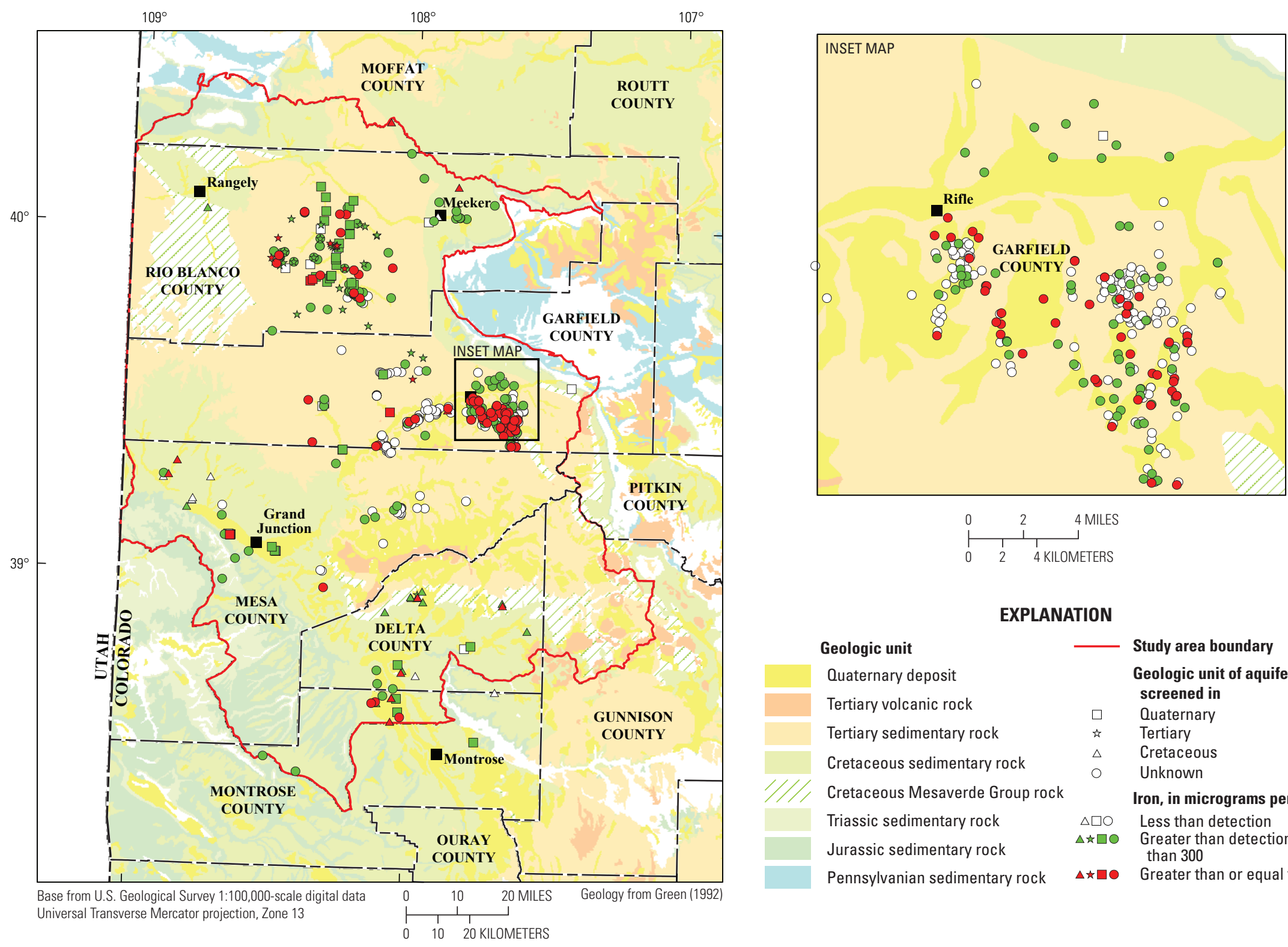

\section{EXPLANATION}

\section{Geologic unit}

Quaternary deposit

Tertiary volcanic rock

Tertiary sedimentary rock

Cretaceous sedimentary rock

Cretaceous Mesaverde Group rock

Triassic sedimentary rock

Jurassic sedimentary rock

Pennsylvanian sedimentary rock

\begin{tabular}{|c|c|}
\hline & Study area boundary \\
\hline & $\begin{array}{l}\text { Geologic unit of aquifer well is } \\
\text { screened in }\end{array}$ \\
\hline$\square$ & Quaternary \\
\hline मे & Tertiary \\
\hline$\Delta$ & Cretaceous \\
\hline ○ & Unknown \\
\hline & Iron, in micrograms per liter \\
\hline $\begin{array}{l}\Delta \square O \\
\Delta \text { 幺 } \square 0\end{array}$ & $\begin{array}{l}\text { Less than detection } \\
\text { Greater than detection and less } \\
\text { than } 300\end{array}$ \\
\hline & Greater than or equal to 300 \\
\hline
\end{tabular}

Figure 18. Concentrations of iron in groundwater from wells in the Piceance Basin, western Colorado. $[<$, less than; $\geq$, greater than or equal to] 

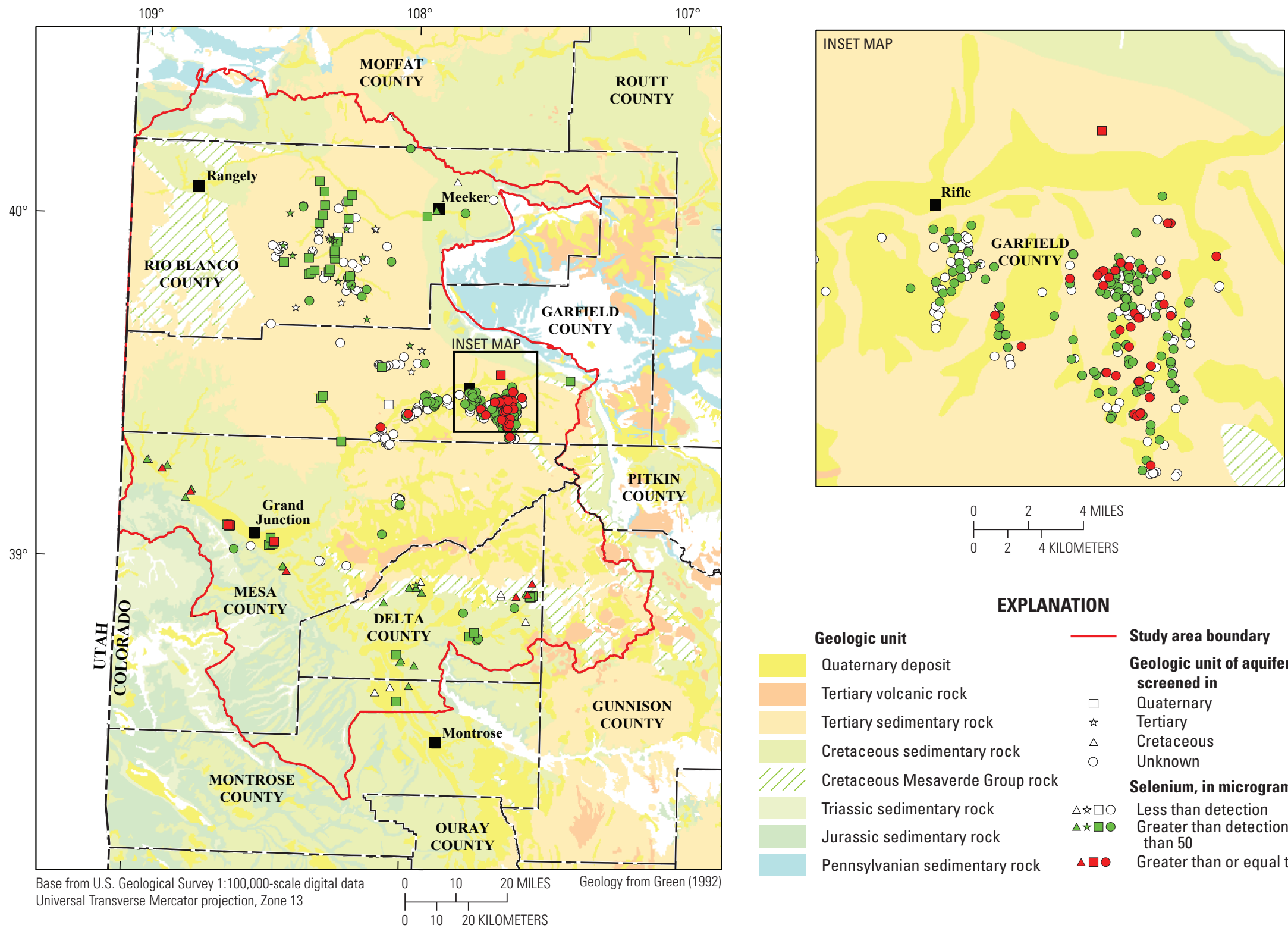

\section{EXPLANATION}

\section{Geologic unit}

Quaternary deposit

Tertiary volcanic rock

Tertiary sedimentary rock

Cretaceous sedimentary rock

Cretaceous Mesaverde Group rock

Triassic sedimentary rock

Jurassic sedimentary rock

Pennsylvanian sedimentary rock
Study area boundary

Geologic unit of aquifer well is

screened in

Quaternary

Tertiary

Cretaceous

Unknown

Selenium, in micrograms per liter

$\triangle \mathrm{r} \square \mathrm{O}$ Less than detection

$\Delta \backsim \square$ Greater than detection and less than 50

$\Delta \square$ - Greater than or equal to 50

Figure 19. Concentrations of selenium in groundwater from wells in the Piceance Basin, western Colorado. [<, less than; $\geq$, greater than or equal to $]$ 

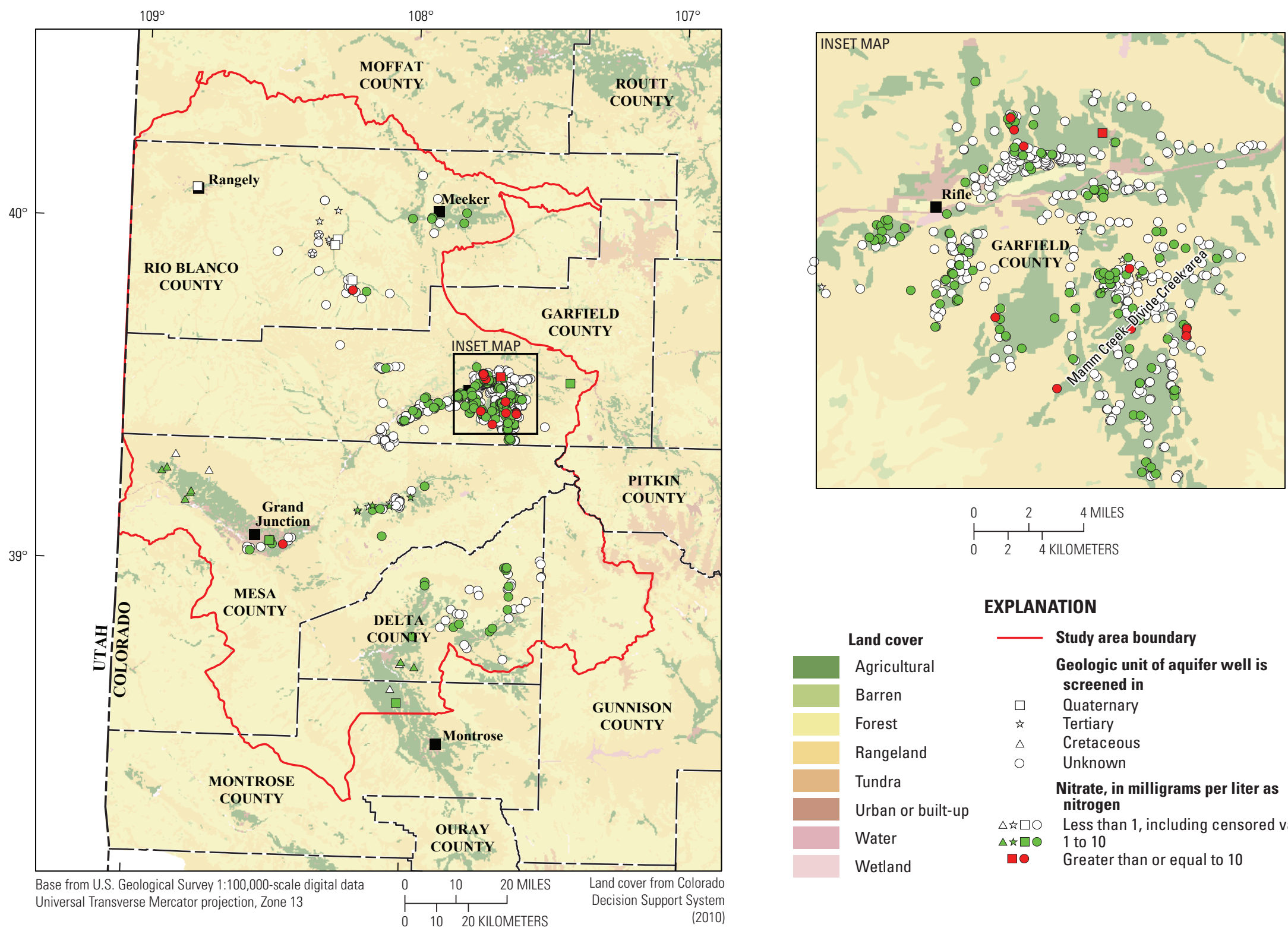

\section{EXPLANATION}

\section{Land cover \\ Study area boundary}

Agricultural

Barren

Forest

Rangeland

Tundra

Urban or built-up

Water

Wetland
Geologic unit of aquifer well is

screened in

Quaternary

Tertiary

Cretaceou

Unknown

Nitrate, in milligrams per liter as

nitrogen

$\triangle i \square O$ Less than 1 , including censored values

- Greater than or equal to 10

Figure 20. Concentrations of nitrate in groundwater from wells in the Piceance Basin, western Colorado. [<, less than; $\geq$, greater than or equal to] 
and 2009 were available for 791 wells, and 13 concentrations (1.6 percent) exceeded the primary standard of $10 \mathrm{mg} / \mathrm{L}$ as nitrogen (table 7). Most of these exceedances were in samples collected from agricultural areas of Garfield County (fig. 20). Nitrate concentrations ranged from less than detection (most commonly about $0.29 \mathrm{mg} / \mathrm{L}$ as nitrogen) to $29.4 \mathrm{mg} / \mathrm{L}$, with a median concentration less than $0.21 \mathrm{mg} / \mathrm{L}$ as nitrogen (table 7). A domestic well in Garfield County had the highest nitrate concentration (geologic unit in which well was screened was not reported) (appendix 1). The Wasatch Formation in Garfield County was the only geologic unit to have at least 10 assigned samples (table 8), and the median nitrate concentration for those samples was $0.4 \mathrm{mg} / \mathrm{L}$ as nitrogen.

McMahon and others (2010) sampled 26 domestic wells completed in the Wasatch Formation in Garfield County and found that nitrate concentrations in oxic groundwater were significantly higher $(\mathrm{p}=0.03)$ than concentrations in anoxic groundwater. This same relation was observed in a national study of nitrate in groundwater (McMahon and Chapelle, 2008). This relation between DO and nitrate was not observed in this study, although nitrate concentrations were significantly higher $(\mathrm{p}=0.015)$ in groundwater containing at least $2 \mathrm{mg} / \mathrm{L}$ DO than in less oxygenated groundwater (table 9). The apparent similarity in nitrate concentrations in oxic and anoxic groundwater may reflect the generally low concentrations of nitrate in the dataset (median nitrate concentration less than $0.21 \mathrm{mg} / \mathrm{L}$ as nitrogen); however, further data collection is needed to verify this hypothesis. Although this DO-nitrate relation differs somewhat from the relation described in previous studies, it still indicates that DO measurements could be a useful indicator of groundwater vulnerability to nitrate contamination.

\section{Benzene, Toluene, Ethylbenzene, Xylene}

Benzene, toluene, ethylbenzene, and xylene (BTEX) are volatile organic compounds typically found in petroleum products such as gasoline and diesel fuel, but these compounds also occur in some natural gas reservoirs. Benzene concentrations measured between 1989 and 2008 were available for 808 wells, but benzene was detected in only 11 wells ( 1.4 percent); 5 concentrations ( 0.6 percent) exceeded the primary standard of $5 \mu \mathrm{g} / \mathrm{L}$ (table7). These exceedances were in samples from wells located in Garfield (3 samples) and Rio Blanco (2 samples) Counties (geologic unit in which the wells were screened not reported) (fig. 21, appendix 1). Toluene concentrations measured between 1997 and 2008 were available for 808 wells. Toluene was detected in 38 wells (4.7 percent), but none of the concentrations exceeded the primary drinking-water standard $(1,000 \mu \mathrm{g} / \mathrm{L})$ (table 7 , fig. 22). Ethylbenzene and xylene were sampled at a frequency similar to that of benzene and toluene, but detections were few and without exceedances of primary standards (table 7).

\section{Methane}

Methane is a combustible gas that can accumulate to explosive levels in well bores and confined spaces in buildings. Methane can be either thermogenic or biogenic in origin. Thermogenic methane is generally produced at depths far below freshwater aquifers, and its presence in water wells can be due to natural conditions, such as migration of gas along naturally occurring fracture zones from underlying gas-bearing reservoirs such as the Mesaverde Group or the Wasatch Formation, or from oil and gas drilling, completion, and production activities. Biogenic methane, which is produced through microbial degradation of organic matter (Whiticar, 1999), generally originates from much shallower depths than thermogenic methane and can occur naturally in shallow anoxic groundwater systems. Methane concentrations measured between 1997 and 2009 were available for 874 wells, in which methane was detected in 207 wells (24 percent) (table 7). Most samples that contained detectable methane concentrations were from Garfield County (fig. 23). Methane concentrations in the study area ranged from less than the detection limit (commonly $0.0008 \mathrm{mg} / \mathrm{L}$ ) to $36.7 \mathrm{mg} / \mathrm{L}$, and 75 values ( 8.5 percent) were greater than $1 \mathrm{mg} / \mathrm{L}$ (high methane). Most methane detections and methane concentrations greater than $1 \mathrm{mg} / \mathrm{L}$ were found in Garfield County in the Mamm Creek-Divide Creek area (fig. 23). A sample from a domestic well in Garfield County had the highest methane concentration (geologic unit in which well was screened was not reported) (appendix 1). The Wasatch Formation in Garfield County was the only one to have at least 10 assigned samples (table 8), and the median methane concentration for those samples was less than $0.0005 \mathrm{mg} / \mathrm{L}$. Methane concentrations in anoxic groundwater were significantly higher $(p=0.004)$ than the concentrations in oxic groundwater, which is consistent with previous results from the study area (McMahon and others, 2010). These findings indicate that dissolved-oxygen measurements could be a useful indicator of groundwater vulnerability to methane enrichment.

Several studies have used methane isotopic compositions (ratio of $13 \mathrm{C}$ to $12 \mathrm{C}$ relative to a standard material) to determine the origin (biogenic or thermogenic) of methane in groundwater (Whiticar, 1999; URS Corporation, 2006; S.S. Papadopoulos \& Associates, Inc., 2008; McMahon and others, 2010). Only about 30 percent of the samples with high methane concentrations (greater than or equal to $1 \mathrm{mg} / \mathrm{L}$ ) had isotopic data for methane (appendix 1). Overall, 37 samples had isotopic data for methane, and all but one sample came from domestic or observation wells located in Garfield County (appendix 1). The isotopic data indicate that methane in the Garfield County water wells was from both biogenic and thermogenic sources (fig. 24) (appendix 1).

For comparison, natural gas from the Mesaverde Group in Garfield County was exclusively thermogenic in origin (fig. 24). Thermogenic methane in domestic wells is likely to have come from much deeper sources than the freshwater aquifer in which the water wells were screened, although the 

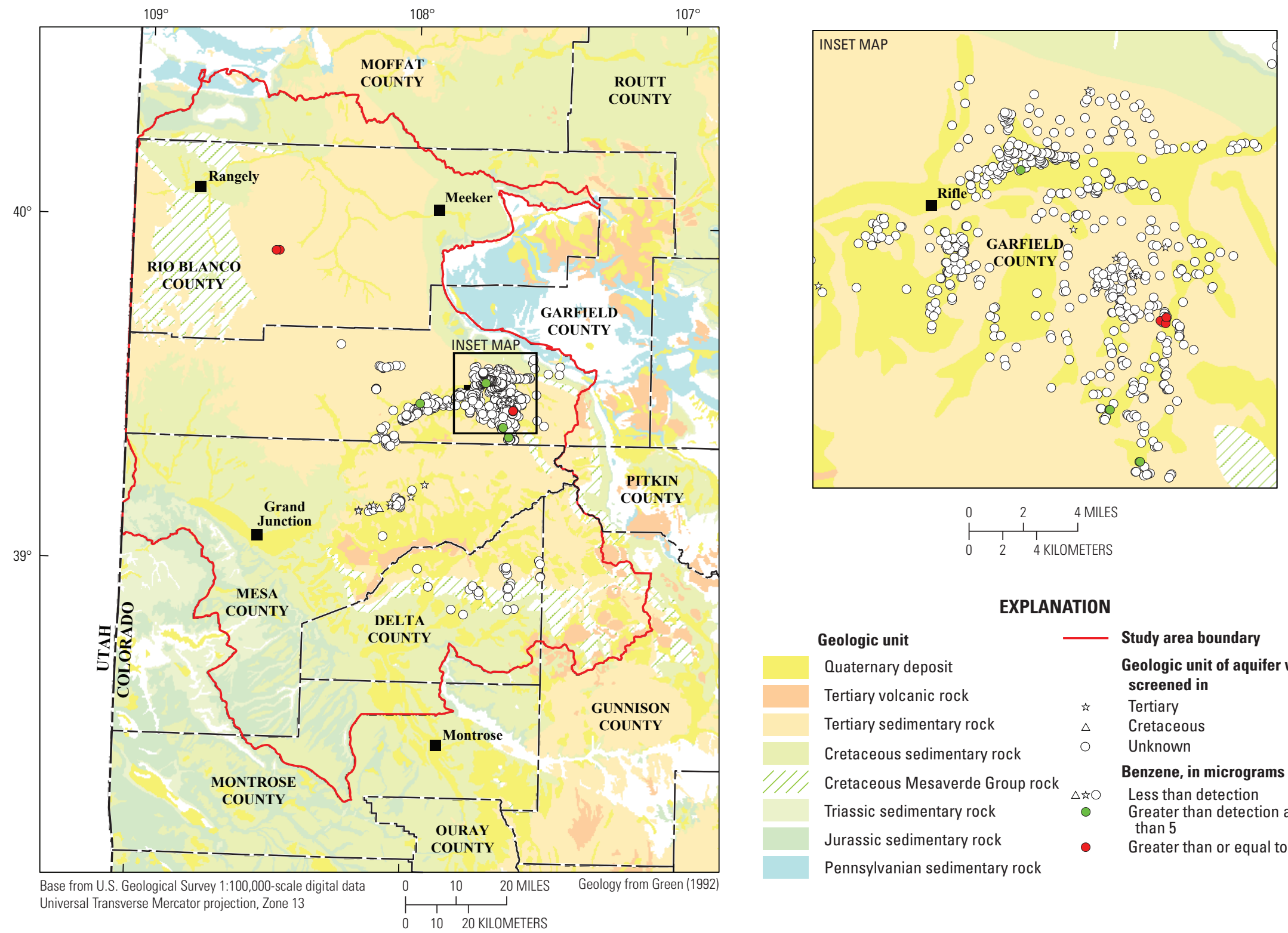

\section{EXPLANATION}

Geologic unit

Quaternary deposit

Tertiary volcanic rock

Tertiary sedimentary rock

Cretaceous sedimentary rock

Cretaceous Mesaverde Group rock

Triassic sedimentary rock

Jurassic sedimentary rock

Pennsylvanian sedimentary rock

Figure 21. Concentrations of benzene in groundwater from wells in the Piceance Basin, western Colorado. $[<$, less than; $\geq$, greater than or equal to $]$ 

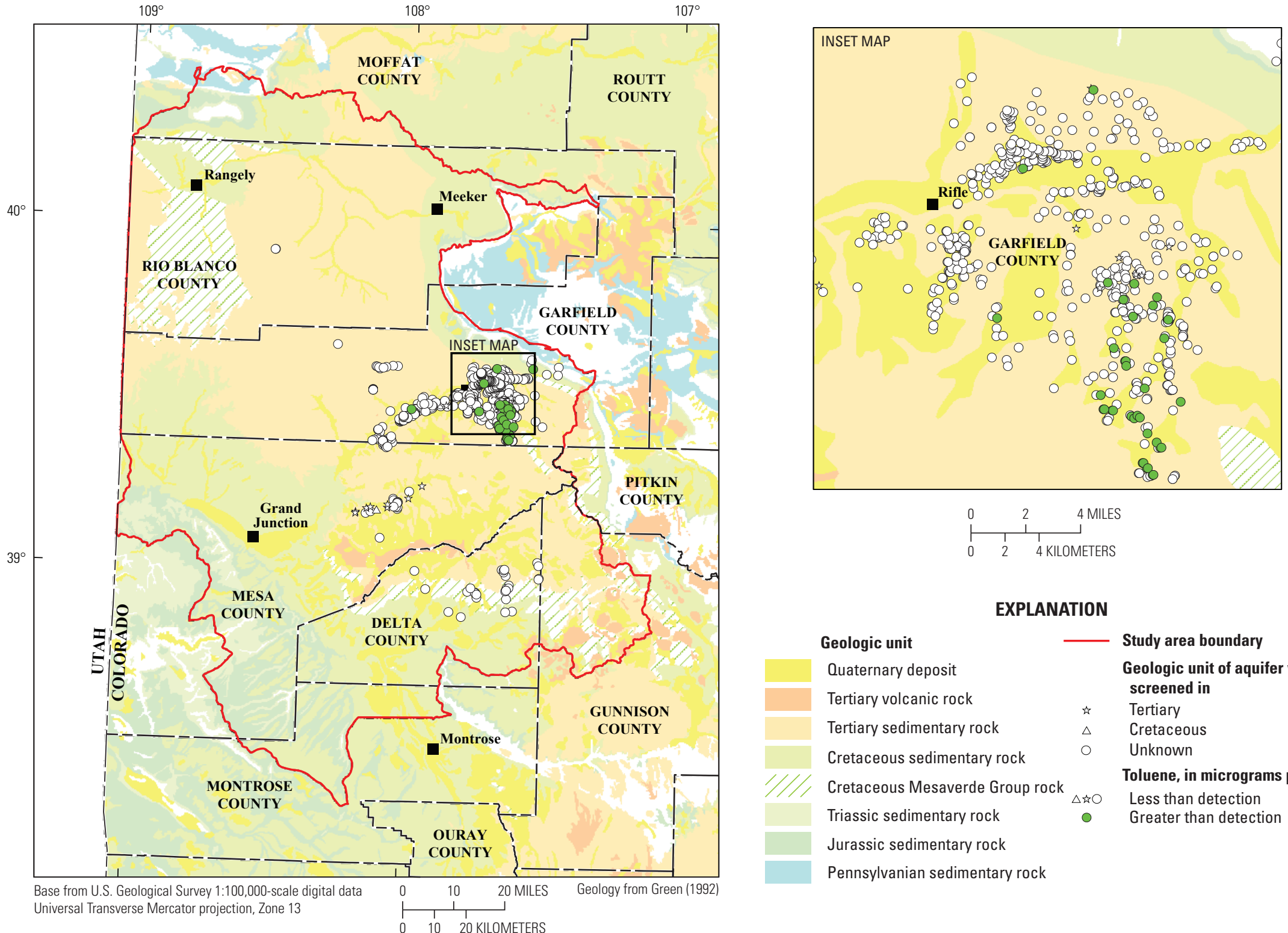

\section{EXPLANATION}

Geologic unit

Quaternary deposit

Tertiary volcanic rock

Tertiary sedimentary rock

Cretaceous sedimentary rock

Cretaceous Mesaverde Group rock

Triassic sedimentary rock

Jurassic sedimentary rock

Pennsylvanian sedimentary rock

Figure 22. Detections of toluene in groundwater from wells in the Piceance Basin, western Colorado. 

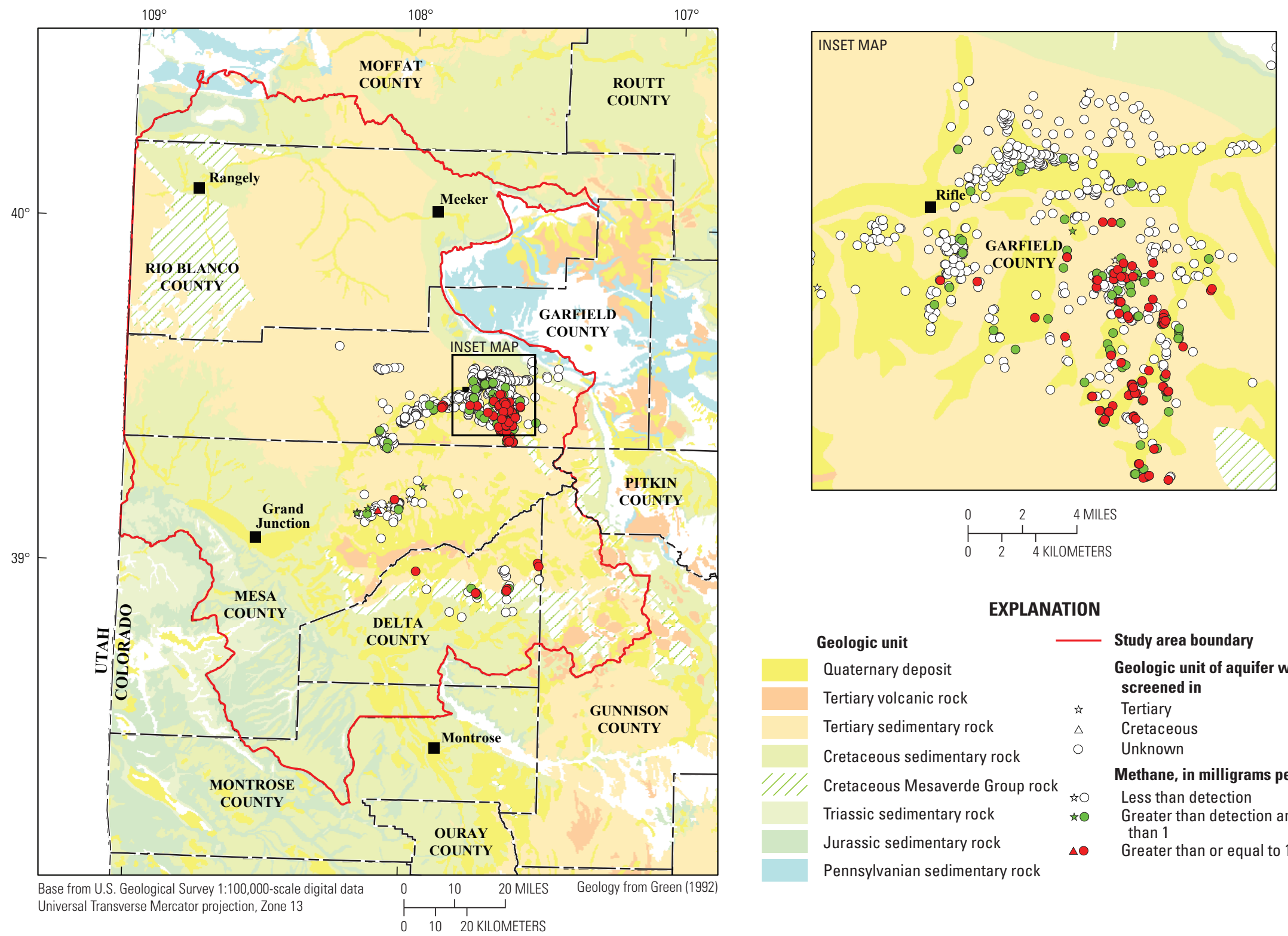

\section{EXPLANATION}

\section{Geologic unit}

Quaternary deposit

Tertiary volcanic rock

Tertiary sedimentary rock

Cretaceous sedimentary rock

Cretaceous Mesaverde Group roc

Triassic sedimentary rock

Jurassic sedimentary rock

Pennsylvanian sedimentary rock

Figure 23. Concentrations of methane in groundwater from wells in the Piceance Basin, western Colorado. $[<$, less than; $\geq$, greater than or equal to] 


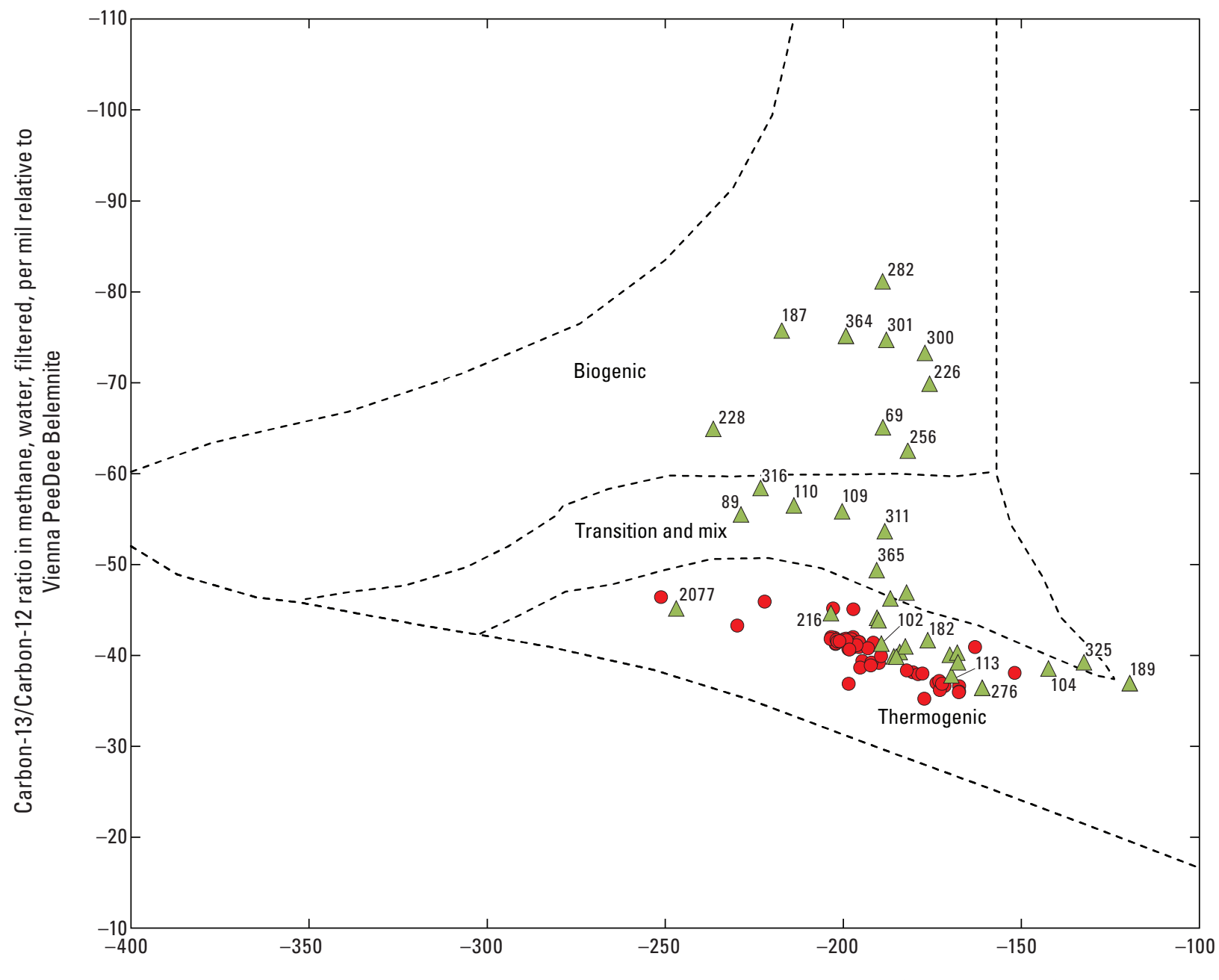

Deuterium/protium ratio in methane, water, filtered, per mil relative to Vienna Standard Mean Ocean Water

\section{EXPLANATION}

$\triangle$ Water-quality data from repository-Methane concentration greater than or equal to 1 milligram per liter

- Produced water-Mesaverde group (S.S. Papadopulos and Associates, Inc., 2008)

164 Report number

Figure 24. Stable hydrogen and carbon isotope compositions of methane in water wells in the Piceance Basin, for water samples with at least $1 \mathrm{mg} / \mathrm{L}$ of methane. The isotopic composition of methane in natural gas from the Mesaverde Group in Garfield Country (Colorado Oil and Gas Conservation Commission, 2010) is shown for comparison (data from submission to common data repository by Colorado Oil and Gas Conservation Commission). The fields for bacterial and thermogenic methane are modified from Whiticar and others, 1986. 
geologic unit in which they were screened was not provided by the data source (appendix 1). In contrast, biogenic methane in the domestic wells could have been produced locally in an aquifer with anoxic groundwater, or it could have come from deeper geologic units underlying the aquifer (but not as deep as the zones of thermogenic methane). In a domestic well completed in the shallow Wasatch Formation in Garfield County, helium-4 concentration data have been used to show that biogenic methane was most likely produced in a deeper part of the Wasatch Formation and subsequently transported to shallower depths (McMahon and others, 2010). The methane transport mechanism was not identified, but possible mechanisms included transport along natural fractures or movement up the uncemented annular space of gas wells.

\section{Limitations of the Common Data Repository for Assessing Groundwater Quality and Opportunities for Future Monitoring}

More than 480 groundwater-quality constituents exist in the common data repository, but only a subset of these has been discussed in this report. Assessing such a large and diverse dataset as the one available through the repository poses unique challenges for assessing groundwater quality in the study area. The repository contains data from several studies that differed widely in purpose and scope. Garfield County is the site of several groundwater studies, which have produced a wealth of information. However, much of the data collection was focused on the effects of energy development on water quality in the Mamm Creek-Divide Creek area. The majority of groundwater-quality data collected in Rio Blanco County is from the 1970s and 1980s and focused on understanding water bearing units of the Green River Formation in relation to oil shale development. Groundwater-quality data in Mesa and Delta Counties are from several small studies and provides a limited understanding of groundwater resources for those counties.

Data gaps exist in the repository. Groundwater-quality data in the repository were not evenly distributed throughout the study area. For example, the repository contained very little data for some parts of the study area that have many oil or gas wells, such as western Garfield, Mesa, and Rio Blanco Counties (fig. 25). Moffat and Montrose Counties contained relatively small amounts of data compared with Garfield and Rio Blanco Counties. Furthermore, the repository did not contain data for many of the water-supply wells (domestic, municipal) in the study area (fig. 26). Delta County, for example, is somewhat underrepresented with respect to groundwater-quality data even though there were many domestic wells in the county (fig. 26).

Several key water-quality constituents or indicators were underrepresented in the repository. Dissolved-oxygen data, for example, were available for only 47 percent of samples even though DO measurements were shown to be useful for assessing the vulnerability of the aquifer to enrichment by or contamination from constituents such as arsenic, manganese, methane, and nitrate. Arsenic and nitrate, in particular, are of concern from a human health standpoint, but they have been measured in fewer than 17 and 51 percent of the samples, respectively. Stable isotopic values of water and methane were available for fewer than 5 percent of the samples even though those data were shown to be useful tracers of water and methane sources, respectively. Other useful tracers that are either underrepresented or absent from the repository include age tracers such as tritium and carbon-14 and gas tracers such as helium-4, the combination of which can be used to examine sources of water and chemicals in aquifers.

Ancillary information, such as well depth, depth to water, and the geologic unit or aquifer in which a well was completed, was missing for more than 50 percent of the samples. This information is crucial for placing the well and its water chemistry in hydrologic context. The majority of wells with completion information were in Quaternary deposits. Quaternary deposits were important sources of groundwater in the study area where population centers were located in river valleys. But many rural household wells are also completed in bedrock aquifers below the Quaternary deposits, and the distribution of wells in the repository in each of those units is not well understood.

One additional aspect of the repository worth noting is the general lack of chemical and isotopic data for produced waters and for other groundwater at depth below the freshwater aquifers. Extensive faulting and fracturing in the study area, as well as drilling for natural gas, provides potential conduits for deep groundwater to move upward into freshwater aquifers. Without adequate chemical and isotopic characterization of that deep water, it can be difficult to recognize its presence in shallow aquifers or, if it is identified, to know from which deep geologic unit it originated.

Future monitoring could avoid several limitations of the current repository by making relatively minor changes to sample-collection and data-reporting protocols.

- Add field measurements for dissolved oxygen to sampling protocols

- Filter cation and trace element samples in the field prior to acidification

- Measure the water level in a well prior to sampling

- Report information on well construction and the geologic unit or aquifer in which a well is completed as part of the water-quality database

Implementation of these types of changes would increase the comparability of data from different monitoring programs and also add value to each program individually and to that of the regional dataset as a whole.

Other changes to monitoring programs could require greater resources. When possible, such changes might include sampling for a basic set of constituents that is relevant to 


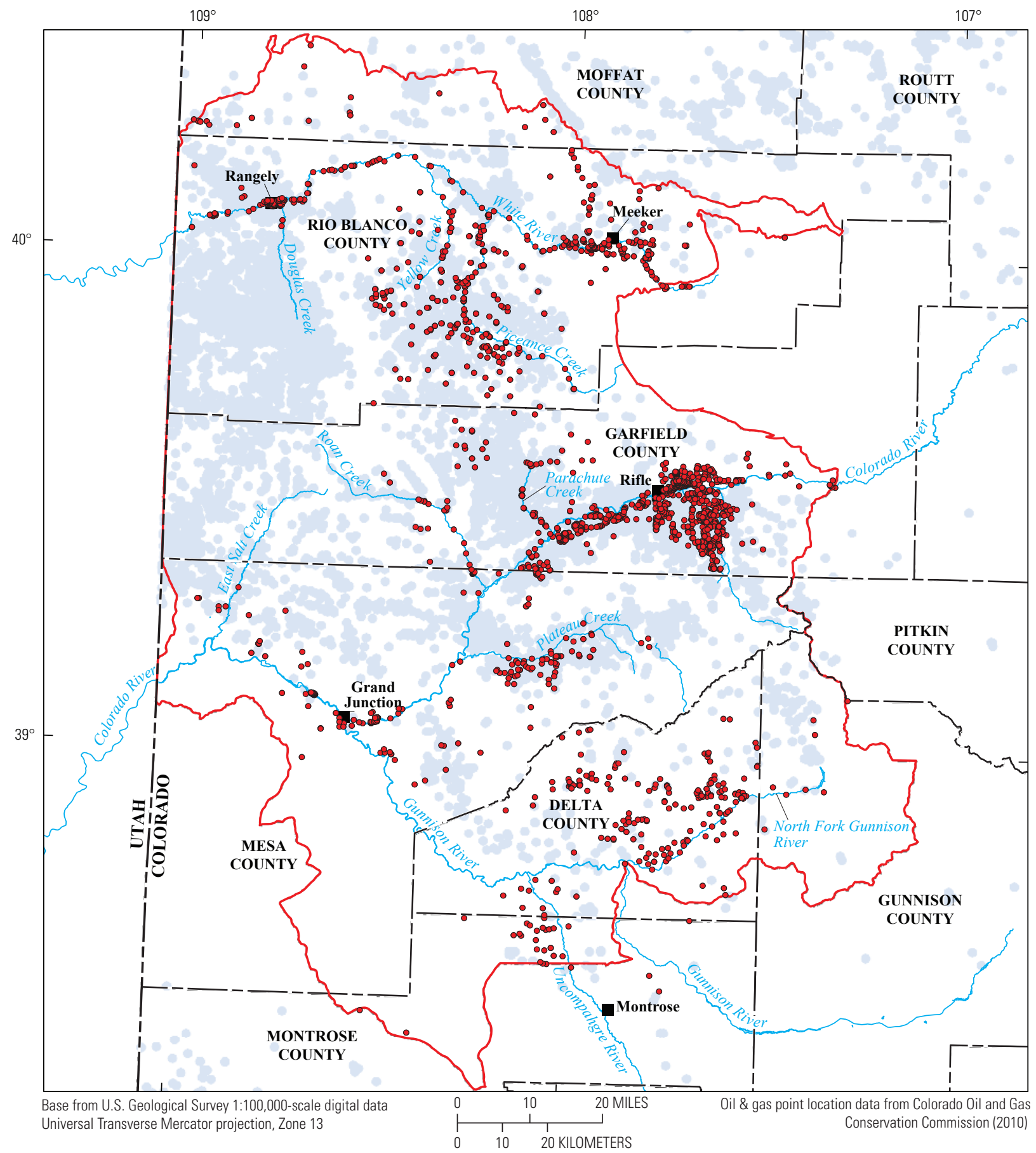

EXPLANATION

Study area boundary

- Repository groundwater-quality site

Colorado Oil and Gas Conservation

Commission oil and gas well

Figure 25. Groundwater-quality sites in the Piceance Basin common data repository and oil and gas wells permitted by Colorado Oil and Gas Conservation Commission permit layer. 


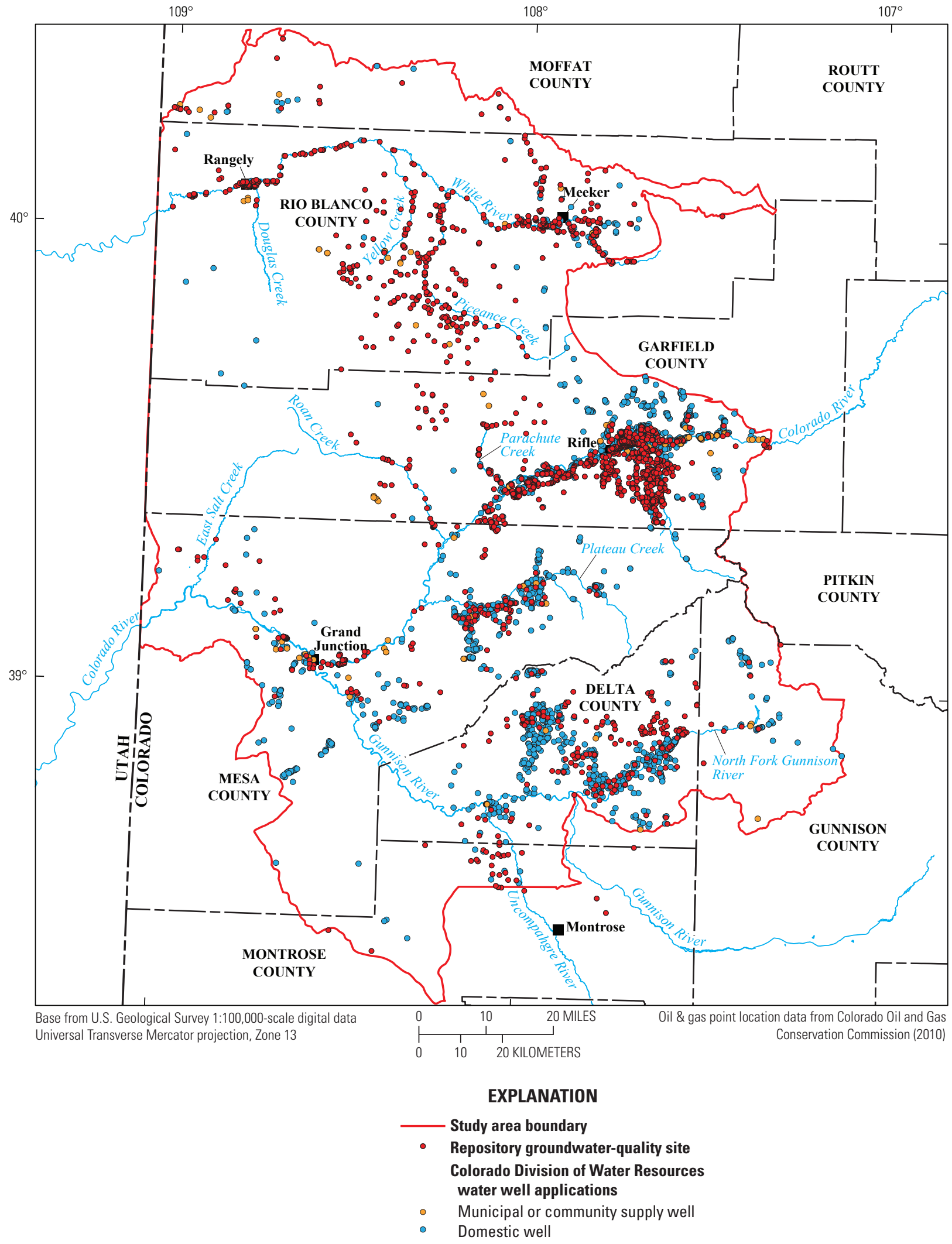

Figure 26. Groundwater-quality sites in the Piceance Basin common data repository and domestic and municipal wells permitted by Colorado Division of Water Resources (2011). 
major water-quality issues in the regional study area. Although some components of the constituent set might not be relevant to the immediate goals of individual monitoring programs, creation of such a dataset for the regional study area would help to provide the kinds of information needed to characterize background conditions and the spatial and temporal variability in constituent concentrations associated with those conditions. Without that information, it becomes difficult to recognize departures from background that might be associated with human activities. That kind of information is likely to be useful to many monitoring programs. In addition to the constituents and ancillary information described above (for example, DO and well completion interval), such a basic set of constituents might include nitrate, arsenic, chloride, fluoride, manganese, selenium, sulfate, methane, and possibly the BTEX compounds and stable isotopic compositions of water and methane.

\section{Summary}

Groundwater-quality data from public and industry sources for the period 1946 to 2009 were compiled and loaded into a common data repository for the Piceance Basin. This report describes the availability of groundwater-quality data in the Piceance Basin in terms of three characteristics: spatial (where sample sites were located), temporal (what is the period of record), and analytical (what constituents were sampled). Spatial variability in constituent concentrations are described and, where possible, examined relative to human and natural factors. The repository is available on the web at http://rmgsc.cr.usgs.gov/cwqdr/Piceance/index.shtml. A subset of groundwater-quality data from this repository was compiled, reviewed, and quality assured for this report. The resulting dataset consists of the most recently collected samples from 1,545 wells, 1,007 (65 percent) of which were domestic wells. Concentration data were compared with primary and secondary drinking-water standards established by the U.S. Environmental Protection Agency. The three principal geologic units discussed in this report-Quaternary deposits, Tertiary sedimentary rocks, and Cretaceous sedimentary rocks - were the primary host materials for aquifers in the Piceance Basin in which water-quality data were collected. From those samples, the following constituents were discussed in this report: dissolved oxygen, dissolved solids, $\mathrm{pH}$, major ions (chloride, sulfate, fluoride), trace elements (arsenic, barium, iron, manganese, selenium), nitrate, BTEX compounds (benzene, toluene, ethylbenzene, xylene), methane, and the stable isotopic compositions of water and methane. Dissolved-oxygen concentration and methane concentrations were also used to stratify concentration data per constituent.

The stable isotopic composition of water was used to identify potential sources of recharge to wells. Recharge to most of the wells for which data were available was derived from precipitation, as indicated by $\delta^{2} \mathrm{H}\left[\mathrm{H}_{2} \mathrm{O}\right]$ and $\delta^{18} \mathrm{O}\left[\mathrm{H}_{2} \mathrm{O}\right]$ values that plot along the Global Meteoric Water Line and near the values of snow samples collected in the study area. Recharge to two wells appeared to be derived from a deeper groundwater source, as indicated by isotopic values that plot near the values for produced water from the Mesaverde Group.

Reduction/oxidation (redox) processes affect the chemical quality of groundwater in the study area by affecting the mobility of potentially toxic metals associated with naturally occurring aquifer materials. These processes influence the degradation or preservation of anthropogenic contaminants such as benzene, toluene, ethylbenzene, and xylene. In addition, these processes can generate undesirable byproducts such as dissolved ferrous iron, hydrogen sulfide, and methane. Dissolved-oxygen concentrations in 93 percent of the samples indicated oxic conditions (less than or equal to 0.5 milligrams per liter). Dissolved-oxygen samples were collected primarily in Garfield County near Rifle and many of the anoxic wells were located in the Mamm Creek-Divide Creek area.

Dissolved solids can be used to infer water sources because deeper sources tend to have higher dissolved-solids concentrations and are also higher in certain constituents like chloride. Dissolved-solids concentrations in 72 percent of samples exceeded the secondary drinking-water standard of 500 milligrams per liter. These exceedances were relatively widespread in the study area. Samples from wells screened in Quaternary, Tertiary, and Cretaceous geologic units each had a distinct suite of major ions. Variations in concentration with depth could not be evaluated because of the general lack of well-depth and water-level data. Chloride concentrations in 10 percent of samples exceeded the chloride standard. Of those samples with geologic unit or aquifer designations, only those from Quaternary units in Mesa County had a median chloride concentration greater than the secondary standard. Sulfate concentration data in 37 percent of samples exceeded the secondary standard of 250 milligrams per liter, and exceedances were relatively widespread in the study area. These data indicate that subsurface sulfate sources were relatively common in the study area. In the absence of additional information, such as stable sulfur isotope data, it is difficult to identify those sources, but likely sources include minerals such as gypsum and pyrite (following oxidation). Produced waters are a less likely source of sulfate to the freshwater aquifers. Fluoride concentrations in 8.4 percent of samples exceeded the primary standard of 4 milligrams per liter. Most of the fluoride exceedances were found in Rio Blanco County, although many exceedances were observed in the Mamm Creek-Divide Creek area of Garfield County.

Trace elements in groundwater are typically derived from minerals in aquifer rocks and sediment that are subsequently mobilized by various processes such as redox processes. Arsenic concentrations were available for 256 wells; 33 concentrations (13 percent) exceeded the primary standard of 10 micrograms per liter, and exceedances occurred primarily from Rio Blanco County. Quaternary and Tertiary units in Rio Blanco County had the highest median arsenic concentrations (5 and 4 micrograms per liter, respectively). Arsenic 
concentrations were significantly higher $(p=0.008)$ in anoxic groundwater than in oxic groundwater, indicating that reductive dissolution of manganese or iron oxide minerals (or both) in the aquifer rock and sediment probably was an important mechanism for producing elevated arsenic concentrations in groundwater. In fact, iron concentrations were significantly higher $(p=0.001)$ in samples with arsenic concentrations above the primary standard of 10 micrograms per liter than in samples with lower arsenic concentrations. The arsenicdissolved oxygen relation also indicates that dissolved-oxygen measurements could be a useful indicator of groundwater vulnerability to arsenic enrichment. Barium concentrations in 4.1 percent of samples exceeded the primary standard of 2,000 micrograms per liter. Barium exceedances were found in samples from Delta, Garfield, and Rio Blanco Counties. The Mesaverde Group in Delta County had the highest median barium concentrations ( 90 micrograms per liter). Manganese concentrations in 21 percent of samples exceeded the secondary standard of 50 micrograms per liter. Exceedances of the secondary standard were relatively widespread in the study area. Quaternary deposits in Mesa County had the highest median manganese concentration (1,410 micrograms per liter). Similar to arsenic concentrations, manganese concentrations were significantly higher $(p=0.001)$ in anoxic groundwater than oxic groundwater. This result indicates that dissolvedoxygen measurements also could be a useful indicator of groundwater vulnerability to manganese enrichment. Iron concentrations were available for 589 wells, and 96 concentrations (16 percent) exceeded the secondary standard of 300 micrograms per liter. Most of the exceedances were in samples from Garfield and Rio Blanco Counties. Unlike arsenic and manganese, iron concentrations were not significantly different between oxic and anoxic water samples, nor were they different at dissolved-oxygen concentration thresholds of 1 and 2 milligrams per liter. The reason for this apparent discordant finding is unclear, but it could be related to the sample collection and preservation techniques used. Selenium concentrations were available for 551 wells, and 51 concentrations ( 9.2 percent) exceeded the primary standard of 50 micrograms per liter. Most of the samples that exceeded the selenium standard were from Garfield County. In this study, selenium concentrations were significantly higher $(p<0.001)$ in samples containing nitrate at a concentration of at least 1 milligram per liter (as nitrogen) than in samples with less nitrate.

Elevated sources of nitrate in groundwater are likely to be associated with septic systems, animal manure, or fertilizers applied to lawns and crops. Nitrate concentrations were available for 791 wells, and 13 concentrations (1.6 percent) exceeded the primary standard of 10 milligrams per liter as nitrogen. Most of the samples that exceeded the standard were from wells located in agricultural areas of Garfield County. Nitrate concentrations ranged from less than detection (most commonly about 0.3 milligrams per liter as nitrogen) to 29.4 milligrams per liter, with a median concentration of 0.21 milligrams per liter as nitrogen. The highest concentration was found in a sample from a domestic well in Garfield County. The Wasatch Formation in Garfield County was the only one to have at least 10 assigned samples, and the median nitrate concentration for those samples was 0.4 milligrams per liter as nitrogen. Nitrate concentrations were significantly higher $(p=0.015)$ in groundwater containing at least 2 milligrams per liter dissolved oxygen than in less oxygenated groundwater. This dissolved oxygen-nitrate relation differs somewhat from the relationship described in previous studies, but it still indicates that dissolved-oxygen measurements could be a useful indicator of groundwater vulnerability to nitrate contamination.

Benzene, toluene, ethylbenzene, and xylene (BTEX) are volatile organic compounds typically found in petroleum products, such as gasoline and diesel fuel, and also occur in some natural gas reservoirs. Benzene concentrations were available for 808 wells, but only 11 wells (1.4 percent) had detections and 5 concentrations ( 0.6 percent $)$ exceeded the primary standard (5 micrograms per liter). Samples that exceeded the standard were from wells located in Garfield and Rio Blanco Counties. Toluene concentrations were available for 808 wells; 38 wells (4.7 percent) had detections but none of the concentrations exceeded the primary drinking-water standard (1,000 micrograms per liter). Ethylbenzene and xylene were sampled at a frequency similar to that of benzene and toluene but they had few detections and no exceedances of primary standards.

Methane is a combustible gas that can accumulate to explosive levels in well bores and confined spaces in buildings. Methane can be either thermogenic or biogenic in origin. Thermogenic methane is generally produced at depths far below freshwater aquifers, and its presence in water wells can be due to natural conditions, or from activities related to oil and gas drilling, completion, and production. Methane concentrations were available for 874 wells primarily in Garfield County. Drinking-water standards have not been established for methane, which was detected in 24 percent of samples (207 wells). Methane concentrations were greater than or equal to 1 milligram per liter in 8.5 percent of samples (75 samples). The majority of methane detections and high methane concentrations were found in Garfield County near the Mamm Creek-Divide Creek area. Methane concentrations in anoxic groundwater were significantly higher $(p=0.004)$ than oxic groundwater, a finding that is consistent with previous results and indicates that dissolved-oxygen measurements could be a useful indicator of groundwater vulnerability to methane enrichment. Isotopic data indicate that methane in the Garfield County water wells was from both biogenic and thermogenic sources. For comparison, natural gas from the Mesaverde Group in Garfield County was exclusively thermogenic in origin. Biogenic methane in domestic wells could have been produced locally in the aquifer if the groundwater was anoxic or it could have come from deeper geologic units underlying the aquifer.

Assessing such a large and diverse dataset as the one available through the repository poses unique challenges for 
assessing groundwater quality in the study area. The repository contains data from several studies that differed widely in purpose and scope. Data gaps exist in the repository. For example, groundwater-quality data in the repository were not evenly distributed throughout the study area. Several key water-quality constituents or indicators were underrepresented in the repository, including dissolved oxygen. Ancillary information, such as well depth, depth to water, and the geologic unit or aquifer in which a well was completed, was missing for more than 50 percent of the samples. Future monitoring could avoid several limitations of the repository by making relatively minor changes to sample-collection and data-reporting protocols. The addition of field measurements for dissolved oxygen to sampling protocols, for example, is one such change. Measuring the water level in a well prior to sampling is another. With respect to data reporting, including information on well construction and the geologic unit or aquifer in which a well is completed as part of the water-quality database would be helpful. Implementing these types of changes would increase the comparability of data from different monitoring programs and also add value to each program individually and to the regional dataset as a whole. Other changes to monitoring programs could require greater resources, such as sampling for a basic set of constituents that is relevant to major waterquality issues in the regional study area. Creation of such a dataset for the regional study area would help to provide the kinds of information needed to characterize background conditions and the spatial and temporal variability in constituent concentrations associated with those conditions. Without that information, it becomes difficult to recognize departures from background that might be associated with human activities.

\section{Acknowledgments}

The authors thank the many agencies that provided data for this report. The authors acknowledge Berry Petroleum Company for their contribution to this project which was undertaken in connection with the settlement of an enforcement action taken by the Colorado Department of Public Health and Environment for violations of the Water Quality Control Act. The author would like to express appreciation to Dennis Risser and Matthew Miller for their technical review of the manuscript, which aided in the overall improvement of this report.

\section{References Cited}

ALL Consulting, 2003, Handbook on coal bed methane produced water-Management and beneficial use alternatives: Tulsa, Oklahoma, 322 p.
Ayers, R.S., and Westcot, D.W., 1985, Water quality for agriculture: FAO Irrigation and Drainage Paper 29 Rev 1, Food and Agriculture Organization of the United Nations; accessed April 3, 2012, at http://www.fao.org/DOCREP/003/ T0234E/T0234E00.HTM

Bradley, W.H., 1931, Origin and microfossils of the oil shale of the Green River Formation of Colorado and Utah: U.S. Geological Survey Professional Paper 168, 58 p.

Brooks, Tom, and Ackerman, D.J., 1985, Reconnaissance of ground-water resources in the Lower Gunnison River Basin, southwestern Colorado: U.S. Geological Survey WaterResources Investigations Report 84-4185, 35 p.

Bureau of Land Management, 2009, Geospatial data and metadata statewide GIS layers for BLM Colorado 1st conflated poly: Bureau of Land Management, Colorado State Office; accessed January 26, 2010, at http://www.blm.gov/co/st/en/ BLM_Programs/geographical_sciences/gis/metadata.html

Bureau of Land Management, 2011a, Oil shale and tar sands: accessed January 3, 2011, at $h t t p: / / w w w . b l m . g o v / w o / s t / e n /$ prog/energy/oilshale_2.html

Bureau of Land Management, 2011b, Reasonable foreseeable development scenario for oil and gas activities in the BLM White River Field Office- - Rio Blanco, Moffat and Garfield Counties, Colorado: accessed January 17, 2011, at http:// www.blm.gov/pgdata/etc/medialib/blm/co/programs/land_ use planning/rmp/white_river/documents.Par.86610.File. dat/RFD_Executuve_Summary.pdf

Butler, D.L., and Osmundson, B.C., 2000, Physical, chemical, and biological data for the Uncompahgre Project area and the Grand Valley, west-central Colorado,1993-98: U.S. Geological Survey Open-File Report 99-453, 216 p.

Butler, D.L., Wright, W.G., Stewart, K.C., Osmundson, B.C., Krueger, R.P., and Crabtree, D.W., 1996, Detailed study of selenium and other constituents in water, bottom sediment, soil, alfalfa, and biota associated with irrigation drainage in the Uncompahgre Project area and in the Grand Valley, west-central Colorado, 1991-93: U.S. Geological Survey Water-Resources Investigations Report 96-4138, 136 p.

Cashion, W.B., and Donnell, J.R., 1974, Revision of nomenclature of the upper part of the Green River Formation, Piceance Creek Basin, Colorado, and eastern Uinta Basin, Utah: U.S. Geological Survey Bulletin 1394-G, 9 p.

Chapelle, F.H., McMahon, P.B., Dubrovsky, N.M., Fujii, R.F., Oaksford, E.T., and Vroblesky, D.A., 1995, Deducing the distribution of terminal electron-accepting processes on hydrologically diverse groundwater systems: Water Resources Research, v. 31, p. 359-371. 
Coffin, D.L., Welder, F.A., and Glanzman, R.K., 1971, Geohydrology of the Piceance Creek structural basin between the White and Colorado Rivers, northwestern Colorado: U.S. Geological Survey Hydrologic Investigations Atlas HA-370; 2 maps on 2 sheets in pocket.

Coffin, D.L., Welder, F.A., Glanzman, R.K., and Dutton, X.W., 1968, Geohydrologic data from Piceance Creek Basin between the White and Colorado Rivers, northwestern Colorado: Colorado Water Conservation Board Water Resources Circular 12, 38 p.

Colorado's Decision Support Systems, 2010, Colorado's Decision Support Systems: accessed February 17, 2010, at http://cdss.state.co.us/

Colorado Division of Water Resources, 2011, GIS data for download-Water well applications: accessed June 9, 2011, at http://water.state.co.us/

Colorado Oil and Gas Conservation Commission, 2010, GIS downloads-Wells: accessed January 26, 2010, at http://cogcc.state.co.us/

Coplen, T.B., 1988, Normalization of oxygen and hydrogen isotope data: Chemical Geology, v. 72, p. 293-297.

Craig, Harmon, 1961, Isotopic variations in meteoric waters: Science, v. 133, p.1702-1703.

Donnell, J.R., 1961, Tertiary geology and oil-shale resources of the Piceance Creek Basin between the Colorado and White Rivers, northwestern Colorado: U.S. Geological Survey Bulletin 1082-L, p. L835-L891.

Dubrovsky, N.M., Burow, K.R., Clark, G.M., Gronberg, J.M., Hamilton, P.A., Hitt, K.J., Mueller, D.K., Munn, M.D., Nolan, B.T., Puckett, L.J., Rupert, M.G., Short, T.M., Spahr, N.E., Sprague, L.A., and Wilber, W.G., 2010, The quality of our Nation's waters-Nutrients in the Nation's streams and groundwater, 1992-2004: U.S. Geological Survey Circular $1350,174 \mathrm{p}$.

Dubrovsky, N.M., Deverel, S.J., and Gilliom, R.J., 1993, Multiscale approach to regional groundwater-quality assessment-Selenium in the San Joaquin Valley, California, in Alley, W.M., ed., Regional groundwater quality: New York, Van Nostrand Reinhold, 26 p.

Green, G.N., 1992, The digital geological map of Colorado in Arc/Info format: U.S. Geological Survey Open-File Report 92-0507, scale 1:500,000.
Hamilton, S.J., Holley, K.M., Buhl, K.J., Bullard, F.A., Weston, L.K., and McDonald, S.F., 2003, Evaluation of flushing of backwater channel-Concentrations of selenium and other inorganic elements in water, sediment, invertebrates, forage fish, and Colorado Pikeminnow: Draft report prepared by the Upper Colorado River Endangered Fish Recovery Program. U.S. Geological Survey, Columbia Environmental Research Center, Yankton Field Research Station, 31247 436th Avenue, Yankton, South Dakota 57078-9214, 149 pages.

Helsel, D.R., 2005a, Insider censoring-distortion of data with nondetects: Human and Ecological Risk Assessment, v. 11, p. 1127-1137.

Helsel, D.R., 2005b, Non-detects and data analysis-Statistics for censored environmental data: New Jersey, John Wiley, $268 \mathrm{p}$.

Helsel, D.R., and Hirsch, R.M., 2002, Statistical methods in water resources: U.S. Geological Survey Techniques of Water-Resources Investigation, book 4, chap. A3, 523 p.

Hem, J.D., 1989, Study and interpretation of the chemical characteristics of natural water, $3 \mathrm{~d}$ edition: U.S. Geological Survey Water-Supply Paper 2254, 225 p.

Johnson, R.C., 1989, Geologic history and hydrocarbon potential of late Cretaceous-age, low permeability reservoirs, Piceance Basin, western Colorado: U.S. Geological Survey Bulletin 1787-E, $51 \mathrm{p}$.

Johnson, R.C., and Flores, R.M., 2003, History of the Piceance Basin from latest Cretaceous through early Eocene and the characterization of lower Tertiary sandstone reservoirs, chap. 3, in Peterson, K.M., Olson, T.M., and Anderson, D.S., eds., Piceance Basin 2003 Guidebook: Denver, Colo., Rocky Mountain Association of Geologists, p. 21-61.

Lohman, S.W., 1965, Geology and artesian water supply, Grand Junction area, Colorado: U.S. Geological Survey Professional Paper 451, 2 plates in pocket, 149 p.

McMahon, P.B., and Chapelle, F.H., 2008, Redox processes and water quality of selected principal aquifer systems: Ground Water, v. 46, p. 259-271.

McMahon, P.B., Thomas, J.C., and Hunt, A.G., 2010, Use of diverse geochemical datasets to enhance groundwaterquality assessments - A case study from Garfield County, Colorado: U.S. Geological Survey Scientific Investigations Report 2010-5215, 40 p.

National Nuclear Security Administration, 2010, Plowshare Program: accessed January 1, 2011, at http://www.nv.doe. gov/library/factsheets/DOENV_766.pdf 
Nelson, P.H., and Santus, S.L., 2010, Gas, water, and oil production from Grand Valley, Parachute, Rulison, and Mamm Creek fields in the Piceance Basin, Colorado: U.S. Geological Survey Open-File Report 2010-1110, 28 p., 6 plates and 6 appendixes.

Reinecke, K.M., Rice, D.D., and Johnson, R.C., 1991, Characteristics and development of fluvial sandstone and coalbed reservoirs of Upper Cretaceous Mesaverde Group, Grand Valley field, Colorado, in Schwochow, S.D., Murray, D.K., and Fahy, M.F., eds., Coalbed methane of Western North America, Rocky Mountain Association of Geologists Fall Conference and Field Trip, September, 17-20, 1991, Guidebook: Rocky Mountain Association of Geologists, p. 209-225.

Smedley, P.L., and Kinniburgh, D.G., 2002, A review of the source, behavior, and distribution of arsenic in natural waters: Applied Geochemistry, v. 17, p. 517-568.

S.S. Papadopulos \& Associates, Inc., 2008, Phase II hydrogeologic characterization of the Mamm Creek Field area, Garfield County, Colorado: (Prepared for the Board of County Commissioners, Garfield County, Colorado), 41 p.; accessed April 5, 2012, at http://www.garfield-county.com/ oil-gas/phase-II-hydrogeologic-characterization-mammcreek.aspx

Taylor, O.J., 1987, Oil shale, water resources, and valuable minerals of the Piceance Basin, Colorado-The challenge and choices of development: U.S. Geological Professional Paper 1310, $143 \mathrm{p}$.

Thomas, J.C., 2009, Analysis of dissolved selenium loading from surface water and groundwater to Sweitzer Lake, Colorado, 2006-07: U.S. Geological Survey Scientific Investigations Report 2009-5048, 19 p.

Tyler, Roger, Kaiser, W.R., McMurry, R.G., Nance, H.S., Scott, A.R., and Zhou, Naijiang, 1995, Geologic characterization and coalbed methane occurrence-Williams Fork Formation, Piceance Basin, northwest Colorado, in The University of Texas at Austin, Bureau of Economic Geology, annual report prepared for Gas Research Institute under contract no. 5091-214-2261, GRI-94/0456, p. 192-197.

URS Corporation, 2006, Phase I hydrogeologic characterization of the Mamm Creek Field area in Garfield County: Prepared for Board of County Commissioners, Garfield County, Colorado, 86 p.: accessed April 5, 2012, at http://www.garfield-county.com/oil-gas/phase-I-hydrogeologic-characterization-mamm-creek.aspx

U.S. Census Bureau, 2011, Population finder, Fact Sheet 2005-2009: accessed January 18, 2011, at http://www.census.gov/
U.S. Department of Energy, 2011a, Naval Petroleum Reserves-Profile: accessed January 1, 2011, at http://fossil.energy.gov/programs/reserves/npr/

U.S. Department of Energy, 2011b, Environmental Management-Rio Blanco and Rulison sites: accessed October 10, 2011, at http://www.em.doe.gov/bemr/BEMRSites/prbs.aspx

U.S. Environmental Protection Agency, 2010, Drinking water contaminants, national primary drinking water regulations and national secondary drinking water regulations: accessed November 9, 2010, at http://water.epa.gov/drink/contaminants/index.cfm

U.S. Geological Survey, 2011, Annual water data reports, 2009: accessed January 5, 2011, at http://wdr.water.usgs. gov/wy2009/search.jsp

U.S. Geological Survey Oil Shale Assessment Team, 2010, Oil shale and nahcolite resources of the Piceance Basin, Colorado: U.S. Geological Survey Digital Data Series DDS-69-Y, 7 chapters, pages variable.

Weeks, J.B., Leavesley, G.H., Welder, F.A., and Saulnier, G.J., Jr., 1975, Simulated effects of oil-shale development on the hydrology of Piceance Basin, Colorado: U.S. Geological Survey Professional Paper 908, 84 p.

Whiticar, M.J., 1999, Carbon and hydrogen isotope systematic of bacterial formation and oxidation of methane: Chemical Geology, v. 161, p. 291-314.

Whiticar, M.J., Faber, E., and Schoell, M., 1986, Biogenic methane formation in marine and freshwater environments$\mathrm{CO}_{2}$ reduction vs. acetate fermentation, isotope evidence: Geochimica and Cosmochimica Acta, v. 50, p. 693-709.

Wright, W.G., and Butler, D.L., 1993, Distribution and mobilization of dissolved selenium in ground water of the irrigated Grand and Uncompahgre Valleys, western Colorado, in Allen, R.G., and Neale, C.M.U., eds., Management of irrigation and drainage systems - Integrated perspectives: American Society of Civil Engineers National Conference on Irrigation and Drainage Engineering: Park City, Utah, July 21-23, 1993, Proceedings, p. 770-777.

Publishing support provided by:

Denver Publishing Service Center

For more information concerning this publication, contact:

Director, USGS Colorado Water Science Center

Box 25046, Mail Stop 415

Denver, CO 80225

(303) 236-4882

Or visit the Colorado Water Science Center Web site at: http://co.water.usgs.gov/ 
Appendix 
Appendix 1. Water Quality Data Used in This Report.

\begin{tabular}{|c|c|c|c|c|c|}
\hline $\begin{array}{c}\text { Report } \\
\text { No. }\end{array}$ & Agency name & $\begin{array}{c}\text { Site } \\
\text { identifier }\end{array}$ & $\begin{array}{c}\text { Site } \\
\text { name }\end{array}$ & $\begin{array}{l}\text { Well } \\
\text { use }\end{array}$ & County \\
\hline 1 & EnCana Oil \& Gas (USA) Inc. & BARE1WW & BARE1WW & DOMESTIC USE & Garfield County \\
\hline 2 & U.S. Geological Survey & 393401107595501 & SC00509536DCA1 & OBSERVATION & Garfield County \\
\hline 3 & U.S. Geological Survey & 393532107583701 & SC00509419DDD1 & OBSERVATION & Garfield County \\
\hline 4 & U.S. Geological Survey & 393533107565701 & SC00509421CDC & OBSERVATION & Garfield County \\
\hline 5 & URS Corporation & WMS-202848 & WMS-202848 & DOMESTIC USE & Garfield County \\
\hline 6 & U.S. Geological Survey & 126150 & 126150 & OBSERVATION & Garfield County \\
\hline 8 & U.S. Geological Survey & 393850108172200 & SC00509704BBD1 & OBSERVATION & Garfield County \\
\hline 9 & EnCana Oil \& Gas (USA) Inc. & OLDL1WW & OLDL1WW & DOMESTIC USE & Garfield County \\
\hline 13 & Colorado Oil and Gas Conservation Commission & 703875 & COGCC Facility ID:703875 & PUBLIC SUPPLY & Garfield County \\
\hline 14 & Colorado Oil and Gas Conservation Commission & 703876 & COGCC Facility ID:703876 & PUBLIC SUPPLY & Garfield County \\
\hline 15 & Colorado Oil and Gas Conservation Commission & 703877 & COGCC Facility ID:703877 & PUBLIC SUPPLY & Garfield County \\
\hline 16 & Colorado Oil and Gas Conservation Commission & 703878 & COGCC Facility ID:703878 & PUBLIC SUPPLY & Garfield County \\
\hline 17 & EnCana Oil \& Gas (USA) Inc. & LCSMW1 & LCSMW1 & OBSERVATION & Garfield County \\
\hline 18 & EnCana Oil \& Gas (USA) Inc. & LCSMW10 & LCSMW10 & OBSERVATION & Garfield County \\
\hline 19 & EnCana Oil \& Gas (USA) Inc. & LCSMW11 & LCSMW11 & OBSERVATION & Garfield County \\
\hline 20 & EnCana Oil \& Gas (USA) Inc. & LCSMW2 & LCSMW2 & OBSERVATION & Garfield County \\
\hline 21 & EnCana Oil \& Gas (USA) Inc. & LCSMW3 & LCSMW3 & OBSERVATION & Garfield County \\
\hline 22 & EnCana Oil \& Gas (USA) Inc. & LCSMW4 & LCSMW4 & OBSERVATION & Garfield County \\
\hline 23 & EnCana Oil \& Gas (USA) Inc. & LCSMW5 & LCSMW5 & OBSERVATION & Garfield County \\
\hline 24 & EnCana Oil \& Gas (USA) Inc. & LCSMW6 & LCSMW6 & OBSERVATION & Garfield County \\
\hline 25 & EnCana Oil \& Gas (USA) Inc. & lcsmw7 & LCSMW7 & OBSERVATION & Garfield County \\
\hline 26 & EnCana Oil \& Gas (USA) Inc. & LCSMW8 & LCSMW8 & OBSERVATION & Garfield County \\
\hline 27 & EnCana Oil \& Gas (USA) Inc. & LCSMW9 & LCSMW9 & OBSERVATION & Garfield County \\
\hline 28 & EnCana Oil \& Gas (USA) Inc. & LCSSUMP & LCSSUMP & AGRICULTURAL USE & Garfield County \\
\hline 29 & U.S. Geological Survey & 127013 & 127013 & OBSERVATION & Garfield County \\
\hline 30 & EnCana Oil \& Gas (USA) Inc. & KEIN2WW & KEIN2WW & DOMESTIC USE & Garfield County \\
\hline 31 & U.S. Geological Survey & 126202 & 126202 & OBSERVATION & Garfield County \\
\hline 33 & U.S. Geological Survey & 393011108234201 & SC00609925CBA1 & AGRICULTURAL USE & Garfield County \\
\hline 34 & U.S. Geological Survey & 393013108234201 & SC00609925CBA2 & DOMESTIC USE & Garfield County \\
\hline 36 & U.S. Geological Survey & 393311108291401 & SC00609907BAC1 & DOMESTIC USE & Garfield County \\
\hline 38 & U.S. Geological Survey & 393442108062901 & SC00509530CCC1 & AGRICULTURAL USE & Garfield County \\
\hline 39 & U.S. Geological Survey & 393637108014301 & SC00509514CBD & OBSERVATION & Garfield County \\
\hline 40 & EnCana Oil \& Gas (USA) Inc. & ENPR10MW & ENPR10MW & OBSERVATION & Garfield County \\
\hline 41 & EnCana Oil \& Gas (USA) Inc. & ENPR11MW & ENPR11MW & OBSERVATION & Garfield County \\
\hline 42 & Colorado Oil and Gas Conservation Commission & 703181 & COGCC Facility ID:703181 & DOMESTIC USE & Garfield County \\
\hline 43 & EnCana Oil \& Gas (USA) Inc. & ENPR3MW & ENPR3MW & OBSERVATION & Garfield County \\
\hline 44 & EnCana Oil \& Gas (USA) Inc. & ENPR4MW & ENPR4MW & OBSERVATION & Garfield County \\
\hline 45 & EnCana Oil \& Gas (USA) Inc. & ENPR5MW & ENPR5MW & OBSERVATION & Garfield County \\
\hline 46 & EnCana Oil \& Gas (USA) Inc. & ENPR6MW & ENPR6MW & OBSERVATION & Garfield County \\
\hline 47 & EnCana Oil \& Gas (USA) Inc. & ENPR7MW & ENPR7MW & OBSERVATION & Garfield County \\
\hline 48 & EnCana Oil \& Gas (USA) Inc. & ENPR8MW & ENPR8MW & OBSERVATION & Garfield County \\
\hline 49 & EnCana Oil \& Gas (USA) Inc. & ENPR9MW & ENPR9MW & OBSERVATION & Garfield County \\
\hline 50 & U.S. Geological Survey & 392524107370301 & SC00709225BADD GRFLDS10 & DOMESTIC USE & Garfield County \\
\hline 51 & Colorado Oil and Gas Conservation Commission & 703200 & COGCC Facility ID:703200 & DOMESTIC USE & Garfield County \\
\hline 52 & Colorado Oil and Gas Conservation Commission & 703248 & COGCC Facility ID:703248 & PUBLIC SUPPLY & Garfield County \\
\hline 53 & Colorado Oil and Gas Conservation Commission & 703269 & COGCC Facility ID:703269 & PUBLIC SUPPLY & Garfield County \\
\hline 54 & Colorado Oil and Gas Conservation Commission & 703022 & COGCC Facility ID:703022 & DOMESTIC USE & Garfield County \\
\hline 55 & Colorado Oil and Gas Conservation Commission & 703149 & COGCC Facility ID:703149 & DOMESTIC USE & Garfield County \\
\hline 56 & Colorado Oil and Gas Conservation Commission & 703977 & COGCC Facility ID:703977 & DOMESTIC USE & Garfield County \\
\hline 57 & Colorado Oil and Gas Conservation Commission & 703978 & COGCC Facility ID:703978 & DOMESTIC USE & Garfield County \\
\hline 58 & Colorado Oil and Gas Conservation Commission & 703979 & COGCC Facility ID:703979 & DOMESTIC USE & Garfield County \\
\hline 59 & Colorado Oil and Gas Conservation Commission & 703980 & COGCC Facility ID:703980 & DOMESTIC USE & Garfield County \\
\hline 60 & Colorado Oil and Gas Conservation Commission & 703998 & COGCC Facility ID:703998 & DOMESTIC USE & Garfield County \\
\hline 61 & Colorado Oil and Gas Conservation Commission & 704115 & COGCC Facility ID:704115 & DOMESTIC USE & Garfield County \\
\hline 62 & Colorado Oil and Gas Conservation Commission & 704150 & COGCC Facility ID:704150 & DOMESTIC USE & Garfield County \\
\hline 63 & Colorado Oil and Gas Conservation Commission & 704166 & COGCC Facility ID:704166 & OBSERVATION & Garfield County \\
\hline 64 & Colorado Oil and Gas Conservation Commission & 703018 & COGCC Facility ID:703018 & DOMESTIC USE & Garfield County \\
\hline 65 & Colorado Oil and Gas Conservation Commission & 704167 & COGCC Facility ID:704167 & OBSERVATION & Garfield County \\
\hline 66 & Colorado Oil and Gas Conservation Commission & 704188 & COGCC Facility ID:704188 & OBSERVATION & Garfield County \\
\hline 67 & Colorado Oil and Gas Conservation Commission & 704337 & COGCC Facility ID:704337 & DOMESTIC USE & Garfield County \\
\hline 68 & Colorado Oil and Gas Conservation Commission & 704380 & COGCC Facility ID:704380 & DOMESTIC USE & Garfield County \\
\hline 69 & EnCana Oil \& Gas (USA) Inc. & EICH2WW & EICH2WW & OBSERVATION & Garfield County \\
\hline 70 & EnCana Oil \& Gas (USA) Inc. & EICH3WW & EICH3WW & OBSERVATION & Garfield County \\
\hline 71 & Colorado Oil and Gas Conservation Commission & 704483 & COGCC Facility ID:704483 & DOMESTIC USE & Garfield County \\
\hline 72 & Colorado Oil and Gas Conservation Commission & 704485 & COGCC Facility ID:704485 & DOMESTIC USE & Garfield County \\
\hline 73 & Colorado Oil and Gas Conservation Commission & 704504 & COGCC Facility ID:704504 & DOMESTIC USE & Garfield County \\
\hline
\end{tabular}


Appendix 1. Water Quality Data Used in This Report.-Continued

\begin{tabular}{|c|c|c|c|c|c|}
\hline $\begin{array}{c}\text { Report } \\
\text { No. }\end{array}$ & Agency name & $\begin{array}{c}\text { Site } \\
\text { identifier }\end{array}$ & $\begin{array}{c}\text { Site } \\
\text { name }\end{array}$ & $\begin{array}{l}\text { Well } \\
\text { use }\end{array}$ & County \\
\hline 74 & Colorado Oil and Gas Conservation Commission & 704505 & COGCC Facility ID:704505 & DOMESTIC USE & Garfield County \\
\hline 75 & Colorado Oil and Gas Conservation Commission & 704506 & COGCC Facility ID:704506 & DOMESTIC USE & Garfield County \\
\hline 76 & Colorado Oil and Gas Conservation Commission & 704530 & COGCC Facility ID:704530 & DOMESTIC USE & Garfield County \\
\hline 77 & Colorado Oil and Gas Conservation Commission & 704321 & COGCC Facility ID:704321 & DOMESTIC USE & Garfield County \\
\hline 78 & Colorado Oil and Gas Conservation Commission & 704336 & COGCC Facility ID:704336 & DOMESTIC USE & Garfield County \\
\hline 79 & EnCana Oil \& Gas (USA) Inc. & DODD1WW & DODD1WW & DOMESTIC USE & Garfield County \\
\hline 80 & EnCana Oil \& Gas (USA) Inc. & EICH1WW & EICH1WW & DOMESTIC USE & Garfield County \\
\hline 81 & EnCana Oil \& Gas (USA) Inc. & HARD2WW & HARD2WW & DOMESTIC USE & Garfield County \\
\hline 82 & EnCana Oil \& Gas (USA) Inc. & LANG6WW & LANG6WW & DOMESTIC USE & Garfield County \\
\hline 83 & EnCana Oil \& Gas (USA) Inc. & LANG7WW & LANG7WW & DOMESTIC USE & Garfield County \\
\hline 84 & EnCana Oil \& Gas (USA) Inc. & LANG8WW & LANG8WW & DOMESTIC USE & Garfield County \\
\hline 85 & EnCana Oil \& Gas (USA) Inc. & LANG9WW & LANG9WW & DOMESTIC USE & Garfield County \\
\hline 86 & EnCana Oil \& Gas (USA) Inc. & MILLWW & MILLWW & DOMESTIC USE & Garfield County \\
\hline 87 & EnCana Oil \& Gas (USA) Inc. & MORG3WW & MORG3WW & AGRICULTURAL USE & Garfield County \\
\hline 88 & EnCana Oil \& Gas (USA) Inc. & MORG4WW & MORG4WW & DOMESTIC USE & Garfield County \\
\hline 89 & EnCana Oil \& Gas (USA) Inc. & SPAU1WW & SPAU1WW & DOMESTIC USE & Garfield County \\
\hline 90 & EnCana Oil \& Gas (USA) Inc. & FAIT1WW & FAIT1WW & DOMESTIC USE & Garfield County \\
\hline 91 & EnCana Oil \& Gas (USA) Inc. & GALL4WW & GALL4WW & DOMESTIC USE & Garfield County \\
\hline 92 & EnCana Oil \& Gas (USA) Inc. & GEOR1WW & GEOR1WW & DOMESTIC USE & Garfield County \\
\hline 93 & EnCana Oil \& Gas (USA) Inc. & HAWK1WW & HAWK1WW & DOMESTIC USE & Garfield County \\
\hline 94 & EnCana Oil \& Gas (USA) Inc. & HAWK2WW & HAWK2WW & DOMESTIC USE & Garfield County \\
\hline 95 & EnCana Oil \& Gas (USA) Inc. & HAWK3WW & HAWK3WW & DOMESTIC USE & Garfield County \\
\hline 96 & EnCana Oil \& Gas (USA) Inc. & HAWK4WW & HAWK4WW & DOMESTIC USE & Garfield County \\
\hline 97 & EnCana Oil \& Gas (USA) Inc. & HAWK5WW & HAWK5WW & DOMESTIC USE & Garfield County \\
\hline 98 & EnCana Oil \& Gas (USA) Inc. & LANG10WW & LANG10WW & OBSERVATION & Garfield County \\
\hline 99 & EnCana Oil \& Gas (USA) Inc. & LANG11WW & LANG11WW & OBSERVATION & Garfield County \\
\hline 100 & EnCana Oil \& Gas (USA) Inc. & LANG12WW & LANG12WW & OBSERVATION & Garfield County \\
\hline 101 & EnCana Oil \& Gas (USA) Inc. & LANG13WW & LANG13WW & OBSERVATION & Garfield County \\
\hline 102 & EnCana Oil \& Gas (USA) Inc. & LANG14WW & LANG14WW & OBSERVATION & Garfield County \\
\hline 103 & EnCana Oil \& Gas (USA) Inc. & LANG15WW & LANG15WW & OBSERVATION & Garfield County \\
\hline 104 & EnCana Oil \& Gas (USA) Inc. & LANG16WW & LANG16WW & OBSERVATION & Garfield County \\
\hline 105 & EnCana Oil \& Gas (USA) Inc. & LANG17WW & LANG17WW & OBSERVATION & Garfield County \\
\hline 106 & EnCana Oil \& Gas (USA) Inc. & LANG18WW & LANG18WW & OBSERVATION & Garfield County \\
\hline 107 & EnCana Oil \& Gas (USA) Inc. & LANG19WW & LANG19WW & OBSERVATION & Garfield County \\
\hline 108 & EnCana Oil \& Gas (USA) Inc. & $\mathrm{MCPH} 2 \mathrm{WW}$ & $\mathrm{MCPH} 2 \mathrm{WW}$ & DOMESTIC USE & Garfield County \\
\hline 109 & EnCana Oil \& Gas (USA) Inc. & MILL5WW & MILL5WW & DOMESTIC USE & Garfield County \\
\hline 110 & EnCana Oil \& Gas (USA) Inc. & MILL6WW & MILL6WW & DOMESTIC USE & Garfield County \\
\hline 111 & EnCana Oil \& Gas (USA) Inc. & MORG1WW & MORG1WW & DOMESTIC USE & Garfield County \\
\hline 112 & EnCana Oil \& Gas (USA) Inc. & MORG2WW & MORG2WW & DOMESTIC USE & Garfield County \\
\hline 113 & EnCana Oil \& Gas (USA) Inc. & LANG20WW & LANG20WW & OBSERVATION & Garfield County \\
\hline 114 & EnCana Oil \& Gas (USA) Inc. & LANG21WW & LANG21WW & OBSERVATION & Garfield County \\
\hline 115 & EnCana Oil \& Gas (USA) Inc. & LANG22WW & LANG22WW & OBSERVATION & Garfield County \\
\hline 116 & EnCana Oil \& Gas (USA) Inc. & LANG23WW & LANG23WW & OBSERVATION & Garfield County \\
\hline 117 & EnCana Oil \& Gas (USA) Inc. & LANG24WW & LANG24WW & OBSERVATION & Garfield County \\
\hline 118 & EnCana Oil \& Gas (USA) Inc. & LANG25WW & LANG25WW & OBSERVATION & Garfield County \\
\hline 119 & EnCana Oil \& Gas (USA) Inc. & LANG26WW & LANG26WW & OBSERVATION & Garfield County \\
\hline 120 & EnCana Oil \& Gas (USA) Inc. & LANG2WW & LANG2WW & OBSERVATION & Garfield County \\
\hline 121 & EnCana Oil \& Gas (USA) Inc. & LANG3WW & LANG3WW & OBSERVATION & Garfield County \\
\hline 122 & EnCana Oil \& Gas (USA) Inc. & LANG4WW & LANG4WW & OBSERVATION & Garfield County \\
\hline 123 & EnCana Oil \& Gas (USA) Inc. & LANG5WW & LANG5WW & OBSERVATION & Garfield County \\
\hline 124 & EnCana Oil \& Gas (USA) Inc. & LIGH1WW & LIGH1WW & DOMESTIC USE & Garfield County \\
\hline 125 & EnCana Oil \& Gas (USA) Inc. & MCPH1WW & MCPH1WW & DOMESTIC USE & Garfield County \\
\hline 126 & EnCana Oil \& Gas (USA) Inc. & MORT1WW & MORT1WW & DOMESTIC USE & Garfield County \\
\hline 127 & EnCana Oil \& Gas (USA) Inc. & ONEI1WW & ONEI1WW & DOMESTIC USE & Garfield County \\
\hline 128 & EnCana Oil \& Gas (USA) Inc. & VANO2WW & VANO2WW & DOMESTIC USE & Garfield County \\
\hline 129 & EnCana Oil \& Gas (USA) Inc. & VANO3WW & VANO3WW & DOMESTIC USE & Garfield County \\
\hline 130 & EnCana Oil \& Gas (USA) Inc. & RIPP1WW & RIPP1WW & DOMESTIC USE & Garfield County \\
\hline 131 & EnCana Oil \& Gas (USA) Inc. & RIPP2WW & RIPP2WW & DOMESTIC USE & Garfield County \\
\hline 132 & EnCana Oil \& Gas (USA) Inc. & STAU1WW & STAU1WW & DOMESTIC USE & Garfield County \\
\hline 133 & EnCana Oil \& Gas (USA) Inc. & STAU2WW & STAU2WW & DOMESTIC USE & Garfield County \\
\hline 134 & EnCana Oil \& Gas (USA) Inc. & THOM2WW & THOM2WW & DOMESTIC USE & Garfield County \\
\hline 135 & EnCana Oil \& Gas (USA) Inc. & THOM3WW & THOM3WW & OBSERVATION & Garfield County \\
\hline 138 & U.S. Geological Survey & 393309107200201 & SC00608909BBD & OBSERVATION & Garfield County \\
\hline 142 & U.S. Geological Survey & 392210108030500 & SC00809612AAC1 & AGRICULTURAL USE & Garfield County \\
\hline 143 & Colorado Oil and Gas Conservation Commission & 704196 & COGCC Facility ID:704196 & DOMESTIC USE & Garfield County \\
\hline
\end{tabular}


Appendix 1. Water Quality Data Used in This Report.-Continued

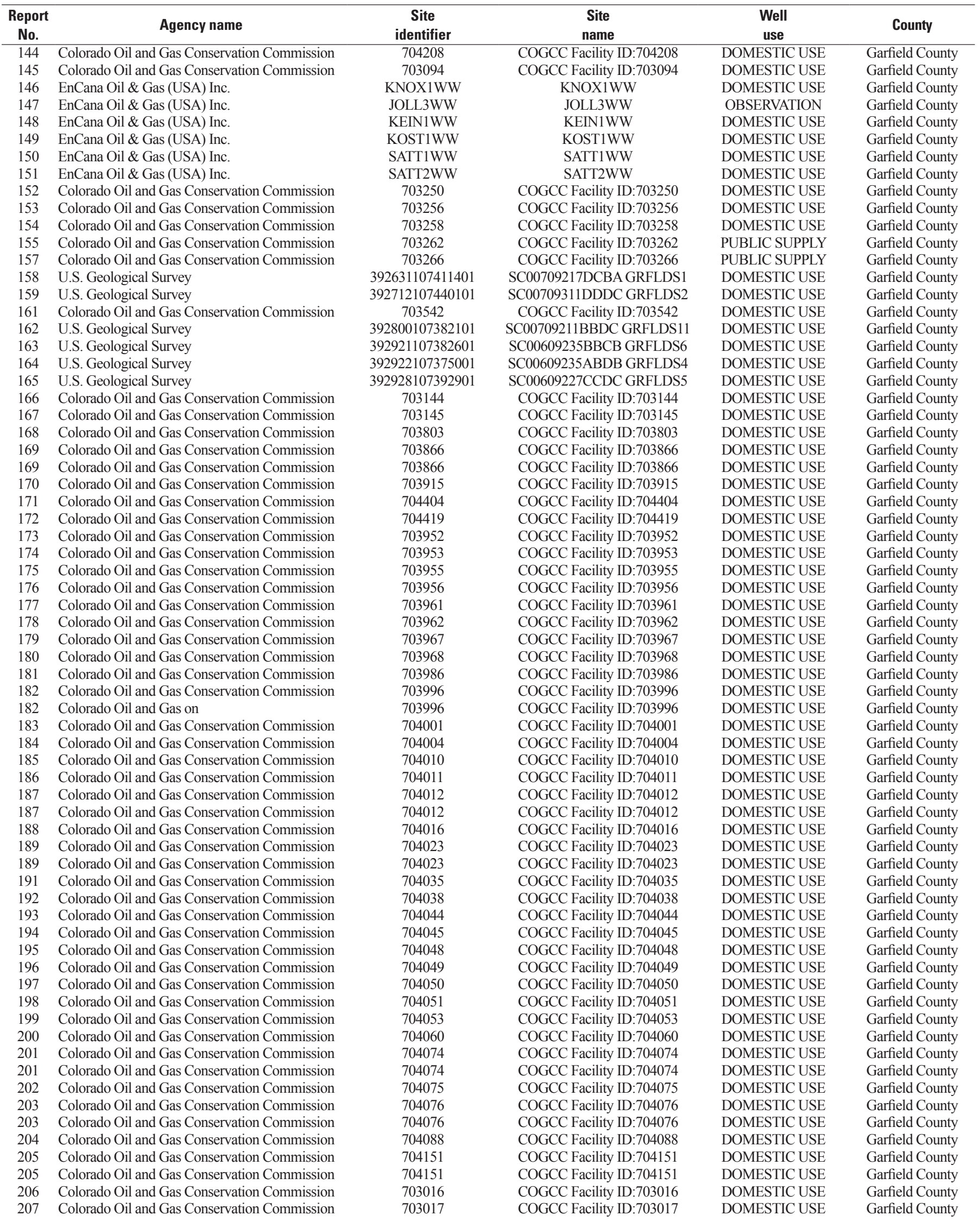


Appendix 1. Water Quality Data Used in This Report.-Continued

\begin{tabular}{|c|c|c|c|c|c|}
\hline $\begin{array}{c}\text { Report } \\
\text { No. }\end{array}$ & Agency name & $\begin{array}{c}\text { Site } \\
\text { identifier }\end{array}$ & $\begin{array}{c}\text { Site } \\
\text { name }\end{array}$ & $\begin{array}{l}\text { Well } \\
\text { use }\end{array}$ & County \\
\hline 208 & Colorado Oil and Gas Conservation Commission & 704164 & COGCC Facility ID:704164 & DOMESTIC USE & Garfield County \\
\hline 209 & Colorado Oil and Gas Conservation Commission & 703019 & COGCC Facility ID:703019 & DOMESTIC USE & Garfield County \\
\hline 211 & Colorado Oil and Gas Conservation Commission & 703021 & COGCC Facility ID:703021 & DOMESTIC USE & Garfield County \\
\hline 213 & Colorado Oil and Gas Conservation Commission & 704197 & COGCC Facility ID:704197 & DOMESTIC USE & Garfield County \\
\hline 214 & Colorado Oil and Gas Conservation Commission & 704222 & COGCC Facility ID:704222 & DOMESTIC USE & Garfield County \\
\hline 214 & Colorado Oil and Gas Conservation Commission & 704222 & COGCC Facility ID:704222 & DOMESTIC USE & Garfield County \\
\hline 215 & Colorado Oil and Gas Conservation Commission & 704329 & COGCC Facility ID:704329 & DOMESTIC USE & Garfield County \\
\hline 216 & Colorado Oil and Gas Conservation Commission & 704330 & COGCC Facility ID:704330 & DOMESTIC USE & Garfield County \\
\hline 216 & Colorado Oil and Gas Conservation Commission & 704330 & COGCC Facility ID:704330 & DOMESTIC USE & Garfield County \\
\hline 217 & Colorado Oil and Gas Conservation Commission & 704338 & COGCC Facility ID:704338 & DOMESTIC USE & Garfield County \\
\hline 218 & Colorado Oil and Gas Conservation Commission & 704343 & COGCC Facility ID:704343 & DOMESTIC USE & Garfield County \\
\hline 219 & Colorado Oil and Gas Conservation Commission & 704374 & COGCC Facility ID:704374 & DOMESTIC USE & Garfield County \\
\hline 220 & Colorado Oil and Gas Conservation Commission & 704392 & COGCC Facility ID:704392 & DOMESTIC USE & Garfield County \\
\hline 221 & Colorado Oil and Gas Conservation Commission & 704430 & COGCC Facility ID:704430 & DOMESTIC USE & Garfield County \\
\hline 222 & Colorado Oil and Gas Conservation Commission & 704434 & COGCC Facility ID:704434 & DOMESTIC USE & Garfield County \\
\hline 223 & Colorado Oil and Gas Conservation Commission & 703082 & COGCC Facility ID:703082 & DOMESTIC USE & Garfield County \\
\hline 224 & EnCana Oil \& Gas (USA) Inc. & DARD1WW & DARD1WW & DOMESTIC USE & Garfield County \\
\hline 225 & EnCana Oil \& Gas (USA) Inc. & DEXT1WW & DEXT1WW & DOMESTIC USE & Garfield County \\
\hline 226 & EnCana Oil \& Gas (USA) Inc. & $\operatorname{diet} 2 w w$ & DIET2WW & DOMESTIC USE & Garfield County \\
\hline 227 & Colorado Oil and Gas Conservation Commission & 703969 & COGCC Facility ID:703969 & DOMESTIC USE & Garfield County \\
\hline 228 & Colorado Oil and Gas Conservation Commission & 703983 & COGCC Facility ID:703983 & DOMESTIC USE & Garfield County \\
\hline 228 & Colorado Oil and Gas Conservation Commission & 703983 & COGCC Facility ID:703983 & DOMESTIC USE & Garfield County \\
\hline 229 & EnCana Oil \& Gas (USA) Inc. & DOLL1WW & DOLL1WW & DOMESTIC USE & Garfield County \\
\hline 230 & EnCana Oil \& Gas (USA) Inc. & DONE1WW & DONE1WW & DOMESTIC USE & Garfield County \\
\hline 231 & EnCana Oil \& Gas (USA) Inc. & DOWN1WW & DOWN1WW & DOMESTIC USE & Garfield County \\
\hline 232 & EnCana Oil \& Gas (USA) Inc. & DROS1WW & DROS1WW & DOMESTIC USE & Garfield County \\
\hline 233 & EnCana Oil \& Gas (USA) Inc. & DUMA1WW & DUMA1WW & DOMESTIC USE & Garfield County \\
\hline 234 & EnCana Oil \& Gas (USA) Inc. & DUNN1WW & DUNN1WW & DOMESTIC USE & Garfield County \\
\hline 235 & Colorado Oil and Gas Conservation Commission & 703084 & COGCC Facility ID:703084 & DOMESTIC USE & Garfield County \\
\hline 236 & Colorado Oil and Gas Conservation Commission & 703086 & COGCC Facility ID:703086 & DOMESTIC USE & Garfield County \\
\hline 237 & EnCana Oil \& Gas (USA) Inc. & EACH1WW & EACH1WW & DOMESTIC USE & Garfield County \\
\hline 238 & EnCana Oil \& Gas (USA) Inc. & ELDE1WW & ELDE1WW & DOMESTIC USE & Garfield County \\
\hline 239 & EnCana Oil \& Gas (USA) Inc. & ELDE2WW & ELDE2WW & DOMESTIC USE & Garfield County \\
\hline 240 & EnCana Oil \& Gas (USA) Inc. & ENRI1WW & ENRI1WW & DOMESTIC USE & Garfield County \\
\hline 241 & Colorado Oil and Gas Conservation Commission & 703147 & COGCC Facility ID:703147 & DOMESTIC USE & Garfield County \\
\hline 242 & Colorado Oil and Gas Conservation Commission & 703148 & COGCC Facility ID:703148 & DOMESTIC USE & Garfield County \\
\hline 243 & Colorado Oil and Gas Conservation Commission & 703182 & COGCC Facility ID:703182 & DOMESTIC USE & Garfield County \\
\hline 244 & Colorado Oil and Gas Conservation Commission & 703184 & COGCC Facility ID:703184 & DOMESTIC USE & Garfield County \\
\hline 245 & Colorado Oil and Gas Conservation Commission & 703187 & COGCC Facility ID:703187 & DOMESTIC USE & Garfield County \\
\hline 246 & EnCana Oil \& Gas (USA) Inc. & SPIN1WW & SPIN1WW & DOMESTIC USE & Garfield County \\
\hline 247 & EnCana Oil \& Gas (USA) Inc. & spin2ww & SPIN2WW & DOMESTIC USE & Garfield County \\
\hline 248 & Colorado Oil and Gas Conservation Commission & 704454 & COGCC Facility ID:704454 & DOMESTIC USE & Garfield County \\
\hline 249 & Colorado Oil and Gas Conservation Commission & 703260 & COGCC Facility ID:703260 & DOMESTIC USE & Garfield County \\
\hline 250 & Colorado Oil and Gas Conservation Commission & 704473 & COGCC Facility ID:704473 & DOMESTIC USE & Garfield County \\
\hline 251 & Colorado Oil and Gas Conservation Commission & 704477 & COGCC Facility ID:704477 & DOMESTIC USE & Garfield County \\
\hline 252 & Colorado Oil and Gas Conservation Commission & 704500 & COGCC Facility ID:704500 & DOMESTIC USE & Garfield County \\
\hline 253 & Colorado Oil and Gas Conservation Commission & 704516 & COGCC Facility ID:704516 & DOMESTIC USE & Garfield County \\
\hline 254 & Colorado Oil and Gas Conservation Commission & 704526 & COGCC Facility ID:704526 & DOMESTIC USE & Garfield County \\
\hline 255 & Colorado Oil and Gas Conservation Commission & 703900 & COGCC Facility ID:703900 & DOMESTIC USE & Garfield County \\
\hline 256 & Colorado Oil and Gas Conservation Commission & 704195 & COGCC Facility ID:704195 & DOMESTIC USE & Garfield County \\
\hline 256 & Colorado Oil and Gas Conservation Commission & 704195 & COGCC Facility ID:704195 & DOMESTIC USE & Garfield County \\
\hline 257 & Colorado Oil and Gas Conservation Commission & 704198 & COGCC Facility ID:704198 & DOMESTIC USE & Garfield County \\
\hline 258 & Colorado Oil and Gas Conservation Commission & 704288 & COGCC Facility ID:704288 & DOMESTIC USE & Garfield County \\
\hline 259 & Colorado Oil and Gas Conservation Commission & 704315 & COGCC Facility ID:704315 & PUBLIC SUPPLY & Garfield County \\
\hline 259 & Colorado Oil and Gas Conservation Commission & 704315 & COGCC Facility ID:704315 & PUBLIC SUPPLY & Garfield County \\
\hline 260 & Colorado Oil and Gas Conservation Commission & 704320 & COGCC Facility ID:704320 & DOMESTIC USE & Garfield County \\
\hline 261 & Colorado Oil and Gas Conservation Commission & 704322 & COGCC Facility ID:704322 & DOMESTIC USE & Garfield County \\
\hline 262 & Colorado Oil and Gas Conservation Commission & 704326 & COGCC Facility ID:704326 & DOMESTIC USE & Garfield County \\
\hline 263 & Colorado Oil and Gas Conservation Commission & 704331 & COGCC Facility ID:704331 & DOMESTIC USE & Garfield County \\
\hline 264 & Colorado Oil and Gas Conservation Commission & 704332 & COGCC Facility ID:704332 & DOMESTIC USE & Garfield County \\
\hline 264 & Colorado Oil and Gas Conservation Commission & 704332 & COGCC Facility ID:704332 & DOMESTIC USE & Garfield County \\
\hline 265 & Colorado Oil and Gas Conservation Commission & 704333 & COGCC Facility ID:704333 & DOMESTIC USE & Garfield County \\
\hline 266 & Colorado Oil and Gas Conservation Commission & 704340 & COGCC Facility ID:704340 & DOMESTIC USE & Garfield County \\
\hline 267 & EnCana Oil \& Gas (USA) Inc. & AABE1WW & AABE1WW & DOMESTIC USE & Garfield County \\
\hline 268 & EnCana Oil \& Gas (USA) Inc. & ANDE2WW & ANDE2WW & DOMESTIC USE & Garfield County \\
\hline 269 & EnCana Oil \& Gas (USA) Inc. & ANGE1WW & ANGE1WW & DOMESTIC USE & Garfield County \\
\hline 270 & EnCana Oil \& Gas (USA) Inc. & ANGE2WW & ANGE2WW & DOMESTIC USE & Garfield County \\
\hline
\end{tabular}


Appendix 1. Water Quality Data Used in This Report.-Continued

\begin{tabular}{|c|c|c|c|c|c|}
\hline $\begin{array}{c}\text { Report } \\
\text { No. }\end{array}$ & Agency name & $\begin{array}{c}\text { Site } \\
\text { identifier }\end{array}$ & $\begin{array}{c}\text { Site } \\
\text { name }\end{array}$ & $\begin{array}{l}\text { Well } \\
\text { use }\end{array}$ & County \\
\hline 271 & EnCana Oil \& Gas (USA) Inc. & ANGE3WW & ANGE3WW & AGRICULTURAL USE & Garfield County \\
\hline 272 & EnCana Oil \& Gas (USA) Inc. & DIET1WW & DIET1WW & DOMESTIC USE & Garfield County \\
\hline 273 & EnCana Oil \& Gas (USA) Inc. & dunc1ww & DUNC1WW & DOMESTIC USE & Garfield County \\
\hline 274 & EnCana Oil \& Gas (USA) Inc. & DUNC2WW & DUNC2WW & DOMESTIC USE & Garfield County \\
\hline 275 & EnCana Oil \& Gas (USA) Inc. & allm1ww & ALLM1WW & DOMESTIC USE & Garfield County \\
\hline 276 & EnCana Oil \& Gas (USA) Inc. & AMOS1WW & AMOS1WW & DOMESTIC USE & Garfield County \\
\hline 277 & EnCana Oil \& Gas (USA) Inc. & ARBA1WW & ARBA1WW & DOMESTIC USE & Garfield County \\
\hline 278 & EnCana Oil \& Gas (USA) Inc. & BELL1WW & BELL1WW & DOMESTIC USE & Garfield County \\
\hline 279 & EnCana Oil \& Gas (USA) Inc. & BELL2WW & BELL2WW & DOMESTIC USE & Garfield County \\
\hline 280 & EnCana Oil \& Gas (USA) Inc. & BELL3WW & BELL3WW & DOMESTIC USE & Garfield County \\
\hline 281 & EnCana Oil \& Gas (USA) Inc. & BUTT1WW & BUTT1WW & DOMESTIC USE & Garfield County \\
\hline 282 & EnCana Oil \& Gas (USA) Inc. & COTT1WW & COTT1WW & DOMESTIC USE & Garfield County \\
\hline 283 & EnCana Oil \& Gas (USA) Inc. & ENCA1ww & ENCA1WW & DOMESTIC USE & Garfield County \\
\hline 284 & EnCana Oil \& Gas (USA) Inc. & ESGA1WW & ESGA1WW & DOMESTIC USE & Garfield County \\
\hline 285 & EnCana Oil \& Gas (USA) Inc. & EUBA1WW & EUBA1WW & DOMESTIC USE & Garfield County \\
\hline 286 & EnCana Oil \& Gas (USA) Inc. & GAGN1WW & GAGN1WW & DOMESTIC USE & Garfield County \\
\hline 287 & EnCana Oil \& Gas (USA) Inc. & griflww & GRIF1WW & DOMESTIC USE & Garfield County \\
\hline 288 & EnCana Oil \& Gas (USA) Inc. & BAKE1WW & BAKE1WW & DOMESTIC USE & Garfield County \\
\hline 289 & EnCana Oil \& Gas (USA) Inc. & BALL1WW & BALL1WW & DOMESTIC USE & Garfield County \\
\hline 290 & EnCana Oil \& Gas (USA) Inc. & BARR1WW & BARR1WW & DOMESTIC USE & Garfield County \\
\hline 291 & EnCana Oil \& Gas (USA) Inc. & BELA1WW & BELA1WW & DOMESTIC USE & Garfield County \\
\hline 292 & EnCana Oil \& Gas (USA) Inc. & BELA2WW & BELA2WW & DOMESTIC USE & Garfield County \\
\hline 293 & EnCana Oil \& Gas (USA) Inc. & BELA3WW & BELA3WW & DOMESTIC USE & Garfield County \\
\hline 294 & EnCana Oil \& Gas (USA) Inc. & BELA4WW & BELA4WW & AGRICULTURAL USE & Garfield County \\
\hline 295 & EnCana Oil \& Gas (USA) Inc. & BELA5WW & BELA5WW & DOMESTIC USE & Garfield County \\
\hline 296 & EnCana Oil \& Gas (USA) Inc. & BENN1WW & BENN1WW & DOMESTIC USE & Garfield County \\
\hline 297 & EnCana Oil \& Gas (USA) Inc. & BILC1WW & BILC1WW & DOMESTIC USE & Garfield County \\
\hline 298 & EnCana Oil \& Gas (USA) Inc. & BILC2WW & BILC2WW & DOMESTIC USE & Garfield County \\
\hline 299 & EnCana Oil \& Gas (USA) Inc. & boullww & BOUL1WW & DOMESTIC USE & Garfield County \\
\hline 300 & EnCana Oil \& Gas (USA) Inc. & LLOY3WW & LLOY3WW & DOMESTIC USE & Garfield County \\
\hline 301 & EnCana Oil \& Gas (USA) Inc. & LLOY4WW & LLOY4WW & DOMESTIC USE & Garfield County \\
\hline 302 & EnCana Oil \& Gas (USA) Inc. & BUER1WW & BUER1WW & DOMESTIC USE & Garfield County \\
\hline 303 & EnCana Oil \& Gas (USA) Inc. & BUER2WW & BUER2WW & DOMESTIC USE & Garfield County \\
\hline 304 & EnCana Oil \& Gas (USA) Inc. & PAVI2WW & PAVI2WW & DOMESTIC USE & Garfield County \\
\hline 305 & EnCana Oil \& Gas (USA) Inc. & PRIC1WW & PRIC1WW & DOMESTIC USE & Garfield County \\
\hline 306 & EnCana Oil \& Gas (USA) Inc. & PRIC2WW & PRIC2WW & DOMESTIC USE & Garfield County \\
\hline 307 & EnCana Oil \& Gas (USA) Inc. & CAMP1WW & CAMP1WW & DOMESTIC USE & Garfield County \\
\hline 308 & EnCana Oil \& Gas (USA) Inc. & CANO1WW & CANO1WW & DOMESTIC USE & Garfield County \\
\hline 309 & EnCana Oil \& Gas (USA) Inc. & CHAP1WW & CHAP1WW & DOMESTIC USE & Garfield County \\
\hline 310 & EnCana Oil \& Gas (USA) Inc. & schwlww & SCHW1WW & DOMESTIC USE & Garfield County \\
\hline 311 & EnCana Oil \& Gas (USA) Inc. & COUE2WW & COUE2WW & DOMESTIC USE & Garfield County \\
\hline 312 & EnCana Oil \& Gas (USA) Inc. & COUE3WW & COUE3WW & DOMESTIC USE & Garfield County \\
\hline 313 & EnCana Oil \& Gas (USA) Inc. & CRAI1WW & CRAI1WW & DOMESTIC USE & Garfield County \\
\hline 314 & EnCana Oil \& Gas (USA) Inc. & CRAI2WW & CRAI2WW & DOMESTIC USE & Garfield County \\
\hline 315 & EnCana Oil \& Gas (USA) Inc. & SHAE3WW & SHAE3WW & DOMESTIC USE & Garfield County \\
\hline 316 & EnCana Oil \& Gas (USA) Inc. & SHAN1WW & SHAN1WW & DOMESTIC USE & Garfield County \\
\hline 317 & EnCana Oil \& Gas (USA) Inc. & CRUZ2WW & CRUZ2WW & DOMESTIC USE & Garfield County \\
\hline 318 & EnCana Oil \& Gas (USA) Inc. & CULL1WW & CULL1WW & DOMESTIC USE & Garfield County \\
\hline 319 & EnCana Oil \& Gas (USA) Inc. & curr1ww & CURR1WW & DOMESTIC USE & Garfield County \\
\hline 320 & EnCana Oil \& Gas (USA) Inc. & DAHL1WW & DAHL1WW & AGRICULTURAL USE & Garfield County \\
\hline 321 & EnCana Oil \& Gas (USA) Inc. & DALE3WW & DALE3WW & DOMESTIC USE & Garfield County \\
\hline 322 & EnCana Oil \& Gas (USA) Inc. & SHER2WW & SHER2WW & DOMESTIC USE & Garfield County \\
\hline 323 & EnCana Oil \& Gas (USA) Inc. & FAZZ1WW & FAZZ1WW & DOMESTIC USE & Garfield County \\
\hline 324 & EnCana Oil \& Gas (USA) Inc. & FERG1WW & FERG1WW & DOMESTIC USE & Garfield County \\
\hline 325 & EnCana Oil \& Gas (USA) Inc. & FILI1WW & FILI1WW & DOMESTIC USE & Garfield County \\
\hline 326 & EnCana Oil \& Gas (USA) Inc. & FILI2WW & FILI2WW & DOMESTIC USE & Garfield County \\
\hline 327 & EnCana Oil \& Gas (USA) Inc. & THOM1WW & THOM1WW & AGRICULTURAL USE & Garfield County \\
\hline 328 & EnCana Oil \& Gas (USA) Inc. & thom 4 ww & THOM4WW & DOMESTIC USE & Garfield County \\
\hline 329 & EnCana Oil \& Gas (USA) Inc. & GALL1WW & GALL1WW & AGRICULTURAL USE & Garfield County \\
\hline 330 & EnCana Oil \& Gas (USA) Inc. & GALL2WW & GALL2WW & DOMESTIC USE & Garfield County \\
\hline 331 & EnCana Oil \& Gas (USA) Inc. & GALL3WW & GALL3WW & DOMESTIC USE & Garfield County \\
\hline 332 & EnCana Oil \& Gas (USA) Inc. & TRUL1WW & TRUL1WW & DOMESTIC USE & Garfield County \\
\hline 333 & EnCana Oil \& Gas (USA) Inc. & vall1ww & VALL1WW & DOMESTIC USE & Garfield County \\
\hline 334 & EnCana Oil \& Gas (USA) Inc. & GARD1WW & GARD1WW & DOMESTIC USE & Garfield County \\
\hline 335 & EnCana Oil \& Gas (USA) Inc. & GEIS1WW & GEIS1WW & DOMESTIC USE & Garfield County \\
\hline
\end{tabular}


Appendix 1. Water Quality Data Used in This Report.-Continued

\begin{tabular}{|c|c|c|c|c|c|}
\hline $\begin{array}{c}\text { Report } \\
\text { No. }\end{array}$ & Agency name & $\begin{array}{c}\text { Site } \\
\text { identifier }\end{array}$ & $\begin{array}{c}\text { Site } \\
\text { name }\end{array}$ & $\begin{array}{c}\text { Well } \\
\text { use }\end{array}$ & County \\
\hline 336 & EnCana Oil \& Gas (USA) Inc. & GEIS2WW & GEIS2WW & DOMESTIC USE & Garfield County \\
\hline 337 & EnCana Oil \& Gas (USA) Inc. & GILK1WW & GILK1WW & DOMESTIC USE & Garfield County \\
\hline 338 & EnCana Oil \& Gas (USA) Inc. & GODA1WW & GODA1WW & DOMESTIC USE & Garfield County \\
\hline 339 & EnCana Oil \& Gas (USA) Inc. & GUTH1WW & GUTH1WW & DOMESTIC USE & Garfield County \\
\hline 340 & EnCana Oil \& Gas (USA) Inc. & HARR1WW & HARR1WW & DOMESTIC USE & Garfield County \\
\hline 341 & EnCana Oil \& Gas (USA) Inc. & HAYE1WW & HAYE1WW & DOMESTIC USE & Garfield County \\
\hline 342 & EnCana Oil \& Gas (USA) Inc. & HILL1WW & HILL1WW & DOMESTIC USE & Garfield County \\
\hline 343 & EnCana Oil \& Gas (USA) Inc. & HILL2WW & HILL2WW & DOMESTIC USE & Garfield County \\
\hline 344 & EnCana Oil \& Gas (USA) Inc. & HOFF1WW & HOFF1WW & DOMESTIC USE & Garfield County \\
\hline 345 & EnCana Oil \& Gas (USA) Inc. & HOFF2WW & HOFF2WW & DOMESTIC USE & Garfield County \\
\hline 346 & EnCana Oil \& Gas (USA) Inc. & HOLG1WW & HOLG1WW & DOMESTIC USE & Garfield County \\
\hline 347 & EnCana Oil \& Gas (USA) Inc. & HOOD1WW & HOOD1WW & DOMESTIC USE & Garfield County \\
\hline 348 & EnCana Oil \& Gas (USA) Inc. & HOWA1WW & HOWA1WW & DOMESTIC USE & Garfield County \\
\hline 349 & EnCana Oil \& Gas (USA) Inc. & KNIG1WW & KNIG1WW & DOMESTIC USE & Garfield County \\
\hline 350 & EnCana Oil \& Gas (USA) Inc. & KNIG2WW & KNIG2WW & DOMESTIC USE & Garfield County \\
\hline 351 & EnCana Oil \& Gas (USA) Inc. & KOCH1WW & KOCH1WW & DOMESTIC USE & Garfield County \\
\hline 352 & EnCana Oil \& Gas (USA) Inc. & KOZA1WW & KOZA1WW & DOMESTIC USE & Garfield County \\
\hline 353 & EnCana Oil \& Gas (USA) Inc. & LAND1WW & LAND1WW & DOMESTIC USE & Garfield County \\
\hline 354 & EnCana Oil \& Gas (USA) Inc. & meas1ww & MEAS1WW & DOMESTIC USE & Garfield County \\
\hline 355 & EnCana Oil \& Gas (USA) Inc. & Meas2ww & MEAS2WW & DOMESTIC USE & Garfield County \\
\hline 356 & EnCana Oil \& Gas (USA) Inc. & MEYE1WW & MEYE1WW & DOMESTIC USE & Garfield County \\
\hline 357 & EnCana Oil \& Gas (USA) Inc. & MILL1WW & MILL1WW & DOMESTIC USE & Garfield County \\
\hline 358 & EnCana Oil \& Gas (USA) Inc. & MILL4WW & MILL4WW & DOMESTIC USE & Garfield County \\
\hline 359 & EnCana Oil \& Gas (USA) Inc. & moon1ww & MOON1WW & OBSERVATION & Garfield County \\
\hline 360 & EnCana Oil \& Gas (USA) Inc. & MOON2WW & MOON2WW & DOMESTIC USE & Garfield County \\
\hline 361 & EnCana Oil \& Gas (USA) Inc. & MOOR1WW & MOOR1WW & DOMESTIC USE & Garfield County \\
\hline 362 & EnCana Oil \& Gas (USA) Inc. & LAYM1WW & LAYM1WW & DOMESTIC USE & Garfield County \\
\hline 363 & EnCana Oil \& Gas (USA) Inc. & LLOY1WW & LLOY1WW & DOMESTIC USE & Garfield County \\
\hline 364 & EnCana Oil \& Gas (USA) Inc. & LLOY2WW & LLOY2WW & DOMESTIC USE & Garfield County \\
\hline 365 & EnCana Oil \& Gas (USA) Inc. & LOUT1WW & LOUT1WW & DOMESTIC USE & Garfield County \\
\hline 366 & EnCana Oil \& Gas (USA) Inc. & MALO1WW & MALO1WW & DOMESTIC USE & Garfield County \\
\hline 367 & EnCana Oil \& Gas (USA) Inc. & MALO2WW & MALO2WW & DOMESTIC USE & Garfield County \\
\hline 368 & EnCana Oil \& Gas (USA) Inc. & MALO3WW & MALO3WW & DOMESTIC USE & Garfield County \\
\hline 369 & EnCana Oil \& Gas (USA) Inc. & MANU1WW & MANU1WW & DOMESTIC USE & Garfield County \\
\hline 370 & EnCana Oil \& Gas (USA) Inc. & MCCR1WW & MCCR1WW & DOMESTIC USE & Garfield County \\
\hline 371 & EnCana Oil \& Gas (USA) Inc. & MCCR2WW & MCCR2WW & DOMESTIC USE & Garfield County \\
\hline 372 & EnCana Oil \& Gas (USA) Inc. & MORR1WW & MORR1WW & DOMESTIC USE & Garfield County \\
\hline 373 & EnCana Oil \& Gas (USA) Inc. & OCON1WW & OCON1WW & DOMESTIC USE & Garfield County \\
\hline 374 & EnCana Oil \& Gas (USA) Inc. & PARA1WW & PARA1WW & DOMESTIC USE & Garfield County \\
\hline 375 & EnCana Oil \& Gas (USA) Inc. & PERI1WW & PERI1WW & DOMESTIC USE & Garfield County \\
\hline 376 & EnCana Oil \& Gas (USA) Inc. & PLAT1WW & PLAT1WW & DOMESTIC USE & Garfield County \\
\hline 377 & EnCana Oil \& Gas (USA) Inc. & PLAT2WW & PLAT2WW & DOMESTIC USE & Garfield County \\
\hline 378 & EnCana Oil \& Gas (USA) Inc. & WAGS1WW & WAGS1WW & DOMESTIC USE & Garfield County \\
\hline 379 & EnCana Oil \& Gas (USA) Inc. & WALL1WW & WALL1WW & DOMESTIC USE & Garfield County \\
\hline 380 & EnCana Oil \& Gas (USA) Inc. & WALT2WW & WALT2WW & DOMESTIC USE & Garfield County \\
\hline 381 & EnCana Oil \& Gas (USA) Inc. & WELL2WW & WELL2WW & DOMESTIC USE & Garfield County \\
\hline 382 & EnCana Oil \& Gas (USA) Inc. & RAIL1WW & RAIL1WW & DOMESTIC USE & Garfield County \\
\hline 383 & EnCana Oil \& Gas (USA) Inc. & RICH1WW & RICH1WW & DOMESTIC USE & Garfield County \\
\hline 384 & EnCana Oil \& Gas (USA) Inc. & RIVE1WW & RIVE1WW & DOMESTIC USE & Garfield County \\
\hline 385 & EnCana Oil \& Gas (USA) Inc. & ROE1WW & ROE1WW & DOMESTIC USE & Garfield County \\
\hline 386 & EnCana Oil \& Gas (USA) Inc. & ROLE1WW & ROLE1WW & DOMESTIC USE & Garfield County \\
\hline 387 & EnCana Oil \& Gas (USA) Inc. & ROWE1WW & ROWE1WW & DOMESTIC USE & Garfield County \\
\hline 388 & EnCana Oil \& Gas (USA) Inc. & ROWE2WW & ROWE2WW & DOMESTIC USE & Garfield County \\
\hline 389 & EnCana Oil \& Gas (USA) Inc. & ROWE3WW & ROWE3WW & DOMESTIC USE & Garfield County \\
\hline 390 & EnCana Oil \& Gas (USA) Inc. & ROWE4WW & ROWE4WW & DOMESTIC USE & Garfield County \\
\hline 391 & EnCana Oil \& Gas (USA) Inc. & SCHE1WW & SCHE1WW & DOMESTIC USE & Garfield County \\
\hline 392 & EnCana Oil \& Gas (USA) Inc. & SCHI1WW & SCHI1WW & DOMESTIC USE & Garfield County \\
\hline 393 & EnCana Oil \& Gas (USA) Inc. & SCHI2WW & SCHI2WW & DOMESTIC USE & Garfield County \\
\hline 394 & EnCana Oil \& Gas (USA) Inc. & SCHO1WW & SCHO1WW & DOMESTIC USE & Garfield County \\
\hline 395 & EnCana Oil \& Gas (USA) Inc. & SCHU1WW & SCHU1WW & DOMESTIC USE & Garfield County \\
\hline 396 & EnCana Oil \& Gas (USA) Inc. & SCHU2WW & SCHU2WW & DOMESTIC USE & Garfield County \\
\hline 397 & EnCana Oil \& Gas (USA) Inc. & SHAE1WW & SHAE1WW & DOMESTIC USE & Garfield County \\
\hline 398 & EnCana Oil \& Gas (USA) Inc. & SHAE2WW & SHAE2WW & AGRICULTURAL USE & Garfield County \\
\hline 399 & EnCana Oil \& Gas (USA) Inc. & SHID1WW & SHID1WW & DOMESTIC USE & Garfield County \\
\hline 400 & EnCana Oil \& Gas (USA) Inc. & SHID2WW & SHID2WW & DOMESTIC USE & Garfield County \\
\hline
\end{tabular}


Appendix 1. Water Quality Data Used in This Report.-Continued

\begin{tabular}{|c|c|c|c|c|c|}
\hline $\begin{array}{c}\text { Report } \\
\text { No. }\end{array}$ & Agency name & $\begin{array}{c}\text { Site } \\
\text { identifier }\end{array}$ & $\begin{array}{c}\text { Site } \\
\text { name }\end{array}$ & $\begin{array}{l}\text { Well } \\
\text { use }\end{array}$ & County \\
\hline 401 & EnCana Oil \& Gas (USA) Inc. & SMIT1WW & SMIT1WW & DOMESTIC USE & Garfield County \\
\hline 402 & EnCana Oil \& Gas (USA) Inc. & SMIT3WW & SMIT3WW & DOMESTIC USE & Garfield County \\
\hline 403 & EnCana Oil \& Gas (USA) Inc. & STAR1WW & STAR1WW & DOMESTIC USE & Garfield County \\
\hline 404 & EnCana Oil \& Gas (USA) Inc. & TURN1WW & TURN1WW & DOMESTIC USE & Garfield County \\
\hline 405 & EnCana Oil \& Gas (USA) Inc. & WILL2WW & WILL2WW & DOMESTIC USE & Garfield County \\
\hline 406 & EnCana Oil \& Gas (USA) Inc. & WILL4WW & WILL4WW & DOMESTIC USE & Garfield County \\
\hline 407 & EnCana Oil \& Gas (USA) Inc. & WING1WW & WING1WW & DOMESTIC USE & Garfield County \\
\hline 408 & U.S. Geological Survey & 392231108153203 & SC00809707ACC1 & DOMESTIC USE & Garfield County \\
\hline 409 & U.S. Geological Survey & 392242108213701 & SC00809807AAD & OBSERVATION & Garfield County \\
\hline 410 & U.S. Geological Survey & 392352108160601 & SC00709731CCB1 & DOMESTIC USE & Garfield County \\
\hline 414 & U.S. Geological Survey & 392859108192101 & SC00609833DCC & OBSERVATION & Garfield County \\
\hline 415 & U.S. Geological Survey & 392859108193901 & SC00609833DDD1 & DOMESTIC USE & Garfield County \\
\hline 416 & U.S. Geological Survey & 392923108191401 & SC00609834BDC1 & DOMESTIC USE & Garfield County \\
\hline 417 & U.S. Geological Survey & 392941108220801 & SC00609831ABD1 & DOMESTIC USE & Garfield County \\
\hline 418 & U.S. Geological Survey & 126166 & 126166 & OBSERVATION & Garfield County \\
\hline 419 & U.S. Geological Survey & 393836108002401 & SC00509501BDC1 & OBSERVATION & Garfield County \\
\hline 420 & U.S. Geological Survey & 393009108191601 & SC00609827CAB & OBSERVATION & Garfield County \\
\hline 428 & Colorado Oil and Gas Conservation Commission & 703240 & COGCC Facility ID:703240 & DOMESTIC USE & Garfield County \\
\hline 429 & Colorado Oil and Gas Conservation Commission & 703078 & COGCC Facility ID:703078 & DOMESTIC USE & Garfield County \\
\hline 430 & Colorado Oil and Gas Conservation Commission & 703104 & COGCC Facility ID:703104 & DOMESTIC USE & Garfield County \\
\hline 432 & Colorado Oil and Gas Conservation Commission & 703141 & COGCC Facility ID:703141 & DOMESTIC USE & Garfield County \\
\hline 433 & Colorado Oil and Gas Conservation Commission & 703142 & COGCC Facility ID:703142 & DOMESTIC USE & Garfield County \\
\hline 434 & Colorado Oil and Gas Conservation Commission & 704406 & COGCC Facility ID:704406 & DOMESTIC USE & Garfield County \\
\hline 435 & Colorado Oil and Gas Conservation Commission & 704407 & COGCC Facility ID:704407 & DOMESTIC USE & Garfield County \\
\hline 436 & Colorado Oil and Gas Conservation Commission & 704410 & COGCC Facility ID:704410 & DOMESTIC USE & Garfield County \\
\hline 437 & Colorado Oil and Gas Conservation Commission & 703023 & COGCC Facility ID:703023 & DOMESTIC USE & Garfield County \\
\hline 438 & Colorado Oil and Gas Conservation Commission & 703028 & COGCC Facility ID:703028 & DOMESTIC USE & Garfield County \\
\hline 439 & Colorado Oil and Gas Conservation Commission & 704267 & COGCC Facility ID:704267 & DOMESTIC USE & Garfield County \\
\hline 440 & Colorado Oil and Gas Conservation Commission & 703085 & COGCC Facility ID:703085 & DOMESTIC USE & Garfield County \\
\hline 441 & Colorado Oil and Gas Conservation Commission & 703140 & COGCC Facility ID:703140 & DOMESTIC USE & Garfield County \\
\hline 442 & Colorado Oil and Gas Conservation Commission & 704444 & COGCC Facility ID:704444 & DOMESTIC USE & Garfield County \\
\hline 443 & Colorado Oil and Gas Conservation Commission & 703907 & COGCC Facility ID:703907 & DOMESTIC USE & Garfield County \\
\hline 444 & Colorado Oil and Gas Conservation Commission & 704540 & COGCC Facility ID:704540 & DOMESTIC USE & Garfield County \\
\hline 445 & Colorado Oil and Gas Conservation Commission & 704555 & COGCC Facility ID:704555 & DOMESTIC USE & Garfield County \\
\hline 446 & Colorado Oil and Gas Conservation Commission & 704737 & COGCC Facility ID:704737 & DOMESTIC USE & Garfield County \\
\hline 447 & Colorado Oil and Gas Conservation Commission & 704738 & COGCC Facility ID:704738 & DOMESTIC USE & Garfield County \\
\hline 447 & Colorado Oil and Gas Conservation Commission & 704738 & COGCC Facility ID:704738 & DOMESTIC USE & Garfield County \\
\hline 448 & Colorado Oil and Gas Conservation Commission & 704739 & COGCC Facility ID:704739 & DOMESTIC USE & Garfield County \\
\hline 448 & Colorado Oil and Gas Conservation Commission & 704739 & COGCC Facility ID:704739 & DOMESTIC USE & Garfield County \\
\hline 449 & Colorado Oil and Gas Conservation Commission & 704736 & COGCC Facility ID:704736 & DOMESTIC USE & Garfield County \\
\hline 450 & Colorado Oil and Gas Conservation Commission & 704740 & COGCC Facility ID:704740 & DOMESTIC USE & Garfield County \\
\hline 451 & Colorado Oil and Gas Conservation Commission & 704741 & COGCC Facility ID:704741 & DOMESTIC USE & Garfield County \\
\hline 452 & Colorado Oil and Gas Conservation Commission & 704742 & COGCC Facility ID:704742 & DOMESTIC USE & Garfield County \\
\hline 453 & Colorado Oil and Gas Conservation Commission & 704743 & COGCC Facility ID:704743 & DOMESTIC USE & Garfield County \\
\hline 453 & Colorado Oil and Gas Conservation Commission & 704743 & COGCC Facility ID:704743 & DOMESTIC USE & Garfield County \\
\hline 454 & Colorado Oil and Gas Conservation Commission & 704744 & COGCC Facility ID:704744 & DOMESTIC USE & Garfield County \\
\hline 454 & Colorado Oil and Gas Conservation Commission & 704744 & COGCC Facility ID:704744 & DOMESTIC USE & Garfield County \\
\hline 455 & Colorado Department of Agriculture & WS-051 & WS-051CDOA & DOMESTIC USE & Garfield County \\
\hline 456 & Colorado Department of Agriculture & WS-049 & WS-049CDOA & DOMESTIC USE & Garfield County \\
\hline 457 & Colorado Oil and Gas Conservation Commission & 703192 & COGCC Facility ID:703192 & DOMESTIC USE & Garfield County \\
\hline 458 & Colorado Oil and Gas Conservation Commission & 703194 & COGCC Facility ID:703194 & DOMESTIC USE & Garfield County \\
\hline 459 & Colorado Oil and Gas Conservation Commission & 703195 & COGCC Facility ID:703195 & DOMESTIC USE & Garfield County \\
\hline 460 & Colorado Oil and Gas Conservation Commission & 703255 & COGCC Facility ID:703255 & DOMESTIC USE & Garfield County \\
\hline 461 & Colorado Oil and Gas Conservation Commission & 703259 & COGCC Facility ID:703259 & DOMESTIC USE & Garfield County \\
\hline 462 & Colorado Oil and Gas Conservation Commission & 703264 & COGCC Facility ID:703264 & DOMESTIC USE & Garfield County \\
\hline 463 & Colorado Oil and Gas Conservation Commission & 703267 & COGCC Facility ID:703267 & PUBLIC SUPPLY & Garfield County \\
\hline 464 & Colorado Oil and Gas Conservation Commission & 703268 & COGCC Facility ID:703268 & PUBLIC SUPPLY & Garfield County \\
\hline 465 & U.S. Geological Survey & 392848107520201 & SC00609434BCD1 (MWX-1) & OBSERVATION & Garfield County \\
\hline 466 & Colorado Oil and Gas Conservation Commission & 703048 & COGCC Facility ID:703048 & DOMESTIC USE & Garfield County \\
\hline 468 & Colorado Oil and Gas Conservation Commission & 703133 & COGCC Facility ID:703133 & DOMESTIC USE & Garfield County \\
\hline 469 & Colorado Oil and Gas Conservation Commission & 704427 & COGCC Facility ID:704427 & DOMESTIC USE & Garfield County \\
\hline 470 & Colorado Oil and Gas Conservation Commission & 703988 & COGCC Facility ID:703988 & DOMESTIC USE & Garfield County \\
\hline 471 & Colorado Oil and Gas Conservation Commission & 703991 & COGCC Facility ID:703991 & DOMESTIC USE & Garfield County \\
\hline 472 & Colorado Oil and Gas Conservation Commission & 703995 & COGCC Facility ID:703995 & DOMESTIC USE & Garfield County \\
\hline 473 & Colorado Oil and Gas Conservation Commission & 704017 & COGCC Facility ID:704017 & DOMESTIC USE & Garfield County \\
\hline 474 & Colorado Oil and Gas Conservation Commission & 704042 & COGCC Facility ID:704042 & DOMESTIC USE & Garfield County \\
\hline 475 & Colorado Oil and Gas Conservation Commission & 704052 & COGCC Facility ID:704052 & DOMESTIC USE & Garfield County \\
\hline
\end{tabular}


Appendix 1. Water Quality Data Used in This Report.-Continued

\begin{tabular}{|c|c|c|c|c|c|}
\hline $\begin{array}{c}\text { Report } \\
\text { No. }\end{array}$ & Agency name & $\begin{array}{c}\text { Site } \\
\text { identifier }\end{array}$ & $\begin{array}{c}\text { Site } \\
\text { name }\end{array}$ & $\begin{array}{l}\text { Well } \\
\text { use }\end{array}$ & County \\
\hline 476 & Colorado Oil and Gas Conservation Commission & 704063 & COGCC Facility ID:704063 & DOMESTIC USE & Garfield County \\
\hline 477 & Colorado Oil and Gas Conservation Commission & 704065 & COGCC Facility ID:704065 & DOMESTIC USE & Garfield County \\
\hline 478 & Colorado Oil and Gas Conservation Commission & 704067 & COGCC Facility ID:704067 & DOMESTIC USE & Garfield County \\
\hline 479 & Colorado Oil and Gas Conservation Commission & 704304 & COGCC Facility ID:704304 & OBSERVATION & Garfield County \\
\hline 480 & Colorado Oil and Gas Conservation Commission & 704306 & COGCC Facility ID:704306 & OBSERVATION & Garfield County \\
\hline 481 & Colorado Oil and Gas Conservation Commission & 704368 & COGCC Facility ID:704368 & DOMESTIC USE & Garfield County \\
\hline 482 & Colorado Oil and Gas Conservation Commission & 704376 & COGCC Facility ID:704376 & DOMESTIC USE & Garfield County \\
\hline 483 & Colorado Oil and Gas Conservation Commission & 704379 & COGCC Facility ID:704379 & DOMESTIC USE & Garfield County \\
\hline 484 & Colorado Oil and Gas Conservation Commission & 704396 & COGCC Facility ID:704396 & DOMESTIC USE & Garfield County \\
\hline 485 & Colorado Oil and Gas Conservation Commission & 704397 & COGCC Facility ID:704397 & DOMESTIC USE & Garfield County \\
\hline 486 & EnCana Oil \& Gas (USA) Inc. & DURN1WW & DURN1WW & DOMESTIC USE & Garfield County \\
\hline 487 & EnCana Oil \& Gas (USA) Inc. & ENLF1MW & ENLF1MW & OBSERVATION & Garfield County \\
\hline 488 & Colorado Oil and Gas Conservation Commission & 703120 & COGCC Facility ID:703120 & DOMESTIC USE & Garfield County \\
\hline 489 & Colorado Oil and Gas Conservation Commission & 703131 & COGCC Facility ID:703131 & DOMESTIC USE & Garfield County \\
\hline 490 & Colorado Oil and Gas Conservation Commission & 703134 & COGCC Facility ID:703134 & DOMESTIC USE & Garfield County \\
\hline 491 & Colorado Oil and Gas Conservation Commission & 703222 & COGCC Facility ID:703222 & DOMESTIC USE & Garfield County \\
\hline 492 & Colorado Oil and Gas Conservation Commission & 704443 & COGCC Facility ID:704443 & DOMESTIC USE & Garfield County \\
\hline 493 & Colorado Oil and Gas Conservation Commission & 703226 & COGCC Facility ID:703226 & DOMESTIC USE & Garfield County \\
\hline 494 & Colorado Oil and Gas Conservation Commission & 703235 & COGCC Facility ID:703235 & PUBLIC SUPPLY & Garfield County \\
\hline 495 & Colorado Oil and Gas Conservation Commission & 703245 & COGCC Facility ID:703245 & DOMESTIC USE & Garfield County \\
\hline 496 & Colorado Oil and Gas Conservation Commission & 704464 & COGCC Facility ID:704464 & DOMESTIC USE & Garfield County \\
\hline 497 & Colorado Oil and Gas Conservation Commission & 704509 & COGCC Facility ID:704509 & DOMESTIC USE & Garfield County \\
\hline 498 & Colorado Oil and Gas Conservation Commission & 704513 & COGCC Facility ID:704513 & DOMESTIC USE & Garfield County \\
\hline 499 & Colorado Oil and Gas Conservation Commission & 704514 & COGCC Facility ID:704514 & DOMESTIC USE & Garfield County \\
\hline 500 & Colorado Oil and Gas Conservation Commission & 704532 & COGCC Facility ID:704532 & DOMESTIC USE & Garfield County \\
\hline 501 & Colorado Oil and Gas Conservation Commission & 704103 & COGCC Facility ID:704103 & DOMESTIC USE & Garfield County \\
\hline 502 & Colorado Oil and Gas Conservation Commission & 704809 & COGCC Facility ID:704809 & DOMESTIC USE & Garfield County \\
\hline 503 & Colorado Oil and Gas Conservation Commission & 705430 & COGCC Facility ID:705430 & DOMESTIC USE & Garfield County \\
\hline 504 & EnCana Oil \& Gas (USA) Inc. & ANDE1WW & ANDE1WW & DOMESTIC USE & Garfield County \\
\hline 505 & EnCana Oil \& Gas (USA) Inc. & HUBB1WW & HUBB1WW & DOMESTIC USE & Garfield County \\
\hline 506 & EnCana Oil \& Gas (USA) Inc. & BARN1WW & BARN1WW & DOMESTIC USE & Garfield County \\
\hline 507 & EnCana Oil \& Gas (USA) Inc. & BECK1WW & BECK1WW & DOMESTIC USE & Garfield County \\
\hline 508 & EnCana Oil \& Gas (USA) Inc. & BEEC1WW & BEEC1WW & DOMESTIC USE & Garfield County \\
\hline 509 & EnCana Oil \& Gas (USA) Inc. & BENJ1WW & BENJ1WW & DOMESTIC USE & Garfield County \\
\hline 510 & EnCana Oil \& Gas (USA) Inc. & BENJ2WW & BENJ2WW & DOMESTIC USE & Garfield County \\
\hline 511 & EnCana Oil \& Gas (USA) Inc. & BLAC1WW & BLAC1WW & DOMESTIC USE & Garfield County \\
\hline 512 & EnCana Oil \& Gas (USA) Inc. & BOLA1WW & BOLA1WW & DOMESTIC USE & Garfield County \\
\hline 513 & EnCana Oil \& Gas (USA) Inc. & broclww & BROC1WW & DOMESTIC USE & Garfield County \\
\hline 514 & EnCana Oil \& Gas (USA) Inc. & ROSE1WW & ROSE1WW & DOMESTIC USE & Garfield County \\
\hline 515 & EnCana Oil \& Gas (USA) Inc. & CAND1WW & CAND1WW & DOMESTIC USE & Garfield County \\
\hline 516 & EnCana Oil \& Gas (USA) Inc. & CAPP1WW & CAPP1WW & DOMESTIC USE & Garfield County \\
\hline 517 & EnCana Oil \& Gas (USA) Inc. & CHAR1WW & CHAR1WW & DOMESTIC USE & Garfield County \\
\hline 518 & EnCana Oil \& Gas (USA) Inc. & CLOS1WW & CLOS1WW & DOMESTIC USE & Garfield County \\
\hline 519 & EnCana Oil \& Gas (USA) Inc. & CLOS2WW & CLOS2WW & DOMESTIC USE & Garfield County \\
\hline 520 & EnCana Oil \& Gas (USA) Inc. & FRAN2WW & FRAN2WW & DOMESTIC USE & Garfield County \\
\hline 521 & EnCana Oil \& Gas (USA) Inc. & HOGU1WW & HOGU1WW & DOMESTIC USE & Garfield County \\
\hline 522 & EnCana Oil \& Gas (USA) Inc. & HUNT1WW & HUNT1WW & DOMESTIC USE & Garfield County \\
\hline 523 & EnCana Oil \& Gas (USA) Inc. & ISLE1WW & ISLE1WW & DOMESTIC USE & Garfield County \\
\hline 524 & EnCana Oil \& Gas (USA) Inc. & JOHN1WW & JOHN1WW & DOMESTIC USE & Garfield County \\
\hline 525 & EnCana Oil \& Gas (USA) Inc. & KEHR1WW & KEHR1WW & DOMESTIC USE & Garfield County \\
\hline 526 & EnCana Oil \& Gas (USA) Inc. & LAKE1MW & LAKE1MW & OBSERVATION & Garfield County \\
\hline 527 & EnCana Oil \& Gas (USA) Inc. & LANG1WW & LANG1WW & DOMESTIC USE & Garfield County \\
\hline 528 & EnCana Oil \& Gas (USA) Inc. & MILL3WW & MILL3WW & DOMESTIC USE & Garfield County \\
\hline 529 & EnCana Oil \& Gas (USA) Inc. & ZILE1WW & ZILE1WW & DOMESTIC USE & Garfield County \\
\hline 530 & EnCana Oil \& Gas (USA) Inc. & ZIMM1WW & ZIMM1WW & DOMESTIC USE & Garfield County \\
\hline 531 & EnCana Oil \& Gas (USA) Inc. & OVER1WW & OVER1WW & DOMESTIC USE & Garfield County \\
\hline 532 & EnCana Oil \& Gas (USA) Inc. & PARL1WW & PARL1WW & DOMESTIC USE & Garfield County \\
\hline 533 & EnCana Oil \& Gas (USA) Inc. & RHIN1WW & RHIN1WW & DOMESTIC USE & Garfield County \\
\hline 534 & EnCana Oil \& Gas (USA) Inc. & SAVA1WW & SAVA1WW & DOMESTIC USE & Garfield County \\
\hline 535 & EnCana Oil \& Gas (USA) Inc. & SAVA3WW & SAVA3WW & AGRICULTURAL USE & Garfield County \\
\hline 536 & EnCana Oil \& Gas (USA) Inc. & SHER1WW & SHER1WW & DOMESTIC USE & Garfield County \\
\hline 537 & EnCana Oil \& Gas (USA) Inc. & SHUS1WW & SHUS1WW & DOMESTIC USE & Garfield County \\
\hline 538 & EnCana Oil \& Gas (USA) Inc. & SIMM1WW & SIMM1WW & DOMESTIC USE & Garfield County \\
\hline 539 & EnCana Oil \& Gas (USA) Inc. & SKEL1WW & SKEL1WW & DOMESTIC USE & Garfield County \\
\hline 540 & EnCana Oil \& Gas (USA) Inc. & STEI1WW & STEI1WW & DOMESTIC USE & Garfield County \\
\hline
\end{tabular}


Appendix 1. Water Quality Data Used in This Report.-Continued

\begin{tabular}{|c|c|c|c|c|c|}
\hline $\begin{array}{c}\text { Report } \\
\text { No. }\end{array}$ & Agency name & $\begin{array}{c}\text { Site } \\
\text { identifier }\end{array}$ & $\begin{array}{c}\text { Site } \\
\text { name }\end{array}$ & $\begin{array}{l}\text { Well } \\
\text { use }\end{array}$ & County \\
\hline 541 & EnCana Oil \& Gas (USA) Inc. & STOK1WW & STOK1WW & DOMESTIC USE & Garfield County \\
\hline 542 & EnCana Oil \& Gas (USA) Inc. & VALD1WW & VALD1WW & DOMESTIC USE & Garfield County \\
\hline 543 & EnCana Oil \& Gas (USA) Inc. & WILS2WW & WILS2WW & DOMESTIC USE & Garfield County \\
\hline 544 & U.S. Geological Survey & 392638108005501 & SC00709517AAB1 & OBSERVATION & Garfield County \\
\hline & & & USGS 392638108005501 & & \\
\hline 558 & U.S. Geological Survey & 392758108043301 & SC00709602DBB1 & DOMESTIC USE & Garfield County \\
\hline 559 & U.S. Geological Survey & 392813108044401 & SC00709602BDB1 & DOMESTIC USE & Garfield County \\
\hline 560 & U.S. Geological Survey & 392835108054900 & SC00609634CDB & OBSERVATION & Garfield County \\
\hline 561 & U.S. Geological Survey & 392838108053900 & SC00609634CAD & OBSERVATION & Garfield County \\
\hline 562 & Colorado Oil and Gas Conservation Commission & 703032 & COGCC Facility ID:703032 & DOMESTIC USE & Garfield County \\
\hline 563 & Colorado Oil and Gas Conservation Commission & 703040 & COGCC Facility ID:703040 & DOMESTIC USE & Garfield County \\
\hline 564 & U.S. Geological Survey & 392859108055300 & SC00609634BDB & OBSERVATION & Garfield County \\
\hline 565 & U.S. Geological Survey & 392900108053900 & SC00609634BDA & OBSERVATION & Garfield County \\
\hline 566 & U.S. Geological Survey & 392920108001400 & SC00609528CDD1 & COMMERCIAL USE & Garfield County \\
\hline 569 & U.S. Geological Survey & 392936108072500 & SC00609629DAA & OBSERVATION & Garfield County \\
\hline 570 & Colorado Oil and Gas Conservation Commission & 703091 & COGCC Facility ID:703091 & DOMESTIC USE & Garfield County \\
\hline 571 & Colorado Oil and Gas Conservation Commission & 703092 & COGCC Facility ID:703092 & DOMESTIC USE & Garfield County \\
\hline 572 & Colorado Oil and Gas Conservation Commission & 703039 & COGCC Facility ID:703039 & DOMESTIC USE & Garfield County \\
\hline 573 & Colorado Oil and Gas Conservation Commission & 703069 & COGCC Facility ID:703069 & DOMESTIC USE & Garfield County \\
\hline 574 & Colorado Oil and Gas Conservation Commission & 703070 & COGCC Facility ID:703070 & DOMESTIC USE & Garfield County \\
\hline 575 & EnCana Oil \& Gas (USA) Inc. & DEKA1 & DEKA1 & DOMESTIC USE & Garfield County \\
\hline 576 & Colorado Oil and Gas Conservation Commission & 703093 & COGCC Facility ID:703093 & DOMESTIC USE & Garfield County \\
\hline 577 & Colorado Oil and Gas Conservation Commission & 703096 & COGCC Facility ID:703096 & DOMESTIC USE & Garfield County \\
\hline 578 & Colorado Oil and Gas Conservation Commission & 703097 & COGCC Facility ID:703097 & DOMESTIC USE & Garfield County \\
\hline 579 & EnCana Oil \& Gas (USA) Inc. & ENYE1WW & ENYE1WW & DOMESTIC USE & Garfield County \\
\hline 580 & Colorado Oil and Gas Conservation Commission & 703164 & COGCC Facility ID:703164 & DOMESTIC USE & Garfield County \\
\hline 581 & Antero Resources & ANT3925381080135 & ANT3925381080135 & DOMESTIC USE & Garfield County \\
\hline 582 & Antero Resources & ANT3925391080145 & ANT3925391080145 & DOMESTIC USE & Garfield County \\
\hline 583 & Antero Resources & ANT3925481080108 & ANT3925481080108 & DOMESTIC USE & Garfield County \\
\hline 584 & Antero Resources & ANT3925481080128 & ANT3925481080128 & DOMESTIC USE & Garfield County \\
\hline 585 & Antero Resources & ANT3925491080052 & ANT3925491080052 & DOMESTIC USE & Garfield County \\
\hline 586 & Antero Resources & ANT3925491080139 & ANT3925491080139 & DOMESTIC USE & Garfield County \\
\hline 587 & Antero Resources & ANT3925501080112 & ANT3925501080112 & DOMESTIC USE & Garfield County \\
\hline 588 & Antero Resources & ANT3925511080108 & ANT3925511080108 & DOMESTIC USE & Garfield County \\
\hline 589 & Antero Resources & ANT3925511080124 & ANT3925511080124 & DOMESTIC USE & Garfield County \\
\hline 590 & Antero Resources & ANT3925531080145 & ANT3925531080145 & DOMESTIC USE & Garfield County \\
\hline 591 & Antero Resources & ANT3925561080144 & ANT3925561080144 & DOMESTIC USE & Garfield County \\
\hline 592 & Antero Resources & ANT3925601080133 & ANT3925601080133 & DOMESTIC USE & Garfield County \\
\hline 593 & Antero Resources & ANT3926031080140 & ANT3926031080140 & DOMESTIC USE & Garfield County \\
\hline 594 & Antero Resources & ANT3926041080145 & ANT3926041080145 & DOMESTIC USE & Garfield County \\
\hline 595 & Antero Resources & ANT3926041080151 & ANT3926041080151 & DOMESTIC USE & Garfield County \\
\hline 596 & Antero Resources & ANT3926221080054 & ANT3926221080054 & DOMESTIC USE & Garfield County \\
\hline 597 & Antero Resources & ANT3926351080051 & ANT3926351080051 & DOMESTIC USE & Garfield County \\
\hline 598 & Antero Resources & ANT3926371080056 & ANT3926371080056 & DOMESTIC USE & Garfield County \\
\hline 599 & Colorado Oil and Gas Conservation Commission & 705029 & COGCC Facility ID:705029 & DOMESTIC USE & Garfield County \\
\hline 600 & Colorado Oil and Gas Conservation Commission & 705051 & COGCC Facility ID:705051 & DOMESTIC USE & Garfield County \\
\hline 601 & EnCana Oil \& Gas (USA) Inc. & Gard3ww & GARD3WW & DOMESTIC USE & Garfield County \\
\hline 602 & EnCana Oil \& Gas (USA) Inc. & BREW1WW & BREW1WW & DOMESTIC USE & Garfield County \\
\hline 603 & EnCana Oil \& Gas (USA) Inc. & CAMP2WW & CAMP2WW & DOMESTIC USE & Garfield County \\
\hline 604 & EnCana Oil \& Gas (USA) Inc. & JOLL2WW & JOLL2WW & OBSERVATION & Garfield County \\
\hline 605 & EnCana Oil \& Gas (USA) Inc. & MCEL1 & MCEL1 & AGRICULTURAL USE & Garfield County \\
\hline 606 & EnCana Oil \& Gas (USA) Inc. & MCEL2 & MCEL2 & DOMESTIC USE & Garfield County \\
\hline 607 & EnCana Oil \& Gas (USA) Inc. & NOCK1WW & NOCK1WW & DOMESTIC USE & Garfield County \\
\hline 608 & EnCana Oil \& Gas (USA) Inc. & PAVI1WW & PAVI1WW & DOMESTIC USE & Garfield County \\
\hline 609 & EnCana Oil \& Gas (USA) Inc. & PAYT1ww & PAYT1WW & DOMESTIC USE & Garfield County \\
\hline 610 & EnCana Oil \& Gas (USA) Inc. & SAVA4WW & SAVA4WW & DOMESTIC USE & Garfield County \\
\hline 611 & EnCana Oil \& Gas (USA) Inc. & TOND1WW & TOND1WW & DOMESTIC USE & Garfield County \\
\hline 612 & EnCana Oil \& Gas (USA) Inc. & TOND2WW & TOND2WW & DOMESTIC USE & Garfield County \\
\hline 613 & EnCana Oil \& Gas (USA) Inc. & TOND3WW & TOND3WW & DOMESTIC USE & Garfield County \\
\hline 614 & EnCana Oil \& Gas (USA) Inc. & WINT1WW & WINT1WW & DOMESTIC USE & Garfield County \\
\hline 615 & Colorado Department of Agriculture & WS-048 & WS-048CDOA & DOMESTIC USE & Garfield County \\
\hline 616 & Colorado Oil and Gas Conservation Commission & 703198 & COGCC Facility ID:703198 & DOMESTIC USE & Garfield County \\
\hline 617 & Colorado Oil and Gas Conservation Commission & 703199 & COGCC Facility ID:703199 & DOMESTIC USE & Garfield County \\
\hline 623 & U.S. Geological Survey & 393237107450901 & SC00609311BCBC GRFLN2 & DOMESTIC USE & Garfield County \\
\hline 624 & U.S. Geological Survey & 393314107455801 & SC0060930CBDC GRFLN7 & DOMESTIC USE & Garfield County \\
\hline
\end{tabular}


Appendix 1. Water Quality Data Used in This Report.-Continued

\begin{tabular}{|c|c|c|c|c|c|}
\hline $\begin{array}{c}\text { Report } \\
\text { No. }\end{array}$ & Agency name & $\begin{array}{c}\text { Site } \\
\text { identifier }\end{array}$ & $\begin{array}{c}\text { Site } \\
\text { name }\end{array}$ & $\begin{array}{l}\text { Well } \\
\text { use }\end{array}$ & County \\
\hline 625 & Colorado Oil and Gas Conservation Commission & 703112 & COGCC Facility ID:703112 & DOMESTIC USE & Garfield County \\
\hline 626 & Colorado Oil and Gas Conservation Commission & 703113 & COGCC Facility ID:703113 & DOMESTIC USE & Garfield County \\
\hline 627 & U.S. Geological Survey & 393528107453601 & SC00509230BADB GRFLN1 & DOMESTIC USE & Garfield County \\
\hline 628 & Colorado Oil and Gas Conservation Commission & 703546 & COGCC Facility ID:703546 & DOMESTIC USE & Garfield County \\
\hline 629 & Colorado Oil and Gas Conservation Commission & 703964 & COGCC Facility ID:703964 & DOMESTIC USE & Garfield County \\
\hline 630 & Colorado Oil and Gas Conservation Commission & 703989 & COGCC Facility ID:703989 & DOMESTIC USE & Garfield County \\
\hline 631 & Colorado Oil and Gas Conservation Commission & 703990 & COGCC Facility ID:703990 & DOMESTIC USE & Garfield County \\
\hline 632 & Colorado Oil and Gas Conservation Commission & 704020 & COGCC Facility ID:704020 & DOMESTIC USE & Garfield County \\
\hline 633 & Colorado Oil and Gas Conservation Commission & 704064 & COGCC Facility ID:704064 & DOMESTIC USE & Garfield County \\
\hline 634 & Colorado Oil and Gas Conservation Commission & 703121 & COGCC Facility ID:703121 & DOMESTIC USE & Garfield County \\
\hline 635 & Colorado Oil and Gas Conservation Commission & 703123 & COGCC Facility ID:703123 & DOMESTIC USE & Garfield County \\
\hline 636 & Colorado Oil and Gas Conservation Commission & 703124 & COGCC Facility ID:703124 & DOMESTIC USE & Garfield County \\
\hline 637 & Colorado Oil and Gas Conservation Commission & 703178 & COGCC Facility ID:703178 & DOMESTIC USE & Garfield County \\
\hline 638 & Colorado Oil and Gas Conservation Commission & 703179 & COGCC Facility ID:703179 & DOMESTIC USE & Garfield County \\
\hline 639 & Colorado Oil and Gas Conservation Commission & 703180 & COGCC Facility ID:703180 & DOMESTIC USE & Garfield County \\
\hline 640 & Colorado Oil and Gas Conservation Commission & 704451 & COGCC Facility ID:704451 & DOMESTIC USE & Garfield County \\
\hline 641 & Colorado Oil and Gas Conservation Commission & 703232 & COGCC Facility ID:703232 & DOMESTIC USE & Garfield County \\
\hline 642 & Colorado Oil and Gas Conservation Commission & 703233 & COGCC Facility ID:703233 & PUBLIC SUPPLY & Garfield County \\
\hline 643 & Colorado Oil and Gas Conservation Commission & 704528 & COGCC Facility ID:704528 & DOMESTIC USE & Garfield County \\
\hline 644 & Colorado Oil and Gas Conservation Commission & 704537 & COGCC Facility ID:704537 & DOMESTIC USE & Garfield County \\
\hline 645 & Colorado Oil and Gas Conservation Commission & 704539 & COGCC Facility ID:704539 & DOMESTIC USE & Garfield County \\
\hline 646 & Colorado Oil and Gas Conservation Commission & 704722 & COGCC Facility ID:704722 & DOMESTIC USE & Garfield County \\
\hline 647 & Colorado Oil and Gas Conservation Commission & 704735 & COGCC Facility ID:704735 & DOMESTIC USE & Garfield County \\
\hline 649 & Colorado Oil and Gas Conservation Commission & 704758 & COGCC Facility ID:704758 & DOMESTIC USE & Garfield County \\
\hline 650 & Colorado Oil and Gas Conservation Commission & 704784 & COGCC Facility ID:704784 & DOMESTIC USE & Garfield County \\
\hline 651 & Colorado Oil and Gas Conservation Commission & 704785 & COGCC Facility ID:704785 & DOMESTIC USE & Garfield County \\
\hline 652 & Colorado Oil and Gas Conservation Commission & 704805 & COGCC Facility ID:704805 & DOMESTIC USE & Garfield County \\
\hline 653 & Colorado Oil and Gas Conservation Commission & 705431 & COGCC Facility ID:705431 & DOMESTIC USE & Garfield County \\
\hline 654 & Antero Resources & ANT3931221074602 & ANT3931221074602 & DOMESTIC USE & Garfield County \\
\hline 655 & Antero Resources & ANT3931231074601 & ANT3931231074601 & DOMESTIC USE & Garfield County \\
\hline 656 & Antero Resources & ANT3931391074513 & ANT3931391074513 & DOMESTIC USE & Garfield County \\
\hline 657 & Antero Resources & ANT3931541074524 & ANT3931541074524 & DOMESTIC USE & Garfield County \\
\hline 658 & Antero Resources & ANT3932021074527 & ANT3932021074527 & DOMESTIC USE & Garfield County \\
\hline 660 & Colorado Oil and Gas Conservation Commission & 704723 & COGCC Facility ID:704723 & DOMESTIC USE & Garfield County \\
\hline 660 & Colorado Oil and Gas Conservation Commission & 704723 & COGCC Facility ID:704723 & DOMESTIC USE & Garfield County \\
\hline 661 & Antero Resources & ANT3932101074552 & ANT3932101074552 & DOMESTIC USE & Garfield County \\
\hline 662 & Colorado Oil and Gas Conservation Commission & 704759 & COGCC Facility ID:704759 & DOMESTIC USE & Garfield County \\
\hline 663 & Colorado Oil and Gas Conservation Commission & 704760 & COGCC Facility ID:704760 & DOMESTIC USE & Garfield County \\
\hline 663 & Colorado Oil and Gas Conservation Commission & 704760 & COGCC Facility ID:704760 & DOMESTIC USE & Garfield County \\
\hline 664 & Antero Resources & ANT3932171074514 & ANT3932171074514 & DOMESTIC USE & Garfield County \\
\hline 665 & Colorado Oil and Gas Conservation Commission & 704783 & COGCC Facility ID:704783 & DOMESTIC USE & Garfield County \\
\hline 666 & Colorado Oil and Gas Conservation Commission & 704795 & COGCC Facility ID:704795 & DOMESTIC USE & Garfield County \\
\hline 667 & Colorado Oil and Gas Conservation Commission & 704798 & COGCC Facility ID:704798 & DOMESTIC USE & Garfield County \\
\hline 668 & Colorado Oil and Gas Conservation Commission & 704799 & COGCC Facility ID:704799 & DOMESTIC USE & Garfield County \\
\hline 669 & Antero Resources & ANT3932191074528 & ANT3932191074528 & DOMESTIC USE & Garfield County \\
\hline 670 & Colorado Oil and Gas Conservation Commission & 704800 & COGCC Facility ID:704800 & DOMESTIC USE & Garfield County \\
\hline 671 & Colorado Oil and Gas Conservation Commission & 704801 & COGCC Facility ID:704801 & DOMESTIC USE & Garfield County \\
\hline 672 & Colorado Oil and Gas Conservation Commission & 704802 & COGCC Facility ID:704802 & DOMESTIC USE & Garfield County \\
\hline 673 & Colorado Oil and Gas Conservation Commission & 704807 & COGCC Facility ID:704807 & DOMESTIC USE & Garfield County \\
\hline 674 & Colorado Oil and Gas Conservation Commission & 704813 & COGCC Facility ID:704813 & DOMESTIC USE & Garfield County \\
\hline 675 & Antero Resources & ANT3932211074530 & ANT3932211074530 & DOMESTIC USE & Garfield County \\
\hline 676 & Antero Resources & ANT3933321074537 & ANT3933321074537 & DOMESTIC USE & Garfield County \\
\hline 677 & EnCana Oil \& Gas (USA) Inc. & HUFF1WW & HUFF1WW & DOMESTIC USE & Garfield County \\
\hline 678 & EnCana Oil \& Gas (USA) Inc. & BUSB1WW & BUSB1WW & DOMESTIC USE & Garfield County \\
\hline 679 & EnCana Oil \& Gas (USA) Inc. & DANI1WW & DANI1WW & DOMESTIC USE & Garfield County \\
\hline 680 & EnCana Oil \& Gas (USA) Inc. & FRAN1WW & FRAN1WW & DOMESTIC USE & Garfield County \\
\hline 681 & EnCana Oil \& Gas (USA) Inc. & HASE1WW & HASE1WW & DOMESTIC USE & Garfield County \\
\hline 682 & EnCana Oil \& Gas (USA) Inc. & JEWE2WW & JEWE2WW & DOMESTIC USE & Garfield County \\
\hline 683 & EnCana Oil \& Gas (USA) Inc. & WUSS1WW & WUSS1WW & DOMESTIC USE & Garfield County \\
\hline 684 & EnCana Oil \& Gas (USA) Inc. & LATR1WW & LATR1WW & DOMESTIC USE & Garfield County \\
\hline 685 & EnCana Oil \& Gas (USA) Inc. & MAY1WW & MAY1WW & DOMESTIC USE & Garfield County \\
\hline 686 & EnCana Oil \& Gas (USA) Inc. & MCKE1WW & MCKE1WW & DOMESTIC USE & Garfield County \\
\hline 687 & EnCana Oil \& Gas (USA) Inc. & PETR1WW & PETR1WW & DOMESTIC USE & Garfield County \\
\hline 688 & EnCana Oil \& Gas (USA) Inc. & WEIN1WW & WEIN1WW & DOMESTIC USE & Garfield County \\
\hline 689 & EnCana Oil \& Gas (USA) Inc. & RENN1WW & RENN1WW & DOMESTIC USE & Garfield County \\
\hline 690 & EnCana Oil \& Gas (USA) Inc. & STEW1WW & STEW1WW & DOMESTIC USE & Garfield County \\
\hline 691 & U.S. Geological Survey & 393746107574801 & SC00509508ACC1 & OBSERVATION & Garfield County \\
\hline
\end{tabular}


Appendix 1. Water Quality Data Used in This Report.-Continued

\begin{tabular}{|c|c|c|c|c|c|}
\hline $\begin{array}{c}\text { Report } \\
\text { No. }\end{array}$ & Agency name & $\begin{array}{c}\text { Site } \\
\text { identifier }\end{array}$ & $\begin{array}{c}\text { Site } \\
\text { name }\end{array}$ & $\begin{array}{c}\text { Well } \\
\text { use }\end{array}$ & County \\
\hline 692 & U.S. Geological Survey & 392421107565200 & R-EX (RULISON & OBSERVATION & Garfield County \\
\hline & & & EXPLORATORY HOLE) & & \\
\hline 693 & Colorado Oil and Gas Conservation Commission & 703190 & COGCC Facility ID:703190 & DOMESTIC USE & Garfield County \\
\hline 694 & Colorado Oil and Gas Conservation Commission & 703201 & COGCC Facility ID:703201 & DOMESTIC USE & Garfield County \\
\hline 695 & Colorado Oil and Gas Conservation Commission & 703224 & COGCC Facility ID:703224 & DOMESTIC USE & Garfield County \\
\hline 696 & Colorado Oil and Gas Conservation Commission & 703225 & COGCC Facility ID:703225 & DOMESTIC USE & Garfield County \\
\hline 699 & Colorado Oil and Gas Conservation Commission & 703528 & COGCC Facility ID:703528 & PUBLIC SUPPLY & Garfield County \\
\hline 704 & U.S. Geological Survey & 392854107560000 & SC00609431BDC1 & COMMERCIAL USE & Garfield County \\
\hline 706 & Colorado Oil and Gas Conservation Commission & 703042 & COGCC Facility ID:703042 & DOMESTIC USE & Garfield County \\
\hline 707 & Colorado Oil and Gas Conservation Commission & 703044 & COGCC Facility ID:703044 & DOMESTIC USE & Garfield County \\
\hline 708 & Colorado Oil and Gas Conservation Commission & 703052 & COGCC Facility ID:703052 & DOMESTIC USE & Garfield County \\
\hline 709 & Colorado Oil and Gas Conservation Commission & 703054 & COGCC Facility ID:703054 & DOMESTIC USE & Garfield County \\
\hline 710 & Colorado Oil and Gas Conservation Commission & 703057 & COGCC Facility ID:703057 & DOMESTIC USE & Garfield County \\
\hline 711 & Colorado Oil and Gas Conservation Commission & 703063 & COGCC Facility ID:703063 & DOMESTIC USE & Garfield County \\
\hline 712 & Colorado Oil and Gas Conservation Commission & 703074 & COGCC Facility ID:703074 & DOMESTIC USE & Garfield County \\
\hline 714 & Colorado Oil and Gas Conservation Commission & 703075 & COGCC Facility ID:703075 & DOMESTIC USE & Garfield County \\
\hline 715 & Colorado Oil and Gas Conservation Commission & 703127 & COGCC Facility ID:703127 & DOMESTIC USE & Garfield County \\
\hline 716 & Colorado Oil and Gas Conservation Commission & 703135 & COGCC Facility ID:703135 & DOMESTIC USE & Garfield County \\
\hline 717 & Colorado Oil and Gas Conservation Commission & 703136 & COGCC Facility ID:703136 & DOMESTIC USE & Garfield County \\
\hline 718 & Colorado Oil and Gas Conservation Commission & 703138 & COGCC Facility ID:703138 & DOMESTIC USE & Garfield County \\
\hline 719 & Colorado Oil and Gas Conservation Commission & 703034 & COGCC Facility ID:703034 & DOMESTIC USE & Garfield County \\
\hline 720 & Colorado Oil and Gas Conservation Commission & 703035 & COGCC Facility ID:703035 & DOMESTIC USE & Garfield County \\
\hline 721 & Colorado Oil and Gas Conservation Commission & 704305 & COGCC Facility ID:704305 & OBSERVATION & Garfield County \\
\hline 722 & Colorado Oil and Gas Conservation Commission & 704307 & COGCC Facility ID:704307 & OBSERVATION & Garfield County \\
\hline 723 & Colorado Oil and Gas Conservation Commission & 704308 & COGCC Facility ID:704308 & OBSERVATION & Garfield County \\
\hline 724 & Colorado Oil and Gas Conservation Commission & 704309 & COGCC Facility ID:704309 & OBSERVATION & Garfield County \\
\hline 725 & Colorado Oil and Gas Conservation Commission & 704312 & COGCC Facility ID:704312 & OBSERVATION & Garfield County \\
\hline 726 & Colorado Oil and Gas Conservation Commission & 704364 & COGCC Facility ID:704364 & AGRICULTURAL USE & Garfield County \\
\hline 727 & Colorado Oil and Gas Conservation Commission & 703041 & COGCC Facility ID:703041 & DOMESTIC USE & Garfield County \\
\hline 728 & Colorado Oil and Gas Conservation Commission & 703043 & COGCC Facility ID:703043 & DOMESTIC USE & Garfield County \\
\hline 729 & Colorado Oil and Gas Conservation Commission & 703045 & COGCC Facility ID:703045 & DOMESTIC USE & Garfield County \\
\hline 730 & Colorado Oil and Gas Conservation Commission & 703046 & COGCC Facility ID:703046 & DOMESTIC USE & Garfield County \\
\hline 731 & Colorado Oil and Gas Conservation Commission & 703049 & COGCC Facility ID:703049 & DOMESTIC USE & Garfield County \\
\hline 732 & Colorado Oil and Gas Conservation Commission & 703050 & COGCC Facility ID:703050 & DOMESTIC USE & Garfield County \\
\hline 733 & Colorado Oil and Gas Conservation Commission & 703051 & COGCC Facility ID:703051 & DOMESTIC USE & Garfield County \\
\hline 734 & Colorado Oil and Gas Conservation Commission & 703053 & COGCC Facility ID:703053 & DOMESTIC USE & Garfield County \\
\hline 735 & Colorado Oil and Gas Conservation Commission & 703055 & COGCC Facility ID:703055 & DOMESTIC USE & Garfield County \\
\hline 736 & Colorado Oil and Gas Conservation Commission & 703056 & COGCC Facility ID:703056 & DOMESTIC USE & Garfield County \\
\hline 737 & Colorado Oil and Gas Conservation Commission & 703058 & COGCC Facility ID:703058 & DOMESTIC USE & Garfield County \\
\hline 738 & Colorado Oil and Gas Conservation Commission & 703059 & COGCC Facility ID:703059 & DOMESTIC USE & Garfield County \\
\hline 739 & Colorado Oil and Gas Conservation Commission & 703060 & COGCC Facility ID:703060 & DOMESTIC USE & Garfield County \\
\hline 740 & Colorado Oil and Gas Conservation Commission & 703061 & COGCC Facility ID:703061 & DOMESTIC USE & Garfield County \\
\hline 741 & Colorado Oil and Gas Conservation Commission & 703062 & COGCC Facility ID:703062 & DOMESTIC USE & Garfield County \\
\hline 742 & Colorado Oil and Gas Conservation Commission & 703064 & COGCC Facility ID:703064 & DOMESTIC USE & Garfield County \\
\hline 743 & Colorado Oil and Gas Conservation Commission & 703098 & COGCC Facility ID:703098 & DOMESTIC USE & Garfield County \\
\hline 744 & Colorado Oil and Gas Conservation Commission & 703099 & COGCC Facility ID:703099 & DOMESTIC USE & Garfield County \\
\hline 745 & Colorado Oil and Gas Conservation Commission & 703100 & COGCC Facility ID:703100 & DOMESTIC USE & Garfield County \\
\hline 746 & Colorado Oil and Gas Conservation Commission & 703114 & COGCC Facility ID:703114 & DOMESTIC USE & Garfield County \\
\hline 747 & Colorado Oil and Gas Conservation Commission & 703115 & COGCC Facility ID:703115 & DOMESTIC USE & Garfield County \\
\hline 748 & Colorado Oil and Gas Conservation Commission & 703116 & COGCC Facility ID:703116 & DOMESTIC USE & Garfield County \\
\hline 749 & Colorado Oil and Gas Conservation Commission & 703117 & COGCC Facility ID:703117 & DOMESTIC USE & Garfield County \\
\hline 750 & Colorado Oil and Gas Conservation Commission & 703118 & COGCC Facility ID:703118 & DOMESTIC USE & Garfield County \\
\hline 751 & Colorado Oil and Gas Conservation Commission & 703119 & COGCC Facility ID:703119 & DOMESTIC USE & Garfield County \\
\hline 752 & Colorado Oil and Gas Conservation Commission & 703122 & COGCC Facility ID:703122 & DOMESTIC USE & Garfield County \\
\hline 753 & Colorado Oil and Gas Conservation Commission & 703125 & COGCC Facility ID:703125 & DOMESTIC USE & Garfield County \\
\hline 754 & Colorado Oil and Gas Conservation Commission & 703126 & COGCC Facility ID:703126 & DOMESTIC USE & Garfield County \\
\hline 755 & Colorado Oil and Gas Conservation Commission & 703128 & COGCC Facility ID:703128 & DOMESTIC USE & Garfield County \\
\hline 756 & Colorado Oil and Gas Conservation Commission & 703129 & COGCC Facility ID:703129 & PUBLIC SUPPLY & Garfield County \\
\hline 757 & Colorado Oil and Gas Conservation Commission & 703130 & COGCC Facility ID:703130 & DOMESTIC USE & Garfield County \\
\hline 758 & Colorado Oil and Gas Conservation Commission & 703137 & COGCC Facility ID:703137 & DOMESTIC USE & Garfield County \\
\hline 759 & Colorado Oil and Gas Conservation Commission & 703139 & COGCC Facility ID:703139 & DOMESTIC USE & Garfield County \\
\hline 760 & Colorado Oil and Gas Conservation Commission & 703161 & COGCC Facility ID:703161 & DOMESTIC USE & Garfield County \\
\hline 761 & Colorado Oil and Gas Conservation Commission & 703162 & COGCC Facility ID:703162 & DOMESTIC USE & Garfield County \\
\hline 762 & Colorado Oil and Gas Conservation Commission & 703163 & COGCC Facility ID:703163 & DOMESTIC USE & Garfield County \\
\hline 763 & Colorado Oil and Gas Conservation Commission & 703165 & COGCC Facility ID:703165 & DOMESTIC USE & Garfield County \\
\hline 764 & Colorado Oil and Gas Conservation Commission & 703166 & COGCC Facility ID:703166 & DOMESTIC USE & Garfield County \\
\hline 765 & Colorado Oil and Gas Conservation Commission & 703193 & COGCC Facility ID:703193 & DOMESTIC USE & Garfield County \\
\hline
\end{tabular}


Appendix 1. Water Quality Data Used in This Report.-Continued

\begin{tabular}{|c|c|c|c|c|c|}
\hline $\begin{array}{c}\text { Report } \\
\text { No. }\end{array}$ & Agency name & $\begin{array}{c}\text { Site } \\
\text { identifier }\end{array}$ & $\begin{array}{c}\text { Site } \\
\text { name }\end{array}$ & $\begin{array}{l}\text { Well } \\
\text { use }\end{array}$ & County \\
\hline 766 & Colorado Oil and Gas Conservation Commission & 704660 & COGCC Facility ID:704660 & DOMESTIC USE & Garfield County \\
\hline 767 & Colorado Oil and Gas Conservation Commission & 704664 & COGCC Facility ID:704664 & DOMESTIC USE & Garfield County \\
\hline 768 & Colorado Oil and Gas Conservation Commission & 704671 & COGCC Facility ID:704671 & DOMESTIC USE & Garfield County \\
\hline 769 & Colorado Oil and Gas Conservation Commission & 704672 & COGCC Facility ID:704672 & DOMESTIC USE & Garfield County \\
\hline 770 & Colorado Oil and Gas Conservation Commission & 704674 & COGCC Facility ID:704674 & DOMESTIC USE & Garfield County \\
\hline 771 & Colorado Oil and Gas Conservation Commission & 704676 & COGCC Facility ID:704676 & DOMESTIC USE & Garfield County \\
\hline 772 & Colorado Oil and Gas Conservation Commission & 704677 & COGCC Facility ID:704677 & DOMESTIC USE & Garfield County \\
\hline 773 & Colorado Oil and Gas Conservation Commission & 704678 & COGCC Facility ID:704678 & DOMESTIC USE & Garfield County \\
\hline 774 & Colorado Oil and Gas Conservation Commission & 704685 & COGCC Facility ID:704685 & DOMESTIC USE & Garfield County \\
\hline 775 & EnCana Oil \& Gas (USA) Inc. & beas1WW & BEAS1WW & DOMESTIC USE & Garfield County \\
\hline 776 & EnCana Oil \& Gas (USA) Inc. & BROD1WW & BROD1WW & DOMESTIC USE & Garfield County \\
\hline 777 & EnCana Oil \& Gas (USA) Inc. & BURD1WW & BURD1WW & DOMESTIC USE & Garfield County \\
\hline 778 & EnCana Oil \& Gas (USA) Inc. & FRAN3WW & FRAN3WW & DOMESTIC USE & Garfield County \\
\hline 779 & URS Corporation & WMS-110187 & WMS-110187 & DOMESTIC USE & Garfield County \\
\hline 780 & URS Corporation & WMS-110699 & WMS-110699 & DOMESTIC USE & Garfield County \\
\hline 781 & URS Corporation & WMS-131809-A & WMS-131809-A & DOMESTIC USE & Garfield County \\
\hline 782 & EnCana Oil \& Gas (USA) Inc. & HANS1WW & HANS1WW & DOMESTIC USE & Garfield County \\
\hline 783 & URS Corporation & WMS-189716 & WMS-189716 & DOMESTIC USE & Garfield County \\
\hline 784 & URS Corporation & WMS-194692 & WMS-194692 & DOMESTIC USE & Garfield County \\
\hline 785 & URS Corporation & WMS-202849 & WMS-202849 & AGRICULTURAL USE & Garfield County \\
\hline 786 & URS Corporation & WMS-205725 & WMS-205725 & DOMESTIC USE & Garfield County \\
\hline 787 & URS Corporation & WMS-218976 & WMS-218976 & DOMESTIC USE & Garfield County \\
\hline 788 & URS Corporation & WMS-55273 & WMS-55273 & DOMESTIC USE & Garfield County \\
\hline 789 & URS Corporation & WMS-62642 & WMS-62642 & DOMESTIC USE & Garfield County \\
\hline 790 & URS Corporation & WMS-71080 & WMS-71080 & DOMESTIC USE & Garfield County \\
\hline 791 & EnCana Oil \& Gas (USA) Inc. & HIRN1WW & HIRN1WW & DOMESTIC USE & Garfield County \\
\hline 792 & EnCana Oil \& Gas (USA) Inc. & LEBO1WW & LEBO1WW & DOMESTIC USE & Garfield County \\
\hline 793 & EnCana Oil \& Gas (USA) Inc. & MAHA1WW & MAHA1WW & DOMESTIC USE & Garfield County \\
\hline 794 & EnCana Oil \& Gas (USA) Inc. & Rill1WW & RILL1WW & DOMESTIC USE & Garfield County \\
\hline 795 & EnCana Oil \& Gas (USA) Inc. & sacc1WW & SACC1WW & DOMESTIC USE & Garfield County \\
\hline 796 & EnCana Oil \& Gas (USA) Inc. & SAPP1WW & SAPP1WW & DOMESTIC USE & Garfield County \\
\hline 797 & EnCana Oil \& Gas (USA) Inc. & SAVA2WW & SAVA2WW & DOMESTIC USE & Garfield County \\
\hline 798 & EnCana Oil \& Gas (USA) Inc. & SCHA1WW & SCHA1WW & DOMESTIC USE & Garfield County \\
\hline 799 & EnCana Oil \& Gas (USA) Inc. & SHOR1WW & SHOR1WW & DOMESTIC USE & Garfield County \\
\hline 800 & Colorado Department of Agriculture & WS-047 & WS-047CDOA & DOMESTIC USE & Garfield County \\
\hline 801 & U.S. Geological Survey & 393135107442301 & SC00609314ACD1 & OBSERVATION & Garfield County \\
\hline 802 & Colorado Oil and Gas Conservation Commission & 703261 & COGCC Facility ID:703261 & DOMESTIC USE & Garfield County \\
\hline 803 & Colorado Oil and Gas Conservation Commission & 703263 & COGCC Facility ID:703263 & DOMESTIC USE & Garfield County \\
\hline 804 & U.S. Geological Survey & 393009107375401 & SC0060925BDAA GRFLDS7 & DOMESTIC USE & Garfield County \\
\hline 805 & U.S. Geological Survey & 393101107391001 & SC00609222BACD GRFLDS8 & DOMESTIC USE & Garfield County \\
\hline 806 & Colorado Oil and Gas Conservation Commission & 703083 & COGCC Facility ID:703083 & DOMESTIC USE & Garfield County \\
\hline 807 & U.S. Geological Survey & 393101107393201 & SC00609222BBDD GRFLDS9 & DOMESTIC USE & Garfield County \\
\hline 811 & U.S. Geological Survey & 393310107422101 & SC00609206DBAC GRFLN4 & DOMESTIC USE & Garfield County \\
\hline 812 & U.S. Geological Survey & 393311107402201 & SC00609204CADD GRFLN9 & DOMESTIC USE & Garfield County \\
\hline 813 & U.S. Geological Survey & 393318107373601 & SC00609202DABA GRFLN3 & DOMESTIC USE & Garfield County \\
\hline 814 & U.S. Geological Survey & 393337107394601 & SC00609203BCBB GRFLN8 & DOMESTIC USE & Garfield County \\
\hline 815 & Colorado Oil and Gas Conservation Commission & 703110 & COGCC Facility ID:703110 & DOMESTIC USE & Garfield County \\
\hline 816 & U.S. Geological Survey & 393407107430901 & SC00509233DBCB GRFLN5 & DOMESTIC USE & Garfield County \\
\hline 817 & U.S. Geological Survey & 393415107415201 & SC00509234DBAC GRFLN10 & DOMESTIC USE & Garfield County \\
\hline 818 & Colorado Oil and Gas Conservation Commission & 703111 & COGCC Facility ID:703111 & DOMESTIC USE & Garfield County \\
\hline 819 & U.S. Geological Survey & 393429107412400 & SC0050923BBD00-BARRIE & DOMESTIC USE & Garfield County \\
\hline 820 & U.S. Geological Survey & 393456107404501 & SC0060920DACB GRFLN6 & DOMESTIC USE & Garfield County \\
\hline 821 & Colorado Oil and Gas Conservation Commission & 703143 & COGCC Facility ID:703143 & DOMESTIC USE & Garfield County \\
\hline 822 & Colorado Oil and Gas Conservation Commission & 704415 & COGCC Facility ID:704415 & DOMESTIC USE & Garfield County \\
\hline 823 & Colorado Oil and Gas Conservation Commission & 704416 & COGCC Facility ID:704416 & DOMESTIC USE & Garfield County \\
\hline 824 & Colorado Oil and Gas Conservation Commission & 704417 & COGCC Facility ID:704417 & DOMESTIC USE & Garfield County \\
\hline 825 & Colorado Oil and Gas Conservation Commission & 704418 & COGCC Facility ID:704418 & DOMESTIC USE & Garfield County \\
\hline 826 & Colorado Oil and Gas Conservation Commission & 704423 & COGCC Facility ID:704423 & DOMESTIC USE & Garfield County \\
\hline 827 & Colorado Oil and Gas Conservation Commission & 704424 & COGCC Facility ID:704424 & DOMESTIC USE & Garfield County \\
\hline 828 & Colorado Oil and Gas Conservation Commission & 703029 & COGCC Facility ID:703029 & DOMESTIC USE & Garfield County \\
\hline 829 & Colorado Oil and Gas Conservation Commission & 703030 & COGCC Facility ID:703030 & DOMESTIC USE & Garfield County \\
\hline 830 & Colorado Oil and Gas Conservation Commission & 703031 & COGCC Facility ID:703031 & DOMESTIC USE & Garfield County \\
\hline 831 & Colorado Oil and Gas Conservation Commission & 704265 & COGCC Facility ID:704265 & DOMESTIC USE & Garfield County \\
\hline 832 & Colorado Oil and Gas Conservation Commission & 704431 & COGCC Facility ID:704431 & DOMESTIC USE & Garfield County \\
\hline 833 & Colorado Oil and Gas Conservation Commission & 704432 & COGCC Facility ID:704432 & DOMESTIC USE & Garfield County \\
\hline 834 & Colorado Oil and Gas Conservation Commission & 703079 & COGCC Facility ID:703079 & DOMESTIC USE & Garfield County \\
\hline
\end{tabular}


Appendix 1. Water Quality Data Used in This Report.-Continued

\begin{tabular}{|c|c|c|c|c|c|}
\hline $\begin{array}{c}\text { Report } \\
\text { No. }\end{array}$ & Agency name & $\begin{array}{c}\text { Site } \\
\text { identifier }\end{array}$ & $\begin{array}{c}\text { Site } \\
\text { name }\end{array}$ & $\begin{array}{l}\text { Well } \\
\text { use }\end{array}$ & County \\
\hline 835 & Colorado Oil and Gas Conservation Commission & 703080 & COGCC Facility ID:703080 & DOMESTIC USE & Garfield County \\
\hline 836 & Colorado Oil and Gas Conservation Commission & 703081 & COGCC Facility ID:703081 & DOMESTIC USE & Garfield County \\
\hline 837 & Colorado Oil and Gas Conservation Commission & 703101 & COGCC Facility ID:703101 & DOMESTIC USE & Garfield County \\
\hline 838 & Colorado Oil and Gas Conservation Commission & 703102 & COGCC Facility ID:703102 & DOMESTIC USE & Garfield County \\
\hline 838 & Colorado Oil and Gas Conservation Commission & 703102 & COGCC Facility ID:703102 & DOMESTIC USE & Garfield County \\
\hline 839 & Colorado Oil and Gas Conservation Commission & 703103 & COGCC Facility ID:703103 & DOMESTIC USE & Garfield County \\
\hline 840 & Colorado Oil and Gas Conservation Commission & 703107 & COGCC Facility ID:703107 & DOMESTIC USE & Garfield County \\
\hline 840 & Colorado Oil and Gas Conservation Commission & 703107 & COGCC Facility ID:703107 & DOMESTIC USE & Garfield County \\
\hline 841 & Colorado Oil and Gas Conservation Commission & 703108 & COGCC Facility ID:703108 & DOMESTIC USE & Garfield County \\
\hline 842 & Colorado Oil and Gas Conservation Commission & 703109 & COGCC Facility ID:703109 & DOMESTIC USE & Garfield County \\
\hline 842 & Colorado Oil and Gas Conservation Commission & 703109 & COGCC Facility ID:703109 & DOMESTIC USE & Garfield County \\
\hline 843 & Colorado Oil and Gas Conservation Commission & 703185 & COGCC Facility ID:703185 & DOMESTIC USE & Garfield County \\
\hline 844 & Colorado Oil and Gas Conservation Commission & 703186 & COGCC Facility ID:703186 & DOMESTIC USE & Garfield County \\
\hline 845 & Colorado Oil and Gas Conservation Commission & 703236 & COGCC Facility ID:703236 & DOMESTIC USE & Garfield County \\
\hline 846 & Colorado Oil and Gas Conservation Commission & 704460 & COGCC Facility ID:704460 & DOMESTIC USE & Garfield County \\
\hline 847 & Colorado Oil and Gas Conservation Commission & 704475 & COGCC Facility ID:704475 & DOMESTIC USE & Garfield County \\
\hline 848 & Colorado Oil and Gas Conservation Commission & 704515 & COGCC Facility ID:704515 & DOMESTIC USE & Garfield County \\
\hline 849 & Colorado Oil and Gas Conservation Commission & 704534 & COGCC Facility ID:704534 & DOMESTIC USE & Garfield County \\
\hline 850 & Colorado Oil and Gas Conservation Commission & 704545 & COGCC Facility ID:704545 & DOMESTIC USE & Garfield County \\
\hline 851 & Colorado Oil and Gas Conservation Commission & 704558 & COGCC Facility ID:704558 & DOMESTIC USE & Garfield County \\
\hline 852 & Colorado Oil and Gas Conservation Commission & 704695 & COGCC Facility ID:704695 & DOMESTIC USE & Garfield County \\
\hline 853 & Colorado Oil and Gas Conservation Commission & 704696 & COGCC Facility ID:704696 & DOMESTIC USE & Garfield County \\
\hline 854 & Colorado Oil and Gas Conservation Commission & 704697 & COGCC Facility ID:704697 & DOMESTIC USE & Garfield County \\
\hline 855 & Colorado Oil and Gas Conservation Commission & 704709 & COGCC Facility ID:704709 & DOMESTIC USE & Garfield County \\
\hline 855 & Colorado Oil and Gas Conservation Commission & 704709 & COGCC Facility ID:704709 & DOMESTIC USE & Garfield County \\
\hline 856 & Colorado Oil and Gas Conservation Commission & 704711 & COGCC Facility ID:704711 & DOMESTIC USE & Garfield County \\
\hline 857 & Colorado Oil and Gas Conservation Commission & 704713 & COGCC Facility ID:704713 & DOMESTIC USE & Garfield County \\
\hline 858 & Colorado Oil and Gas Conservation Commission & 704715 & COGCC Facility ID:704715 & DOMESTIC USE & Garfield County \\
\hline 859 & Colorado Oil and Gas Conservation Commission & 704717 & COGCC Facility ID:704717 & DOMESTIC USE & Garfield County \\
\hline 860 & Colorado Oil and Gas Conservation Commission & 704720 & COGCC Facility ID:704720 & DOMESTIC USE & Garfield County \\
\hline 860 & Colorado Oil and Gas Conservation Commission & 704720 & COGCC Facility ID:704720 & DOMESTIC USE & Garfield County \\
\hline 861 & Colorado Oil and Gas Conservation Commission & 704725 & COGCC Facility ID:704725 & DOMESTIC USE & Garfield County \\
\hline 862 & Colorado Oil and Gas Conservation Commission & 704732 & COGCC Facility ID:704732 & DOMESTIC USE & Garfield County \\
\hline 862 & Colorado Oil and Gas Conservation Commission & 704732 & COGCC Facility ID:704732 & DOMESTIC USE & Garfield County \\
\hline 863 & Colorado Oil and Gas Conservation Commission & 704234 & COGCC Facility ID:704234 & DOMESTIC USE & Garfield County \\
\hline 864 & Colorado Oil and Gas Conservation Commission & 704748 & COGCC Facility ID:704748 & DOMESTIC USE & Garfield County \\
\hline 865 & Colorado Oil and Gas Conservation Commission & 704750 & COGCC Facility ID:704750 & DOMESTIC USE & Garfield County \\
\hline 866 & Colorado Oil and Gas Conservation Commission & 704754 & COGCC Facility ID:704754 & DOMESTIC USE & Garfield County \\
\hline 866 & Colorado Oil and Gas Conservation Commission & 704754 & COGCC Facility ID:704754 & DOMESTIC USE & Garfield County \\
\hline 867 & Colorado Oil and Gas Conservation Commission & 704755 & COGCC Facility ID:704755 & DOMESTIC USE & Garfield County \\
\hline 868 & Colorado Oil and Gas Conservation Commission & 704756 & COGCC Facility ID:704756 & DOMESTIC USE & Garfield County \\
\hline 869 & Colorado Oil and Gas Conservation Commission & 704763 & COGCC Facility ID:704763 & DOMESTIC USE & Garfield County \\
\hline 870 & Antero Resources & ANT3930451074016 & ANT3930451074016 & DOMESTIC USE & Garfield County \\
\hline 871 & Antero Resources & ANT3931001074241 & ANT3931001074241 & DOMESTIC USE & Garfield County \\
\hline 872 & Antero Resources & ANT3931011073910 & ANT3931011073910 & DOMESTIC USE & Garfield County \\
\hline 873 & Antero Resources & ANT3931021073954 & ANT3931021073954 & DOMESTIC USE & Garfield County \\
\hline 874 & Antero Resources & ANT3931111074058 & ANT3931111074058 & DOMESTIC USE & Garfield County \\
\hline 875 & Antero Resources & ANT3931121074228 & ANT3931121074228 & DOMESTIC USE & Garfield County \\
\hline 876 & Antero Resources & ANT3931151074158 & ANT3931151074158 & DOMESTIC USE & Garfield County \\
\hline 877 & Antero Resources & ANT3931401074214 & ANT3931401074214 & DOMESTIC USE & Garfield County \\
\hline 878 & Antero Resources & ANT3931451074446 & ANT3931451074446 & DOMESTIC USE & Garfield County \\
\hline 879 & Antero Resources & ANT3931501073746 & ANT3931501073746 & DOMESTIC USE & Garfield County \\
\hline 880 & Antero Resources & ANT3931511074137 & ANT3931511074137 & DOMESTIC USE & Garfield County \\
\hline 881 & Antero Resources & ANT3931511074140 & ANT3931511074140 & DOMESTIC USE & Garfield County \\
\hline 882 & Antero Resources & ANT3931511074144 & ANT3931511074144 & DOMESTIC USE & Garfield County \\
\hline 883 & Antero Resources & ANT3931511074206 & ANT3931511074206 & DOMESTIC USE & Garfield County \\
\hline 884 & Antero Resources & ANT3931521074050 & ANT3931521074050 & DOMESTIC USE & Garfield County \\
\hline 885 & Antero Resources & ANT3931531074013 & ANT3931531074013 & DOMESTIC USE & Garfield County \\
\hline 886 & Colorado Oil and Gas Conservation Commission & 704556 & COGCC Facility ID:704556 & DOMESTIC USE & Garfield County \\
\hline 887 & Antero Resources & ANT3931531074017 & ANT3931531074017 & DOMESTIC USE & Garfield County \\
\hline 888 & Antero Resources & ANT3931531074127 & ANT3931531074127 & DOMESTIC USE & Garfield County \\
\hline 889 & Antero Resources & ANT3931561074055 & ANT3931561074055 & DOMESTIC USE & Garfield County \\
\hline 890 & Antero Resources & ANT3931581074011 & ANT3931581074011 & DOMESTIC USE & Garfield County \\
\hline 891 & Antero Resources & ANT3931591073750 & ANT3931591073750 & DOMESTIC USE & Garfield County \\
\hline 892 & Antero Resources & ANT3931591074017 & ANT3931591074017 & DOMESTIC USE & Garfield County \\
\hline 893 & Antero Resources & ANT3931601074012 & ANT3931601074012 & DOMESTIC USE & Garfield County \\
\hline
\end{tabular}


Appendix 1. Water Quality Data Used in This Report.-Continued

\begin{tabular}{|c|c|c|c|c|c|}
\hline $\begin{array}{c}\text { Report } \\
\text { No. }\end{array}$ & Agency name & $\begin{array}{c}\text { Site } \\
\text { identifier }\end{array}$ & $\begin{array}{c}\text { Site } \\
\text { name }\end{array}$ & $\begin{array}{l}\text { Well } \\
\text { use }\end{array}$ & County \\
\hline 894 & Antero Resources & ANT3932021074034 & ANT3932021074034 & DOMESTIC USE & Garfield County \\
\hline 895 & Antero Resources & ANT3932031074454 & ANT3932031074454 & DOMESTIC USE & Garfield County \\
\hline 896 & Antero Resources & ANT3932041073917 & ANT3932041073917 & DOMESTIC USE & Garfield County \\
\hline 897 & Colorado Oil and Gas Conservation Commission & 704710 & COGCC Facility ID:704710 & DOMESTIC USE & Garfield County \\
\hline 898 & Colorado Oil and Gas Conservation Commission & 704712 & COGCC Facility ID:704712 & DOMESTIC USE & Garfield County \\
\hline 898 & Colorado Oil and Gas Conservation Commission & 704712 & COGCC Facility ID:704712 & DOMESTIC USE & Garfield County \\
\hline 899 & Colorado Oil and Gas Conservation Commission & 704714 & COGCC Facility ID:704714 & DOMESTIC USE & Garfield County \\
\hline 899 & Colorado Oil and Gas Conservation Commission & 704714 & COGCC Facility ID:704714 & DOMESTIC USE & Garfield County \\
\hline 900 & Antero Resources & ANT3932041073919 & ANT3932041073919 & DOMESTIC USE & Garfield County \\
\hline 901 & Antero Resources & ANT3932041074019 & ANT3932041074019 & DOMESTIC USE & Garfield County \\
\hline 902 & Antero Resources & ANT3932041074035 & ANT3932041074035 & DOMESTIC USE & Garfield County \\
\hline 903 & Antero Resources & ANT3932061073907 & ANT3932061073907 & DOMESTIC USE & Garfield County \\
\hline 904 & Antero Resources & ANT3932061073911 & ANT3932061073911 & DOMESTIC USE & Garfield County \\
\hline 905 & Colorado Oil and Gas Conservation Commission & 704716 & COGCC Facility ID:704716 & DOMESTIC USE & Garfield County \\
\hline 905 & Colorado Oil and Gas Conservation Commission & 704716 & COGCC Facility ID:704716 & DOMESTIC USE & Garfield County \\
\hline 906 & Colorado Oil and Gas Conservation Commission & 704718 & COGCC Facility ID:704718 & DOMESTIC USE & Garfield County \\
\hline 907 & Colorado Oil and Gas Conservation Commission & 704719 & COGCC Facility ID:704719 & DOMESTIC USE & Garfield County \\
\hline 907 & Colorado Oil and Gas Conservation Commission & 704719 & COGCC Facility ID:704719 & DOMESTIC USE & Garfield County \\
\hline 908 & Colorado Oil and Gas Conservation Commission & 704724 & COGCC Facility ID:704724 & DOMESTIC USE & Garfield County \\
\hline 908 & Colorado Oil and Gas Conservation Commission & 704724 & COGCC Facility ID:704724 & DOMESTIC USE & Garfield County \\
\hline 909 & Antero Resources & ANT3932061074012 & ANT3932061074012 & DOMESTIC USE & Garfield County \\
\hline 910 & Colorado Oil and Gas Conservation Commission & 704726 & COGCC Facility ID:704726 & DOMESTIC USE & Garfield County \\
\hline 911 & Colorado Oil and Gas Conservation Commission & 704727 & COGCC Facility ID:704727 & DOMESTIC USE & Garfield County \\
\hline 912 & Colorado Oil and Gas Conservation Commission & 704728 & COGCC Facility ID:704728 & DOMESTIC USE & Garfield County \\
\hline 913 & Colorado Oil and Gas Conservation Commission & 704729 & COGCC Facility ID:704729 & DOMESTIC USE & Garfield County \\
\hline 913 & Colorado Oil and Gas Conservation Commission & 704729 & COGCC Facility ID:704729 & DOMESTIC USE & Garfield County \\
\hline 915 & Colorado Oil and Gas Conservation Commission & 704731 & COGCC Facility ID:704731 & DOMESTIC USE & Garfield County \\
\hline 916 & Colorado Oil and Gas Conservation Commission & 704733 & COGCC Facility ID:704733 & DOMESTIC USE & Garfield County \\
\hline 916 & Colorado Oil and Gas Conservation Commission & 704733 & COGCC Facility ID:704733 & DOMESTIC USE & Garfield County \\
\hline 917 & Antero Resources & ANT3932111073945 & ANT3932111073945 & DOMESTIC USE & Garfield County \\
\hline 918 & Colorado Oil and Gas Conservation Commission & 704734 & COGCC Facility ID:704734 & DOMESTIC USE & Garfield County \\
\hline 918 & Colorado Oil and Gas Conservation Commission & 704734 & COGCC Facility ID:704734 & DOMESTIC USE & Garfield County \\
\hline 919 & Antero Resources & ANT3932121073847 & ANT3932121073847 & DOMESTIC USE & Garfield County \\
\hline 920 & Antero Resources & ANT3932131073818 & ANT3932131073818 & DOMESTIC USE & Garfield County \\
\hline 921 & Antero Resources & ANT3932131073842 & ANT3932131073842 & DOMESTIC USE & Garfield County \\
\hline 922 & Colorado Oil and Gas Conservation Commission & 704745 & COGCC Facility ID:704745 & DOMESTIC USE & Garfield County \\
\hline 923 & Colorado Oil and Gas Conservation Commission & 704746 & COGCC Facility ID:704746 & DOMESTIC USE & Garfield County \\
\hline 924 & Colorado Oil and Gas Conservation Commission & 704747 & COGCC Facility ID:704747 & DOMESTIC USE & Garfield County \\
\hline 924 & Colorado Oil and Gas Conservation Commission & 704747 & COGCC Facility ID:704747 & DOMESTIC USE & Garfield County \\
\hline 925 & Colorado Oil and Gas Conservation Commission & 704749 & COGCC Facility ID:704749 & DOMESTIC USE & Garfield County \\
\hline 925 & Colorado Oil and Gas Conservation Commission & 704749 & COGCC Facility ID:704749 & DOMESTIC USE & Garfield County \\
\hline 926 & Colorado Oil and Gas Conservation Commission & 704751 & COGCC Facility ID:704751 & DOMESTIC USE & Garfield County \\
\hline 927 & Antero Resources & ANT3932131074453 & ANT3932131074453 & DOMESTIC USE & Garfield County \\
\hline 928 & Colorado Oil and Gas Conservation Commission & 704752 & COGCC Facility ID:704752 & DOMESTIC USE & Garfield County \\
\hline 928 & Colorado Oil and Gas Conservation Commission & 704752 & COGCC Facility ID:704752 & DOMESTIC USE & Garfield County \\
\hline 930 & Antero Resources & ANT3932141074421 & ANT3932141074421 & DOMESTIC USE & Garfield County \\
\hline 931 & Antero Resources & ANT3932171074038 & ANT3932171074038 & DOMESTIC USE & Garfield County \\
\hline 932 & Antero Resources & ANT3932181073847 & ANT3932181073847 & DOMESTIC USE & Garfield County \\
\hline 933 & Colorado Oil and Gas Conservation Commission & 704761 & COGCC Facility ID:704761 & DOMESTIC USE & Garfield County \\
\hline 934 & Colorado Oil and Gas Conservation Commission & 704762 & COGCC Facility ID:704762 & DOMESTIC USE & Garfield County \\
\hline 934 & Colorado Oil and Gas Conservation Commission & 704762 & COGCC Facility ID:704762 & DOMESTIC USE & Garfield County \\
\hline 935 & Antero Resources & ANT3932201074446 & ANT3932201074446 & DOMESTIC USE & Garfield County \\
\hline 936 & Antero Resources & ANT3932221073940 & ANT3932221073940 & DOMESTIC USE & Garfield County \\
\hline 937 & Antero Resources & ANT3932221074413 & ANT3932221074413 & DOMESTIC USE & Garfield County \\
\hline 938 & Antero Resources & ANT3932231073941 & ANT3932231073941 & DOMESTIC USE & Garfield County \\
\hline 939 & Antero Resources & ANT3932251074410 & ANT3932251074410 & DOMESTIC USE & Garfield County \\
\hline 940 & Antero Resources & ANT3932251074431 & ANT3932251074431 & DOMESTIC USE & Garfield County \\
\hline 941 & Antero Resources & ANT3932261074419 & ANT3932261074419 & DOMESTIC USE & Garfield County \\
\hline 942 & Antero Resources & ANT3932291074416 & ANT3932291074416 & DOMESTIC USE & Garfield County \\
\hline 943 & Antero Resources & ANT3932311074422 & ANT3932311074422 & DOMESTIC USE & Garfield County \\
\hline 944 & Antero Resources & ANT3932311074441 & ANT3932311074441 & DOMESTIC USE & Garfield County \\
\hline 945 & Antero Resources & ANT3932341073950 & ANT3932341073950 & DOMESTIC USE & Garfield County \\
\hline 946 & Antero Resources & ANT3932341073954 & ANT3932341073954 & DOMESTIC USE & Garfield County \\
\hline 947 & Antero Resources & ANT3932351074324 & ANT3932351074324 & DOMESTIC USE & Garfield County \\
\hline 948 & Antero Resources & ANT3932371074360 & ANT3932371074360 & DOMESTIC USE & Garfield County \\
\hline 949 & Antero Resources & ANT3932391074433 & ANT3932391074433 & DOMESTIC USE & Garfield County \\
\hline
\end{tabular}


Appendix 1. Water Quality Data Used in This Report.-Continued

\begin{tabular}{|c|c|c|c|c|c|}
\hline $\begin{array}{c}\text { Report } \\
\text { No. }\end{array}$ & Agency name & $\begin{array}{c}\text { Site } \\
\text { identifier }\end{array}$ & $\begin{array}{c}\text { Site } \\
\text { name }\end{array}$ & $\begin{array}{l}\text { Well } \\
\text { use }\end{array}$ & County \\
\hline 950 & Antero Resources & ANT3932401074311 & ANT3932401074311 & DOMESTIC USE & Garfield County \\
\hline 951 & Antero Resources & ANT3932401074351 & ANT3932401074351 & DOMESTIC USE & Garfield County \\
\hline 952 & Antero Resources & ANT3932411074410 & ANT3932411074410 & DOMESTIC USE & Garfield County \\
\hline 953 & Antero Resources & ANT3932421074359 & ANT3932421074359 & DOMESTIC USE & Garfield County \\
\hline 954 & Antero Resources & ANT3932451074320 & ANT3932451074320 & DOMESTIC USE & Garfield County \\
\hline 955 & Antero Resources & ANT3932461074234 & ANT3932461074234 & DOMESTIC USE & Garfield County \\
\hline 956 & Antero Resources & ANT3932471074356 & ANT3932471074356 & DOMESTIC USE & Garfield County \\
\hline 957 & Antero Resources & ANT3932481074110 & ANT3932481074110 & DOMESTIC USE & Garfield County \\
\hline 958 & Antero Resources & ANT3932481074229 & ANT3932481074229 & DOMESTIC USE & Garfield County \\
\hline 959 & Antero Resources & ANT3932481074309 & ANT3932481074309 & DOMESTIC USE & Garfield County \\
\hline 960 & Antero Resources & ANT3932491074226 & ANT3932491074226 & DOMESTIC USE & Garfield County \\
\hline 961 & Antero Resources & ANT3932491074242 & ANT3932491074242 & DOMESTIC USE & Garfield County \\
\hline 962 & Antero Resources & ANT3932511074229 & ANT3932511074229 & DOMESTIC USE & Garfield County \\
\hline 963 & Antero Resources & ANT3932511074253 & ANT3932511074253 & DOMESTIC USE & Garfield County \\
\hline 964 & Antero Resources & ANT3932511074258 & ANT3932511074258 & DOMESTIC USE & Garfield County \\
\hline 965 & Antero Resources & ANT3932531074234 & ANT3932531074234 & DOMESTIC USE & Garfield County \\
\hline 966 & Antero Resources & ANT3932531074237 & ANT3932531074237 & DOMESTIC USE & Garfield County \\
\hline 967 & Antero Resources & ANT3932541074350 & ANT3932541074350 & DOMESTIC USE & Garfield County \\
\hline 968 & Antero Resources & ANT3932551074340 & ANT3932551074340 & DOMESTIC USE & Garfield County \\
\hline 969 & Antero Resources & ANT3932561074156 & ANT3932561074156 & DOMESTIC USE & Garfield County \\
\hline 970 & Antero Resources & ANT3932561074158 & ANT3932561074158 & DOMESTIC USE & Garfield County \\
\hline 971 & Antero Resources & ANT3932581074236 & ANT3932581074236 & DOMESTIC USE & Garfield County \\
\hline 972 & Antero Resources & ANT3932581074408 & ANT3932581074408 & DOMESTIC USE & Garfield County \\
\hline 973 & Antero Resources & ANT3932581074415 & ANT3932581074415 & DOMESTIC USE & Garfield County \\
\hline 974 & Antero Resources & ANT3932591074113 & ANT3932591074113 & DOMESTIC USE & Garfield County \\
\hline 975 & Antero Resources & ANT3932591074203 & ANT3932591074203 & DOMESTIC USE & Garfield County \\
\hline 976 & Antero Resources & ANT3932601074160 & ANT3932601074160 & DOMESTIC USE & Garfield County \\
\hline 977 & Antero Resources & ANT3933001074148 & ANT3933001074148 & DOMESTIC USE & Garfield County \\
\hline 978 & Antero Resources & ANT3933011074118 & ANT3933011074118 & DOMESTIC USE & Garfield County \\
\hline 979 & Antero Resources & ANT3933011074200 & ANT3933011074200 & DOMESTIC USE & Garfield County \\
\hline 980 & Antero Resources & ANT3933011074305 & ANT3933011074305 & DOMESTIC USE & Garfield County \\
\hline 981 & Antero Resources & ANT3933011074355 & ANT3933011074355 & DOMESTIC USE & Garfield County \\
\hline 982 & Antero Resources & ANT3933021074114 & ANT3933021074114 & DOMESTIC USE & Garfield County \\
\hline 983 & Antero Resources & ANT3933021074302 & ANT3933021074302 & DOMESTIC USE & Garfield County \\
\hline 984 & Antero Resources & ANT3933031074112 & ANT3933031074112 & DOMESTIC USE & Garfield County \\
\hline 985 & Antero Resources & ANT3933031074129 & ANT3933031074129 & DOMESTIC USE & Garfield County \\
\hline 986 & Antero Resources & ANT3933031074136 & ANT3933031074136 & DOMESTIC USE & Garfield County \\
\hline 987 & Antero Resources & ANT3933031074236 & ANT3933031074236 & DOMESTIC USE & Garfield County \\
\hline 988 & Antero Resources & ANT3933041074110 & ANT3933041074110 & DOMESTIC USE & Garfield County \\
\hline 989 & Antero Resources & ANT3933041074147 & ANT3933041074147 & DOMESTIC USE & Garfield County \\
\hline 990 & Antero Resources & ANT3933051074203 & ANT3933051074203 & DOMESTIC USE & Garfield County \\
\hline 991 & Antero Resources & ANT3933051074311 & ANT3933051074311 & DOMESTIC USE & Garfield County \\
\hline 992 & Antero Resources & ANT3933051074315 & ANT3933051074315 & DOMESTIC USE & Garfield County \\
\hline 993 & Antero Resources & ANT3933051074333 & ANT3933051074333 & DOMESTIC USE & Garfield County \\
\hline 994 & Antero Resources & ANT3933051074418 & ANT3933051074418 & DOMESTIC USE & Garfield County \\
\hline 995 & Antero Resources & ANT3933061074344 & ANT3933061074344 & DOMESTIC USE & Garfield County \\
\hline 996 & Antero Resources & ANT3933071074210 & ANT3933071074210 & DOMESTIC USE & Garfield County \\
\hline 997 & Antero Resources & ANT3933071074348 & ANT3933071074348 & DOMESTIC USE & Garfield County \\
\hline 998 & Antero Resources & ANT3933081074212 & ANT3933081074212 & DOMESTIC USE & Garfield County \\
\hline 999 & Antero Resources & ANT3933081074341 & ANT3933081074341 & DOMESTIC USE & Garfield County \\
\hline 1000 & Antero Resources & ANT3933081074356 & ANT3933081074356 & DOMESTIC USE & Garfield County \\
\hline 1001 & Antero Resources & ANT3933091074220 & ANT3933091074220 & DOMESTIC USE & Garfield County \\
\hline 1002 & Antero Resources & ANT3933091074232 & ANT3933091074232 & DOMESTIC USE & Garfield County \\
\hline 1003 & Antero Resources & ANT3933101074246 & ANT3933101074246 & DOMESTIC USE & Garfield County \\
\hline 1004 & Antero Resources & ANT3933121074249 & ANT3933121074249 & DOMESTIC USE & Garfield County \\
\hline 1005 & Antero Resources & ANT3933121074353 & ANT3933121074353 & DOMESTIC USE & Garfield County \\
\hline 1006 & Antero Resources & ANT3933151074302 & ANT3933151074302 & DOMESTIC USE & Garfield County \\
\hline 1007 & Antero Resources & ANT3933181074357 & ANT3933181074357 & DOMESTIC USE & Garfield County \\
\hline 1008 & Antero Resources & ANT3933201074320 & ANT3933201074320 & DOMESTIC USE & Garfield County \\
\hline 1009 & Antero Resources & ANT3933211074354 & ANT3933211074354 & DOMESTIC USE & Garfield County \\
\hline 1010 & Antero Resources & ANT3933241074332 & ANT3933241074332 & DOMESTIC USE & Garfield County \\
\hline 1011 & Antero Resources & ANT3933511074360 & ANT3933511074360 & DOMESTIC USE & Garfield County \\
\hline 1012 & Antero Resources & ANT3933521074445 & ANT3933521074445 & DOMESTIC USE & Garfield County \\
\hline 1013 & Antero Resources & ANT3933561074357 & ANT3933561074357 & DOMESTIC USE & Garfield County \\
\hline
\end{tabular}


Appendix 1. Water Quality Data Used in This Report.-Continued

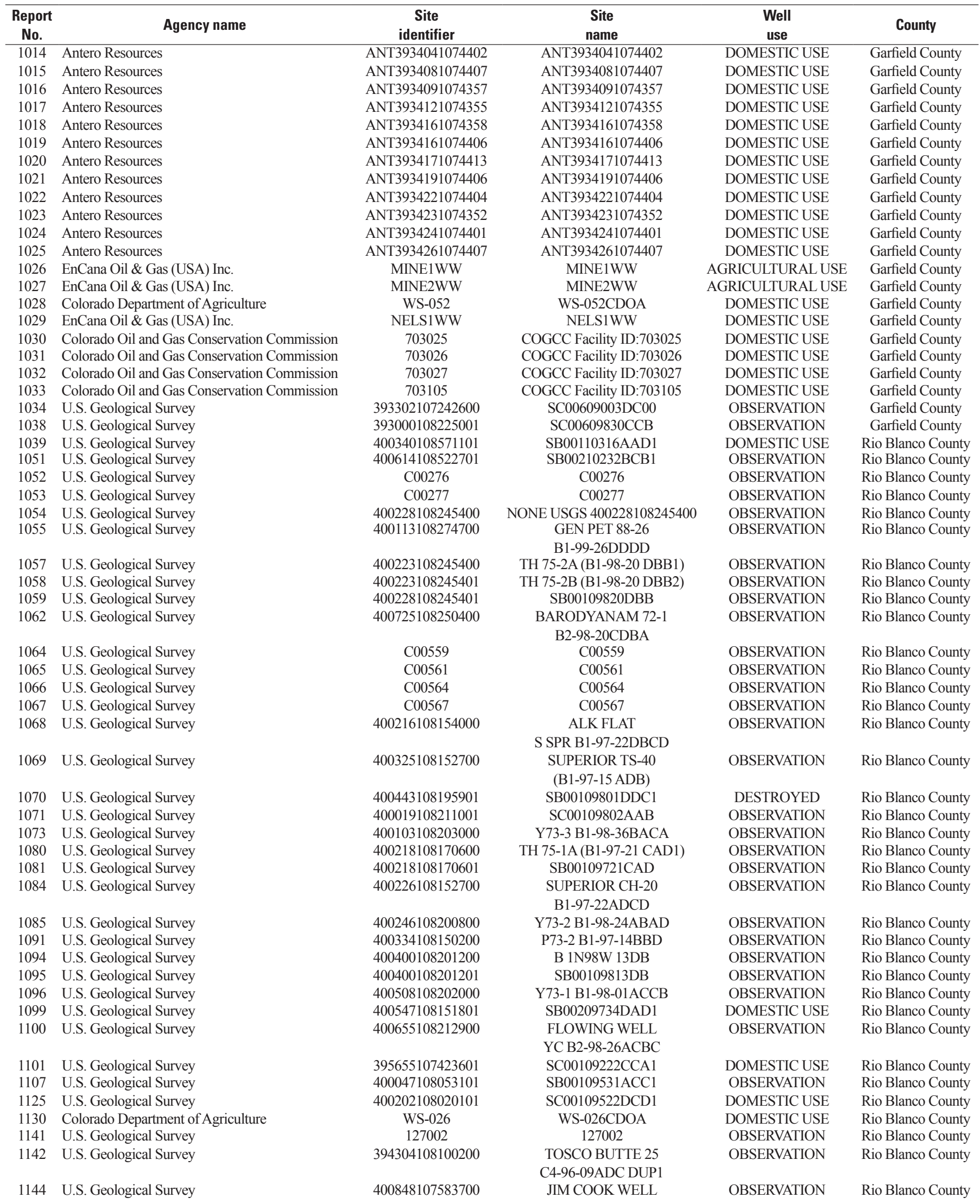


Appendix 1. Water Quality Data Used in This Report.-Continued

\begin{tabular}{|c|c|c|c|c|c|}
\hline $\begin{array}{l}\text { Report } \\
\text { No. }\end{array}$ & Agency name & $\begin{array}{c}\text { Site } \\
\text { identifier }\end{array}$ & $\begin{array}{c}\text { Site } \\
\text { name }\end{array}$ & $\begin{array}{l}\text { Well } \\
\text { use }\end{array}$ & County \\
\hline 1147 & U.S. Geological Survey & 400943107594701 & SB00209512ABD1 & DOMESTIC USE & Rio Blanco County \\
\hline 1149 & U.S. Geological Survey & 401035108331701 & SB00209906BBC1 & AGRICULTURAL USE & Rio Blanco County \\
\hline 1154 & U.S. Geological Survey & 401119108313201 & SB00309932ACA1 & AGRICULTURAL USE & Rio Blanco County \\
\hline 1160 & U.S. Geological Survey & 394444108255400 & $\begin{array}{l}\text { ARCO-MOBIL 31-1 } \\
\text { C3-98-31ACCD }\end{array}$ & OBSERVATION & Rio Blanco County \\
\hline 1174 & U.S. Geological Survey & 395239108133900 & $\begin{array}{c}\text { MOBIL } 5413 \text { C2 } 97 \\
\text { 13ACCC }\end{array}$ & OBSERVATION & Rio Blanco County \\
\hline 1175 & U.S. Geological Survey & 395322108104800 & $\begin{array}{l}\text { SC00209609CBB } \\
\text { MOBIL WELL }\end{array}$ & OBSERVATION & Rio Blanco County \\
\hline 1176 & Colorado Oil and Gas Conservation Commission & 701448 & COGCC Facility ID:701448 & PUBLIC SUPPLY & Rio Blanco County \\
\hline 1177 & Colorado Oil and Gas Conservation Commission & 701449 & COGCC Facility ID:701449 & PUBLIC SUPPLY & Rio Blanco County \\
\hline 1186 & U.S. Geological Survey & 395852108084400 & $\begin{array}{l}\text { L HILLS IR WELL C1- } \\
\text { 96-10DACA }\end{array}$ & OBSERVATION & Rio Blanco County \\
\hline 1187 & U.S. Geological Survey & 126120 & 126120 & OBSERVATION & Rio Blanco County \\
\hline 1188 & U.S. Geological Survey & 394751108145201 & SC00309711CDD1 (W-2) & OBSERVATION & Rio Blanco County \\
\hline 1189 & U.S. Geological Survey & 394801108124300 & ARCO AT 1 C3 967 CAD & OBSERVATION & Rio Blanco County \\
\hline 1190 & U.S. Geological Survey & 394806108124301 & AT-1C-3 & OBSERVATION & Rio Blanco County \\
\hline 1191 & U.S. Geological Survey & 394812108102101 & C-B ALLUVIUM WELL A-9 & OBSERVATION & Rio Blanco County \\
\hline 1192 & U.S. Geological Survey & 394826108132301 & $32 \mathrm{Y} 12$ & OBSERVATION & Rio Blanco County \\
\hline 1193 & U.S. Geological Survey & 394826108133001 & $32 \times 12$ & OBSERVATION & Rio Blanco County \\
\hline 1194 & U.S. Geological Survey & 394828108132704 & $\begin{array}{c}\text { SC00309712ACB1 } \\
\text { USGS 394828108132704 }\end{array}$ & OBSERVATION & Rio Blanco County \\
\hline 1195 & U.S. Geological Survey & 394836108134001 & 21X12-01 & OBSERVATION & Rio Blanco County \\
\hline 1196 & U.S. Geological Survey & 394659108112402 & C-B ALLUVIUM WELLA-12 & OBSERVATION & Rio Blanco County \\
\hline 1206 & U.S. Geological Survey & 394900108142101 & WELL 43 X2-1 & OBSERVATION & Rio Blanco County \\
\hline 1207 & U.S. Geological Survey & 394900108142102 & $43 \times 2-2$ & OBSERVATION & Rio Blanco County \\
\hline 1208 & U.S. Geological Survey & 394903108135002 & $22 \times 1-2$ & OBSERVATION & Rio Blanco County \\
\hline 1209 & U.S. Geological Survey & 394903108135003 & $22 \times 1-3$ & OBSERVATION & Rio Blanco County \\
\hline 1210 & U.S. Geological Survey & 395012108143702 & C-B ALLUVIUM WELL A-2 & OBSERVATION & Rio Blanco County \\
\hline 1211 & U.S. Geological Survey & 395034108135700 & C-B ALLUVIUM WELL A-1 & OBSERVATION & Rio Blanco County \\
\hline 1213 & U.S. Geological Survey & 394824108133500 & OCCY $32 X-12(C 3-97-12 A C B B)$ & OBSERVATION & Rio Blanco County \\
\hline 1230 & U.S. Geological Survey & 395010108132901 & SC00209736ACA1 & OBSERVATION & Rio Blanco County \\
\hline 1237 & U.S. Geological Survey & 395029108140400 & P73-5 C2-97-25CCDA & OBSERVATION & Rio Blanco County \\
\hline 1239 & U.S. Geological Survey & 395155108123100 & TH 75-10A (C2-96-19 ACBA1) & OBSERVATION & Rio Blanco County \\
\hline 1244 & Colorado Department of Agriculture & WS-021 & WS-021CDOA & DOMESTIC USE & Rio Blanco County \\
\hline 1250 & U.S. Geological Survey & 400131107561400 & SCHULTZ NO. 1 & OBSERVATION & Rio Blanco County \\
\hline 1251 & U.S. Geological Survey & 400137107574701 & SB00109429BDD1 & DOMESTIC USE & Rio Blanco County \\
\hline 1252 & U.S. Geological Survey & 400140107570001 & SB00109428ADD1 & DOMESTIC USE & Rio Blanco County \\
\hline 1253 & U.S. Geological Survey & 400447107550700 & SULPHUR C CO WELL L & OBSERVATION & Rio Blanco County \\
\hline 1254 & U.S. Geological Survey & 400643107551901 & SB00209427DBB1 & AGRICULTURAL USE & Rio Blanco County \\
\hline 1256 & U.S. Geological Survey & 400009107525801 & SC00109306BBC1 & DOMESTIC USE & Rio Blanco County \\
\hline 1260 & U.S. Geological Survey & 400036107580301 & SB00109432CBA1 & AGRICULTURAL USE & Rio Blanco County \\
\hline 1265 & U.S. Geological Survey & 400044107530601 & SB00109436DBB1 & DOMESTIC USE & Rio Blanco County \\
\hline 1267 & U.S. Geological Survey & 400053107583901 & SB00109431ACA1 & AGRICULTURAL USE & Rio Blanco County \\
\hline 1272 & U.S. Geological Survey & 400108107581901 & SB00109431AAA1 & DOMESTIC USE & Rio Blanco County \\
\hline 1273 & U.S. Geological Survey & 400109107562101 & SB00109433ABA1 & OBSERVATION & Rio Blanco County \\
\hline 1277 & U.S. Geological Survey & 400115107572101 & SB00109429AAC1 & PUBLIC SUPPLY & Rio Blanco County \\
\hline
\end{tabular}


Appendix 1. Water Quality Data Used in This Report.-Continued

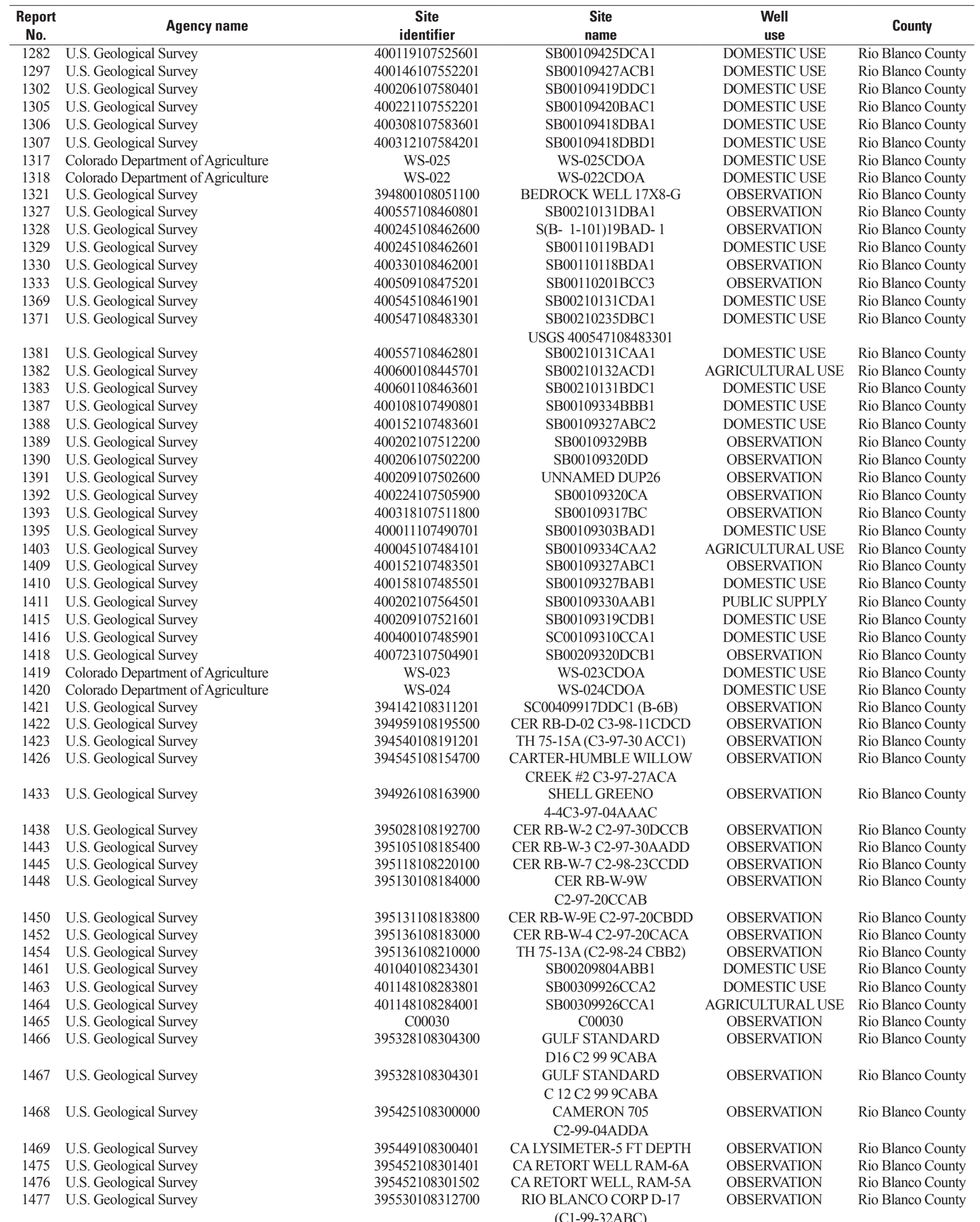


Appendix 1. Water Quality Data Used in This Report.-Continued

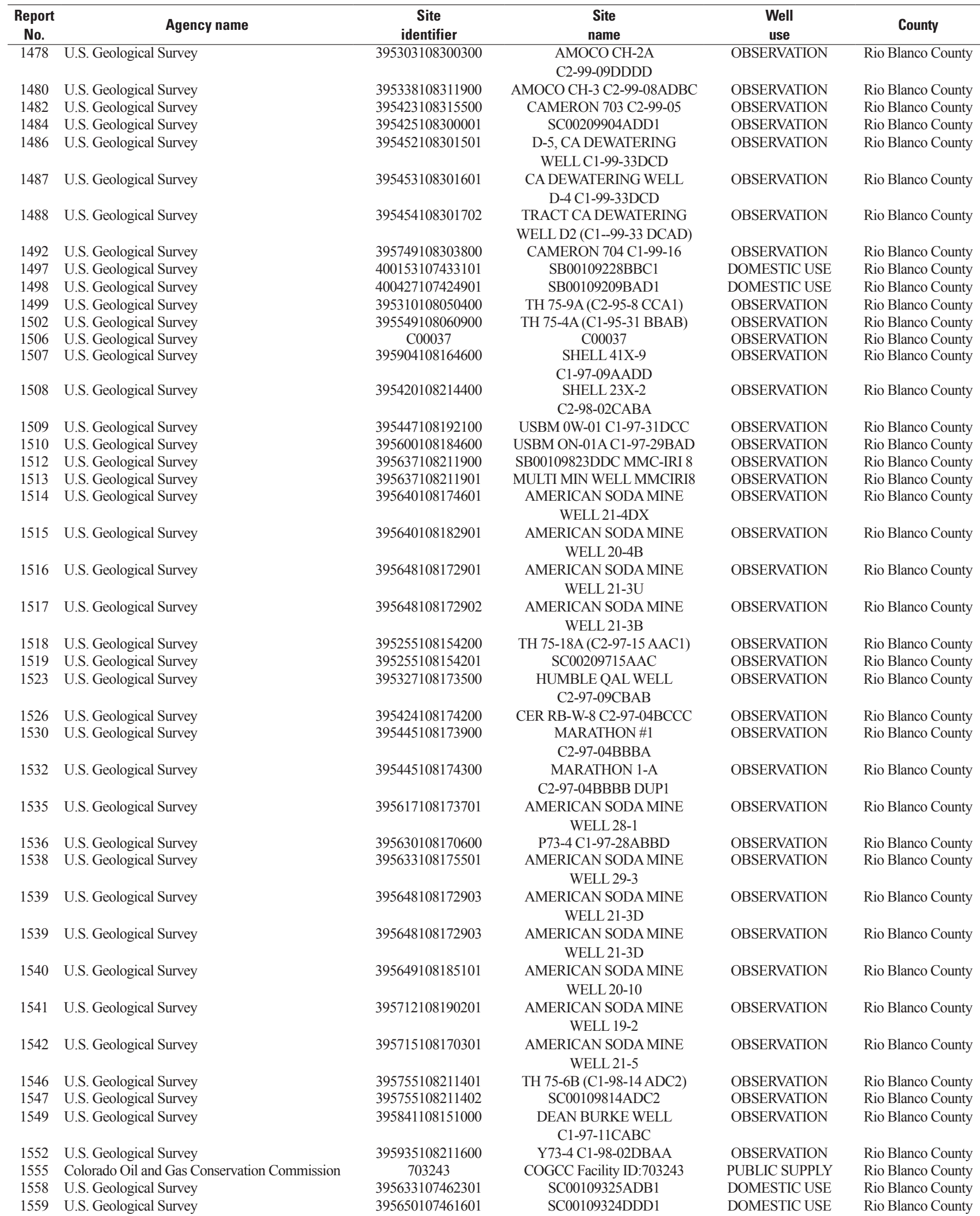


Appendix 1. Water Quality Data Used in This Report.-Continued

\begin{tabular}{|c|c|c|c|c|c|}
\hline $\begin{array}{c}\text { Report } \\
\text { No. }\end{array}$ & Agency name & $\begin{array}{c}\text { Site } \\
\text { identifier }\end{array}$ & $\begin{array}{c}\text { Site } \\
\text { name }\end{array}$ & $\begin{array}{l}\text { Well } \\
\text { use }\end{array}$ & County \\
\hline 1561 & U.S. Geological Survey & 395741107474401 & SC00109323ABA1 & DOMESTIC USE & Rio Blanco County \\
\hline 1565 & U.S. Geological Survey & 395832107483901 & SC00109315AAB1 & DOMESTIC USE & Rio Blanco County \\
\hline 1567 & U.S. Geological Survey & 395922107493301 & SC00109310BBB1 & DOMESTIC USE & Rio Blanco County \\
\hline 1568 & U.S. Geological Survey & 395953107495301 & SC00109304DAB1 & DOMESTIC USE & Rio Blanco County \\
\hline 1571 & U.S. Geological Survey & 400019108112500 & LITTLE HILLS HQ B1-96-32 & OBSERVATION & Rio Blanco County \\
\hline 1581 & U.S. Geological Survey & 400045108131401 & $\begin{array}{l}\text { TH 75-17B, CR2, } \\
\text { (B1-97-36 ADCC) }\end{array}$ & OBSERVATION & Rio Blanco County \\
\hline 1587 & U.S. Geological Survey & 400439108141000 & P73-1 B1-97-02DDDD & OBSERVATION & Rio Blanco County \\
\hline 1592 & U.S. Geological Survey & 400525108132601 & SB00109701ABB1 & DOMESTIC USE & Rio Blanco County \\
\hline 1593 & U.S. Geological Survey & $\mathrm{C} 00028$ & $\mathrm{C} 00028$ & OBSERVATION & Rio Blanco County \\
\hline 1594 & U.S. Geological Survey & 401209108011101 & SB00309526BAD1 & AGRICULTURAL USE & Rio Blanco County \\
\hline 1595 & U.S. Geological Survey & 401038108002801 & SB00309501BBB1 & AGRICULTURAL USE & Rio Blanco County \\
\hline 1599 & U.S. Geological Survey & C00795 & C00795 & OBSERVATION & Rio Blanco County \\
\hline 1600 & U.S. Geological Survey & 126281 & 126281 & OBSERVATION & Rio Blanco County \\
\hline 1601 & U.S. Geological Survey & 126139 & 126139 & OBSERVATION & Rio Blanco County \\
\hline 1602 & U.S. Geological Survey & 395347108270000 & UNNAMED DUP25 & OBSERVATION & Rio Blanco County \\
\hline 1603 & U.S. Geological Survey & 395516108294200 & GULF STND C 45 C1 99 BDC & OBSERVATION & Rio Blanco County \\
\hline 1604 & U.S. Geological Survey & 395524108290301 & $\begin{array}{l}\text { RIO BLANCO CORP } \\
\text { D-18 (C1-99-34A) }\end{array}$ & OBSERVATION & Rio Blanco County \\
\hline 1605 & U.S. Geological Survey & 395524108290302 & $\begin{array}{l}\text { GULF STANDARD } \\
\text { CA } 3 \text { C1 } 9934 \text { ADB }\end{array}$ & OBSERVATION & Rio Blanco County \\
\hline 1607 & U.S. Geological Survey & 395238108284400 & $\begin{array}{l}\text { BLM QAL SSD } \\
\text { C2-99-14BCDA }\end{array}$ & OBSERVATION & Rio Blanco County \\
\hline 1609 & U.S. Geological Survey & 395327108232000 & $\begin{array}{l}\text { BLM QAL WELL } \\
\text { C2-98-09DACC }\end{array}$ & OBSERVATION & Rio Blanco County \\
\hline 1614 & U.S. Geological Survey & 395439108223302 & SC00209803ABD2 & OBSERVATION & Rio Blanco County \\
\hline 1617 & U.S. Geological Survey & 395524108290300 & $\begin{array}{l}\text { CAMERON } 702 \\
\text { C1-99-34ADBA }\end{array}$ & OBSERVATION & Rio Blanco County \\
\hline 1622 & U.S. Geological Survey & 394557108225300 & $\begin{array}{l}\text { CARTER HUMBLE FAWN CR } \\
2 \text { C3 } 98 \text { 27BAD }\end{array}$ & OBSERVATION & Rio Blanco County \\
\hline 1623 & U.S. Geological Survey & 395046108224301 & SC00209827DBB1 (CER 5) & OBSERVATION & Rio Blanco County \\
\hline 1626 & U.S. Geological Survey & 395034108231300 & CER RB-W-5 C2-98-27CCAB & OBSERVATION & Rio Blanco County \\
\hline 1628 & U.S. Geological Survey & 395053108223900 & CER RB-W-6 C2-98-27ACDB & OBSERVATION & Rio Blanco County \\
\hline 1631 & Colorado Department of Agriculture & WS-M-005 & WS-M-005CDOA & OBSERVATION & Mesa County \\
\hline 1632 & U.S. Geological Survey & 391629108540101 & 034 OLD SCHOOL WELL & OBSERVATION & Mesa County \\
\hline 1633 & U.S. Geological Survey & 391559108540001 & SC00910321BBB-080Q9 & OBSERVATION & Mesa County \\
\hline 1634 & U.S. Geological Survey & 391630108525701 & SC00910316ADD-090R5 & OBSERVATION & Mesa County \\
\hline 1635 & U.S. Geological Survey & 391723108570501 & SC00910412BDC1 (VT1) & OBSERVATION & Mesa County \\
\hline 1636 & U.S. Geological Survey & 391723108572101 & SC00910412BCC1 (VT2) & OBSERVATION & Mesa County \\
\hline 1639 & U.S. Geological Survey & 390312108290101 & $\begin{array}{l}\text { OBSERVATION WELL } \\
\text { OMW1 AT ORCHARD } \\
\text { MESA W. AREA }\end{array}$ & OBSERVATION & Mesa County \\
\hline 1640 & U.S. Geological Survey & 390339108285701 & $\begin{array}{l}\text { COLO.R.WILDLIFE } \\
\text { AREA,EAST POOL, } \\
\text { TEST SITE 1,(SW) }\end{array}$ & OBSERVATION & Mesa County \\
\hline 1641 & U.S. Geological Survey & 390257108295301 & $\begin{array}{l}\text { OBSERVATION WELL OMW9, } \\
\text { ORCHARD } \\
\text { MESA W. AREA }\end{array}$ & OBSERVATION & Mesa County \\
\hline 1642 & U.S. Geological Survey & 390303108295501 & $\begin{array}{l}\text { OBSERVATION WELL } \\
\text { OMW10, ORCHARD }\end{array}$ & OBSERVATION & Mesa County \\
\hline 1643 & U.S. Geological Survey & 390305108294101 & $\begin{array}{c}\text { MESA W. AREA } \\
\text { OBSERVATION WELL OMW7, } \\
\text { ORCHARD } \\
\text { MESA W. AREA }\end{array}$ & OBSERVATION & Mesa County \\
\hline
\end{tabular}


Appendix 1. Water Quality Data Used in This Report.-Continued

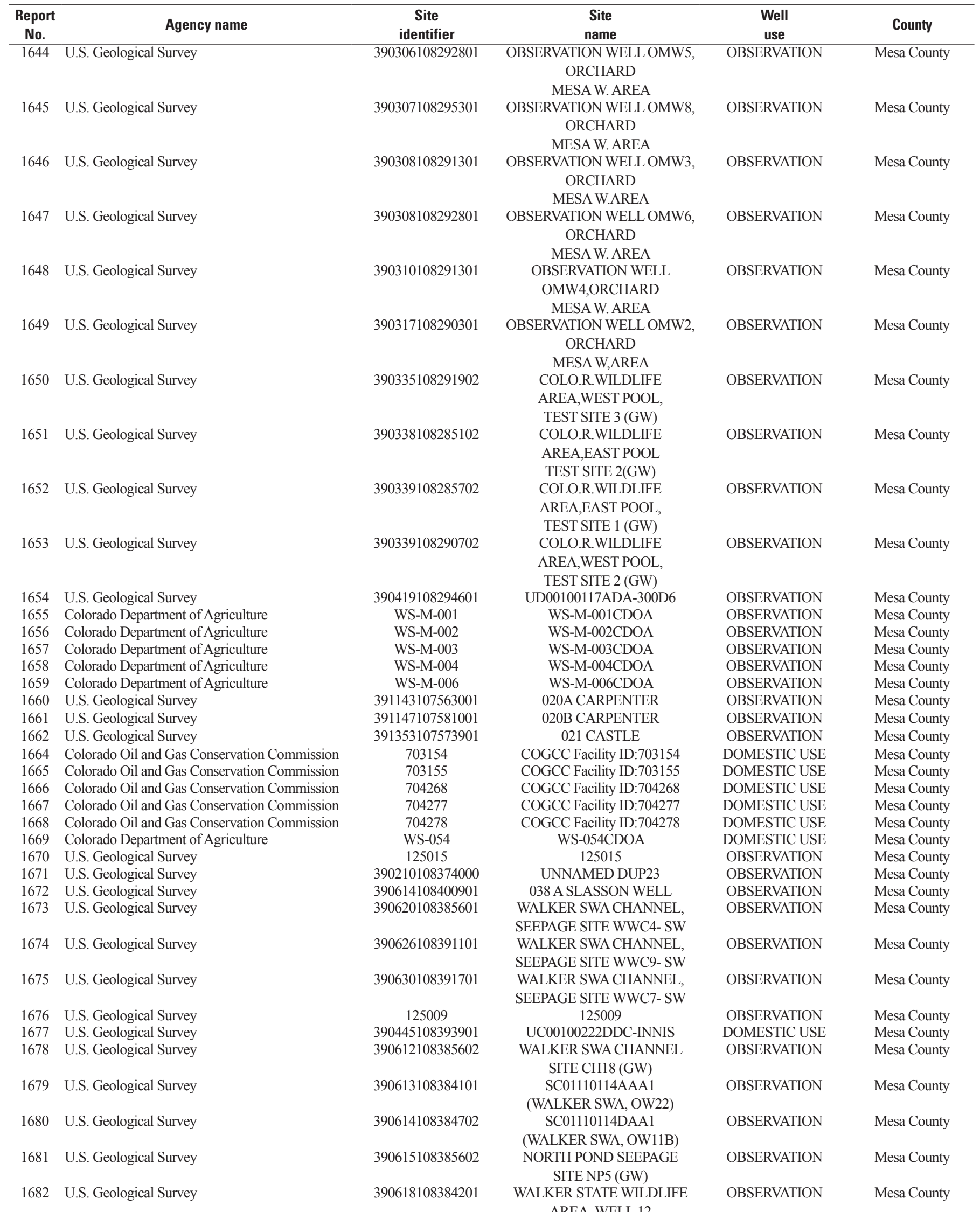


Appendix 1. Water Quality Data Used in This Report.-Continued

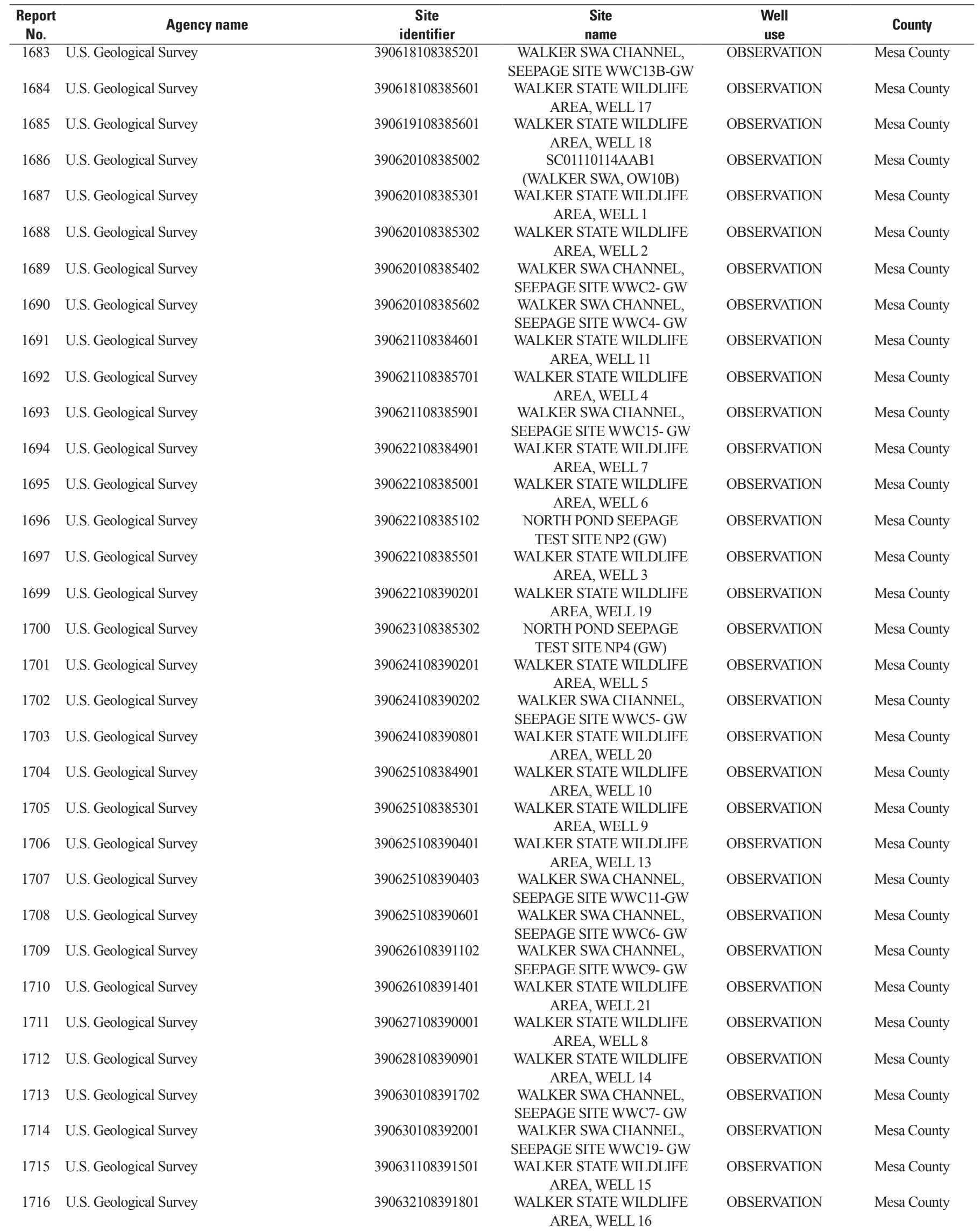


Appendix 1. Water Quality Data Used in This Report.-Continued

\begin{tabular}{|c|c|c|c|c|c|}
\hline $\begin{array}{c}\text { Report } \\
\text { No. }\end{array}$ & Agency name & $\begin{array}{c}\text { Site } \\
\text { identifier }\end{array}$ & $\begin{array}{c}\text { Site } \\
\text { name }\end{array}$ & $\begin{array}{l}\text { Well } \\
\text { use }\end{array}$ & County \\
\hline 1723 & U.S. Geological Survey & 392132108145301 & SC00809717CBA4 & OBSERVATION & Mesa County \\
\hline 1727 & U.S. Geological Survey & 390933108405701 & 035 ARS18 & OBSERVATION & Mesa County \\
\hline 1728 & U.S. Geological Survey & 391119108405301 & 036 ARS13 & OBSERVATION & Mesa County \\
\hline 1730 & U.S. Geological Survey & C16217 & $\mathrm{C} 16217$ & OBSERVATION & Mesa County \\
\hline 1731 & U.S. Geological Survey & 390232108325201 & UC00100125BCD1 & DOMESTIC USE & Mesa County \\
\hline 1732 & U.S. Geological Survey & 390246108340301 & UC00100126BBD1 & OBSERVATION & Mesa County \\
\hline 1733 & U.S. Geological Survey & 390255108311201 & UD00100119DCD1 & AGRICULTURAL USE & Mesa County \\
\hline 1734 & U.S. Geological Survey & 390301108300101 & $\begin{array}{c}\text { OBSERVATION WELL } \\
\text { OMW13, ORCHARD } \\
\text { MESA W. AREA }\end{array}$ & OBSERVATION & Mesa County \\
\hline 1735 & U.S. Geological Survey & 390302108295901 & $\begin{array}{c}\text { OBSERVATION WELL } \\
\text { OMW11, ORCHARD } \\
\text { MESA W. AREA }\end{array}$ & OBSERVATION & Mesa County \\
\hline 1736 & U.S. Geological Survey & 390306108295901 & $\begin{array}{l}\text { OBSERVATION WELL } \\
\text { OMW12, ORCHARD } \\
\text { MESA W. AREA }\end{array}$ & OBSERVATION & Mesa County \\
\hline 1738 & U.S. Geological Survey & 390328108344701 & UC00100122BAA1 & OBSERVATION & Mesa County \\
\hline 1741 & Colorado Department of Agriculture & WS-M-007 & WS-M-007CDOA & OBSERVATION & Mesa County \\
\hline 1742 & Colorado Department of Agriculture & WS-M-008 & WS-M-008CDOA & OBSERVATION & Mesa County \\
\hline 1743 & Colorado Department of Agriculture & WS-M-009 & WS-M-009CDOA & OBSERVATION & Mesa County \\
\hline 1744 & Colorado Department of Agriculture & WS-M-010 & WS-M-010CDOA & OBSERVATION & Mesa County \\
\hline 1745 & Colorado Oil and Gas Conservation Commission & 703089 & COGCC Facility ID:703089 & DOMESTIC USE & Mesa County \\
\hline 1747 & U.S. Geological Survey & 391853108510401 & SC00810335DBD-108U0 & OBSERVATION & Mesa County \\
\hline 1752 & EnCana Oil \& Gas (USA) Inc. & BAIL1WW & BAIL1WW & DOMESTIC USE & Mesa County \\
\hline 1753 & EnCana Oil \& Gas (USA) Inc. & BRAD1WW & BRAD1WW & DOMESTIC USE & Mesa County \\
\hline 1754 & EnCana Oil \& Gas (USA) Inc. & CROM1WW & CROM1WW & DOMESTIC USE & Mesa County \\
\hline 1755 & EnCana Oil \& Gas (USA) Inc. & HONA1WW & HONA1WW & DOMESTIC USE & Mesa County \\
\hline 1756 & EnCana Oil \& Gas (USA) Inc. & LAKE1WW & LAKE1WW & DOMESTIC USE & Mesa County \\
\hline 1757 & EnCana Oil \& Gas (USA) Inc. & MACK1WW & MACK1WW & DOMESTIC USE & Mesa County \\
\hline 1758 & EnCana Oil \& Gas (USA) Inc. & RADE1WW & RADE1WW & DOMESTIC USE & Mesa County \\
\hline 1759 & EnCana Oil \& Gas (USA) Inc. & STAN1WW & STAN1WW & DOMESTIC USE & Mesa County \\
\hline 1760 & EnCana Oil \& Gas (USA) Inc. & WHIT1WW & WHIT1WW & DOMESTIC USE & Mesa County \\
\hline 1761 & City of Grand Junction & GWSOMR3 & Sommerville Well 3 & OBSERVATION & Mesa County \\
\hline 1762 & U.S. Geological Survey & 385623108255401 & UD00200234DBC1 & OBSERVATION & Mesa County \\
\hline 1763 & U.S. Geological Survey & $\mathrm{C} 16582$ & $\mathrm{C} 16582$ & OBSERVATION & Mesa County \\
\hline 1765 & U.S. Geological Survey & 391057108484301 & UB00100303CCB-130L3 & OBSERVATION & Mesa County \\
\hline 1766 & U.S. Geological Survey & 391203108473801 & UB00200334ADD01-RW1 & OBSERVATION & Mesa County \\
\hline 1768 & U.S. Geological Survey & 391208108473801 & UB00200334ADD03-RW3 & OBSERVATION & Mesa County \\
\hline 1769 & U.S. Geological Survey & 391226108472801 & UB00200335BBA-143N0 & OBSERVATION & Mesa County \\
\hline 1770 & U.S. Geological Survey & 125788 & 125788 & OBSERVATION & Mesa County \\
\hline 1771 & U.S. Geological Survey & 390931108093601 & 059 HARVEY WELL & OBSERVATION & Mesa County \\
\hline 1772 & U.S. Geological Survey & 391059108075601 & 016 HIGHWAY SPRING & OBSERVATION & Mesa County \\
\hline 1773 & Colorado Oil and Gas Conservation Commission & 703157 & COGCC Facility ID:703157 & DOMESTIC USE & Mesa County \\
\hline 1774 & Colorado Oil and Gas Conservation Commission & 703076 & COGCC Facility ID:703076 & DOMESTIC USE & Mesa County \\
\hline 1776 & Colorado Oil and Gas Conservation Commission & 703090 & COGCC Facility ID:703090 & DOMESTIC USE & Mesa County \\
\hline 1777 & Colorado Oil and Gas Conservation Commission & 703158 & COGCC Facility ID:703158 & DOMESTIC USE & Mesa County \\
\hline 1778 & Colorado Oil and Gas Conservation Commission & 703167 & COGCC Facility ID:703167 & DOMESTIC USE & Mesa County \\
\hline 1779 & Colorado Oil and Gas Conservation Commission & 703168 & COGCC Facility ID:703168 & DOMESTIC USE & Mesa County \\
\hline 1780 & Colorado Oil and Gas Conservation Commission & 703170 & COGCC Facility ID:703170 & DOMESTIC USE & Mesa County \\
\hline 1781 & Colorado Oil and Gas Conservation Commission & 703171 & COGCC Facility ID:703171 & DOMESTIC USE & Mesa County \\
\hline 1782 & Colorado Oil and Gas Conservation Commission & 704270 & COGCC Facility ID:704270 & DOMESTIC USE & Mesa County \\
\hline 1783 & Colorado Oil and Gas Conservation Commission & 704271 & COGCC Facility ID:704271 & DOMESTIC USE & Mesa County \\
\hline 1784 & Colorado Oil and Gas Conservation Commission & 704272 & COGCC Facility ID:704272 & DOMESTIC USE & Mesa County \\
\hline 1785 & Colorado Oil and Gas Conservation Commission & 704273 & COGCC Facility ID:704273 & DOMESTIC USE & Mesa County \\
\hline 1786 & Colorado Oil and Gas Conservation Commission & 704274 & COGCC Facility ID:704274 & DOMESTIC USE & Mesa County \\
\hline 1787 & U.S. Geological Survey & 125808 & 125808 & OBSERVATION & Mesa County \\
\hline 1788 & EnCana Oil \& Gas (USA) Inc. & LIND1WW & LIND1WW & DOMESTIC USE & Mesa County \\
\hline 1789 & U.S. Geological Survey & 125619 & 125619 & OBSERVATION & Mesa County \\
\hline 1790 & U.S. Geological Survey & 125772 & 125772 & OBSERVATION & Mesa County \\
\hline 1791 & U.S. Geological Survey & 125773 & 125773 & OBSERVATION & Mesa County \\
\hline 1792 & U.S. Geological Survey & 125774 & 125774 & OBSERVATION & Mesa County \\
\hline 1793 & U.S. Geological Survey & 125775 & 125775 & OBSERVATION & Mesa County \\
\hline 1794 & U.S. Geological Survey & 390958108071701 & 058 TRAHERN WELL & OBSERVATION & Mesa County \\
\hline 1795 & U.S. Geological Survey & 391119108031301 & 017 CHURCH & OBSERVATION & Mesa County \\
\hline 1796 & Colorado Oil and Gas Conservation Commission & 703152 & COGCC Facility ID:703152 & DOMESTIC USE & Mesa County \\
\hline 1797 & Colorado Oil and Gas Conservation Commission & 703150 & COGCC Facility ID:703150 & DOMESTIC USE & Mesa County \\
\hline 1798 & Colorado Oil and Gas Conservation Commission & 703151 & COGCC Facility ID:703151 & DOMESTIC USE & Mesa County \\
\hline 1799 & Colorado Oil and Gas Conservation Commission & 703153 & COGCC Facility ID:703153 & DOMESTIC USE & Mesa County \\
\hline
\end{tabular}


Appendix 1. Water Quality Data Used in This Report.-Continued

\begin{tabular}{|c|c|c|c|c|c|}
\hline $\begin{array}{c}\text { Report } \\
\text { No. }\end{array}$ & Agency name & $\begin{array}{c}\text { Site } \\
\text { identifier }\end{array}$ & $\begin{array}{c}\text { Site } \\
\text { name }\end{array}$ & $\begin{array}{l}\text { Well } \\
\text { use }\end{array}$ & County \\
\hline 1800 & Colorado Oil and Gas Conservation Commission & 703156 & COGCC Facility ID:703156 & DOMESTIC USE & Mesa County \\
\hline 1801 & Colorado Oil and Gas Conservation Commission & 703159 & COGCC Facility ID:703159 & DOMESTIC USE & Mesa County \\
\hline 1802 & Colorado Oil and Gas Conservation Commission & 703172 & COGCC Facility ID:703172 & DOMESTIC USE & Mesa County \\
\hline 1803 & Colorado Oil and Gas Conservation Commission & 703173 & COGCC Facility ID:703173 & DOMESTIC USE & Mesa County \\
\hline 1804 & Colorado Oil and Gas Conservation Commission & 703174 & COGCC Facility ID:703174 & DOMESTIC USE & Mesa County \\
\hline 1805 & Colorado Oil and Gas Conservation Commission & 703175 & COGCC Facility ID:703175 & DOMESTIC USE & Mesa County \\
\hline 1806 & Colorado Oil and Gas Conservation Commission & 703176 & COGCC Facility ID:703176 & DOMESTIC USE & Mesa County \\
\hline 1807 & Colorado Oil and Gas Conservation Commission & 703805 & COGCC Facility ID:703805 & DOMESTIC USE & Mesa County \\
\hline 1807 & Colorado Oil and Gas Conservation Commission & 703805 & COGCC Facility ID:703805 & DOMESTIC USE & Mesa County \\
\hline 1808 & Colorado Oil and Gas Conservation Commission & 704269 & COGCC Facility ID:704269 & DOMESTIC USE & Mesa County \\
\hline 1809 & Colorado Oil and Gas Conservation Commission & 704275 & COGCC Facility ID:704275 & DOMESTIC USE & Mesa County \\
\hline 1810 & Colorado Oil and Gas Conservation Commission & 704276 & COGCC Facility ID:704276 & DOMESTIC USE & Mesa County \\
\hline 1811 & EnCana Oil \& Gas (USA) Inc. & brit1ww & BRIT1WW & DOMESTIC USE & Mesa County \\
\hline 1812 & EnCana Oil \& Gas (USA) Inc. & curr2ww & CURR2WW & DOMESTIC USE & Mesa County \\
\hline 1813 & EnCana Oil \& Gas (USA) Inc. & FIFI1WW & FIFI1WW & DOMESTIC USE & Mesa County \\
\hline 1814 & EnCana Oil \& Gas (USA) Inc. & hall1ww & HALL1WW & DOMESTIC USE & Mesa County \\
\hline 1815 & Colorado Department of Agriculture & WS-055 & WS-055CDOA & DOMESTIC USE & Mesa County \\
\hline 1816 & EnCana Oil \& Gas (USA) Inc. & HITT1WW & HITT1WW & DOMESTIC USE & Mesa County \\
\hline 1817 & EnCana Oil \& Gas (USA) Inc. & hitt2ww & HITT2WW & DOMESTIC USE & Mesa County \\
\hline 1818 & EnCana Oil \& Gas (USA) Inc. & KITC1WW & KITC1WW & DOMESTIC USE & Mesa County \\
\hline 1819 & Colorado Department of Agriculture & WS-056 & WS-056CDOA & DOMESTIC USE & Mesa County \\
\hline 1820 & EnCana Oil \& Gas (USA) Inc. & ZENT1WW & ZENT1WW & DOMESTIC USE & Mesa County \\
\hline 1821 & EnCana Oil \& Gas (USA) Inc. & VANZ1 & VANZ1 & DOMESTIC USE & Mesa County \\
\hline 1822 & EnCana Oil \& Gas (USA) Inc. & REID1WW & REID1WW & DOMESTIC USE & Mesa County \\
\hline 1827 & City of Grand Junction & GWSOMR1 & Sommerville Well 1 & OBSERVATION & Mesa County \\
\hline 1828 & City of Grand Junction & GWSOMR2 & Sommerville Well 2 & OBSERVATION & Mesa County \\
\hline 1829 & U.S. Geological Survey & 391613108434301 & $174 \mathrm{R} 4$ & OBSERVATION & Mesa County \\
\hline 1830 & U.S. Geological Survey & 391310107472801 & 023 VEGA LAKE WELL & OBSERVATION & Mesa County \\
\hline 1832 & Colorado Oil and Gas Conservation Commission & 703088 & COGCC Facility ID:703088 & DOMESTIC USE & Mesa County \\
\hline 1833 & U.S. Geological Survey & 391906108161801 & 044 WEB PLACE WELL & OBSERVATION & Mesa County \\
\hline 1837 & U.S. Geological Survey & 392129108145701 & SC00809717CBA8 & OBSERVATION & Mesa County \\
\hline 1842 & U.S. Geological Survey & 385835108260701 & UC00200113CCC1 (WELL 3) & OBSERVATION & Mesa County \\
\hline 1843 & U.S. Geological Survey & 385918108263901 & UC00200114AAC1 (WELL 5) & OBSERVATION & Mesa County \\
\hline 1845 & U.S. Geological Survey & 385924108314101 & UD00200115BAB1 & AGRICULTURAL USE & Mesa County \\
\hline 1847 & U.S. Geological Survey & 385930108265701 & UC00200111DCC1（WELL6) & OBSERVATION & Mesa County \\
\hline 1855 & Colorado Oil and Gas Conservation Commission & 703831 & COGCC Facility ID:703831 & PUBLIC SUPPLY & Delta County \\
\hline 1856 & Colorado Oil and Gas Conservation Commission & 703832 & COGCC Facility ID:703832 & PUBLIC SUPPLY & Delta County \\
\hline 1857 & Colorado Oil and Gas Conservation Commission & 703839 & COGCC Facility ID:703839 & PUBLIC SUPPLY & Delta County \\
\hline 1858 & Colorado Oil and Gas Conservation Commission & 703845 & COGCC Facility ID:703845 & PUBLIC SUPPLY & Delta County \\
\hline 1859 & Colorado Oil and Gas Conservation Commission & 703846 & COGCC Facility ID:703846 & PUBLIC SUPPLY & Delta County \\
\hline 1860 & Colorado Oil and Gas Conservation Commission & 703847 & COGCC Facility ID:703847 & PUBLIC SUPPLY & Delta County \\
\hline 1861 & Colorado Oil and Gas Conservation Commission & 703856 & COGCC Facility ID:703856 & PUBLIC SUPPLY & Delta County \\
\hline 1862 & Colorado Oil and Gas Conservation Commission & 703837 & COGCC Facility ID:703837 & PUBLIC SUPPLY & Delta County \\
\hline 1863 & Colorado Oil and Gas Conservation Commission & 703838 & COGCC Facility ID:703838 & PUBLIC SUPPLY & Delta County \\
\hline 1864 & Mine Consultant & DH-15-Bowie \#2 & DH-15-Bowie \#2 & OBSERVATION & Delta County \\
\hline 1865 & Mine Consultant & DH-25-Bowie \#2 & DH-25-Bowie \#2 & OBSERVATION & Delta County \\
\hline 1866 & Mine Consultant & DH-38-Bowie \#2 & DH-38-Bowie \#2 & OBSERVATION & Delta County \\
\hline 1867 & Mine Consultant & DH-39-Bowie \#2 & DH-39-Bowie \#2 & OBSERVATION & Delta County \\
\hline 1868 & Mine Consultant & DH-49-Bowie \#2 & DH-49-Bowie \#2 & OBSERVATION & Delta County \\
\hline 1869 & Mine Consultant & DH-58-Bowie \#2 & DH-58-Bowie \#2 & OBSERVATION & Delta County \\
\hline 1870 & Mine Consultant & DH-67B-Bowie \#2 & DH-67B-Bowie \#2 & OBSERVATION & Delta County \\
\hline 1871 & Mine Consultant & AW-11-Bowie \#2 & AW-11-Bowie \#2 & OBSERVATION & Delta County \\
\hline 1872 & Mine Consultant & AW-14-Bowie \#2 & AW-14-Bowie \#2 & OBSERVATION & Delta County \\
\hline 1873 & Mine Consultant & AW-16-Bowie \#2 & AW-16-Bowie \#2 & OBSERVATION & Delta County \\
\hline 1874 & Mine Consultant & AW-17-Bowie \#2 & AW-17-Bowie \#2 & OBSERVATION & Delta County \\
\hline 1875 & Mine Consultant & AW-1-Bowie \#2 & AW-1-Bowie \#2 & OBSERVATION & Delta County \\
\hline 1876 & Mine Consultant & AW-2-Bowie \#2 & AW-2-Bowie \#2 & OBSERVATION & Delta County \\
\hline 1877 & Mine Consultant & AW-3-Bowie \#2 & AW-3-Bowie \#2 & OBSERVATION & Delta County \\
\hline 1878 & Mine Consultant & AW-4-Bowie \#2 & AW-4-Bowie \#2 & OBSERVATION & Delta County \\
\hline 1879 & Mine Consultant & AW-5-Bowie \#2 & AW-5-Bowie \#2 & OBSERVATION & Delta County \\
\hline 1880 & Mine Consultant & AW-6-Bowie \#2 & AW-6-Bowie \#2 & OBSERVATION & Delta County \\
\hline 1881 & Mine Consultant & AW-7-Bowie \#2 & AW-7-Bowie \#2 & OBSERVATION & Delta County \\
\hline 1882 & Mine Consultant & AW-8-Bowie \#2 & AW-8-Bowie \#2 & OBSERVATION & Delta County \\
\hline 1883 & Mine Consultant & AW-9-Bowie \#2 & AW-9-Bowie \#2 & OBSERVATION & Delta County \\
\hline 1884 & Mine Consultant & B07-Bowie \#1 & B07-Bowie \#1 & OBSERVATION & Delta County \\
\hline 1885 & Mine Consultant & MW01-Bowie \#1 & MW01-Bowie \#1 & OBSERVATION & Delta County \\
\hline 1886 & Mine Consultant & MW03-Bowie \#1 & MW03-Bowie \#1 & OBSERVATION & Delta County \\
\hline 1887 & Colorado Department of Agriculture & WS-063 & WS-063CDOA & DOMESTIC USE & Delta County \\
\hline
\end{tabular}


Appendix 1. Water Quality Data Used in This Report.-Continued

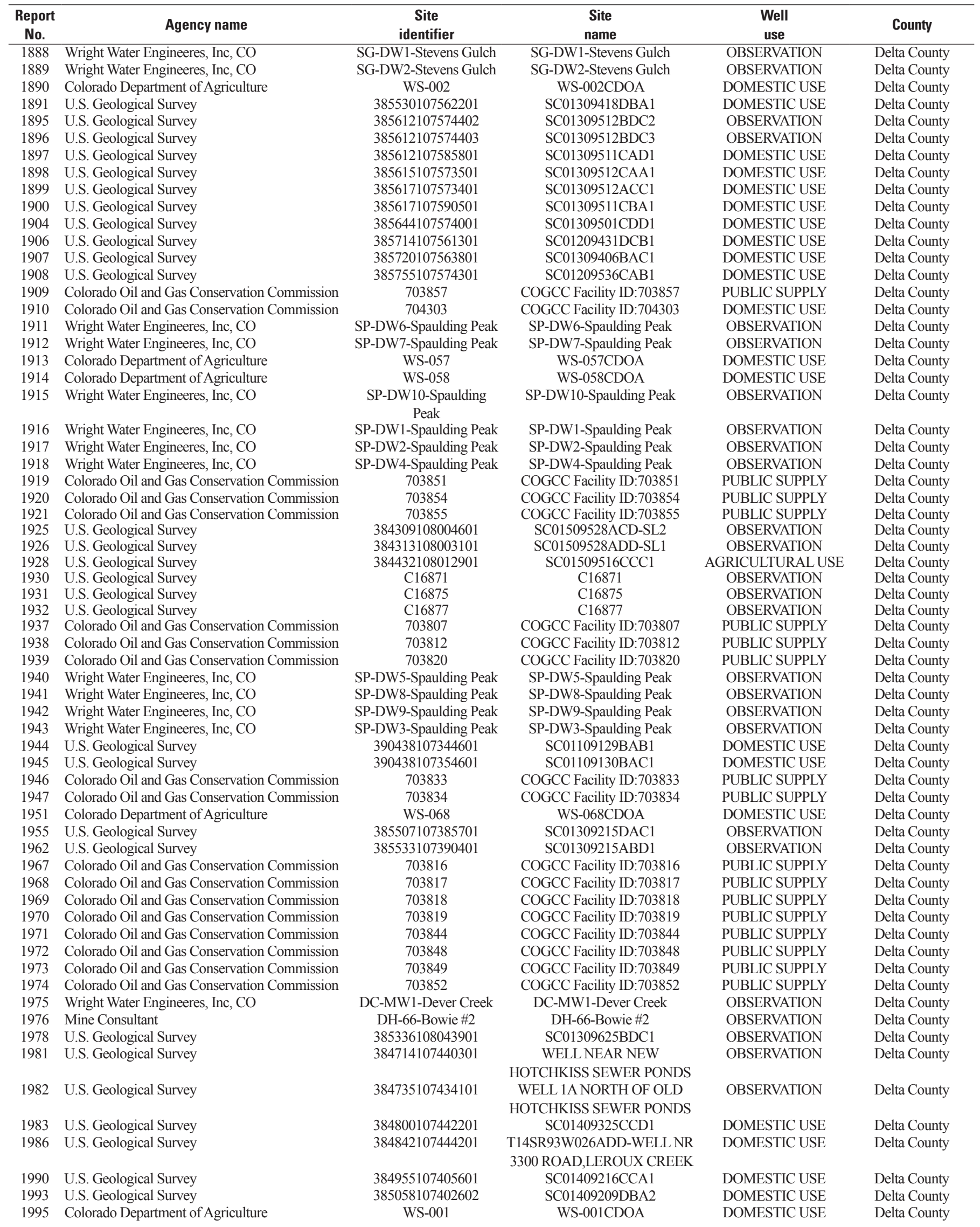


Appendix 1. Water Quality Data Used in This Report.-Continued

\begin{tabular}{|c|c|c|c|c|c|}
\hline $\begin{array}{c}\text { Report } \\
\text { No. }\end{array}$ & Agency name & $\begin{array}{c}\text { Site } \\
\text { identifier }\end{array}$ & $\begin{array}{c}\text { Site } \\
\text { name }\end{array}$ & $\begin{array}{c}\text { Well } \\
\text { use }\end{array}$ & County \\
\hline 1996 & Colorado Department of Agriculture & WS-008 & WS-008CDOA & DOMESTIC USE & Delta County \\
\hline 2001 & U.S. Geological Survey & 384735107470501 & SC01409333ADC1 & OBSERVATION & Delta County \\
\hline 2002 & U.S. Geological Survey & 384801107454201 & SC01409326CCC1 & DOMESTIC USE & Delta County \\
\hline 2003 & U.S. Geological Survey & 384809107464501 & SC01409327CCC1 & DOMESTIC USE & Delta County \\
\hline 2011 & U.S. Geological Survey & 385215107473201 & SC01409304BAD1 & OBSERVATION & Delta County \\
\hline 2012 & U.S. Geological Survey & 385221107500101 & SC01409306BCD1 & DOMESTIC USE & Delta County \\
\hline 2013 & Wright Water Engineeres, Inc, CO & DC-DW1-Dever Creek & DC-DW1-Dever Creek & OBSERVATION & Delta County \\
\hline 2014 & Wright Water Engineeres, Inc, CO & DC-DW2-Dever Creek & DC-DW2-Dever Creek & OBSERVATION & Delta County \\
\hline 2015 & Colorado Oil and Gas Conservation Commission & 703809 & COGCC Facility ID:703809 & PUBLIC SUPPLY & Delta County \\
\hline 2016 & Colorado Department of Agriculture & WS-059 & WS-059CDOA & DOMESTIC USE & Delta County \\
\hline 2017 & Colorado Department of Agriculture & WS-062 & WS-062CDOA & DOMESTIC USE & Delta County \\
\hline 2018 & Colorado Department of Agriculture & WS-061 & WS-061CDOA & DOMESTIC USE & Delta County \\
\hline 2019 & Colorado Department of Agriculture & WS-004 & WS-004CDOA & DOMESTIC USE & Delta County \\
\hline 2020 & Colorado Department of Agriculture & WS-005 & WS-005CDOA & DOMESTIC USE & Delta County \\
\hline 2021 & Colorado Department of Agriculture & WS-006 & WS-006CDOA & DOMESTIC USE & Delta County \\
\hline 2022 & Colorado Department of Agriculture & WS-007 & WS-007CDOA & DOMESTIC USE & Delta County \\
\hline 2023 & Colorado Department of Agriculture & WS-009 & WS-009CDOA & DOMESTIC USE & Delta County \\
\hline 2024 & Colorado Department of Agriculture & WS-011 & WS-011CDOA & DOMESTIC USE & Delta County \\
\hline 2025 & U.S. Forest Service & 390021107505801 & LEON LAKE 5 MW & OBSERVATION & Delta County \\
\hline 2027 & U.S. Geological Survey & 384243107573701 & SC01509525DCC-B254334 & OBSERVATION & Delta County \\
\hline 2029 & U.S. Geological Survey & 384953107540601 & SC01409416DCA1 & DOMESTIC USE & Delta County \\
\hline 2030 & Colorado Department of Agriculture & WS-012 & WS-012CDOA & DOMESTIC USE & Delta County \\
\hline 2033 & U.S. Geological Survey & 385047107332801 & SC01409109DAC1 & DOMESTIC USE & Delta County \\
\hline 2034 & Colorado Department of Agriculture & WS-003 & WS-003CDOA & DOMESTIC USE & Delta County \\
\hline 2035 & Colorado Department of Agriculture & WS-013 & WS-013CDOA & DOMESTIC USE & Delta County \\
\hline 2037 & U.S. Geological Survey & 390256107214001 & SC01208905BAA1 & DOMESTIC USE & Gunnison County \\
\hline 2040 & U.S. Geological Survey & $\mathrm{C} 00132$ & $\mathrm{C} 00132$ & OBSERVATION & Moffat County \\
\hline 2041 & U.S. Geological Survey & 383746108055601 & NB05001104ADC1 & DOMESTIC USE & Montrose County \\
\hline 2044 & U.S. Geological Survey & 383839108013801 & NB05101031ACD & AGRICULTURAL USE & Montrose County \\
\hline 2046 & U.S. Geological Survey & 383847108024001 & NB05101136ABD-KRAMER & AGRICULTURAL USE & Montrose County \\
\hline 2046 & U.S. Geological Survey & 383847108024001 & NB05101136ABD-KRAMER & AGRICULTURAL USE & Montrose County \\
\hline 2049 & U.S. Geological Survey & $\mathrm{C} 16867$ & C16867 & OBSERVATION & Montrose County \\
\hline 2050 & U.S. Geological Survey & C16868 & C16868 & OBSERVATION & Montrose County \\
\hline 2051 & U.S. Geological Survey & C16869 & C16869 & OBSERVATION & Montrose County \\
\hline 2052 & U.S. Geological Survey & $\mathrm{C} 00106$ & $\mathrm{C} 00106$ & OBSERVATION & Moffat County \\
\hline 2053 & U.S. Geological Survey & 384005107401001 & NB05100720DBD1 & OBSERVATION & Montrose County \\
\hline 2054 & U.S. Geological Survey & 383126107443801 & NB04900811CBD & DOMESTIC USE & Montrose County \\
\hline 2055 & U.S. Geological Survey & $\mathrm{C} 00268$ & $\mathrm{C} 00268$ & OBSERVATION & Moffat County \\
\hline 2056 & U.S. Geological Survey & 383424108015801 & NB05001030BAD1 & DOMESTIC USE & Montrose County \\
\hline 2058 & U.S. Geological Survey & 383438108025401 & NB05001124DCC & DOMESTIC USE & Montrose County \\
\hline 2061 & U.S. Geological Survey & 383621108012001 & NB05001007DDD-WEBB & AGRICULTURAL USE & Montrose County \\
\hline 2063 & U.S. Geological Survey & 383715108033901 & NB05001111AAB1 & OBSERVATION & Montrose County \\
\hline 2064 & U.S. Geological Survey & $\mathrm{C} 16856$ & $\mathrm{C} 16856$ & OBSERVATION & Montrose County \\
\hline 2065 & U.S. Geological Survey & 401545108514200 & $\mathrm{~S}(\mathrm{~B}-3-102) 5 \mathrm{ADB}-1$ & OBSERVATION & Moffat County \\
\hline 2066 & U.S. Geological Survey & 401545108514201 & SB00310205ADB1 & PUBLIC SUPPLY & Moffat County \\
\hline 2067 & U.S. Geological Survey & $\mathrm{C} 00337$ & $\mathrm{C} 00337$ & OBSERVATION & Moffat County \\
\hline 2068 & U.S. Geological Survey & 401648108362701 & SB00410034BBB1 & DOMESTIC USE & Moffat County \\
\hline 2069 & U.S. Geological Survey & $\mathrm{C} 00200$ & $\mathrm{C} 00200$ & OBSERVATION & Moffat County \\
\hline 2070 & U.S. Geological Survey & $\mathrm{C} 00204$ & C00204 & OBSERVATION & Moffat County \\
\hline 2071 & U.S. Geological Survey & 401449108540101 & SB00310312ACA1 & AGRICULTURAL USE & Moffat County \\
\hline 2072 & U.S. Geological Survey & $\mathrm{C} 00220$ & $\mathrm{C} 00220$ & OBSERVATION & Moffat County \\
\hline 2076 & U.S. Geological Survey & 383901107584401 & NB05101027CDC1 & AGRICULTURAL USE & Montrose County \\
\hline & & & (STOCK WELL,BANNER RD) & & \\
\hline 2077 & Colorado Oil and Gas Conservation Commission & 704194 & COGCC Facility ID:704194 & DOMESTIC USE & Gunnison County \\
\hline 2078 & U.S. Geological Survey & 401505108584201 & S(B- 3-103) 5DCC- 1 & OBSERVATION & Moffat County \\
\hline 2079 & U.S. Geological Survey & 401505108594501 & SB00310307ABA1 & PUBLIC SUPPLY & Moffat County \\
\hline 2080 & U.S. Geological Survey & 401506108584201 & SB00310305DCC1 & PUBLIC SUPPLY & Moffat County \\
\hline 2082 & U.S. Geological Survey & $\mathrm{C} 00251$ & $\mathrm{C} 00251$ & OBSERVATION & Moffat County \\
\hline 2083 & U.S. Geological Survey & 401511108042701 & SB00509533CCB & AGRICULTURAL USE & Moffat County \\
\hline 2084 & U.S. Geological Survey & 401640108055101 & SB00409531BAC & OBSERVATION & Moffat County \\
\hline 2085 & U.S. Geological Survey & 401825108061201 & SB00409624AAD1 & OBSERVATION & Moffat County \\
\hline 2088 & U.S. Geological Survey & 401539108424601 & SB00310103ACD1 & OBSERVATION & Moffat County \\
\hline 2089 & U.S. Geological Survey & $\mathrm{C} 00344$ & $\mathrm{C} 00344$ & OBSERVATION & Moffat County \\
\hline 2090 & U.S. Geological Survey & 401509109001001 & SB00310306CDC1 & PUBLIC SUPPLY & Moffat County \\
\hline 2091 & U.S. Geological Survey & 401517109004701 & SB00310401DCA2 & COMMERCIAL USE & Moffat County \\
\hline 2094 & Oxbow Mining & EC-14-Elk Creek Mine & EC-14-Elk Creek Mine & OBSERVATION & Gunnison County \\
\hline 2095 & U.S. Geological Survey & C16477 & C16477 & OBSERVATION & Montrose County \\
\hline 2096 & U.S. Geological Survey & $\mathrm{C} 16510$ & $\mathrm{C} 16510$ & OBSERVATION & Montrose County \\
\hline
\end{tabular}


Appendix 1. Water Quality Data Used in This Report.-Continued

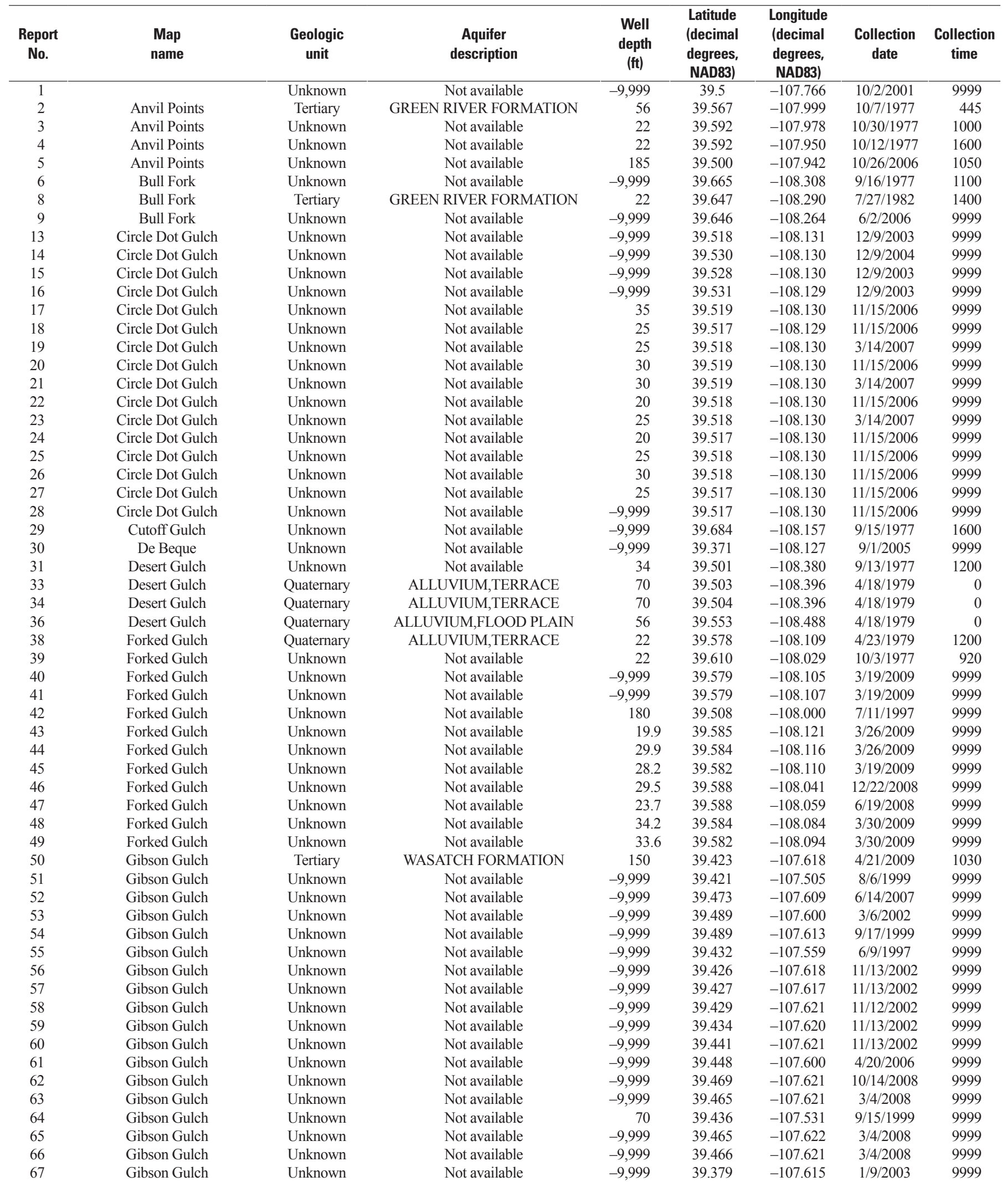


Appendix 1. Water Quality Data Used in This Report.-Continued

\begin{tabular}{|c|c|c|c|c|c|c|c|c|}
\hline $\begin{array}{c}\text { Report } \\
\text { No. }\end{array}$ & $\begin{array}{l}\text { Map } \\
\text { name }\end{array}$ & $\begin{array}{c}\text { Geologic } \\
\text { unit }\end{array}$ & $\begin{array}{c}\text { Aquifer } \\
\text { description }\end{array}$ & $\begin{array}{c}\text { Well } \\
\text { depth } \\
\text { (ft) }\end{array}$ & $\begin{array}{l}\text { Latitude } \\
\text { (decimal } \\
\text { degrees, } \\
\text { NAD83) }\end{array}$ & $\begin{array}{c}\text { Longitude } \\
\text { (decimal } \\
\text { degrees, } \\
\text { NAD83) }\end{array}$ & $\begin{array}{c}\text { Collection } \\
\text { date }\end{array}$ & $\begin{array}{c}\text { Collection } \\
\text { time }\end{array}$ \\
\hline 68 & Gibson Gulch & Unknown & Not available & $-9,999$ & 39.492 & -107.592 & $2 / 16 / 2005$ & 9999 \\
\hline 69 & Gibson Gulch & Unknown & Not available & $-9,999$ & 39.466 & -107.621 & $9 / 29 / 2008$ & 9999 \\
\hline 70 & Gibson Gulch & Unknown & Not available & $-9,999$ & 39.468 & -107.621 & $9 / 29 / 2008$ & 9999 \\
\hline 71 & Gibson Gulch & Unknown & Not available & $-9,999$ & 39.496 & -107.601 & $4 / 29 / 2005$ & 9999 \\
\hline 72 & Gibson Gulch & Unknown & Not available & $-9,999$ & 39.489 & -107.598 & $4 / 29 / 2005$ & 9999 \\
\hline 73 & Gibson Gulch & Unknown & Not available & $-9,999$ & 39.492 & -107.611 & $2 / 1 / 2005$ & 9999 \\
\hline 74 & Gibson Gulch & Unknown & Not available & $-9,999$ & 39.491 & -107.610 & $4 / 29 / 2005$ & 9999 \\
\hline 75 & Gibson Gulch & Unknown & Not available & $-9,999$ & 39.487 & -107.607 & $4 / 29 / 2005$ & 9999 \\
\hline 76 & Gibson Gulch & Unknown & Not available & $-9,999$ & 39.500 & -107.575 & $7 / 21 / 2005$ & 9999 \\
\hline 77 & Gibson Gulch & Unknown & Not available & 320 & 39.396 & -107.624 & $1 / 9 / 2003$ & 9999 \\
\hline 78 & Gibson Gulch & Unknown & Not available & 260 & 39.381 & -107.613 & $1 / 9 / 2003$ & 9999 \\
\hline 79 & Gibson Gulch & Unknown & Not available & 15 & 39.416 & -107.616 & $8 / 11 / 2004$ & 9999 \\
\hline 80 & Gibson Gulch & Unknown & Not available & 18 & 39.467 & -107.620 & $12 / 1 / 2008$ & 9999 \\
\hline 81 & Gibson Gulch & Unknown & Not available & 150 & 39.453 & -107.619 & $5 / 1 / 2006$ & 9999 \\
\hline 82 & Gibson Gulch & Unknown & Not available & 90 & 39.462 & -107.613 & $6 / 5 / 2006$ & 9999 \\
\hline 83 & Gibson Gulch & Unknown & Not available & 140 & 39.459 & -107.611 & $5 / 2 / 2006$ & 9999 \\
\hline 84 & Gibson Gulch & Unknown & Not available & 120 & 39.460 & -107.610 & $5 / 1 / 2006$ & 9999 \\
\hline 85 & Gibson Gulch & Unknown & Not available & 120 & 39.461 & -107.611 & $6 / 5 / 2006$ & 9999 \\
\hline 86 & Gibson Gulch & Unknown & Not available & 140 & 39.474 & -107.609 & $12 / 1 / 2008$ & 9999 \\
\hline 87 & Gibson Gulch & Unknown & Not available & 98 & 39.456 & -107.610 & $5 / 1 / 2006$ & 9999 \\
\hline 88 & Gibson Gulch & Unknown & Not available & 12 & 39.455 & -107.611 & $5 / 1 / 2006$ & 9999 \\
\hline 89 & Gibson Gulch & Unknown & Not available & 117 & 39.451 & -107.607 & $5 / 2 / 2006$ & 9999 \\
\hline 90 & Gibson Gulch & Unknown & Not available & $-9,999$ & 39.446 & -107.619 & $4 / 28 / 2004$ & 9999 \\
\hline 91 & Gibson Gulch & Unknown & Not available & $-9,999$ & 39.396 & -107.623 & $1 / 9 / 2003$ & 9999 \\
\hline 92 & Gibson Gulch & Unknown & Not available & $-9,999$ & 39.440 & -107.620 & $5 / 2 / 2005$ & 9999 \\
\hline 93 & Gibson Gulch & Unknown & Not available & $-9,999$ & 39.436 & -107.618 & $11 / 12 / 2002$ & 9999 \\
\hline 94 & Gibson Gulch & Unknown & Not available & $-9,999$ & 39.426 & -107.617 & $11 / 13 / 2002$ & 9999 \\
\hline 95 & Gibson Gulch & Unknown & Not available & $-9,999$ & 39.427 & -107.617 & $11 / 13 / 2002$ & 9999 \\
\hline 96 & Gibson Gulch & Unknown & Not available & $-9,999$ & 39.434 & -107.619 & $11 / 13 / 2002$ & 9999 \\
\hline 97 & Gibson Gulch & Unknown & Not available & $-9,999$ & 39.429 & -107.620 & $11 / 12 / 2002$ & 9999 \\
\hline 98 & Gibson Gulch & Unknown & Not available & $-9,999$ & 39.464 & -107.621 & 9/30/2008 & 9999 \\
\hline 99 & Gibson Gulch & Unknown & Not available & $-9,999$ & 39.464 & -107.621 & $10 / 1 / 2008$ & 9999 \\
\hline 100 & Gibson Gulch & Unknown & Not available & $-9,999$ & 39.464 & -107.621 & $10 / 1 / 2008$ & 9999 \\
\hline 101 & Gibson Gulch & Unknown & Not available & $-9,999$ & 39.464 & -107.621 & $9 / 30 / 2008$ & 9999 \\
\hline 102 & Gibson Gulch & Unknown & Not available & $-9,999$ & 39.463 & -107.621 & 9/30/2008 & 9999 \\
\hline 103 & Gibson Gulch & Unknown & Not available & $-9,999$ & 39.463 & -107.621 & 9/30/2008 & 9999 \\
\hline 104 & Gibson Gulch & Unknown & Not available & $-9,999$ & 39.463 & -107.622 & 9/30/2008 & 9999 \\
\hline 105 & Gibson Gulch & Unknown & Not available & $-9,999$ & 39.463 & -107.623 & $9 / 12 / 2007$ & 9999 \\
\hline 106 & Gibson Gulch & Unknown & Not available & $-9,999$ & 39.463 & -107.621 & 9/30/2008 & 9999 \\
\hline 107 & Gibson Gulch & Unknown & Not available & $-9,999$ & 39.464 & -107.620 & $6 / 17 / 2008$ & 9999 \\
\hline 108 & Gibson Gulch & Unknown & Not available & $-9,999$ & 39.379 & -107.615 & $1 / 25 / 2005$ & 9999 \\
\hline 109 & Gibson Gulch & Unknown & Not available & $-9,999$ & 39.482 & -107.589 & $5 / 1 / 2006$ & 9999 \\
\hline 110 & Gibson Gulch & Unknown & Not available & $-9,999$ & 39.483 & -107.589 & $5 / 1 / 2006$ & 9999 \\
\hline 111 & Gibson Gulch & Unknown & Not available & $-9,999$ & 39.448 & -107.619 & $11 / 19 / 2002$ & 9999 \\
\hline 112 & Gibson Gulch & Unknown & Not available & $-9,999$ & 39.455 & -107.610 & $7 / 8 / 2004$ & 9999 \\
\hline 113 & Gibson Gulch & Unknown & Not available & $-9,999$ & 39.464 & -107.621 & $9 / 30 / 2008$ & 9999 \\
\hline 114 & Gibson Gulch & Unknown & Not available & $-9,999$ & 39.464 & -107.621 & 9/30/2008 & 9999 \\
\hline 115 & Gibson Gulch & Unknown & Not available & $-9,999$ & 39.465 & -107.620 & 9/30/2008 & 9999 \\
\hline 116 & Gibson Gulch & Unknown & Not available & $-9,999$ & 39.463 & -107.621 & $6 / 12 / 2006$ & 9999 \\
\hline 117 & Gibson Gulch & Unknown & Not available & $-9,999$ & 39.465 & -107.621 & 9/30/2008 & 9999 \\
\hline 118 & Gibson Gulch & Unknown & Not available & $-9,999$ & 39.463 & -107.621 & $6 / 17 / 2008$ & 9999 \\
\hline 119 & Gibson Gulch & Unknown & Not available & $-9,999$ & 39.465 & -107.620 & $10 / 1 / 2008$ & 9999 \\
\hline 120 & Gibson Gulch & Unknown & Not available & $-9,999$ & 39.464 & -107.621 & $6 / 17 / 2008$ & 9999 \\
\hline 121 & Gibson Gulch & Unknown & Not available & $-9,999$ & 39.465 & -107.621 & 9/30/2008 & 9999 \\
\hline 122 & Gibson Gulch & Unknown & Not available & $-9,999$ & 39.465 & -107.620 & $9 / 30 / 2008$ & 9999 \\
\hline 123 & Gibson Gulch & Unknown & Not available & $-9,999$ & 39.465 & -107.621 & 9/30/2008 & 9999 \\
\hline 124 & Gibson Gulch & Unknown & Not available & $-9,999$ & 39.441 & -107.620 & $11 / 13 / 2002$ & 9999 \\
\hline 125 & Gibson Gulch & Unknown & Not available & $-9,999$ & 39.380 & -107.614 & $1 / 25 / 2005$ & 9999 \\
\hline 126 & Gibson Gulch & Unknown & Not available & $-9,999$ & 39.420 & -107.611 & $5 / 22 / 2003$ & 9999 \\
\hline 127 & Gibson Gulch & Unknown & Not available & $-9,999$ & 39.452 & -107.619 & $5 / 2 / 2006$ & 9999 \\
\hline 128 & Gibson Gulch & Unknown & Not available & $-9,999$ & 39.499 & -107.591 & $12 / 1 / 2005$ & 9999 \\
\hline 129 & Gibson Gulch & Unknown & Not available & $-9,999$ & 39.499 & -107.591 & $5 / 1 / 2006$ & 9999 \\
\hline 130 & Gibson Gulch & Unknown & Not available & $-9,999$ & 39.427 & -107.617 & $1 / 20 / 2005$ & 9999 \\
\hline
\end{tabular}


Appendix 1. Water Quality Data Used in This Report.-Continued

\begin{tabular}{|c|c|c|c|c|c|c|c|c|}
\hline $\begin{array}{c}\text { Report } \\
\text { No. }\end{array}$ & $\begin{array}{l}\text { Map } \\
\text { name }\end{array}$ & $\begin{array}{c}\text { Geologic } \\
\text { unit }\end{array}$ & $\begin{array}{c}\text { Aquifer } \\
\text { description }\end{array}$ & $\begin{array}{c}\text { Well } \\
\text { depth } \\
\text { (ft) }\end{array}$ & $\begin{array}{c}\text { Latitude } \\
\text { (decimal } \\
\text { degrees, } \\
\text { NAD83) }\end{array}$ & $\begin{array}{c}\text { Longitude } \\
\text { (decimal } \\
\text { degrees, } \\
\text { NAD83) }\end{array}$ & $\begin{array}{c}\text { Collection } \\
\text { date }\end{array}$ & $\begin{array}{l}\text { Collection } \\
\text { time }\end{array}$ \\
\hline 131 & Gibson Gulch & Unknown & Not available & $-9,999$ & 39.427 & -107.617 & $3 / 16 / 2005$ & 9999 \\
\hline 132 & Gibson Gulch & Unknown & Not available & $-9,999$ & 39.454 & -107.623 & $1 / 24 / 2003$ & 9999 \\
\hline 133 & Gibson Gulch & Unknown & Not available & $-9,999$ & 39.455 & -107.623 & $5 / 2 / 2006$ & 9999 \\
\hline 134 & Gibson Gulch & Unknown & Not available & $-9,999$ & 39.466 & -107.621 & $4 / 12 / 2006$ & 9999 \\
\hline 135 & Gibson Gulch & Unknown & Not available & $-9,999$ & 39.466 & -107.620 & $10 / 1 / 2008$ & 9999 \\
\hline 138 & Glenwood Springs & Quaternary & ALLUVIUM,TERRACE & $-9,999$ & 39.552 & -107.334 & $11 / 16 / 1984$ & 0 \\
\hline 142 & Housetop Mountain & Unknown & Not available & 160 & 39.369 & -108.052 & $10 / 21 / 1969$ & 0 \\
\hline 143 & Housetop Mountain & Unknown & Not available & $-9,999$ & 39.368 & -108.053 & $9 / 15 / 2004$ & 9999 \\
\hline 144 & Housetop Mountain & Unknown & Not available & $-9,999$ & 39.369 & -108.076 & $10 / 12 / 2004$ & 9999 \\
\hline 145 & Housetop Mountain & Unknown & Not available & 220 & 39.369 & -108.052 & $6 / 12 / 1997$ & 9999 \\
\hline 146 & Housetop Mountain & Unknown & Not available & 100 & 39.372 & -108.087 & 7/7/2006 & 9999 \\
\hline 147 & Housetop Mountain & Unknown & Not available & $-9,999$ & 39.374 & -108.106 & $6 / 23 / 2006$ & 9999 \\
\hline 148 & Housetop Mountain & Unknown & Not available & $-9,999$ & 39.374 & -108.122 & $9 / 1 / 2005$ & 9999 \\
\hline 149 & Housetop Mountain & Unknown & Not available & $-9,999$ & 39.369 & -108.087 & $7 / 6 / 2006$ & 9999 \\
\hline 150 & Housetop Mountain & Unknown & Not available & $-9,999$ & 39.371 & -108.088 & $7 / 7 / 2006$ & 9999 \\
\hline 151 & Housetop Mountain & Unknown & Not available & $-9,999$ & 39.371 & -108.089 & $7 / 13 / 2006$ & 9999 \\
\hline 152 & Hunter Mesa & Unknown & Not available & $-9,999$ & 39.478 & -107.691 & $5 / 17 / 2004$ & 9999 \\
\hline 153 & Hunter Mesa & Unknown & Not available & $-9,999$ & 39.476 & -107.657 & $6 / 12 / 2007$ & 9999 \\
\hline 154 & Hunter Mesa & Unknown & Not available & $-9,999$ & 39.464 & -107.691 & $3 / 4 / 2003$ & 1200 \\
\hline 155 & Hunter Mesa & Unknown & Not available & $-9,999$ & 39.454 & -107.689 & $3 / 5 / 2002$ & 9999 \\
\hline 157 & Hunter Mesa & Unknown & Not available & $-9,999$ & 39.453 & -107.734 & $9 / 5 / 2007$ & 9999 \\
\hline 158 & Hunter Mesa & Tertiary & WASATCH FORMATION & 120 & 39.442 & -107.687 & $4 / 21 / 2009$ & 1400 \\
\hline 159 & Hunter Mesa & Tertiary & WASATCH FORMATION & 180 & 39.453 & -107.734 & $4 / 25 / 2009$ & 910 \\
\hline 161 & Hunter Mesa & Unknown & Not available & $-9,999$ & 39.441 & -107.650 & $1 / 21 / 2003$ & 9999 \\
\hline 162 & Hunter Mesa & Tertiary & WASATCH FORMATION & 190 & 39.467 & -107.639 & 4/23/2009 & 1230 \\
\hline 163 & Hunter Mesa & Tertiary & WASATCH FORMATION & 200 & 39.489 & -107.641 & $4 / 23 / 2009$ & 930 \\
\hline 164 & Hunter Mesa & Tertiary & WASATCH FORMATION & 495 & 39.489 & -107.631 & $4 / 26 / 2009$ & 940 \\
\hline 165 & Hunter Mesa & Tertiary & WASATCH FORMATION & 143 & 39.491 & -107.658 & $4 / 27 / 2009$ & 1010 \\
\hline 166 & Hunter Mesa & Unknown & Not available & $-9,999$ & 39.438 & -107.658 & $6 / 4 / 1997$ & 9999 \\
\hline 167 & Hunter Mesa & Unknown & Not available & $-9,999$ & 39.480 & -107.653 & 6/4/1997 & 9999 \\
\hline 168 & Hunter Mesa & Unknown & Not available & $-9,999$ & 39.434 & -107.664 & $9 / 5 / 2007$ & 9999 \\
\hline 169 & Hunter Mesa & Unknown & Not available & $-9,999$ & 39.432 & -107.635 & $9 / 6 / 2007$ & 1200 \\
\hline 169 & Hunter Mesa & Unknown & Not available & $-9,999$ & 39.432 & -107.635 & $9 / 6 / 2007$ & 1215 \\
\hline 170 & Hunter Mesa & Unknown & Not available & $-9,999$ & 39.483 & -107.645 & $8 / 3 / 2005$ & 9999 \\
\hline 171 & Hunter Mesa & Unknown & Not available & $-9,999$ & 39.490 & -107.743 & $7 / 7 / 2007$ & 9999 \\
\hline 172 & Hunter Mesa & Unknown & Not available & $-9,999$ & 39.488 & -107.653 & $4 / 14 / 2005$ & 9999 \\
\hline 173 & Hunter Mesa & Unknown & Not available & $-9,999$ & 39.488 & -107.665 & $5 / 5 / 2005$ & 9999 \\
\hline 174 & Hunter Mesa & Unknown & Not available & $-9,999$ & 39.451 & -107.659 & $1 / 16 / 2003$ & 9999 \\
\hline 175 & Hunter Mesa & Unknown & Not available & $-9,999$ & 39.478 & -107.670 & $7 / 13 / 2004$ & 9999 \\
\hline 176 & Hunter Mesa & Unknown & Not available & $-9,999$ & 39.476 & -107.653 & $6 / 13 / 2007$ & 9999 \\
\hline 177 & Hunter Mesa & Unknown & Not available & $-9,999$ & 39.489 & -107.669 & $6 / 14 / 2007$ & 9999 \\
\hline 178 & Hunter Mesa & Unknown & Not available & $-9,999$ & 39.489 & -107.671 & $8 / 25 / 2004$ & 9999 \\
\hline 179 & Hunter Mesa & Unknown & Not available & $-9,999$ & 39.486 & -107.660 & $9 / 5 / 2007$ & 9999 \\
\hline 180 & Hunter Mesa & Unknown & Not available & $-9,999$ & 39.489 & -107.657 & $8 / 5 / 2005$ & 9999 \\
\hline 181 & Hunter Mesa & Unknown & Not available & $-9,999$ & 39.487 & -107.664 & $8 / 25 / 2004$ & 9999 \\
\hline 182 & Hunter Mesa & Unknown & Not available & $-9,999$ & 39.425 & -107.638 & $7 / 12 / 2007$ & 1215 \\
\hline 182 & Hunter Mesa & Unknown & Not available & $-9,999$ & 39.425 & -107.638 & $7 / 12 / 2007$ & 1200 \\
\hline 183 & Hunter Mesa & Unknown & Not available & $-9,999$ & 39.483 & -107.660 & $7 / 12 / 2007$ & 9999 \\
\hline 184 & Hunter Mesa & Unknown & Not available & $-9,999$ & 39.483 & -107.657 & $8 / 31 / 2005$ & 9999 \\
\hline 185 & Hunter Mesa & Unknown & Not available & $-9,999$ & 39.430 & -107.642 & $7 / 13 / 2007$ & 9999 \\
\hline 186 & Hunter Mesa & Unknown & Not available & $-9,999$ & 39.498 & -107.690 & $9 / 5 / 2007$ & 9999 \\
\hline 187 & Hunter Mesa & Unknown & Not available & $-9,999$ & 39.486 & -107.668 & $7 / 20 / 2005$ & 1215 \\
\hline 187 & Hunter Mesa & Unknown & Not available & $-9,999$ & 39.486 & -107.668 & $7 / 20 / 2005$ & 1200 \\
\hline 188 & Hunter Mesa & Unknown & Not available & $-9,999$ & 39.409 & -107.661 & $11 / 6 / 2003$ & 9999 \\
\hline 189 & Hunter Mesa & Unknown & Not available & $-9,999$ & 39.428 & -107.641 & $6 / 25 / 2007$ & 1215 \\
\hline 189 & Hunter Mesa & Unknown & Not available & $-9,999$ & 39.428 & -107.641 & $6 / 25 / 2007$ & 1200 \\
\hline 191 & Hunter Mesa & Unknown & Not available & $-9,999$ & 39.483 & -107.664 & $7 / 13 / 2004$ & 9999 \\
\hline 192 & Hunter Mesa & Unknown & Not available & $-9,999$ & 39.464 & -107.710 & $10 / 7 / 2002$ & 9999 \\
\hline 193 & Hunter Mesa & Unknown & Not available & $-9,999$ & 39.475 & -107.659 & $7 / 13 / 2004$ & 9999 \\
\hline 194 & Hunter Mesa & Unknown & Not available & $-9,999$ & 39.476 & -107.657 & $7 / 13 / 2004$ & 9999 \\
\hline 195 & Hunter Mesa & Unknown & Not available & $-9,999$ & 39.454 & -107.699 & $8 / 8 / 2001$ & 9999 \\
\hline 196 & Hunter Mesa & Unknown & Not available & $-9,999$ & 39.486 & -107.651 & $4 / 14 / 2005$ & 9999 \\
\hline 197 & Hunter Mesa & Unknown & Not available & $-9,999$ & 39.487 & -107.647 & $9 / 6 / 2007$ & 9999 \\
\hline 198 & Hunter Mesa & Unknown & Not available & $-9,999$ & 39.486 & -107.658 & $6 / 12 / 2007$ & 9999 \\
\hline
\end{tabular}


Appendix 1. Water Quality Data Used in This Report.-Continued

\begin{tabular}{|c|c|c|c|c|c|c|c|c|}
\hline $\begin{array}{c}\text { Report } \\
\text { No. }\end{array}$ & $\begin{array}{l}\text { Map } \\
\text { name }\end{array}$ & $\begin{array}{c}\text { Geologic } \\
\text { unit }\end{array}$ & $\begin{array}{c}\text { Aquifer } \\
\text { description }\end{array}$ & $\begin{array}{c}\text { Well } \\
\text { depth } \\
\text { (ft) }\end{array}$ & $\begin{array}{l}\text { Latitude } \\
\text { (decimal } \\
\text { degrees, } \\
\text { NAD83) }\end{array}$ & $\begin{array}{c}\text { Longitude } \\
\text { (decimal } \\
\text { degrees, } \\
\text { NAD83) }\end{array}$ & $\begin{array}{c}\text { Collection } \\
\text { date }\end{array}$ & $\begin{array}{l}\text { Collection } \\
\text { time }\end{array}$ \\
\hline 199 & Hunter Mesa & Unknown & Not available & $-9,999$ & 39.477 & -107.666 & $7 / 12 / 2007$ & 9999 \\
\hline 200 & Hunter Mesa & Unknown & Not available & $-9,999$ & 39.486 & -107.660 & $6 / 29 / 2004$ & 9999 \\
\hline 201 & Hunter Mesa & Unknown & Not available & $-9,999$ & 39.424 & -107.644 & $7 / 13 / 2007$ & 1200 \\
\hline 201 & Hunter Mesa & Unknown & Not available & $-9,999$ & 39.424 & -107.644 & $7 / 13 / 2007$ & 1215 \\
\hline 202 & Hunter Mesa & Unknown & Not available & $-9,999$ & 39.480 & -107.652 & $7 / 14 / 2004$ & 9999 \\
\hline 203 & Hunter Mesa & Unknown & Not available & $-9,999$ & 39.430 & -107.642 & $7 / 13 / 2007$ & 1215 \\
\hline 203 & Hunter Mesa & Unknown & Not available & $-9,999$ & 39.430 & -107.642 & $7 / 13 / 2007$ & 1200 \\
\hline 204 & Hunter Mesa & Unknown & Not available & $-9,999$ & 39.498 & -107.652 & $4 / 7 / 2005$ & 9999 \\
\hline 205 & Hunter Mesa & Unknown & Not available & $-9,999$ & 39.413 & -107.640 & $6 / 26 / 2007$ & 1215 \\
\hline 205 & Hunter Mesa & Unknown & Not available & $-9,999$ & 39.413 & -107.640 & $6 / 26 / 2007$ & 1200 \\
\hline 206 & Hunter Mesa & Unknown & Not available & 500 & 39.406 & -107.634 & $12 / 18 / 2002$ & 9999 \\
\hline 207 & Hunter Mesa & Unknown & Not available & 320 & 39.395 & -107.625 & 9/15/1999 & 9999 \\
\hline 208 & Hunter Mesa & Unknown & Not available & $-9,999$ & 39.467 & -107.657 & $8 / 26 / 2004$ & 9999 \\
\hline 209 & Hunter Mesa & Unknown & Not available & 70 & 39.469 & -107.687 & 9/16/1999 & 9999 \\
\hline 211 & Hunter Mesa & Unknown & Not available & 76 & 39.426 & -107.698 & 9/16/1999 & 9999 \\
\hline 213 & Hunter Mesa & Unknown & Not available & $-9,999$ & 39.446 & -107.657 & $9 / 15 / 2004$ & 9999 \\
\hline 214 & Hunter Mesa & Unknown & Not available & $-9,999$ & 39.474 & -107.653 & $6 / 6 / 2006$ & 1215 \\
\hline 214 & Hunter Mesa & Unknown & Not available & $-9,999$ & 39.474 & -107.653 & $6 / 6 / 2006$ & 1200 \\
\hline 215 & Hunter Mesa & Unknown & Not available & $-9,999$ & 39.415 & -107.657 & $1 / 14 / 2003$ & 9999 \\
\hline 216 & Hunter Mesa & Unknown & Not available & $-9,999$ & 39.411 & -107.639 & $6 / 23 / 2007$ & 1215 \\
\hline 216 & Hunter Mesa & Unknown & Not available & $-9,999$ & 39.411 & -107.639 & $6 / 23 / 2007$ & 1200 \\
\hline 217 & Hunter Mesa & Unknown & Not available & $-9,999$ & 39.423 & -107.666 & $1 / 14 / 2003$ & 9999 \\
\hline 218 & Hunter Mesa & Unknown & Not available & $-9,999$ & 39.381 & -107.629 & $12 / 18 / 2002$ & 9999 \\
\hline 219 & Hunter Mesa & Unknown & Not available & $-9,999$ & 39.453 & -107.739 & $6 / 22 / 2007$ & 9999 \\
\hline 220 & Hunter Mesa & Unknown & Not available & $-9,999$ & 39.492 & -107.654 & $7 / 12 / 2007$ & 9999 \\
\hline 221 & Hunter Mesa & Unknown & Not available & $-9,999$ & 39.486 & -107.657 & $8 / 4 / 2005$ & 9999 \\
\hline 222 & Hunter Mesa & Unknown & Not available & $-9,999$ & 39.479 & -107.644 & $6 / 15 / 2007$ & 9999 \\
\hline 223 & Hunter Mesa & Unknown & Not available & 300 & 39.430 & -107.674 & 9/14/1999 & 9999 \\
\hline 224 & Hunter Mesa & Unknown & Not available & $-9,999$ & 39.481 & -107.653 & $6 / 8 / 2006$ & 9999 \\
\hline 225 & Hunter Mesa & Unknown & Not available & $-9,999$ & 39.438 & -107.634 & $7 / 8 / 2004$ & 9999 \\
\hline 226 & Hunter Mesa & Unknown & Not available & $-9,999$ & 39.465 & -107.645 & $1 / 16 / 2008$ & 9999 \\
\hline 227 & Hunter Mesa & Unknown & Not available & 400 & 39.490 & -107.661 & $6 / 30 / 2004$ & 9999 \\
\hline 228 & Hunter Mesa & Unknown & Not available & 135 & 39.488 & -107.649 & $6 / 14 / 2007$ & 1215 \\
\hline 228 & Hunter Mesa & Unknown & Not available & 135 & 39.488 & -107.649 & $6 / 14 / 2007$ & 1200 \\
\hline 229 & Hunter Mesa & Unknown & Not available & $-9,999$ & 39.497 & -107.658 & $9 / 2 / 2004$ & 9999 \\
\hline 230 & Hunter Mesa & Unknown & Not available & $-9,999$ & 39.483 & -107.645 & $6 / 14 / 2006$ & 9999 \\
\hline 231 & Hunter Mesa & Unknown & Not available & $-9,999$ & 39.451 & -107.687 & $1 / 31 / 2002$ & 9999 \\
\hline 232 & Hunter Mesa & Unknown & Not available & $-9,999$ & 39.411 & -107.659 & $11 / 6 / 2003$ & 9999 \\
\hline 233 & Hunter Mesa & Unknown & Not available & $-9,999$ & 39.441 & -107.740 & $2 / 16 / 2005$ & 9999 \\
\hline 234 & Hunter Mesa & Unknown & Not available & $-9,999$ & 39.474 & -107.678 & $8 / 8 / 2001$ & 9999 \\
\hline 235 & Hunter Mesa & Unknown & Not available & 340 & 39.410 & -107.659 & 9/14/1999 & 9999 \\
\hline 236 & Hunter Mesa & Unknown & Not available & 100 & 39.489 & -107.653 & $3 / 13 / 2003$ & 9999 \\
\hline 237 & Hunter Mesa & Unknown & Not available & $-9,999$ & 39.381 & -107.637 & $12 / 18 / 2002$ & 9999 \\
\hline 238 & Hunter Mesa & Unknown & Not available & $-9,999$ & 39.488 & -107.652 & $6 / 19 / 2006$ & 9999 \\
\hline 239 & Hunter Mesa & Unknown & Not available & $-9,999$ & 39.446 & -107.732 & 7/19/2004 & 9999 \\
\hline 240 & Hunter Mesa & Unknown & Not available & $-9,999$ & 39.411 & -107.642 & $12 / 20 / 2002$ & 9999 \\
\hline 241 & Hunter Mesa & Unknown & Not available & 180 & 39.410 & -107.636 & 6/9/1997 & 9999 \\
\hline 242 & Hunter Mesa & Unknown & Not available & 30 & 39.430 & -107.635 & 6/9/1997 & 9999 \\
\hline 243 & Hunter Mesa & Unknown & Not available & 160 & 39.453 & -107.734 & 7/11/1997 & 9999 \\
\hline 244 & Hunter Mesa & Unknown & Not available & 120 & 39.412 & -107.645 & $1 / 15 / 2003$ & 9999 \\
\hline 245 & Hunter Mesa & Unknown & Not available & 5 & 39.440 & -107.654 & 7/12/1997 & 9999 \\
\hline 246 & Hunter Mesa & Unknown & Not available & $-9,999$ & 39.475 & -107.659 & $6 / 6 / 2006$ & 9999 \\
\hline 247 & Hunter Mesa & Unknown & Not available & $-9,999$ & 39.476 & -107.657 & $7 / 15 / 2008$ & 9999 \\
\hline 248 & Hunter Mesa & Unknown & Not available & $-9,999$ & 39.494 & -107.645 & $6 / 14 / 2007$ & 9999 \\
\hline 249 & Hunter Mesa & Unknown & Not available & 190 & 39.482 & -107.663 & $6 / 12 / 2007$ & 9999 \\
\hline 250 & Hunter Mesa & Unknown & Not available & $-9,999$ & 39.498 & -107.630 & $6 / 29 / 2005$ & 9999 \\
\hline 251 & Hunter Mesa & Unknown & Not available & $-9,999$ & 39.495 & -107.651 & $9 / 5 / 2007$ & 9999 \\
\hline 252 & Hunter Mesa & Unknown & Not available & $-9,999$ & 39.491 & -107.642 & $6 / 14 / 2007$ & 9999 \\
\hline 253 & Hunter Mesa & Unknown & Not available & $-9,999$ & 39.489 & -107.641 & $6 / 13 / 2007$ & 9999 \\
\hline 254 & Hunter Mesa & Unknown & Not available & $-9,999$ & 39.494 & -107.657 & $6 / 25 / 2007$ & 9999 \\
\hline 255 & Hunter Mesa & Tertiary & WASATCH FORMATION & $-9,999$ & 39.487 & -107.640 & $4 / 9 / 2004$ & 9999 \\
\hline 256 & Hunter Mesa & Tertiary & WASATCH FORMATION & $-9,999$ & 39.488 & -107.644 & $4 / 14 / 2005$ & 1215 \\
\hline 256 & Hunter Mesa & Tertiary & WASATCH FORMATION & $-9,999$ & 39.488 & -107.644 & $4 / 14 / 2005$ & 1200 \\
\hline
\end{tabular}


Appendix 1. Water Quality Data Used in This Report.-Continued

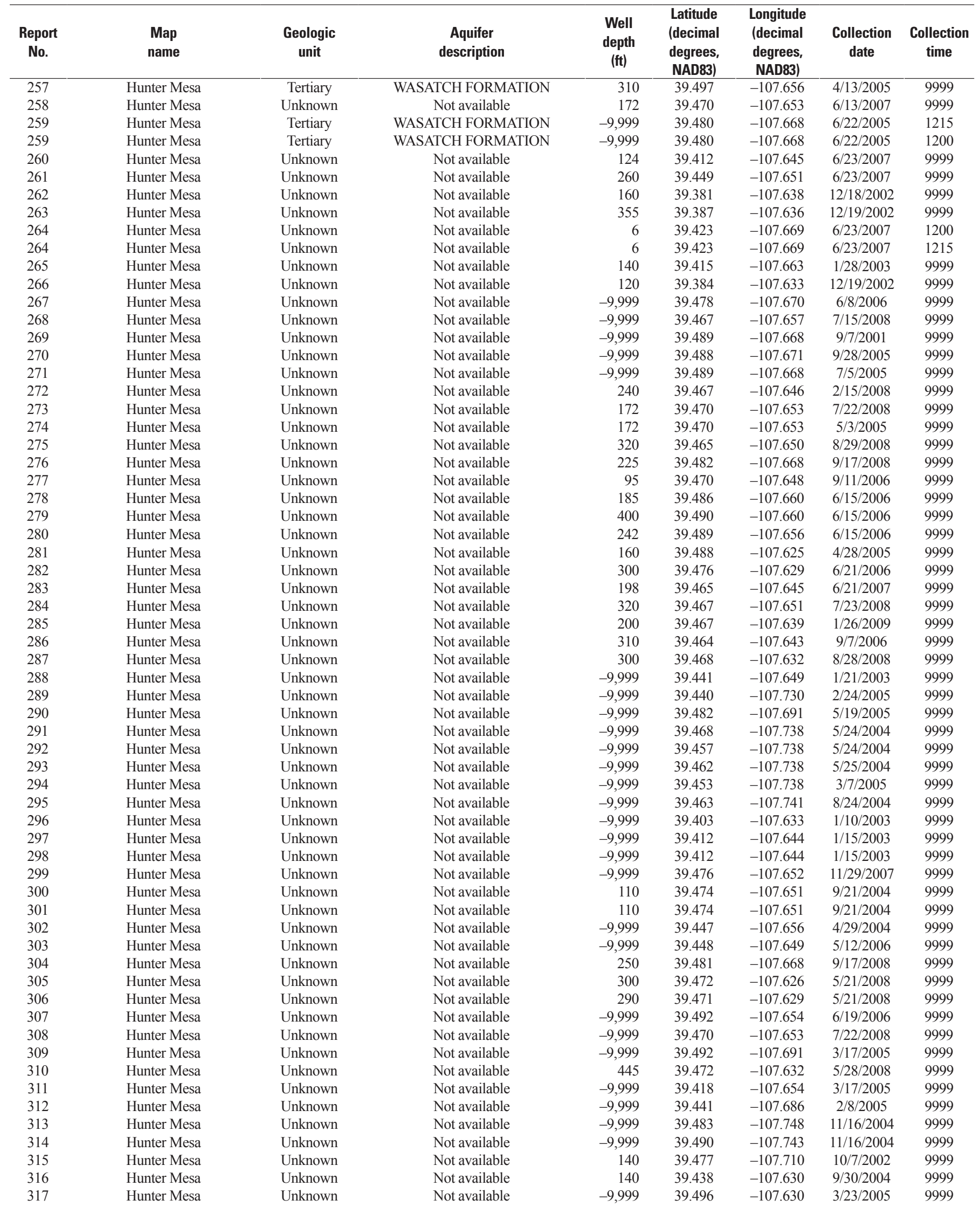


Appendix 1. Water Quality Data Used in This Report.-Continued

\begin{tabular}{|c|c|c|c|c|c|c|c|c|}
\hline $\begin{array}{c}\text { Report } \\
\text { No. }\end{array}$ & $\begin{array}{l}\text { Map } \\
\text { name }\end{array}$ & $\begin{array}{l}\text { Geologic } \\
\text { unit }\end{array}$ & $\begin{array}{c}\text { Aquifer } \\
\text { description }\end{array}$ & $\begin{array}{c}\text { Well } \\
\text { depth } \\
\text { (ft) }\end{array}$ & $\begin{array}{l}\text { Latitude } \\
\text { (decimal } \\
\text { degrees, } \\
\text { NAD83) }\end{array}$ & $\begin{array}{l}\text { Longitude } \\
\text { (decimal } \\
\text { degrees, } \\
\text { NAD83) }\end{array}$ & $\begin{array}{c}\text { Collection } \\
\text { date }\end{array}$ & $\begin{array}{l}\text { Collection } \\
\text { time }\end{array}$ \\
\hline 318 & Hunter Mesa & Unknown & Not available & $-9,999$ & 39.424 & -107.643 & $9 / 11 / 2003$ & 9999 \\
\hline 319 & Hunter Mesa & Unknown & Not available & $-9,999$ & 39.480 & -107.651 & $7 / 30 / 2008$ & 9999 \\
\hline 320 & Hunter Mesa & Unknown & Not available & $-9,999$ & 39.485 & -107.635 & $6 / 8 / 2006$ & 9999 \\
\hline 321 & Hunter Mesa & Unknown & Not available & $-9,999$ & 39.430 & -107.642 & $8 / 2 / 2002$ & 9999 \\
\hline 322 & Hunter Mesa & Unknown & Not available & 140 & 39.469 & -107.642 & $8 / 29 / 2008$ & 9999 \\
\hline 323 & Hunter Mesa & Unknown & Not available & $-9,999$ & 39.412 & -107.64 & $1 / 15 / 2003$ & 9999 \\
\hline 324 & Hunter Mesa & Unknown & Not available & $-9,999$ & 39.492 & -107.651 & $6 / 8 / 2006$ & 9999 \\
\hline 325 & Hunter Mesa & Unknown & Not available & $-9,999$ & 39.422 & -107.634 & $3 / 8 / 2005$ & 9999 \\
\hline 326 & Hunter Mesa & Unknown & Not available & $-9,999$ & 39.421 & -107.634 & $3 / 8 / 2005$ & 9999 \\
\hline 327 & Hunter Mesa & Unknown & Not available & 300 & 39.465 & -107.631 & $8 / 28 / 2008$ & 9999 \\
\hline 328 & Hunter Mesa & Unknown & Not available & 110 & 39.474 & -107.651 & $7 / 15 / 2008$ & 9999 \\
\hline 329 & Hunter Mesa & Unknown & Not available & $-9,999$ & 39.397 & -107.635 & $1 / 25 / 2005$ & 9999 \\
\hline 330 & Hunter Mesa & Unknown & Not available & $-9,999$ & 39.398 & -107.626 & $12 / 19 / 2002$ & 9999 \\
\hline 331 & Hunter Mesa & Unknown & Not available & $-9,999$ & 39.387 & -107.635 & $12 / 19 / 2002$ & 9999 \\
\hline 332 & Hunter Mesa & Unknown & Not available & 180 & 39.488 & -107.664 & $6 / 15 / 2006$ & 9999 \\
\hline 333 & Hunter Mesa & Unknown & Not available & 180 & 39.468 & -107.652 & $7 / 23 / 2008$ & 9999 \\
\hline 334 & Hunter Mesa & Unknown & Not available & $-9,999$ & 39.486 & -107.656 & $6 / 20 / 2006$ & 9999 \\
\hline 335 & Hunter Mesa & Unknown & Not available & $-9,999$ & 39.431 & -107.634 & $12 / 20 / 2005$ & 9999 \\
\hline 336 & Hunter Mesa & Unknown & Not available & $-9,999$ & 39.431 & -107.634 & $12 / 20 / 2005$ & 9999 \\
\hline 337 & Hunter Mesa & Unknown & Not available & $-9,999$ & 39.448 & -107.658 & $1 / 17 / 2003$ & 9999 \\
\hline 338 & Hunter Mesa & Unknown & Not available & $-9,999$ & 39.415 & -107.657 & $1 / 14 / 2003$ & 9999 \\
\hline 339 & Hunter Mesa & Unknown & Not available & $-9,999$ & 39.481 & -107.658 & $6 / 8 / 2006$ & 9999 \\
\hline 340 & Hunter Mesa & Unknown & Not available & $-9,999$ & 39.411 & -107.638 & $1 / 15 / 2003$ & 9999 \\
\hline 341 & Hunter Mesa & Unknown & Not available & $-9,999$ & 39.483 & -107.749 & $7 / 15 / 2004$ & 9999 \\
\hline 342 & Hunter Mesa & Unknown & Not available & $-9,999$ & 39.464 & -107.661 & $7 / 13 / 2004$ & 9999 \\
\hline 343 & Hunter Mesa & Unknown & Not available & $-9,999$ & 39.455 & -107.659 & $7 / 13 / 2004$ & 9999 \\
\hline 344 & Hunter Mesa & Unknown & Not available & $-9,999$ & 39.488 & -107.651 & $6 / 14 / 2006$ & 9999 \\
\hline 345 & Hunter Mesa & Unknown & Not available & $-9,999$ & 39.484 & -107.651 & $8 / 22 / 2008$ & 9999 \\
\hline 346 & Hunter Mesa & Unknown & Not available & $-9,999$ & 39.486 & -107.664 & $6 / 26 / 2006$ & 9999 \\
\hline 347 & Hunter Mesa & Unknown & Not available & $-9,999$ & 39.439 & -107.649 & $1 / 23 / 2003$ & 9999 \\
\hline 348 & Hunter Mesa & Unknown & Not available & $-9,999$ & 39.478 & -107.657 & $7 / 13 / 2004$ & 9999 \\
\hline 349 & Hunter Mesa & Unknown & Not available & $-9,999$ & 39.433 & -107.657 & $1 / 31 / 2003$ & 9999 \\
\hline 350 & Hunter Mesa & Unknown & Not available & $-9,999$ & 39.434 & -107.664 & $1 / 31 / 2003$ & 9999 \\
\hline 351 & Hunter Mesa & Unknown & Not available & $-9,999$ & 39.487 & -107.672 & $9 / 3 / 2004$ & 9999 \\
\hline 352 & Hunter Mesa & Unknown & Not available & $-9,999$ & 39.440 & -107.646 & $10 / 25 / 2004$ & 9999 \\
\hline 353 & Hunter Mesa & Unknown & Not available & $-9,999$ & 39.479 & -107.644 & 3/7/2003 & 9999 \\
\hline 354 & Hunter Mesa & Unknown & Not available & $-9,999$ & 39.477 & -107.660 & $7 / 22 / 2008$ & 9999 \\
\hline 355 & Hunter Mesa & Unknown & Not available & $-9,999$ & 39.478 & -107.657 & $7 / 22 / 2008$ & 9999 \\
\hline 356 & Hunter Mesa & Unknown & Not available & $-9,999$ & 39.430 & -107.641 & $8 / 2 / 2002$ & 9999 \\
\hline 357 & Hunter Mesa & Unknown & Not available & $-9,999$ & 39.498 & -107.689 & $6 / 13 / 2003$ & 9999 \\
\hline 358 & Hunter Mesa & Unknown & Not available & $-9,999$ & 39.498 & -107.689 & $9 / 23 / 2004$ & 9999 \\
\hline 359 & Hunter Mesa & Unknown & Not available & $-9,999$ & 39.467 & -107.646 & $9 / 16 / 2008$ & 9999 \\
\hline 360 & Hunter Mesa & Unknown & Not available & $-9,999$ & 39.467 & -107.646 & $9 / 26 / 2008$ & 9999 \\
\hline 361 & Hunter Mesa & Unknown & Not available & $-9,999$ & 39.486 & -107.667 & $6 / 19 / 2006$ & 9999 \\
\hline 362 & Hunter Mesa & Unknown & Not available & $-9,999$ & 39.425 & -107.640 & $7 / 1 / 2003$ & 9999 \\
\hline 363 & Hunter Mesa & Unknown & Not available & $-9,999$ & 39.474 & -107.652 & $12 / 1 / 2004$ & 9999 \\
\hline 364 & Hunter Mesa & Unknown & Not available & $-9,999$ & 39.474 & -107.652 & $6 / 6 / 2006$ & 9999 \\
\hline 365 & Hunter Mesa & Unknown & Not available & $-9,999$ & 39.494 & -107.644 & $6 / 7 / 2006$ & 9999 \\
\hline 366 & Hunter Mesa & Unknown & Not available & $-9,999$ & 39.433 & -107.671 & $12 / 9 / 2002$ & 9999 \\
\hline 367 & Hunter Mesa & Unknown & Not available & $-9,999$ & 39.423 & -107.668 & $1 / 28 / 2003$ & 9999 \\
\hline 368 & Hunter Mesa & Unknown & Not available & $-9,999$ & 39.415 & -107.663 & $1 / 28 / 2003$ & 9999 \\
\hline 369 & Hunter Mesa & Unknown & Not available & $-9,999$ & 39.478 & -107.690 & $10 / 16 / 2008$ & 9999 \\
\hline 370 & Hunter Mesa & Unknown & Not available & $-9,999$ & 39.444 & -107.627 & $5 / 9 / 2003$ & 9999 \\
\hline 371 & Hunter Mesa & Unknown & Not available & $-9,999$ & 39.427 & -107.636 & $1 / 16 / 2003$ & 9999 \\
\hline 372 & Hunter Mesa & Unknown & Not available & $-9,999$ & 39.457 & -107.656 & $11 / 18 / 2004$ & 9999 \\
\hline 373 & Hunter Mesa & Unknown & Not available & $-9,999$ & 39.409 & -107.660 & $11 / 6 / 2003$ & 9999 \\
\hline 374 & Hunter Mesa & Unknown & Not available & $-9,999$ & 39.468 & -107.739 & $2 / 16 / 2005$ & 9999 \\
\hline 375 & Hunter Mesa & Unknown & Not available & $-9,999$ & 39.384 & -107.632 & $12 / 19 / 2002$ & 9999 \\
\hline 376 & Hunter Mesa & Unknown & Not available & $-9,999$ & 39.497 & -107.655 & $6 / 19 / 2006$ & 9999 \\
\hline 377 & Hunter Mesa & Unknown & Not available & $-9,999$ & 39.495 & -107.650 & $6 / 19 / 2006$ & 9999 \\
\hline 378 & Hunter Mesa & Unknown & Not available & $-9,999$ & 39.452 & -107.699 & 8/8/2001 & 9999 \\
\hline 379 & Hunter Mesa & Unknown & Not available & $-9,999$ & 39.480 & -107.749 & $8 / 11 / 2005$ & 9999 \\
\hline 380 & Hunter Mesa & Unknown & Not available & $-9,999$ & 39.464 & -107.643 & $8 / 28 / 2008$ & 9999 \\
\hline 381 & Hunter Mesa & Unknown & Not available & $-9,999$ & 39.486 & -107.651 & $3 / 11 / 2003$ & 9999 \\
\hline
\end{tabular}


Appendix 1. Water Quality Data Used in This Report.-Continued

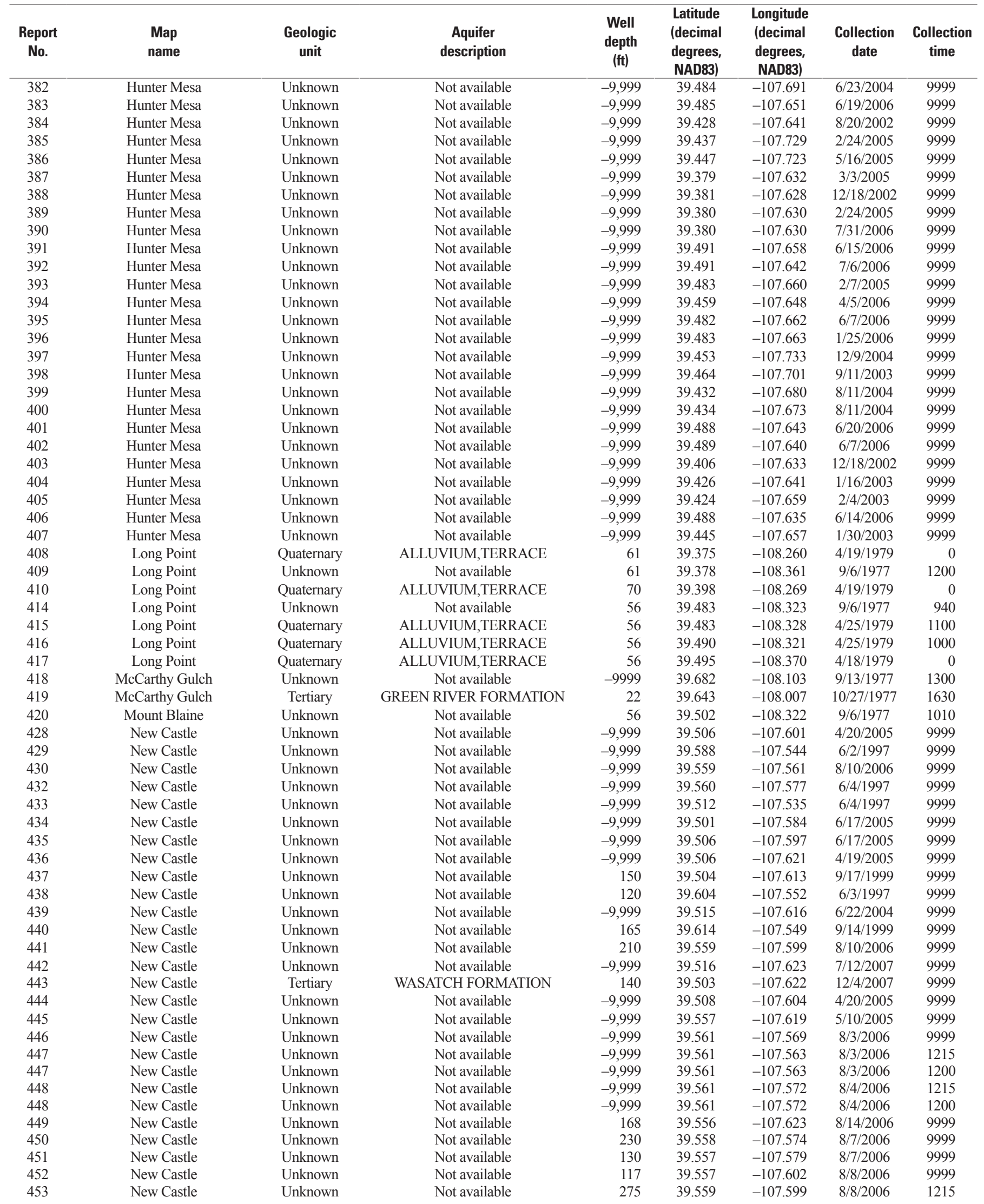


Appendix 1. Water Quality Data Used in This Report.-Continued

\begin{tabular}{|c|c|c|c|c|c|c|c|c|}
\hline $\begin{array}{c}\text { Report } \\
\text { No. }\end{array}$ & $\begin{array}{l}\text { Map } \\
\text { name }\end{array}$ & $\begin{array}{l}\text { Geologic } \\
\text { unit }\end{array}$ & $\begin{array}{c}\text { Aquifer } \\
\text { description }\end{array}$ & $\begin{array}{l}\text { Well } \\
\text { depth } \\
\text { (ft) }\end{array}$ & $\begin{array}{l}\text { Latitude } \\
\text { (decimal } \\
\text { degrees, } \\
\text { NAD83) }\end{array}$ & $\begin{array}{c}\text { Longitude } \\
\text { (decimal } \\
\text { degrees, } \\
\text { NAD83) }\end{array}$ & $\begin{array}{c}\text { Collection } \\
\text { date }\end{array}$ & $\begin{array}{l}\text { Collection } \\
\text { time }\end{array}$ \\
\hline 453 & New Castle & Unknown & Not available & 275 & 39.559 & -107.599 & $8 / 8 / 2006$ & 1200 \\
\hline 454 & New Castle & Unknown & Not available & 110 & 39.558 & -107.610 & $8 / 1 / 2006$ & 1215 \\
\hline 454 & New Castle & Unknown & Not available & 110 & 39.558 & -107.610 & $8 / 1 / 2006$ & 1200 \\
\hline 455 & New Castle & Unknown & Not available & $-9,999$ & 39.56 & -107.62 & 8/12/1998 & 9999 \\
\hline 456 & New Castle & Unknown & Not available & $-9,999$ & 39.55 & -107.59 & 8/12/1998 & 9999 \\
\hline 457 & North Mamm Peak & Unknown & Not available & $-9,999$ & 39.485 & -107.868 & $10 / 23 / 1997$ & 9999 \\
\hline 458 & North Mamm Peak & Unknown & Not available & $-9,999$ & 39.499 & -107.864 & $10 / 23 / 1997$ & 9999 \\
\hline 459 & North Mamm Peak & Unknown & Not available & $-9,999$ & 39.497 & -107.863 & $10 / 23 / 1997$ & 9999 \\
\hline 460 & North Mamm Peak & Unknown & Not available & $-9,999$ & 39.487 & -107.772 & $4 / 19 / 2004$ & 9999 \\
\hline 461 & North Mamm Peak & Unknown & Not available & $-9,999$ & 39.483 & -107.750 & $7 / 9 / 2002$ & 9999 \\
\hline 462 & North Mamm Peak & Unknown & Not available & $-9,999$ & 39.475 & -107.777 & $6 / 25 / 2007$ & 9999 \\
\hline 463 & North Mamm Peak & Unknown & Not available & $-9,999$ & 39.475 & -107.782 & $3 / 4 / 2002$ & 9999 \\
\hline 464 & North Mamm Peak & Unknown & Not available & $-9,999$ & 39.487 & -107.772 & $3 / 4 / 2002$ & 9999 \\
\hline 465 & North Mamm Peak & Cretaceous & MESAVERDE GROUP & $-9,999$ & 39.480 & -107.868 & $4 / 18 / 1986$ & 1300 \\
\hline 466 & North Mamm Peak & Unknown & Not available & $-9,999$ & 39.499 & -107.862 & $6 / 12 / 1998$ & 9999 \\
\hline 468 & North Mamm Peak & Unknown & Not available & $-9,999$ & 39.479 & -107.874 & $10 / 23 / 1997$ & 9999 \\
\hline 469 & North Mamm Peak & Unknown & Not available & $-9,999$ & 39.498 & -107.761 & $5 / 11 / 2004$ & 9999 \\
\hline 470 & North Mamm Peak & Unknown & Not available & $-9,999$ & 39.457 & -107.782 & $7 / 19 / 2004$ & 9999 \\
\hline 471 & North Mamm Peak & Unknown & Not available & $-9,999$ & 39.484 & -107.762 & $4 / 6 / 2004$ & 9999 \\
\hline 472 & North Mamm Peak & Unknown & Not available & $-9,999$ & 39.476 & -107.779 & $6 / 8 / 2004$ & 9999 \\
\hline 473 & North Mamm Peak & Unknown & Not available & $-9,999$ & 39.498 & -107.762 & $10 / 2 / 2001$ & 9999 \\
\hline 474 & North Mamm Peak & Unknown & Not available & $-9,999$ & 39.497 & -107.762 & $10 / 2 / 2001$ & 9999 \\
\hline 475 & North Mamm Peak & Unknown & Not available & $-9,999$ & 39.492 & -107.773 & $10 / 2 / 2001$ & 9999 \\
\hline 476 & North Mamm Peak & Unknown & Not available & $-9,999$ & 39.483 & -107.765 & $7 / 6 / 2007$ & 9999 \\
\hline 477 & North Mamm Peak & Unknown & Not available & $-9,999$ & 39.465 & -107.779 & $6 / 8 / 2004$ & 9999 \\
\hline 478 & North Mamm Peak & Unknown & Not available & $-9,999$ & 39.499 & -107.772 & $3 / 23 / 2004$ & 9999 \\
\hline 479 & North Mamm Peak & Unknown & Not available & $-9,999$ & 39.479 & -107.769 & $1 / 30 / 2004$ & 9999 \\
\hline 480 & North Mamm Peak & Unknown & Not available & $-9,999$ & 39.481 & -107.872 & $4 / 27 / 2001$ & 9999 \\
\hline 481 & North Mamm Peak & Unknown & Not available & $-9,999$ & 39.486 & -107.771 & $4 / 8 / 2004$ & 9999 \\
\hline 482 & North Mamm Peak & Unknown & Not available & $-9,999$ & 39.491 & -107.767 & $4 / 8 / 2004$ & 9999 \\
\hline 483 & North Mamm Peak & Unknown & Not available & $-9,999$ & 39.488 & -107.770 & $4 / 7 / 2004$ & 9999 \\
\hline 484 & North Mamm Peak & Unknown & Not available & $-9,999$ & 39.464 & -107.783 & $7 / 21 / 2005$ & 9999 \\
\hline 485 & North Mamm Peak & Unknown & Not available & $-9,999$ & 39.468 & -107.783 & $12 / 29 / 2004$ & 9999 \\
\hline 486 & North Mamm Peak & Unknown & Not available & $-9,999$ & 39.470 & -107.779 & $3 / 26 / 2003$ & 9999 \\
\hline 487 & North Mamm Peak & Unknown & Not available & $-9,999$ & 39.455 & -107.781 & $11 / 17 / 2005$ & 9999 \\
\hline 488 & North Mamm Peak & Unknown & Not available & 102 & 39.495 & -107.865 & 7/8/1997 & 9999 \\
\hline 489 & North Mamm Peak & Unknown & Not available & 120 & 39.483 & -107.873 & $10 / 22 / 1997$ & 9999 \\
\hline 490 & North Mamm Peak & Unknown & Not available & 140 & 39.485 & -107.873 & $10 / 23 / 1997$ & 9999 \\
\hline 491 & North Mamm Peak & Unknown & Not available & 300 & 39.471 & -107.768 & $6 / 14 / 2001$ & 9999 \\
\hline 492 & North Mamm Peak & Unknown & Not available & $-9,999$ & 39.489 & -107.754 & $7 / 22 / 2004$ & 9999 \\
\hline 493 & North Mamm Peak & Tertiary & WASATCH FORMATION & 100 & 39.491 & -107.754 & $6 / 21 / 2007$ & 9999 \\
\hline 494 & North Mamm Peak & Unknown & Not available & 282 & 39.500 & -107.766 & $9 / 28 / 2001$ & 9999 \\
\hline 495 & North Mamm Peak & Unknown & Not available & 300 & 39.473 & -107.768 & $1 / 31 / 2002$ & 9999 \\
\hline 496 & North Mamm Peak & Unknown & Not available & $-9,999$ & 39.488 & -107.763 & $4 / 6 / 2004$ & 9999 \\
\hline 497 & North Mamm Peak & Unknown & Not available & $-9,999$ & 39.483 & -107.776 & $5 / 17 / 2004$ & 9999 \\
\hline 498 & North Mamm Peak & Unknown & Not available & $-9,999$ & 39.494 & -107.765 & $4 / 20 / 2004$ & 9999 \\
\hline 499 & North Mamm Peak & Unknown & Not available & $-9,999$ & 39.491 & -107.763 & $4 / 6 / 2004$ & 9999 \\
\hline 500 & North Mamm Peak & Unknown & Not available & $-9,999$ & 39.499 & -107.771 & $3 / 23 / 2004$ & 9999 \\
\hline 501 & North Mamm Peak & Tertiary & WASATCH FORMATION & 160 & 39.477 & -107.860 & $2 / 3 / 2004$ & 9999 \\
\hline 502 & North Mamm Peak & Unknown & Not available & 235 & 39.500 & -107.824 & $1 / 9 / 2008$ & 9999 \\
\hline 503 & North Mamm Peak & Unknown & Not available & 180 & 39.477 & -107.834 & $7 / 16 / 2008$ & 9999 \\
\hline 504 & North Mamm Peak & Unknown & Not available & 100 & 39.491 & -107.752 & $11 / 20 / 2008$ & 9999 \\
\hline 505 & North Mamm Peak & Unknown & Not available & 180 & 39.457 & -107.781 & $6 / 6 / 2008$ & 9999 \\
\hline 506 & North Mamm Peak & Unknown & Not available & $-9,999$ & 39.486 & -107.771 & $4 / 8 / 2004$ & 9999 \\
\hline 507 & North Mamm Peak & Unknown & Not available & $-9,999$ & 39.480 & -107.782 & $7 / 20 / 2004$ & 9999 \\
\hline 508 & North Mamm Peak & Unknown & Not available & $-9,999$ & 39.481 & -107.773 & $6 / 8 / 2004$ & 9999 \\
\hline 509 & North Mamm Peak & Unknown & Not available & $-9,999$ & 39.497 & -107.783 & $7 / 21 / 2004$ & 9999 \\
\hline 510 & North Mamm Peak & Unknown & Not available & $-9,999$ & 39.500 & -107.784 & $7 / 14 / 2006$ & 9999 \\
\hline 511 & North Mamm Peak & Unknown & Not available & $-9,999$ & 39.491 & -107.767 & $4 / 8 / 2004$ & 9999 \\
\hline 512 & North Mamm Peak & Unknown & Not available & $-9,999$ & 39.483 & -107.764 & $7 / 14 / 2004$ & 9999 \\
\hline 513 & North Mamm Peak & Unknown & Not available & $-9,999$ & 39.488 & -107.770 & $2 / 6 / 2007$ & 9999 \\
\hline 514 & North Mamm Peak & Unknown & Not available & 180 & 39.480 & -107.770 & $4 / 14 / 2003$ & 9999 \\
\hline 515 & North Mamm Peak & Unknown & Not available & $-9,999$ & 39.465 & -107.778 & $12 / 3 / 2008$ & 9999 \\
\hline 516 & North Mamm Peak & Unknown & Not available & $-9,999$ & 39.499 & -107.772 & $3 / 23 / 2004$ & 9999 \\
\hline 517 & North Mamm Peak & Unknown & Not available & $-9,999$ & 39.476 & -107.772 & $7 / 20 / 2004$ & 9999 \\
\hline 518 & North Mamm Peak & Unknown & Not available & $-9,999$ & 39.464 & -107.783 & $12 / 3 / 2008$ & 9999 \\
\hline
\end{tabular}


Appendix 1. Water Quality Data Used in This Report.-Continued

\begin{tabular}{|c|c|c|c|c|c|c|c|c|}
\hline $\begin{array}{c}\text { Report } \\
\text { No. }\end{array}$ & $\begin{array}{l}\text { Map } \\
\text { name }\end{array}$ & $\begin{array}{l}\text { Geologic } \\
\text { unit }\end{array}$ & $\begin{array}{c}\text { Aquifer } \\
\text { description }\end{array}$ & $\begin{array}{c}\text { Well } \\
\text { depth } \\
\text { (ft) }\end{array}$ & $\begin{array}{l}\text { Latitude } \\
\text { (decimal } \\
\text { degrees, } \\
\text { NAD83) }\end{array}$ & $\begin{array}{c}\text { Longitude } \\
\text { (decimal } \\
\text { degrees, } \\
\text { NAD83) }\end{array}$ & $\begin{array}{c}\text { Collection } \\
\text { date }\end{array}$ & $\begin{array}{c}\text { Collection } \\
\text { time }\end{array}$ \\
\hline 519 & North Mamm Peak & Unknown & Not available & $-9,999$ & 39.468 & -107.782 & $12 / 3 / 2008$ & 9999 \\
\hline 520 & North Mamm Peak & Unknown & Not available & $-9,999$ & 39.498 & -107.760 & 4/13/2009 & 9999 \\
\hline 521 & North Mamm Peak & Unknown & Not available & $-9,999$ & 39.488 & -107.758 & $4 / 20 / 2005$ & 9999 \\
\hline 522 & North Mamm Peak & Unknown & Not available & $-9,999$ & 39.497 & -107.761 & $3 / 24 / 2005$ & 9999 \\
\hline 523 & North Mamm Peak & Unknown & Not available & $-9,999$ & 39.473 & -107.857 & $6 / 16 / 2005$ & 9999 \\
\hline 524 & North Mamm Peak & Unknown & Not available & $-9,999$ & 39.477 & -107.781 & $7 / 14 / 2004$ & 9999 \\
\hline 525 & North Mamm Peak & Unknown & Not available & $-9,999$ & 39.484 & -107.761 & $4 / 6 / 2004$ & 9999 \\
\hline 526 & North Mamm Peak & Unknown & Not available & $-9,999$ & 39.455 & -107.781 & 5/11/2006 & 9999 \\
\hline 527 & North Mamm Peak & Unknown & Not available & $-9,999$ & 39.476 & -107.778 & $11 / 20 / 2008$ & 9999 \\
\hline 528 & North Mamm Peak & Unknown & Not available & $-9,999$ & 39.488 & -107.762 & 4/6/2004 & 9999 \\
\hline 529 & North Mamm Peak & Unknown & Not available & $-9,999$ & 39.492 & -107.772 & $11 / 20 / 2008$ & 9999 \\
\hline 530 & North Mamm Peak & Unknown & Not available & $-9,999$ & 39.477 & -107.799 & 6/7/2006 & 9999 \\
\hline 531 & North Mamm Peak & Unknown & Not available & $-9,999$ & 39.499 & -107.762 & $10 / 2 / 2001$ & 9999 \\
\hline 532 & North Mamm Peak & Unknown & Not available & $-9,999$ & 39.488 & -107.766 & $4 / 26 / 2003$ & 9999 \\
\hline 533 & North Mamm Peak & Unknown & Not available & $-9,999$ & 39.482 & -107.776 & $6 / 8 / 2004$ & 9999 \\
\hline 534 & North Mamm Peak & Unknown & Not available & $-9,999$ & 39.486 & -107.867 & $12 / 22 / 2004$ & 9999 \\
\hline 535 & North Mamm Peak & Unknown & Not available & $-9,999$ & 39.491 & -107.866 & $12 / 22 / 2004$ & 9999 \\
\hline 536 & North Mamm Peak & Unknown & Not available & $-9,999$ & 39.483 & -107.776 & 2/19/2009 & 9999 \\
\hline 537 & North Mamm Peak & Unknown & Not available & $-9,999$ & 39.495 & -107.764 & $9 / 1 / 2005$ & 9999 \\
\hline 538 & North Mamm Peak & Unknown & Not available & $-9,999$ & 39.497 & -107.761 & $10 / 2 / 2001$ & 9999 \\
\hline 539 & North Mamm Peak & Unknown & Not available & $-9,999$ & 39.491 & -107.762 & $11 / 20 / 2008$ & 9999 \\
\hline 540 & North Mamm Peak & Unknown & Not available & $-9,999$ & 39.495 & -107.767 & $5 / 23 / 2005$ & 9999 \\
\hline 541 & North Mamm Peak & Unknown & Not available & $-9,999$ & 39.472 & -107.767 & $6 / 8 / 2004$ & 9999 \\
\hline 542 & North Mamm Peak & Unknown & Not available & $-9,999$ & 39.499 & -107.770 & $3 / 23 / 2004$ & 9999 \\
\hline 543 & North Mamm Peak & Unknown & Not available & $-9,999$ & 39.462 & -107.780 & $12 / 3 / 2008$ & 9999 \\
\hline 544 & Parachute & Unknown & Not available & $-9,999$ & 39.444 & -108.016 & $8 / 3 / 1978$ & 0 \\
\hline 558 & Parachute & Quaternary & ALLUVIUM,TERRACE & 152 & 39.466 & -108.076 & 4/23/1979 & 0 \\
\hline 559 & Parachute & Quaternary & ALLUVIUM,TERRACE & 152 & 39.470 & -108.080 & $4 / 24 / 1979$ & 930 \\
\hline 560 & Parachute & Unknown & Not available & 56 & 39.476 & -108.098 & $3 / 20 / 1969$ & 0 \\
\hline 561 & Parachute & Unknown & Not available & 56 & 39.477 & -108.095 & $3 / 20 / 1969$ & 0 \\
\hline 562 & Parachute & Unknown & Not available & $-9,999$ & 39.411 & -108.092 & 8/5/1999 & 9999 \\
\hline 563 & Parachute & Unknown & Not available & $-9,999$ & 39.459 & -108.067 & 6/11/1998 & 9999 \\
\hline 564 & Parachute & Unknown & Not available & 56 & 39.483 & -108.099 & 3/20/1969 & 0 \\
\hline 565 & Parachute & Unknown & Not available & 56 & 39.483 & -108.095 & $3 / 20 / 1969$ & 0 \\
\hline 566 & Parachute & Unknown & Not available & 180 & 39.489 & -108.005 & $3 / 26 / 1969$ & 0 \\
\hline 569 & Parachute & Unknown & Not available & 56 & 39.493 & -108.124 & 3/20/1969 & 0 \\
\hline 570 & Parachute & Unknown & Not available & $-9,999$ & 39.483 & -108.100 & 6/12/1997 & 9999 \\
\hline 571 & Parachute & Unknown & Not available & $-9,999$ & 39.465 & -108.077 & 6/12/1997 & 9999 \\
\hline 572 & Parachute & Unknown & Not available & 60 & 39.457 & -108.064 & $6 / 11 / 1998$ & 9999 \\
\hline 573 & Parachute & Unknown & Not available & 150 & 39.467 & -108.009 & $6 / 10 / 1998$ & 9999 \\
\hline 574 & Parachute & Unknown & Not available & 170 & 39.467 & -108.009 & 9/17/1999 & 9999 \\
\hline 575 & Parachute & Unknown & Not available & $-9,999$ & 39.396 & -108.100 & $11 / 13 / 2006$ & 9999 \\
\hline 576 & Parachute & Unknown & Not available & 70 & 39.383 & -108.078 & $6 / 12 / 1997$ & 9999 \\
\hline 577 & Parachute & Unknown & Not available & 87 & 39.432 & -108.023 & 6/12/1997 & 9999 \\
\hline 578 & Parachute & Unknown & Not available & 220 & 39.443 & -108.017 & $6 / 12 / 1997$ & 9999 \\
\hline 579 & Parachute & Unknown & Not available & $-9,999$ & 39.445 & -108.008 & $7 / 5 / 2006$ & 9999 \\
\hline 580 & Parachute & Unknown & Not available & 75 & 39.427 & -108.018 & $7 / 9 / 1997$ & 9999 \\
\hline 581 & Parachute & Unknown & Not available & $-9,999$ & 39.427 & -108.026 & 7/14/2009 & 1335 \\
\hline 582 & Parachute & Unknown & Not available & $-9,999$ & 39.428 & -108.029 & 7/14/2009 & 1058 \\
\hline 583 & Parachute & Unknown & Not available & $-9,999$ & 39.430 & -108.019 & 7/16/2009 & 856 \\
\hline 584 & Parachute & Unknown & Not available & $-9,999$ & 39.430 & -108.024 & 7/15/2009 & 1337 \\
\hline 585 & Parachute & Unknown & Not available & $-9,999$ & 39.430 & -108.014 & 7/16/2009 & 932 \\
\hline 586 & Parachute & Unknown & Not available & $-9,999$ & 39.430 & -108.027 & 7/14/2009 & 1155 \\
\hline 587 & Parachute & Unknown & Not available & $-9,999$ & 39.431 & -108.020 & 7/15/2009 & 951 \\
\hline 588 & Parachute & Unknown & Not available & $-9,999$ & 39.431 & -108.019 & 7/15/2009 & 1129 \\
\hline 589 & Parachute & Unknown & Not available & $-9,999$ & 39.431 & -108.023 & 7/15/2009 & 1223 \\
\hline 590 & Parachute & Unknown & Not available & $-9,999$ & 39.431 & -108.029 & 7/14/2009 & 1010 \\
\hline 591 & Parachute & Unknown & Not available & $-9,999$ & 39.432 & -108.029 & 7/14/2009 & 929 \\
\hline 592 & Parachute & Unknown & Not available & $-9,999$ & 39.433 & -108.026 & 7/16/2009 & 1330 \\
\hline 593 & Parachute & Unknown & Not available & $-9,999$ & 39.434 & -108.028 & 7/15/2009 & 850 \\
\hline 594 & Parachute & Unknown & Not available & $-9,999$ & 39.434 & -108.029 & $7 / 15 / 2009$ & 1058 \\
\hline 595 & Parachute & Unknown & Not available & $-9,999$ & 39.434 & -108.031 & 7/14/2009 & 840 \\
\hline 596 & Parachute & Unknown & Not available & $-9,999$ & 39.440 & -108.015 & 7/16/2009 & 1027 \\
\hline 597 & Parachute & Unknown & Not available & $-9,999$ & 39.443 & -108.014 & 7/16/2009 & 1119 \\
\hline 598 & Parachute & Unknown & Not available & $-9,999$ & 39.444 & -108.016 & 7/16/2009 & 1204 \\
\hline 599 & Parachute & Unknown & Not available & 340 & 39.377 & -108.058 & $7 / 13 / 2007$ & 9999 \\
\hline
\end{tabular}


Appendix 1. Water Quality Data Used in This Report.-Continued

\begin{tabular}{|c|c|c|c|c|c|c|c|c|}
\hline $\begin{array}{c}\text { Report } \\
\text { No. }\end{array}$ & $\begin{array}{l}\text { Map } \\
\text { name }\end{array}$ & $\begin{array}{c}\text { Geologic } \\
\text { unit }\end{array}$ & $\begin{array}{c}\text { Aquifer } \\
\text { description }\end{array}$ & $\begin{array}{c}\text { Well } \\
\text { depth } \\
\text { (ft) }\end{array}$ & $\begin{array}{l}\text { Latitude } \\
\text { (decimal } \\
\text { degrees, } \\
\text { NAD83) }\end{array}$ & $\begin{array}{l}\text { Longitude } \\
\text { (decimal } \\
\text { degrees, } \\
\text { NAD83) }\end{array}$ & $\begin{array}{c}\text { Collection } \\
\text { date }\end{array}$ & $\begin{array}{c}\text { Collection } \\
\text { time }\end{array}$ \\
\hline 600 & Parachute & Unknown & Not available & 200 & 39.378 & -108.068 & $7 / 13 / 2007$ & 9999 \\
\hline 601 & Parachute & Unknown & Not available & 112 & 39.438 & -108.012 & $5 / 1 / 2007$ & 9999 \\
\hline 602 & Parachute & Unknown & Not available & $-9,999$ & 39.376 & -108.092 & 7/7/2006 & 9999 \\
\hline 603 & Parachute & Unknown & Not available & $-9,999$ & 39.437 & -108.000 & $12 / 5 / 2008$ & 9999 \\
\hline 604 & Parachute & Unknown & Not available & $-9,999$ & 39.378 & -108.109 & $6 / 23 / 2006$ & 9999 \\
\hline 605 & Parachute & Unknown & Not available & $-9,999$ & 39.403 & -108.108 & $10 / 19 / 2006$ & 9999 \\
\hline 606 & Parachute & Unknown & Not available & $-9,999$ & 39.403 & -108.107 & $10 / 19 / 2006$ & 9999 \\
\hline 607 & Parachute & Unknown & Not available & $-9,999$ & 39.385 & -108.059 & $4 / 28 / 2006$ & 9999 \\
\hline 608 & Parachute & Unknown & Not available & $-9,999$ & 39.445 & -108.006 & $9 / 17 / 2008$ & 9999 \\
\hline 609 & Parachute & Unknown & Not available & $-9,999$ & 39.430 & -108.014 & $11 / 8 / 2007$ & 9999 \\
\hline 610 & Parachute & Unknown & Not available & $-9,999$ & 39.440 & -108.015 & $6 / 29 / 2006$ & 9999 \\
\hline 611 & Parachute & Unknown & Not available & $-9,999$ & 39.443 & -108.014 & $6 / 30 / 2006$ & 9999 \\
\hline 612 & Parachute & Unknown & Not available & $-9,999$ & 39.444 & -108.015 & $7 / 5 / 2006$ & 9999 \\
\hline 613 & Parachute & Unknown & Not available & $-9,999$ & 39.442 & -108.015 & $7 / 6 / 2006$ & 9999 \\
\hline 614 & Parachute & Unknown & Not available & $-9,999$ & 39.444 & -108.016 & $6 / 29 / 2006$ & 9999 \\
\hline 615 & Parachute & Unknown & Not available & $-9,999$ & 39.48 & -108.1 & $8 / 12 / 1998$ & 9999 \\
\hline 616 & Rifle & Unknown & Not available & $-9,999$ & 39.504 & -107.822 & 8/30/1999 & 9999 \\
\hline 617 & Rifle & Unknown & Not available & $-9,999$ & 39.504 & -107.823 & 8/30/1999 & 9999 \\
\hline 623 & Rifle & Tertiary & WASATCH FORMATION & 200 & 39.544 & -107.753 & $4 / 24 / 2009$ & 1620 \\
\hline 624 & Rifle & Tertiary & WASATCH FORMATION & 110 & 39.554 & -107.766 & $4 / 26 / 2009$ & 1240 \\
\hline 625 & Rifle & Unknown & Not available & $-9,999$ & 39.559 & -107.760 & $8 / 15 / 2006$ & 9999 \\
\hline 626 & Rifle & Unknown & Not available & $-9,999$ & 39.536 & -107.756 & $7 / 7 / 1997$ & 9999 \\
\hline 627 & Rifle & Tertiary & WASATCH FORMATION & 100 & 39.591 & -107.76 & $4 / 26 / 2009$ & 1640 \\
\hline 628 & Rifle & Unknown & Not available & $-9,999$ & 39.511 & -107.821 & $10 / 3 / 2007$ & 9999 \\
\hline 629 & Rifle & Unknown & Not available & $-9,999$ & 39.500 & -107.766 & $10 / 2 / 2001$ & 9999 \\
\hline 630 & Rifle & Unknown & Not available & $-9,999$ & 39.508 & -107.756 & $6 / 8 / 2004$ & 9999 \\
\hline 631 & Rifle & Unknown & Not available & $-9,999$ & 39.512 & -107.760 & $10 / 15 / 2001$ & 9999 \\
\hline 632 & Rifle & Unknown & Not available & $-9,999$ & 39.505 & -107.762 & $2 / 17 / 2005$ & 9999 \\
\hline 633 & Rifle & Unknown & Not available & $-9,999$ & 39.501 & -107.760 & $2 / 17 / 2005$ & 9999 \\
\hline 634 & Rifle & Unknown & Not available & 325 & 39.506 & -107.845 & 7/8/1997 & 9999 \\
\hline 635 & Rifle & Unknown & Not available & 150 & 39.512 & -107.824 & $10 / 1 / 2007$ & 9999 \\
\hline 636 & Rifle & Unknown & Not available & 150 & 39.511 & -107.821 & $10 / 2 / 2007$ & 9999 \\
\hline 637 & Rifle & Unknown & Not available & 120 & 39.510 & -107.804 & $2 / 12 / 2008$ & 9999 \\
\hline 638 & Rifle & Unknown & Not available & 300 & 39.505 & -107.808 & $2 / 12 / 2008$ & 9999 \\
\hline 639 & Rifle & Unknown & Not available & 200 & 39.504 & -107.823 & 7/11/1997 & 9999 \\
\hline 640 & Rifle & Unknown & Not available & $-9,999$ & 39.500 & -107.767 & $3 / 23 / 2004$ & 9999 \\
\hline 641 & Rifle & Unknown & Not available & 194 & 39.503 & -107.763 & $5 / 11 / 2004$ & 9999 \\
\hline 642 & Rifle & Unknown & Not available & 185 & 39.508 & -107.751 & $9 / 28 / 2001$ & 9999 \\
\hline 643 & Rifle & Unknown & Not available & $-9,999$ & 39.508 & -107.774 & $3 / 23 / 2004$ & 9999 \\
\hline 644 & Rifle & Unknown & Not available & $-9,999$ & 39.501 & -107.833 & $1 / 9 / 2008$ & 9999 \\
\hline 645 & Rifle & Unknown & Not available & $-9,999$ & 39.501 & -107.773 & $3 / 23 / 2004$ & 9999 \\
\hline 646 & Rifle & Unknown & Not available & $-9,999$ & 39.587 & -107.768 & $8 / 16 / 2006$ & 9999 \\
\hline 647 & Rifle & Unknown & Not available & $-9,999$ & 39.566 & -107.772 & $8 / 15 / 2006$ & 9999 \\
\hline 649 & Rifle & Unknown & Not available & $-9,999$ & 39.534 & -107.757 & $8 / 14 / 2006$ & 9999 \\
\hline 650 & Rifle & Unknown & Not available & $-9,999$ & 39.504 & -107.820 & $11 / 14 / 2006$ & 9999 \\
\hline 651 & Rifle & Unknown & Not available & $-9,999$ & 39.504 & -107.820 & $11 / 14 / 2006$ & 9999 \\
\hline 652 & Rifle & Unknown & Not available & $-9,999$ & 39.503 & -107.812 & $10 / 2 / 2007$ & 9999 \\
\hline 653 & Rifle & Unknown & Not available & $-9,999$ & 39.504 & -107.818 & $7 / 16 / 2008$ & 9999 \\
\hline 654 & Rifle & Unknown & Not available & $-9,999$ & 39.523 & -107.767 & $9 / 26 / 2007$ & 1124 \\
\hline 655 & Rifle & Unknown & Not available & $-9,999$ & 39.523 & -107.767 & $9 / 26 / 2007$ & 1156 \\
\hline 656 & Rifle & Unknown & Not available & $-9,999$ & 39.528 & -107.754 & $8 / 23 / 2006$ & 1244 \\
\hline 657 & Rifle & Unknown & Not available & $-9,999$ & 39.532 & -107.757 & $8 / 23 / 2006$ & 1154 \\
\hline 658 & Rifle & Unknown & Not available & $-9,999$ & 39.534 & -107.757 & $8 / 23 / 2006$ & 930 \\
\hline 660 & Rifle & Unknown & Not available & 80 & 39.578 & -107.763 & $8 / 1 / 2006$ & 1215 \\
\hline 660 & Rifle & Unknown & Not available & 80 & 39.578 & -107.763 & $8 / 1 / 2006$ & 1200 \\
\hline 661 & Rifle & Unknown & Not available & $-9,999$ & 39.536 & -107.765 & $9 / 25 / 2006$ & 1116 \\
\hline 662 & Rifle & Unknown & Not available & 75 & 39.539 & -107.758 & $8 / 2 / 2006$ & 9999 \\
\hline 663 & Rifle & Unknown & Not available & 200 & 39.542 & -107.753 & $8 / 1 / 2006$ & 1215 \\
\hline 663 & Rifle & Unknown & Not available & 200 & 39.542 & -107.753 & $8 / 1 / 2006$ & 1200 \\
\hline 664 & Rifle & Unknown & Not available & $-9,999$ & 39.538 & -107.754 & $8 / 23 / 2006$ & 1012 \\
\hline 665 & Rifle & Unknown & Not available & 250 & 39.504 & -107.820 & $1 / 8 / 2008$ & 9999 \\
\hline 666 & Rifle & Unknown & Not available & 240 & 39.511 & -107.816 & $10 / 4 / 2007$ & 9999 \\
\hline 667 & Rifle & Unknown & Not available & 160 & 39.514 & -107.818 & $10 / 4 / 2007$ & 9999 \\
\hline 668 & Rifle & Unknown & Not available & 145 & 39.514 & -107.817 & $10 / 1 / 2007$ & 9999 \\
\hline 669 & Rifle & Unknown & Not available & $-9,999$ & 39.539 & -107.758 & $9 / 25 / 2006$ & 826 \\
\hline 670 & Rifle & Unknown & Not available & 200 & 39.508 & -107.820 & $10 / 3 / 2007$ & 9999 \\
\hline
\end{tabular}


Appendix 1. Water Quality Data Used in This Report.-Continued

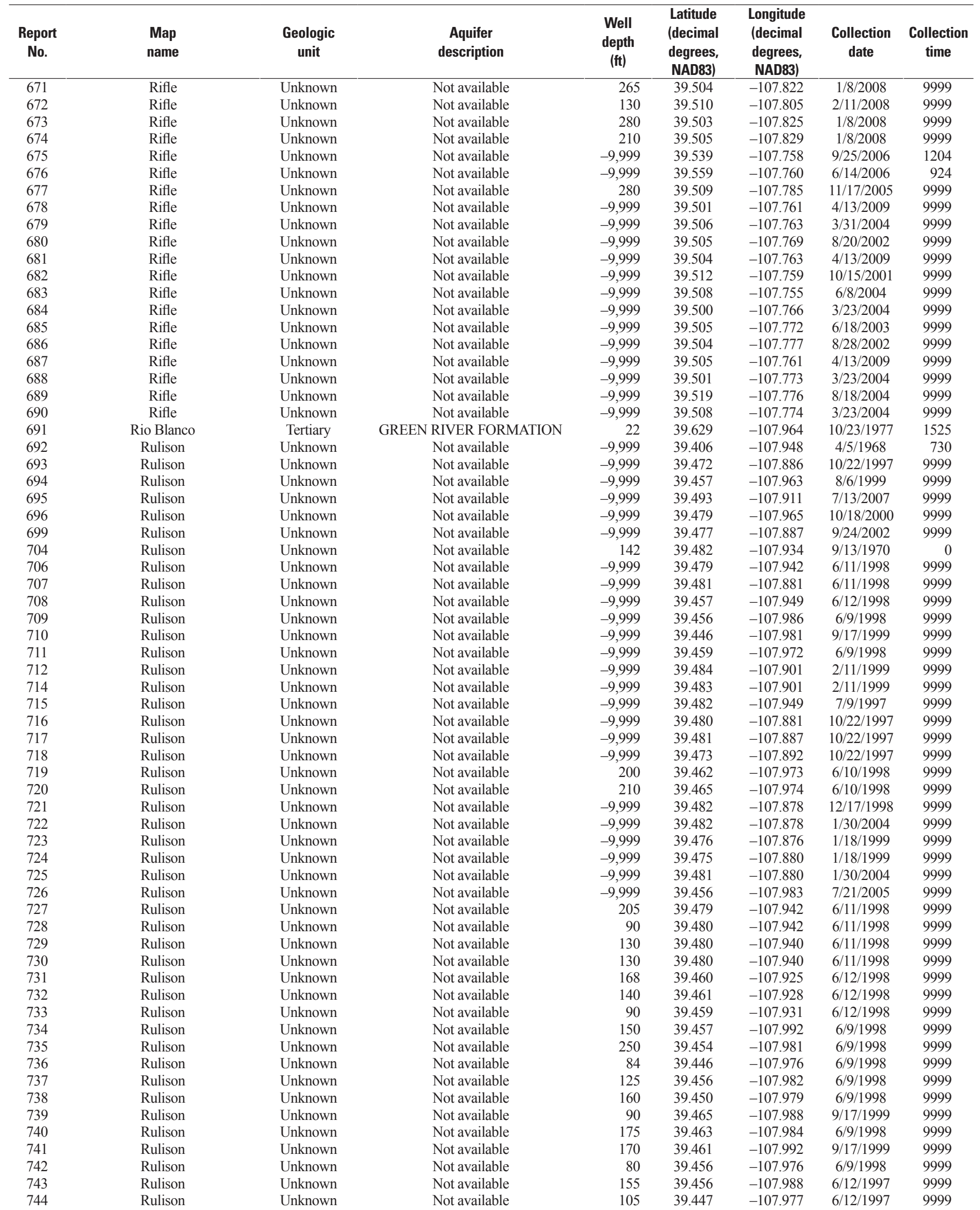


Appendix 1. Water Quality Data Used in This Report.-Continued

\begin{tabular}{|c|c|c|c|c|c|c|c|c|}
\hline $\begin{array}{c}\text { Report } \\
\text { No. }\end{array}$ & $\begin{array}{l}\text { Map } \\
\text { name }\end{array}$ & $\begin{array}{c}\text { Geologic } \\
\text { unit }\end{array}$ & $\begin{array}{c}\text { Aquifer } \\
\text { description }\end{array}$ & $\begin{array}{c}\text { Well } \\
\text { depth } \\
\text { (ft) }\end{array}$ & $\begin{array}{l}\text { Latitude } \\
\text { (decimal } \\
\text { degrees, } \\
\text { NAD83) }\end{array}$ & $\begin{array}{c}\text { Longitude } \\
\text { (decimal } \\
\text { degrees, } \\
\text { NAD83) }\end{array}$ & $\begin{array}{c}\text { Collection } \\
\text { date }\end{array}$ & $\begin{array}{c}\text { Collection } \\
\text { time }\end{array}$ \\
\hline 745 & Rulison & Unknown & Not available & 200 & 39.463 & -107.973 & $6 / 12 / 1997$ & 9999 \\
\hline 746 & Rulison & Unknown & Not available & 50 & 39.492 & -107.937 & 7/8/1997 & 9999 \\
\hline 747 & Rulison & Unknown & Not available & 110 & 39.485 & -107.937 & $7 / 8 / 1997$ & 9999 \\
\hline 748 & Rulison & Unknown & Not available & 130 & 39.485 & -107.935 & 7/8/1997 & 9999 \\
\hline 749 & Rulison & Unknown & Not available & 52 & 39.480 & -107.928 & $7 / 8 / 1997$ & 9999 \\
\hline 750 & Rulison & Unknown & Not available & 130 & 39.485 & -107.901 & $7 / 8 / 1997$ & 9999 \\
\hline 751 & Rulison & Unknown & Not available & 6 & 39.480 & -107.882 & $7 / 8 / 1997$ & 9999 \\
\hline 752 & Rulison & Unknown & Not available & 130 & 39.480 & -107.878 & 7/8/1997 & 9999 \\
\hline 753 & Rulison & Unknown & Not available & 180 & 39.479 & -107.943 & 7/9/1997 & 9999 \\
\hline 754 & Rulison & Unknown & Not available & 80 & 39.480 & -107.952 & $7 / 9 / 1997$ & 9999 \\
\hline 755 & Rulison & Unknown & Not available & 155 & 39.455 & -107.990 & $7 / 9 / 1997$ & 9999 \\
\hline 756 & Rulison & Unknown & Not available & 270 & 39.478 & -107.887 & $10 / 22 / 1997$ & 9999 \\
\hline 757 & Rulison & Unknown & Not available & 130 & 39.481 & -107.875 & $10 / 23 / 1997$ & 9999 \\
\hline 758 & Rulison & Unknown & Not available & 150 & 39.472 & -107.891 & $10 / 22 / 1997$ & 9999 \\
\hline 759 & Rulison & Unknown & Not available & 160 & 39.440 & -107.891 & $10 / 22 / 1997$ & 9999 \\
\hline 760 & Rulison & Unknown & Not available & 170 & 39.458 & -107.983 & 7/9/1997 & 9999 \\
\hline 761 & Rulison & Unknown & Not available & 124 & 39.458 & -107.979 & $7 / 9 / 1997$ & 9999 \\
\hline 762 & Rulison & Unknown & Not available & 250 & 39.424 & -107.959 & $7 / 9 / 1997$ & 9999 \\
\hline 763 & Rulison & Unknown & Not available & 140 & 39.482 & -107.934 & $7 / 9 / 1997$ & 9999 \\
\hline 764 & Rulison & Unknown & Not available & 120 & 39.482 & -107.926 & $7 / 9 / 1997$ & 9999 \\
\hline 765 & Rulison & Unknown & Not available & 130 & 39.479 & -107.879 & 10/23/1997 & 9999 \\
\hline 766 & Rulison & Unknown & Not available & $-9,999$ & 39.455 & -107.973 & $6 / 20 / 2006$ & 9999 \\
\hline 767 & Rulison & Unknown & Not available & $-9,999$ & 39.454 & -107.976 & $6 / 20 / 2006$ & 9999 \\
\hline 768 & Rulison & Unknown & Not available & $-9,999$ & 39.464 & -107.977 & $6 / 20 / 2006$ & 9999 \\
\hline 769 & Rulison & Unknown & Not available & $-9,999$ & 39.455 & -107.974 & $6 / 20 / 2006$ & 9999 \\
\hline 770 & Rulison & Unknown & Not available & $-9,999$ & 39.449 & -107.978 & $7 / 19 / 2006$ & 9999 \\
\hline 771 & Rulison & Unknown & Not available & $-9,999$ & 39.466 & -107.974 & $7 / 25 / 2006$ & 9999 \\
\hline 772 & Rulison & Unknown & Not available & $-9,999$ & 39.459 & -107.979 & $7 / 19 / 2006$ & 9999 \\
\hline 773 & Rulison & Unknown & Not available & $-9,999$ & 39.458 & -107.963 & $7 / 19 / 2006$ & 9999 \\
\hline 774 & Rulison & Unknown & Not available & 275 & 39.461 & -107.986 & $7 / 18 / 2006$ & 9999 \\
\hline 775 & Rulison & Unknown & Not available & $-9,999$ & 39.455 & -107.973 & $10 / 5 / 2006$ & 9999 \\
\hline 776 & Rulison & Unknown & Not available & $-9,999$ & 39.455 & -107.972 & $10 / 5 / 2006$ & 9999 \\
\hline 777 & Rulison & Unknown & Not available & $-9,999$ & 39.444 & -107.983 & $5 / 3 / 2005$ & 9999 \\
\hline 778 & Rulison & Unknown & Not available & $-9,999$ & 39.454 & -107.972 & $10 / 10 / 2006$ & 9999 \\
\hline 779 & Rulison & Unknown & Not available & 170 & 39.485 & -107.897 & $10 / 9 / 2006$ & 1145 \\
\hline 780 & Rulison & Unknown & Not available & 60 & 39.474 & -107.907 & $9 / 1 / 2006$ & 1020 \\
\hline 781 & Rulison & Unknown & Not available & 135 & 39.482 & -107.935 & $11 / 1 / 2006$ & 1500 \\
\hline 782 & Rulison & Unknown & Not available & $-9,999$ & 39.465 & -107.972 & $3 / 27 / 2008$ & 9999 \\
\hline 783 & Rulison & Unknown & Not available & 170 & 39.469 & -107.909 & $9 / 13 / 2006$ & 903 \\
\hline 784 & Rulison & Unknown & Not available & 220 & 39.467 & -107.912 & $9 / 1 / 2006$ & 1250 \\
\hline 785 & Rulison & Unknown & Not available & 85 & 39.496 & -107.948 & $10 / 26 / 2006$ & 1200 \\
\hline 786 & Rulison & Unknown & Not available & 240 & 39.470 & -107.913 & $8 / 30 / 2006$ & 1515 \\
\hline 787 & Rulison & Unknown & Not available & 130 & 39.476 & -107.911 & $10 / 6 / 2008$ & 1515 \\
\hline 788 & Rulison & Unknown & Not available & 130 & 39.482 & -107.926 & $10 / 18 / 2006$ & 1205 \\
\hline 789 & Rulison & Unknown & Not available & 100 & 39.459 & -107.935 & $10 / 17 / 2006$ & 1510 \\
\hline 790 & Rulison & Unknown & Not available & 74 & 39.480 & -107.940 & $10 / 6 / 2008$ & 1615 \\
\hline 791 & Rulison & Unknown & Not available & $-9,999$ & 39.452 & -107.976 & $10 / 5 / 2006$ & 9999 \\
\hline 792 & Rulison & Unknown & Not available & $-9,999$ & 39.456 & -107.977 & $10 / 5 / 2006$ & 9999 \\
\hline 793 & Rulison & Unknown & Not available & $-9,999$ & 39.480 & -107.951 & $8 / 7 / 2006$ & 9999 \\
\hline 794 & Rulison & Unknown & Not available & $-9,999$ & 39.455 & -107.976 & $10 / 10 / 2006$ & 9999 \\
\hline 795 & Rulison & Unknown & Not available & $-9,999$ & 39.452 & -107.974 & $10 / 5 / 2006$ & 9999 \\
\hline 796 & Rulison & Unknown & Not available & $-9,999$ & 39.453 & -107.986 & $7 / 9 / 2004$ & 9999 \\
\hline 797 & Rulison & Unknown & Not available & $-9,999$ & 39.483 & -107.904 & $12 / 22 / 2004$ & 9999 \\
\hline 798 & Rulison & Unknown & Not available & $-9,999$ & 39.450 & -107.975 & $10 / 10 / 2006$ & 9999 \\
\hline 799 & Rulison & Unknown & Not available & $-9,999$ & 39.439 & -107.999 & $12 / 5 / 2008$ & 9999 \\
\hline 800 & Rulison & Unknown & Not available & $-9,999$ & 39.46 & -107.99 & $8 / 12 / 1998$ & 9999 \\
\hline 801 & Silt & Unknown & Not available & $-9,999$ & 39.526 & -107.740 & $8 / 3 / 1978$ & 0 \\
\hline 802 & Silt & Unknown & Not available & $-9,999$ & 39.501 & -107.689 & $1 / 20 / 2005$ & 9999 \\
\hline 803 & Silt & Unknown & Not available & $-9,999$ & 39.505 & -107.631 & $7 / 14 / 2005$ & 9999 \\
\hline 804 & Silt & Tertiary & WASATCH FORMATION & 140 & 39.503 & -107.632 & $4 / 23 / 2009$ & 1550 \\
\hline 805 & Silt & Tertiary & WASATCH FORMATION & 150 & 39.517 & -107.653 & $4 / 25 / 2009$ & 1510 \\
\hline 806 & Silt & Unknown & Not available & $-9,999$ & 39.503 & -107.659 & $6 / 8 / 2007$ & 9999 \\
\hline 807 & Silt & Tertiary & WASATCH FORMATION & 440 & 39.517 & -107.659 & $4 / 25 / 2009$ & 1210 \\
\hline 811 & Silt & Tertiary & WASATCH FORMATION & 80 & 39.553 & -107.706 & $4 / 21 / 2009$ & 1700 \\
\hline 812 & Silt & Tertiary & WASATCH FORMATION & 52 & 39.553 & -107.673 & $4 / 22 / 2009$ & 1600 \\
\hline 813 & Silt & Tertiary & WASATCH FORMATION & 80 & 39.555 & -107.627 & $4 / 20 / 2009$ & 1430 \\
\hline
\end{tabular}


Appendix 1. Water Quality Data Used in This Report.-Continued

\begin{tabular}{|c|c|c|c|c|c|c|c|c|}
\hline $\begin{array}{c}\text { Report } \\
\text { No. }\end{array}$ & $\begin{array}{l}\text { Map } \\
\text { name }\end{array}$ & $\begin{array}{c}\text { Geologic } \\
\text { unit }\end{array}$ & $\begin{array}{c}\text { Aquifer } \\
\text { description }\end{array}$ & $\begin{array}{c}\text { Well } \\
\text { depth } \\
\text { (ft) }\end{array}$ & $\begin{array}{c}\text { Latitude } \\
\text { (decimal } \\
\text { degrees, } \\
\text { NAD83) }\end{array}$ & $\begin{array}{c}\text { Longitude } \\
\text { (decimal } \\
\text { degrees, } \\
\text { NAD83) }\end{array}$ & $\begin{array}{c}\text { Collection } \\
\text { date }\end{array}$ & $\begin{array}{l}\text { Collection } \\
\text { time }\end{array}$ \\
\hline 814 & Silt & Tertiary & WASATCH FORMATION & 145 & 39.560 & -107.663 & $4 / 24 / 2009$ & 1100 \\
\hline 815 & Silt & Unknown & Not available & $-9,999$ & 39.544 & -107.733 & $8 / 14 / 2006$ & 9999 \\
\hline 816 & Silt & Tertiary & WASATCH FORMATION & 100 & 39.569 & -107.719 & $4 / 24 / 2009$ & 900 \\
\hline 817 & Silt & Tertiary & WASATCH FORMATION & 138 & 39.571 & -107.698 & $4 / 22 / 2009$ & 900 \\
\hline 818 & Silt & Unknown & Not available & $-9,999$ & 39.542 & -107.747 & $8 / 14 / 2006$ & 9999 \\
\hline 819 & Silt & Quaternary & VALLEY-FILL DEPOSITS & 50 & 39.565 & -107.672 & 9/11/1997 & 1015 \\
\hline 820 & Silt & Tertiary & WASATCH FORMATION & 200 & 39.582 & -107.679 & $4 / 22 / 2009$ & 1200 \\
\hline 821 & Silt & Unknown & Not available & $-9,999$ & 39.537 & -107.639 & 6/4/1997 & 9999 \\
\hline 822 & Silt & Unknown & Not available & $-9,999$ & 39.519 & -107.684 & $6 / 28 / 2005$ & 9999 \\
\hline 823 & Silt & Unknown & Not available & $-9,999$ & 39.518 & -107.685 & $6 / 28 / 2005$ & 9999 \\
\hline 824 & Silt & Unknown & Not available & $-9,999$ & 39.520 & -107.678 & $6 / 30 / 2005$ & 9999 \\
\hline 825 & Silt & Unknown & Not available & $-9,999$ & 39.520 & -107.683 & $7 / 12 / 2005$ & 9999 \\
\hline 826 & Silt & Unknown & Not available & $-9,999$ & 39.515 & -107.628 & $6 / 14 / 2007$ & 9999 \\
\hline 827 & Silt & Unknown & Not available & $-9,999$ & 39.509 & -107.639 & $4 / 20 / 2005$ & 9999 \\
\hline 828 & Silt & Unknown & Not available & 100 & 39.580 & -107.660 & 8/9/2006 & 9999 \\
\hline 829 & Silt & Unknown & Not available & 120 & 39.574 & -107.639 & 6/3/1997 & 9999 \\
\hline 830 & Silt & Unknown & Not available & 140 & 39.566 & -107.733 & $8 / 15 / 2006$ & 9999 \\
\hline 831 & Silt & Unknown & Not available & $-9,999$ & 39.516 & -107.661 & $1 / 31 / 1995$ & 9999 \\
\hline 832 & Silt & Unknown & Not available & $-9,999$ & 39.518 & -107.691 & $7 / 12 / 2005$ & 9999 \\
\hline 833 & Silt & Unknown & Not available & $-9,999$ & 39.504 & -107.628 & $7 / 14 / 2005$ & 9999 \\
\hline 834 & Silt & Unknown & Not available & 70 & 39.534 & -107.672 & 6/4/1997 & 9999 \\
\hline 835 & Silt & Unknown & Not available & 100 & 39.564 & -107.675 & $8 / 1 / 2006$ & 9999 \\
\hline 836 & Silt & Unknown & Not available & 160 & 39.573 & -107.635 & $8 / 15 / 2006$ & 9999 \\
\hline 837 & Silt & Unknown & Not available & 80 & 39.564 & -107.701 & $8 / 10 / 2006$ & 9999 \\
\hline 838 & Silt & Unknown & Not available & 78 & 39.555 & -107.677 & $8 / 8 / 2006$ & 1215 \\
\hline 838 & Silt & Unknown & Not available & 78 & 39.555 & -107.677 & $8 / 8 / 2006$ & 1200 \\
\hline 839 & Silt & Unknown & Not available & 250 & 39.535 & -107.625 & $6 / 13 / 1997$ & 9999 \\
\hline 840 & Silt & Unknown & Not available & 100 & 39.558 & -107.730 & $7 / 31 / 2006$ & 1215 \\
\hline 840 & Silt & Unknown & Not available & 100 & 39.558 & -107.730 & $7 / 31 / 2006$ & 1200 \\
\hline 841 & Silt & Unknown & Not available & 97 & 39.552 & -107.726 & $8 / 14 / 2006$ & 9999 \\
\hline 842 & Silt & Unknown & Not available & 80 & 39.550 & -107.718 & $7 / 31 / 2006$ & 1215 \\
\hline 842 & Silt & Unknown & Not available & 80 & 39.550 & -107.718 & $7 / 31 / 2006$ & 1200 \\
\hline 843 & Silt & Unknown & Not available & 100 & 39.528 & -107.661 & 7/12/1997 & 9999 \\
\hline 844 & Silt & Unknown & Not available & 50 & 39.534 & -107.656 & $7 / 12 / 1997$ & 9999 \\
\hline 845 & Silt & Unknown & Not available & 430 & 39.581 & -107.670 & $8 / 15 / 2006$ & 9999 \\
\hline 846 & Silt & Unknown & Not available & $-9,999$ & 39.516 & -107.625 & $6 / 14 / 2007$ & 9999 \\
\hline 847 & Silt & Unknown & Not available & $-9,999$ & 39.510 & -107.647 & $7 / 13 / 2007$ & 9999 \\
\hline 848 & Silt & Unknown & Not available & $-9,999$ & 39.513 & -107.629 & $5 / 5 / 2005$ & 9999 \\
\hline 849 & Silt & Unknown & Not available & $-9,999$ & 39.501 & -107.629 & $7 / 6 / 2007$ & 9999 \\
\hline 850 & Silt & Unknown & Not available & $-9,999$ & 39.512 & -107.633 & $5 / 5 / 2005$ & 9999 \\
\hline 851 & Silt & Unknown & Not available & $-9,999$ & 39.556 & -107.700 & $9 / 21 / 2005$ & 9999 \\
\hline 852 & Silt & Unknown & Not available & $-9,999$ & 39.543 & -107.724 & $10 / 17 / 2006$ & 9999 \\
\hline 853 & Silt & Unknown & Not available & $-9,999$ & 39.544 & -107.720 & $10 / 17 / 2006$ & 9999 \\
\hline 854 & Silt & Unknown & Not available & $-9,999$ & 39.546 & -107.721 & $10 / 17 / 2006$ & 9999 \\
\hline 855 & Silt & Unknown & Not available & $-9,999$ & 39.585 & -107.653 & $8 / 7 / 2006$ & 1215 \\
\hline 855 & Silt & Unknown & Not available & $-9,999$ & 39.585 & -107.653 & $8 / 7 / 2006$ & 1200 \\
\hline 856 & Silt & Unknown & Not available & $-9,999$ & 39.576 & -107.638 & $8 / 2 / 2006$ & 9999 \\
\hline 857 & Silt & Unknown & Not available & $-9,999$ & 39.569 & -107.653 & $8 / 9 / 2006$ & 9999 \\
\hline 858 & Silt & Unknown & Not available & $-9,999$ & 39.564 & -107.649 & $8 / 2 / 2006$ & 9999 \\
\hline 859 & Silt & Unknown & Not available & $-9,999$ & 39.586 & -107.677 & $8 / 16 / 2006$ & 9999 \\
\hline 860 & Silt & Unknown & Not available & $-9,999$ & 39.584 & -107.716 & $8 / 15 / 2006$ & 1215 \\
\hline 860 & Silt & Unknown & Not available & $-9,999$ & 39.584 & -107.716 & $8 / 15 / 2006$ & 1200 \\
\hline 861 & Silt & Unknown & Not available & $-9,999$ & 39.565 & -107.746 & $8 / 16 / 2006$ & 9999 \\
\hline 862 & Silt & Unknown & Not available & $-9,999$ & 39.572 & -107.680 & $7 / 31 / 2006$ & 1215 \\
\hline 862 & Silt & Unknown & Not available & $-9,999$ & 39.572 & -107.680 & $7 / 31 / 2006$ & 1200 \\
\hline 863 & Silt & Tertiary & WASATCH FORMATION & 215 & 39.512 & -107.686 & $3 / 31 / 2005$ & 9999 \\
\hline 864 & Silt & Unknown & Not available & $-9,999$ & 39.560 & -107.648 & $8 / 15 / 2006$ & 9999 \\
\hline 865 & Silt & Unknown & Not available & $-9,999$ & 39.560 & -107.664 & $8 / 8 / 2006$ & 9999 \\
\hline 866 & Silt & Unknown & Not available & $-9,999$ & 39.558 & -107.706 & $8 / 2 / 2006$ & 1215 \\
\hline 866 & Silt & Unknown & Not available & $-9,999$ & 39.558 & -107.706 & $8 / 2 / 2006$ & 1200 \\
\hline 867 & Silt & Unknown & Not available & $-9,999$ & 39.548 & -107.673 & $8 / 15 / 2006$ & 9999 \\
\hline 868 & Silt & Unknown & Not available & $-9,999$ & 39.553 & -107.740 & $8 / 14 / 2006$ & 9999 \\
\hline 869 & Silt & Unknown & Not available & $-9,999$ & 39.544 & -107.731 & $8 / 2 / 2006$ & 9999 \\
\hline 870 & Silt & Unknown & Not available & $-9,999$ & 39.512 & -107.671 & $6 / 12 / 2007$ & 1014 \\
\hline 871 & Silt & Unknown & Not available & $-9,999$ & 39.517 & -107.711 & $6 / 27 / 2006$ & 813 \\
\hline 872 & Silt & Unknown & Not available & $-9,999$ & 39.517 & -107.653 & $9 / 24 / 2008$ & 1156 \\
\hline
\end{tabular}


Appendix 1. Water Quality Data Used in This Report.-Continued

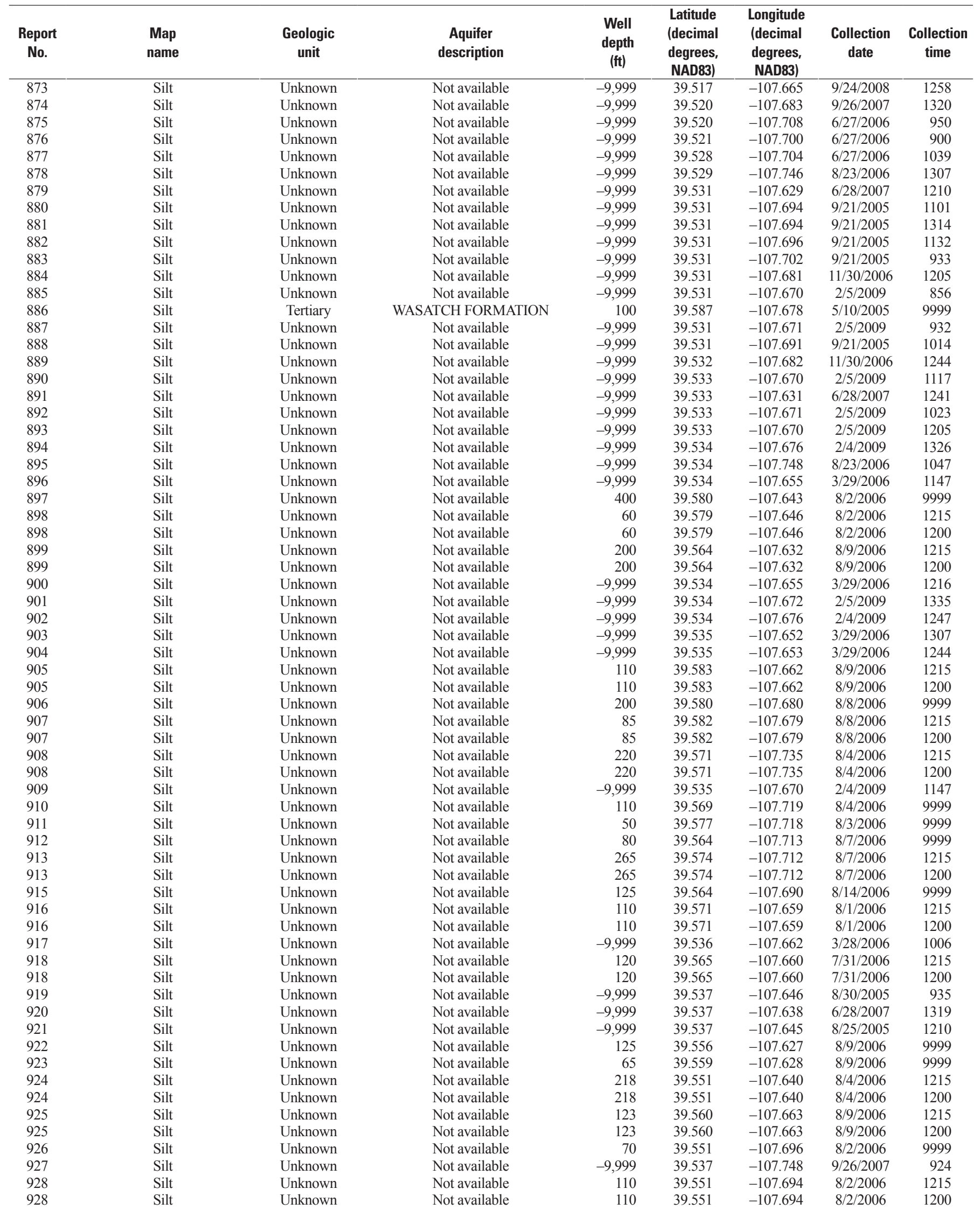


Appendix 1. Water Quality Data Used in This Report.-Continued

\begin{tabular}{|c|c|c|c|c|c|c|c|c|}
\hline $\begin{array}{c}\text { Report } \\
\text { No. }\end{array}$ & $\begin{array}{l}\text { Map } \\
\text { name }\end{array}$ & $\begin{array}{c}\text { Geologic } \\
\text { unit }\end{array}$ & $\begin{array}{c}\text { Aquifer } \\
\text { description }\end{array}$ & $\begin{array}{c}\text { Well } \\
\text { depth } \\
\text { (ft) }\end{array}$ & $\begin{array}{l}\text { Latitude } \\
\text { (decimal } \\
\text { degrees, } \\
\text { NAD83) }\end{array}$ & $\begin{array}{c}\text { Longitude } \\
\text { (decimal } \\
\text { degrees, } \\
\text { NAD83) }\end{array}$ & $\begin{array}{c}\text { Collection } \\
\text { date }\end{array}$ & $\begin{array}{l}\text { Collection } \\
\text { time }\end{array}$ \\
\hline 930 & Silt & Unknown & Not available & $-9,999$ & 39.537 & -107.739 & $1 / 31 / 2006$ & 924 \\
\hline 931 & Silt & Unknown & Not available & $-9,999$ & 39.538 & -107.677 & $8 / 30 / 2005$ & 1204 \\
\hline 932 & Silt & Unknown & Not available & $-9,999$ & 39.538 & -107.646 & $8 / 30 / 2005$ & 1236 \\
\hline 933 & Silt & Unknown & Not available & 60 & 39.542 & -107.739 & $8 / 2 / 2006$ & 9999 \\
\hline 934 & Silt & Unknown & Not available & 105 & 39.545 & -107.736 & $8 / 2 / 2006$ & 1215 \\
\hline 934 & Silt & Unknown & Not available & 105 & 39.545 & -107.736 & $8 / 2 / 2006$ & 1200 \\
\hline 935 & Silt & Unknown & Not available & $-9,999$ & 39.539 & -107.746 & $2 / 1 / 2006$ & 1233 \\
\hline 936 & Silt & Unknown & Not available & $-9,999$ & 39.539 & -107.661 & $3 / 28 / 2006$ & 906 \\
\hline 937 & Silt & Unknown & Not available & $-9,999$ & 39.539 & -107.737 & $1 / 31 / 2006$ & 1052 \\
\hline 938 & Silt & Unknown & Not available & $-9,999$ & 39.540 & -107.662 & $3 / 28 / 2006$ & 845 \\
\hline 939 & Silt & Unknown & Not available & $-9,999$ & 39.540 & -107.736 & $1 / 31 / 2006$ & 1015 \\
\hline 940 & Silt & Unknown & Not available & $-9,999$ & 39.540 & -107.742 & $2 / 1 / 2006$ & 1105 \\
\hline 941 & Silt & Unknown & Not available & $-9,999$ & 39.541 & -107.739 & $2 / 1 / 2006$ & 1001 \\
\hline 942 & Silt & Unknown & Not available & $-9,999$ & 39.541 & -107.738 & $2 / 1 / 2006$ & 846 \\
\hline 943 & Silt & Unknown & Not available & $-9,999$ & 39.542 & -107.739 & $2 / 1 / 2006$ & 930 \\
\hline 944 & Silt & Unknown & Not available & $-9,999$ & 39.542 & -107.745 & $2 / 2 / 2006$ & 847 \\
\hline 945 & Silt & Unknown & Not available & $-9,999$ & 39.543 & -107.664 & $11 / 30 / 2006$ & 1044 \\
\hline 946 & Silt & Unknown & Not available & $-9,999$ & 39.543 & -107.665 & $11 / 30 / 2006$ & 1030 \\
\hline 947 & Silt & Unknown & Not available & $-9,999$ & 39.543 & -107.723 & $12 / 6 / 2005$ & 1037 \\
\hline 948 & Silt & Unknown & Not available & $-9,999$ & 39.544 & -107.733 & $4 / 10 / 2007$ & 1045 \\
\hline 949 & Silt & Unknown & Not available & $-9,999$ & 39.544 & -107.743 & $2 / 1 / 2006$ & 1146 \\
\hline 950 & Silt & Unknown & Not available & $-9,999$ & 39.544 & -107.720 & $12 / 6 / 2005$ & 936 \\
\hline 951 & Silt & Unknown & Not available & $-9,999$ & 39.544 & -107.731 & $4 / 10 / 2007$ & 1120 \\
\hline 952 & Silt & Unknown & Not available & $-9,999$ & 39.545 & -107.736 & $1 / 31 / 2006$ & 1201 \\
\hline 953 & Silt & Unknown & Not available & $-9,999$ & 39.545 & -107.733 & $4 / 10 / 2007$ & 1007 \\
\hline 954 & Silt & Unknown & Not available & $-9,999$ & 39.546 & -107.722 & $12 / 6 / 2005$ & 1213 \\
\hline 955 & Silt & Unknown & Not available & $-9,999$ & 39.546 & -107.709 & $3 / 8 / 2006$ & 1318 \\
\hline 956 & Silt & Unknown & Not available & $-9,999$ & 39.546 & -107.732 & $4 / 10 / 2007$ & 930 \\
\hline 957 & Silt & Unknown & Not available & $-9,999$ & 39.547 & -107.686 & $11 / 15 / 2006$ & 1346 \\
\hline 958 & Silt & Unknown & Not available & $-9,999$ & 39.547 & -107.708 & $3 / 7 / 2006$ & 1334 \\
\hline 959 & Silt & Unknown & Not available & $-9,999$ & 39.547 & -107.719 & $12 / 6 / 2005$ & 1121 \\
\hline 960 & Silt & Unknown & Not available & $-9,999$ & 39.547 & -107.707 & $3 / 7 / 2006$ & 1306 \\
\hline 961 & Silt & Unknown & Not available & $-9,999$ & 39.547 & -107.712 & $5 / 9 / 2006$ & 1312 \\
\hline 962 & Silt & Unknown & Not available & $-9,999$ & 39.548 & -107.708 & $3 / 7 / 2006$ & 948 \\
\hline 963 & Silt & Unknown & Not available & $-9,999$ & 39.547 & -107.715 & $5 / 9 / 2006$ & 1232 \\
\hline 964 & Silt & Unknown & Not available & $-9,999$ & 39.547 & -107.716 & $12 / 6 / 2005$ & 840 \\
\hline 965 & Silt & Unknown & Not available & $-9,999$ & 39.548 & -107.709 & $3 / 7 / 2006$ & 855 \\
\hline 966 & Silt & Unknown & Not available & $-9,999$ & 39.548 & -107.710 & $3 / 7 / 2006$ & 1026 \\
\hline 967 & Silt & Unknown & Not available & $-9,999$ & 39.548 & -107.730 & $4 / 10 / 2007$ & 815 \\
\hline 968 & Silt & Unknown & Not available & $-9,999$ & 39.549 & -107.728 & $4 / 10 / 2007$ & 857 \\
\hline 969 & Silt & Unknown & Not available & $-9,999$ & 39.549 & -107.699 & $11 / 15 / 2006$ & 1203 \\
\hline 970 & Silt & Unknown & Not available & $-9,999$ & 39.549 & -107.700 & $3 / 8 / 2006$ & 1137 \\
\hline 971 & Silt & Unknown & Not available & $-9,999$ & 39.549 & -107.710 & $3 / 7 / 2006$ & 1138 \\
\hline 972 & Silt & Unknown & Not available & $-9,999$ & 39.549 & -107.736 & $10 / 16 / 2008$ & 1020 \\
\hline 973 & Silt & Unknown & Not available & $-9,999$ & 39.550 & -107.738 & $10 / 16 / 2008$ & 930 \\
\hline 974 & Silt & Unknown & Not available & $-9,999$ & 39.550 & -107.687 & $11 / 15 / 2006$ & 906 \\
\hline 975 & Silt & Unknown & Not available & $-9,999$ & 39.550 & -107.701 & $3 / 8 / 2006$ & 959 \\
\hline 976 & Silt & Unknown & Not available & $-9,999$ & 39.550 & -107.700 & $3 / 8 / 2006$ & 1057 \\
\hline 977 & Silt & Unknown & Not available & $-9,999$ & 39.550 & -107.697 & $11 / 15 / 2006$ & 1052 \\
\hline 978 & Silt & Unknown & Not available & $-9,999$ & 39.550 & -107.688 & $11 / 14 / 2006$ & 1326 \\
\hline 979 & Silt & Unknown & Not available & $-9,999$ & 39.550 & -107.700 & $3 / 8 / 2006$ & 844 \\
\hline 980 & Silt & Unknown & Not available & $-9,999$ & 39.550 & -107.718 & $5 / 9 / 2006$ & 955 \\
\hline 981 & Silt & Unknown & Not available & $-9,999$ & 39.550 & -107.732 & $10 / 15 / 2008$ & 1251 \\
\hline 982 & Silt & Unknown & Not available & $-9,999$ & 39.551 & -107.687 & $11 / 14 / 2006$ & 1238 \\
\hline 983 & Silt & Unknown & Not available & $-9,999$ & 39.551 & -107.717 & $5 / 9 / 2006$ & 1034 \\
\hline 984 & Silt & Unknown & Not available & $-9,999$ & 39.551 & -107.687 & $11 / 14 / 2006$ & 1154 \\
\hline 985 & Silt & Unknown & Not available & $-9,999$ & 39.551 & -107.691 & $11 / 14 / 2006$ & 1044 \\
\hline 986 & Silt & Unknown & Not available & $-9,999$ & 39.551 & -107.693 & $11 / 14 / 2006$ & 947 \\
\hline 987 & Silt & Unknown & Not available & $-9,999$ & 39.551 & -107.710 & $5 / 9 / 2006$ & 1116 \\
\hline 988 & Silt & Unknown & Not available & $-9,999$ & 39.551 & -107.686 & $11 / 15 / 2006$ & 1010 \\
\hline 989 & Silt & Unknown & Not available & $-9,999$ & 39.551 & -107.696 & $11 / 14 / 2006$ & 906 \\
\hline 990 & Silt & Unknown & Not available & $-9,999$ & 39.551 & -107.701 & $3 / 8 / 2006$ & 917 \\
\hline 991 & Silt & Unknown & Not available & $-9,999$ & 39.551 & -107.720 & $5 / 9 / 2006$ & 832 \\
\hline 992 & Silt & Unknown & Not available & $-9,999$ & 39.551 & -107.721 & $5 / 9 / 2006$ & 913 \\
\hline 993 & Silt & Unknown & Not available & $-9,999$ & 39.552 & -107.726 & $4 / 10 / 2007$ & 1202 \\
\hline 994 & Silt & Unknown & Not available & $-9,999$ & 39.551 & -107.738 & $10 / 16 / 2008$ & 1103 \\
\hline
\end{tabular}


Appendix 1. Water Quality Data Used in This Report.-Continued

\begin{tabular}{|c|c|c|c|c|c|c|c|c|}
\hline $\begin{array}{c}\text { Report } \\
\text { No. }\end{array}$ & $\begin{array}{l}\text { Map } \\
\text { name }\end{array}$ & $\begin{array}{c}\text { Geologic } \\
\text { unit }\end{array}$ & $\begin{array}{c}\text { Aquifer } \\
\text { description }\end{array}$ & $\begin{array}{c}\text { Well } \\
\text { depth } \\
\text { (ft) }\end{array}$ & $\begin{array}{l}\text { Latitude } \\
\text { (decimal } \\
\text { degrees, } \\
\text { NAD83) }\end{array}$ & $\begin{array}{c}\text { Longitude } \\
\text { (decimal } \\
\text { degrees, } \\
\text { NAD83) }\end{array}$ & $\begin{array}{c}\text { Collection } \\
\text { date }\end{array}$ & $\begin{array}{c}\text { Collection } \\
\text { time }\end{array}$ \\
\hline 995 & Silt & Unknown & Not available & $-9,999$ & 39.552 & -107.729 & $4 / 10 / 2007$ & 1222 \\
\hline 996 & Silt & Unknown & Not available & $-9,999$ & 39.552 & -107.703 & $3 / 9 / 2006$ & 916 \\
\hline 997 & Silt & Unknown & Not available & $-9,999$ & 39.552 & -107.730 & $10 / 15 / 2008$ & 911 \\
\hline 998 & Silt & Unknown & Not available & $-9,999$ & 39.552 & -107.703 & $3 / 9 / 2006$ & 846 \\
\hline 999 & Silt & Unknown & Not available & $-9,999$ & 39.552 & -107.728 & $10 / 14 / 2008$ & 1130 \\
\hline 1000 & Silt & Unknown & Not available & $-9,999$ & 39.552 & -107.732 & $10 / 15 / 2008$ & 953 \\
\hline 1001 & Silt & Unknown & Not available & $-9,999$ & 39.552 & -107.706 & $6 / 18 / 2008$ & 1122 \\
\hline 1002 & Silt & Unknown & Not available & $-9,999$ & 39.552 & -107.709 & $6 / 18 / 2008$ & 1039 \\
\hline 1003 & Silt & Unknown & Not available & $-9,999$ & 39.553 & -107.713 & $6 / 18 / 2008$ & 934 \\
\hline 1004 & Silt & Unknown & Not available & $-9,999$ & 39.553 & -107.714 & $10 / 14 / 2008$ & 851 \\
\hline 1005 & Silt & Unknown & Not available & $-9,999$ & 39.553 & -107.731 & $10 / 15 / 2008$ & 1030 \\
\hline 1006 & Silt & Unknown & Not available & $-9,999$ & 39.554 & -107.717 & $10 / 14 / 2008$ & 951 \\
\hline 1007 & Silt & Unknown & Not available & $-9,999$ & 39.555 & -107.732 & $10 / 15 / 2008$ & 1127 \\
\hline 1008 & Silt & Unknown & Not available & $-9,999$ & 39.556 & -107.722 & $10 / 14 / 2008$ & 1201 \\
\hline 1009 & Silt & Unknown & Not available & $-9,999$ & 39.556 & -107.732 & $10 / 14 / 2008$ & 1313 \\
\hline 1010 & Silt & Unknown & Not available & $-9,999$ & 39.557 & -107.726 & $10 / 14 / 2008$ & 1404 \\
\hline 1011 & Silt & Unknown & Not available & $-9,999$ & 39.564 & -107.733 & $6 / 15 / 2006$ & 903 \\
\hline 1012 & Silt & Unknown & Not available & $-9,999$ & 39.565 & -107.746 & $6 / 14 / 2006$ & 902 \\
\hline 1013 & Silt & Unknown & Not available & $-9,999$ & 39.566 & -107.732 & $6 / 13 / 2006$ & 1102 \\
\hline 1014 & Silt & Unknown & Not available & $-9,999$ & 39.568 & -107.734 & $6 / 14 / 2006$ & 1140 \\
\hline 1015 & Silt & Unknown & Not available & $-9,999$ & 39.569 & -107.735 & $6 / 14 / 2006$ & 1216 \\
\hline 1016 & Silt & Unknown & Not available & $-9,999$ & 39.569 & -107.732 & $6 / 14 / 2006$ & 1342 \\
\hline 1017 & Silt & Unknown & Not available & $-9,999$ & 39.570 & -107.732 & $6 / 14 / 2006$ & 1312 \\
\hline 1018 & Silt & Unknown & Not available & $-9,999$ & 39.571 & -107.733 & $6 / 13 / 2006$ & 1143 \\
\hline 1019 & Silt & Unknown & Not available & $-9,999$ & 39.571 & -107.735 & $6 / 13 / 2006$ & 1222 \\
\hline 1020 & Silt & Unknown & Not available & $-9,999$ & 39.571 & -107.737 & $6 / 13 / 2006$ & 1337 \\
\hline 1021 & Silt & Unknown & Not available & $-9,999$ & 39.572 & -107.735 & $6 / 13 / 2006$ & 1307 \\
\hline 1022 & Silt & Unknown & Not available & $-9,999$ & 39.573 & -107.734 & $6 / 15 / 2006$ & 950 \\
\hline 1023 & Silt & Unknown & Not available & $-9,999$ & 39.573 & -107.731 & $6 / 13 / 2006$ & 957 \\
\hline 1024 & Silt & Unknown & Not available & $-9,999$ & 39.573 & -107.734 & $6 / 13 / 2006$ & 916 \\
\hline 1025 & Silt & Unknown & Not available & $-9,999$ & 39.574 & -107.735 & $6 / 13 / 2006$ & 837 \\
\hline 1026 & Silt & Unknown & Not available & $-9,999$ & 39.531 & -107.630 & $9 / 16 / 2004$ & 9999 \\
\hline 1027 & Silt & Unknown & Not available & $-9,999$ & 39.518 & -107.632 & $9 / 16 / 2004$ & 9999 \\
\hline 1028 & Silt & Unknown & Not available & $-9,999$ & 39.53 & -107.67 & $8 / 12 / 1998$ & 9999 \\
\hline 1029 & Silt & Unknown & Not available & $-9,999$ & 39.531 & -107.630 & 9/16/2004 & 9999 \\
\hline 1030 & Storm King Mountain & Unknown & Not available & $-9,999$ & 39.594 & -107.450 & $6 / 2 / 1997$ & 9999 \\
\hline 1031 & Storm King Mountain & Unknown & Not available & $-9,999$ & 39.571 & -107.452 & 6/2/1997 & 9999 \\
\hline 1032 & Storm King Mountain & Unknown & Not available & $-9,999$ & 39.572 & -107.494 & $6 / 2 / 1997$ & 9999 \\
\hline 1033 & Storm King Mountain & Unknown & Not available & $-9,999$ & 39.568 & -107.492 & $6 / 12 / 1997$ & 9999 \\
\hline 1034 & Storm King Mountain & Quaternary & VALLEY-FILL DEPOSITS & 32 & 39.550 & -107.415 & 9/10/1997 & 1500 \\
\hline 1038 & The Saddle & Unknown & Not available & 56 & 39.500 & -108.381 & $8 / 2 / 1977$ & 940 \\
\hline 1039 & Banty Point & Unknown & Not available & 30 & 40.061 & -108.954 & $7 / 21 / 1981$ & 0 \\
\hline 1051 & Banty Point & Pennsylvanian & WEBER FORMATION & 7,500 & 40.104 & -108.875 & $5 / 8 / 1973$ & 0 \\
\hline 1052 & Banty Point & Unknown & Not available & $-9,999$ & 40.101 & -108.881 & 9/19/1977 & 800 \\
\hline 1053 & Banty Point & Unknown & Not available & $-9,999$ & 40.121 & -108.883 & 9/19/1977 & 800 \\
\hline 1054 & Barcus Creek & Unknown & Not available & $-9,999$ & 40.041 & -108.416 & 9/13/1979 & 1130 \\
\hline 1055 & Barcus Creek & Tertiary & GREEN RIVER FORMATION & $-9,999$ & 40.020 & -108.464 & 9/12/1973 & 1400 \\
\hline 1057 & Barcus Creek & Unknown & Not available & 1,122 & 40.040 & -108.416 & $11 / 12 / 1975$ & 930 \\
\hline 1058 & Barcus Creek & Unknown & Not available & 1,510 & 40.040 & -108.416 & $11 / 16 / 1975$ & 2100 \\
\hline 1059 & Barcus Creek & Unknown & Not available & 2,008 & 40.041 & -108.416 & $9 / 11 / 1979$ & 1700 \\
\hline 1062 & Barcus Creek & Tertiary & GREEN RIVER FORMATION & $-9,999$ & 40.124 & -108.418 & $6 / 21 / 1973$ & 1330 \\
\hline 1064 & Barcus Creek & Unknown & Not available & $-9,999$ & 40.065 & -108.417 & $9 / 17 / 1977$ & 1400 \\
\hline 1065 & Barcus Creek & Unknown & Not available & $-9,999$ & 40.044 & -108.436 & $9 / 17 / 1977$ & 1400 \\
\hline 1066 & Barcus Creek & Unknown & Not available & $-9,999$ & 40.020 & -108.463 & 9/17/1977 & 1400 \\
\hline 1067 & Barcus Creek & Unknown & Not available & $-9,999$ & 40.089 & -108.470 & 9/17/1977 & 1600 \\
\hline 1068 & Barcus Creek SE & Unknown & Not available & $-9,999$ & 40.038 & -108.262 & $8 / 30 / 1972$ & 1100 \\
\hline 1069 & Barcus Creek SE & Unknown & Not available & $-9,999$ & 40.057 & -108.258 & $8 / 18 / 1975$ & 1415 \\
\hline 1070 & Barcus Creek SE & Unknown & Not available & $-9,999$ & 40.079 & -108.334 & $10 / 2 / 1982$ & 0 \\
\hline 1071 & Barcus Creek SE & Tertiary & GREEN RIVER FORMATION & $-9,999$ & 40.005 & -108.353 & $10 / 14 / 1982$ & 1445 \\
\hline 1073 & Barcus Creek SE & Quaternary & ALLUVIUM,FLOOD PLAIN & $-9,999$ & 40.017 & -108.342 & $9 / 12 / 1973$ & 1130 \\
\hline 1080 & Barcus Creek SE & Tertiary & GREEN RIVER FORMATION & 1,060 & 40.038 & -108.286 & $10 / 13 / 1982$ & 1445 \\
\hline 1081 & Barcus Creek SE & Unknown & Not available & 950 & 40.038 & -108.286 & $12 / 8 / 1975$ & 1800 \\
\hline 1084 & Barcus Creek SE & Tertiary & GREEN RIVER FORMATION & $-9,999$ & 40.041 & -108.258 & $2 / 20 / 1973$ & 1415 \\
\hline 1085 & Barcus Creek SE & Quaternary & ALLUVIUM,FLOOD PLAIN & $-9,999$ & 40.046 & -108.336 & 9/12/1973 & 1600 \\
\hline 1091 & Barcus Creek SE & Quaternary & ALLUVIUM,FLOOD PLAIN & $-9,999$ & 40.059 & -108.251 & 9/7/1973 & 930 \\
\hline 1094 & Barcus Creek SE & Unknown & Not available & 3,140 & 40.067 & -108.337 & $5 / 24 / 1966$ & 0 \\
\hline
\end{tabular}


Appendix 1. Water Quality Data Used in This Report.-Continued

\begin{tabular}{|c|c|c|c|c|c|c|c|c|}
\hline $\begin{array}{c}\text { Report } \\
\text { No. }\end{array}$ & $\begin{array}{l}\text { Map } \\
\text { name }\end{array}$ & $\begin{array}{c}\text { Geologic } \\
\text { unit }\end{array}$ & $\begin{array}{c}\text { Aquifer } \\
\text { description }\end{array}$ & $\begin{array}{c}\text { Well } \\
\text { depth } \\
\text { (ft) }\end{array}$ & $\begin{array}{l}\text { Latitude } \\
\text { (decimal } \\
\text { degrees, } \\
\text { NAD83) }\end{array}$ & $\begin{array}{c}\text { Longitude } \\
\text { (decimal } \\
\text { degrees, } \\
\text { NAD83) }\end{array}$ & $\begin{array}{c}\text { Collection } \\
\text { date }\end{array}$ & $\begin{array}{c}\text { Collection } \\
\text { time }\end{array}$ \\
\hline 1095 & Barcus Creek SE & Unknown & Not available & 3,140 & 40.067 & -108.337 & $5 / 24 / 1966$ & 0 \\
\hline 1096 & Barcus Creek SE & Quaternary & ALLUVIUM,FLOOD PLAIN & $-9,999$ & 40.086 & -108.340 & $9 / 13 / 1973$ & 1730 \\
\hline 1099 & Barcus Creek SE & Quaternary & ALLUVIUM,TERRACE & $-9,999$ & 40.096 & -108.256 & $7 / 13 / 1982$ & 0 \\
\hline 1100 & Barcus Creek SE & Quaternary & ALLUVIUM,FLOOD PLAIN & $-9,999$ & 40.115 & -108.359 & $8 / 30 / 1973$ & 1100 \\
\hline 1101 & Big Beaver Reservoir & Unknown & Not available & $-9,999$ & 39.949 & -107.711 & $8 / 17 / 1981$ & 0 \\
\hline 1107 & Buckskin Point & Quaternary & ALLUVIUM,FLOOD PLAIN & 17.9 & 40.013 & -108.093 & $3 / 30 / 1983$ & 0 \\
\hline 1125 & Buckskin Point & Unknown & Not available & 60 & 40.034 & -108.034 & $1 / 23 / 1981$ & 0 \\
\hline 1130 & Buckskin Point & Unknown & Not available & $-9,999$ & 40.02 & -108.01 & $7 / 8 / 1998$ & 9999 \\
\hline 1141 & Cutoff Gulch & Unknown & Not available & $-9,999$ & 39.718 & -108.226 & 9/15/1977 & 1100 \\
\hline 1142 & Cutoff Gulch & Tertiary & GREEN RIVER FORMATION & $-9,999$ & 39.718 & -108.168 & 9/5/1973 & 1230 \\
\hline 1144 & Devils Hole Gulch & Unknown & Not available & $-9,999$ & 40.147 & -107.978 & 9/4/1981 & 1455 \\
\hline 1146 & Devils Hole Gulch & Tertiary & WASATCH FORMATION & 100 & 40.149 & -107.965 & $7 / 1 / 1975$ & 0 \\
\hline 1147 & Devils Hole Gulch & Tertiary & WASATCH FORMATION & 40 & 40.162 & -107.997 & $7 / 1 / 1975$ & 0 \\
\hline 1149 & Divide Creek & Unknown & Not available & 65 & 40.176 & -108.555 & $7 / 17 / 1981$ & 0 \\
\hline 1153 & Divide Creek & Unknown & Not available & 25 & 40.185 & -108.533 & $7 / 19 / 1981$ & 0 \\
\hline 1154 & Divide Creek & Unknown & Not available & 35 & 40.189 & -108.526 & $7 / 19 / 1981$ & 0 \\
\hline 1160 & Figure Four Spring & Tertiary & GREEN RIVER FORMATION & 2,389 & 39.746 & -108.432 & $7 / 12 / 1973$ & 1700 \\
\hline 1164 & Gillam Draw & Unknown & Not available & 25 & 40.097 & -108.722 & $7 / 20 / 1981$ & 0 \\
\hline 1173 & Greasewood Gulch & Unknown & Not available & $-9,999$ & 39.889 & -108.181 & 9/16/1977 & 1700 \\
\hline 1174 & Greasewood Gulch & Unknown & Not available & $-9,999$ & 39.877 & -108.228 & $6 / 6 / 1975$ & 1430 \\
\hline 1175 & Greasewood Gulch & Unknown & Not available & $-9,999$ & 39.889 & -108.181 & $6 / 6 / 1975$ & 1700 \\
\hline 1176 & Greasewood Gulch & Unknown & Not available & $-9,999$ & 39.912 & -108.208 & $9 / 12 / 1977$ & 9999 \\
\hline 1177 & Greasewood Gulch & Unknown & Not available & $-9,999$ & 39.897 & -108.182 & $9 / 12 / 1977$ & 9999 \\
\hline 1178 & Greasewood Gulch & Tertiary & GREEN RIVER FORMATION & $-9,999$ & 39.897 & -108.192 & $8 / 29 / 1973$ & 1200 \\
\hline 1182 & Greasewood Gulch & Tertiary & GREEN RIVER FORMATION & $-9,999$ & 39.979 & -108.146 & $7 / 2 / 1973$ & 1030 \\
\hline 1185 & Greasewood Gulch & Quaternary & ALLUVIUM,FLOOD PLAIN & $-9,999$ & 39.980 & -108.247 & $7 / 11 / 1973$ & 930 \\
\hline 1186 & Greasewood Gulch & Tertiary & GREEN RIVER FORMATION & $-9,999$ & 39.981 & -108.146 & $6 / 27 / 1973$ & 1630 \\
\hline 1187 & Jessup Gulch & Unknown & Not available & $-9,999$ & 39.753 & -108.233 & 9/13/1977 & 1300 \\
\hline 1188 & Jessup Gulch & Unknown & Not available & $-9,999$ & 39.797 & -108.248 & $10 / 30 / 1980$ & 1015 \\
\hline 1189 & Jessup Gulch & Unknown & Not available & $-9,999$ & 39.800 & -108.213 & $2 / 25 / 1975$ & 1330 \\
\hline 1190 & Jessup Gulch & Unknown & Not available & $-9,999$ & 39.802 & -108.213 & 4/9/1991 & 1005 \\
\hline 1191 & Jessup Gulch & Unknown & Not available & $-9,999$ & 39.803 & -108.173 & $5 / 22 / 1981$ & 1300 \\
\hline 1192 & Jessup Gulch & Unknown & Not available & $-9,999$ & 39.807 & -108.224 & 4/2/1991 & 1235 \\
\hline 1193 & Jessup Gulch & Unknown & Not available & $-9,999$ & 39.807 & -108.226 & $4 / 3 / 1991$ & 1425 \\
\hline 1194 & Jessup Gulch & Unknown & Not available & $-9,999$ & 39.808 & -108.225 & $3 / 24 / 1983$ & 1230 \\
\hline 1195 & Jessup Gulch & Unknown & Not available & $-9,999$ & 39.810 & -108.228 & $4 / 1 / 1991$ & 1515 \\
\hline 1196 & Jessup Gulch & Unknown & Not available & $-9,999$ & 39.783 & -108.191 & $5 / 22 / 1981$ & 1030 \\
\hline 1197 & Jessup Gulch & Unknown & Not available & $-9,999$ & 39.786 & -108.198 & $12 / 4 / 1972$ & 1535 \\
\hline 1198 & Jessup Gulch & Unknown & Not available & $-9,999$ & 39.797 & -108.203 & $10 / 9 / 1975$ & 1430 \\
\hline 1199 & Jessup Gulch & Unknown & Not available & $-9,999$ & 39.810 & -108.228 & 4/1/1991 & 1530 \\
\hline 1200 & Jessup Gulch & Unknown & Not available & $-9,999$ & 39.812 & -108.225 & 4/1/1991 & 1330 \\
\hline 1201 & Jessup Gulch & Unknown & Not available & $-9,999$ & 39.814 & -108.192 & $12 / 6 / 1972$ & 1335 \\
\hline 1202 & Jessup Gulch & Unknown & Not available & $-9,999$ & 39.814 & -108.233 & $11 / 12 / 1972$ & 2015 \\
\hline 1203 & Jessup Gulch & Unknown & Not available & $-9,999$ & 39.815 & -108.244 & 1/31/1975 & 1130 \\
\hline 1204 & Jessup Gulch & Unknown & Not available & $-9,999$ & 39.816 & -108.206 & $11 / 13 / 1972$ & 1435 \\
\hline 1205 & Jessup Gulch & Unknown & Not available & $-9,999$ & 39.816 & -108.231 & 3/17/1977 & 800 \\
\hline 1206 & Jessup Gulch & Unknown & Not available & $-9,999$ & 39.817 & -108.240 & 4/1/1991 & 1010 \\
\hline 1207 & Jessup Gulch & Unknown & Not available & $-9,999$ & 39.817 & -108.240 & 4/1/1991 & 1050 \\
\hline 1208 & Jessup Gulch & Unknown & Not available & $-9,999$ & 39.817 & -108.231 & 4/2/1991 & 1020 \\
\hline 1209 & Jessup Gulch & Unknown & Not available & $-9,999$ & 39.817 & -108.231 & 4/2/1991 & 1030 \\
\hline 1210 & Jessup Gulch & Unknown & Not available & $-9,999$ & 39.837 & -108.244 & $5 / 21 / 1981$ & 1130 \\
\hline 1211 & Jessup Gulch & Unknown & Not available & $-9,999$ & 39.843 & -108.233 & $5 / 21 / 1981$ & 1345 \\
\hline 1213 & Jessup Gulch & Tertiary & GREEN RIVER FORMATION & $-9,999$ & 39.807 & -108.227 & $4 / 13 / 1977$ & 1230 \\
\hline 1230 & Jessup Gulch & Quaternary & VALLEY-FILL DEPOSITS & 75.4 & 39.836 & -108.225 & 4/3/1991 & 1200 \\
\hline 1237 & Jessup Gulch & Quaternary & ALLUVIUM,FLOOD PLAIN & $-9,999$ & 39.841 & -108.235 & $9 / 6 / 1973$ & 1000 \\
\hline 1239 & Jessup Gulch & Unknown & Not available & 495 & 39.865 & -108.209 & $5 / 12 / 1975$ & 1900 \\
\hline 1244 & LO 7 Hill & Unknown & Not available & $-9,999$ & 39.98 & -107.93 & $7 / 7 / 1998$ & 9999 \\
\hline 1250 & Meeker & Unknown & Not available & $-9,999$ & 40.025 & -107.938 & $6 / 29 / 1981$ & 1400 \\
\hline 1251 & Meeker & Unknown & Not available & $-9,999$ & 40.027 & -107.964 & $6 / 26 / 1980$ & 0 \\
\hline 1252 & Meeker & Unknown & Not available & $-9,999$ & 40.028 & -107.935 & $7 / 26 / 1979$ & 0 \\
\hline 1253 & Meeker & Unknown & Not available & $-9,999$ & 40.080 & -107.919 & $6 / 9 / 1981$ & 1100 \\
\hline 1254 & Meeker & Unknown & Not available & $-9,999$ & 40.112 & -107.923 & $7 / 1 / 1975$ & 0 \\
\hline 1256 & Meeker & Quaternary & ALLUVIUM,TERRACE & 60 & 40.002 & -107.883 & $12 / 27 / 1978$ & 0 \\
\hline 1260 & Meeker & Unknown & Not available & 175 & 40.010 & -107.968 & $7 / 1 / 1975$ & 0 \\
\hline 1265 & Meeker & Unknown & Not available & 22.5 & 40.012 & -107.886 & $1 / 19 / 1979$ & 0 \\
\hline 1267 & Meeker & Unknown & Not available & 20 & 40.015 & -107.978 & $7 / 1 / 1975$ & 0 \\
\hline
\end{tabular}


Appendix 1. Water Quality Data Used in This Report.-Continued

\begin{tabular}{|c|c|c|c|c|c|c|c|c|}
\hline $\begin{array}{c}\text { Report } \\
\text { No. }\end{array}$ & $\begin{array}{l}\text { Map } \\
\text { name }\end{array}$ & $\begin{array}{c}\text { Geologic } \\
\text { unit }\end{array}$ & $\begin{array}{c}\text { Aquifer } \\
\text { description }\end{array}$ & $\begin{array}{c}\text { Well } \\
\text { depth } \\
\text { (ft) }\end{array}$ & $\begin{array}{c}\text { Latitude } \\
\text { (decimal } \\
\text { degrees, } \\
\text { NAD83) }\end{array}$ & $\begin{array}{c}\text { Longitude } \\
\text { (decimal } \\
\text { degrees, } \\
\text { NAD83) }\end{array}$ & $\begin{array}{l}\text { Collection } \\
\text { date }\end{array}$ & $\begin{array}{c}\text { Collection } \\
\text { time }\end{array}$ \\
\hline 1272 & Meeker & Unknown & Not available & 16 & 40.019 & -107.973 & $7 / 11 / 1979$ & 0 \\
\hline 1273 & Meeker & Quaternary & ALLUVIUM,FLOOD PLAIN & 120 & 40.019 & -107.940 & $7 / 1 / 1975$ & 0 \\
\hline 1277 & Meeker & Quaternary & ALLUVIUM,FLOOD PLAIN & 25 & 40.021 & -107.956 & $7 / 29 / 1975$ & 0 \\
\hline 1282 & Meeker & Unknown & Not available & 80 & 40.022 & -107.883 & $7 / 26 / 1979$ & 0 \\
\hline 1297 & Meeker & Unknown & Not available & 35 & 40.029 & -107.923 & 8/1/1979 & 0 \\
\hline 1302 & Meeker & Unknown & Not available & 240 & 40.035 & -107.968 & $1 / 14 / 1981$ & 0 \\
\hline 1305 & Meeker & Cretaceous & MESAVERDE GROUP & 180 & 40.039 & -107.923 & $7 / 29 / 1975$ & 0 \\
\hline 1306 & Meeker & Tertiary & WASATCH FORMATION & 280 & 40.052 & -107.977 & $7 / 1 / 1975$ & 0 \\
\hline 1307 & Meeker & Tertiary & WASATCH FORMATION & 530 & 40.053 & -107.979 & $7 / 16 / 1979$ & 0 \\
\hline 1317 & Meeker & Unknown & Not available & 40 & 40.02 & -107.94 & 7/7/1998 & 9999 \\
\hline 1318 & Meeker & Unknown & Not available & $-9,999$ & 40.01 & -107.91 & 7/7/1998 & 9999 \\
\hline 1321 & No Name Ridge & Unknown & Not available & $-9,999$ & 39.800 & -108.087 & $8 / 3 / 1983$ & 915 \\
\hline 1327 & Rangely & Unknown & Not available & $-9,999$ & 40.099 & -108.770 & $6 / 18 / 1981$ & 0 \\
\hline 1328 & Rangely & Cretaceous & MESAVERDE GROUP & 665 & 40.046 & -108.775 & $10 / 17 / 1972$ & 0 \\
\hline 1329 & Rangely & Cretaceous & MESAVERDE GROUP & 665 & 40.046 & -108.775 & 10/1/1972 & 0 \\
\hline 1330 & Rangely & Cretaceous & MESAVERDE GROUP & 908 & 40.058 & -108.773 & $3 / 29 / 1951$ & 0 \\
\hline 1333 & Rangely & Unknown & Not available & 18 & 40.086 & -108.798 & $6 / 19 / 1981$ & 0 \\
\hline 1369 & Rangely & Unknown & Not available & 46 & 40.096 & -108.773 & $7 / 17 / 1981$ & 0 \\
\hline 1371 & Rangely & Quaternary & ALLUVIUM & 12 & 40.096 & -108.810 & $7 / 14 / 1958$ & 0 \\
\hline 1381 & Rangely & Unknown & Not available & 16 & 40.099 & -108.775 & 6/9/1981 & 0 \\
\hline 1382 & Rangely & Unknown & Not available & 15 & 40.100 & -108.750 & $6 / 19 / 1980$ & 0 \\
\hline 1383 & Rangely & Unknown & Not available & 11 & 40.100 & -108.777 & 6/9/1981 & 0 \\
\hline 1387 & Rattlesnake Mesa & Unknown & Not available & $-9,999$ & 40.019 & -107.820 & $8 / 12 / 1981$ & 0 \\
\hline 1388 & Rattlesnake Mesa & Unknown & Not available & $-9,999$ & 40.031 & -107.811 & $7 / 2 / 1982$ & 0 \\
\hline 1389 & Rattlesnake Mesa & Unknown & Not available & $-9,999$ & 40.034 & -107.857 & $7 / 11 / 1972$ & 1430 \\
\hline 1390 & Rattlesnake Mesa & Unknown & Not available & $-9,999$ & 40.035 & -107.840 & $7 / 11 / 1972$ & 1500 \\
\hline 1391 & Rattlesnake Mesa & Unknown & Not available & $-9,999$ & 40.036 & -107.841 & $4 / 28 / 1973$ & 1445 \\
\hline 1392 & Rattlesnake Mesa & Unknown & Not available & $-9,999$ & 40.040 & -107.850 & $7 / 11 / 1972$ & 1200 \\
\hline 1393 & Rattlesnake Mesa & Unknown & Not available & $-9,999$ & 40.055 & -107.856 & $7 / 11 / 1972$ & 1730 \\
\hline 1395 & Rattlesnake Mesa & Quaternary & ALLUVIUM,FLOOD PLAIN & $-9,999$ & 40.003 & -107.819 & $7 / 1 / 1975$ & 0 \\
\hline 1403 & Rattlesnake Mesa & Unknown & Not available & 72 & 40.012 & -107.812 & $8 / 13 / 1981$ & 0 \\
\hline 1409 & Rattlesnake Mesa & Unknown & Not available & 57.5 & 40.031 & -107.810 & $7 / 2 / 1982$ & 0 \\
\hline 1410 & Rattlesnake Mesa & Unknown & Not available & 100 & 40.033 & -107.816 & $6 / 18 / 1975$ & 0 \\
\hline 1411 & Rattlesnake Mesa & Quaternary & ALLUVIUM,FLOOD PLAIN & 5.3 & 40.034 & -107.863 & $7 / 20 / 1979$ & 0 \\
\hline 1415 & Rattlesnake Mesa & Unknown & Not available & 20 & 40.036 & -107.872 & $8 / 17 / 1981$ & 0 \\
\hline 1416 & Rattlesnake Mesa & Unknown & Not available & 52 & 40.067 & -107.817 & $1 / 23 / 1981$ & 0 \\
\hline 1418 & Rattlesnake Mesa & Cretaceous & MESAVERDE GROUP & 1,200 & 40.123 & -107.848 & $6 / 4 / 1975$ & 0 \\
\hline 1419 & Rattlesnake Mesa & Unknown & Not available & $-9,999$ & 40.01 & -107.82 & 7/7/1998 & 9999 \\
\hline 1420 & Rattlesnake Mesa & Unknown & Not available & $-9,999$ & 40.04 & -107.81 & 7/7/1998 & 9999 \\
\hline 1421 & Razorback Ridge & Unknown & Not available & $-9,999$ & 39.695 & -108.521 & 10/6/1976 & 1030 \\
\hline 1422 & Rock School & Unknown & Not available & $-9,999$ & 39.833 & -108.333 & 12/19/1971 & 1415 \\
\hline 1423 & Rock School & Unknown & Not available & 655 & 39.761 & -108.321 & 10/5/1982 & 1230 \\
\hline 1426 & Rock School & Tertiary & GREEN RIVER FORMATION & $-9,999$ & 39.762 & -108.264 & $10 / 18 / 1974$ & 400 \\
\hline 1433 & Rock School & Tertiary & GREEN RIVER FORMATION & $-9,999$ & 39.824 & -108.278 & 8/14/1973 & 1500 \\
\hline 1438 & Rock School & Quaternary & ALLUVIUM,FLOOD PLAIN & $-9,999$ & 39.841 & -108.325 & $8 / 2 / 1973$ & 1200 \\
\hline 1443 & Rock School & Quaternary & ALLUVIUM,FLOOD PLAIN & $-9,999$ & 39.851 & -108.316 & $8 / 2 / 1973$ & 1300 \\
\hline 1445 & Rock School & Quaternary & ALLUVIUM,FLOOD PLAIN & 80 & 39.855 & -108.368 & $8 / 2 / 1973$ & 1630 \\
\hline 1448 & Rock School & Quaternary & ALLUVIUM,FLOOD PLAIN & $-9,999$ & 39.858 & -108.312 & $8 / 2 / 1973$ & 1530 \\
\hline 1450 & Rock School & Quaternary & ALLUVIUM,FLOOD PLAIN & $-9,999$ & 39.859 & -108.311 & $8 / 2 / 1973$ & 1500 \\
\hline 1452 & Rock School & Quaternary & ALLUVIUM,FLOOD PLAIN & $-9,999$ & 39.860 & -108.309 & $8 / 2 / 1973$ & 1600 \\
\hline 1454 & Rock School & Unknown & Not available & 640 & 39.860 & -108.351 & 10/7/1982 & 1630 \\
\hline 1461 & Rough Gulch & Unknown & Not available & 10 & 40.178 & -108.396 & $7 / 20 / 1981$ & 0 \\
\hline 1463 & Rough Gulch & Unknown & Not available & 27 & 40.197 & -108.478 & $7 / 20 / 1981$ & 0 \\
\hline 1464 & Rough Gulch & Unknown & Not available & 60 & 40.197 & -108.478 & $7 / 20 / 1981$ & 0 \\
\hline 1465 & Rough Gulch & Unknown & Not available & $-9,999$ & 40.177 & -108.392 & 9/17/1977 & 1000 \\
\hline 1466 & Sagebrush Hill & Unknown & Not available & $-9,999$ & 39.891 & -108.513 & $2 / 20 / 1975$ & 1515 \\
\hline 1467 & Sagebrush Hill & Unknown & Not available & $-9,999$ & 39.891 & -108.513 & $4 / 3 / 1975$ & 1300 \\
\hline 1468 & Sagebrush Hill & Unknown & Not available & $-9,999$ & 39.907 & -108.501 & $3 / 7 / 1975$ & 1445 \\
\hline 1469 & Sagebrush Hill & Unknown & Not available & $-9,999$ & 39.914 & -108.502 & $8 / 26 / 1980$ & 1635 \\
\hline 1475 & Sagebrush Hill & Unknown & Not available & $-9,999$ & 39.914 & -108.505 & 9/13/1989 & 1000 \\
\hline 1476 & Sagebrush Hill & Unknown & Not available & $-9,999$ & 39.914 & -108.505 & 9/13/1989 & 830 \\
\hline 1477 & Sagebrush Hill & Unknown & Not available & $-9,999$ & 39.925 & -108.525 & $1 / 29 / 1975$ & 1100 \\
\hline 1478 & Sagebrush Hill & Tertiary & GREEN RIVER FORMATION & $-9,999$ & 39.884 & -108.501 & $9 / 26 / 1973$ & 1300 \\
\hline 1480 & Sagebrush Hill & Tertiary & GREEN RIVER FORMATION & $-9,999$ & 39.894 & -108.523 & 10/9/1973 & 1700 \\
\hline 1482 & Sagebrush Hill & Tertiary & GREEN RIVER FORMATION & $-9,999$ & 39.906 & -108.533 & $6 / 8 / 1973$ & 1200 \\
\hline 1484 & Sagebrush Hill & Tertiary & GREEN RIVER FORMATION & 1,736 & 39.907 & -108.501 & $4 / 10 / 1975$ & 1545 \\
\hline
\end{tabular}


Appendix 1. Water Quality Data Used in This Report.-Continued

\begin{tabular}{|c|c|c|c|c|c|c|c|c|}
\hline $\begin{array}{c}\text { Report } \\
\text { No. }\end{array}$ & $\begin{array}{l}\text { Map } \\
\text { name }\end{array}$ & $\begin{array}{c}\text { Geologic } \\
\text { unit }\end{array}$ & $\begin{array}{c}\text { Aquifer } \\
\text { description }\end{array}$ & $\begin{array}{c}\text { Well } \\
\text { depth } \\
\text { (ft) }\end{array}$ & $\begin{array}{c}\text { Latitude } \\
\text { (decimal } \\
\text { degrees, } \\
\text { NAD83) }\end{array}$ & $\begin{array}{c}\text { Longitude } \\
\text { (decimal } \\
\text { degrees, } \\
\text { NAD83) }\end{array}$ & $\begin{array}{l}\text { Collection } \\
\text { date }\end{array}$ & $\begin{array}{l}\text { Collection } \\
\text { time }\end{array}$ \\
\hline 1486 & Sagebrush Hill & Tertiary & GREEN RIVER FORMATION & $-9,999$ & 39.914 & -108.505 & $7 / 19 / 1978$ & 1030 \\
\hline 1487 & Sagebrush Hill & Tertiary & GREEN RIVER FORMATION & $-9,999$ & 39.915 & -108.505 & $7 / 19 / 1978$ & 1230 \\
\hline 1488 & Sagebrush Hill & Tertiary & GREEN RIVER FORMATION & $-9,999$ & 39.915 & -108.505 & $3 / 22 / 1978$ & 1500 \\
\hline 1492 & Sagebrush Hill & Tertiary & GREEN RIVER FORMATION & $-9,999$ & 39.964 & -108.511 & $6 / 26 / 1973$ & 1000 \\
\hline 1497 & Sawmill Mountain & Unknown & Not available & 35 & 40.031 & -107.726 & $8 / 28 / 1981$ & 0 \\
\hline 1498 & Sawmill Mountain & Unknown & Not available & 80 & 40.074 & -107.714 & $7 / 31 / 1975$ & 0 \\
\hline 1499 & Segar Mountain & Unknown & Not available & 1,182 & 39.886 & -108.085 & $9 / 18 / 1975$ & 2000 \\
\hline 1502 & Segar Mountain & Unknown & Not available & 790 & 39.930 & -108.103 & $1 / 26 / 1976$ & 1700 \\
\hline 1506 & Smizer Gulch & Unknown & Not available & $-9,999$ & 40.147 & -108.335 & 9/16/1977 & 1700 \\
\hline 1507 & Square S Ranch & Unknown & Not available & $-9,999$ & 39.984 & -108.280 & $8 / 14 / 1972$ & 1130 \\
\hline 1508 & Square S Ranch & Unknown & Not available & $-9,999$ & 39.906 & -108.363 & $8 / 28 / 1972$ & 1400 \\
\hline 1509 & Square S Ranch & Unknown & Not available & $-9,999$ & 39.913 & -108.323 & $10 / 28 / 1976$ & 600 \\
\hline 1510 & Square S Ranch & Unknown & Not available & $-9,999$ & 39.933 & -108.313 & 11/7/1976 & 410 \\
\hline 1512 & Square S Ranch & Unknown & Not available & $-9,999$ & 39.944 & -108.356 & 11/13/1981 & 1030 \\
\hline 1513 & Square S Ranch & Unknown & Not available & $-9,999$ & 39.944 & -108.356 & 7/17/1981 & 1500 \\
\hline 1514 & Square S Ranch & Tertiary & EOCENE SERIES & 1,442 & 39.944 & -108.297 & $7 / 13 / 2000$ & 1531 \\
\hline 1515 & Square S Ranch & Tertiary & EOCENE SERIES & 1,305 & 39.944 & -108.309 & $7 / 12 / 2000$ & 1551 \\
\hline 1516 & Square S Ranch & Tertiary & EOCENE SERIES & 490 & 39.947 & -108.292 & $7 / 11 / 2000$ & 901 \\
\hline 1517 & Square S Ranch & Tertiary & EOCENE SERIES & 919 & 39.947 & -108.292 & $7 / 10 / 2000$ & 1201 \\
\hline 1518 & Square S Ranch & Tertiary & GREEN RIVER FORMATION & 810 & 39.882 & -108.262 & 9/9/1975 & 1000 \\
\hline 1519 & Square S Ranch & Unknown & Not available & 5,182 & 39.882 & -108.262 & $6 / 15 / 1979$ & 710 \\
\hline 1523 & Square S Ranch & Quaternary & ALLUVIUM,FLOOD PLAIN & $-9,999$ & 39.891 & -108.294 & $8 / 3 / 1973$ & 1130 \\
\hline 1526 & Square S Ranch & Quaternary & ALLUVIUM,FLOOD PLAIN & $-9,999$ & 39.907 & -108.296 & $8 / 3 / 1973$ & 1200 \\
\hline 1530 & Square S Ranch & Tertiary & GREEN RIVER FORMATION & $-9,999$ & 39.912 & -108.295 & $6 / 21 / 1973$ & 1700 \\
\hline 1532 & Square S Ranch & Quaternary & ALLUVIUM,FLOOD PLAIN & $-9,999$ & 39.912 & -108.296 & $8 / 3 / 1973$ & 1300 \\
\hline 1535 & Square S Ranch & Quaternary & ALLUVIUM,TERRACE & 34.5 & 39.938 & -108.294 & $7 / 12 / 2000$ & 1811 \\
\hline 1536 & Square S Ranch & Quaternary & ALLUVIUM,FLOOD PLAIN & 42 & 39.942 & -108.286 & $9 / 6 / 1973$ & 1230 \\
\hline 1538 & Square S Ranch & Tertiary & EOCENE SERIES & 1,028 & 39.942 & -108.299 & $7 / 13 / 2000$ & 1131 \\
\hline 1539 & Square S Ranch & Tertiary & EOCENE SERIES & 1,283 & 39.947 & -108.292 & $7 / 11 / 2000$ & 1001 \\
\hline 1539 & Square S Ranch & Tertiary & EOCENE SERIES & 1,283 & 39.947 & -108.292 & $7 / 11 / 2000$ & 1000 \\
\hline 1540 & Square S Ranch & Tertiary & EOCENE SERIES & 1,000 & 39.947 & -108.315 & $7 / 12 / 2000$ & 1211 \\
\hline 1541 & Square S Ranch & Tertiary & EOCENE SERIES & 1,280 & 39.953 & -108.318 & $7 / 12 / 2000$ & 1101 \\
\hline 1542 & Square S Ranch & Quaternary & ALLUVIUM,TERRACE & 111 & 39.954 & -108.285 & $7 / 11 / 2000$ & 1711 \\
\hline 1546 & Square S Ranch & Unknown & Not available & 1,755 & 39.965 & -108.355 & $10 / 6 / 1983$ & 1100 \\
\hline 1547 & Square S Ranch & Tertiary & GREEN RIVER FORMATION & 1,755 & 39.965 & -108.355 & $10 / 4 / 1983$ & 1800 \\
\hline 1549 & Square S Ranch & Tertiary & GREEN RIVER FORMATION & $-9,999$ & 39.978 & -108.253 & $7 / 11 / 1973$ & 1030 \\
\hline 1552 & Square S Ranch & Quaternary & ALLUVIUM,FLOOD PLAIN & $-9,999$ & 39.993 & -108.355 & $9 / 12 / 1973$ & 930 \\
\hline 1555 & Square S Ranch & Cretaceous & MESAVERDE GROUP & 12,700 & 39.878 & -108.294 & $1 / 30 / 2002$ & 9999 \\
\hline 1558 & Veatch Gulch & Quaternary & ALLUVIUM,TERRACE & 50 & 39.942 & -107.774 & $8 / 2 / 1979$ & 0 \\
\hline 1559 & Veatch Gulch & Pennsylvanian & UPPER PENNSYLVANIAN SERIES & 86 & 39.947 & -107.772 & 7/8/1982 & 0 \\
\hline 1560 & Veatch Gulch & Pennsylvanian & PERMIAN & 85 & 39.949 & -107.781 & $7 / 8 / 1982$ & 0 \\
\hline 1561 & Veatch Gulch & Quaternary & $\begin{array}{l}\text { PENNSYLVANIAN SYSTEMS } \\
\text { ALLUVIUM,TERRACE }\end{array}$ & 112 & 39.961 & -107.796 & $8 / 2 / 1979$ & 0 \\
\hline 1565 & Veatch Gulch & Quaternary & ALLUVIUM,FLOOD PLAIN & 50 & 39.976 & -107.811 & $7 / 8 / 1982$ & 0 \\
\hline 1566 & Veatch Gulch & Unknown & Not available & 45 & 39.983 & -107.821 & $8 / 14 / 1981$ & 0 \\
\hline 1567 & Veatch Gulch & Unknown & Not available & 30 & 39.989 & -107.826 & $8 / 15 / 1981$ & 0 \\
\hline 1568 & Veatch Gulch & Unknown & Not available & 35 & 39.998 & -107.832 & $8 / 12 / 1981$ & 0 \\
\hline 1571 & White River City & Tertiary & GREEN RIVER FORMATION & $-9,999$ & 40.005 & -108.191 & $6 / 27 / 1973$ & 1300 \\
\hline 1576 & White River City & Quaternary & ALLUVIUM,FLOOD PLAIN & $-9,999$ & 40.008 & -108.248 & 9/6/1973 & 1430 \\
\hline 1580 & White River City & Tertiary & GREEN RIVER FORMATION & $-9,999$ & 40.009 & -108.210 & $6 / 27 / 1973$ & 1430 \\
\hline 1581 & White River City & Unknown & Not available & 2,400 & 40.012 & -108.221 & $7 / 17 / 1980$ & 735 \\
\hline 1587 & White River City & Quaternary & ALLUVIUM,FLOOD PLAIN & $-9,999$ & 40.077 & -108.237 & 9/6/1973 & 1730 \\
\hline 1592 & White River City & Quaternary & ALLUVIUM,TERRACE & 60 & 40.090 & -108.225 & $7 / 10 / 1982$ & 0 \\
\hline 1593 & White River City & Unknown & Not available & $-9,999$ & 40.006 & -108.191 & 9/16/1977 & 1200 \\
\hline 1594 & White Rock & Unknown & Not available & $-9,999$ & 40.202 & -108.020 & $7 / 1 / 1975$ & 0 \\
\hline 1595 & White Rock & Unknown & Not available & 40 & 40.177 & -108.008 & $7 / 1 / 1975$ & 0 \\
\hline 1596 & White Rock & Quaternary & ALLUVIUM,FLOOD PLAIN & 14 & 40.185 & -108.009 & $7 / 1 / 1975$ & 0 \\
\hline 1597 & White Rock & Quaternary & ALLUVIUM,FLOOD PLAIN & 30 & 40.211 & -108.027 & $7 / 1 / 1975$ & 0 \\
\hline 1598 & White Rock & Unknown & Not available & 60 & 40.218 & -108.025 & $7 / 28 / 1975$ & 0 \\
\hline 1599 & White Rock & Unknown & Not available & $-9,999$ & 40.164 & -108.080 & $9 / 20 / 1977$ & 1300 \\
\hline 1600 & Wolf Ridge & Unknown & Not available & $-9,999$ & 39.981 & -108.443 & $9 / 16 / 1977$ & 1600 \\
\hline 1601 & Wolf Ridge & Unknown & Not available & 600 & 39.891 & -108.386 & 9/15/1977 & 1100 \\
\hline 1602 & Wolf Ridge & Unknown & Not available & $-9,999$ & 39.896 & -108.451 & $11 / 28 / 1971$ & 1000 \\
\hline 1603 & Wolf Ridge & Unknown & Not available & $-9,999$ & 39.921 & -108.496 & $5 / 7 / 1975$ & 1530 \\
\hline 1604 & Wolf Ridge & Unknown & Not available & $-9,999$ & 39.923 & -108.485 & $5 / 27 / 1975$ & 1400 \\
\hline 1605 & Wolf Ridge & Unknown & Not available & $-9,999$ & 39.923 & -108.485 & $1 / 8 / 1975$ & 1305 \\
\hline
\end{tabular}


Appendix 1. Water Quality Data Used in This Report.-Continued

\begin{tabular}{|c|c|c|c|c|c|c|c|c|}
\hline $\begin{array}{l}\text { Report } \\
\text { No. }\end{array}$ & $\begin{array}{l}\text { Map } \\
\text { name }\end{array}$ & $\begin{array}{l}\text { Geologic } \\
\text { unit }\end{array}$ & $\begin{array}{c}\text { Aquifer } \\
\text { description }\end{array}$ & $\begin{array}{c}\text { Well } \\
\text { depth } \\
\text { (ft) }\end{array}$ & $\begin{array}{l}\text { Latitude } \\
\text { (decimal } \\
\text { degrees, } \\
\text { NAD83) }\end{array}$ & $\begin{array}{l}\text { Longitude } \\
\text { (decimal } \\
\text { degrees, } \\
\text { NAD83) }\end{array}$ & $\begin{array}{l}\text { Collection } \\
\text { date }\end{array}$ & $\begin{array}{l}\text { Collection } \\
\text { time }\end{array}$ \\
\hline 1607 & Wolf Ridge & Quaternary & ALLUVIUM,FLOOD PLAIN & $-9,999$ & 39.877 & -108.480 & $8 / 31 / 1973$ & 1130 \\
\hline 1609 & Wolf Ridge & Quaternary & ALLUVIUM,FLOOD PLAIN & $-9,999$ & 39.891 & -108.390 & $8 / 31 / 1973$ & 930 \\
\hline 1610 & Wolf Ridge & Tertiary & GREEN RIVER FORMATION & $-9,999$ & 39.893 & -108.488 & 9/9/1973 & 2050 \\
\hline 1612 & Wolf Ridge & Tertiary & GREEN RIVER FORMATION & $-9,999$ & 39.897 & -108.450 & $8 / 2 / 1973$ & 1700 \\
\hline 1613 & Wolf Ridge & Unknown & Not available & 3,100 & 39.911 & -108.376 & $8 / 26 / 1983$ & 1245 \\
\hline 1614 & Wolf Ridge & Tertiary & GREEN RIVER FORMATION & 1,185 & 39.911 & -108.376 & $8 / 30 / 1983$ & 1545 \\
\hline 1617 & Wolf Ridge & Tertiary & GREEN RIVER FORMATION & $-9,999$ & 39.923 & -108.485 & 8/9/1973 & 1500 \\
\hline 1622 & Yankee Gulch & Unknown & Not available & $-9,999$ & 39.766 & -108.382 & $11 / 21 / 1974$ & 2200 \\
\hline 1623 & Yankee Gulch & Unknown & Not available & $-9,999$ & 39.846 & -108.379 & $11 / 5 / 1976$ & 1030 \\
\hline 1626 & Yankee Gulch & Quaternary & ALLUVIUM,FLOOD PLAIN & $-9,999$ & 39.843 & -108.388 & $8 / 3 / 1973$ & 1030 \\
\hline 1628 & Yankee Gulch & Quaternary & ALLUVIUM,FLOOD PLAIN & $-9,999$ & 39.848 & -108.378 & $8 / 3 / 1973$ & 1100 \\
\hline 1631 & & Unknown & Not available & $-9,999$ & 39.07 & -108.5 & $10 / 18 / 2000$ & 9999 \\
\hline 1632 & Badger Wash & Unknown & Not available & $-9,999$ & 39.275 & -108.901 & 9/20/1977 & 1515 \\
\hline 1633 & Badger Wash & Cretaceous & MANCOS SHALE & 35 & 39.266 & -108.901 & $2 / 21 / 1992$ & 900 \\
\hline 1634 & Badger Wash & Cretaceous & MANCOS SHALE & 19 & 39.275 & -108.883 & 2/21/1992 & 1000 \\
\hline 1635 & Badger Wash & Cretaceous & MANCOS SHALE & 40 & 39.290 & -108.952 & $3 / 27 / 2001$ & 1040 \\
\hline 1636 & Badger Wash & Cretaceous & MANCOS SHALE & 37 & 39.290 & -108.956 & $3 / 27 / 2001$ & 1215 \\
\hline 1639 & Clifton & Unknown & Not available & $-9,999$ & 39.053 & -108.484 & $5 / 16 / 2000$ & 900 \\
\hline 1640 & Clifton & Unknown & Not available & $-9,999$ & 39.061 & -108.483 & $7 / 29 / 1998$ & 1000 \\
\hline 1641 & Clifton & Quaternary & QUATERNARY SYSTEM & $-9,999$ & 39.049 & -108.499 & $3 / 30 / 2000$ & 1325 \\
\hline 1642 & Clifton & Quaternary & QUATERNARY SYSTEM & $-9,999$ & 39.051 & -108.499 & $5 / 16 / 2000$ & 1050 \\
\hline 1643 & Clifton & Quaternary & QUATERNARY SYSTEM & $-9,999$ & 39.051 & -108.495 & $5 / 16 / 2000$ & 1000 \\
\hline 1644 & Clifton & Quaternary & QUATERNARY SYSTEM & $-9,999$ & 39.052 & -108.492 & $5 / 16 / 2000$ & 940 \\
\hline 1645 & Clifton & Quaternary & QUATERNARY SYSTEM & $-9,999$ & 39.052 & -108.499 & $3 / 30 / 2000$ & 1300 \\
\hline 1646 & Clifton & Quaternary & QUATERNARY SYSTEM & $-9,999$ & 39.052 & -108.488 & $3 / 30 / 2000$ & 1035 \\
\hline 1647 & Clifton & Quaternary & QUATERNARY SYSTEM & $-9,999$ & 39.052 & -108.492 & $3 / 30 / 2000$ & 1220 \\
\hline 1648 & Clifton & Quaternary & QUATERNARY SYSTEM & $-9,999$ & 39.053 & -108.488 & $5 / 16 / 2000$ & 925 \\
\hline 1649 & Clifton & Quaternary & QUATERNARY SYSTEM & $-9,999$ & 39.055 & -108.485 & $3 / 30 / 2000$ & 1025 \\
\hline 1650 & Clifton & Quaternary & QUATERNARY SYSTEM & $-9,999$ & 39.060 & -108.489 & $5 / 5 / 1998$ & 1115 \\
\hline 1651 & Clifton & Quaternary & QUATERNARY SYSTEM & $-9,999$ & 39.061 & -108.481 & $7 / 29 / 1998$ & 910 \\
\hline 1652 & Clifton & Quaternary & QUATERNARY SYSTEM & $-9,999$ & 39.061 & -108.483 & $7 / 29 / 1998$ & 950 \\
\hline 1653 & Clifton & Quaternary & QUATERNARY SYSTEM & $-9,999$ & 39.061 & -108.486 & $7 / 29 / 1998$ & 1025 \\
\hline 1654 & Clifton & Quaternary & QUATERNARY SYSTEM & 46.8 & 39.072 & -108.497 & 3/10/1992 & 900 \\
\hline 1655 & Clifton & Unknown & Not available & $-9,999$ & 39.07 & -108.43 & $10 / 17 / 2000$ & 9999 \\
\hline 1656 & Clifton & Unknown & Not available & $-9,999$ & 39.08 & -108.42 & $10 / 17 / 2000$ & 9999 \\
\hline 1657 & Clifton & Unknown & Not available & $-9,999$ & 39.08 & -108.43 & $10 / 17 / 2000$ & 9999 \\
\hline 1658 & Clifton & Unknown & Not available & $-9,999$ & 39.06 & -108.45 & $10 / 18 / 2000$ & 9999 \\
\hline 1659 & Clifton & Unknown & Not available & $-9,999$ & 39.06 & -108.49 & $10 / 18 / 2000$ & 9999 \\
\hline 1660 & Collbran & Unknown & Not available & $-9,999$ & 39.195 & -107.942 & 9/7/1977 & 1455 \\
\hline 1661 & Collbran & Unknown & Not available & $-9,999$ & 39.196 & -107.970 & 9/7/1977 & 1520 \\
\hline 1662 & Collbran & Unknown & Not available & $-9,999$ & 39.231 & -107.961 & 8/1/1977 & 1445 \\
\hline 1664 & Collbran & Unknown & Not available & 60 & 39.200 & -107.972 & 6/10/1997 & 9999 \\
\hline 1665 & Collbran & Unknown & Not available & 45 & 39.196 & -107.943 & $6 / 10 / 1997$ & 9999 \\
\hline 1666 & Collbran & Unknown & Not available & 80 & 39.226 & -107.987 & $2 / 25 / 2004$ & 9999 \\
\hline 1667 & Collbran & Tertiary & WASATCH FORMATION & 110 & 39.207 & -107.990 & $2 / 25 / 2004$ & 9999 \\
\hline 1668 & Collbran & Tertiary & WASATCH FORMATION & 400 & 39.243 & -107.941 & $2 / 26 / 2004$ & 9999 \\
\hline 1669 & Collbran & Unknown & Not available & 200 & 39.24 & -107.94 & 8/19/1998 & 9999 \\
\hline 1670 & Colorado National Monument & Unknown & Not available & $-9,999$ & 39.104 & -108.730 & $8 / 13 / 1977$ & 1400 \\
\hline 1671 & Colorado National Monument & Unknown & Not available & $-9,999$ & 39.036 & -108.628 & 5/30/1972 & 1120 \\
\hline 1672 & Colorado National Monument & Unknown & Not available & $-9,999$ & 39.104 & -108.670 & 9/20/1977 & 1800 \\
\hline 1673 & Colorado National Monument & Unknown & Not available & $-9,999$ & 39.104 & -108.650 & 3/3/1997 & 1300 \\
\hline 1674 & Colorado National Monument & Unknown & Not available & $-9,999$ & 39.106 & -108.654 & 2/11/1998 & 900 \\
\hline 1675 & Colorado National Monument & Unknown & Not available & $-9,999$ & 39.107 & -108.655 & $6 / 6 / 2002$ & 940 \\
\hline 1676 & Colorado National Monument & Unknown & Not available & 350 & 39.035 & -108.628 & $8 / 13 / 1977$ & 1200 \\
\hline 1677 & Colorado National Monument & Jurrassic & $\begin{array}{c}\text { BRUSHY BASIN SHALE MEMBER } \\
\text { OF MORRISON FORMATION }\end{array}$ & 210 & 39.079 & -108.661 & $5 / 8 / 1991$ & 900 \\
\hline 1678 & Colorado National Monument & Quaternary & QUATERNARY SYSTEM & $-9,999$ & 39.103 & -108.650 & 2/11/1998 & 1105 \\
\hline 1679 & Colorado National Monument & Quaternary & QUATERNARY SYSTEM & 22.5 & 39.104 & -108.645 & $4 / 1 / 2003$ & 1610 \\
\hline 1680 & Colorado National Monument & Quaternary & QUATERNARY SYSTEM & 18 & 39.104 & -108.646 & $4 / 1 / 2003$ & 1520 \\
\hline 1681 & Colorado National Monument & Quaternary & QUATERNARY SYSTEM & $-9,999$ & 39.104 & -108.650 & $2 / 18 / 1998$ & 800 \\
\hline 1682 & Colorado National Monument & Quaternary & QUATERNARY SYSTEM & $-9,999$ & 39.103 & -108.646 & $7 / 24 / 1998$ & 950 \\
\hline 1683 & Colorado National Monument & Quaternary & QUATERNARY SYSTEM & $-9,999$ & 39.102 & -108.648 & $7 / 17 / 1997$ & 1215 \\
\hline 1684 & Colorado National Monument & Quaternary & QUATERNARY SYSTEM & $-9,999$ & 39.103 & -108.650 & $4 / 1 / 1998$ & 1120 \\
\hline 1685 & Colorado National Monument & Quaternary & QUATERNARY SYSTEM & $-9,999$ & 39.104 & -108.650 & $4 / 1 / 1998$ & 1145 \\
\hline 1686 & Colorado National Monument & Quaternary & QUATERNARY SYSTEM & 17 & 39.106 & -108.647 & $4 / 1 / 2003$ & 1425 \\
\hline 1687 & Colorado National Monument & Quaternary & QUATERNARY SYSTEM & $-9,999$ & 39.103 & -108.649 & $4 / 2 / 1998$ & 950 \\
\hline
\end{tabular}


Appendix 1. Water Quality Data Used in This Report.-Continued

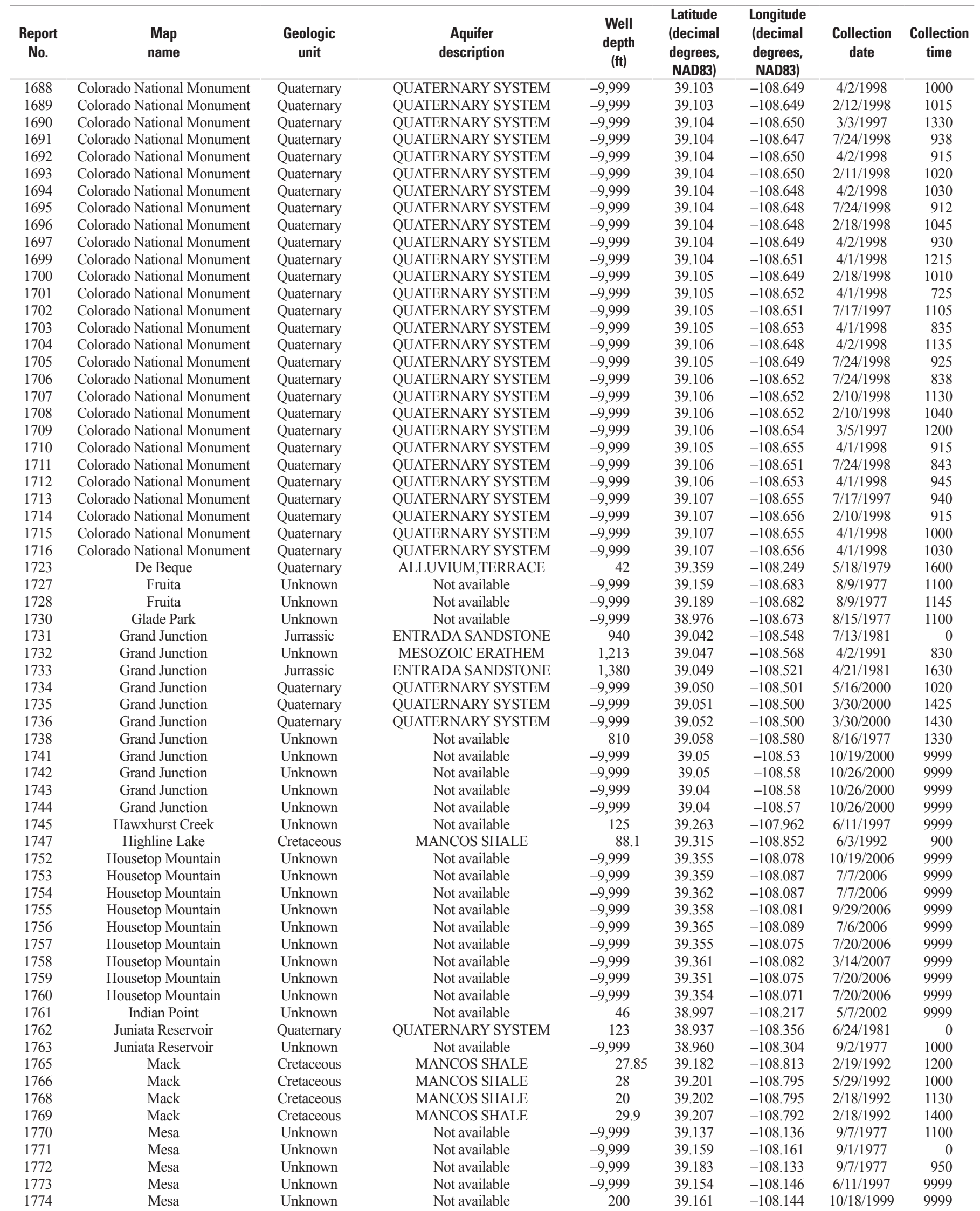


Appendix 1. Water Quality Data Used in This Report.-Continued

\begin{tabular}{|c|c|c|c|c|c|c|c|c|}
\hline $\begin{array}{c}\text { Report } \\
\text { No. }\end{array}$ & $\begin{array}{l}\text { Map } \\
\text { name }\end{array}$ & $\begin{array}{c}\text { Geologic } \\
\text { unit }\end{array}$ & $\begin{array}{c}\text { Aquifer } \\
\text { description }\end{array}$ & $\begin{array}{c}\text { Well } \\
\text { depth } \\
\text { (ft) }\end{array}$ & $\begin{array}{c}\text { Latitude } \\
\text { (decimal } \\
\text { degrees, } \\
\text { NAD83) }\end{array}$ & $\begin{array}{c}\text { Longitude } \\
\text { (decimal } \\
\text { degrees, } \\
\text { NAD83) }\end{array}$ & $\begin{array}{l}\text { Collection } \\
\text { date }\end{array}$ & $\begin{array}{c}\text { Collection } \\
\text { time }\end{array}$ \\
\hline 1776 & Mesa & Unknown & Not available & 350 & 39.217 & -108.156 & $6 / 11 / 1997$ & 9999 \\
\hline 1777 & Mesa & Unknown & Not available & 300 & 39.159 & -108.174 & $6 / 11 / 1997$ & 9999 \\
\hline 1778 & Mesa & Unknown & Not available & 100 & 39.148 & -108.150 & $7 / 10 / 1997$ & 9999 \\
\hline 1779 & Mesa & Unknown & Not available & 85 & 39.137 & -108.153 & $7 / 10 / 1997$ & 9999 \\
\hline 1780 & Mesa & Unknown & Not available & 30 & 39.153 & -108.133 & $7 / 10 / 1997$ & 9999 \\
\hline 1781 & Mesa & Unknown & Not available & 35 & 39.155 & -108.128 & $7 / 10 / 1997$ & 9999 \\
\hline 1782 & Mesa & Tertiary & WASATCH FORMATION & 500 & 39.165 & -108.181 & $2 / 23 / 2004$ & 9999 \\
\hline 1783 & Mesa & Tertiary & WASATCH FORMATION & 500 & 39.162 & -108.182 & $2 / 24 / 2004$ & 9999 \\
\hline 1784 & Mesa & Tertiary & WASATCH FORMATION & 480 & 39.176 & -108.142 & $2 / 25 / 2004$ & 9999 \\
\hline 1785 & Mesa & Unknown & Not available & 60 & 39.166 & -108.127 & $2 / 23 / 2004$ & 9999 \\
\hline 1786 & Mesa & Tertiary & WASATCH FORMATION & 125 & 39.180 & -108.128 & $2 / 24 / 2004$ & 9999 \\
\hline 1787 & Mesa Lakes & Unknown & Not available & $-9,999$ & 39.050 & -108.091 & 9/7/1977 & 9999 \\
\hline 1788 & Mesa Lakes & Unknown & Not available & $-9,999$ & 39.091 & -108.091 & $4 / 19 / 2006$ & 9999 \\
\hline 1789 & Molina & Unknown & Not available & $-9,999$ & 39.232 & -108.005 & 9/5/1977 & 1900 \\
\hline 1790 & Molina & Unknown & Not available & $-9,999$ & 39.163 & -108.031 & $9 / 6 / 1977$ & 1000 \\
\hline 1791 & Molina & Unknown & Not available & $-9,999$ & 39.131 & -108.033 & 9/6/1977 & 1000 \\
\hline 1792 & Molina & Unknown & Not available & $-9,999$ & 39.196 & -108.017 & $9 / 6 / 1977$ & 1100 \\
\hline 1793 & Molina & Unknown & Not available & $-9,999$ & 39.189 & -108.062 & $9 / 6 / 1977$ & 1200 \\
\hline 1794 & Molina & Unknown & Not available & $-9,999$ & 39.166 & -108.122 & 9/7/1977 & 1030 \\
\hline 1795 & Molina & Unknown & Not available & $-9,999$ & 39.189 & -108.054 & $7 / 28 / 1977$ & 1245 \\
\hline 1796 & Molina & Unknown & Not available & $-9,999$ & 39.155 & -108.033 & $6 / 10 / 1997$ & 9999 \\
\hline 1797 & Molina & Unknown & Not available & 40 & 39.184 & -108.113 & 6/10/1997 & 9999 \\
\hline 1798 & Molina & Unknown & Not available & 20 & 39.183 & -108.042 & $6 / 10 / 1997$ & 9999 \\
\hline 1799 & Molina & Unknown & Not available & 31 & 39.196 & -108.025 & $6 / 10 / 1997$ & 9999 \\
\hline 1800 & Molina & Unknown & Not available & 35 & 39.190 & -108.092 & $6 / 10 / 1997$ & 9999 \\
\hline 1801 & Molina & Unknown & Not available & 30 & 39.166 & -108.123 & $6 / 11 / 1997$ & 9999 \\
\hline 1802 & Molina & Unknown & Not available & 40 & 39.178 & -108.113 & $7 / 10 / 1997$ & 9999 \\
\hline 1803 & Molina & Unknown & Not available & 60 & 39.167 & -108.093 & $7 / 10 / 1997$ & 9999 \\
\hline 1804 & Molina & Unknown & Not available & 89 & 39.157 & -108.069 & $7 / 10 / 1997$ & 9999 \\
\hline 1805 & Molina & Unknown & Not available & 150 & 39.147 & -108.048 & $7 / 10 / 1997$ & 9999 \\
\hline 1806 & Molina & Unknown & Not available & 120 & 39.139 & -108.047 & $7 / 10 / 1997$ & 9999 \\
\hline 1807 & Molina & Cretaceous & FOX HILLS FORMATION & 700 & 39.173 & -108.106 & $2 / 18 / 2003$ & 1215 \\
\hline 1807 & Molina & Cretaceous & FOX HILLS FORMATION & 700 & 39.173 & -108.106 & $2 / 18 / 2003$ & 1200 \\
\hline 1808 & Molina & Unknown & Not available & 46 & 39.205 & -108.045 & $2 / 23 / 2004$ & 9999 \\
\hline 1809 & Molina & Tertiary & WASATCH FORMATION & 127 & 39.180 & -108.066 & $2 / 24 / 2004$ & 9999 \\
\hline 1810 & Molina & Tertiary & WASATCH FORMATION & 108 & 39.188 & -108.065 & $2 / 25 / 2004$ & 9999 \\
\hline 1811 & Molina & Unknown & Not available & $-9,999$ & 39.178 & -108.030 & $1 / 19 / 2007$ & 9999 \\
\hline 1812 & Molina & Unknown & Not available & $-9,999$ & 39.176 & -108.030 & $1 / 19 / 2007$ & 9999 \\
\hline 1813 & Molina & Unknown & Not available & $-9,999$ & 39.200 & -108.043 & $4 / 26 / 2006$ & 9999 \\
\hline 1814 & Molina & Unknown & Not available & $-9,999$ & 39.196 & -108.028 & $4 / 24 / 2006$ & 9999 \\
\hline 1815 & Molina & Unknown & Not available & 150 & 39.17 & -108.1 & 8/20/1998 & 9999 \\
\hline 1816 & Molina & Unknown & Not available & $-9,999$ & 39.180 & -108.028 & $1 / 19 / 2007$ & 9999 \\
\hline 1817 & Molina & Unknown & Not available & $-9,999$ & 39.181 & -108.033 & $1 / 19 / 2007$ & 9999 \\
\hline 1818 & Molina & Unknown & Not available & $-9,999$ & 39.195 & -108.027 & $2 / 28 / 2006$ & 9999 \\
\hline 1819 & Molina & Unknown & Not available & $-9,999$ & 39.17 & -108.09 & 8/20/1998 & 9999 \\
\hline 1820 & Molina & Unknown & Not available & $-9,999$ & 39.182 & -108.042 & $4 / 10 / 2007$ & 9999 \\
\hline 1821 & Molina & Unknown & Not available & $-9,999$ & 39.193 & -108.030 & $4 / 24 / 2006$ & 9999 \\
\hline 1822 & Molina & Unknown & Not available & $-9,999$ & 39.195 & -108.030 & 4/24/2006 & 9999 \\
\hline 1827 & Palisade & Unknown & Not available & 50 & 39.008 & -108.311 & $10 / 28 / 2008$ & 9999 \\
\hline 1828 & Palisade & Unknown & Not available & 80 & 39.009 & -108.317 & $10 / 28 / 2008$ & 9999 \\
\hline 1829 & Ruby Lee Reservoir & Cretaceous & MANCOS SHALE & 100 & 39.270 & -108.729 & $6 / 3 / 1992$ & 1200 \\
\hline 1830 & Vega Reservoir & Unknown & Not available & $-9,999$ & 39.219 & -107.792 & 9/7/1977 & 1605 \\
\hline 1832 & Vega Reservoir & Unknown & Not available & $-9,999$ & 39.227 & -107.812 & $6 / 11 / 1997$ & 9999 \\
\hline 1833 & Wagon Track Ridge & Unknown & Not available & $-9,999$ & 39.318 & -108.272 & $8 / 18 / 1977$ & 1420 \\
\hline 1837 & Wagon Track Ridge & Quaternary & ALLUVIUM,TERRACE & 47 & 39.358 & -108.250 & $5 / 15 / 1979$ & 1430 \\
\hline 1842 & Whitewater & Cretaceous & MANCOS SHALE & 25 & 38.977 & -108.435 & $11 / 15 / 2001$ & 1315 \\
\hline 1843 & Whitewater & Cretaceous & MANCOS SHALE & 23 & 38.988 & -108.444 & $11 / 15 / 2001$ & 1200 \\
\hline 1845 & Whitewater & Unknown & MESOZOIC ERATHEM & 1,100 & 38.990 & -108.473 & 6/18/1981 & 0 \\
\hline 1847 & Whitewater & Cretaceous & MANCOS SHALE & 29.6 & 38.992 & -108.449 & $11 / 15 / 2001$ & 935 \\
\hline 1855 & Bowie & Unknown & Not available & $-9,999$ & 38.984 & -107.507 & $5 / 29 / 2002$ & 9999 \\
\hline 1856 & Bowie & Unknown & Not available & $-9,999$ & 38.982 & -107.508 & $5 / 29 / 2002$ & 9999 \\
\hline 1857 & Bowie & Unknown & Not available & $-9,999$ & 38.963 & -107.616 & $5 / 17 / 2002$ & 9999 \\
\hline 1858 & Bowie & Unknown & Not available & $-9,999$ & 38.954 & -107.623 & $5 / 17 / 2002$ & 9999 \\
\hline 1859 & Bowie & Unknown & Not available & $-9,999$ & 38.955 & -107.616 & $5 / 17 / 2002$ & 9999 \\
\hline 1860 & Bowie & Unknown & Not available & $-9,999$ & 38.948 & -107.624 & $6 / 23 / 2000$ & 9999 \\
\hline 1861 & Bowie & Unknown & Not available & $-9,999$ & 38.948 & -107.625 & $6 / 23 / 2002$ & 9999 \\
\hline
\end{tabular}


Appendix 1. Water Quality Data Used in This Report.-Continued

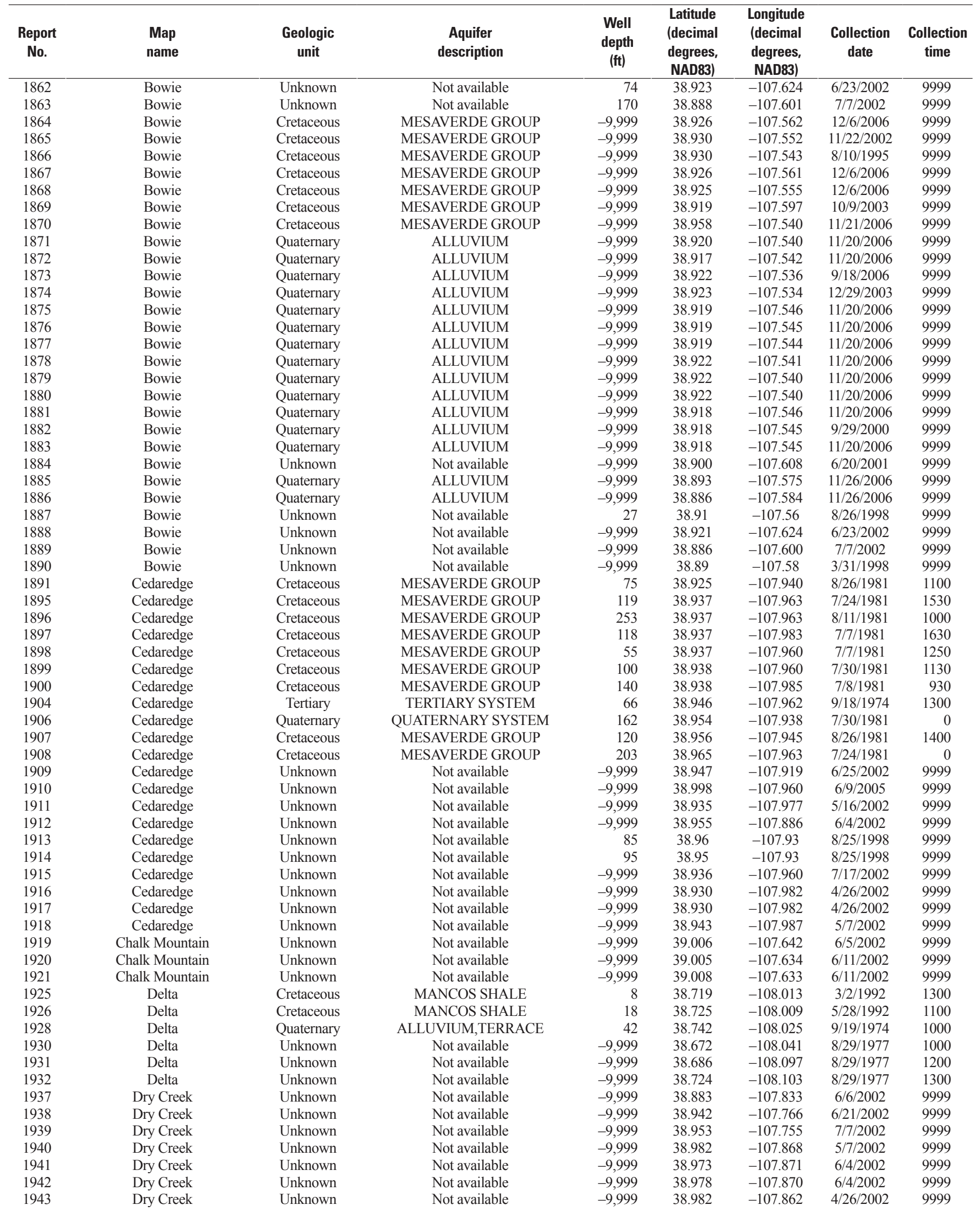


Appendix 1. Water Quality Data Used in This Report.-Continued

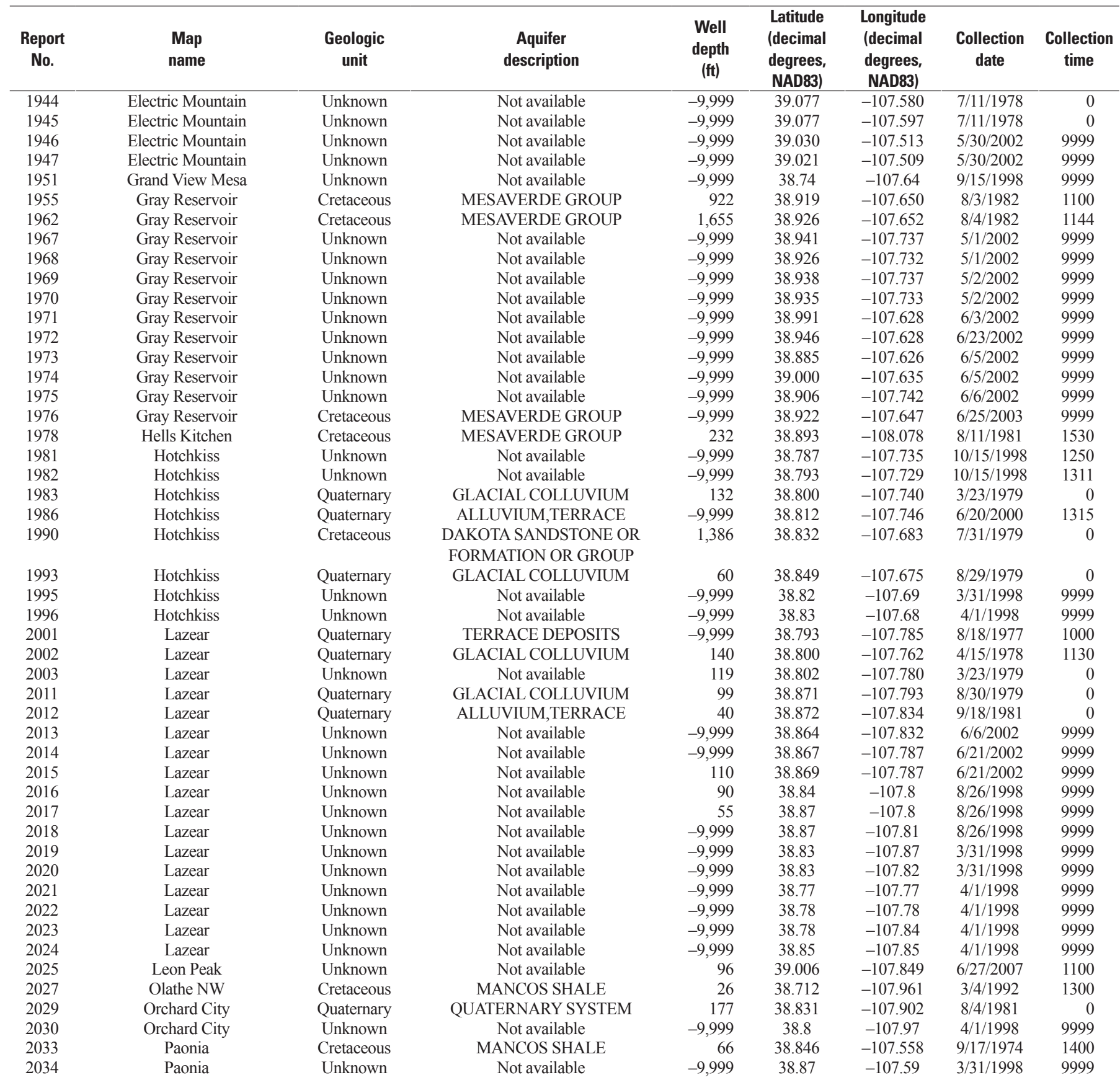


Appendix 1. Water Quality Data Used in This Report.-Continued

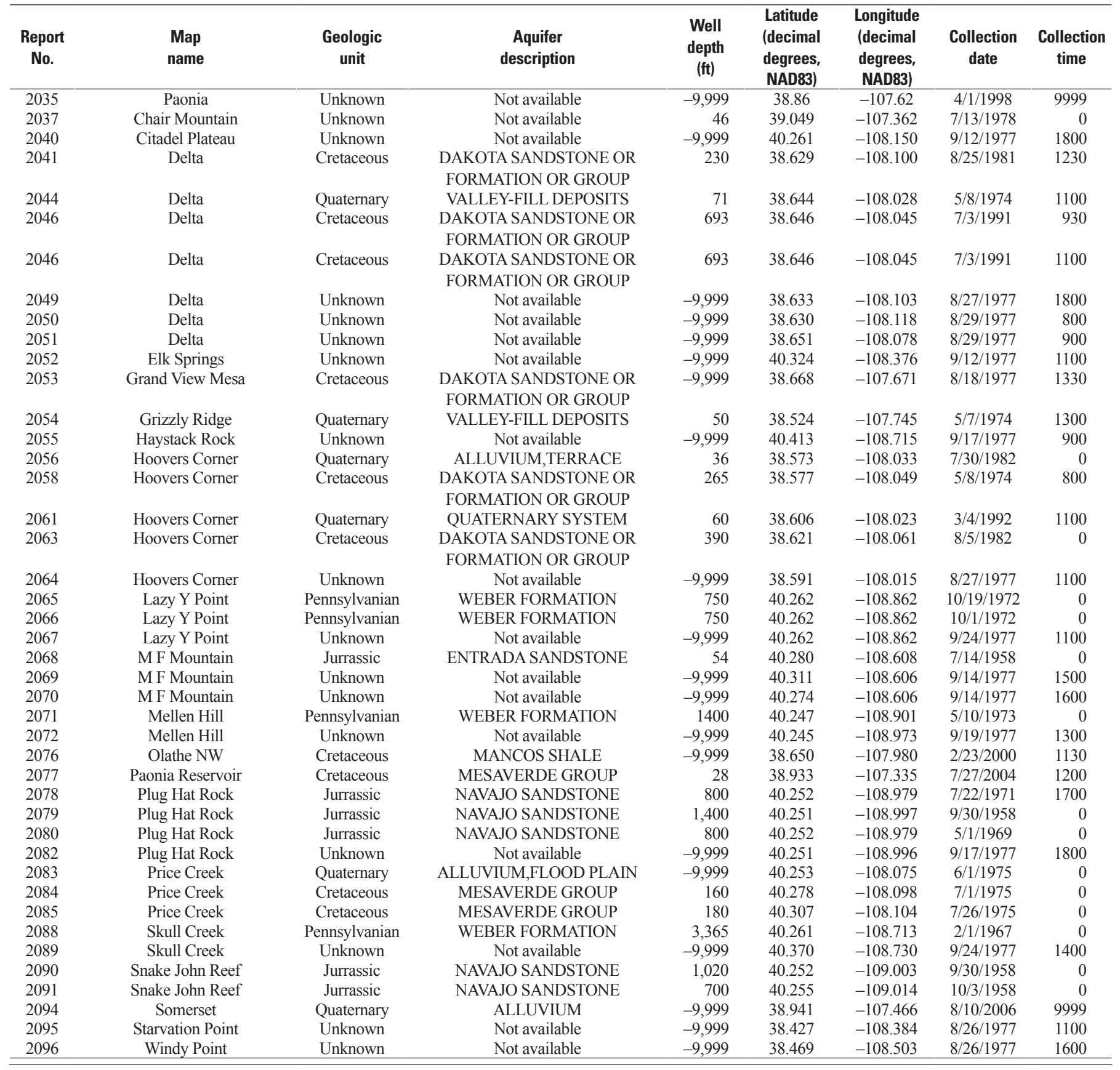


Appendix 1. Water Quality Data Used in This Report.-Continued

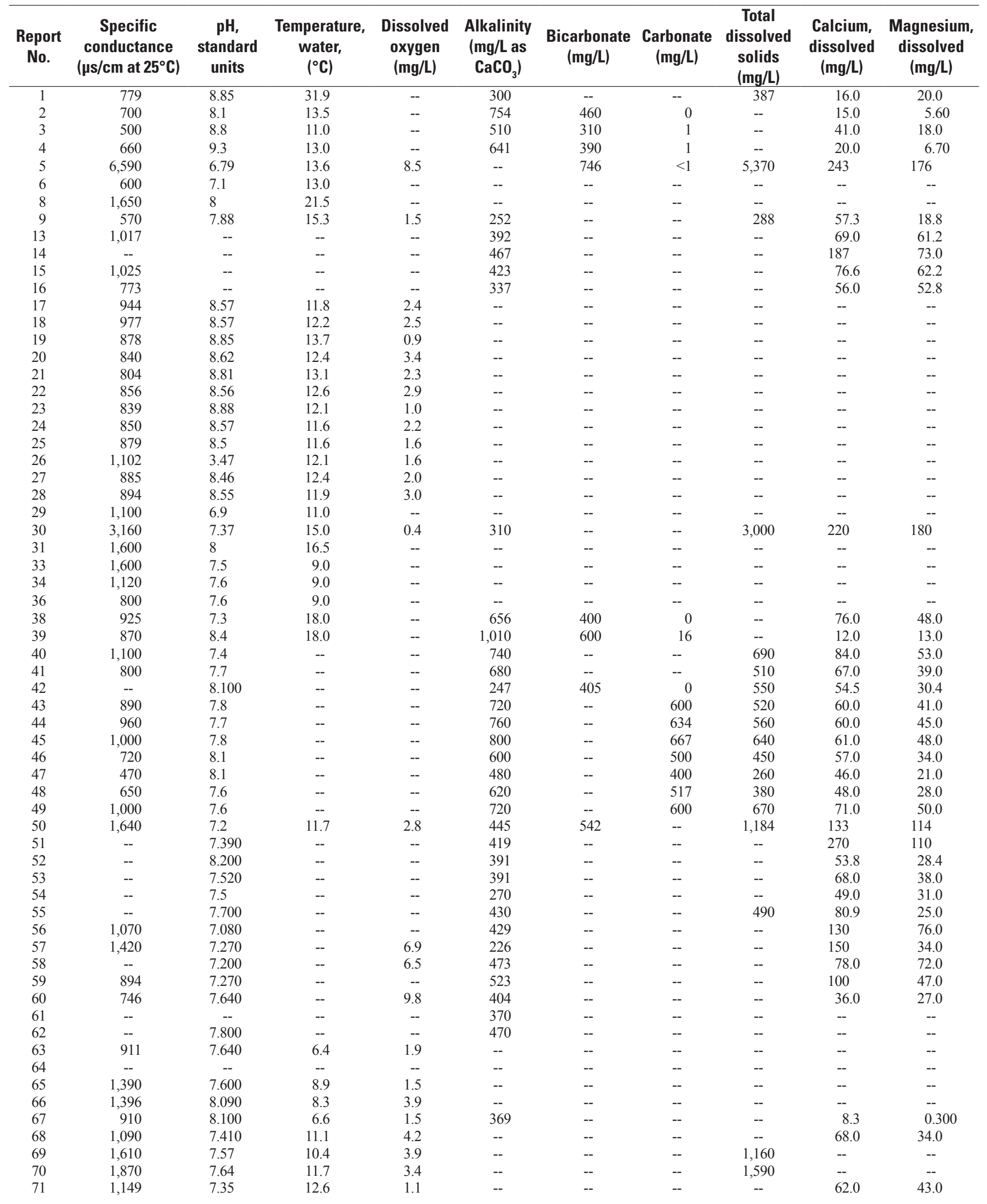


Appendix 1. Water Quality Data Used in This Report.-Continued

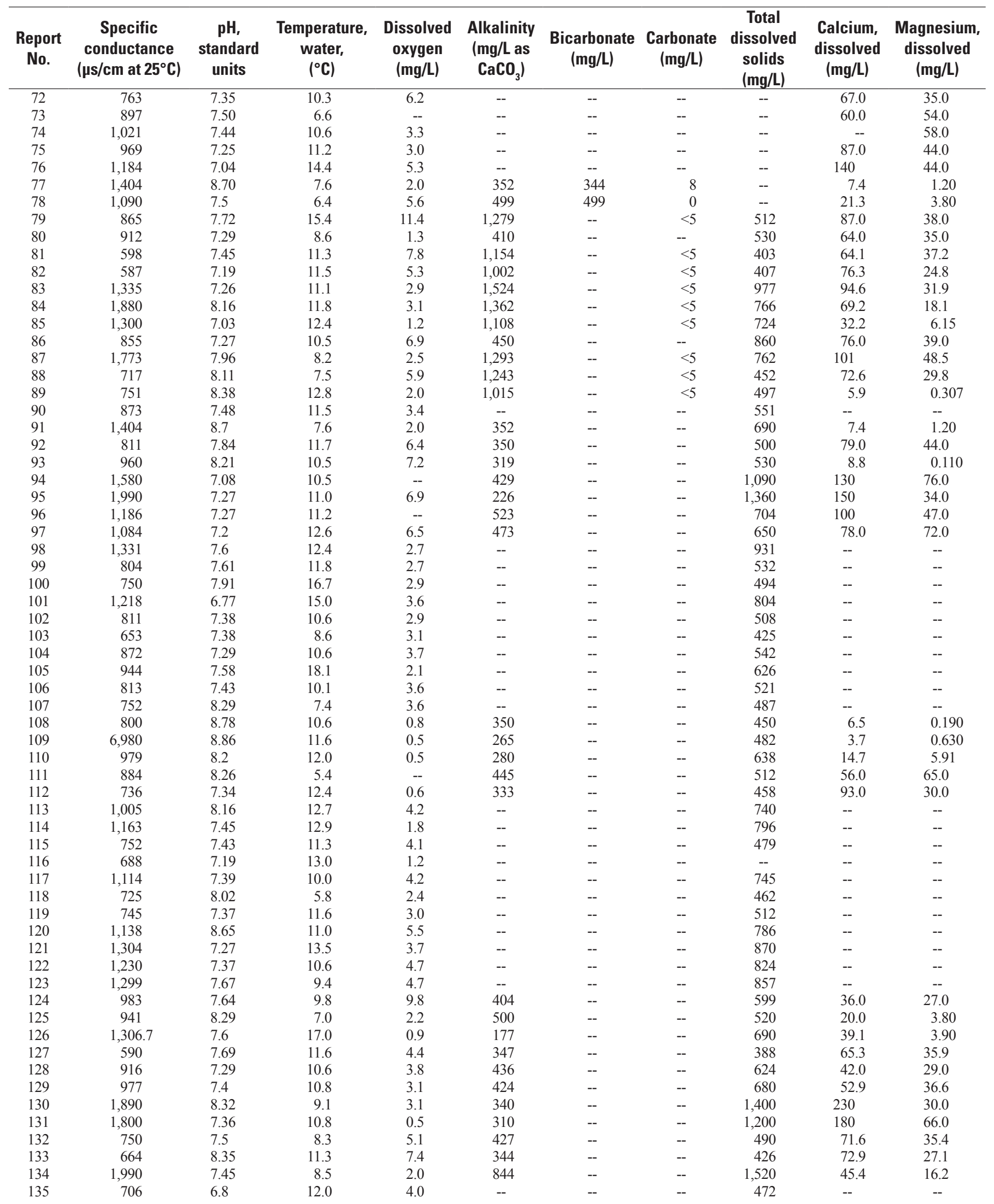


Appendix 1. Water Quality Data Used in This Report.-Continued

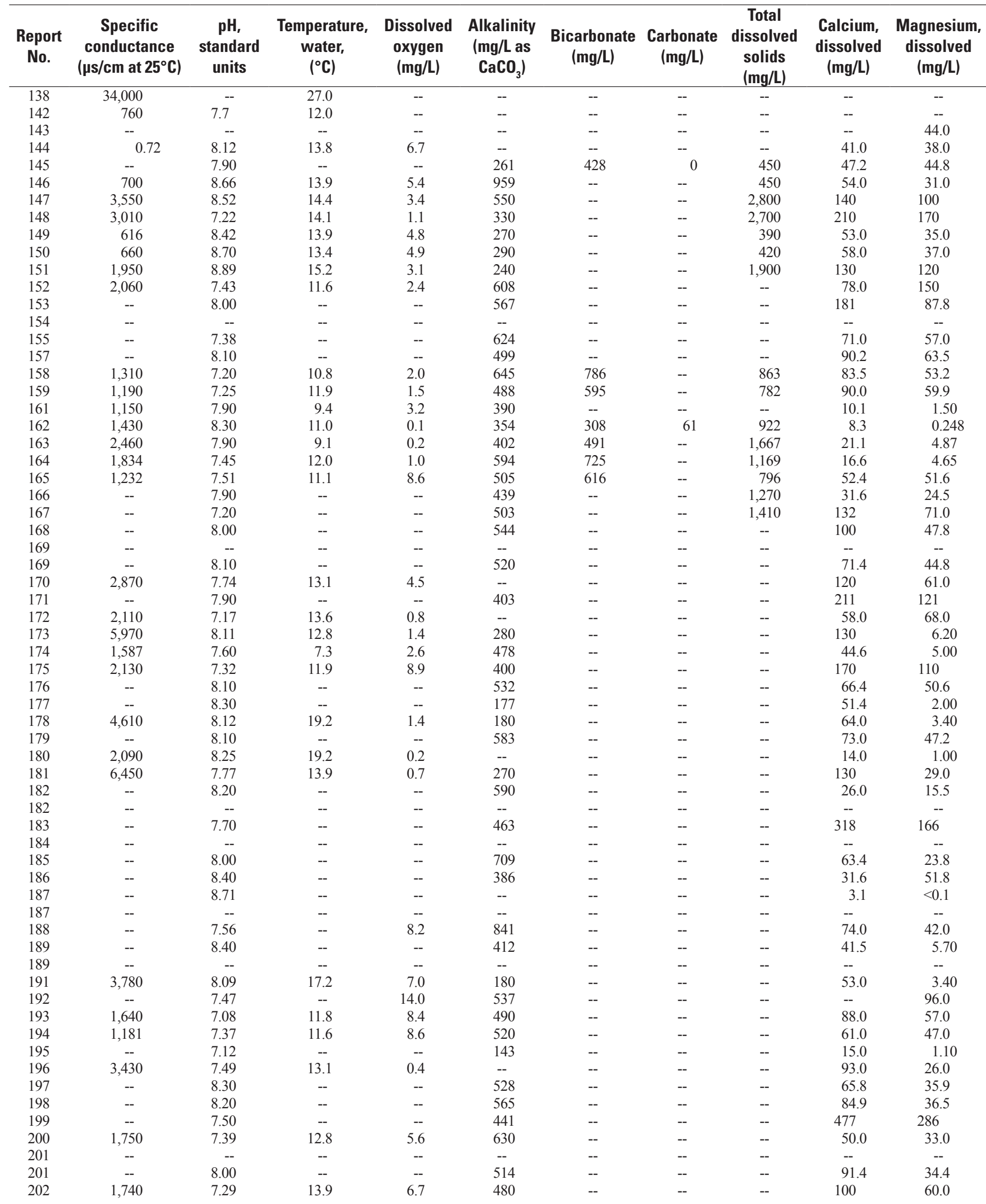


Appendix 1. Water Quality Data Used in This Report.-Continued

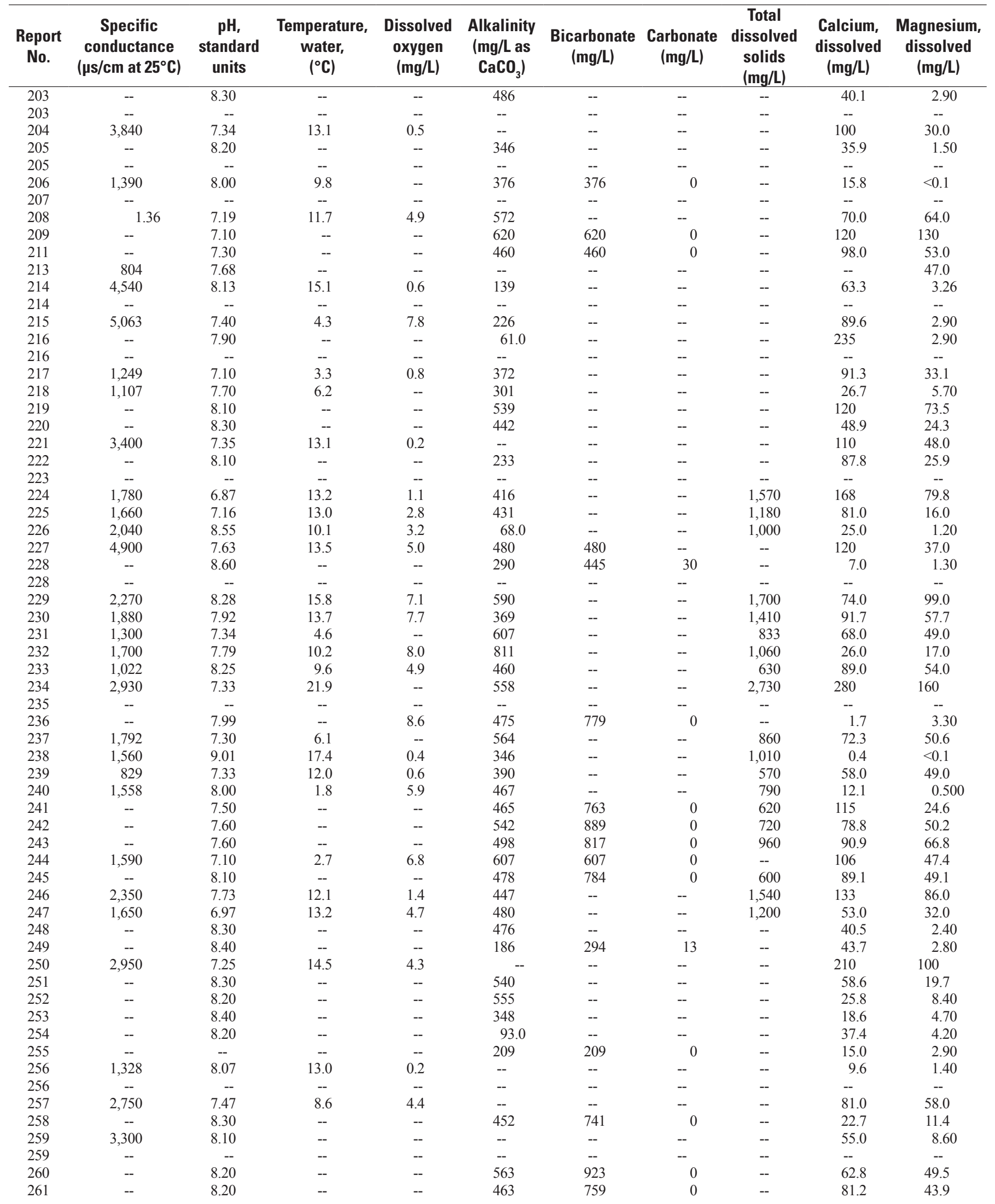


Appendix 1. Water Quality Data Used in This Report.-Continued

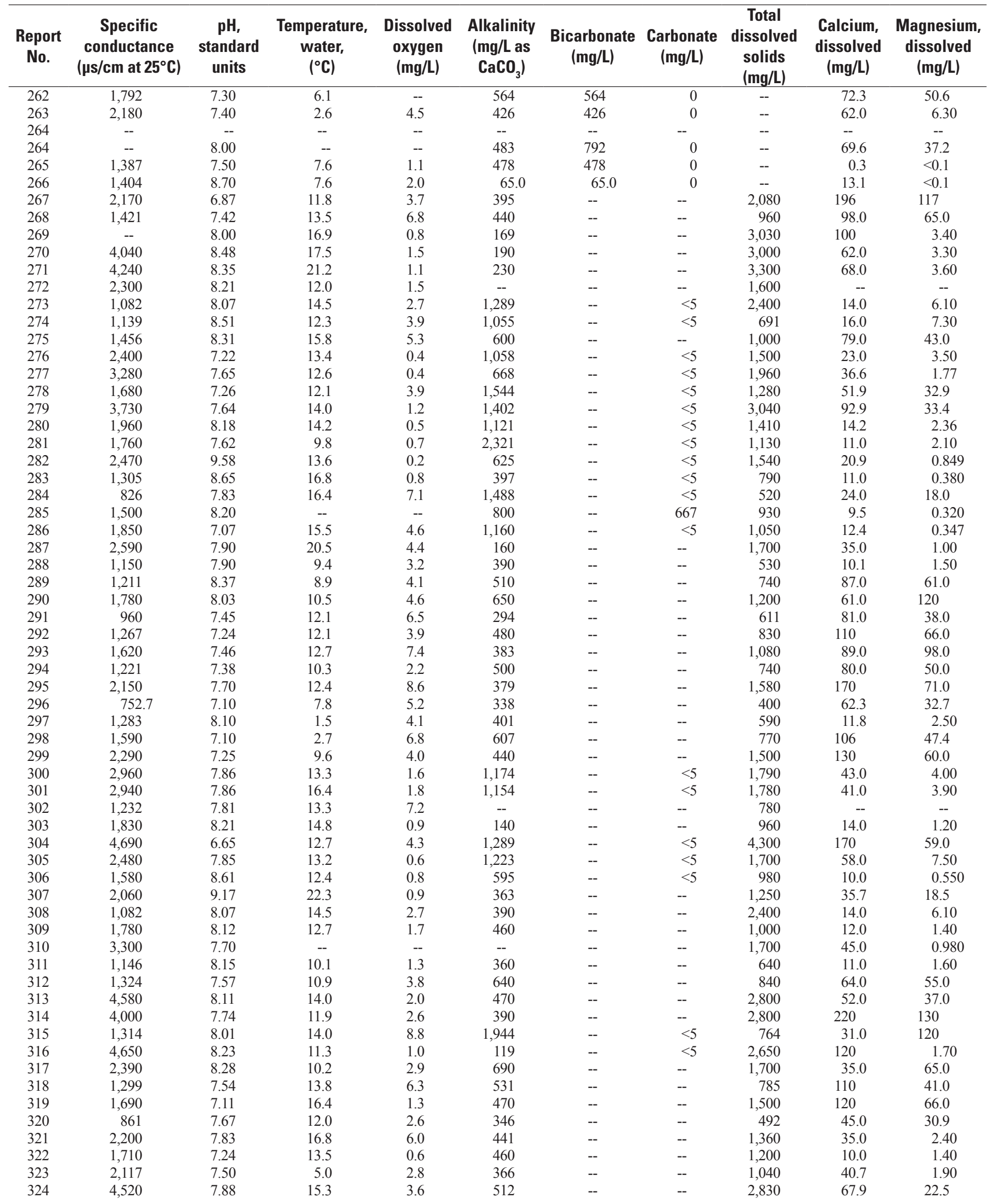


Appendix 1. Water Quality Data Used in This Report.-Continued

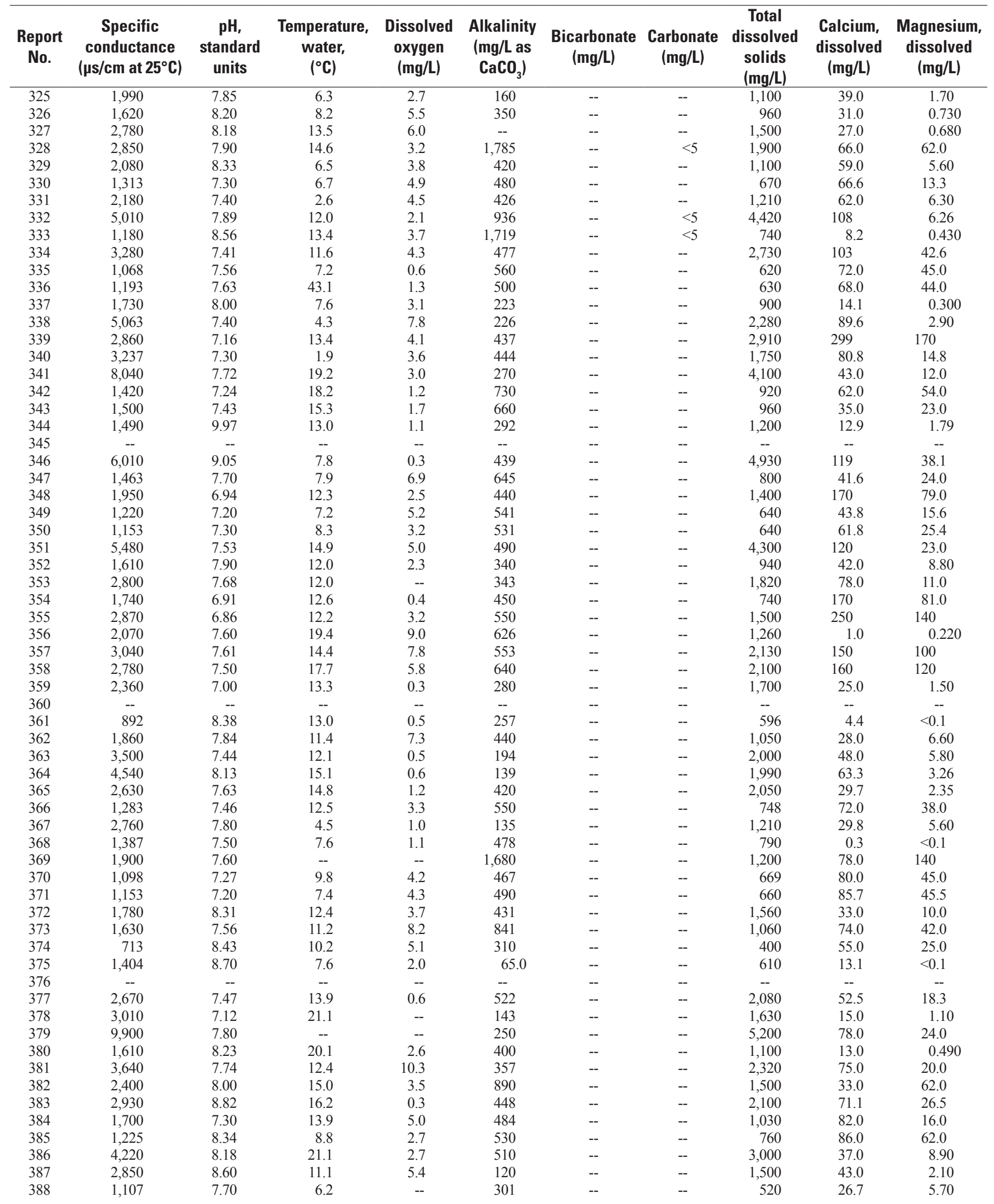


Appendix 1. Water Quality Data Used in This Report.-Continued

\begin{tabular}{|c|c|c|c|c|c|c|c|c|c|c|}
\hline $\begin{array}{l}\text { Report } \\
\text { No. }\end{array}$ & $\begin{array}{c}\text { Specific } \\
\text { conductance } \\
\left(\mu \mathrm{s} / \mathrm{cm} \text { at } 25^{\circ} \mathrm{C}\right)\end{array}$ & $\begin{array}{c}\text { pH, } \\
\text { standard } \\
\text { units }\end{array}$ & $\begin{array}{c}\text { Temperature, } \\
\text { water, } \\
\left({ }^{\circ} \mathrm{C}\right)\end{array}$ & $\begin{array}{l}\text { Dissolved } \\
\text { oxygen } \\
\text { (mg/L) }\end{array}$ & $\begin{array}{c}\text { Alkalinity } \\
\text { (mg/L as } \\
\mathrm{CaCO}_{3} \text { ) }\end{array}$ & $\begin{array}{c}\text { Bicarbonate } \\
\text { (mg/L) }\end{array}$ & $\begin{array}{c}\text { Carbonate } \\
\text { (mg/L) }\end{array}$ & $\begin{array}{c}\text { Total } \\
\text { dissolved } \\
\text { solids } \\
\text { (mq/L) }\end{array}$ & $\begin{array}{l}\text { Calcium, } \\
\text { dissolved } \\
\text { (mg/L) }\end{array}$ & $\begin{array}{c}\text { Magnesium, } \\
\text { dissolved } \\
\text { (mg/L) }\end{array}$ \\
\hline 390 & 692 & 7.12 & 15.4 & 3.5 & 300 & -- & -- & 340 & 37.0 & 10.0 \\
\hline 391 & 906 & 8.76 & 14.6 & -- & 411 & -- & -- & 704 & 37.6 & 43.1 \\
\hline 392 & 1,830 & 8.08 & 13.2 & 0.6 & 439 & --- & -- & 4,930 & 119 & 38.1 \\
\hline 393 & 3,250 & 7.10 & 10.7 & 0.8 & 160 & -- & -- & 2,700 & 310 & 170 \\
\hline 396 & 3,310 & 8.20 & 9.1 & 3.5 & 193 & -- & -- & 2,500 & 49.1 & 3.00 \\
\hline 397 & 1,054 & 7.50 & 10.8 & 2.0 & 510 & -- & -- & 700 & 80.0 & 55.0 \\
\hline 398 & 1,350 & 7.53 & 15.0 & 5.1 & 514 & -- & -- & 844 & 69.0 & 96.0 \\
\hline 399 & 1,296 & 7.37 & 15.5 & 14.0 & 660 & -- & -- & 770 & 75.0 & 48.0 \\
\hline 400 & 1,276 & 7.42 & 14.6 & 3.9 & 590 & -- & -- & 750 & 69.0 & 44.0 \\
\hline 401 & 1,142 & 8.40 & 15.6 & 0.4 & 340 & --- & -- & 743 & 6.2 & 0.491 \\
\hline 402 & 1,830 & 8.24 & 11.7 & 0.1 & 232 & -- & -- & 1,410 & 12.6 & 2.79 \\
\hline 407 & 1,510 & 7.90 & 7.2 & 6.2 & 577 & -- & -- & 880 & 18.5 & 7.70 \\
\hline 408 & 3,000 & 7.10 & 17.0 & -- & - & -- & -- & -- & -- & -- \\
\hline 409 & 1,200 & 7.20 & 18.0 & -- & 967 & 590 & 0 & -- & 82.0 & 88.0 \\
\hline 410 & 1,550 & 7.40 & 15.0 & -- & -- & -- & -- & -- & -- & -- \\
\hline 414 & 1,250 & 7.50 & 12.5 & -- & 951 & 580 & 0 & -- & 83.0 & 66.0 \\
\hline 415 & 975 & 7.20 & 20.0 & -- & 836 & 510 & 0 & -- & 76.0 & 55.0 \\
\hline 416 & 1,225 & 7.10 & 12.0 & -- & 787 & 480 & 0 & -- & 78.0 & 60.0 \\
\hline 417 & 1,820 & 7.00 & 9.5 & -- & -- & -- & -- & -- & -- & -- \\
\hline 418 & -- & 7.80 & 18.0 & -- & -- & -- & -- & -- & -- & -- \\
\hline 419 & 1,550 & 8.40 & 17.0 & -- & 1879 & 1,130 & 16 & _- & 9.0 & 7.60 \\
\hline 420 & 220 & 7.70 & 14.8 & -- & 197 & 120 & 0 & -- & 25.0 & 8.00 \\
\hline 428 & 12,310 & 7.15 & 10.9 & 2.3 & - & - & -- & -- & 110 & 43.0 \\
\hline 429 & -- & 7.60 & -- & -- & 198 & -- & -- & 1,870 & 487 & 49.0 \\
\hline 430 & 690 & 7.20 & 19.4 & 5.8 & -- & -- & -- & -- & 90.0 & 30.0 \\
\hline 441 & 900 & 7.90 & 13.7 & -- & -- & 591 & 0 & -- & 19.0 & 1.30 \\
\hline 442 & -- & 8.00 & -- & -- & 275 & -- & -- & -- & 256 & 153 \\
\hline 443 & -- & 7.70 & -- & -- & 350 & 574 & -- & -- & 70.0 & $<0.1$ \\
\hline 444 & 1,430 & 7.08 & 7.6 & 0.8 & - & - & -- & -- & 27.0 & 5.80 \\
\hline 445 & - & 7.79 & -- & -- & -- & -- & -- & .- & 44.0 & 18.0 \\
\hline 446 & 510 & 7.20 & 13.3 & 1.7 & -- & -- & -- & - & 79.0 & 38.0 \\
\hline 447 & 570 & 7.40 & 19.3 & 6.4 & -- & -- & -- & -- & 85.0 & 29.0 \\
\hline 447 & -- & -- & -- & -- & -- & -- & -- & -- & -- & -- \\
\hline 448 & 410 & 7.40 & 15.0 & 8.1 & -- & -- & -- & -- & 87.0 & 13.0 \\
\hline 448 & - & -- & -- & -- & -- & -- & -- & -- & -- & - \\
\hline 449 & 1,810 & 8.00 & 12.1 & 0.6 & -- & 771 & 0 & -- & 38.0 & 16.0 \\
\hline 450 & 530 & 7.30 & 14.1 & 4.0 & -- & 476 & 0 & -- & 77.0 & 17.0 \\
\hline 451 & 630 & 7.30 & 19.9 & 8.9 & -- & 558 & 0 & -- & 100 & 21.0 \\
\hline 452 & 800 & 7.60 & 13.6 & -- & -- & 738 & 0 & -- & 43.0 & 15.0 \\
\hline 453 & 780 & 7.30 & 13.0 & -- & -- & 689 & 0 & -- & 80.0 & 32.0 \\
\hline 453 & - & -- & -- & -- & -- & -- & -- & -- & -- & -- \\
\hline 454 & 890 & 7.10 & 13.7 & 0.2 & -- & 689 & 0 & -- & 50.0 & 16.0 \\
\hline 454 & -- & - & - & -- & -- & -- & -- & -- & -- & - \\
\hline 455 & 812 & 8.10 & 14.0 & -- & -- & -- & -- & 733 & 6.0 & 3.10 \\
\hline 456 & 541 & 7.50 & 14.6 & -- & -- & -- & -- & 444 & 32.8 & 44.1 \\
\hline 457 & -- & 7.60 & - & -- & -- & -- & -- & -- & -- & -- \\
\hline 458 & -- & 7.60 & -- & -- & -- & -- & -- & -- & -- & -- \\
\hline 459 & -- & 7.60 & -- & -- & -- & -- & -- & -- & -- & -- \\
\hline
\end{tabular}


Appendix 1. Water Quality Data Used in This Report.-Continued

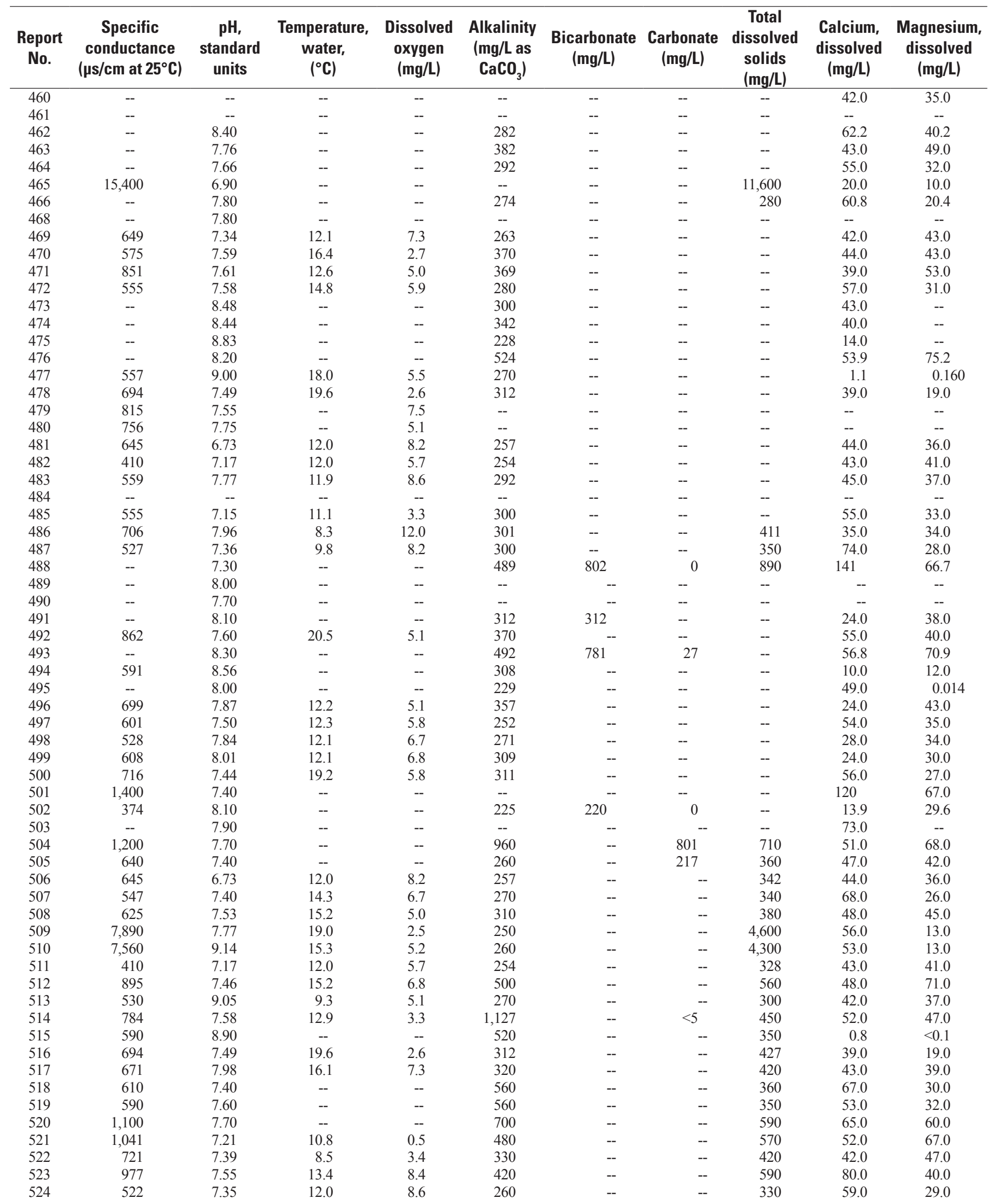


Appendix 1. Water Quality Data Used in This Report.-Continued

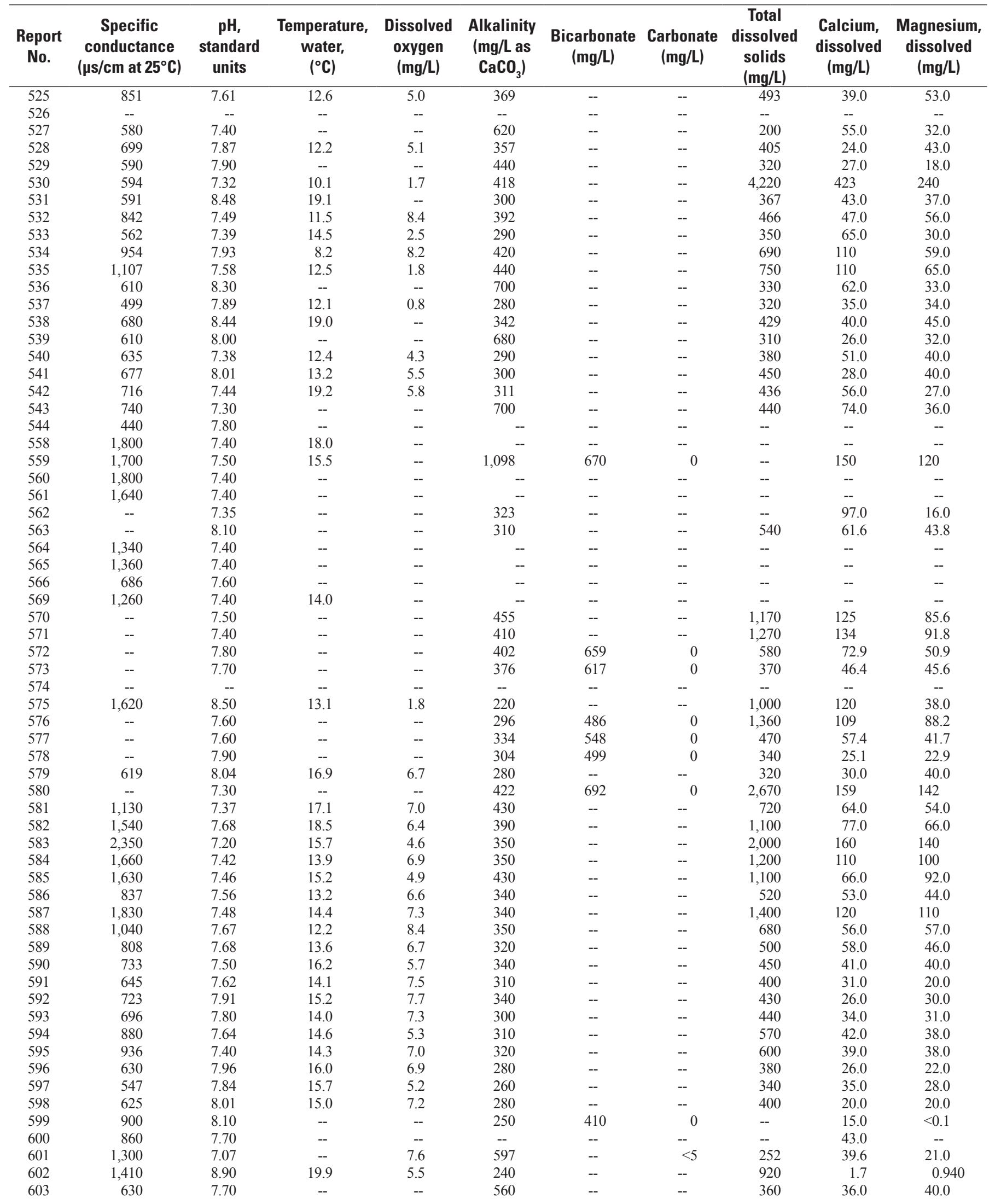


Appendix 1. Water Quality Data Used in This Report.-Continued

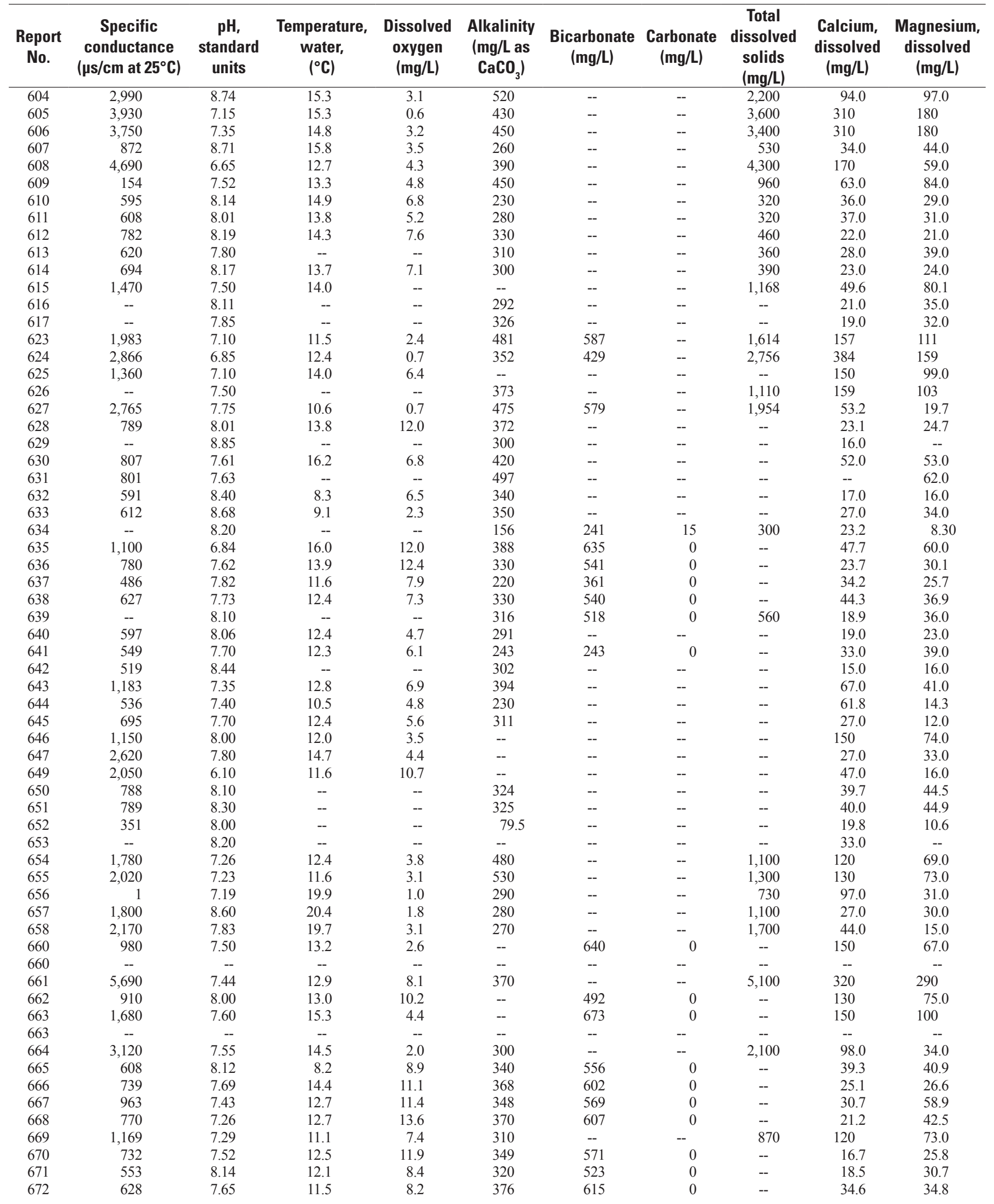


Appendix 1. Water Quality Data Used in This Report.-Continued

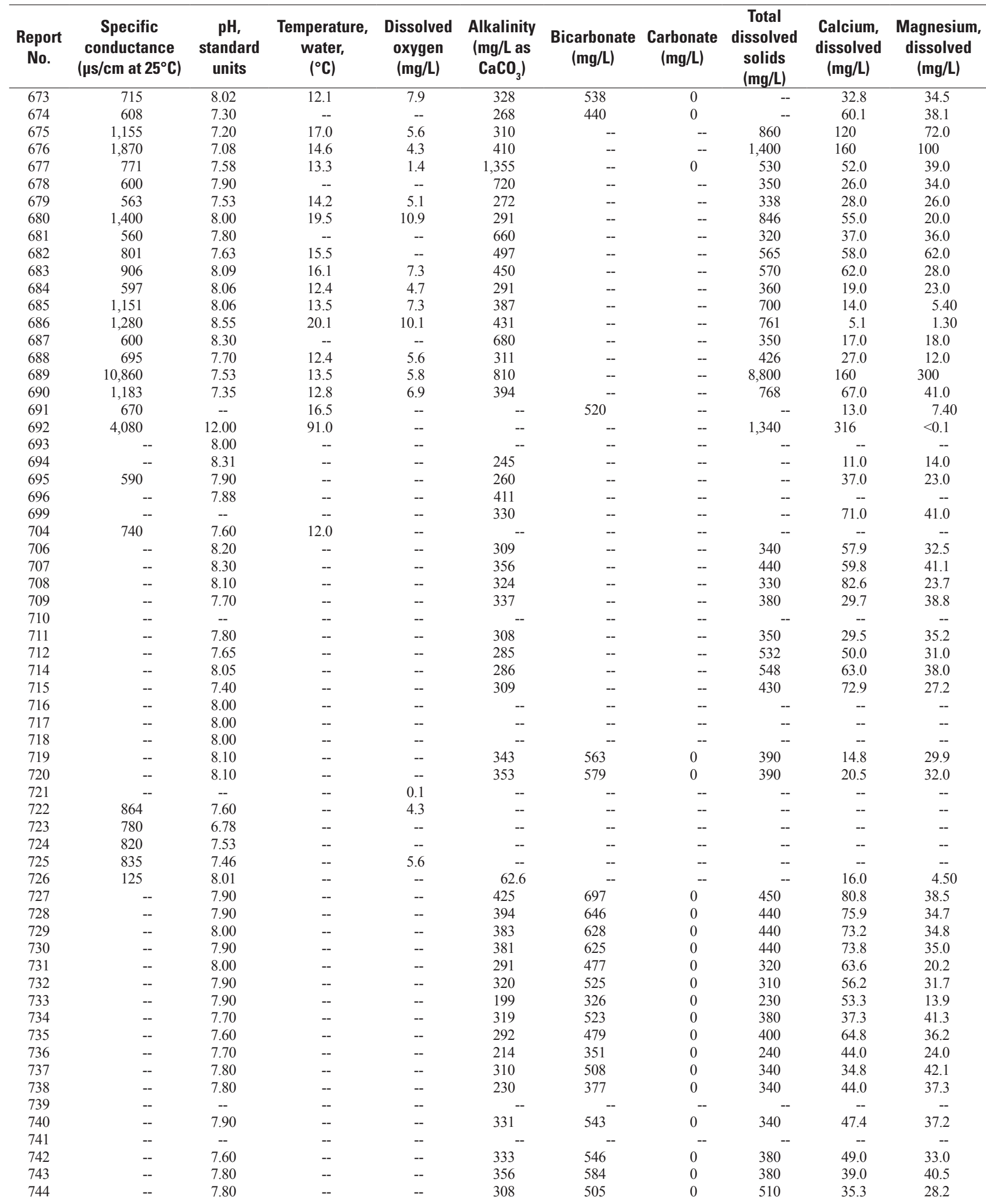


Appendix 1. Water Quality Data Used in This Report.-Continued

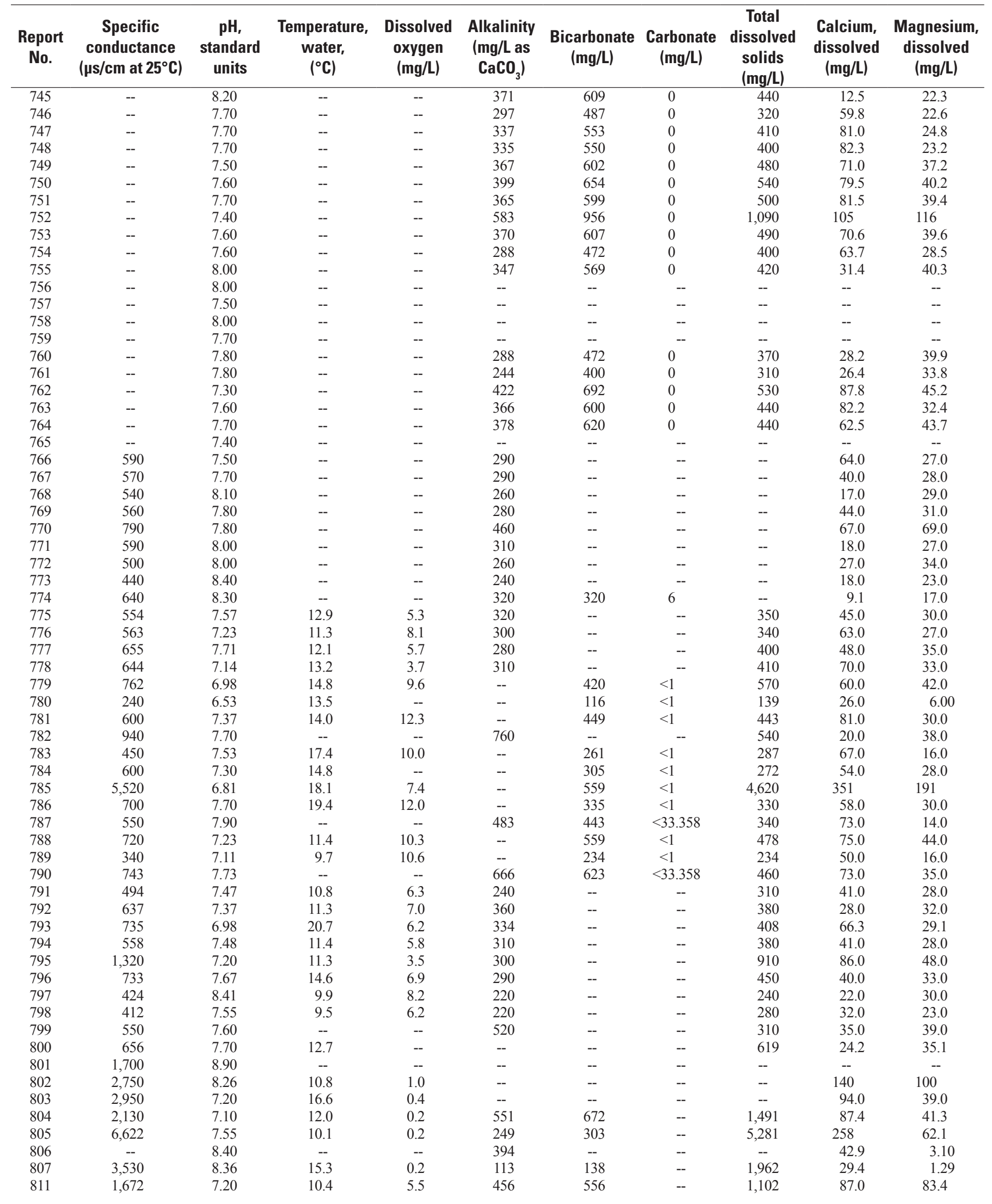


Appendix 1. Water Quality Data Used in This Report.-Continued

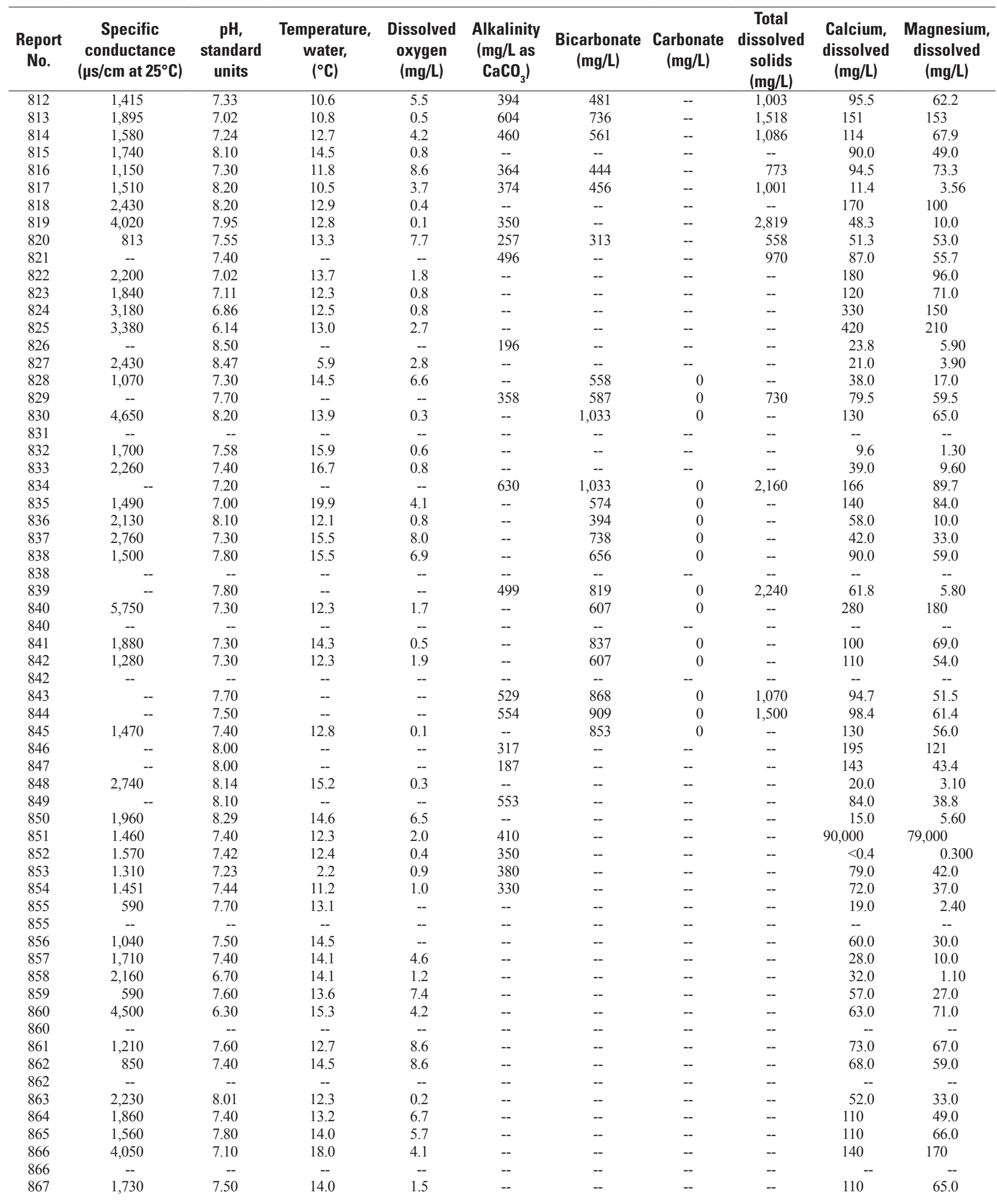


Appendix 1. Water Quality Data Used in This Report.-Continued

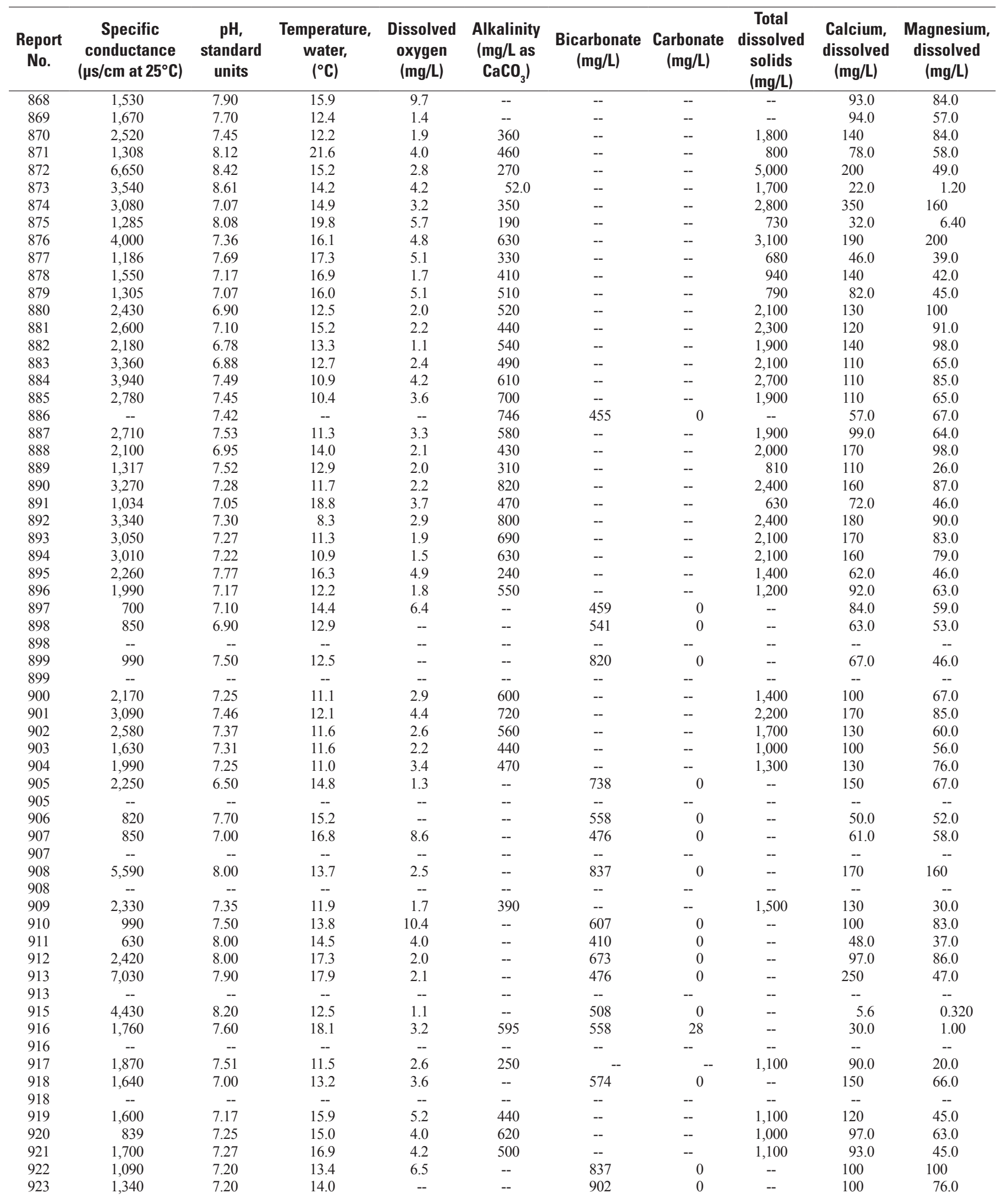


Appendix 1. Water Quality Data Used in This Report.-Continued

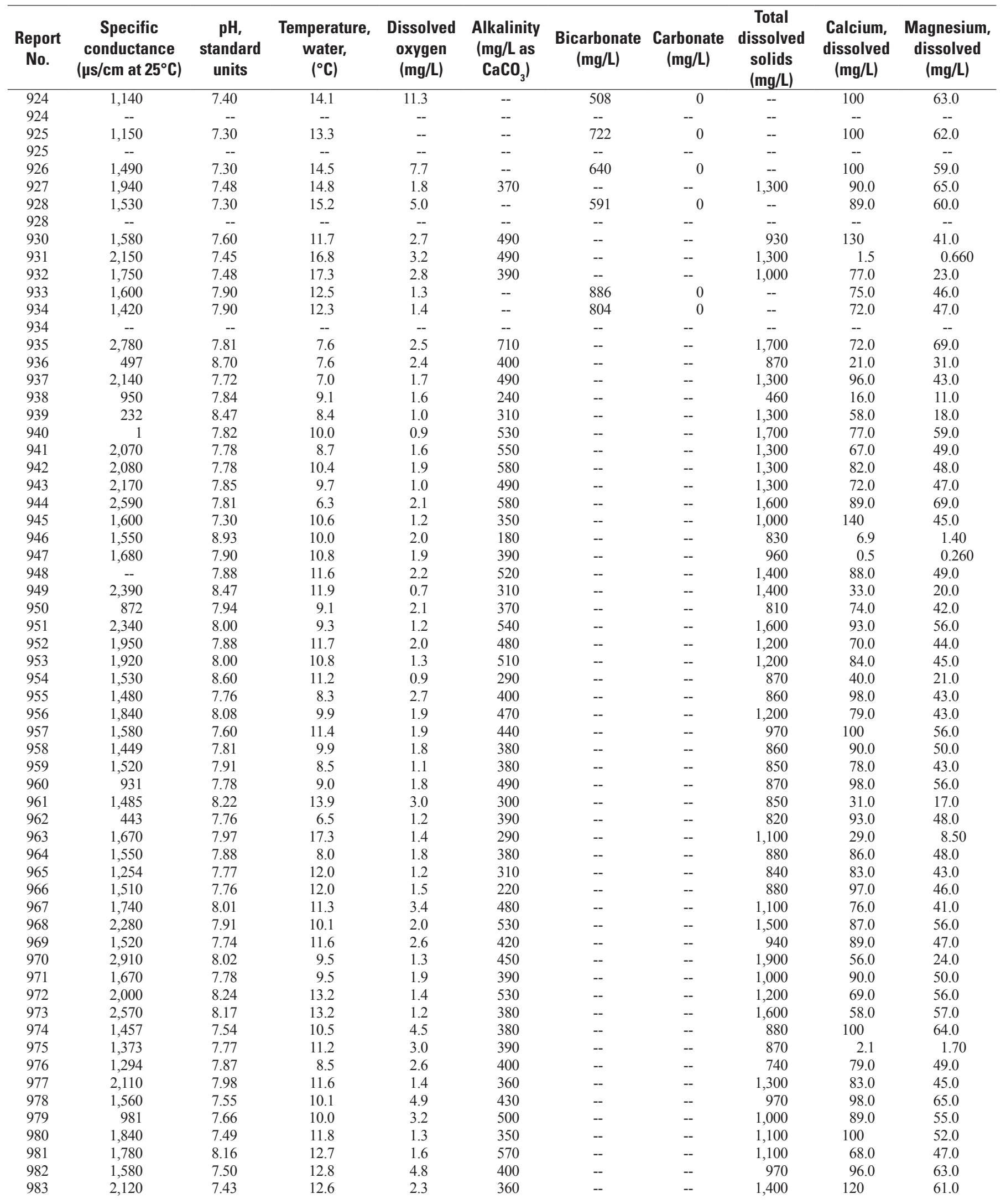


Appendix 1. Water Quality Data Used in This Report.-Continued

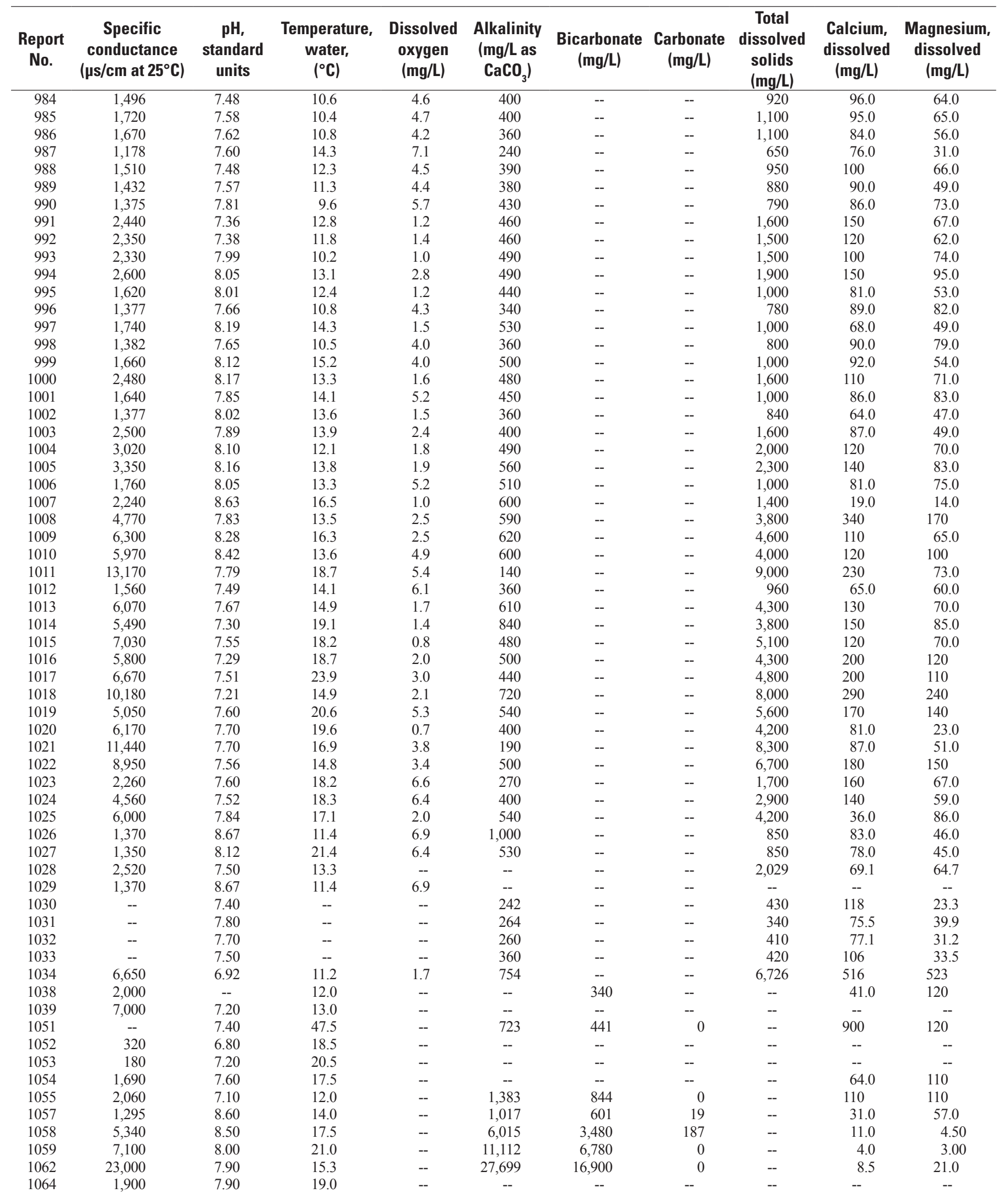


Appendix 1. Water Quality Data Used in This Report.-Continued

\begin{tabular}{|c|c|c|c|c|c|c|c|c|c|c|}
\hline $\begin{array}{l}\text { Report } \\
\text { No. }\end{array}$ & $\begin{array}{c}\text { Specific } \\
\text { conductance } \\
\left(\mu \mathrm{s} / \mathrm{cm} \text { at } 25^{\circ} \mathrm{C}\right)\end{array}$ & $\begin{array}{c}\text { pH, } \\
\text { standard } \\
\text { units }\end{array}$ & $\begin{array}{l}\text { Temperature, } \\
\text { water, } \\
\left({ }^{\circ} \mathrm{C}\right)\end{array}$ & $\begin{array}{c}\text { Dissolved } \\
\text { oxygen } \\
\text { (mg/L) }\end{array}$ & $\begin{array}{c}\text { Alkalinity } \\
\text { (mg/L as } \\
\mathrm{CaCO}_{3} \text { ) }\end{array}$ & $\begin{array}{c}\text { Bicarbonate } \\
(\mathrm{mg} / \mathrm{L})\end{array}$ & $\begin{array}{l}\text { Carbonate } \\
\text { (mg/L) }\end{array}$ & $\begin{array}{c}\text { Total } \\
\text { dissolved } \\
\text { solids } \\
\text { (mg/L) }\end{array}$ & $\begin{array}{l}\text { Calcium, } \\
\text { dissolved } \\
\text { (mg/L) }\end{array}$ & $\begin{array}{c}\text { Magnesium, } \\
\text { dissolved } \\
\text { (mg/L) }\end{array}$ \\
\hline 1066 & 1,950 & 7.80 & 18.0 & -- & -- & -- & -- & -- & -- & -- \\
\hline 1067 & 1,500 & 8.40 & 21.0 & -- & -- & -- & -- & -- & -- & -- \\
\hline 1068 & 9,190 & 8.90 & 20.0 & -- & -- & -- & -- & -- & 18.0 & 64.0 \\
\hline 1069 & 8,550 & 7.70 & 16.0 & -- & -- & -- & -- & -- & 5.0 & 6.90 \\
\hline 1073 & 2,300 & 8.20 & 11.0 & -- & 1,069 & 652 & 0 & -- & 77.0 & 150 \\
\hline 1080 & 1,330 & 7.90 & 14.0 & -- & -- & -- & -- & .- & 26.0 & 54.0 \\
\hline 1081 & 38,300 & 8.30 & 20.5 & -- & 50,937 & 29,400 & 1,650 & 33,300 & 4.2 & 2.80 \\
\hline 1084 & 30,000 & 7.70 & 17.0 & -- & 43,270 & 26400 & 0 & -- & 6.3 & 14.0 \\
\hline 1085 & 669 & 7.80 & 11.0 & -- & 397 & 242 & 0 & -- & 32.0 & 40.0 \\
\hline 1091 & 4,664 & 8.40 & 9.0 & -- & 4,852 & 2,660 & 295 & -- & 14.0 & 67.0 \\
\hline 1094 & 29,400 & 8.30 & 25.6 & -- & 4,423 & 2,200 & 490 & 24,700 & 4.7 & 6.20 \\
\hline 1101 & 595 & 7.00 & 13.0 & -- & -- & -- & -- & -- & -- & -- \\
\hline 1107 & 680 & -- & 6.0 & -- & -- & -- & -- & -- & -- & -- \\
\hline 1125 & 5,000 & 7.70 & 9.0 & -- & -- & -- & -- & -- & -- & -- \\
\hline 1130 & 1,080 & 7.50 & 13.2 & -- & -- & -- & -- & 764 & 47.5 & 29.2 \\
\hline 1141 & 950 & 6.80 & 14.0 & -- & -- & -- & -- & -- & -- & -- \\
\hline 1142 & 827 & 8.10 & 19.0 & -- & 836 & 510 & 0 & -- & 5.4 & 3.90 \\
\hline 1144 & 4,170 & 7.40 & 12.8 & -- & -- & -- & -- & -- & 220 & 240 \\
\hline 1146 & -- & -- & 9.0 & -- & -- & -- & -- & -- & -- & -- \\
\hline 1147 & -- & -- & 18.0 & -- & -- & -- & -- & -- & -- & -- \\
\hline 1149 & 5,600 & 8.20 & 15.0 & -- & -- & -- & -- & -- & -- & -- \\
\hline 1153 & 2,100 & 7.50 & 12.0 & -- & -- & -- & -- & -- & -- & -- \\
\hline 1154 & 3,250 & 7.70 & 12.0 & -- & -- & -- & -- & -- & -- & -- \\
\hline 1160 & 1,340 & 7.60 & 14.0 & -- & 654 & 399 & 0 & -- & 30.0 & 27.0 \\
\hline 1164 & 1,300 & 7.40 & 19.0 & -- & -- & -- & -- & -- & -- & -- \\
\hline 1187 & 1,600 & 6.70 & -- & -- & -- & -- & -- & -- & -- & -- \\
\hline 1188 & 1,390 & 7.60 & 9.0 & -- & -- & -- & -- & -- & 97.0 & 71.0 \\
\hline 1189 & 1,180 & 8.20 & 29.0 & -- & -- & -- & -- & -- & 4.4 & 2.50 \\
\hline 1190 & 1,250 & 8.50 & - & -- & -- & -- & -- & -- & 21.0 & 48.0 \\
\hline 1191 & 1,200 & 7.70 & 9.8 & -- & -- & -- & -- & -- & 74.0 & 63.0 \\
\hline 1192 & 1,470 & 7.80 & - & -- & -- & -- & -- & -- & 96.0 & 65.0 \\
\hline 1193 & 962 & 8.40 & -- & -- & -- & -- & -- & -- & 10.0 & 8.00 \\
\hline 1194 & 1,940 & 8.30 & 25.5 & -- & -- & -- & -- & -- & 5.0 & 3.20 \\
\hline 1195 & 2,400 & 8.10 & -- & -- & -- & -- & -- & -- & 8.8 & 8.40 \\
\hline 1196 & 1,550 & 7.60 & 8.5 & -- & -- & -- & -- & -- & 100 & 91.0 \\
\hline 1197 & 1,010 & 8.20 & 18.5 & -- & -- & -- & -- & -- & 23.0 & 16.0 \\
\hline 1198 & 38,000 & 7.70 & 26.0 & -- & -- & -- & -- & -- & 9.5 & 4.30 \\
\hline 1199 & 1,790 & 7.60 & -- & -- & -- & -- & -- & -- & 100 & 110 \\
\hline 1200 & 3,050 & 6.40 & -- & -- & -- & -- & -- & -- & 57.0 & 250 \\
\hline 1201 & 3,040 & 8.40 & 29.0 & -- & -- & -- & -- & -- & 6.3 & 3.10 \\
\hline 1202 & 2,520 & 8.40 & 25.0 & -- & -- & -- & -- & -- & 9.2 & 7.40 \\
\hline 1203 & 1,800 & 8.30 & 16.0 & -- & -- & -- & -- & -- & 4.7 & 3.80 \\
\hline 1204 & 2,180 & 8.40 & 23.0 & -- & -- & -- & -- & -- & 13.0 & 4.50 \\
\hline 1205 & 3,900 & 7.40 & 29.0 & -- & -- & -- & -- & -- & 11.0 & 11.0 \\
\hline 1206 & 2,540 & 8.10 & - & -- & -- & -- & -- & -- & 8.3 & 7.20 \\
\hline 1207 & 1,230 & 8.30 & -- & -- & -- & -- & -- & -- & 4.7 & 3.00 \\
\hline
\end{tabular}


Appendix 1. Water Quality Data Used in This Report.-Continued

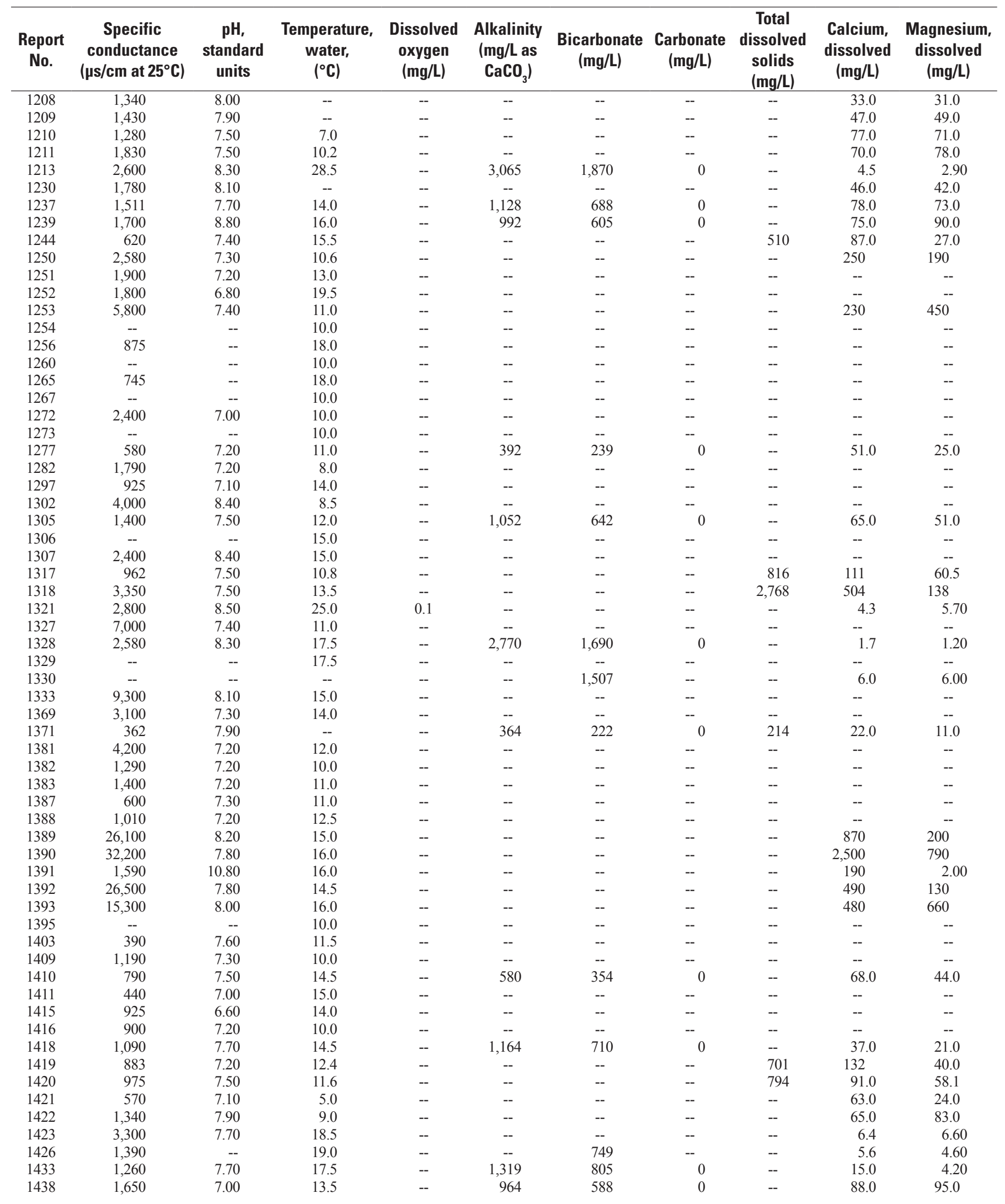


Appendix 1. Water Quality Data Used in This Report.-Continued

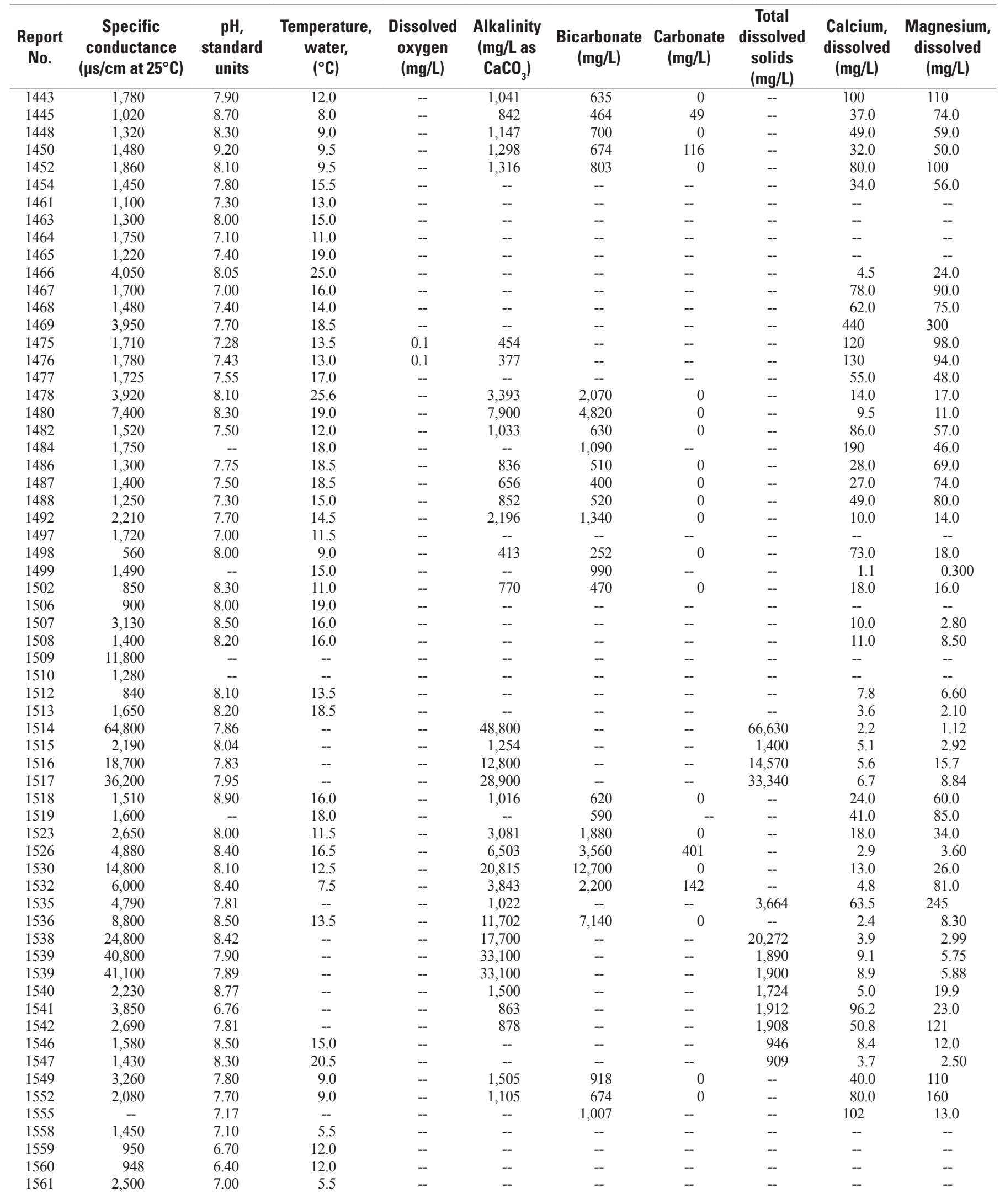


Appendix 1. Water Quality Data Used in This Report.-Continued

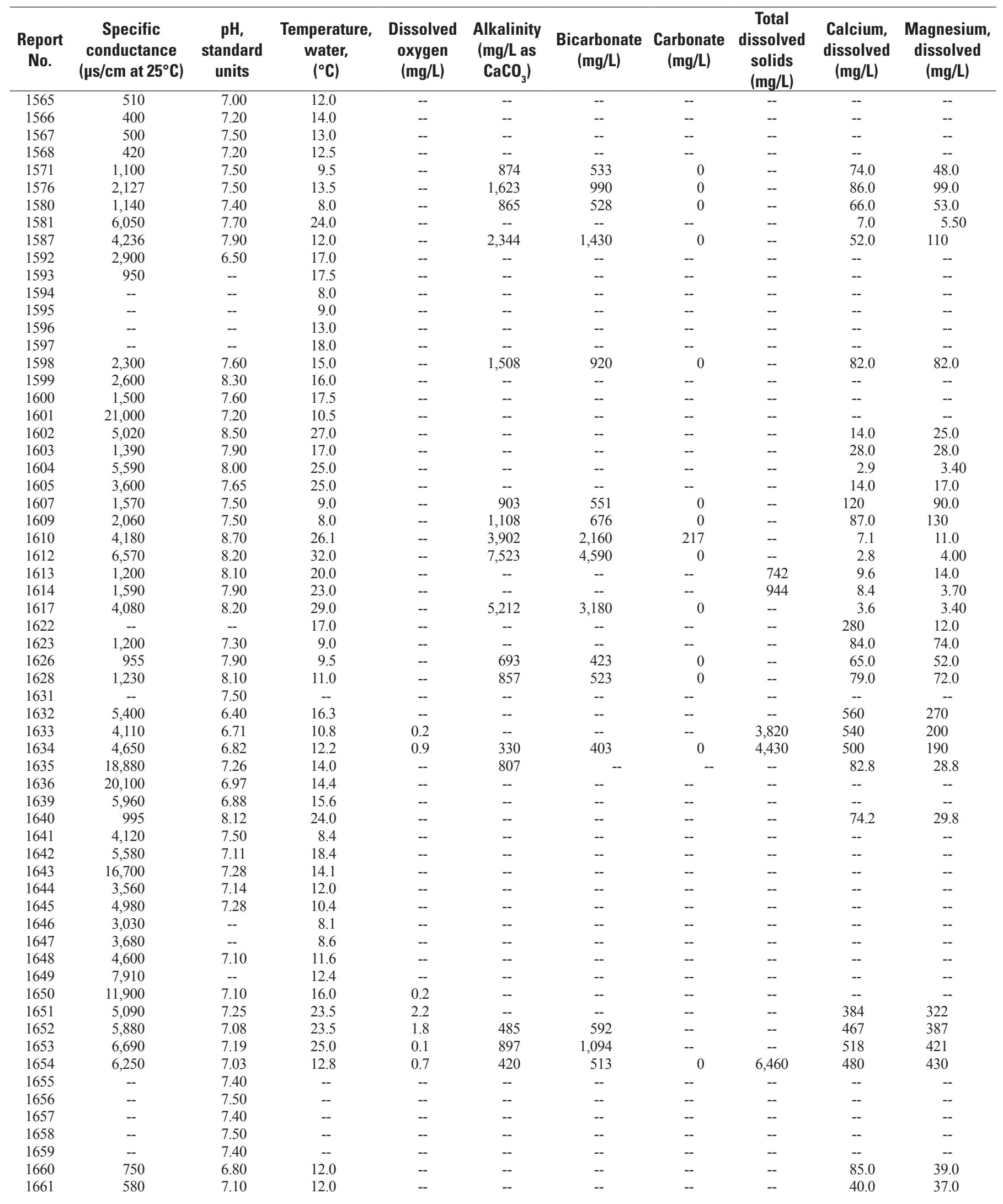


Appendix 1. Water Quality Data Used in This Report.-Continued

\begin{tabular}{|c|c|c|c|c|c|c|c|c|c|c|}
\hline $\begin{array}{l}\text { Report } \\
\text { No. }\end{array}$ & $\begin{array}{c}\text { Specific } \\
\text { conductance } \\
\left(\mu \mathrm{s} / \mathrm{cm} \text { at } 25^{\circ} \mathrm{C}\right)\end{array}$ & $\begin{array}{c}\text { pH, } \\
\text { standard } \\
\text { units }\end{array}$ & $\begin{array}{c}\text { Temperature, } \\
\text { water, } \\
\left({ }^{\circ} \mathrm{C}\right)\end{array}$ & $\begin{array}{c}\text { Dissolved } \\
\text { oxygen } \\
\text { (mg/L) }\end{array}$ & $\begin{array}{c}\text { Alkalinity } \\
\text { (mg/L as } \\
\mathrm{CaCO}_{3} \text { ) }\end{array}$ & $\begin{array}{c}\text { Bicarbonate } \\
\text { (mg/L) }\end{array}$ & $\begin{array}{c}\text { Carbonate } \\
\text { (mg/L) }\end{array}$ & $\begin{array}{c}\text { Total } \\
\text { dissolved } \\
\text { solids } \\
\text { (mg/L) }\end{array}$ & $\begin{array}{c}\text { Calcium, } \\
\text { dissolved } \\
\text { (mg/L) }\end{array}$ & $\begin{array}{c}\text { Magnesium, } \\
\text { dissolved } \\
\text { (mg/L) }\end{array}$ \\
\hline 1664 & -- & 7.80 & -- & -- & 366 & 600 & 0 & 410 & 65.6 & 39.0 \\
\hline 1665 & -- & 8.00 & -- & -- & 342 & 561 & 0 & 380 & 65.7 & 35.4 \\
\hline 1666 & -- & 7.77 & -- & -- & -- & -- & -- & -- & 38.0 & 38.0 \\
\hline 1667 & -- & 7.85 & -- & -- & -- & -- & -- & -- & 57.0 & 34.0 \\
\hline 1670 & 150 & 7.00 & 27.0 & -- & -- & -- & -- & -- & -- & -- \\
\hline 1671 & 503 & 7.90 & 18.0 & -- & -- & -- & -- & -- & 37.0 & 10.0 \\
\hline 1672 & 950 & 8.80 & 18.0 & -- & -- & -- & -- & -- & 20.0 & 0.300 \\
\hline 1673 & 980 & 8.66 & 6.5 & -- & -- & -- & -- & -- & -- & -- \\
\hline 1674 & 907 & 8.13 & 2.5 & -- & -- & -- & -- & -- & 65.3 & 23.1 \\
\hline 1675 & 3,420 & 8.30 & -- & -- & -- & -- & -- & -- & -- & -- \\
\hline 1681 & 10,640 & 7.44 & 2.5 & -- & -- & -- & -- & -- & -- & -- \\
\hline 1682 & 19,580 & 6.24 & 20.0 & -- & -- & -- & -- & -- & -- & -- \\
\hline 1683 & 10,200 & 7.25 & 25.0 & -- & -- & -- & -- & -- & 494 & 425 \\
\hline 1684 & 1,090 & 7.81 & 11.0 & 2.3 & -- & -- & -- & -- & -- & -- \\
\hline 1685 & 7,070 & 8.63 & 10.0 & 2.1 & -- & -- & -- & -- & -- & -- \\
\hline 1686 & 4,600 & 7.04 & 12.7 & 0.8 & 419 & -- & -- & -- & 338 & 220 \\
\hline 1687 & 9,290 & 10.96 & 7.0 & -- & -- & -- & -- & -- & -- & -- \\
\hline 1688 & 7,440 & 7.46 & 7.5 & 0.6 & -- & -- & -- & -- & -- & -- \\
\hline 1689 & 8,220 & 7.35 & 4.5 & 0.2 & 525 & 641 & -- & -- & 608 & 606 \\
\hline 1690 & 2,330 & 8.17 & 10.0 & -- & -- & -- & -- & -- & -- & -- \\
\hline 1691 & 13,820 & 6.91 & 17.5 & -- & -- & -- & -- & -- & -- & -- \\
\hline 1692 & 9,390 & 7.50 & 8.5 & 0.9 & -- & -- & -- & -- & -- & -- \\
\hline 1693 & 4,230 & 7.55 & 4.5 & 1.2 & 459 & 560 & -- & -- & 316 & 234 \\
\hline 1694 & 13,600 & 6.88 & 8.0 & 0.1 & -- & -- & -- & -- & -- & -- \\
\hline 1705 & 6,780 & 6.63 & 17.5 & -- & -- & -- & -- & -- & -- & -- \\
\hline 1706 & 11,210 & 6.94 & 21.5 & -- & -- & -- & -- & -- & -- & -- \\
\hline 1707 & 9,060 & 7.33 & 8.0 & 2.3 & 588 & 717 & -- & -- & 465 & 556 \\
\hline 1708 & 5,220 & 7.69 & 7.0 & 2.0 & -- & -- & -- & -- & 307 & 268 \\
\hline 1709 & 9,510 & 7.31 & 8.5 & -- & -- & -- & -- & -- & -- & -- \\
\hline 1710 & 11,100 & 7.14 & 7.0 & 0.2 & -- & -- & -- & -- & -- & -- \\
\hline 1711 & 10,840 & 6.64 & 18.5 & -- & -- & -- & -- & -- & -- & -- \\
\hline 1712 & 9,530 & 7.26 & 8.5 & 0.9 & -- & -- & -- & -- & -- & -- \\
\hline 1713 & 7,760 & 7.14 & 22.5 & -- & -- & -- & -- & -- & 430 & 396 \\
\hline 1714 & 8,040 & 7.21 & 5.0 & 2.2 & 516 & 630 & -- & -- & 522 & 473 \\
\hline 1715 & 9,020 & 7.23 & 9.5 & 1.4 & -- & -- & -- & -- & -- & -- \\
\hline 1716 & 9,540 & 6.96 & 10.0 & -- & -- & -- & -- & -- & -- & -- \\
\hline 1723 & 2,900 & 7.20 & 10.0 & -- & 951 & 580 & 0 & -- & 140 & 130 \\
\hline 1727 & 8,500 & -- & 14.0 & -- & -- & -- & -- & -- & 300 & 160 \\
\hline 1728 & 5,500 & -- & 16.0 & -- & -- & -- & -- & -- & 540 & 160 \\
\hline 1730 & 650 & 8.40 & 20.0 & -- & -- & -- & -- & -- & 86.6 & 16.5 \\
\hline 1731 & 629 & -- & 18.5 & -- & -- & -- & -- & -- & -- & -- \\
\hline 1732 & 560 & 8.02 & 19.2 & 1.4 & 225 & -- & -- & 316 & 16.0 & 8.10 \\
\hline 1733 & 806 & 9.00 & 25.5 & -- & -- & -- & -- & -- & 2.0 & 0.300 \\
\hline 1734 & 817 & 7.58 & 19.1 & -- & -- & -- & -- & -- & -- & -- \\
\hline 1735 & 6,440 & 7.26 & 8.2 & -- & -- & -- & -- & -- & -- & -- \\
\hline 1736 & 1,260 & 7.88 & 7.8 & -- & -- & -- & -- & -- & -- & -- \\
\hline
\end{tabular}


Appendix 1. Water Quality Data Used in This Report.-Continued

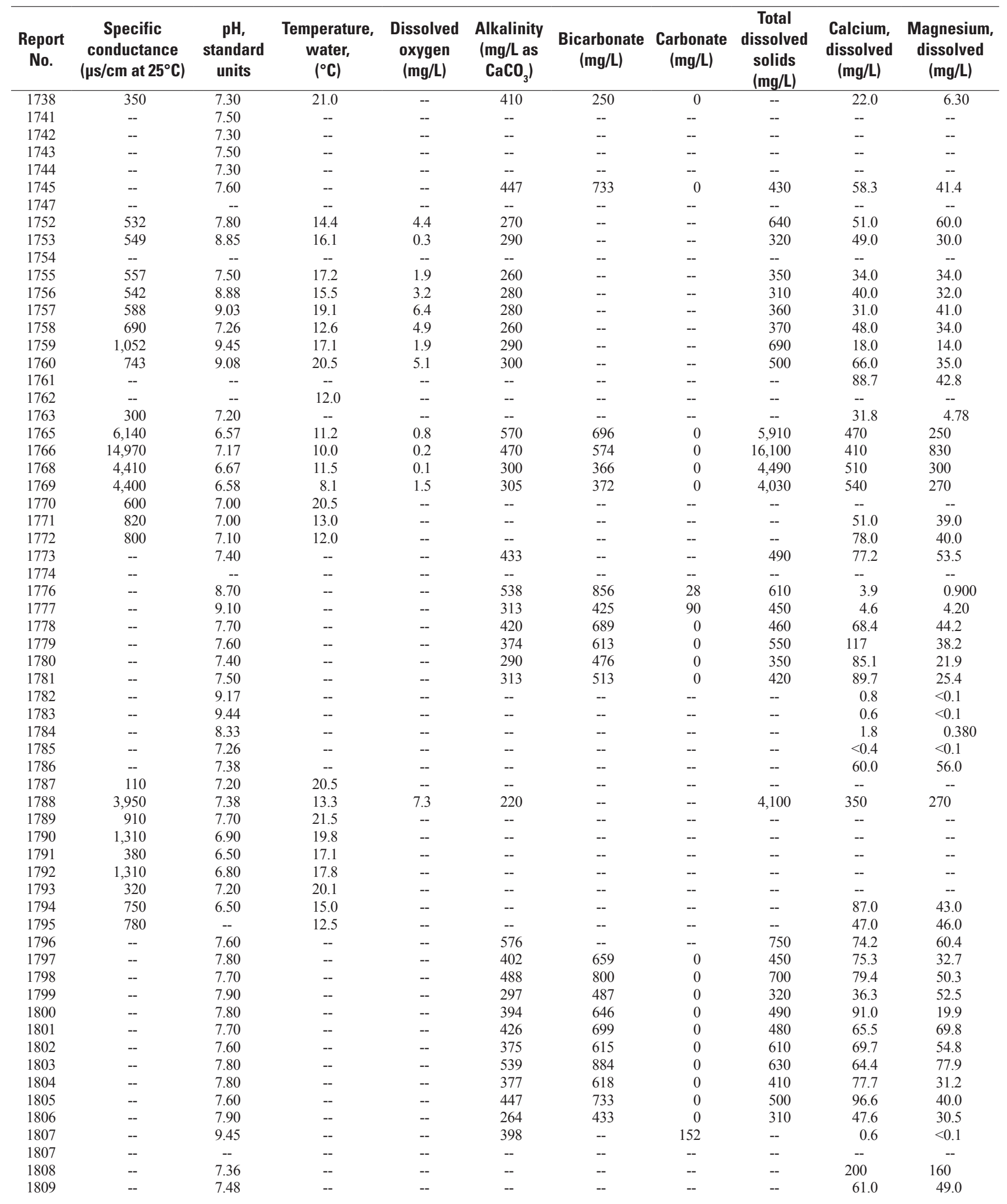


Appendix 1. Water Quality Data Used in This Report.-Continued

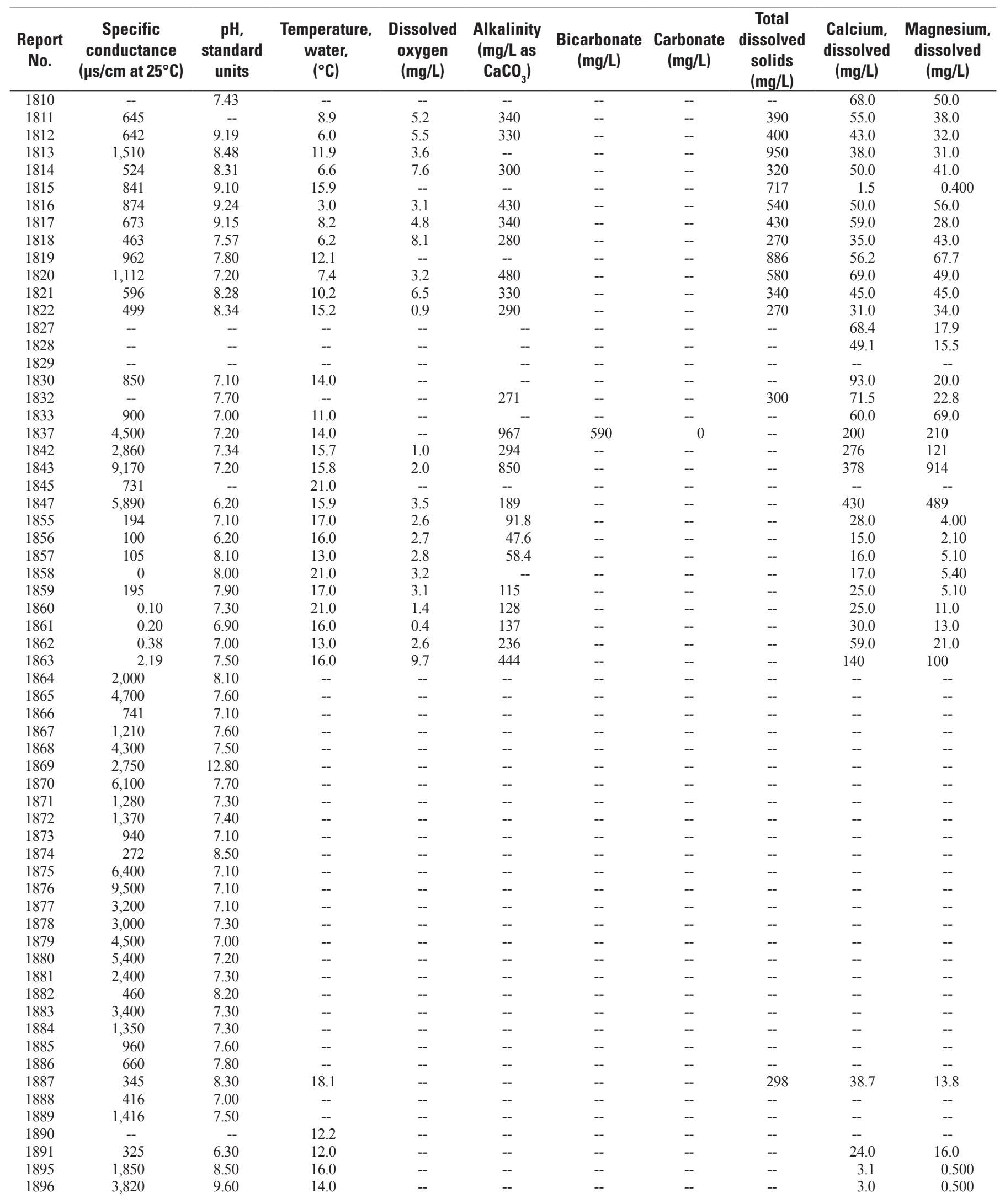


Appendix 1. Water Quality Data Used in This Report.-Continued

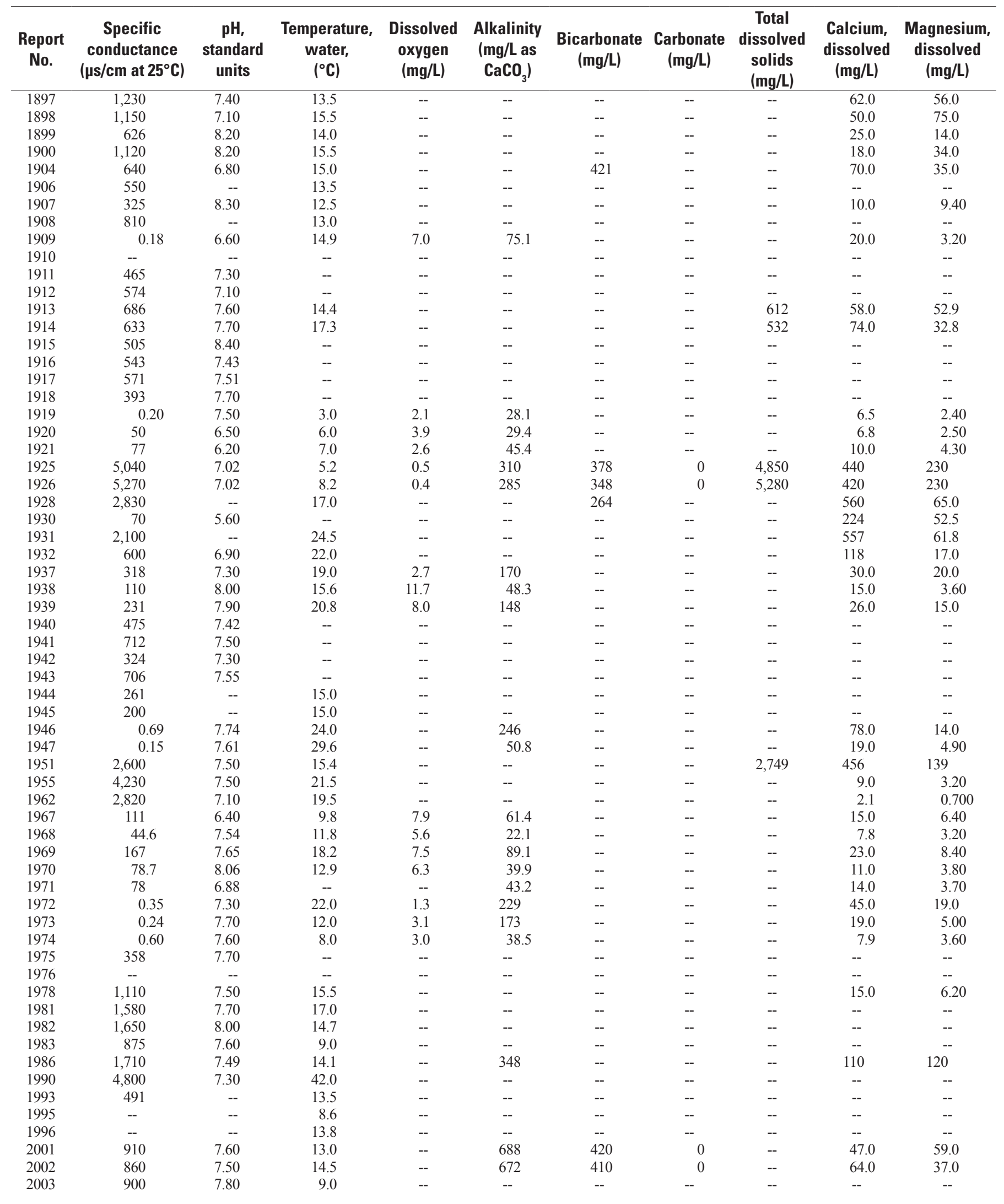


Appendix 1. Water Quality Data Used in This Report.-Continued

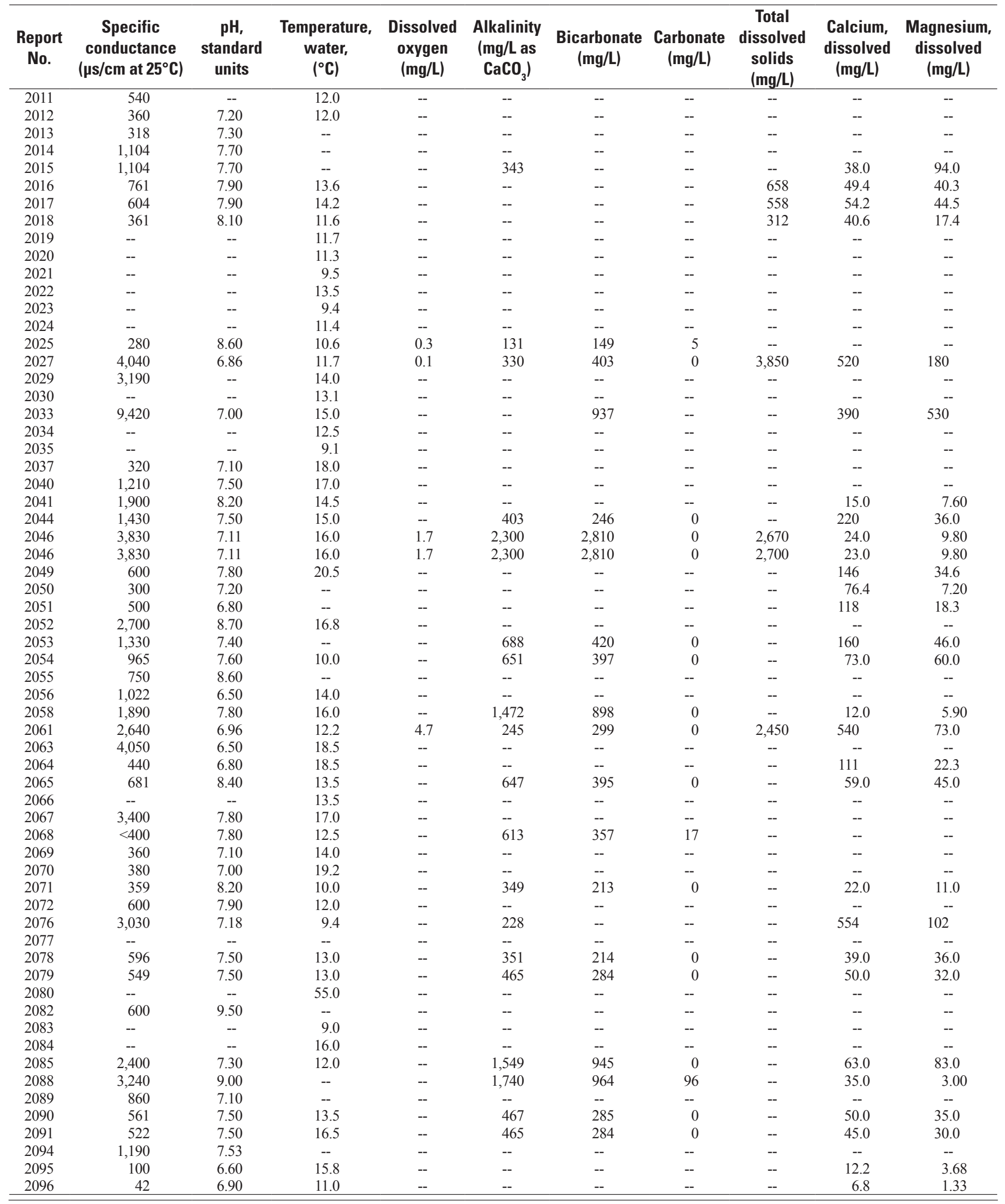


Appendix 1. Water Quality Data Used in This Report.-Continued

\begin{tabular}{|c|c|c|c|c|c|c|c|c|c|c|}
\hline $\begin{array}{c}\text { Report } \\
\text { No. }\end{array}$ & $\begin{array}{c}\text { Sodium, } \\
\text { dissolved } \\
\text { (mg/L) }\end{array}$ & $\begin{array}{l}\text { Potassium, } \\
\text { dissolved } \\
\text { (mg/L) }\end{array}$ & $\begin{array}{c}\text { Sulfate, } \\
\text { dissolved } \\
\text { (mg/L) }\end{array}$ & $\begin{array}{c}\text { Chloride, } \\
\text { dissolved } \\
\text { (mg/L) }\end{array}$ & $\begin{array}{c}\text { Fluoride, } \\
\text { dissolved } \\
\text { (mg/L) }\end{array}$ & $\begin{array}{c}\text { Bromide, } \\
\text { dissolved } \\
\text { (mg/L) }\end{array}$ & $\begin{array}{c}\text { Nitrite+Nitrate, } \\
\text { dissolved } \\
\text { (mg/L as } \mathrm{N})\end{array}$ & $\begin{array}{c}\text { Iron, } \\
\text { dissolved } \\
\text { (ug/L) }\end{array}$ & $\begin{array}{l}\text { Manganese, } \\
\text { dissolved } \\
\text { (ug/L) }\end{array}$ & $\begin{array}{c}\text { Arsenic, } \\
\text { dissolved } \\
\text { (ug/L) }\end{array}$ \\
\hline 1 & 110 & 2.50 & 24.8 & 20.7 & -- & -- & -- & $<70$ & $<5$ & -- \\
\hline 3 & 57.0 & 0.300 & 25.0 & 1.80 & 0.900 & -- & -- & $<10$ & $<10$ & 2.00 \\
\hline 4 & 140 & 1.40 & 30.0 & 7.20 & 2.20 & -- & -- & 130 & 20.0 & 1,100 \\
\hline 5 & 1,290 & 9.00 & 3,200 & 157 & 0.680 & 0.15 & 2.70 & $<50$ & 170 & $<3$ \\
\hline 6 & -- & -- & -- & -- & -- & -- & -- & -- & -- & -- \\
\hline 13 & 89.6 & 4.49 & 236 & 63.1 & 0.813 & -- & -- & -- & -- & -- \\
\hline 14 & 99.6 & 7.85 & 369 & 31.6 & 0.209 & -- & -- & -- & -- & -- \\
\hline 15 & 80.6 & 9.00 & 384 & 47.3 & 0.249 & -- & -- & -- & -- & -- \\
\hline 16 & 61.2 & 2.69 & 369 & 15.8 & 0.222 & -- & -- & -- & -- & -- \\
\hline 17 & -- & -- & -- & -- & -- & -- & -- & $<70$ & -- & -- \\
\hline 18 & -- & -- & -- & -- & -- & -- & -- & $<70$ & -- & -- \\
\hline 23 & -- & -- & -- & -- & -- & -- & -- & $<70$ & -- & -- \\
\hline 24 & -- & -- & -- & -- & -- & -- & -- & $<70$ & -- & -- \\
\hline 25 & -- & -- & -- & -- & -- & -- & -- & $<70$ & -- & -- \\
\hline 26 & -- & -- & -- & -- & -- & -- & -- & $<70$ & -- & -- \\
\hline 27 & -- & -- & -- & -- & -- & -- & -- & $<70$ & -- & -- \\
\hline 28 & -- & -- & -- & -- & -- & -- & -- & $<70$ & -- & -- \\
\hline 29 & -- & -- & -- & -- & -- & -- & -- & -- & -- & -- \\
\hline 30 & 350 & 6.30 & 1,700 & 14.0 & 0.340 & -- & $<0.29$ & 650 & 140 & -- \\
\hline 31 & -- & -- & -- & -- & -- & -- & -- & -- & -- & -- \\
\hline 33 & -- & -- & -- & -- & -- & -- & -- & -- & -- & -- \\
\hline 34 & -- & -- & -- & -- & -- & -- & -- & -- & -- & -- \\
\hline 36 & -- & -- & -- & -- & -- & -- & -- & -- & -- & -- \\
\hline 38 & 80.0 & 1.40 & 190 & 12.0 & 0.700 & -- & -- & 170 & $<10$ & 3.00 \\
\hline 39 & 220 & 0.700 & 8.70 & 10.0 & 5.60 & -- & -- & 20 & 20.0 & 8.00 \\
\hline 48 & 39.0 & 2.00 & 60.0 & 16.0 & 0.300 & $<0.1$ & 0.16 & $<70$ & $<10$ & $<20$ \\
\hline 49 & 64.0 & 2.80 & 190 & 44.0 & 0.330 & $<0.1$ & 1.06 & $<70$ & $<10$ & $<20$ \\
\hline 50 & 75.5 & 8.99 & 362 & 108 & 0.929 & 0.24 & 1.20 & 9.1 & 2.12 & -- \\
\hline 51 & 420 & 4.00 & 11.2 & 19.0 & 0.640 & -- & 0.45 & -- & $<5$ & -- \\
\hline 52 & 81.0 & 4.80 & 29.0 & 8.80 & 1.40 & -- & -- & -- & -- & -- \\
\hline 53 & 67.0 & 1.90 & 31.8 & 6.00 & 1.10 & -- & 0.61 & -- & -- & -- \\
\hline 54 & 18.0 & 1.70 & 9.50 & 7.80 & 0.250 & -- & 0.55 & -- & -- & -- \\
\hline 55 & 74.3 & 5.00 & 20.0 & 10.0 & 0.600 & -- & -- & -- & 8.00 & -- \\
\hline 56 & 96.0 & 4.70 & 401 & 40.6 & 1.60 & -- & 0.079 & -- & -- & -- \\
\hline 57 & 230 & 2.10 & 622 & 102 & 5.60 & -- & 0.081 & -- & -- & -- \\
\hline 58 & 51.0 & 5.30 & 117 & 6.30 & 1.30 & -- & 0.14 & -- & -- & -- \\
\hline 59 & 90.0 & 6.20 & 72.2 & 30.6 & 1.40 & -- & 0.10 & -- & -- & -- \\
\hline 60 & 130 & 14.0 & 60.0 & 28.3 & 0.980 & -- & 0.17 & -- & -- & -- \\
\hline 61 & 30.0 & -- & 14.0 & 5.30 & 1.00 & -- & 0.21 & -- & -- & -- \\
\hline 62 & -- & -- & 33.0 & 12.0 & 0.600 & -- & $<0.29$ & -- & -- & $<1$ \\
\hline 63 & 93.0 & -- & -- & 18.7 & -- & -- & -- & -- & -- & -- \\
\hline 64 & -- & -- & -- & -- & -- & -- & -- & -- & -- & -- \\
\hline 65 & 260 & -- & -- & 30.4 & -- & -- & -- & -- & -- & -- \\
\hline 66 & 320 & -- & -- & 64.4 & -- & -- & -- & -- & -- & -- \\
\hline 67 & 195 & 0.500 & 30.0 & 35.0 & 5.10 & -- & $<0.29$ & -- & -- & -- \\
\hline 68 & 140 & 1.30 & 140 & 21.0 & 1.10 & -- & 0.23 & -- & -- & -- \\
\hline 69 & 350 & -- & -- & -- & -- & -- & -- & -- & -- & -- \\
\hline
\end{tabular}


Appendix 1. Water Quality Data Used in This Report.-Continued

\begin{tabular}{|c|c|c|c|c|c|c|c|c|c|c|}
\hline $\begin{array}{c}\text { Report } \\
\text { No. }\end{array}$ & $\begin{array}{c}\text { Sodium, } \\
\text { dissolved } \\
\text { (mg/L) }\end{array}$ & $\begin{array}{c}\text { Potassium, } \\
\text { dissolved } \\
\text { (mg/L) }\end{array}$ & $\begin{array}{c}\text { Sulfate, } \\
\text { dissolved } \\
\text { (mg/L) }\end{array}$ & $\begin{array}{c}\text { Chloride, } \\
\text { dissolved } \\
(\mathrm{mg} / \mathrm{L})\end{array}$ & $\begin{array}{c}\text { Fluoride, } \\
\text { dissolved } \\
\text { (mg/L) } \\
\end{array}$ & $\begin{array}{c}\text { Bromide, } \\
\text { dissolved } \\
\text { (mg/L) }\end{array}$ & $\begin{array}{c}\text { Nitrite+Nitrate, } \\
\text { dissolved } \\
\text { (mg/L as } \mathrm{N})\end{array}$ & $\begin{array}{c}\text { Iron, } \\
\text { dissolved } \\
\text { (ug/L) } \\
\end{array}$ & $\begin{array}{c}\text { Manganese, } \\
\text { dissolved } \\
\text { (ug/L) }\end{array}$ & $\begin{array}{c}\text { Arsenic, } \\
\text { dissolved } \\
\text { (ug/L) } \\
\end{array}$ \\
\hline 70 & 440 & -- & -- & -- & -- & -- & -- & -- & -- & -- \\
\hline 72 & 61.0 & 1.90 & 27.0 & 5.20 & 0.870 & -- & 0.27 & -- & -- & -- \\
\hline 73 & 95.0 & 3.60 & 42.0 & 5.80 & 1.30 & -- & 0.34 & -- & -- & -- \\
\hline 74 & 98.0 & 3.60 & 45.0 & 6.10 & 1.20 & -- & 0.32 & -- & -- & -- \\
\hline 75 & 120 & 7.20 & 38.0 & 7.30 & 0.440 & -- & 0.27 & -- & -- & -- \\
\hline 78 & 205 & 1.10 & 20.0 & 7.00 & 1.70 & $<0.1$ & 0.21 & -- & -- & -- \\
\hline 79 & 60.0 & 0.810 & 45.4 & 5.67 & 1.20 & 0.080 & $<0.29$ & $<70$ & 35.0 & -- \\
\hline 80 & 80.0 & 4.70 & 25.0 & 10.0 & 1.00 & $<0.1$ & 0.29 & $<70$ & $<10$ & -- \\
\hline 81 & 28.6 & 2.94 & 16.8 & 3.51 & 0.820 & $<0.1$ & 0.41 & $<70$ & $<5$ & -- \\
\hline 82 & 24.8 & 1.99 & 24.2 & 7.11 & 0.680 & 0.050 & 3.91 & $<70$ & $<5$ & -- \\
\hline 83 & 200 & 1.07 & 114 & 93.1 & 0.700 & 0.52 & 19.45 & 392 & $<5$ & -- \\
\hline 88 & 45.9 & 3.11 & 18.8 & 5.42 & 0.890 & 0.090 & $<0.29$ & $<70$ & 459 & -- \\
\hline 89 & 197 & 0.415 & 8.30 & 69.3 & 3.30 & 0.33 & 0.11 & $<70$ & $<5$ & -- \\
\hline 90 & -- & -- & -- & 12.7 & -- & -- & -- & -- & -- & -- \\
\hline 91 & 249 & 0.600 & 20.0 & 151 & 3.50 & -- & $<0.29$ & $<70$ & 19.0 & 0.003 \\
\hline 92 & 55.0 & 5.20 & 68.0 & 9.90 & 0.880 & -- & 0.34 & $<70$ & $<5$ & -- \\
\hline 93 & 200 & 0.380 & 1.10 & 105 & 3.00 & -- & $<0.29$ & 420 & 27.0 & -- \\
\hline 94 & 96.0 & 4.70 & 401 & 40.6 & 1.60 & -- & 0.079 & 2,200 & 48.0 & -- \\
\hline 95 & 230 & 2.10 & 622 & 102 & 5.60 & -- & 0.081 & 210 & 140 & -- \\
\hline 96 & 90.0 & 6.20 & 72.2 & 30.6 & 1.40 & -- & 0.10 & 1,300 & 220 & -- \\
\hline 97 & 51.0 & 5.30 & 117 & 6.30 & 1.30 & -- & 0.14 & 930 & 14.0 & -- \\
\hline 98 & 260 & -- & -- & -- & -- & -- & -- & -- & -- & -- \\
\hline 99 & 130 & -- & -- & -- & -- & -- & -- & -- & -- & -- \\
\hline 100 & 130 & -- & -- & -- & -- & -- & -- & -- & -- & -- \\
\hline 110 & 219 & 0.816 & 49.0 & 137 & 5.50 & -- & $<0.29$ & $<70$ & 6.60 & -- \\
\hline 111 & 43.0 & 1.40 & 40.0 & 3.59 & 1.40 & -- & 0.19 & $<70$ & $<5$ & -- \\
\hline 112 & 33.0 & 3.20 & 31.1 & 6.32 & 0.620 & -- & $<0.29$ & 410 & 2,100 & -- \\
\hline 113 & 260 & -- & -- & -- & -- & -- & -- & -- & -- & -- \\
\hline 114 & 260 & -- & -- & -- & -- & -- & -- & -- & -- & -- \\
\hline 115 & 68.0 & -- & -- & -- & -- & -- & -- & -- & -- & -- \\
\hline 116 & -- & -- & -- & -- & -- & -- & -- & -- & -- & -- \\
\hline 117 & 160 & -- & -- & -- & -- & -- & -- & -- & -- & -- \\
\hline 118 & 37.0 & -- & -- & 32.5 & -- & -- & -- & -- & -- & -- \\
\hline 119 & 86.0 & -- & -- & -- & -- & -- & -- & -- & -- & -- \\
\hline 120 & 140 & -- & -- & 35.6 & -- & -- & -- & -- & -- & -- \\
\hline 121 & 190 & -- & -- & -- & -- & -- & -- & -- & -- & -- \\
\hline 122 & 130 & -- & -- & -- & -- & -- & -- & -- & -- & -- \\
\hline 123 & 280 & -- & -- & -- & -- & -- & -- & -- & -- & -- \\
\hline 124 & 130 & 14.0 & 60.0 & 28.3 & 0.980 & -- & 0.17 & 270 & $<5$ & -- \\
\hline 125 & 210 & 1.00 & 19.0 & 5.70 & 1.80 & -- & 0.21 & $<70$ & $<5$ & -- \\
\hline 126 & 217 & 1.10 & 310 & 46.0 & 2.40 & -- & -- & 30 & 6.00 & 0.001 \\
\hline 127 & 32.5 & 3.44 & 11.5 & 4.21 & 0.740 & -- & 0.095 & 242 & 55.2 & -- \\
\hline 128 & 150 & 1.80 & 98.8 & 25.3 & 1.50 & -- & 1.33 & 290 & $<5$ & -- \\
\hline 129 & 143 & 1.69 & 113 & 35.7 & 1.30 & -- & 1.72 & $<70$ & $<5$ & -- \\
\hline 130 & 180 & 3.30 & 660 & 49.0 & 2.50 & -- & $<0.29$ & $<70$ & 220 & -- \\
\hline 131 & 140 & 4.80 & 570 & 55.0 & 2.70 & -- & $<0.29$ & 6,000 & 270 & -- \\
\hline 132 & 85.8 & 3.10 & 50.0 & 8.00 & 0.800 & -- & 1.63 & 40 & $<5$ & 0.001 \\
\hline
\end{tabular}


Appendix 1. Water Quality Data Used in This Report.-Continued

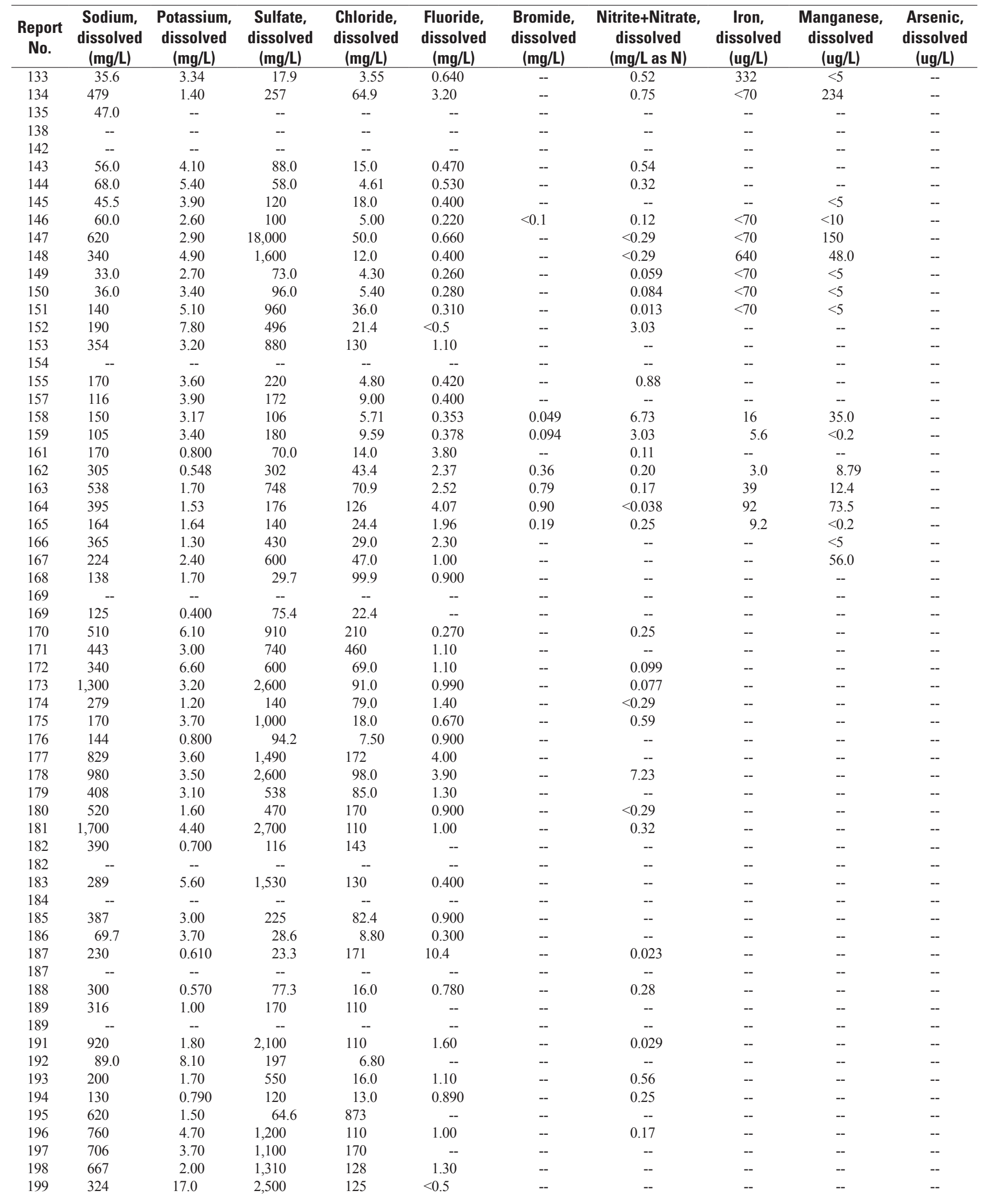


Appendix 1. Water Quality Data Used in This Report.-Continued

\begin{tabular}{|c|c|c|c|c|c|c|c|c|c|c|}
\hline $\begin{array}{c}\text { Report } \\
\text { No. }\end{array}$ & $\begin{array}{c}\text { Sodium, } \\
\text { dissolved } \\
\text { (mg/L) } \\
\end{array}$ & $\begin{array}{c}\text { Potassium, } \\
\text { dissolved } \\
\text { (mg/L) }\end{array}$ & $\begin{array}{c}\text { Sulfate, } \\
\text { dissolved } \\
\text { (mg/L) } \\
\end{array}$ & $\begin{array}{c}\text { Chloride, } \\
\text { dissolved } \\
(\mathrm{mg} / \mathrm{L}) \\
\end{array}$ & $\begin{array}{c}\text { Fluoride, } \\
\text { dissolved } \\
\text { (mg/L) } \\
\end{array}$ & $\begin{array}{c}\text { Bromide, } \\
\text { dissolved } \\
\text { (mg/L) }\end{array}$ & $\begin{array}{c}\text { Nitrite+Nitrate, } \\
\text { dissolved } \\
\text { (mg/L as } \mathrm{N}) \\
\end{array}$ & $\begin{array}{c}\text { Iron, } \\
\text { dissolved } \\
\text { (ug/L) } \\
\end{array}$ & $\begin{array}{c}\text { Manganese, } \\
\text { dissolved } \\
\text { (ug/L) }\end{array}$ & $\begin{array}{c}\text { Arsenic, } \\
\text { dissolved } \\
\text { (ug/L) }\end{array}$ \\
\hline 200 & 340 & 2.40 & 390 & 48.0 & 1.40 & -- & 0.099 & -- & -- & -- \\
\hline 201 & 122 & 0.700 & 71.6 & 10.6 & -- & -- & -- & -- & -- & -- \\
\hline 202 & 190 & 2.60 & 660 & 34.0 & 0.810 & -- & 0.16 & -- & -- & -- \\
\hline 203 & 492 & 1.50 & 479 & 111 & 1.50 & -- & -- & -- & -- & -- \\
\hline 203 & -- & -- & -- & -- & -- & -- & -- & -- & -- & -- \\
\hline 205 & -- & -- & -- & -- & -- & -- & -- & -- & -- & -- \\
\hline 206 & 275 & 0.700 & 240 & 30.0 & 1.90 & $<0.1$ & 0.14 & -- & -- & -- \\
\hline 207 & -- & -- & -- & -- & -- & -- & -- & -- & -- & -- \\
\hline 208 & 200 & 1.50 & 229 & 12.6 & -- & -- & 1.93 & -- & -- & -- \\
\hline 209 & 250 & 3.70 & -- & 32.0 & 0.360 & -- & 6.00 & -- & -- & -- \\
\hline 211 & 110 & 1.80 & 130 & 29.0 & 0.220 & -- & 23.00 & -- & -- & -- \\
\hline 216 & 909 & 2.10 & 40.0 & 1,790 & -- & -- & -- & -- & -- & -- \\
\hline 216 & -- & -- & -- & -- & -- & -- & -- & -- & -- & -- \\
\hline 217 & 81.5 & 1.40 & 160 & 49.0 & 1.20 & -- & 0.12 & -- & -- & -- \\
\hline 218 & 190 & 1.20 & 20.0 & 115 & 2.90 & -- & 0.009 & -- & -- & -- \\
\hline 219 & 162 & 3.50 & 300 & 70.0 & $<0.5$ & -- & -- & -- & -- & -- \\
\hline 220 & 438 & 1.70 & 265 & 277 & 3.50 & -- & -- & -- & -- & -- \\
\hline 221 & 690 & 2.20 & 1,300 & 150 & 1.00 & -- & 0.027 & -- & -- & -- \\
\hline 222 & 451 & 2.60 & 468 & 389 & 2.30 & -- & -- & -- & -- & -- \\
\hline 223 & -- & -- & -- & -- & -- & -- & -- & -- & -- & -- \\
\hline 224 & 170 & 2.48 & 638 & 77.5 & 0.710 & -- & $<0.29$ & $<70$ & 364 & -- \\
\hline 225 & 340 & 3.60 & 383 & 46.7 & 1.50 & -- & 1.86 & 490 & $<5$ & -- \\
\hline 226 & 450 & 1.30 & 6.00 & 560 & 6.00 & -- & $<0.29$ & $<70$ & 280 & -- \\
\hline 227 & 1,000 & 5.40 & 1,600 & 400 & 1.20 & -- & 0.43 & -- & -- & -- \\
\hline 236 & 480 & 160 & 600 & 136 & 2.30 & -- & 0.50 & -- & -- & -- \\
\hline 237 & 213 & 4.10 & 80.0 & 117 & 1.10 & -- & -- & 20 & $<5$ & 0.001 \\
\hline 238 & 365 & 6.67 & 220 & 154 & 5.50 & -- & $<0.29$ & $<70$ & 35.3 & -- \\
\hline 239 & 74.0 & 3.90 & 120 & 3.70 & 0.240 & -- & 0.072 & 71 & 12.0 & -- \\
\hline 240 & 215 & 0.800 & 130 & 65.0 & 1.30 & -- & -- & 180 & $<5$ & 0.004 \\
\hline 241 & 78.3 & 0.800 & 90.0 & 11.0 & 0.500 & -- & 0.39 & -- & $<5$ & -- \\
\hline 242 & 144 & 0.600 & 90.0 & 24.0 & 1.10 & -- & $<0.29$ & -- & 493 & -- \\
\hline 243 & 122 & 3.60 & 200 & 9.00 & 0.400 & -- & -- & -- & $<5$ & -- \\
\hline 244 & 149 & 3.00 & 110 & 24.0 & 0.500 & $<0.1$ & 0.032 & -- & -- & -- \\
\hline 245 & 59.5 & 0.900 & 20.0 & 14.0 & 0.900 & -- & -- & -- & 31.0 & -- \\
\hline 246 & 219 & 1.68 & 687 & 49.9 & 1.00 & -- & 2.18 & $<70$ & 6.10 & -- \\
\hline 247 & 170 & 2.60 & 74.0 & 15.0 & 1.00 & -- & 0.027 & $<70$ & 13.0 & $<1$ \\
\hline 248 & 745 & 1.80 & 1,040 & 140 & 1.70 & -- & -- & -- & -- & -- \\
\hline 249 & 717 & 1.50 & 1,510 & 130 & 1.70 & 2.00 & -- & -- & -- & -- \\
\hline 250 & 340 & 5.00 & 830 & 290 & 0.510 & -- & 3.39 & -- & -- & -- \\
\hline 251 & 578 & 3.20 & 820 & 190 & 2.00 & -- & -- & -- & -- & -- \\
\hline 252 & 349 & 3.20 & 204 & 73.5 & 2.30 & -- & -- & -- & -- & -- \\
\hline 253 & 576 & 1.90 & 750 & 80.0 & 2.70 & -- & -- & -- & -- & -- \\
\hline 254 & 677 & 1.60 & 1,080 & 250 & 4.20 & -- & -- & -- & -- & -- \\
\hline 255 & 490 & 1.20 & 747 & 86.5 & 3.40 & -- & $<0.29$ & -- & 14.0 & -- \\
\hline 256 & 330 & 0.560 & 77.0 & 150 & 5.10 & 1.10 & 0.093 & -- & -- & -- \\
\hline 256 & -- & -- & -- & -- & -- & -- & -- & -- & -- & -- \\
\hline 257 & 500 & 3.70 & 660 & 98.0 & 1.60 & $<0.1$ & $<0.29$ & -- & -- & -- \\
\hline 258 & 266 & 1.20 & 79.1 & 69.3 & 3.10 & -- & -- & -- & -- & -- \\
\hline
\end{tabular}


Appendix 1. Water Quality Data Used in This Report.-Continued

\begin{tabular}{|c|c|c|c|c|c|c|c|c|c|c|}
\hline $\begin{array}{c}\text { Report } \\
\text { No. }\end{array}$ & $\begin{array}{c}\text { Sodium, } \\
\text { dissolved } \\
\text { (mg/L) }\end{array}$ & $\begin{array}{c}\text { Potassium, } \\
\text { dissolved } \\
\text { (mg/L) }\end{array}$ & $\begin{array}{c}\text { Sulfate, } \\
\text { dissolved } \\
\text { (mg/L) }\end{array}$ & $\begin{array}{c}\text { Chloride, } \\
\text { dissolved } \\
\text { (mg/L) }\end{array}$ & $\begin{array}{c}\text { Fluoride, } \\
\text { dissolved } \\
\text { (mg/L) }\end{array}$ & $\begin{array}{c}\text { Bromide, } \\
\text { dissolved } \\
\text { (mg/L) }\end{array}$ & $\begin{array}{c}\text { Nitrite+Nitrate, } \\
\text { dissolved } \\
(\mathrm{mg} / \mathrm{L} \text { as } \mathrm{N})\end{array}$ & $\begin{array}{c}\text { Iron, } \\
\text { dissolved } \\
\text { (ug/L) }\end{array}$ & $\begin{array}{c}\text { Manganese, } \\
\text { dissolved } \\
\text { (ug/L) }\end{array}$ & $\begin{array}{c}\text { Arsenic, } \\
\text { dissolved } \\
\text { (ug/L) }\end{array}$ \\
\hline 259 & 700 & 3.00 & 1,300 & 73.0 & 1.50 & $<0.1$ & 0.075 & -- & -- & -- \\
\hline 260 & 134 & 1.60 & 38.3 & 28.1 & 2.50 & -- & -- & -- & -- & -- \\
\hline 261 & 57.5 & 1.60 & 6.10 & 8.80 & 0.900 & -- & -- & -- & -- & -- \\
\hline 262 & 213 & 4.10 & 80.0 & 117 & 1.10 & 0.80 & 1.38 & -- & -- & -- \\
\hline 263 & 378 & 1.50 & $<5$ & 321 & 2.70 & 1.00 & 0.009 & -- & -- & -- \\
\hline 265 & 319 & 1.50 & 160 & 26.0 & 1.00 & 0.20 & $<0.29$ & -- & -- & -- \\
\hline 266 & 238 & 0.500 & 60.0 & 260 & 9.50 & 2.00 & 0.23 & -- & -- & -- \\
\hline 267 & 218 & 3.81 & 1,110 & 20.2 & 0.660 & -- & 2.06 & $<70$ & 5.97 & -- \\
\hline 268 & 220 & 1.80 & 430 & 15.0 & 0.930 & -- & -- & $<70$ & 11.0 & $<1$ \\
\hline 269 & 1,500 & 3.10 & 1,720 & 151 & -- & -- & -- & 730 & 19.0 & -- \\
\hline 270 & 1,300 & 3.10 & -- & 97.0 & 4.00 & -- & 4.74 & $<70$ & $<5$ & -- \\
\hline 275 & 270 & 4.20 & 140 & 78.0 & 2.60 & $<0.1$ & 0.27 & $<70$ & 800 & -- \\
\hline 276 & 520 & 1.60 & 830 & 62.0 & 1.20 & $<0.1$ & $<0.29$ & $<70$ & 22.0 & -- \\
\hline 277 & 633 & 1.53 & 911 & 263 & 3.30 & 2.40 & 2.01 & $<70$ & 27.5 & -- \\
\hline 278 & 312 & 2.17 & 459 & 65.5 & 1.80 & 0.80 & 0.59 & $<70$ & $<5$ & -- \\
\hline 279 & 756 & 4.64 & 1,380 & 287 & 1.20 & 3.14 & 2.78 & $<70$ & $<5$ & -- \\
\hline 280 & 439 & 1.07 & 514 & 173 & 1.20 & 1.10 & $<0.29$ & $<70$ & 5.93 & -- \\
\hline 281 & 490 & 2.40 & 168 & 47.4 & 2.80 & 0.30 & 0.67 & $<70$ & $<10$ & -- \\
\hline 282 & 510 & 1.30 & 299 & 492 & 2.20 & 2.76 & $<0.29$ & $<70$ & 113 & -- \\
\hline 283 & 300 & 1.00 & 11.0 & 390 & 8.00 & 1.80 & $<0.29$ & -- & 14.0 & -- \\
\hline 284 & 170 & 0.950 & -- & -- & -- & -- & 0.063 & $<70$ & $<10$ & -- \\
\hline 285 & 320 & 0.660 & 320 & 29.0 & 1.70 & $<0.1$ & $<0.29$ & $<70$ & 11.0 & $<20$ \\
\hline 286 & 374 & 0.644 & 316 & 111 & 4.00 & 0.69 & 3.73 & $<70$ & $<5$ & -- \\
\hline 287 & 560 & 1.50 & 600 & 380 & 2.50 & 3.80 & 0.036 & $<70$ & $<10$ & -- \\
\hline 297 & 238 & 0.700 & 70.0 & 45.0 & 2.60 & -- & -- & $<70$ & $<5$ & 0.004 \\
\hline 298 & 149 & 3.00 & 110 & 24.0 & 0.500 & -- & -- & $<70$ & 191 & 0.001 \\
\hline 299 & 340 & 2.60 & 560 & 97.0 & 1.00 & -- & $<0.29$ & $<70$ & 1,600 & -- \\
\hline 300 & 660 & 1.30 & 190 & 663 & 4.60 & 2.75 & $<0.29$ & 220 & 73.0 & -- \\
\hline 301 & 640 & 1.30 & 189 & 661 & 4.70 & 2.74 & $<0.29$ & 210 & 69.0 & -- \\
\hline 302 & -- & -- & -- & 6.90 & -- & -- & -- & -- & -- & -- \\
\hline 303 & 320 & 2.00 & 310 & 180 & 3.90 & -- & 2.10 & 2,000 & 20.0 & -- \\
\hline 304 & 990 & -- & 2,700 & 150 & 1.20 & 1.50 & 0.79 & $<70$ & 130 & -- \\
\hline 305 & 550 & 2.30 & 870 & -- & 0.730 & $<0.1$ & $<0.29$ & $<70$ & 14.0 & -- \\
\hline 306 & 340 & 1.00 & 260 & -- & 2.80 & $<0.1$ & $<0.29$ & $<70$ & 48.0 & -- \\
\hline 307 & 388 & 1.10 & 196 & 348 & 5.40 & -- & $<0.29$ & $<70$ & 51.8 & -- \\
\hline 308 & 250 & 0.810 & 80.0 & 97.0 & 3.50 & -- & 0.084 & $<70$ & $<5$ & $<1$ \\
\hline 309 & 400 & 1.40 & 200 & 150 & 1.30 & -- & 0.54 & $<70$ & $<5$ & -- \\
\hline 310 & 610 & 1.70 & $<2.5$ & 1,000 & 4.70 & 6.00 & $<0.29$ & $<70$ & 54.0 & -- \\
\hline 311 & 240 & 0.580 & 77.0 & 100 & 1.50 & -- & $<0.29$ & 110 & 18.0 & -- \\
\hline 312 & 170 & 5.80 & 130 & 5.00 & 0.330 & -- & 0.27 & $<70$ & 280 & -- \\
\hline 313 & 940 & 5.50 & 820 & 750 & 1.10 & -- & 0.047 & 1,600 & 53.0 & -- \\
\hline 314 & 520 & 3.60 & 930 & 810 & 0.990 & -- & 0.99 & 260 & $<5$ & -- \\
\hline 315 & 95.0 & 11.0 & 142 & 9.90 & -- & -- & -- & 14,000 & 74.0 & -- \\
\hline 316 & 930 & 1.90 & $<2.5$ & 1,560 & 2.40 & 4.00 & $<0.29$ & 300 & 200 & -- \\
\hline 317 & 490 & 2.70 & 530 & 110 & 1.10 & -- & $<0.29$ & 160 & 16.0 & -- \\
\hline 318 & 130 & 0.810 & 110 & 42.6 & 1.10 & -- & 0.10 & 840 & 30.0 & -- \\
\hline 319 & 220 & 2.40 & -- & -- & -- & -- & 0.32 & $<70$ & 42.0 & -- \\
\hline 320 & 91.0 & 2.05 & 58.0 & 11.1 & 1.80 & -- & 0.25 & $<70$ & $<5$ & -- \\
\hline
\end{tabular}


Appendix 1. Water Quality Data Used in This Report.-Continued

\begin{tabular}{|c|c|c|c|c|c|c|c|c|c|c|}
\hline $\begin{array}{c}\text { Report } \\
\text { No. }\end{array}$ & $\begin{array}{c}\text { Sodium, } \\
\text { dissolved } \\
\text { (mg/L) } \\
\end{array}$ & $\begin{array}{c}\text { Potassium, } \\
\text { dissolved } \\
\text { (mg/L) }\end{array}$ & $\begin{array}{c}\text { Sulfate, } \\
\text { dissolved } \\
\text { (mg/L) } \\
\end{array}$ & $\begin{array}{c}\text { Chloride, } \\
\text { dissolved } \\
\text { (mg/L) } \\
\end{array}$ & $\begin{array}{c}\text { Fluoride, } \\
\text { dissolved } \\
\text { (mg/L) } \\
\end{array}$ & $\begin{array}{c}\text { Bromide, } \\
\text { dissolved } \\
(\mathrm{mg} / \mathrm{L}) \\
\end{array}$ & $\begin{array}{c}\text { Nitrite+Nitrate, } \\
\text { dissolved } \\
\text { (mg/L as N) } \\
\end{array}$ & $\begin{array}{c}\text { Iron, } \\
\text { dissolved } \\
\text { (ug/L) } \\
\end{array}$ & $\begin{array}{c}\text { Manganese, } \\
\text { dissolved } \\
\text { (ug/L) }\end{array}$ & $\begin{array}{c}\text { Arsenic, } \\
\text { dissolved } \\
\text { (ug/L) } \\
\end{array}$ \\
\hline 321 & 480 & 1.40 & 452 & 127 & 2.30 & -- & 3.34 & 220 & 14.0 & -- \\
\hline 323 & 348 & 1.30 & 150 & 195 & 1.20 & -- & 6.37 & $<70$ & 13.0 & 0.002 \\
\hline 324 & 758 & 3.25 & 1,070 & 293 & 0.910 & -- & 16.85 & $<70$ & $<5$ & -- \\
\hline 325 & 380 & 0.750 & 44.0 & 500 & 4.40 & -- & $<0.29$ & 160 & 45.0 & -- \\
\hline 326 & 350 & 0.910 & 180 & 170 & 2.60 & -- & 0.86 & 300 & 20.0 & -- \\
\hline 329 & 380 & 1.40 & 150 & 280 & 2.10 & -- & 0.25 & 130 & 25.0 & -- \\
\hline 330 & 191 & 2.40 & 130 & 12.0 & 1.00 & -- & -- & $<70$ & $<5$ & 0.001 \\
\hline 331 & 378 & 1.50 & $<2.5$ & 321 & 2.70 & -- & 0.040 & $<70$ & 37.0 & 0.002 \\
\hline 332 & 1,100 & 3.09 & 2,670 & 92.7 & 1.50 & 1.13 & 1.01 & $<70$ & 59.1 & -- \\
\hline 333 & 290 & 0.590 & -- & -- & -- & -- & $<0.29$ & $<70$ & 43.0 & $<20$ \\
\hline 334 & 702 & 1.96 & 1,350 & 154 & 1.50 & -- & $<0.29$ & $<70$ & 136 & -- \\
\hline 339 & 244 & 5.43 & 1,520 & 114 & 0.490 & -- & 0.39 & $<70$ & 25.0 & -- \\
\hline 340 & 468 & 2.20 & 730 & 158 & 1.40 & -- & $<0.29$ & 160 & 404 & 0.002 \\
\hline 341 & 2,100 & 4.40 & 160 & 3,500 & 1.90 & -- & -- & 260 & 27.0 & -- \\
\hline 342 & 200 & 1.50 & 140 & 18.0 & -- & -- & -- & 110 & -- & -- \\
\hline 343 & 300 & 1.60 & 140 & 24.0 & 1.80 & -- & -- & -- & -- & -- \\
\hline 344 & 304 & 0.975 & 246 & 132 & 5.40 & -- & $<0.29$ & $<70$ & 16.9 & -- \\
\hline 345 & -- & -- & -- & -- & -- & -- & -- & -- & -- & -- \\
\hline 346 & 1,300 & 4.08 & 2,890 & 115 & 1.80 & -- & 1.56 & $<70$ & 6.61 & -- \\
\hline 347 & 232 & 0.500 & 80.0 & 20.0 & 1.20 & -- & $<0.29$ & 70 & 155 & 0.002 \\
\hline 348 & 160 & 2.20 & 760 & 28.0 & 0.510 & -- & -- & 470 & 510 & -- \\
\hline 349 & 210 & 2.20 & 30.0 & 14.0 & 1.60 & -- & -- & 30 & $<5$ & 0.001 \\
\hline 350 & 190 & 1.60 & 40.0 & 25.0 & 1.40 & -- & -- & $<70$ & $<5$ & 0.002 \\
\hline 351 & 1,400 & 6.70 & 3,200 & 84.0 & 0.860 & -- & 0.13 & -- & -- & -- \\
\hline 361 & 211 & 0.411 & 17.6 & 135 & 11.0 & -- & $<0.29$ & $<70$ & 10.4 & -- \\
\hline 362 & 390 & 0.470 & 186 & 174 & 4.10 & -- & 0.099 & $<70$ & 190 & -- \\
\hline 363 & 660 & 1.60 & 101 & 1,030 & 4.00 & -- & $<0.29$ & 440 & 170 & -- \\
\hline 364 & 783 & 1.31 & 96.2 & 1,280 & 3.50 & -- & $<0.29$ & $<70$ & 79.8 & -- \\
\hline 365 & 650 & 1.35 & 882 & 137 & 1.90 & -- & 0.70 & $<70$ & 41.6 & -- \\
\hline 366 & 170 & 2.80 & 91.6 & 12.6 & 0.650 & -- & 0.99 & 2,100 & 31.0 & -- \\
\hline 367 & 433 & 1.80 & $<2.5$ & 590 & 4.20 & -- & $<0.29$ & 30 & 51.0 & 0.009 \\
\hline 368 & 319 & 1.50 & 160 & 26.0 & 1.00 & -- & $<0.29$ & $<70$ & $<5$ & 0.001 \\
\hline 369 & 190 & 7.90 & 420 & 20.0 & 0.350 & -- & 0.38 & $<70$ & 64.0 & $<1$ \\
\hline 370 & 110 & 3.00 & 101 & 12.0 & 1.10 & -- & 0.18 & $<70$ & $<5$ & -- \\
\hline 371 & 106 & 3.00 & 110 & 16.0 & 0.900 & -- & -- & $<70$ & 10.0 & 0.001 \\
\hline 372 & 430 & 1.80 & 689 & 48.3 & 2.50 & -- & 2.89 & 840 & 22.0 & -- \\
\hline 373 & 300 & 0.570 & 77.3 & 16.0 & 0.780 & -- & 0.28 & 370 & 110 & -- \\
\hline 374 & 68.0 & 1.30 & 77.0 & 20.0 & 0.610 & -- & 0.38 & $<70$ & $<5$ & -- \\
\hline 375 & 238 & 0.500 & 60.0 & 260 & 9.50 & -- & 1.04 & 130 & 20.0 & 0.009 \\
\hline 376 & -- & -- & -- & -- & -- & -- & -- & -- & -- & -- \\
\hline 377 & 629 & 2.69 & 842 & 172 & 2.40 & -- & $<0.29$ & $<70$ & 19.5 & -- \\
\hline 378 & 620 & 1.50 & 64.6 & 873 & -- & -- & -- & 220 & $<5$ & -- \\
\hline 379 & 1,600 & 6.70 & 130 & 3,100 & $<0.5$ & -- & $<0.29$ & 800 & 40.0 & -- \\
\hline 380 & 360 & 1.10 & 320 & 81.0 & 2.60 & -- & 0.77 & $<70$ & $<5$ & -- \\
\hline 381 & 690 & 4.50 & 1,290 & 151 & 2.60 & -- & 0.74 & 270 & $<5$ & -- \\
\hline 382 & 470 & 2.80 & 450 & 26.0 & 0.950 & -- & -- & 130 & 72.0 & -- \\
\hline 383 & 583 & 4.72 & 884 & 181 & 1.30 & -- & 0.68 & $<70$ & $<5$ & -- \\
\hline
\end{tabular}


Appendix 1. Water Quality Data Used in This Report.-Continued

\begin{tabular}{|c|c|c|c|c|c|c|c|c|c|c|}
\hline $\begin{array}{c}\text { Report } \\
\text { No. }\end{array}$ & $\begin{array}{c}\text { Sodium, } \\
\text { dissolved } \\
(\mathrm{mg} / \mathrm{L})\end{array}$ & $\begin{array}{c}\text { Potassium, } \\
\text { dissolved } \\
\text { (mg/L) }\end{array}$ & $\begin{array}{c}\text { Sulfate, } \\
\text { dissolved } \\
(\mathrm{mg} / \mathrm{L})\end{array}$ & $\begin{array}{l}\text { Chloride, } \\
\text { dissolved } \\
\text { (mg/L) }\end{array}$ & $\begin{array}{c}\text { Fluoride, } \\
\text { dissolved } \\
\text { (mg/L) }\end{array}$ & $\begin{array}{c}\text { Bromide, } \\
\text { dissolved } \\
\text { (mg/L) }\end{array}$ & $\begin{array}{c}\text { Nitrite+Nitrate, } \\
\text { dissolved } \\
\text { (mg/L as } \mathrm{N})\end{array}$ & $\begin{array}{c}\text { Iron, } \\
\text { dissolved } \\
\text { (ug/L) }\end{array}$ & $\begin{array}{c}\text { Manganese, } \\
\text { dissolved } \\
\text { (ug/L) }\end{array}$ & $\begin{array}{c}\text { Arsenic, } \\
\text { dissolved } \\
\text { (ug/L) }\end{array}$ \\
\hline 384 & 260 & 1.70 & 236 & 82.9 & 2.40 & -- & 0.070 & $<70$ & 38.0 & -- \\
\hline 386 & 1,100 & 4.40 & 1,200 & 220 & 0.580 & -- & 9.04 & 520 & 8,900 & -- \\
\hline 387 & 520 & 2.30 & 17.0 & 820 & 2.90 & -- & $<0.29$ & 4,000 & 51.0 & -- \\
\hline 388 & 190 & 1.20 & 20.0 & 115 & 2.90 & -- & -- & 20 & 16.0 & 0.004 \\
\hline 389 & -- & 0.920 & 17.0 & 24.0 & 1.30 & -- & $<0.29$ & $<70$ & $<5$ & -- \\
\hline 392 & 1,300 & 4.08 & 2,890 & 115 & 1.80 & -- & 1.56 & $<70$ & 6.61 & -- \\
\hline 393 & 280 & 6.30 & 1,500 & 94.0 & 0.410 & -- & 0.11 & $<70$ & 140 & -- \\
\hline 394 & 460 & 1.44 & 292 & 98.3 & 2.30 & -- & 23.72 & $<70$ & 8.76 & -- \\
\hline 395 & 834 & 1.85 & 1,780 & 118 & 0.440 & -- & 0.33 & $<70$ & 7.19 & -- \\
\hline 396 & 762 & 1.90 & 1,480 & 114 & 1.90 & -- & 0.034 & 60 & 50.0 & -- \\
\hline 397 & 110 & 3.30 & 160 & 6.00 & 0.370 & -- & 0.38 & 67 & 39.0 & -- \\
\hline 403 & 275 & 0.700 & 240 & 30.0 & 1.90 & -- & -- & $<70$ & $<5$ & 0.002 \\
\hline 404 & 279 & 1.20 & 140 & 79.0 & 1.40 & -- & $<0.29$ & 30 & 72.0 & 0.001 \\
\hline 405 & 84.5 & 1.20 & 40.0 & 15.0 & 0.700 & -- & -- & 170 & $<5$ & 0.001 \\
\hline 406 & 334 & 1.03 & 230 & 55.0 & 3.70 & -- & $<0.29$ & $<70$ & 5.23 & -- \\
\hline 407 & 329 & 1.30 & 150 & 30.0 & 1.80 & -- & $<0.29$ & 40 & 28.0 & 0.002 \\
\hline 408 & -- & -- & -- & -- & -- & -- & -- & -- & -- & -- \\
\hline 409 & 540 & 1.80 & 1,200 & 15.0 & 0.600 & -- & -- & 310 & 80.0 & -- \\
\hline 410 & -- & -- & -- & -- & -- & -- & -- & -- & -- & -- \\
\hline 414 & 100 & 3.00 & 190 & 19.0 & 1.00 & -- & -- & 30 & $<10$ & -- \\
\hline 415 & 84.0 & 2.90 & 170 & 19.0 & 1.00 & -- & -- & $<10$ & $<10$ & 2.00 \\
\hline 416 & 120 & 3.50 & 260 & 22.0 & 1.20 & -- & -- & $<10$ & $<10$ & 3.00 \\
\hline 417 & -- & -- & -- & -- & -- & -- & -- & -- & -- & -- \\
\hline 418 & -- & -- & -- & -- & -- & -- & -- & -- & -- & -- \\
\hline 435 & 110 & 1.70 & 90.0 & 12.0 & 1.00 & -- & 0.50 & -- & -- & -- \\
\hline 436 & 630 & 3.10 & 1,200 & 140 & 0.840 & -- & 0.043 & -- & -- & -- \\
\hline 437 & 33.0 & 1.50 & 80.0 & 13.0 & $<0.1$ & -- & 1.60 & -- & -- & -- \\
\hline 438 & 8.10 & 1.30 & 90.0 & 3.00 & 0.100 & -- & -- & -- & $<5$ & -- \\
\hline 439 & 180 & 3.10 & 210 & 115 & 1.30 & -- & 0.83 & -- & -- & -- \\
\hline 440 & -- & -- & -- & -- & -- & -- & -- & -- & -- & -- \\
\hline 441 & 270 & $<0.5$ & 130 & 70.0 & 3.40 & $<0.1$ & $<0.29$ & -- & -- & -- \\
\hline 442 & 546 & 8.20 & 2,310 & 200 & 0.700 & -- & -- & -- & -- & -- \\
\hline 443 & 230 & 3.90 & 390 & 29.0 & 1.00 & 0.21 & -- & -- & -- & -- \\
\hline 444 & 330 & 3.20 & 190 & 30.0 & 0.360 & -- & 0.23 & -- & -- & -- \\
\hline 445 & 380 & 1.30 & 508 & 31.8 & 3.10 & -- & 0.10 & -- & -- & -- \\
\hline 446 & 15.0 & $<0.5$ & 51.0 & $<1$ & $<0.5$ & -- & $<0.29$ & -- & -- & -- \\
\hline 447 & 18.0 & $<0.5$ & 49.0 & $<1$ & $<0.5$ & -- & $<0.29$ & -- & -- & -- \\
\hline 447 & -- & -- & -- & -- & -- & -- & -- & -- & -- & -- \\
\hline 448 & 9.60 & $<0.5$ & 25.0 & $<1$ & $<0.5$ & -- & $<0.29$ & -- & -- & -- \\
\hline 448 & -- & -- & -- & -- & -- & -- & -- & -- & -- & -- \\
\hline 449 & 380 & $<0.5$ & 430 & 25.0 & 2.50 & $<0.1$ & -- & -- & -- & -- \\
\hline 450 & 57.0 & $<0.5$ & 74.0 & 3.80 & 0.590 & $<0.1$ & $<0.29$ & -- & -- & -- \\
\hline 451 & 29.0 & $<0.5$ & 51.0 & 7.80 & 0.650 & $<0.1$ & -- & -- & -- & -- \\
\hline 452 & 200 & $<0.5$ & 110 & 9.50 & 1.40 & $<0.1$ & -- & -- & -- & -- \\
\hline 453 & 120 & $<0.5$ & 130 & 26.0 & 1.20 & $<0.1$ & -- & -- & -- & -- \\
\hline 453 & -- & -- & -- & -- & -- & -- & -- & -- & -- & -- \\
\hline 454 & 210 & $<0.5$ & 150 & 19.0 & 1.70 & $<0.1$ & $<0.29$ & -- & -- & -- \\
\hline
\end{tabular}


Appendix 1. Water Quality Data Used in This Report.-Continued

\begin{tabular}{|c|c|c|c|c|c|c|c|c|c|c|}
\hline $\begin{array}{c}\text { Report } \\
\text { No. }\end{array}$ & $\begin{array}{c}\text { Sodium, } \\
\text { dissolved } \\
\text { (mg/L) }\end{array}$ & $\begin{array}{l}\text { Potassium, } \\
\text { dissolved } \\
\text { (mg/L) }\end{array}$ & $\begin{array}{c}\text { Sulfate, } \\
\text { dissolved } \\
\text { (mg/L) }\end{array}$ & $\begin{array}{c}\text { Chloride, } \\
\text { dissolved } \\
\text { (mg/L) }\end{array}$ & $\begin{array}{c}\text { Fluoride, } \\
\text { dissolved } \\
\text { (mg/L) }\end{array}$ & $\begin{array}{c}\text { Bromide, } \\
\text { dissolved } \\
\text { (mg/L) }\end{array}$ & $\begin{array}{c}\text { Nitrite+Nitrate, } \\
\text { dissolved } \\
(\mathrm{mg} / \mathrm{L} \text { as } \mathrm{N})\end{array}$ & $\begin{array}{c}\text { Iron, } \\
\text { dissolved } \\
\text { (ug/L) }\end{array}$ & $\begin{array}{c}\text { Manganese, } \\
\text { dissolved } \\
\text { (ug/L) }\end{array}$ & $\begin{array}{c}\text { Arsenic, } \\
\text { dissolved } \\
\text { (ug/L) }\end{array}$ \\
\hline 454 & -- & -- & -- & -- & -- & -- & -- & -- & -- & -- \\
\hline 456 & 15.0 & 2.80 & 58.0 & 3.70 & -- & -- & $<0.01$ & -- & -- & -- \\
\hline 457 & -- & -- & -- & -- & -- & -- & -- & -- & -- & -- \\
\hline 458 & -- & -- & -- & -- & -- & -- & -- & -- & -- & -- \\
\hline 459 & -- & -- & -- & -- & -- & -- & -- & -- & -- & -- \\
\hline 462 & 57.5 & 3.40 & 64.0 & 43.9 & 0.400 & -- & -- & -- & -- & -- \\
\hline 463 & 46.0 & 3.80 & 34.2 & 14.4 & 0.430 & -- & 1.13 & -- & -- & -- \\
\hline 464 & 21.0 & 2.70 & 15.7 & 11.4 & $<0.5$ & -- & 1.56 & -- & -- & -- \\
\hline 465 & 4,000 & 590 & 99.0 & 4,500 & 2.30 & 15.00 & -- & 3,000 & 40.0 & 160 \\
\hline 466 & 22.6 & 3.30 & 20.0 & $<1$ & 0.300 & -- & -- & -- & $<5$ & -- \\
\hline 468 & -- & -- & -- & -- & -- & -- & -- & -- & -- & -- \\
\hline 473 & -- & 3.00 & 20.5 & 12.0 & -- & -- & -- & -- & -- & -- \\
\hline 474 & -- & 3.40 & 34.4 & 14.9 & -- & -- & -- & -- & -- & -- \\
\hline 475 & -- & 2.80 & 14.7 & 4.70 & -- & -- & -- & -- & -- & -- \\
\hline 476 & 70.4 & 5.10 & 32.6 & 12.9 & 0.200 & -- & -- & -- & -- & -- \\
\hline 477 & 150 & 0.720 & 24.0 & 4.50 & 0.390 & -- & -- & -- & -- & -- \\
\hline 478 & 90.0 & 2.50 & 54.4 & 9.30 & $<0.5$ & -- & 0.32 & -- & -- & -- \\
\hline 479 & -- & -- & -- & -- & -- & -- & -- & -- & -- & -- \\
\hline 480 & -- & -- & -- & -- & -- & -- & -- & -- & -- & -- \\
\hline 481 & 26.0 & 2.80 & 16.3 & 10.4 & $<0.5$ & -- & 2.01 & -- & -- & -- \\
\hline 482 & 29.0 & 3.00 & 15.5 & 8.58 & $<0.5$ & -- & 1.59 & -- & -- & -- \\
\hline 483 & 27.0 & 2.80 & 15.1 & 7.33 & $<0.5$ & -- & 0.77 & -- & -- & -- \\
\hline 484 & -- & -- & -- & -- & -- & -- & -- & -- & -- & -- \\
\hline 485 & 25.0 & 3.00 & 14.0 & 9.00 & 0.410 & -- & 0.11 & -- & -- & -- \\
\hline 486 & 66.0 & 3.70 & 24.0 & 20.5 & $<0.5$ & -- & 2.91 & $<70$ & $<5$ & -- \\
\hline 495 & 110 & 4.90 & 92.8 & 119 & 0.750 & -- & 2.46 & -- & -- & -- \\
\hline 496 & 64.0 & 2.90 & 29.7 & 7.56 & $<0.5$ & -- & $<0.29$ & -- & -- & -- \\
\hline 497 & 24.0 & 2.70 & 15.7 & 11.6 & $<0.5$ & -- & 1.40 & -- & -- & -- \\
\hline 498 & 35.0 & 2.50 & 14.3 & 7.71 & 0.440 & -- & 0.52 & -- & -- & -- \\
\hline 499 & 69.0 & 2.40 & 20.4 & 7.08 & 0.460 & -- & 0.13 & -- & -- & -- \\
\hline 500 & 64.0 & 2.50 & 67.2 & 8.50 & $<0.5$ & -- & 0.41 & -- & -- & -- \\
\hline 501 & 100 & 7.40 & 240 & $<1$ & 0.580 & -- & 0.72 & -- & -- & -- \\
\hline 502 & 26.1 & 10.0 & 48.0 & 6.50 & 0.570 & $<0.1$ & 0.40 & -- & -- & -- \\
\hline 503 & -- & -- & 56.0 & 16.0 & $<0.5$ & $<0.1$ & 0.14 & -- & -- & -- \\
\hline 504 & 130 & 2.60 & 64.0 & 92.0 & 0.400 & $<0.1$ & 0.68 & $<70$ & $<10$ & $<20$ \\
\hline 505 & 28.0 & 4.10 & 14.0 & 8.50 & 0.140 & $<0.1$ & 0.081 & $<70$ & $<10$ & $<20$ \\
\hline 506 & 26.0 & 2.80 & 16.3 & 10.4 & $<0.5$ & -- & 2.01 & $<70$ & $<5$ & -- \\
\hline 507 & 19.0 & 2.20 & 14.0 & 10.0 & 0.240 & -- & 0.14 & -- & -- & -- \\
\hline 508 & 28.0 & 3.30 & 17.0 & 15.0 & 0.370 & -- & 0.50 & -- & -- & -- \\
\hline 509 & 1,600 & 5.50 & 260 & 2,800 & 0.360 & -- & -- & 61 & -- & -- \\
\hline 510 & 1,600 & 5.30 & 250 & 2,400 & 0.670 & -- & 0.38 & $<70$ & 10.0 & -- \\
\hline 511 & 29.0 & 3.00 & 15.5 & 8.58 & $<0.5$ & -- & 1.59 & 250 & $<5$ & -- \\
\hline 512 & 67.0 & 5.80 & 31.0 & 10.0 & 0.360 & -- & 0.63 & 120 & 18.0 & -- \\
\hline 513 & 28.0 & 2.80 & 13.0 & 4.70 & 0.250 & -- & 0.029 & $<70$ & $<5$ & -- \\
\hline 514 & 34.0 & 3.10 & 26.0 & 22.4 & $<0.4$ & -- & 3.46 & 260 & 18.0 & -- \\
\hline 515 & 130 & 0.930 & 24.0 & 5.60 & 0.350 & -- & $<0.29$ & $<70$ & $<5$ & $<1$ \\
\hline 516 & 90.0 & 2.50 & 54.4 & 9.30 & $<0.5$ & -- & 0.32 & $<70$ & $<5$ & -- \\
\hline 517 & 54.0 & 3.70 & 38.0 & 19.0 & 0.410 & -- & 0.54 & -- & -- & -- \\
\hline 518 & 21.0 & 2.90 & 17.0 & 15.0 & 0.220 & -- & 0.14 & $<70$ & $<5$ & $<1$ \\
\hline 519 & 25.0 & 3.20 & 14.0 & 10.0 & 0.200 & -- & 0.13 & $<70$ & $<5$ & $<1$ \\
\hline
\end{tabular}


Appendix 1. Water Quality Data Used in This Report.-Continued

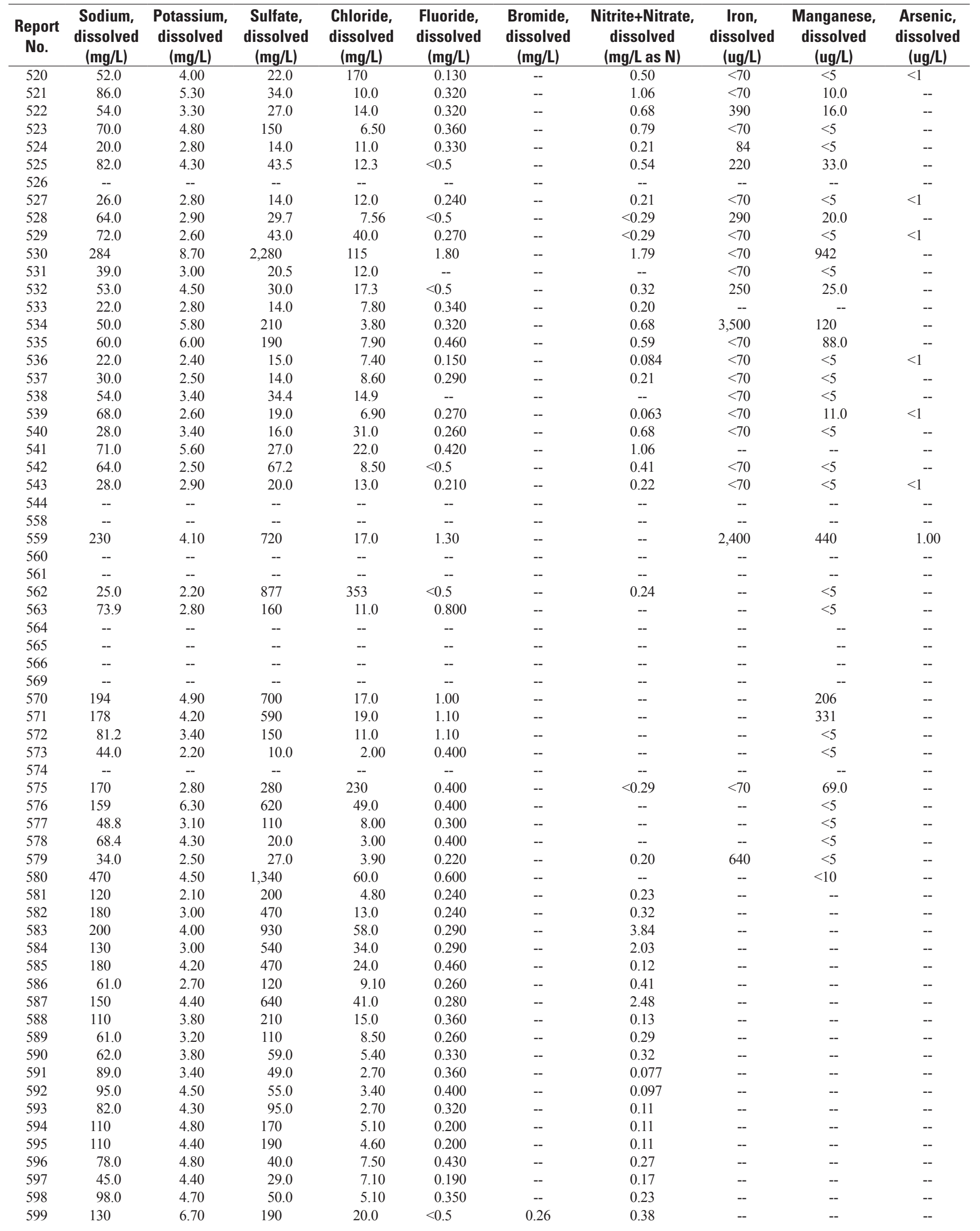


Appendix 1. Water Quality Data Used in This Report.-Continued

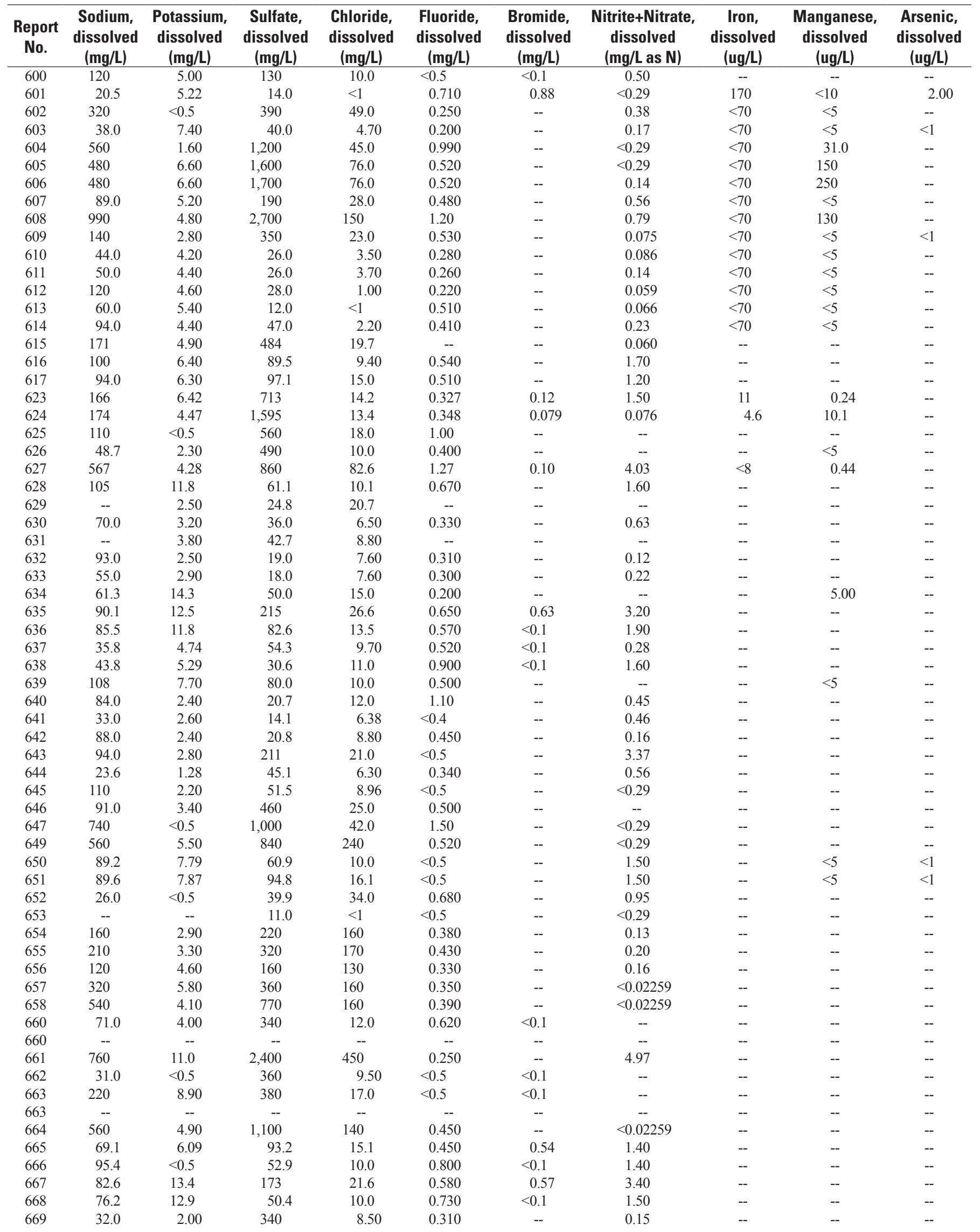


Appendix 1. Water Quality Data Used in This Report.-Continued

\begin{tabular}{|c|c|c|c|c|c|c|c|c|c|c|}
\hline $\begin{array}{c}\text { Report } \\
\text { No. }\end{array}$ & $\begin{array}{c}\text { Sodium, } \\
\text { dissolved } \\
\text { (mg/L) }\end{array}$ & $\begin{array}{l}\text { Potassium, } \\
\text { dissolved } \\
\text { (mg/L) }\end{array}$ & $\begin{array}{c}\text { Sulfate, } \\
\text { dissolved } \\
(\mathrm{mg} / \mathrm{L})\end{array}$ & $\begin{array}{l}\text { Chloride, } \\
\text { dissolved } \\
\text { (mg/L) }\end{array}$ & $\begin{array}{c}\text { Fluoride, } \\
\text { dissolved } \\
\text { (mg/L) }\end{array}$ & $\begin{array}{l}\text { Bromide, } \\
\text { dissolved } \\
\text { (mg/L) }\end{array}$ & $\begin{array}{c}\text { Nitrite+Nitrate, } \\
\text { dissolved } \\
\text { (mg/L as N) }\end{array}$ & $\begin{array}{l}\text { Iron, } \\
\text { dissolved } \\
\text { (ug/L) }\end{array}$ & $\begin{array}{c}\text { Manganese, } \\
\text { dissolved } \\
\text { (ug/L) }\end{array}$ & $\begin{array}{c}\text { Arsenic, } \\
\text { dissolved } \\
\text { (ug/L) }\end{array}$ \\
\hline 670 & 98.3 & $<0.5$ & 40.7 & 9.00 & 0.780 & $<0.1$ & 1.30 & -- & -- & -- \\
\hline 672 & 97.4 & 6.80 & 82.3 & 10.9 & 0.640 & $<0.1$ & 1.30 & -- & -- & -- \\
\hline 673 & 63.5 & 8.27 & 56.6 & 7.10 & 0.560 & $<0.1$ & 1.40 & -- & -- & -- \\
\hline 674 & 46.0 & 4.51 & 138 & 12.0 & 0.190 & 0.53 & 1.80 & -- & -- & -- \\
\hline 675 & 31.0 & 1.90 & 360 & 8.30 & 0.320 & -- & 0.14 & -- & -- & -- \\
\hline 678 & 60.0 & 3.70 & 19.0 & 10.0 & 0.160 & -- & 0.25 & $<70$ & $<5$ & $<1$ \\
\hline 679 & 34.0 & 2.10 & 15.2 & 6.67 & 0.420 & -- & 0.71 & $<70$ & 74.0 & -- \\
\hline 680 & 210 & 4.00 & 264 & 99.2 & -- & -- & -- & 210 & $<5$ & -- \\
\hline 681 & 30.0 & 3.10 & 14.0 & 7.90 & 0.160 & -- & 0.27 & $<70$ & $<5$ & $<1$ \\
\hline 682 & 85.0 & 3.80 & 42.7 & 8.80 & -- & -- & -- & 350 & $<5$ & -- \\
\hline 683 & 180 & 10.0 & 65.0 & 7.40 & 0.390 & -- & 0.17 & 17,000 & 530 & -- \\
\hline 689 & 3,200 & 11.0 & 3,600 & 1,500 & 2.40 & -- & 1.08 & 1,900 & 15.0 & -- \\
\hline 690 & 94.0 & 2.80 & 211 & 21.0 & $<0.5$ & -- & 3.37 & 1,500 & 16.0 & -- \\
\hline 691 & 180 & 0.700 & 11.0 & 6.20 & 4.90 & -- & -- & 40 & $<10$ & 65.0 \\
\hline 692 & 114 & 79.0 & 112 & 20.0 & 3.70 & -- & 0.25 & 100 & 10.0 & -- \\
\hline 693 & -- & -- & -- & -- & -- & -- & -- & -- & -- & -- \\
\hline 694 & 44.0 & 2.60 & -- & -- & $<0.5$ & -- & -- & -- & $<5$ & -- \\
\hline 695 & 68.0 & 7.00 & 47.0 & 5.80 & 0.650 & -- & -- & -- & $<5$ & 12.0 \\
\hline 696 & -- & -- & 32.2 & 2.70 & 0.440 & -- & $<0.29$ & -- & -- & -- \\
\hline 699 & 40.0 & 3.40 & -- & 12.8 & 0.550 & -- & 1.83 & -- & -- & -- \\
\hline 704 & -- & -- & -- & -- & -- & -- & -- & -- & -- & -- \\
\hline 706 & 23.3 & 2.70 & 10.0 & 2.00 & 0.400 & -- & -- & -- & $<5$ & -- \\
\hline 707 & 54.5 & 3.80 & 60.0 & 11.0 & 0.400 & -- & -- & -- & $<5$ & -- \\
\hline 708 & 24.6 & 1.80 & 20.0 & 6.00 & 0.500 & -- & -- & -- & $<5$ & -- \\
\hline 718 & -- & -- & -- & -- & -- & -- & -- & -- & -- & -- \\
\hline 719 & 108 & 2.40 & 30.0 & 1.00 & 0.500 & -- & -- & -- & $<5$ & -- \\
\hline 720 & 99.0 & 2.30 & 30.0 & $<1$ & 0.400 & -- & -- & -- & $<5$ & -- \\
\hline 721 & - & - & -- & - & -- & -- & -- & -- & -- & -- \\
\hline 722 & -- & -- & -- & -- & -- & -- & -- & -- & -- & -- \\
\hline 723 & -- & -- & -- & -- & -- & -- & -- & -- & -- & -- \\
\hline 724 & -- & -- & -- & -- & -- & -- & -- & -- & -- & -- \\
\hline 725 & -- & -- & -- & -- & -- & -- & -- & -- & -- & -- \\
\hline 726 & 5.90 & 1.50 & 6.80 & 1.10 & $<0.5$ & -- & $<0.29$ & -- & -- & -- \\
\hline 727 & 44.1 & 2.30 & 30.0 & 5.00 & 0.500 & -- & - & -- & $<5$ & -- \\
\hline 728 & 49.4 & 2.80 & 40.0 & 6.00 & 0.500 & -- & -- & -- & $<5$ & -- \\
\hline 729 & 52.4 & 3.20 & 50.0 & 10.0 & 0.400 & -- & -- & -- & $<5$ & -- \\
\hline 730 & 52.9 & 3.10 & 40.0 & 10.0 & 0.400 & -- & -- & -- & $<5$ & -- \\
\hline 731 & 34.4 & 3.20 & 20.0 & 4.00 & 0.300 & -- & -- & -- & $<5$ & -- \\
\hline 732 & 30.0 & 2.30 & 20.0 & 2.00 & 0.300 & -- & -- & -- & $<5$ & -- \\
\hline 733 & 10.7 & 1.10 & $<5$ & $<1$ & 0.400 & -- & -- & -- & $<5$ & -- \\
\hline 734 & 57.6 & 2.60 & 30.0 & 6.00 & 0.200 & -- & -- & -- & $<5$ & -- \\
\hline 735 & 38.4 & 1.20 & 50.0 & 5.00 & 0.400 & -- & -- & -- & $<5$ & -- \\
\hline 736 & 17.3 & 1.00 & 20.0 & $<1$ & 0.600 & -- & $<0.29$ & -- & $<5$ & -- \\
\hline 737 & 49.1 & 1.90 & 30.0 & 3.00 & 0.300 & -- & -- & -- & $<5$ & -- \\
\hline 738 & 29.5 & 1.40 & 50.0 & 5.00 & 0.600 & -- & -- & -- & $<5$ & -- \\
\hline 739 & -- & -- & -- & -- & -- & -- & -- & -- & -- & -- \\
\hline 740 & 41.9 & 1.10 & 20.0 & $<1$ & 0.500 & -- & -- & -- & $<5$ & -- \\
\hline 741 & -- & -- & -- & -- & -- & -- & -- & -- & -- & -- \\
\hline 742 & 58.5 & 1.50 & 20.0 & 6.00 & 0.400 & -- & -- & -- & $<5$ & -- \\
\hline
\end{tabular}


Appendix 1. Water Quality Data Used in This Report.-Continued

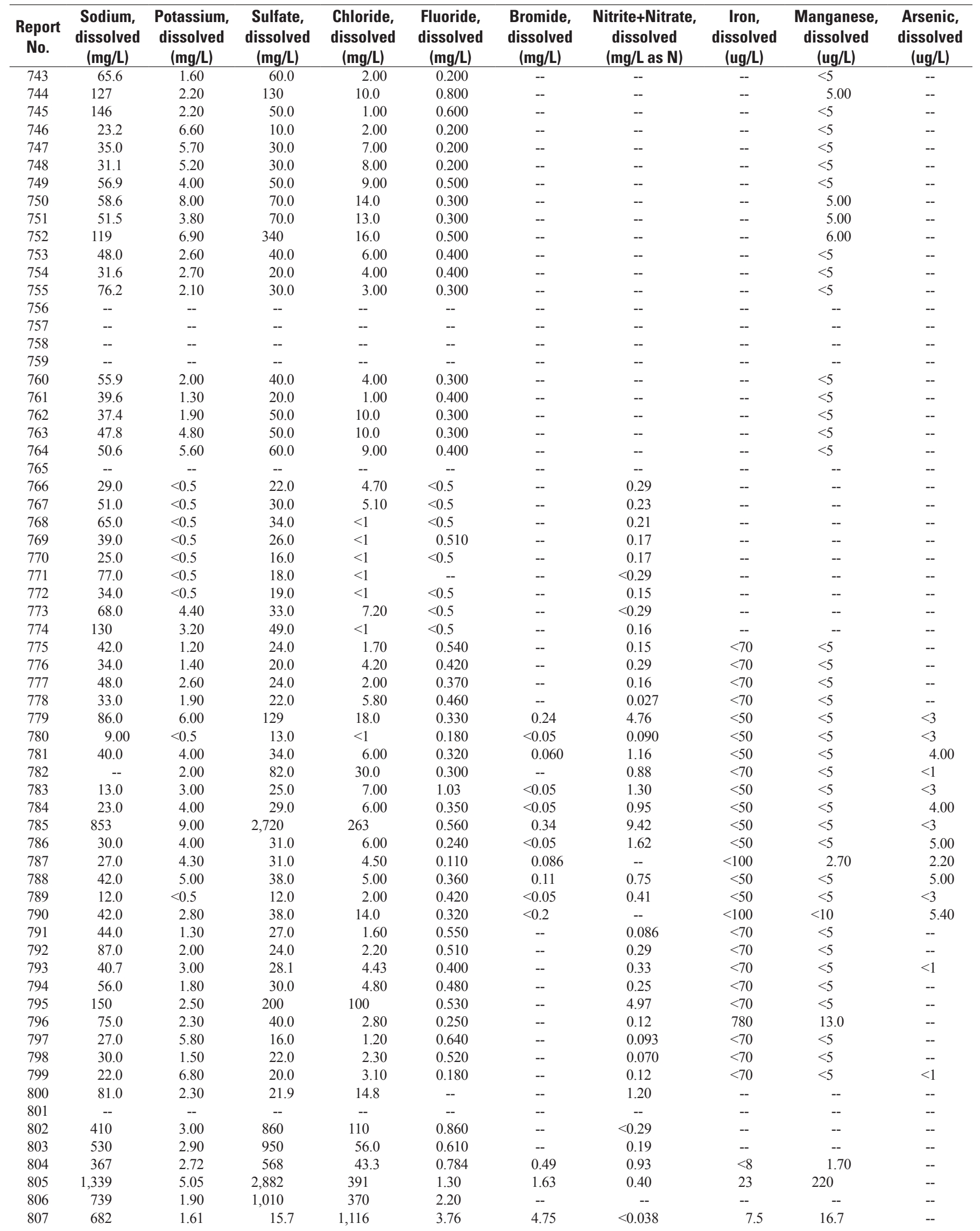


Appendix 1. Water Quality Data Used in This Report.-Continued

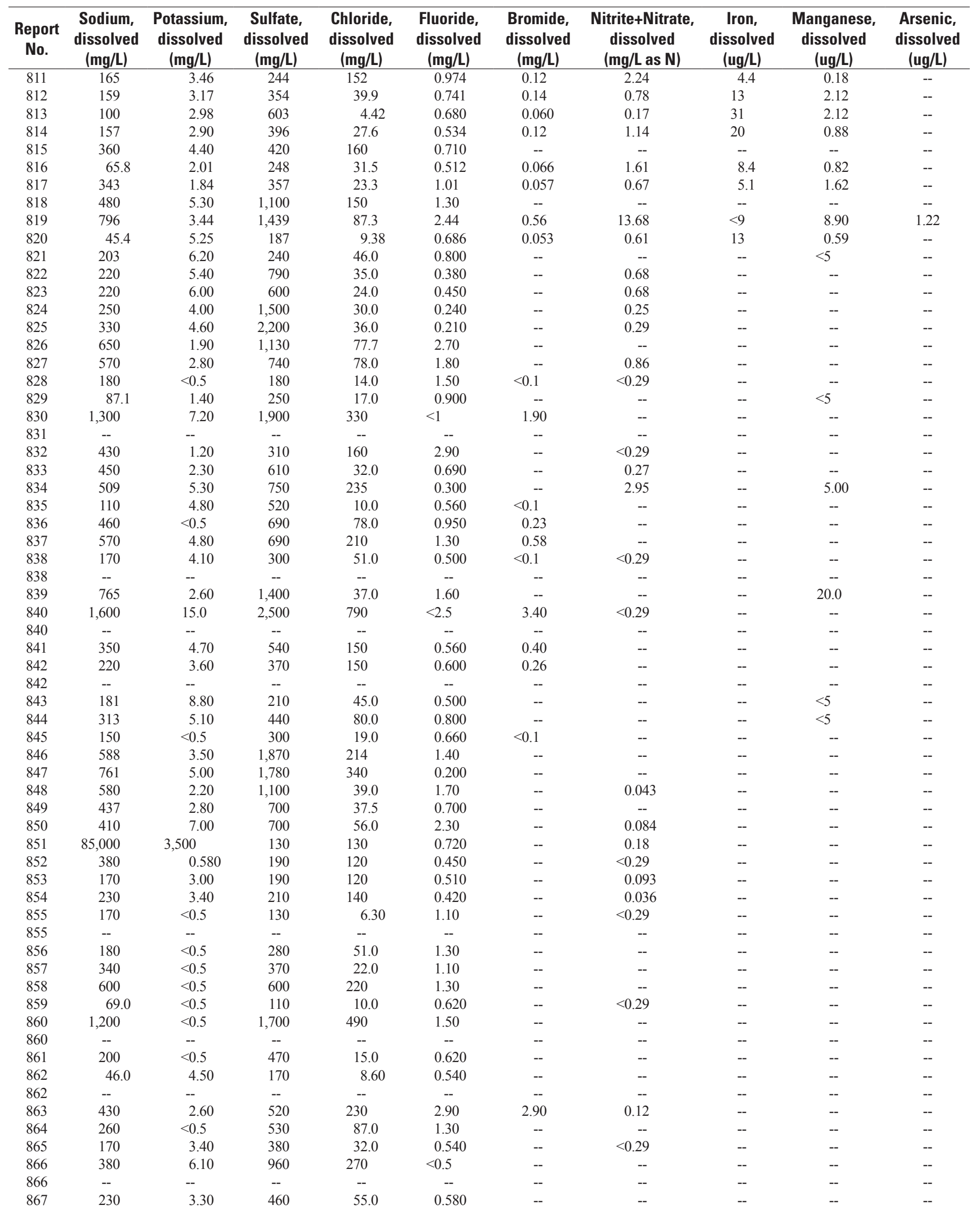


Appendix 1. Water Quality Data Used in This Report.-Continued

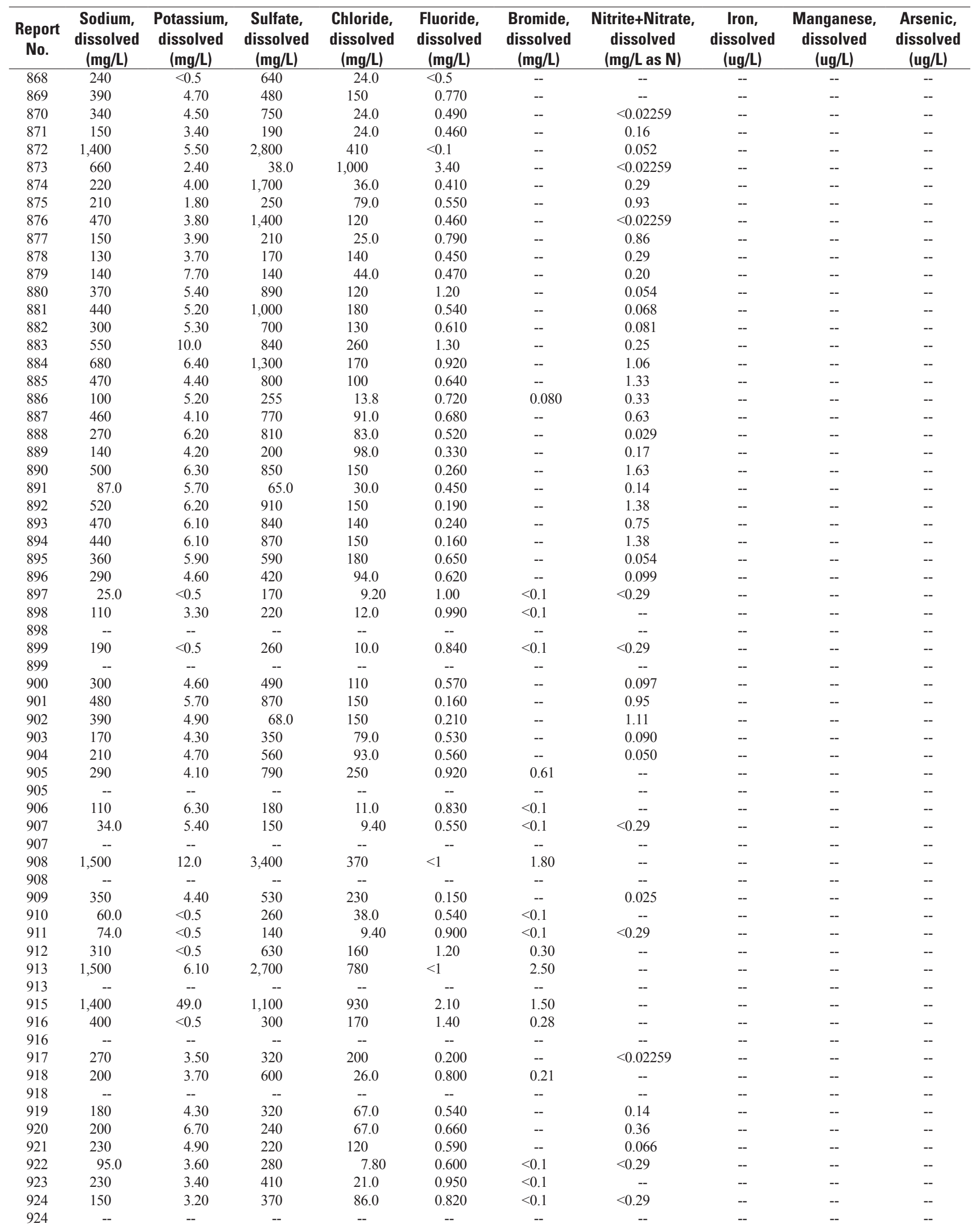


Appendix 1. Water Quality Data Used in This Report.-Continued

\begin{tabular}{|c|c|c|c|c|c|c|c|c|c|c|}
\hline $\begin{array}{c}\text { Report } \\
\text { No. }\end{array}$ & $\begin{array}{c}\text { Sodium, } \\
\text { dissolved } \\
\text { (mg/L) } \\
\end{array}$ & $\begin{array}{c}\text { Potassium, } \\
\text { dissolved } \\
(\mathrm{mg} / \mathrm{L})\end{array}$ & $\begin{array}{c}\text { Sulfate, } \\
\text { dissolved } \\
\text { (mg/L) } \\
\end{array}$ & $\begin{array}{c}\text { Chloride, } \\
\text { dissolved } \\
\text { (mg/L) } \\
\end{array}$ & $\begin{array}{c}\text { Fluoride, } \\
\text { dissolved } \\
\text { (mg/L) }\end{array}$ & $\begin{array}{c}\text { Bromide, } \\
\text { dissolved } \\
\text { (mg/L) } \\
\end{array}$ & $\begin{array}{c}\text { Nitrite+Nitrate, } \\
\text { dissolved } \\
\text { (mg/L as N) } \\
\end{array}$ & $\begin{array}{c}\text { Iron, } \\
\text { dissolved } \\
\text { (ug/L) }\end{array}$ & $\begin{array}{c}\text { Manganese, } \\
\text { dissolved } \\
\text { (ug/L) }\end{array}$ & $\begin{array}{c}\text { Arsenic, } \\
\text { dissolved } \\
\text { (ug/L) }\end{array}$ \\
\hline 925 & 180 & 3.10 & 360 & 30.0 & 0.540 & $<0.1$ & -- & -- & -- & -- \\
\hline 925 & -- & -- & -- & -- & -- & -- & -- & -- & -- & -- \\
\hline 926 & 170 & $<0.5$ & 280 & 99.0 & 0.770 & $<0.1$ & -- & -- & -- & -- \\
\hline 927 & 240 & 3.20 & 480 & 120 & 0.600 & -- & $<0.02259$ & -- & -- & -- \\
\hline 928 & 200 & 3.70 & 370 & 79.0 & 0.800 & $<0.1$ & -- & -- & -- & -- \\
\hline 928 & -- & -- & -- & -- & -- & -- & -- & -- & -- & -- \\
\hline 930 & 150 & 4.40 & 210 & 140 & 0.410 & -- & 0.045 & -- & -- & -- \\
\hline 931 & 290 & 270 & 350 & 110 & 0.330 & -- & $<0.02259$ & -- & -- & -- \\
\hline 932 & 270 & 2.60 & 260 & 170 & 1.00 & -- & 0.068 & -- & -- & -- \\
\hline 933 & 360 & 3.80 & 390 & 160 & 0.850 & 0.25 & $<0.29$ & -- & -- & -- \\
\hline 934 & 310 & 3.90 & 330 & 140 & 0.740 & 0.21 & $<0.29$ & -- & -- & -- \\
\hline 934 & -- & -- & -- & -- & -- & -- & -- & -- & -- & -- \\
\hline 935 & 530 & 12.0 & 64.0 & 180 & 0.920 & -- & 0.093 & -- & -- & -- \\
\hline 936 & 260 & 4.20 & 180 & 110 & 0.240 & -- & $<0.02259$ & -- & -- & -- \\
\hline 937 & 320 & 3.50 & 330 & 170 & 0.600 & -- & 0.15 & -- & -- & -- \\
\hline 938 & 140 & 2.80 & $<5$ & 200 & $<0.1$ & -- & $<0.02259$ & -- & -- & -- \\
\hline 939 & 390 & 3.30 & 240 & 370 & 0.990 & -- & $<0.02259$ & -- & -- & -- \\
\hline 940 & 450 & 3.70 & 630 & 190 & 0.840 & -- & 0.12 & -- & -- & -- \\
\hline 941 & 360 & 3.90 & 35.0 & 170 & 0.640 & -- & 0.17 & -- & -- & -- \\
\hline 942 & 360 & 3.90 & 34.0 & 170 & 0.640 & -- & 0.19 & -- & -- & -- \\
\hline 943 & 390 & 3.90 & 38.0 & 160 & 0.760 & -- & 0.10 & -- & -- & -- \\
\hline 944 & 390 & 4.00 & 620 & 180 & 0.730 & -- & 0.14 & -- & -- & -- \\
\hline 945 & 140 & 3.20 & 230 & 150 & 0.320 & -- & $<0.02259$ & -- & -- & -- \\
\hline 946 & 300 & 1.00 & 120 & 280 & 2.10 & -- & $<0.02259$ & -- & -- & -- \\
\hline 947 & 350 & 0.500 & 200 & 120 & 0.490 & -- & 0.023 & -- & -- & -- \\
\hline 948 & 340 & 4.10 & 390 & 160 & 0.650 & -- & 0.50 & -- & -- & -- \\
\hline 949 & 480 & 3.10 & 42.0 & 24.0 & 0.960 & -- & $<0.02259$ & -- & -- & -- \\
\hline 950 & 160 & 2.40 & 190 & 110 & 0.540 & -- & 0.10 & -- & -- & -- \\
\hline 951 & 370 & 4.40 & 480 & 170 & 0.700 & -- & 0.54 & -- & -- & -- \\
\hline 952 & 290 & 3.20 & 300 & 150 & 0.690 & -- & 0.086 & -- & -- & -- \\
\hline 953 & 310 & 3.40 & 340 & 140 & 0.600 & -- & 0.36 & -- & -- & -- \\
\hline 954 & 240 & 2.50 & 230 & 140 & 0.400 & -- & $<0.02259$ & -- & -- & -- \\
\hline 955 & 160 & 3.00 & 210 & 150 & 0.490 & -- & 0.099 & -- & -- & -- \\
\hline 956 & 270 & 3.70 & 290 & 130 & 0.550 & -- & 0.38 & -- & -- & -- \\
\hline 957 & 180 & 2.50 & 240 & 120 & 0.590 & -- & 0.029 & -- & -- & -- \\
\hline 958 & 170 & 2.90 & 200 & 130 & 0.520 & -- & 0.045 & -- & -- & -- \\
\hline 959 & 170 & 2.50 & 220 & 110 & 0.570 & -- & 0.12 & -- & -- & -- \\
\hline 960 & 150 & 3.10 & 190 & 130 & 0.450 & -- & 0.041 & -- & -- & -- \\
\hline 961 & 240 & 2.10 & 220 & 140 & 0.600 & -- & $<0.02259$ & -- & -- & -- \\
\hline 962 & 160 & 2.90 & 180 & 130 & 0.490 & -- & 0.043 & -- & -- & -- \\
\hline 963 & 280 & 1.90 & 350 & 170 & 0.870 & -- & $<0.02259$ & -- & -- & -- \\
\hline 964 & 180 & 2.90 & 220 & 120 & 0.480 & -- & 0.12 & -- & -- & -- \\
\hline 965 & 180 & 2.70 & 230 & 150 & 0.600 & -- & 0.070 & -- & -- & -- \\
\hline 966 & 160 & 3.00 & 190 & 150 & 0.470 & -- & 0.10 & -- & -- & -- \\
\hline 967 & 270 & 3.30 & 260 & 130 & 0.500 & -- & 0.27 & -- & -- & -- \\
\hline 968 & 350 & 4.20 & 540 & 170 & 0.670 & -- & 0.43 & -- & -- & -- \\
\hline 969 & 190 & 2.60 & 250 & 110 & 0.740 & -- & $<0.02259$ & -- & -- & -- \\
\hline 970 & 580 & 3.40 & 770 & 180 & 0.670 & -- & $<0.02259$ & -- & -- & -- \\
\hline 971 & 220 & 2.90 & 260 & 160 & 0.640 & -- & 0.11 & -- & -- & -- \\
\hline 972 & 310 & 3.70 & 390 & 150 & 0.650 & -- & 0.054 & -- & -- & -- \\
\hline 973 & 440 & 4.20 & 480 & 180 & 0.810 & -- & 0.21 & -- & -- & -- \\
\hline 974 & 140 & 3.60 & 260 & 93.0 & 0.550 & -- & 0.29 & -- & -- & -- \\
\hline 975 & 340 & 0.570 & 180 & 110 & 1.00 & -- & 0.20 & -- & -- & -- \\
\hline 976 & 140 & 2.40 & 170 & 110 & 0.800 & -- & 0.063 & -- & -- & -- \\
\hline 977 & 340 & 3.80 & 450 & 180 & 0.720 & -- & $<0.02259$ & -- & -- & -- \\
\hline 978 & 160 & 4.70 & 230 & 110 & 0.540 & -- & 0.36 & -- & -- & -- \\
\hline 979 & 200 & 2.50 & 270 & 91.0 & 0.790 & -- & 0.15 & -- & -- & -- \\
\hline 980 & 200 & 2.90 & 380 & 140 & 0.560 & -- & 0.17 & -- & -- & -- \\
\hline 981 & 260 & 2.90 & 260 & 140 & 0.590 & -- & 0.11 & -- & -- & -- \\
\hline 982 & 160 & 4.90 & 300 & 96.0 & 0.550 & -- & 0.32 & -- & -- & -- \\
\hline 983 & 230 & 3.40 & 560 & 150 & 0.520 & -- & 0.27 & -- & -- & -- \\
\hline 984 & 150 & 4.60 & 270 & 99.0 & 0.540 & -- & 0.29 & -- & -- & -- \\
\hline 985 & 190 & 4.30 & 420 & 71.0 & 0.590 & -- & 0.36 & -- & -- & -- \\
\hline 986 & 200 & 3.30 & 390 & 96.0 & 0.800 & -- & 0.23 & -- & -- & -- \\
\hline 987 & 100 & 1.80 & 170 & 130 & 0.590 & -- & $<0.02259$ & -- & -- & -- \\
\hline
\end{tabular}


Appendix 1. Water Quality Data Used in This Report.-Continued

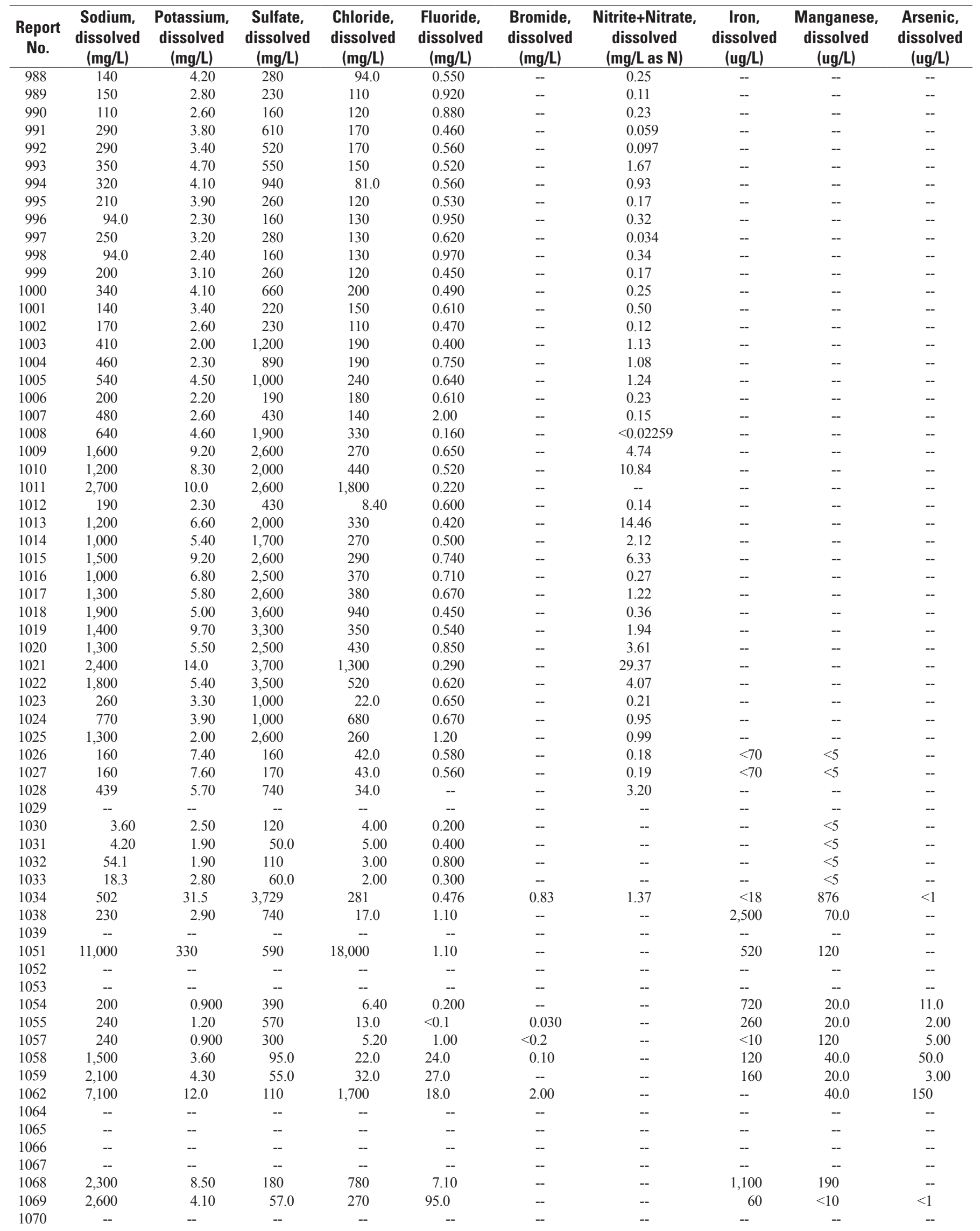


Appendix 1. Water Quality Data Used in This Report.-Continued

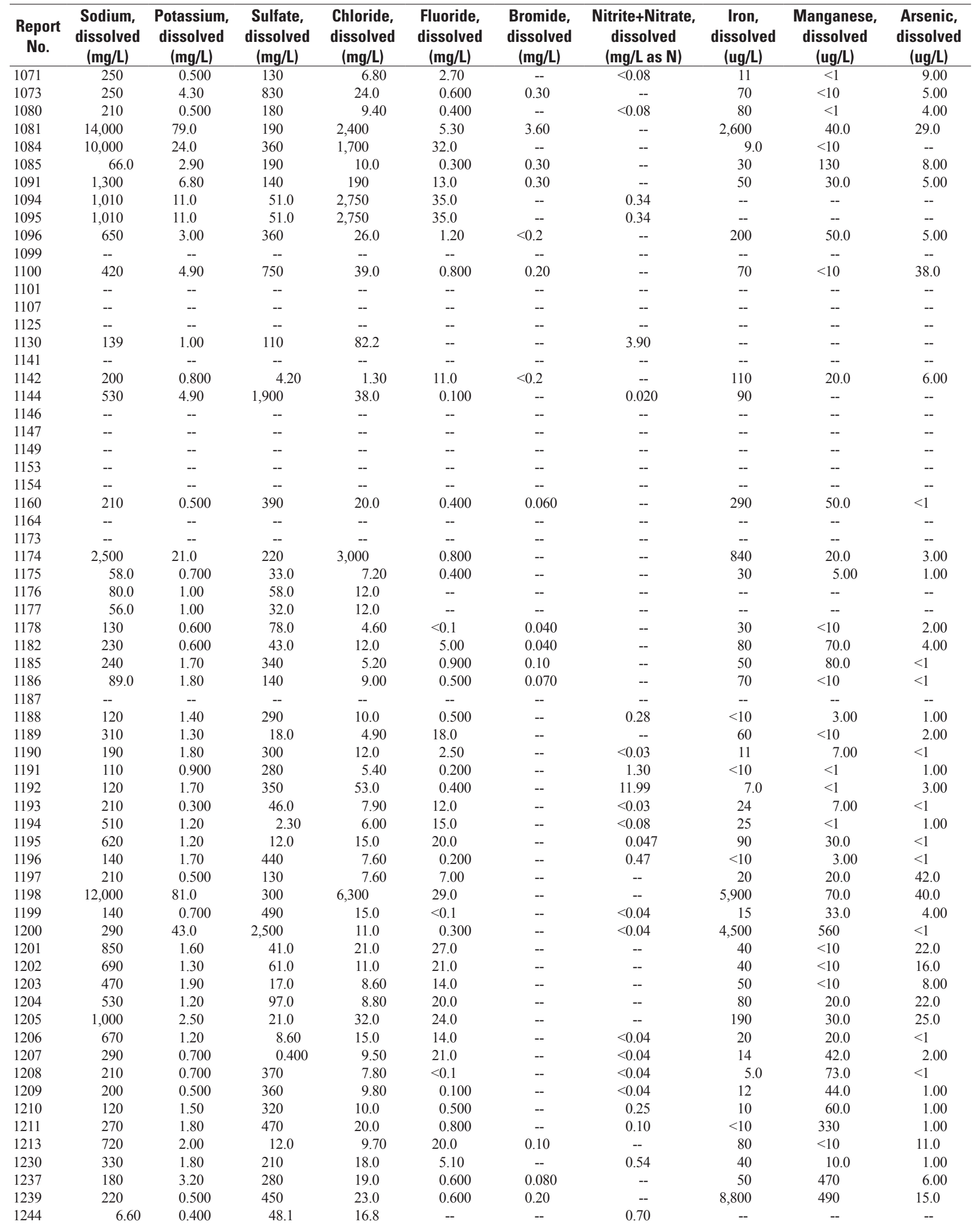


Appendix 1. Water Quality Data Used in This Report.-Continued

\begin{tabular}{|c|c|c|c|c|c|c|c|c|c|c|}
\hline $\begin{array}{c}\text { Report } \\
\text { No. }\end{array}$ & $\begin{array}{c}\text { Sodium, } \\
\text { dissolved } \\
\text { (mg/L) } \\
\end{array}$ & $\begin{array}{c}\text { Potassium, } \\
\text { dissolved } \\
(\mathrm{mg} / \mathrm{L}) \\
\end{array}$ & $\begin{array}{c}\text { Sulfate, } \\
\text { dissolved } \\
\text { (mg/L) }\end{array}$ & $\begin{array}{c}\text { Chloride, } \\
\text { dissolved } \\
\text { (mg/L) } \\
\end{array}$ & $\begin{array}{c}\text { Fluoride, } \\
\text { dissolved } \\
\text { (mg/L) }\end{array}$ & $\begin{array}{c}\text { Bromide, } \\
\text { dissolved } \\
\text { (mg/L) }\end{array}$ & $\begin{array}{c}\text { Nitrite+Nitrate, } \\
\text { dissolved } \\
(\mathrm{mg} / \mathrm{L} \text { as } \mathrm{N}) \\
\end{array}$ & $\begin{array}{c}\text { Iron, } \\
\text { dissolved } \\
\text { (ug/L) } \\
\end{array}$ & $\begin{array}{c}\text { Manganese, } \\
\text { dissolved } \\
\text { (ug/L) }\end{array}$ & $\begin{array}{c}\text { Arsenic, } \\
\text { dissolved } \\
\text { (ug/L) }\end{array}$ \\
\hline 1250 & 170 & 3.80 & 1,200 & 70.0 & 0.200 & -- & 7.79 & 120 & -- & -- \\
\hline 1252 & -- & -- & -- & -- & -- & -- & -- & -- & -- & -- \\
\hline 1253 & 710 & 21.0 & 3,200 & 43.0 & 0.600 & -- & 0.030 & 50 & -- & -- \\
\hline 1254 & -- & -- & -- & -- & -- & -- & -- & -- & -- & -- \\
\hline 1256 & -- & -- & -- & -- & -- & -- & -- & -- & -- & -- \\
\hline 1267 & -- & -- & -- & -- & -- & -- & -- & -- & -- & -- \\
\hline 1272 & -- & -- & -- & -- & -- & -- & -- & -- & -- & -- \\
\hline 1273 & -- & -- & -- & -- & -- & -- & -- & -- & -- & -- \\
\hline 1277 & 31.0 & 1.40 & 78.0 & 12.0 & 0.500 & -- & -- & $<10$ & $<10$ & $<1$ \\
\hline 1282 & -- & -- & -- & -- & -- & -- & -- & -- & -- & -- \\
\hline 1297 & -- & -- & -- & -- & -- & -- & -- & -- & -- & -- \\
\hline 1317 & 19.1 & 4.20 & 121 & 16.0 & -- & -- & 1.30 & -- & -- & -- \\
\hline 1318 & 66.1 & 2.00 & 1,617 & 6.50 & -- & -- & $<0.05$ & -- & -- & -- \\
\hline 1321 & 660 & 1.50 & 0.700 & 340 & 22.0 & -- & $<0.08$ & 70 & 10.0 & $<1$ \\
\hline 1327 & -- & -- & -- & -- & -- & -- & -- & -- & -- & -- \\
\hline 1328 & 710 & 2.40 & 5.30 & 67.0 & 2.50 & -- & -- & 180 & $<10$ & -- \\
\hline 1329 & -- & -- & -- & -- & -- & -- & -- & -- & -- & -- \\
\hline 1330 & -- & -- & 16.0 & 90.0 & -- & -- & -- & -- & -- & -- \\
\hline 1333 & -- & -- & -- & -- & -- & -- & -- & -- & -- & -- \\
\hline 1369 & -- & -- & -- & -- & -- & -- & -- & -- & -- & -- \\
\hline 1371 & -- & -- & 13.0 & 4.00 & 1.00 & -- & 0.36 & -- & -- & -- \\
\hline 1381 & -- & -- & -- & -- & -- & -- & -- & -- & -- & -- \\
\hline 1382 & -- & -- & -- & -- & -- & -- & -- & -- & -- & -- \\
\hline 1383 & -- & -- & -- & -- & -- & -- & -- & -- & -- & -- \\
\hline 1409 & -- & -- & -- & -- & -- & -- & -- & -- & -- & -- \\
\hline 1410 & 44.0 & 1.50 & 110 & 16.0 & 0.600 & -- & -- & 60 & 20.0 & $<1$ \\
\hline 1411 & -- & -- & -- & -- & -- & -- & -- & -- & -- & -- \\
\hline 1415 & -- & -- & -- & -- & -- & -- & -- & -- & -- & -- \\
\hline 1416 & -- & -- & -- & -- & -- & -- & -- & -- & -- & -- \\
\hline 1418 & 200 & 13.0 & 39.0 & 3.80 & 0.800 & -- & -- & 2,200 & 80.0 & $<1$ \\
\hline 1419 & 11.5 & 1.70 & 163 & 14.8 & -- & -- & 1.40 & -- & -- & -- \\
\hline 1420 & 48.2 & 1.40 & 112 & 20.0 & -- & -- & 2.60 & -- & -- & -- \\
\hline 1421 & 28.0 & 0.500 & 40.0 & 1.80 & 0.200 & -- & -- & 30 & $<5$ & 4.00 \\
\hline 1422 & 140 & 1.20 & 390 & 7.00 & 0.600 & -- & -- & -- & -- & 1.00 \\
\hline 1423 & 820 & 2.60 & 6.00 & 230 & 21.0 & -- & $<0.08$ & 110 & 20.0 & $<1$ \\
\hline 1426 & 330 & 2.00 & 18.0 & 68.0 & $<0.1$ & -- & -- & 50 & $<10$ & 9.00 \\
\hline 1433 & 310 & 0.900 & 5.70 & 14.0 & 17.0 & 0.020 & -- & 90 & 40.0 & 4.00 \\
\hline 1438 & 190 & 0.900 & 490 & 8.70 & 0.800 & 0.020 & -- & 280 & 530 & 5.00 \\
\hline 1443 & 170 & 1.60 & 560 & 8.70 & 0.400 & 0.020 & -- & 110 & 60.0 & 4.00 \\
\hline 1445 & 120 & 1.80 & 170 & 7.70 & 0.200 & 0.070 & -- & 80 & 180 & 2.00 \\
\hline 1448 & 230 & 1.10 & 220 & 9.90 & 1.50 & 0.10 & -- & 160 & 20.0 & $<1$ \\
\hline 1450 & 280 & 0.800 & 41.0 & 62.0 & 30.0 & 0.20 & -- & 260 & $<10$ & $<1$ \\
\hline 1452 & 280 & 1.60 & 460 & 16.0 & 1.10 & 0.10 & -- & 250 & 50.0 & $<1$ \\
\hline 1454 & 220 & 0.400 & 320 & 7.30 & 2.20 & -- & $<0.08$ & 1,000 & 56.0 & $<1$ \\
\hline 1461 & -- & -- & -- & -- & -- & -- & -- & -- & -- & -- \\
\hline 1463 & -- & -- & -- & -- & -- & -- & -- & -- & -- & -- \\
\hline 1464 & -- & -- & -- & -- & -- & -- & -- & -- & -- & -- \\
\hline 1465 & -- & -- & -- & -- & -- & -- & -- & -- & -- & -- \\
\hline 1466 & 1,200 & 1.90 & 48.0 & 130 & 60.0 & -- & -- & 80 & $<10$ & 6.00 \\
\hline
\end{tabular}


Appendix 1. Water Quality Data Used in This Report.-Continued

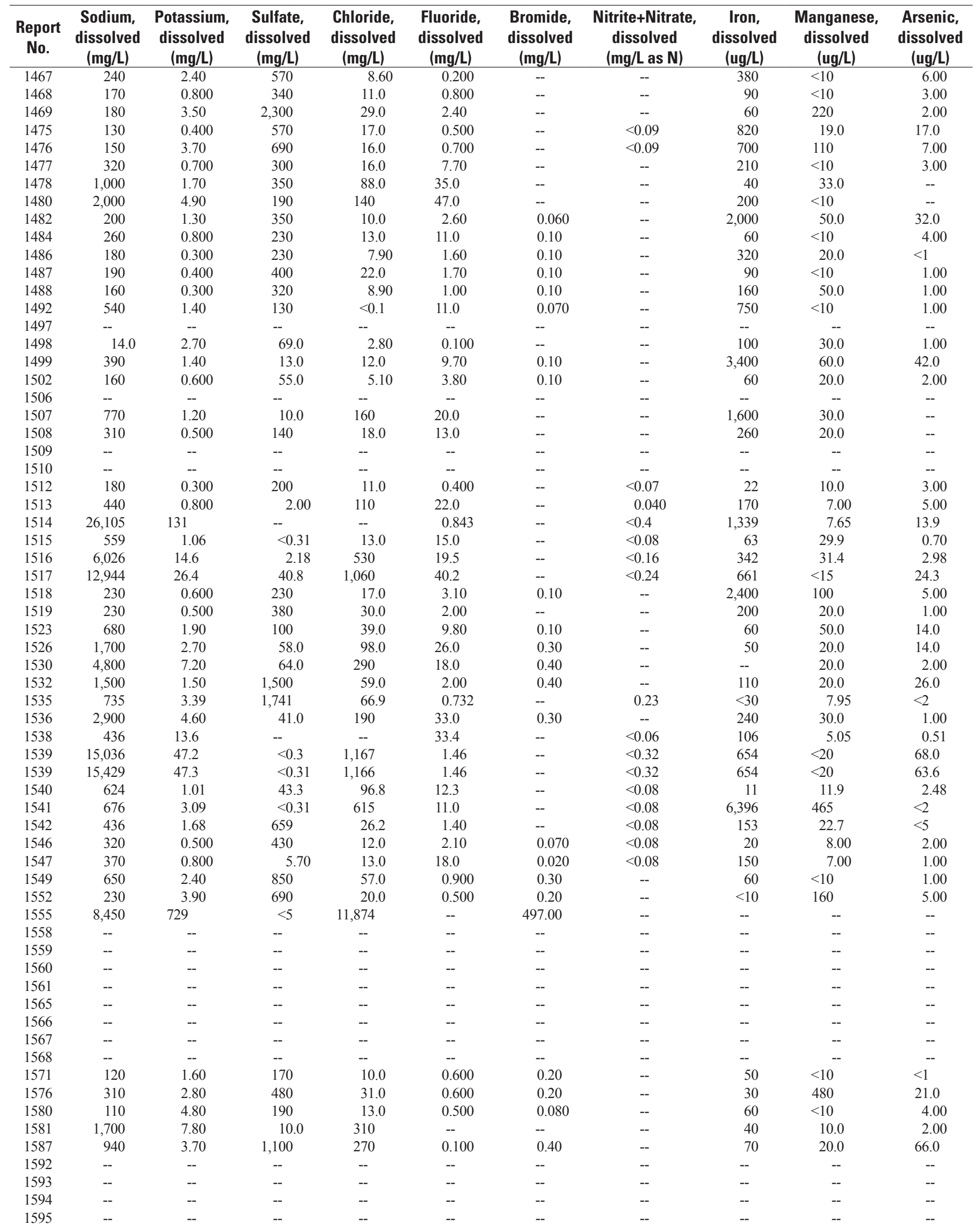


Appendix 1. Water Quality Data Used in This Report.-Continued

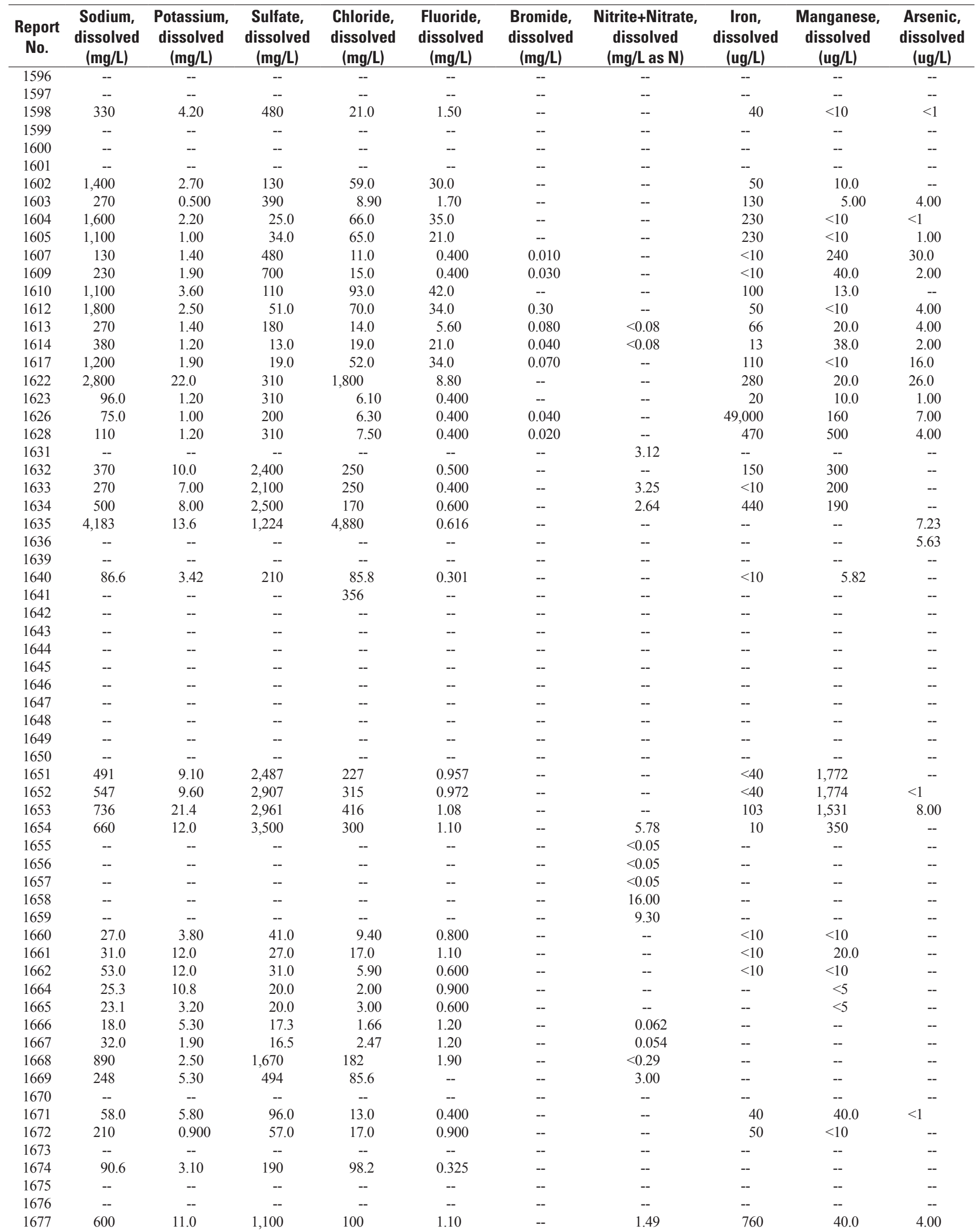


Appendix 1. Water Quality Data Used in This Report.-Continued

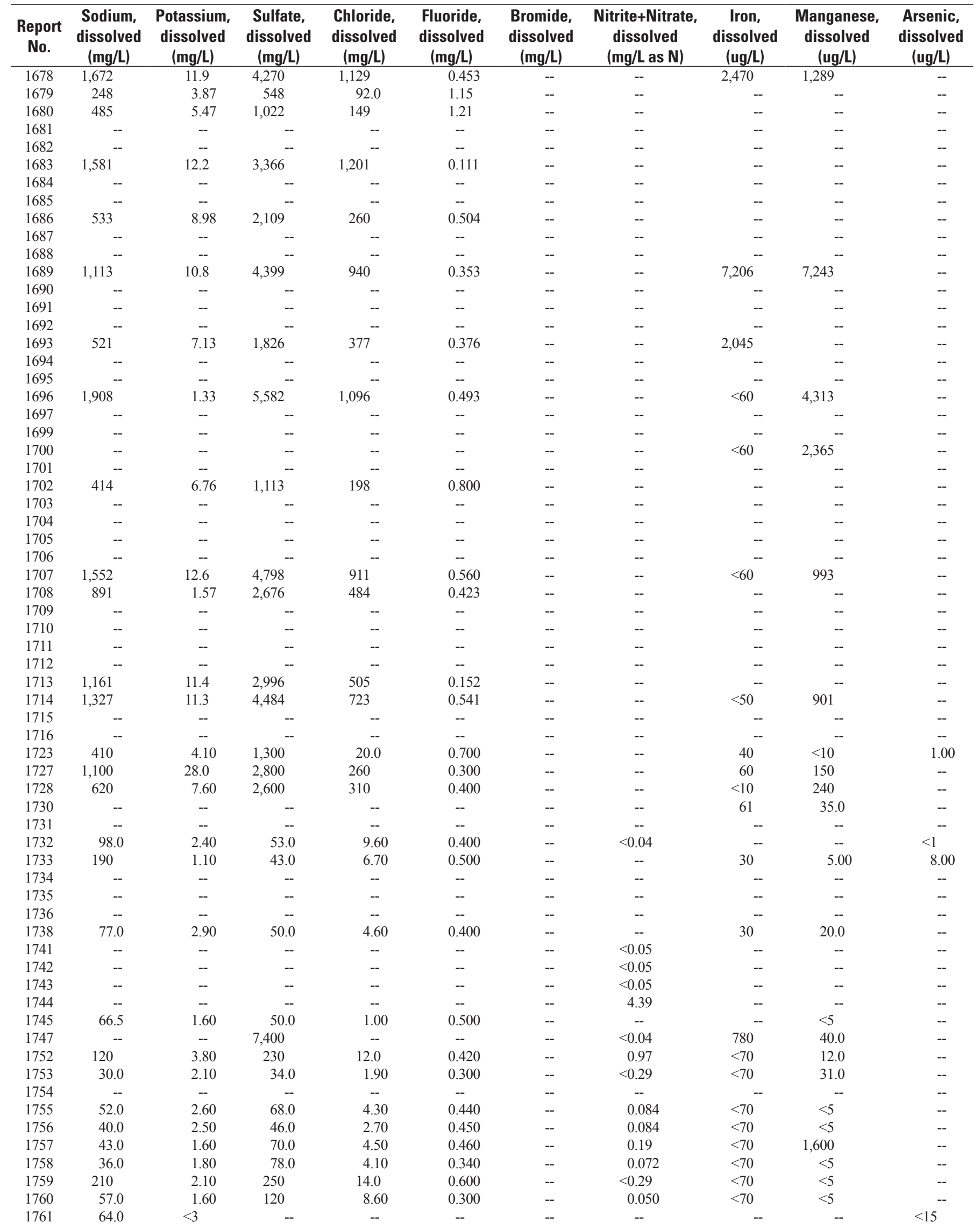


Appendix 1. Water Quality Data Used in This Report.-Continued

\begin{tabular}{|c|c|c|c|c|c|c|c|c|c|c|}
\hline $\begin{array}{c}\text { Report } \\
\text { No. }\end{array}$ & $\begin{array}{c}\text { Sodium, } \\
\text { dissolved } \\
\text { (mg/L) }\end{array}$ & $\begin{array}{l}\text { Potassium, } \\
\text { dissolved } \\
\text { (mg/L) }\end{array}$ & $\begin{array}{c}\text { Sulfate, } \\
\text { dissolved } \\
\text { (mg/L) }\end{array}$ & $\begin{array}{c}\text { Chloride, } \\
\text { dissolved } \\
\text { (mg/L) }\end{array}$ & $\begin{array}{c}\text { Fluoride, } \\
\text { dissolved } \\
\text { (mg/L) }\end{array}$ & $\begin{array}{c}\text { Bromide, } \\
\text { dissolved } \\
\text { (mg/L) }\end{array}$ & $\begin{array}{c}\text { Nitrite+Nitrate, } \\
\text { dissolved } \\
\text { (mg/L as } \mathrm{N})\end{array}$ & $\begin{array}{c}\text { Iron, } \\
\text { dissolved } \\
\text { (ug/L) }\end{array}$ & $\begin{array}{c}\text { Manganese, } \\
\text { dissolved } \\
\text { (ug/L) }\end{array}$ & $\begin{array}{c}\text { Arsenic, } \\
\text { dissolved } \\
\text { (ug/L) }\end{array}$ \\
\hline 1762 & -- & -- & -- & -- & -- & -- & -- & -- & -- & -- \\
\hline 1765 & 830 & 12.0 & 3,100 & 240 & 1.50 & -- & 3.81 & 260 & 540 & -- \\
\hline 1766 & 2,600 & 16.0 & 7,700 & 500 & 0.800 & -- & -- & $<10$ & 110 & -- \\
\hline 1768 & 310 & 2.90 & 2,500 & 190 & 0.300 & -- & 2.15 & $<10$ & 860 & -- \\
\hline 1769 & 250 & 2.10 & 2,000 & 270 & 0.300 & -- & 7.58 & $<10$ & 450 & -- \\
\hline 1772 & 46.0 & 4.10 & 40.0 & 6.00 & 0.400 & -- & -- & $<10$ & $<10$ & -- \\
\hline 1773 & 43.8 & 1.10 & 50.0 & 6.00 & 0.700 & -- & -- & -- & 9.00 & -- \\
\hline 1774 & -- & -- & -- & -- & -- & -- & -- & -- & -- & -- \\
\hline 1776 & 275 & 0.600 & 20.0 & 3.00 & 1.70 & -- & -- & -- & $<5$ & -- \\
\hline 1777 & 168 & 0.600 & 80.0 & 3.00 & 0.800 & -- & -- & -- & $<5$ & -- \\
\hline 1778 & 42.9 & 1.60 & 30.0 & 5.00 & 0.700 & -- & -- & -- & $<5$ & -- \\
\hline 1783 & 170 & 0.390 & 57.6 & 1.16 & 0.860 & -- & $<0.29$ & -- & -- & -- \\
\hline 1784 & 140 & 0.740 & 32.5 & 3.21 & $<0.5$ & -- & $<0.29$ & -- & -- & -- \\
\hline 1785 & 160 & 0.600 & 20.2 & 9.66 & $<0.5$ & -- & 0.035 & -- & -- & -- \\
\hline 1786 & 30.0 & 5.10 & 37.5 & 4.99 & 0.830 & -- & 0.078 & -- & -- & -- \\
\hline 1787 & -- & -- & -- & -- & -- & -- & -- & -- & -- & -- \\
\hline 1788 & 450 & 8.30 & 2,000 & 150 & $<0.5$ & -- & 2.06 & $<70$ & $<5$ & -- \\
\hline 1789 & -- & -- & -- & -- & -- & -- & -- & -- & -- & -- \\
\hline 1790 & -- & -- & -- & -- & -- & -- & -- & -- & -- & -- \\
\hline 1791 & -- & -- & -- & -- & -- & -- & -- & -- & -- & -- \\
\hline 1792 & -- & -- & -- & -- & -- & -- & -- & -- & -- & -- \\
\hline 1793 & -- & -- & -- & -- & -- & -- & -- & -- & -- & -- \\
\hline 1794 & 19.0 & 2.30 & 21.0 & 3.90 & 0.600 & -- & -- & 20 & $<10$ & -- \\
\hline 1795 & 47.0 & 17.0 & 49.0 & 9.20 & 0.800 & -- & -- & 20 & $<10$ & -- \\
\hline 1805 & 42.3 & 5.00 & 40.0 & 5.00 & 0.400 & -- & -- & -- & $<5$ & -- \\
\hline 1806 & 28.0 & 4.20 & 30.0 & 5.00 & 0.200 & -- & -- & -- & $<5$ & -- \\
\hline 1807 & 170 & 0.580 & 0.680 & 2.10 & 1.40 & -- & $<0.29$ & -- & -- & -- \\
\hline 1807 & -- & -- & -- & -- & -- & -- & -- & -- & -- & -- \\
\hline 1808 & 200 & 7.60 & 827 & 26.5 & 0.660 & -- & $<0.29$ & -- & -- & -- \\
\hline 1809 & 68.0 & 1.00 & 48.2 & 4.38 & 1.60 & -- & 0.080 & -- & -- & -- \\
\hline 1810 & 39.0 & 11.0 & 31.1 & 6.73 & 0.790 & -- & 0.25 & -- & -- & -- \\
\hline 1811 & 42.0 & 9.50 & 24.0 & 2.70 & 0.680 & -- & 0.070 & $<70$ & $<5$ & -- \\
\hline 1812 & 59.0 & 14.0 & 34.0 & 3.50 & 0.760 & -- & 0.086 & $<70$ & $<5$ & -- \\
\hline 1813 & 280 & 2.50 & 190 & 9.20 & 3.00 & -- & $<0.29$ & 130 & $<5$ & -- \\
\hline 1814 & 5.40 & 0.620 & 5.30 & 4.10 & 1.40 & -- & 0.070 & $<70$ & $<5$ & -- \\
\hline 1815 & 189 & 0.700 & 17.8 & 16.4 & -- & -- & 4.90 & -- & -- & -- \\
\hline 1816 & 79.0 & 4.50 & 54.0 & 11.0 & 1.20 & -- & 0.54 & $<70$ & $<5$ & -- \\
\hline 1817 & 63.0 & -- & 35.0 & 4.10 & 0.630 & -- & 0.14 & $<70$ & $<5$ & -- \\
\hline 1818 & 15.0 & 0.880 & 6.90 & 2.00 & 2.00 & -- & 0.14 & $<70$ & $<5$ & -- \\
\hline 1819 & 69.3 & 1.10 & 72.7 & 6.00 & -- & -- & 0.50 & -- & -- & -- \\
\hline 1820 & 97.0 & 3.50 & 68.0 & 9.00 & 0.740 & -- & 0.043 & $<70$ & 17.0 & -- \\
\hline 1821 & 24.0 & 1.10 & 6.40 & 5.80 & 1.20 & -- & 0.15 & $<70$ & $<5$ & -- \\
\hline 1822 & 30.0 & 1.60 & 7.30 & 1.50 & 1.50 & -- & 0.052 & $<70$ & $<5$ & -- \\
\hline 1827 & 17.7 & 1.50 & -- & -- & -- & -- & -- & $<100$ & 16.8 & 1.54 \\
\hline 1828 & 16.4 & 1.59 & -- & -- & -- & -- & -- & $<100$ & $<1$ & $<1$ \\
\hline 1829 & -- & -- & 8,400 & -- & -- & -- & $<0.04$ & $<10$ & 30.0 & -- \\
\hline 1830 & 75.0 & 1.40 & 70.0 & 33.0 & 0.400 & -- & -- & $<10$ & $<10$ & -- \\
\hline 1832 & 12.7 & 2.60 & 20.0 & 2.00 & 0.200 & -- & -- & -- & $<5$ & -- \\
\hline 1833 & 110 & 9.80 & 200 & 18.0 & 1.10 & -- & -- & 20 & $<10$ & -- \\
\hline
\end{tabular}


Appendix 1. Water Quality Data Used in This Report.-Continued

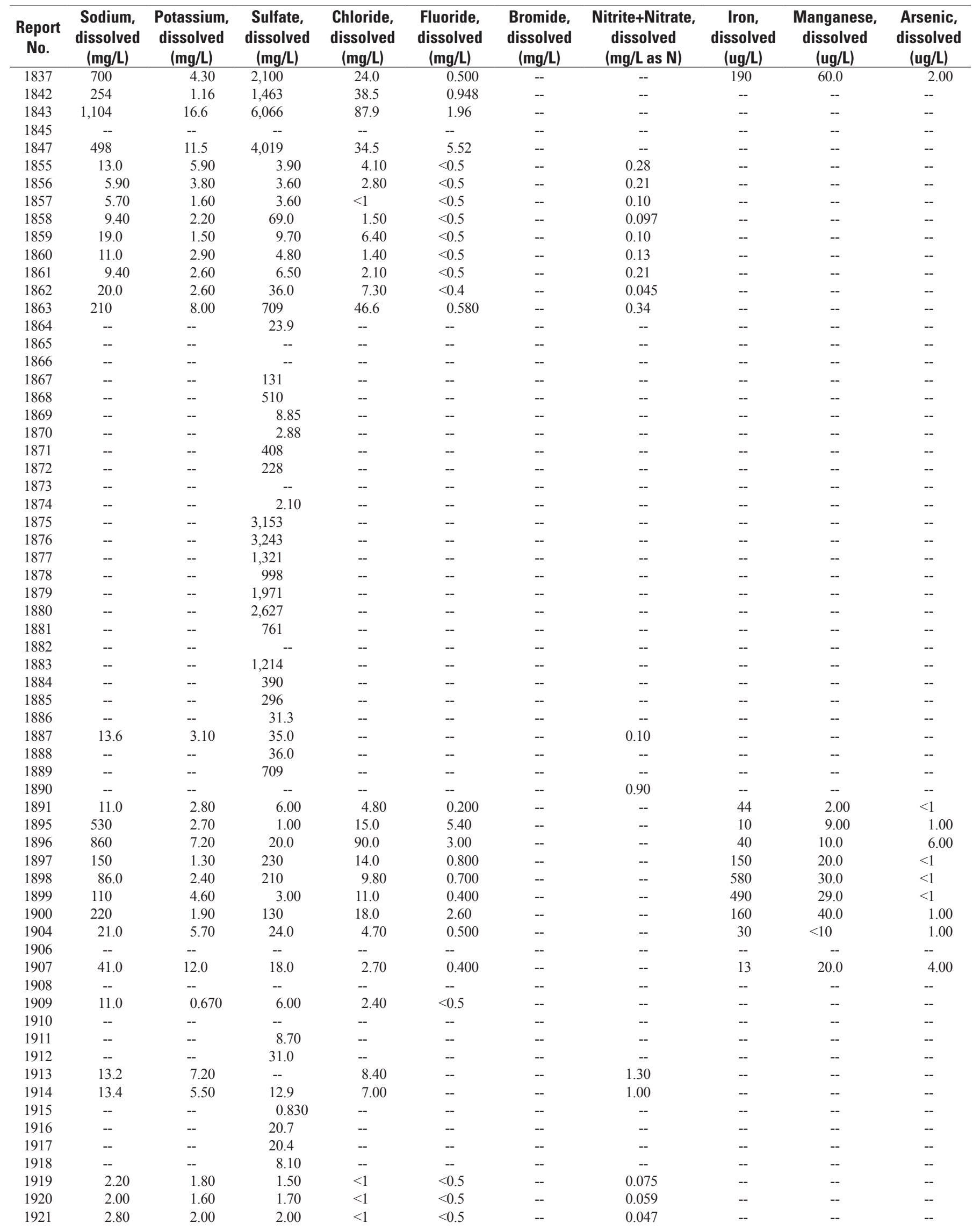


Appendix 1. Water Quality Data Used in This Report.-Continued

\begin{tabular}{|c|c|c|c|c|c|c|c|c|c|c|}
\hline $\begin{array}{c}\text { Report } \\
\text { No. }\end{array}$ & $\begin{array}{c}\text { Sodium, } \\
\text { dissolved } \\
(\mathrm{mg} / \mathrm{L})\end{array}$ & $\begin{array}{c}\text { Potassium, } \\
\text { dissolved } \\
\text { (mg/L) }\end{array}$ & $\begin{array}{c}\text { Sulfate, } \\
\text { dissolved } \\
(\mathrm{mg} / \mathrm{L})\end{array}$ & $\begin{array}{c}\text { Chloride, } \\
\text { dissolved } \\
\text { (mg/L) }\end{array}$ & $\begin{array}{c}\text { Fluoride, } \\
\text { dissolved } \\
(\mathrm{mg} / \mathrm{L})\end{array}$ & $\begin{array}{c}\text { Bromide, } \\
\text { dissolved } \\
(\mathrm{mg} / \mathrm{L})\end{array}$ & $\begin{array}{c}\text { Nitrite+Nitrate, } \\
\text { dissolved } \\
(\mathrm{mg} / \mathrm{L} \text { as } \mathrm{N})\end{array}$ & $\begin{array}{c}\text { Iron, } \\
\text { dissolved } \\
\text { (ug/L) }\end{array}$ & $\begin{array}{c}\text { Manganese, } \\
\text { dissolved } \\
\text { (ug/L) }\end{array}$ & $\begin{array}{c}\text { Arsenic, } \\
\text { dissolved } \\
\text { (ug/L) }\end{array}$ \\
\hline 1925 & 600 & 2.60 & 3,000 & 24.0 & 0.300 & -- & 0.96 & 1,200 & 330 & -- \\
\hline 1928 & 110 & 4.30 & 1,600 & 11.0 & 1.50 & -- & -- & 60 & 20.0 & $<1$ \\
\hline 1930 & -- & -- & -- & -- & -- & -- & -- & 123 & 20.0 & -- \\
\hline 1931 & -- & -- & -- & -- & -- & -- & -- & 168 & 41.0 & -- \\
\hline 1932 & -- & -- & -- & -- & -- & -- & -- & 172 & 11.0 & -- \\
\hline 1939 & 8.90 & 1.00 & $<5$ & $<1$ & $<0.5$ & -- & -- & -- & -- & -- \\
\hline 1940 & -- & -- & 7.10 & -- & -- & -- & -- & -- & -- & -- \\
\hline 1941 & -- & -- & 19.9 & -- & -- & -- & -- & -- & -- & -- \\
\hline 1942 & -- & -- & 5.10 & -- & -- & -- & -- & -- & -- & -- \\
\hline 1943 & -- & -- & 44.9 & -- & -- & -- & -- & -- & -- & -- \\
\hline 1944 & -- & -- & -- & -- & -- & -- & -- & -- & -- & -- \\
\hline 1962 & 750 & 6.50 & $<5$ & 56.0 & 3.50 & -- & -- & 100 & 40.0 & 1.00 \\
\hline 1967 & 3.70 & 3.00 & 1.70 & 1.50 & $<0.5$ & -- & 0.22 & -- & -- & -- \\
\hline 1968 & 1.40 & 1.80 & 2.30 & 1.10 & $<0.5$ & -- & 0.42 & -- & -- & -- \\
\hline 1969 & 6.40 & 1.10 & 2.70 & 1.60 & $<0.5$ & -- & -- & -- & -- & -- \\
\hline 1970 & 2.00 & 4.60 & 1.80 & 1.60 & $<0.5$ & -- & -- & -- & -- & -- \\
\hline 1971 & 2.20 & 1.20 & 4.20 & $<1$ & $<0.5$ & -- & 0.16 & -- & -- & -- \\
\hline 1972 & 11.0 & 10.0 & 2.20 & 3.40 & $<0.5$ & -- & 0.62 & -- & -- & -- \\
\hline 1973 & 55.0 & 1.30 & 3.90 & 1.40 & $<0.5$ & -- & 0.036 & -- & -- & -- \\
\hline 1974 & 2.90 & 1.60 & 1.60 & $<1$ & $<0.5$ & -- & 0.050 & -- & -- & -- \\
\hline 1975 & -- & -- & 4.70 & -- & -- & -- & -- & -- & -- & -- \\
\hline 1976 & -- & -- & 1.23 & -- & -- & -- & -- & -- & -- & -- \\
\hline 1978 & 190 & 5.40 & 150 & 7.90 & 0.500 & -- & -- & 150 & 13.0 & 1.00 \\
\hline 1981 & -- & -- & -- & -- & -- & -- & -- & -- & -- & -- \\
\hline 2002 & 110 & 12.0 & 210 & 6.40 & 0.500 & -- & -- & 40 & $<10$ & 5.00 \\
\hline 2003 & -- & -- & -- & -- & -- & -- & -- & --- & -- & -- \\
\hline 2011 & -- & -- & -- & -- & -- & -- & -- & -- & -- & -- \\
\hline 2012 & -- & -- & -- & -- & -- & -- & -- & -- & -- & -- \\
\hline 2013 & -- & -- & 12.7 & -- & -- & -- & -- & -- & -- & -- \\
\hline 2014 & -- & -- & 393 & -- & -- & -- & -- & -- & -- & -- \\
\hline 2015 & 100 & 25.0 & 393 & 19.7 & 0.710 & -- & 0.42 & -- & -- & -- \\
\hline 2016 & 60.7 & 8.10 & 113 & 12.7 & -- & -- & 1.30 & -- & -- & -- \\
\hline 2017 & 20.5 & 1.60 & 29.2 & 12.9 & -- & -- & 0.60 & -- & -- & -- \\
\hline 2018 & 10.2 & 2.30 & 11.3 & 2.60 & -- & -- & 0.50 & -- & -- & -- \\
\hline 2019 & -- & -- & -- & -- & -- & -- & 0.90 & -- & -- & -- \\
\hline 2020 & -- & -- & -- & -- & -- & -- & 3.10 & -- & -- & -- \\
\hline
\end{tabular}


Appendix 1. Water Quality Data Used in This Report.-Continued

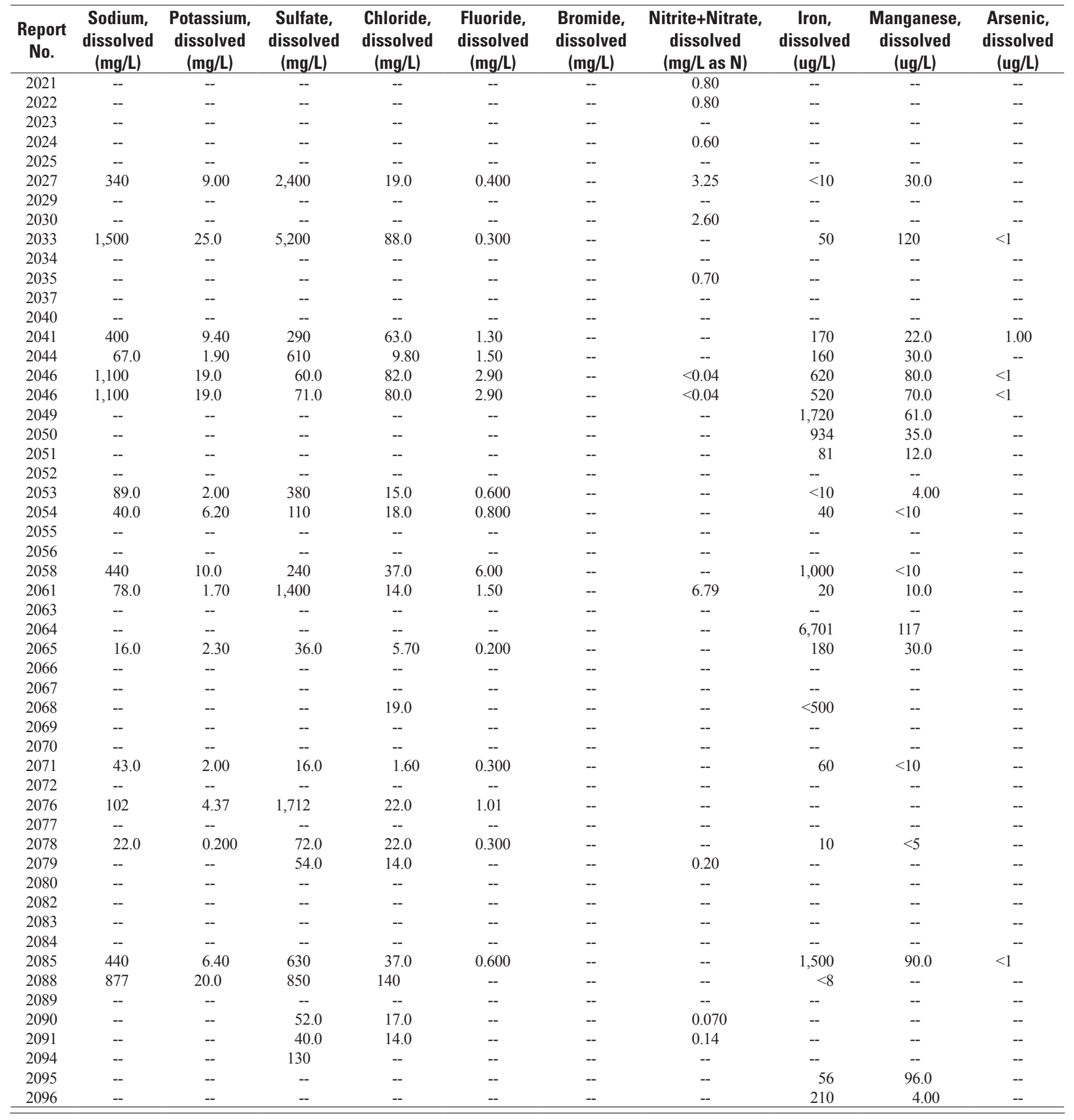


Appendix 1. Water Quality Data Used in This Report.-Continued

\begin{tabular}{|c|c|c|c|c|c|c|c|c|c|c|}
\hline $\begin{array}{c}\text { Report } \\
\text { No. }\end{array}$ & $\begin{array}{c}\text { Barium, } \\
\text { dissolved } \\
\text { (ug/L) }\end{array}$ & $\begin{array}{c}\text { Selenium, } \\
\text { dissolved } \\
\text { (ug/L) }\end{array}$ & $\begin{array}{l}\text { Methane } \\
\text { (mg/L) }\end{array}$ & $\begin{array}{c}\text { Benzene } \\
\text { (ug/L) }\end{array}$ & $\begin{array}{c}\text { Toluene } \\
\text { (ug/L) }\end{array}$ & $\begin{array}{l}\text { Ethylbenzene } \\
\text { (ug/L) }\end{array}$ & $\begin{array}{c}\text { Xylene } \\
\text { (all isomers) } \\
\text { (ug/L) }\end{array}$ & $\begin{array}{c}\text { Methane } \\
(\mathrm{mol} \%)\end{array}$ & $\begin{array}{l}\text { Ethane } \\
\text { (mol \%) }\end{array}$ & $\begin{array}{r}\text { Propane } \\
\text { (mol \%) }\end{array}$ \\
\hline 1 & -- & -- & $<0.0008$ & $<1$ & $<5$ & $<2$ & -- & -- & -- & -- \\
\hline 3 & 600 & $<1$ & -- & -- & -- & -- & -- & -- & -- & -- \\
\hline 4 & $<100$ & 6.00 & -- & -- & -- & -- & -- & -- & -- & -- \\
\hline 5 & 7.0 & 19.0 & $<0.001$ & $<0.5$ & $<0.5$ & $<0.5$ & $<1.5$ & -- & -- & -- \\
\hline 6 & -- & -- & -- & -- & -- & -- & -- & -- & -- & -- \\
\hline 13 & -- & -- & -- & -- & -- & -- & -- & -- & -- & -- \\
\hline 14 & -- & -- & -- & -- & -- & -- & -- & -- & -- & -- \\
\hline 15 & -- & -- & -- & -- & -- & -- & -- & -- & -- & -- \\
\hline 16 & -- & -- & -- & -- & -- & -- & -- & -- & -- & -- \\
\hline 17 & -- & -- & -- & $<0.5$ & $<5$ & $<2$ & $<3$ & -- & -- & -- \\
\hline 18 & -- & -- & -- & $<0.5$ & $<5$ & $<2$ & $<3$ & -- & -- & -- \\
\hline 23 & -- & -- & -- & $<0.001$ & $<0.005$ & $<2$ & $<3$ & -- & -- & -- \\
\hline 24 & -- & -- & -- & $<0.5$ & $<5$ & $<2$ & $<3$ & -- & -- & -- \\
\hline 25 & -- & -- & -- & $<0.5$ & $<5$ & $<2$ & $<3$ & -- & -- & -- \\
\hline 26 & -- & -- & -- & $<0.5$ & $<5$ & $<2$ & $<3$ & -- & -- & -- \\
\hline 27 & -- & -- & -- & $<0.5$ & $<5$ & $<2$ & $<3$ & -- & -- & -- \\
\hline 28 & -- & -- & -- & $<1$ & $<5$ & $<2$ & $<3$ & -- & -- & -- \\
\hline 29 & -- & -- & -- & -- & -- & -- & -- & -- & -- & -- \\
\hline 30 & -- & $<20$ & $<0.0008$ & $<1$ & $<5$ & $<2$ & $<3$ & -- & -- & -- \\
\hline 31 & -- & -- & -- & -- & -- & -- & -- & -- & -- & -- \\
\hline 33 & -- & -- & -- & -- & -- & -- & -- & -- & -- & -- \\
\hline 34 & -- & -- & -- & -- & -- & -- & -- & -- & -- & -- \\
\hline 36 & -- & -- & -- & -- & -- & -- & -- & -- & -- & -- \\
\hline 38 & $<100$ & 2.00 & -- & -- & -- & -- & -- & -- & -- & -- \\
\hline 48 & 59 & $<20$ & $<0.0008$ & $<1$ & $<5$ & $<2$ & -- & -- & -- & -- \\
\hline 49 & 63 & $<20$ & $<0.0008$ & $<1$ & $<5$ & 0.001 & -- & -- & -- & -- \\
\hline 50 & -- & -- & 0.002 & -- & -- & -- & -- & -- & -- & -- \\
\hline 51 & -- & -- & $<0.0008$ & $<1$ & $<5$ & $<2$ & $<3$ & -- & -- & -- \\
\hline 52 & -- & 3.50 & -- & -- & -- & -- & -- & -- & -- & -- \\
\hline 53 & -- & -- & $<0.0008$ & $<1$ & $<5$ & $<2$ & -- & -- & -- & -- \\
\hline 54 & -- & -- & $<0.0008$ & $<1$ & $<5$ & $<2$ & $<3$ & -- & -- & -- \\
\hline 55 & -- & -- & $<0.0008$ & -- & -- & -- & -- & -- & -- & -- \\
\hline 56 & -- & -- & 0.14 & $<1$ & $<5$ & $<2$ & -- & -- & -- & -- \\
\hline 57 & -- & -- & 2.90 & $<1$ & $<5$ & $<2$ & -- & -- & -- & -- \\
\hline 58 & -- & -- & 0.002 & $<1$ & $<5$ & $<2$ & -- & -- & -- & -- \\
\hline 59 & -- & -- & 0.22 & $<1$ & $<5$ & $<2$ & -- & -- & -- & -- \\
\hline 60 & -- & -- & $<0.0008$ & $<1$ & $<5$ & $<2$ & -- & -- & -- & -- \\
\hline 61 & -- & -- & $<0.0008$ & $<1$ & $<5$ & $<2$ & $<3$ & -- & -- & -- \\
\hline 62 & 210 & $<20$ & 0.028 & $<1$ & $<5$ & $<2$ & $<3$ & -- & -- & -- \\
\hline 63 & -- & -- & $<0.0008$ & $<1$ & $<5$ & $<2$ & -- & -- & -- & -- \\
\hline 64 & -- & -- & 0.58 & $<0.2$ & $<0.2$ & $<2$ & $<3$ & -- & -- & -- \\
\hline 65 & -- & -- & $<0.0008$ & $<1$ & $<5$ & $<2$ & -- & -- & -- & -- \\
\hline 66 & -- & -- & 1.30 & $<1$ & $<5$ & $<2$ & -- & -- & -- & -- \\
\hline 67 & -- & -- & 1.00 & $<1$ & $<5$ & $<2$ & -- & -- & -- & -- \\
\hline 68 & -- & -- & $<0.0008$ & $<1$ & $<5$ & $<2$ & $<3$ & -- & -- & -- \\
\hline 69 & -- & -- & 0.72 & $<1$ & $<5$ & $<2$ & -- & 12.7 & 0.19 & 0.013 \\
\hline 70 & -- & -- & $<0.0008$ & $<1$ & $<5$ & $<2$ & -- & 0.025 & 0 & 0 \\
\hline 71 & -- & -- & $<0.0008$ & $<1$ & $<5$ & $<2$ & $<3$ & -- & -- & -- \\
\hline
\end{tabular}


Appendix 1. Water Quality Data Used in This Report.-Continued

\begin{tabular}{|c|c|c|c|c|c|c|c|c|c|c|}
\hline $\begin{array}{c}\text { Report } \\
\text { No. }\end{array}$ & $\begin{array}{c}\text { Barium, } \\
\text { dissolved } \\
\text { (ug/L) }\end{array}$ & $\begin{array}{c}\text { Selenium, } \\
\text { dissolved } \\
\text { (ug/L) }\end{array}$ & $\begin{array}{c}\text { Methane } \\
\text { (mg/L) }\end{array}$ & $\begin{array}{c}\text { Benzene } \\
\text { (ug/L) }\end{array}$ & $\begin{array}{c}\text { Toluene } \\
\text { (ug/L) }\end{array}$ & $\begin{array}{c}\text { Ethylbenzene } \\
\text { (ug/L) }\end{array}$ & $\begin{array}{c}\text { Xylene } \\
\text { (all isomers) } \\
\text { (ug/L) }\end{array}$ & $\begin{array}{c}\text { Methane } \\
\text { (mol \%) }\end{array}$ & $\begin{array}{l}\text { Ethane } \\
\text { (mol \%) }\end{array}$ & $\begin{array}{r}\text { Propane } \\
\text { (mol \%) }\end{array}$ \\
\hline 72 & -- & -- & $<0.0008$ & $<1$ & $<5$ & $<2$ & $<3$ & -- & -- & -- \\
\hline 74 & -- & -- & $<0.0008$ & $<1$ & $<5$ & $<2$ & $<3$ & -- & -- & -- \\
\hline 75 & -- & -- & $<0.0008$ & $<1$ & $<5$ & $<2$ & $<3$ & -- & -- & -- \\
\hline 76 & -- & -- & $<0.0008$ & $<1$ & $<5$ & $<2$ & $<3$ & -- & -- & -- \\
\hline 77 & -- & -- & 0.86 & $<0.3$ & 1.30 & $<2$ & -- & -- & -- & -- \\
\hline 80 & -- & $<20$ & $<0.0008$ & $<0.5$ & $<5$ & $<2$ & $<3$ & -- & -- & -- \\
\hline 81 & -- & $<5$ & $<0.0008$ & $<1$ & $<2$ & $<2$ & -- & -- & -- & -- \\
\hline 82 & -- & $<5$ & $<0.0008$ & $<1$ & $<2$ & $<2$ & -- & -- & -- & -- \\
\hline 83 & -- & 17.5 & 0.007 & $<1$ & $<2$ & $<2$ & -- & -- & -- & -- \\
\hline 84 & -- & 13.0 & 0.010 & $<1$ & $<2$ & $<2$ & -- & -- & -- & -- \\
\hline 85 & -- & 7.30 & 0.053 & $<1$ & $<2$ & $<2$ & -- & -- & -- & -- \\
\hline 90 & -- & -- & $<0.0008$ & $<1$ & $<5$ & $<2$ & -- & -- & -- & -- \\
\hline 91 & 142 & $<20$ & 0.86 & $<1$ & 1.30 & $<2$ & -- & -- & -- & -- \\
\hline 92 & -- & 22.0 & $<0.0008$ & $<1$ & $<5$ & $<2$ & $<3$ & -- & -- & -- \\
\hline 93 & -- & $<20$ & 6.90 & $<1$ & $<5$ & $<2$ & -- & -- & -- & -- \\
\hline 94 & -- & 6.70 & 0.14 & $<1$ & $<5$ & $<2$ & -- & -- & -- & -- \\
\hline 95 & -- & $<20$ & 2.90 & $<1$ & $<5$ & $<2$ & -- & -- & -- & -- \\
\hline 96 & -- & 9.30 & 0.22 & $<1$ & $<5$ & $<2$ & -- & -- & -- & -- \\
\hline 97 & -- & 6.20 & 0.002 & $<1$ & $<5$ & $<2$ & -- & -- & -- & -- \\
\hline 98 & -- & -- & $<0.0008$ & $<1$ & 4.10 & $<2$ & -- & -- & -- & -- \\
\hline 99 & -- & -- & 4.70 & 160 & $<5$ & 4.60 & -- & 52.2 & 6.95 & 1.99 \\
\hline 100 & -- & -- & 6.20 & 110 & $<5$ & $<2$ & -- & 59.1 & 7.51 & 2.79 \\
\hline 101 & -- & -- & $<0.0008$ & $<1$ & $<5$ & $<2$ & -- & -- & -- & -- \\
\hline 102 & -- & -- & 5.00 & $<1$ & $<5$ & $<2$ & -- & 46.9 & 5.23 & 1.92 \\
\hline 112 & -- & $<20$ & 0.054 & $<1$ & $<5$ & $<2$ & -- & -- & -- & -- \\
\hline 113 & -- & -- & 1.30 & $<1$ & $<5$ & $<2$ & -- & 13.3 & 0.80 & 0 \\
\hline 114 & -- & -- & 1.90 & 31.0 & $<5$ & $<2$ & -- & 28.0 & 3.39 & 0.51 \\
\hline 115 & -- & -- & $<0.0008$ & $<1$ & $<5$ & $<2$ & -- & -- & -- & -- \\
\hline 116 & -- & -- & 1.30 & $<1$ & $<5$ & $<2$ & -- & -- & -- & -- \\
\hline 117 & -- & -- & $<0.0008$ & $<1$ & $<5$ & $<2$ & -- & -- & -- & -- \\
\hline 118 & -- & -- & 0.003 & $<1$ & $<5$ & $<2$ & -- & -- & -- & -- \\
\hline 119 & -- & -- & 1.00 & $<1$ & $<5$ & $<2$ & -- & -- & -- & -- \\
\hline 120 & -- & -- & $<0.0008$ & $<1$ & $<5$ & $<2$ & -- & -- & -- & -- \\
\hline 121 & -- & -- & $<0.0008$ & $<1$ & $<5$ & $<2$ & -- & -- & -- & -- \\
\hline 122 & -- & -- & 0.004 & $<1$ & $<5$ & $<2$ & -- & -- & -- & -- \\
\hline 123 & -- & -- & 0.002 & $<1$ & $<5$ & $<2$ & -- & -- & -- & -- \\
\hline 124 & -- & 5.40 & $<0.0008$ & $<1$ & $<5$ & $<2$ & -- & -- & -- & -- \\
\hline 125 & -- & $<20$ & $<0.0008$ & $<1$ & $<5$ & $<2$ & $<3$ & -- & -- & -- \\
\hline 126 & 42 & 47.0 & $<0.0008$ & $<1$ & 0.40 & $<2$ & -- & -- & -- & -- \\
\hline 127 & -- & $<20$ & $<0.0008$ & $<1$ & $<5$ & $<2$ & -- & -- & -- & -- \\
\hline 128 & -- & 41.0 & 0.001 & $<1$ & $<5$ & $<2$ & -- & -- & -- & -- \\
\hline 129 & -- & 67.0 & $<0.0008$ & $<1$ & $<5$ & $<2$ & -- & -- & -- & -- \\
\hline 130 & -- & $<20$ & 3.10 & $<1$ & $<5$ & $<2$ & $<3$ & -- & -- & -- \\
\hline 131 & -- & $<20$ & 7.80 & $<1$ & $<5$ & $<2$ & $<3$ & 34.8 & 0.039 & 0 \\
\hline 132 & 97 & 3.00 & $<0.0008$ & $<1$ & 0.20 & $<2$ & -- & -- & -- & -- \\
\hline 133 & -- & $<20$ & $<0.0008$ & $<1$ & $<5$ & $<2$ & -- & -- & -- & -- \\
\hline 134 & -- & 61.0 & 0.001 & $<1$ & $<5$ & $<2$ & -- & -- & -- & -- \\
\hline 135 & -- & -- & 0.004 & $<1$ & $<5$ & $<2$ & -- & -- & -- & -- \\
\hline
\end{tabular}


Appendix 1. Water Quality Data Used in This Report.-Continued

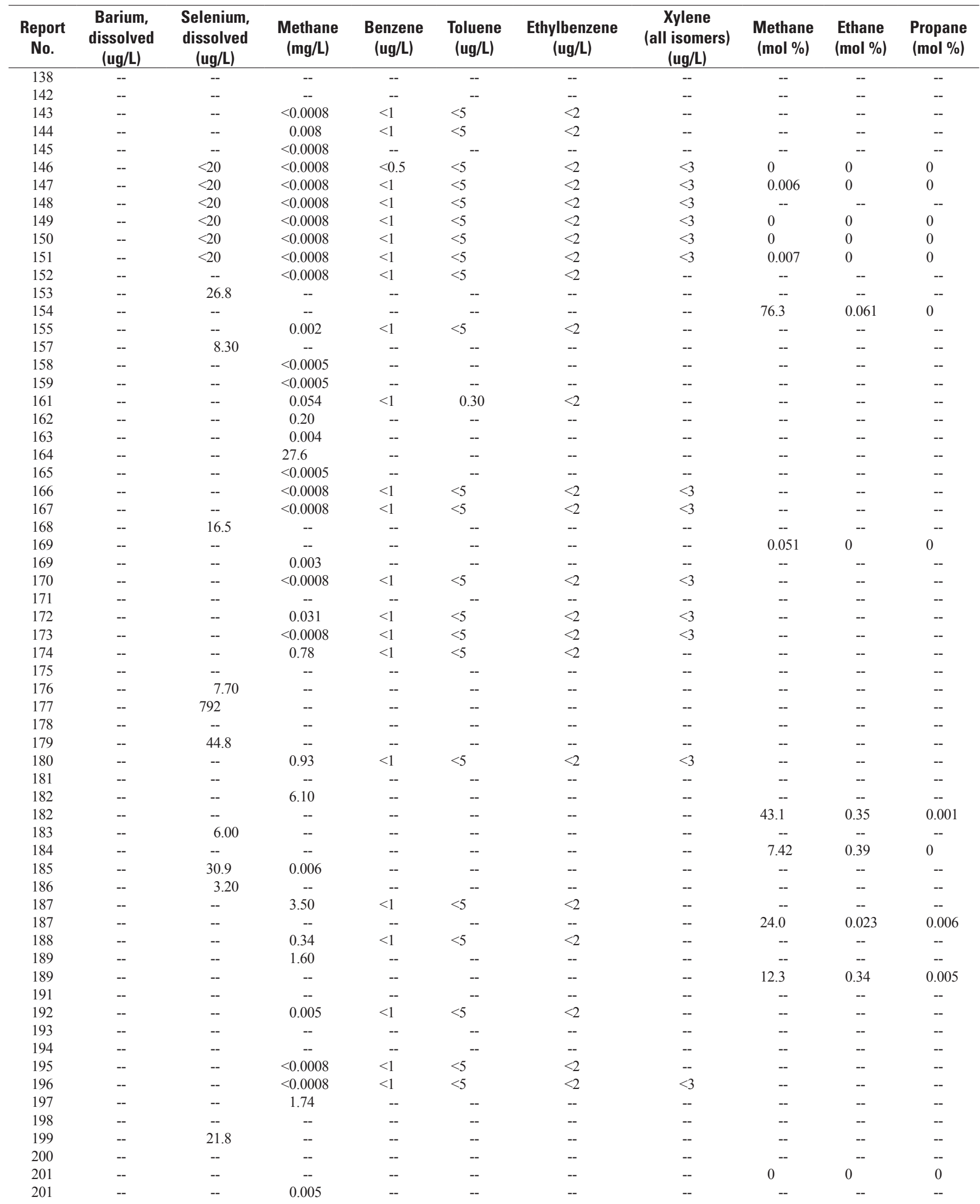


Appendix 1. Water Quality Data Used in This Report.-Continued

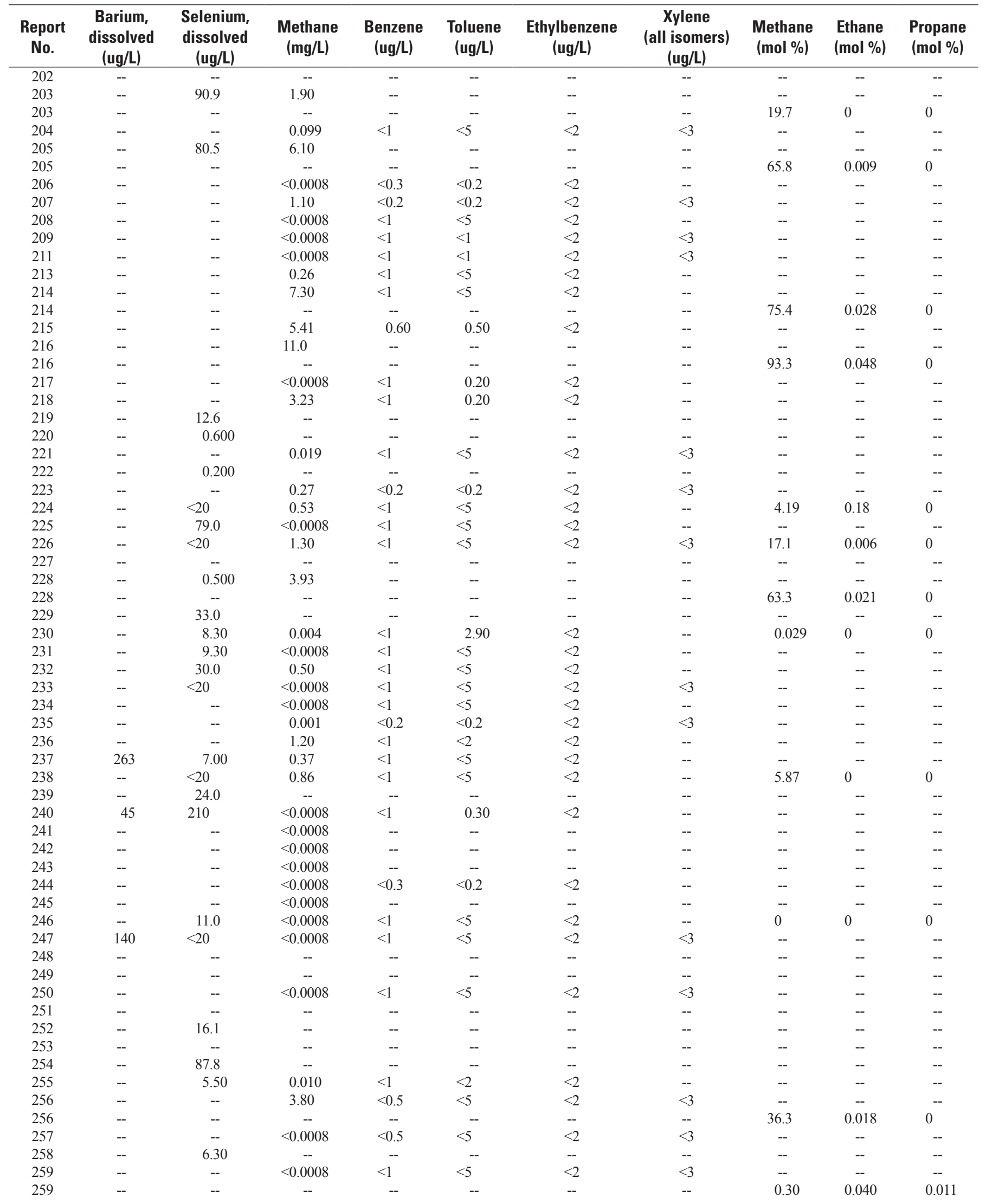


Appendix 1. Water Quality Data Used in This Report.-Continued

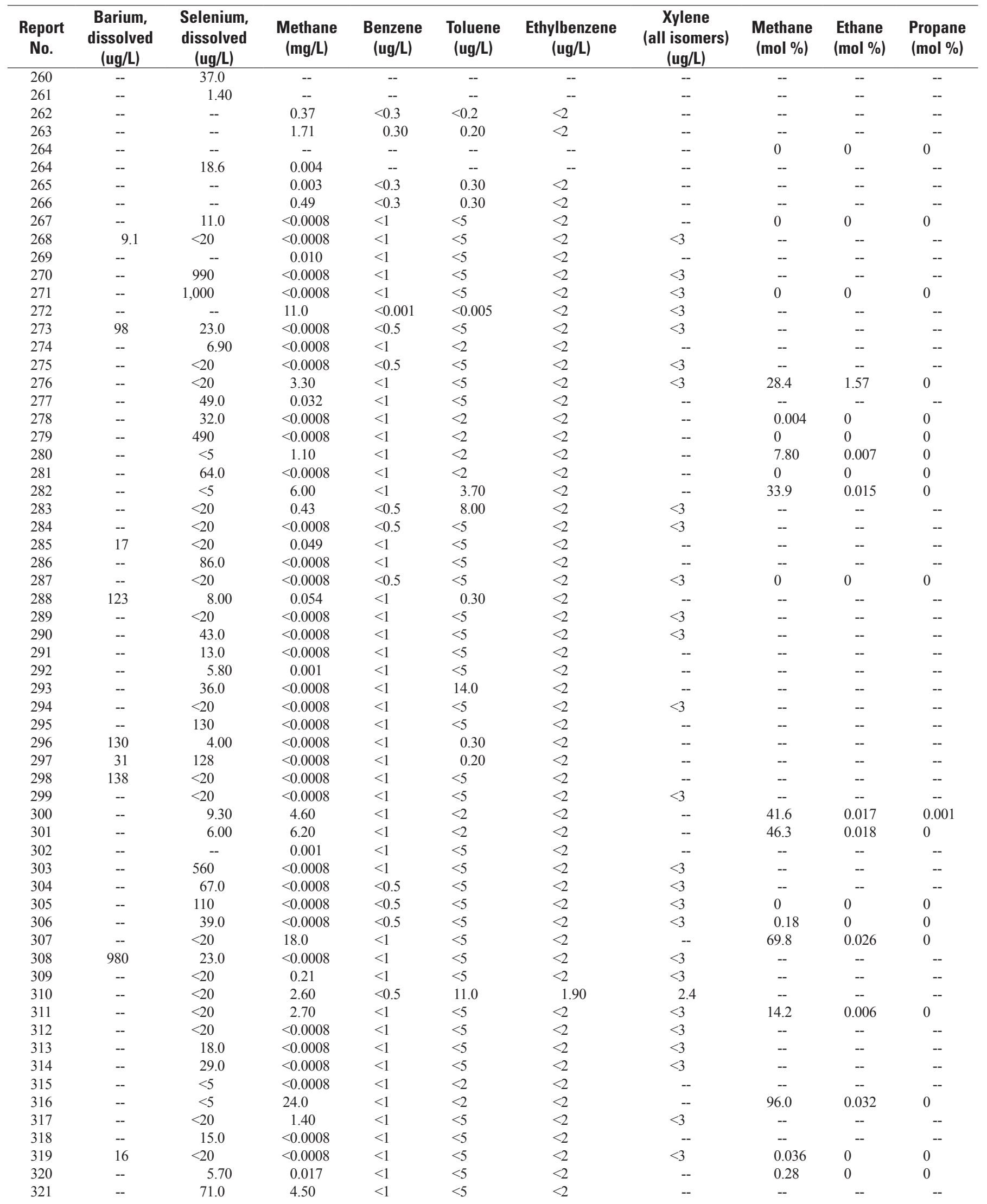


Appendix 1. Water Quality Data Used in This Report.-Continued

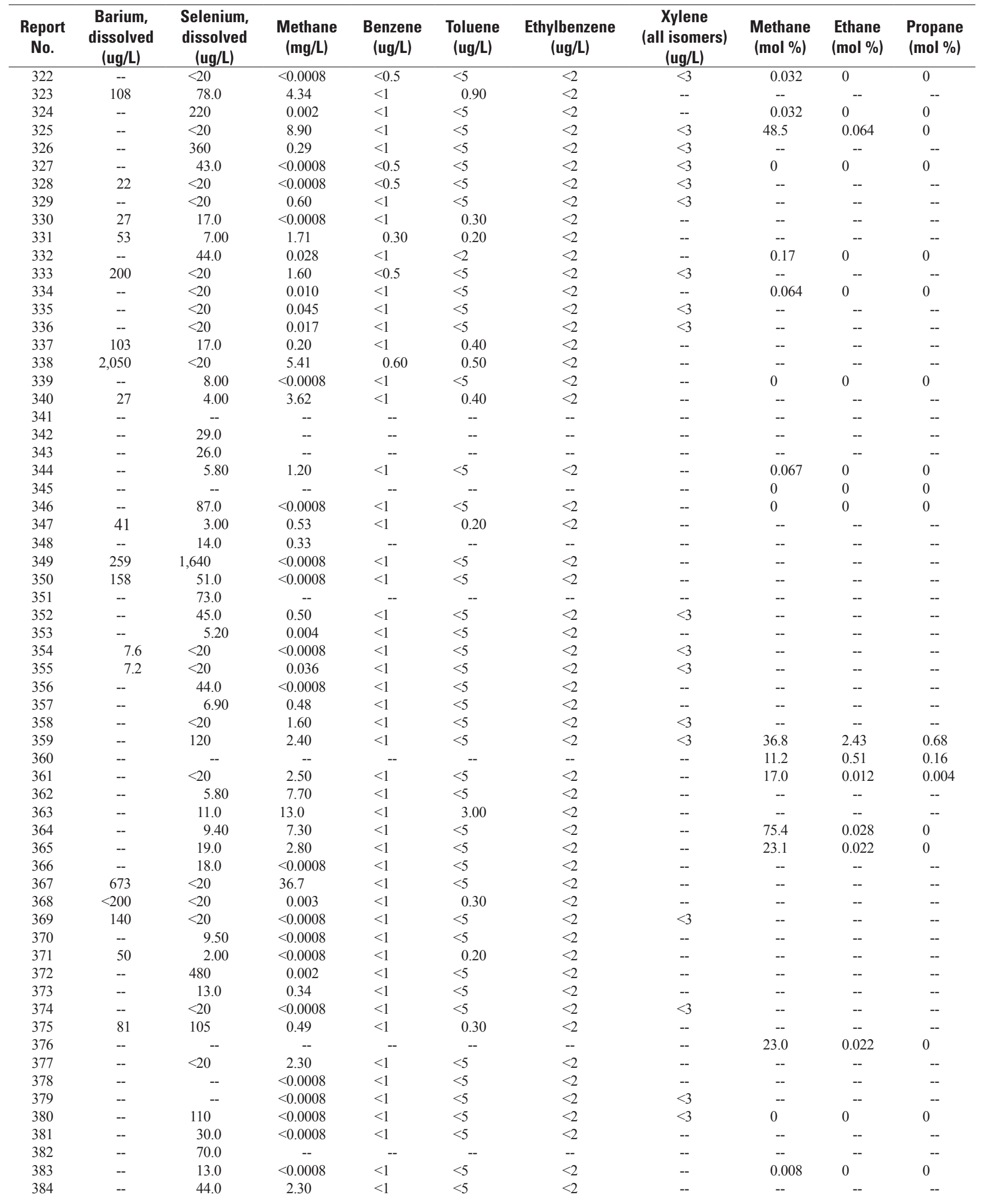


Appendix 1. Water Quality Data Used in This Report.-Continued

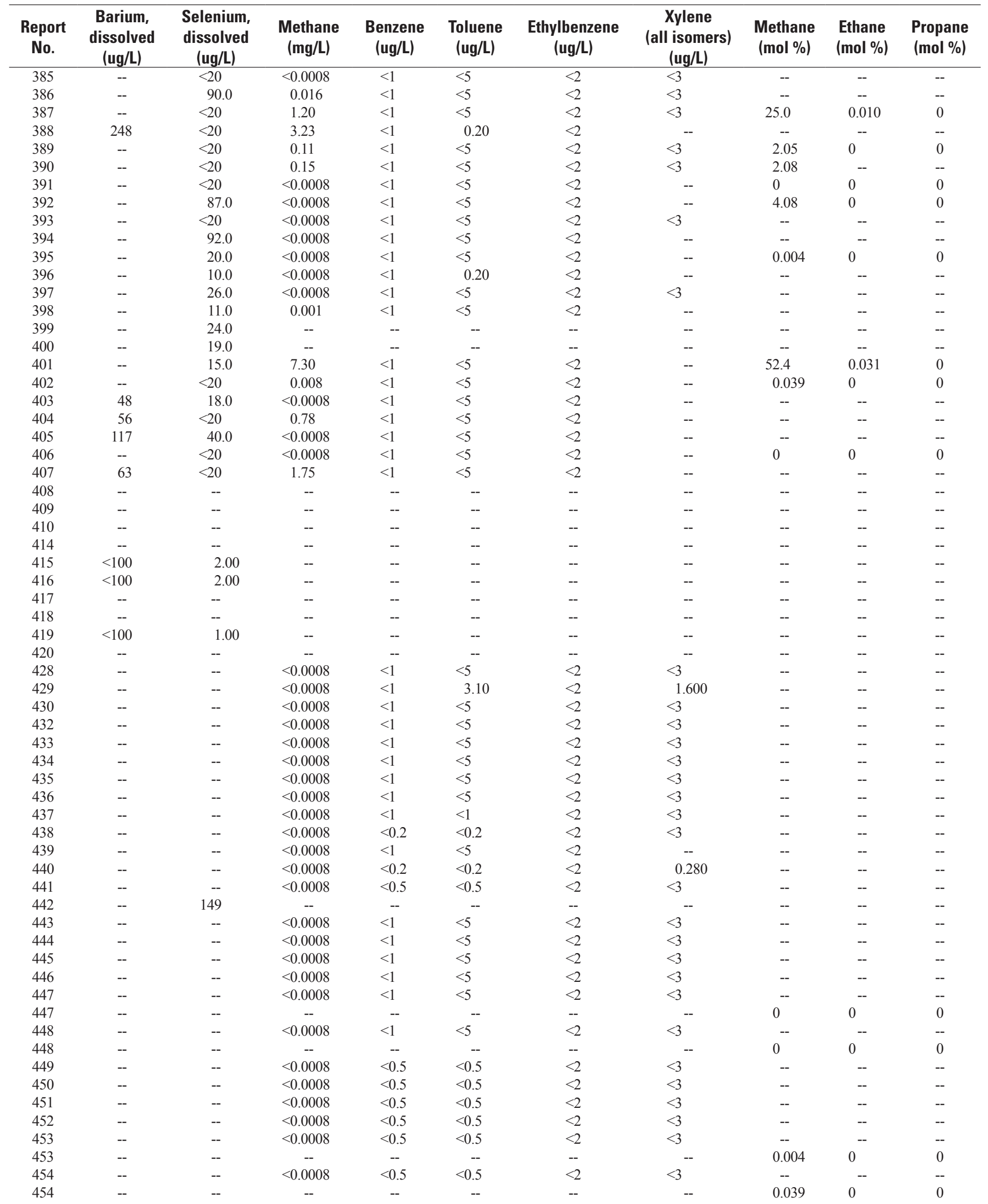


Appendix 1. Water Quality Data Used in This Report.-Continued

\begin{tabular}{|c|c|c|c|c|c|c|c|c|c|c|}
\hline $\begin{array}{l}\text { Report } \\
\text { No. }\end{array}$ & $\begin{array}{c}\text { Barium, } \\
\text { dissolved } \\
\text { (ug/L) }\end{array}$ & $\begin{array}{c}\text { Selenium, } \\
\text { dissolved } \\
\text { (ug/L) }\end{array}$ & $\begin{array}{c}\text { Methane } \\
\text { (mg/L) }\end{array}$ & $\begin{array}{c}\text { Benzene } \\
\text { (ug/L) }\end{array}$ & $\begin{array}{c}\text { Toluene } \\
\text { (ug/L) }\end{array}$ & $\begin{array}{c}\text { Ethylbenzene } \\
\text { (ug/L) }\end{array}$ & $\begin{array}{c}\text { Xylene } \\
\text { (all isomers) } \\
\text { (ug/L) }\end{array}$ & $\begin{array}{c}\text { Methane } \\
(\mathrm{mol} \%)\end{array}$ & $\begin{array}{l}\text { Ethane } \\
\text { (mol \%) }\end{array}$ & $\begin{array}{r}\text { Propane } \\
\text { (mol \%) }\end{array}$ \\
\hline 455 & -- & -- & -- & -- & -- & -- & -- & -- & -- & -- \\
\hline 457 & -- & -- & -- & -- & -- & -- & -- & -- & -- & -- \\
\hline 458 & -- & -- & -- & -- & -- & -- & -- & -- & -- & -- \\
\hline 459 & -- & -- & -- & -- & -- & -- & -- & -- & -- & -- \\
\hline 460 & -- & -- & $<0.0008$ & $<1$ & $<5$ & $<2$ & -- & -- & -- & -- \\
\hline 463 & -- & -- & $<0.0008$ & $<1$ & $<5$ & $<2$ & -- & -- & -- & -- \\
\hline 464 & -- & -- & $<0.0008$ & $<1$ & $<5$ & $<2$ & -- & -- & -- & -- \\
\hline 465 & 2,900 & $<1$ & -- & -- & -- & -- & -- & -- & -- & -- \\
\hline 466 & - & -- & -- & -- & -- & -- & -- & -- & -- & -- \\
\hline 468 & -- & -- & -- & -- & -- & -- & -- & -- & -- & -- \\
\hline 469 & -- & -- & 0.008 & $<1$ & $<5$ & $<2$ & -- & -- & -- & -- \\
\hline 474 & -- & -- & $<0.0008$ & $<1$ & $<5$ & $<2$ & -- & -- & -- & -- \\
\hline 475 & -- & -- & $<0.0008$ & $<1$ & $<5$ & $<2$ & -- & -- & -- & -- \\
\hline 476 & -- & 16.2 & -- & -- & -- & -- & -- & -- & -- & -- \\
\hline 477 & -- & -- & -- & -- & -- & -- & -- & -- & -- & -- \\
\hline 478 & -- & -- & $<0.0008$ & $<1$ & $<5$ & $<2$ & -- & -- & -- & -- \\
\hline 479 & -- & -- & 0.10 & $<1$ & $<5$ & $<2$ & $<3$ & -- & -- & -- \\
\hline 480 & -- & -- & 1.75 & $<1$ & $<5$ & $<2$ & $<3$ & -- & -- & -- \\
\hline 481 & -- & -- & $<0.0008$ & $<1$ & $<5$ & $<2$ & -- & -- & -- & -- \\
\hline 482 & -- & -- & $<0.0008$ & $<1$ & $<5$ & $<2$ & -- & -- & -- & -- \\
\hline 483 & -- & -- & $<0.0008$ & $<1$ & $<5$ & $<2$ & -- & -- & -- & -- \\
\hline 484 & -- & -- & -- & -- & -- & -- & -- & 0 & 0 & 0 \\
\hline 485 & -- & -- & $<0.0008$ & $<1$ & $<5$ & $<2$ & $<3$ & -- & -- & -- \\
\hline 486 & -- & 8.50 & 0.002 & $<1$ & $<5$ & $<2$ & -- & -- & -- & -- \\
\hline 496 & -- & -- & $<0.0008$ & $<1$ & $<5$ & $<2$ & -- & -- & -- & -- \\
\hline 497 & -- & -- & 0.003 & $<1$ & $<5$ & $<2$ & -- & -- & -- & -- \\
\hline 498 & -- & -- & $<0.0008$ & $<1$ & $<5$ & $<2$ & -- & -- & -- & -- \\
\hline 499 & -- & -- & $<0.0008$ & $<1$ & $<5$ & $<2$ & -- & -- & -- & -- \\
\hline 500 & -- & -- & $<0.0008$ & $<1$ & $<5$ & $<2$ & -- & -- & -- & -- \\
\hline 501 & -- & -- & $<0.0008$ & $<1$ & $<5$ & $<2$ & $<3$ & -- & -- & -- \\
\hline 502 & -- & -- & $<0.0008$ & $<1$ & $<5$ & $<2$ & $<3$ & -- & -- & -- \\
\hline 503 & -- & -- & $<0.0008$ & $<1$ & $<5$ & $<2$ & $<3$ & -- & -- & -- \\
\hline 504 & 100 & $<20$ & $<0.0008$ & $<1$ & $<5$ & $<2$ & $<3$ & -- & -- & -- \\
\hline 505 & 140 & $<20$ & $<0.0008$ & $<1$ & $<5$ & $<2$ & $<3$ & -- & -- & -- \\
\hline 506 & -- & 5.60 & $<0.0008$ & $<1$ & $<5$ & $<2$ & -- & -- & -- & -- \\
\hline 507 & -- & 20.0 & -- & -- & -- & -- & -- & -- & -- & -- \\
\hline 508 & -- & -- & -- & -- & -- & -- & -- & -- & -- & -- \\
\hline 509 & -- & -- & -- & -- & -- & -- & -- & -- & -- & -- \\
\hline 510 & -- & $<20$ & $<0.0008$ & $<1$ & $<5$ & $<2$ & $<3$ & 0.008 & -- & -- \\
\hline 511 & -- & 6.30 & $<0.0008$ & $<1$ & $<5$ & $<2$ & -- & -- & -- & -- \\
\hline 512 & -- & -- & -- & -- & -- & -- & -- & -- & -- & -- \\
\hline 513 & -- & $<20$ & $<0.0008$ & $<1$ & $<5$ & $<2$ & $<3$ & 0 & 0 & 0 \\
\hline 514 & -- & 13.0 & $<0.0008$ & $<1$ & $<2$ & $<2$ & -- & -- & -- & -- \\
\hline 515 & 34 & $<20$ & $<0.0008$ & $<1$ & $<5$ & $<2$ & $<3$ & -- & -- & -- \\
\hline 516 & -- & 5.80 & $<0.0008$ & $<1$ & $<5$ & $<2$ & -- & -- & -- & -- \\
\hline 517 & -- & 24.0 & -- & -- & -- & - & -- & -- & -- & -- \\
\hline 518 & 140 & $<20$ & $<0.0008$ & $<1$ & $<5$ & $<2$ & $<3$ & -- & -- & -- \\
\hline
\end{tabular}


Appendix 1. Water Quality Data Used in This Report.-Continued

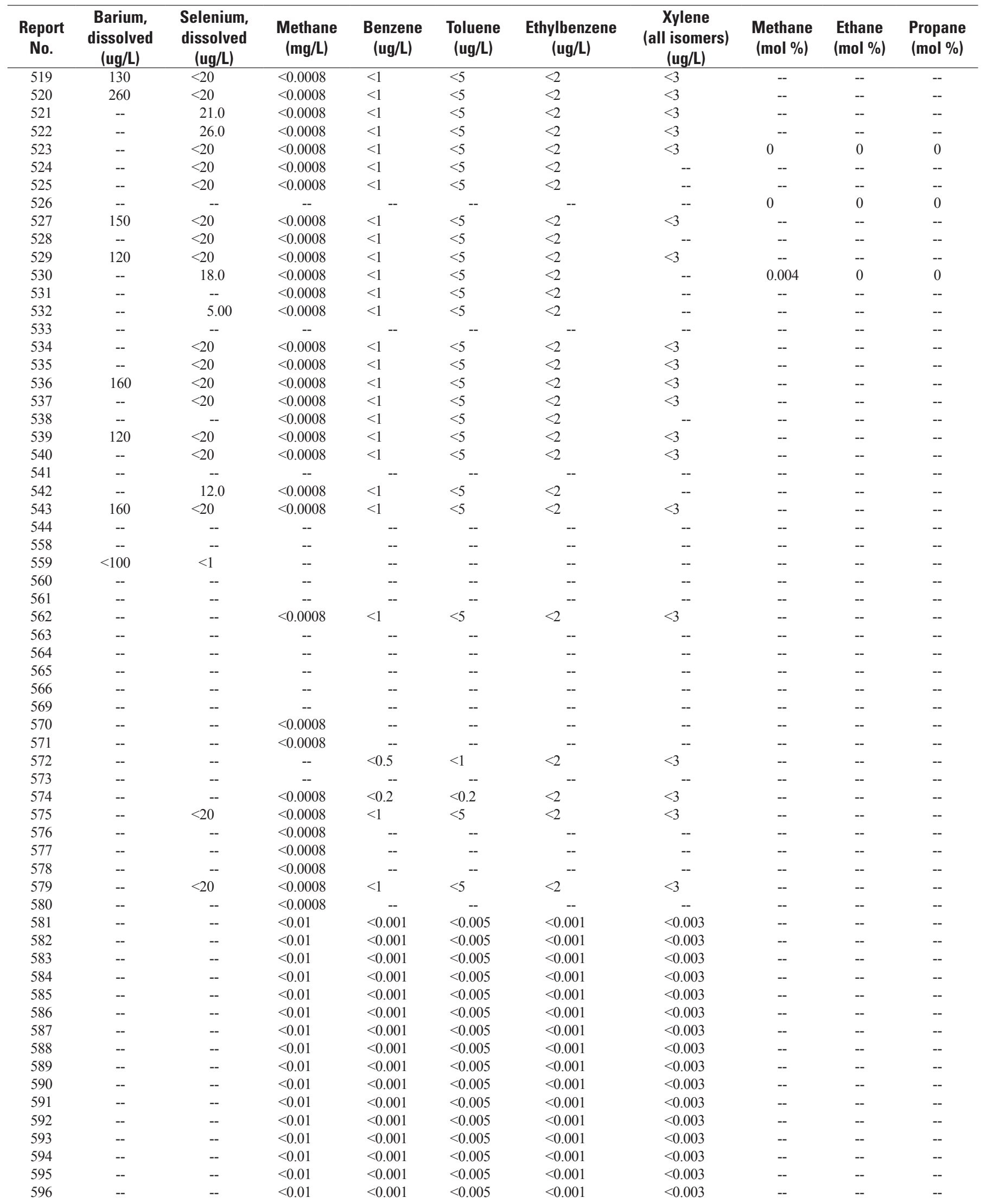


Appendix 1. Water Quality Data Used in This Report.-Continued

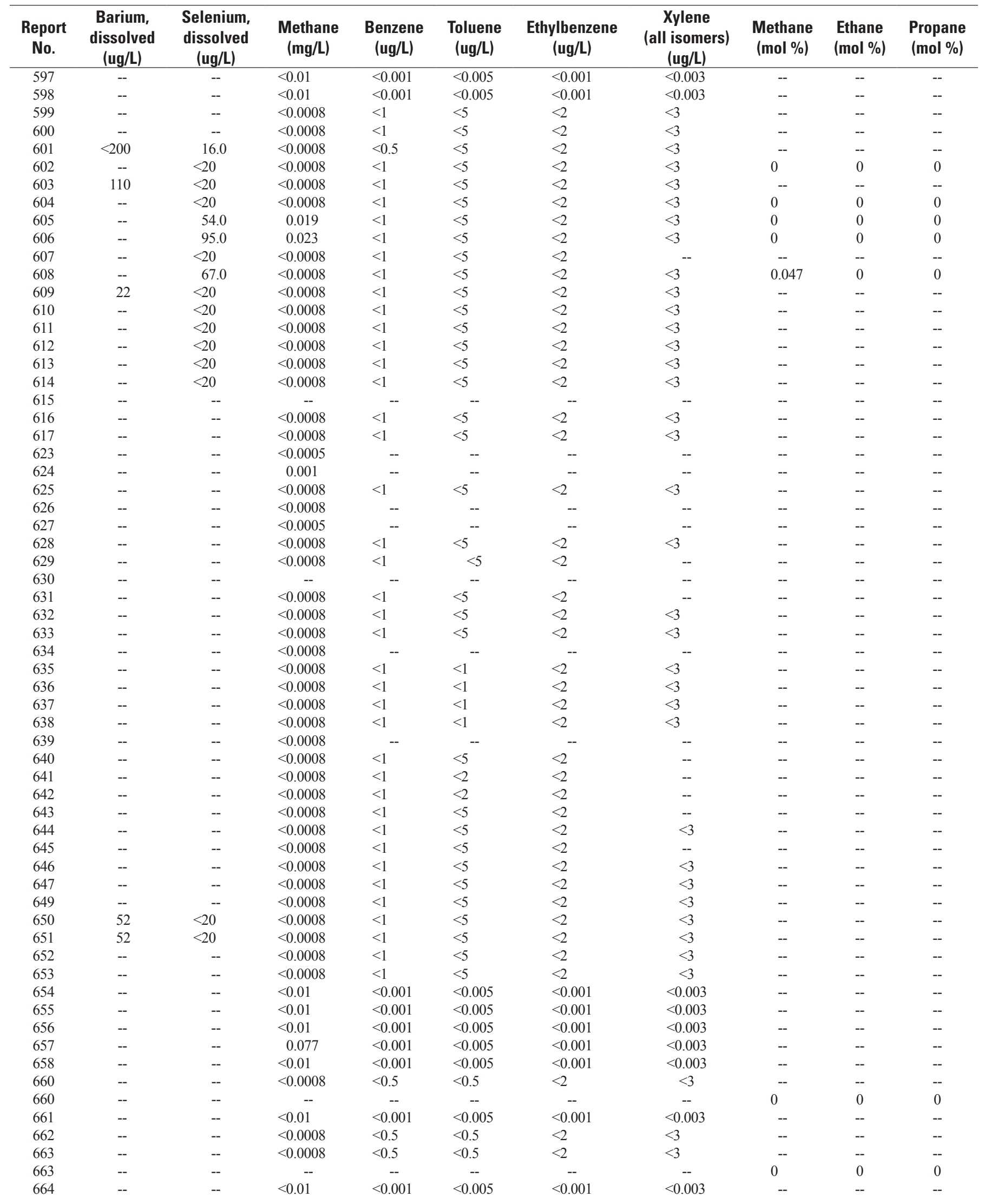


Appendix 1. Water Quality Data Used in This Report.-Continued

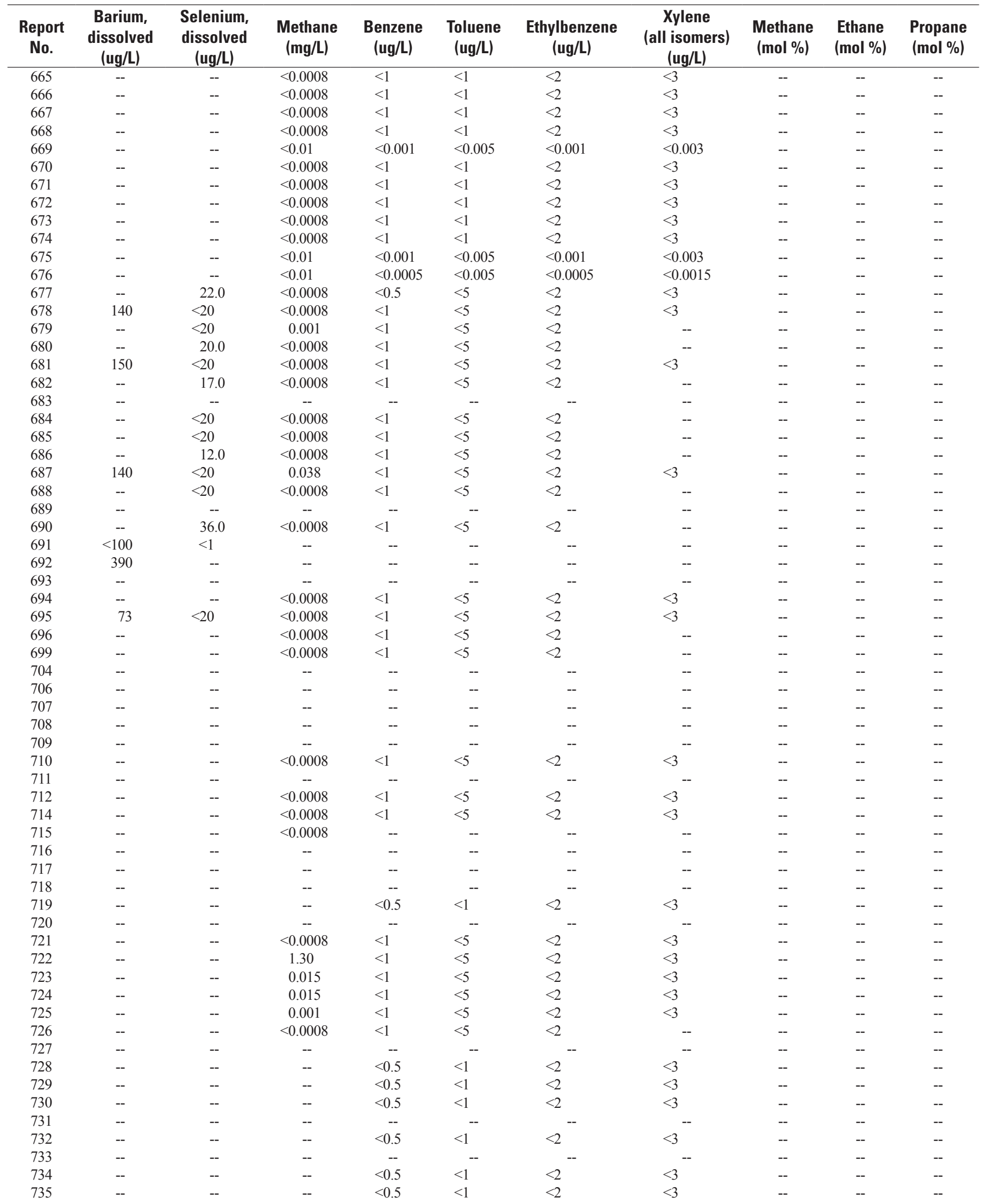


Appendix 1. Water Quality Data Used in This Report.-Continued

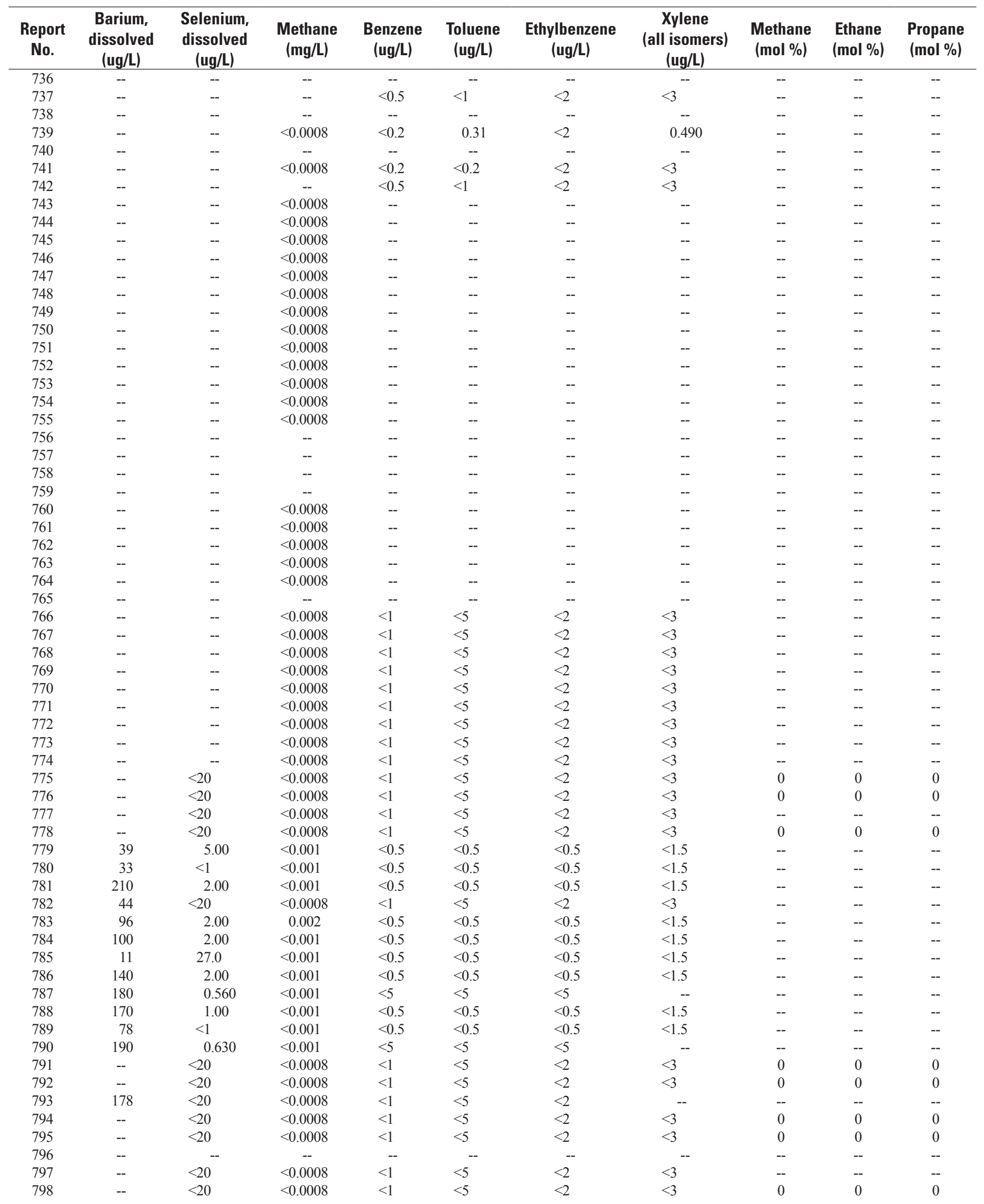


Appendix 1. Water Quality Data Used in This Report.-Continued

\begin{tabular}{|c|c|c|c|c|c|c|c|c|c|c|}
\hline $\begin{array}{l}\text { Report } \\
\text { No. }\end{array}$ & $\begin{array}{c}\text { Barium, } \\
\text { dissolved } \\
\text { (ug/L) }\end{array}$ & $\begin{array}{c}\text { Selenium, } \\
\text { dissolved } \\
\text { (ug/L) }\end{array}$ & $\begin{array}{l}\text { Methane } \\
\text { (mg/L) }\end{array}$ & $\begin{array}{l}\text { Benzene } \\
\text { (ug/L) }\end{array}$ & $\begin{array}{l}\text { Toluene } \\
\text { (ug/L) }\end{array}$ & $\begin{array}{c}\text { Ethylbenzene } \\
\text { (ug/L) }\end{array}$ & $\begin{array}{c}\text { Xylene } \\
\text { (all isomers) } \\
\text { (ug/L) }\end{array}$ & $\begin{array}{c}\text { Methane } \\
\text { (mol \%) }\end{array}$ & $\begin{array}{l}\text { Ethane } \\
\text { (mol \%) }\end{array}$ & $\begin{array}{r}\text { Propane } \\
\text { (mol \%) }\end{array}$ \\
\hline 799 & 110 & $<20$ & $<0.0008$ & $<1$ & $<5$ & $<2$ & $<3$ & -- & -- & -- \\
\hline 801 & -- & -- & -- & -- & -- & -- & -- & -- & -- & -- \\
\hline 802 & -- & -- & 0.23 & $<1$ & $<5$ & $<2$ & $<3$ & -- & -- & -- \\
\hline 803 & -- & -- & $<0.0008$ & $<1$ & $<5$ & $<2$ & $<3$ & -- & -- & -- \\
\hline 804 & -- & -- & $<0.0005$ & -- & -- & -- & -- & -- & -- & -- \\
\hline 807 & -- & -- & 32.5 & -- & -- & -- & -- & -- & -- & -- \\
\hline 811 & -- & -- & $<0.0005$ & -- & -- & -- & -- & -- & -- & -- \\
\hline 812 & -- & -- & $<0.0005$ & -- & -- & -- & -- & -- & -- & -- \\
\hline 813 & -- & -- & $<0.0005$ & -- & -- & -- & -- & -- & -- & -- \\
\hline 814 & -- & -- & $<0.0005$ & -- & -- & -- & -- & -- & -- & -- \\
\hline 815 & -- & -- & $<0.0008$ & $<1$ & $<5$ & $<2$ & $<3$ & -- & -- & -- \\
\hline 820 & -- & -- & $<0.0005$ & -- & -- & -- & -- & -- & -- & -- \\
\hline 821 & -- & -- & $<0.0008$ & $<1$ & $<5$ & $<2$ & $<3$ & -- & -- & -- \\
\hline 822 & -- & -- & $<0.0008$ & $<1$ & $<5$ & $<2$ & $<3$ & -- & -- & -- \\
\hline 823 & -- & -- & $<0.0008$ & $<1$ & $<5$ & $<2$ & $<3$ & -- & -- & -- \\
\hline 824 & -- & -- & $<0.0008$ & $<1$ & $<5$ & $<2$ & $<3$ & -- & -- & -- \\
\hline 825 & -- & -- & $<0.0008$ & $<1$ & $<5$ & $<2$ & $<3$ & -- & -- & -- \\
\hline 826 & -- & -- & -- & -- & -- & -- & -- & -- & -- & -- \\
\hline 827 & -- & -- & $<0.0008$ & $<1$ & $<5$ & $<2$ & $<3$ & -- & -- & -- \\
\hline 828 & -- & -- & $<0.0008$ & $<0.5$ & $<0.5$ & $<2$ & $<3$ & -- & -- & -- \\
\hline 829 & -- & -- & $<0.0008$ & $<0.2$ & $<0.2$ & $<2$ & $<3$ & -- & -- & -- \\
\hline 830 & -- & -- & $<0.0008$ & $<0.5$ & $<0.5$ & $<2$ & $<3$ & -- & -- & -- \\
\hline 831 & -- & -- & -- & -- & -- & -- & -- & 65.5 & 0.16 & 0.010 \\
\hline 832 & -- & -- & 0.68 & $<1$ & $<5$ & $<2$ & $<3$ & -- & -- & -- \\
\hline 840 & -- & -- & -- & -- & -- & -- & -- & 0 & 0 & 0 \\
\hline 841 & -- & -- & $<0.0008$ & $<0.5$ & $<0.5$ & $<2$ & $<3$ & -- & -- & -- \\
\hline 842 & -- & -- & $<0.0008$ & $<0.5$ & $<0.5$ & $<2$ & $<3$ & -- & -- & -- \\
\hline 842 & -- & -- & -- & -- & -- & -- & -- & 0 & 0 & 0 \\
\hline 843 & -- & -- & $<0.0008$ & -- & -- & -- & -- & -- & -- & -- \\
\hline 844 & -- & -- & $<0.0008$ & -- & -- & -- & -- & -- & -- & -- \\
\hline 845 & -- & -- & $<0.0008$ & $<0.5$ & $<0.5$ & $<2$ & $<3$ & -- & -- & -- \\
\hline 846 & -- & 189 & -- & -- & -- & -- & -- & -- & -- & -- \\
\hline 847 & -- & 39.7 & -- & -- & -- & -- & -- & -- & -- & -- \\
\hline 848 & -- & -- & $<0.0008$ & $<1$ & $<5$ & $<2$ & $<3$ & -- & -- & -- \\
\hline 849 & -- & -- & $<0.0008$ & -- & -- & -- & -- & -- & -- & -- \\
\hline 850 & -- & -- & $<0.0008$ & $<1$ & $<5$ & $<2$ & $<3$ & -- & -- & -- \\
\hline 851 & -- & -- & $<0.0008$ & $<1$ & $<5$ & $<2$ & $<3$ & -- & -- & -- \\
\hline 852 & -- & -- & -- & $<1$ & $<5$ & $<2$ & $<3$ & -- & -- & -- \\
\hline 853 & -- & -- & -- & $<1$ & $<5$ & $<2$ & $<3$ & -- & -- & -- \\
\hline 854 & -- & -- & -- & $<1$ & $<5$ & $<2$ & $<3$ & -- & -- & -- \\
\hline 855 & -- & -- & $<0.0008$ & $<1$ & $<5$ & $<2$ & $<3$ & -- & -- & -- \\
\hline 855 & -- & -- & -- & -- & -- & -- & -- & 0.005 & 0 & 0 \\
\hline 856 & -- & -- & $<0.0008$ & $<1$ & $<5$ & $<2$ & $<3$ & -- & -- & -- \\
\hline 857 & -- & -- & $<0.0008$ & $<1$ & $<5$ & $<2$ & $<3$ & -- & -- & -- \\
\hline 858 & -- & -- & $<0.0008$ & $<1$ & $<5$ & $<2$ & $<3$ & -- & -- & -- \\
\hline 859 & -- & -- & $<0.0008$ & $<1$ & 2.40 & $<2$ & $<3$ & -- & -- & -- \\
\hline 860 & -- & -- & $<0.0008$ & $<1$ & $<5$ & $<2$ & $<3$ & -- & -- & -- \\
\hline 860 & -- & -- & -- & -- & -- & -- & -- & 0.009 & 0 & 0 \\
\hline
\end{tabular}


Appendix 1. Water Quality Data Used in This Report.-Continued

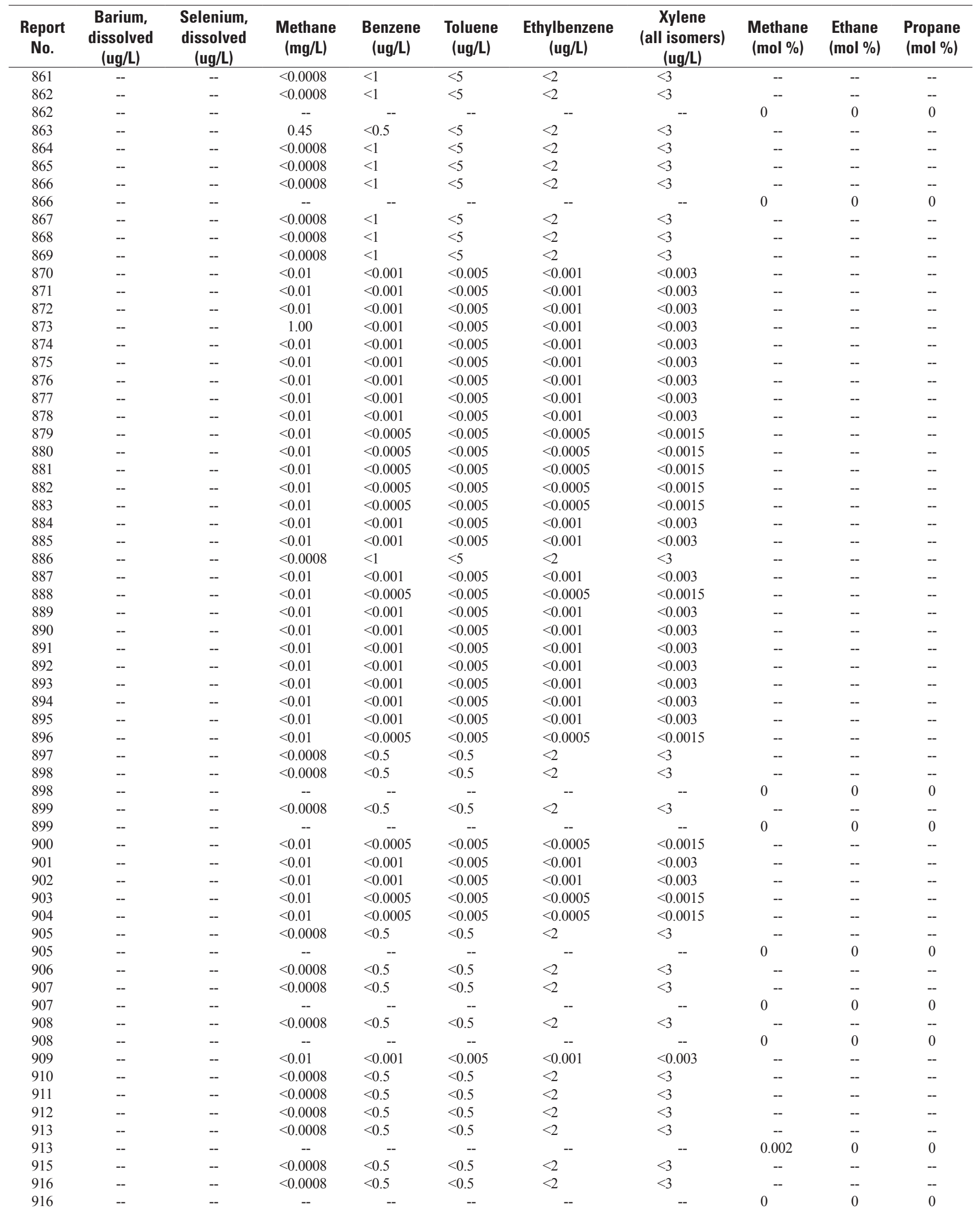


Appendix 1. Water Quality Data Used in This Report.-Continued

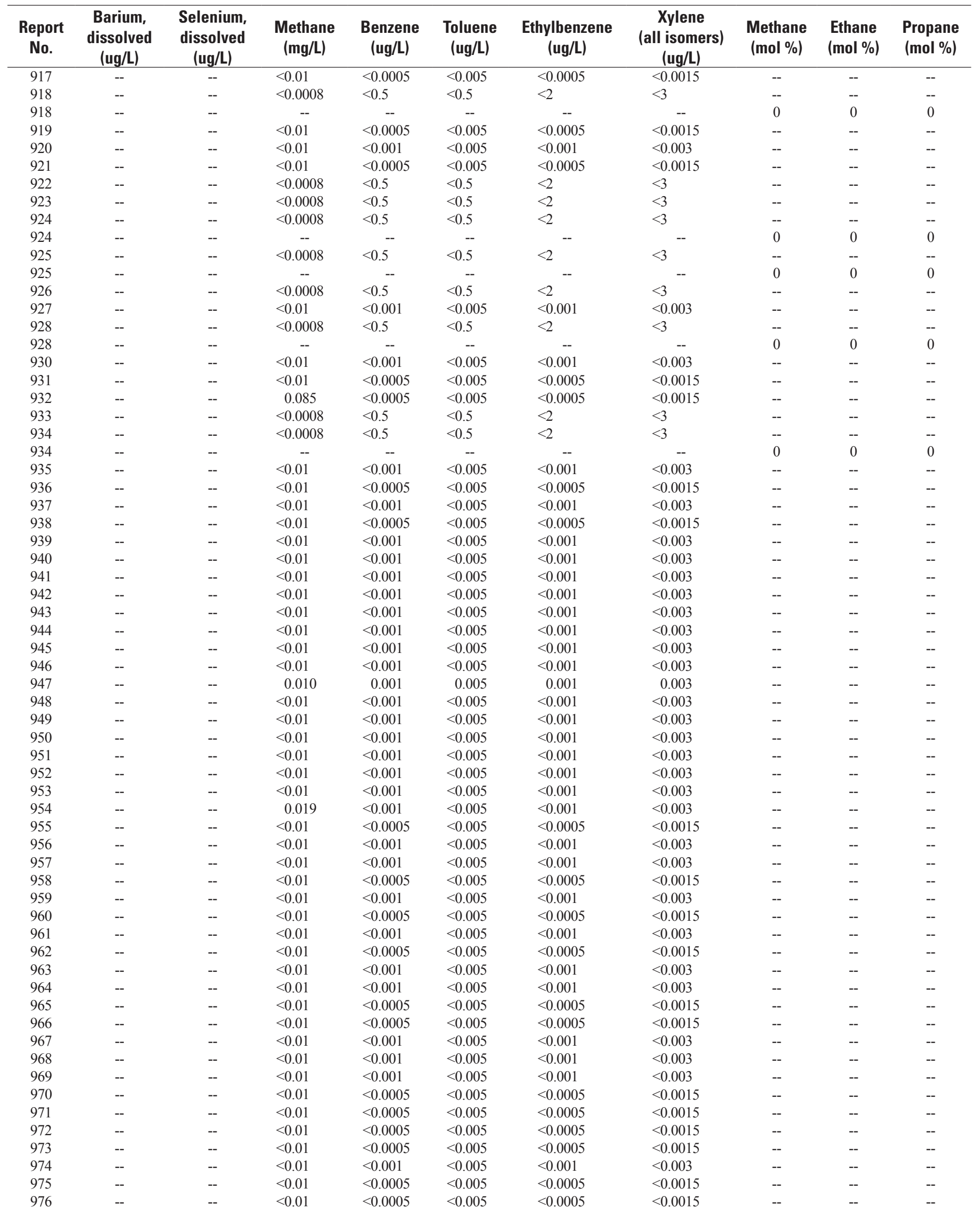


Appendix 1. Water Quality Data Used in This Report.-Continued

\begin{tabular}{|c|c|c|c|c|c|c|c|c|c|c|}
\hline $\begin{array}{l}\text { Report } \\
\text { No. }\end{array}$ & $\begin{array}{c}\text { Barium, } \\
\text { dissolved } \\
\text { (ug/L) }\end{array}$ & $\begin{array}{c}\text { Selenium, } \\
\text { dissolved } \\
\text { (ug/L) }\end{array}$ & $\begin{array}{c}\text { Methane } \\
\text { (mg/L) }\end{array}$ & $\begin{array}{c}\text { Benzene } \\
\text { (ug/L) }\end{array}$ & $\begin{array}{c}\text { Toluene } \\
\text { (ug/L) }\end{array}$ & $\begin{array}{c}\text { Ethylbenzene } \\
\text { (ug/L) }\end{array}$ & $\begin{array}{c}\text { Xylene } \\
\text { (all isomers) } \\
\text { (ug/L) }\end{array}$ & $\begin{array}{c}\text { Methane } \\
(\mathrm{mol} \%)\end{array}$ & $\begin{array}{l}\text { Ethane } \\
\text { (mol \%) }\end{array}$ & $\begin{array}{r}\text { Propane } \\
\text { (mol \%) }\end{array}$ \\
\hline 977 & -- & -- & $<0.01$ & $<0.001$ & $<0.005$ & $<0.001$ & $<0.003$ & -- & -- & -- \\
\hline 979 & -- & -- & $<0.01$ & $<0.0005$ & $<0.005$ & $<0.0005$ & $<0.0015$ & -- & -- & -- \\
\hline 980 & -- & -- & $<0.01$ & $<0.001$ & $<0.005$ & $<0.001$ & $<0.003$ & -- & -- & -- \\
\hline 981 & -- & -- & $<0.01$ & $<0.001$ & $<0.005$ & $<0.001$ & $<0.003$ & -- & -- & -- \\
\hline 982 & -- & -- & $<0.01$ & $<0.001$ & $<0.005$ & $<0.001$ & $<0.003$ & -- & -- & -- \\
\hline 985 & -- & -- & $<0.01$ & $<0.001$ & $<0.005$ & $<0.001$ & $<0.003$ & -- & -- & -- \\
\hline 986 & -- & -- & 0.017 & $<0.001$ & $<0.005$ & $<0.001$ & $<0.003$ & -- & -- & -- \\
\hline 987 & -- & -- & $<0.01$ & $<0.001$ & $<0.005$ & $<0.001$ & $<0.003$ & -- & -- & -- \\
\hline 988 & -- & -- & $<0.01$ & $<0.001$ & $<0.005$ & $<0.001$ & $<0.003$ & -- & -- & -- \\
\hline 989 & -- & -- & $<0.01$ & $<0.001$ & $<0.005$ & $<0.001$ & $<0.003$ & -- & -- & -- \\
\hline 990 & -- & -- & $<0.01$ & $<0.0005$ & $<0.005$ & $<0.0005$ & $<0.0015$ & -- & -- & -- \\
\hline 995 & -- & -- & $<0.01$ & $<0.001$ & $<0.005$ & $<0.001$ & $<0.003$ & -- & -- & -- \\
\hline 996 & -- & -- & $<0.01$ & $<0.0005$ & $<0.005$ & $<0.0005$ & $<0.0015$ & -- & -- & -- \\
\hline 997 & -- & -- & $<0.01$ & $<0.001$ & $<0.005$ & $<0.001$ & $<0.003$ & -- & -- & -- \\
\hline 998 & -- & -- & $<0.01$ & $<0.0005$ & $<0.005$ & $<0.0005$ & $<0.0015$ & -- & -- & -- \\
\hline 999 & -- & -- & $<0.01$ & $<0.001$ & $<0.005$ & $<0.001$ & $<0.003$ & -- & -- & -- \\
\hline 1000 & -- & -- & $<0.01$ & $<0.001$ & $<0.005$ & $<0.001$ & $<0.003$ & -- & -- & -- \\
\hline 1001 & -- & -- & $<0.01$ & $<0.001$ & $<0.005$ & $<0.001$ & $<0.003$ & -- & -- & -- \\
\hline 1002 & -- & -- & $<0.01$ & $<0.001$ & $<0.005$ & $<0.001$ & $<0.003$ & -- & -- & -- \\
\hline 1003 & -- & -- & $<0.01$ & $<0.001$ & $<0.005$ & $<0.001$ & $<0.003$ & -- & -- & -- \\
\hline 1004 & -- & -- & $<0.01$ & $<0.001$ & $<0.005$ & $<0.001$ & $<0.003$ & -- & -- & -- \\
\hline 1005 & -- & -- & $<0.01$ & $<0.001$ & $<0.005$ & $<0.001$ & $<0.003$ & -- & -- & -- \\
\hline 1006 & -- & -- & $<0.01$ & $<0.001$ & $<0.005$ & $<0.001$ & $<0.003$ & -- & -- & -- \\
\hline 1007 & -- & -- & $<0.01$ & $<0.001$ & $<0.005$ & $<0.001$ & $<0.003$ & -- & -- & -- \\
\hline 1017 & -- & -- & $<0.01$ & $<0.0005$ & $<0.005$ & $<0.0005$ & $<0.0015$ & -- & -- & -- \\
\hline 1018 & -- & -- & $<0.01$ & $<0.0005$ & $<0.005$ & $<0.0005$ & $<0.0015$ & -- & -- & -- \\
\hline 1019 & -- & -- & $<0.01$ & $<0.0005$ & $<0.005$ & $<0.0005$ & $<0.0015$ & -- & -- & -- \\
\hline 1020 & -- & -- & $<0.01$ & $<0.0005$ & $<0.005$ & $<0.0005$ & $<0.0015$ & -- & -- & -- \\
\hline 1021 & -- & -- & $<0.01$ & $<0.0005$ & $<0.005$ & $<0.0005$ & $<0.0015$ & -- & -- & -- \\
\hline 1022 & -- & -- & $<0.01$ & $<0.0005$ & $<0.005$ & $<0.0005$ & $<0.0015$ & -- & -- & -- \\
\hline 1023 & -- & -- & $<0.01$ & $<0.0005$ & $<0.005$ & $<0.0005$ & $<0.0015$ & -- & -- & -- \\
\hline 1024 & -- & -- & $<0.01$ & $<0.0005$ & $<0.005$ & $<0.0005$ & $<0.0015$ & -- & -- & -- \\
\hline 1025 & -- & -- & $<0.01$ & $<0.0005$ & $<0.005$ & $<0.0005$ & $<0.0015$ & -- & -- & -- \\
\hline 1026 & -- & 21.0 & $<0.0008$ & $<1$ & $<5$ & $<2$ & $<3$ & -- & -- & -- \\
\hline 1027 & -- & $<20$ & $<0.0008$ & $<1$ & $<5$ & $<2$ & $<3$ & -- & -- & -- \\
\hline 1028 & -- & -- & -- & -- & -- & -- & -- & -- & -- & -- \\
\hline 1029 & -- & -- & -- & -- & -- & -- & -- & -- & -- & -- \\
\hline 1030 & -- & -- & $<0.0008$ & $<1$ & $<5$ & $<2$ & $<3$ & -- & -- & -- \\
\hline 1031 & -- & -- & $<0.0008$ & $<1$ & $<5$ & $<2$ & $<3$ & -- & -- & -- \\
\hline 1032 & -- & -- & $<0.0008$ & $<1$ & $<5$ & $<2$ & $<3$ & -- & -- & -- \\
\hline 1033 & -- & -- & $<0.0008$ & -- & -- & -- & -- & -- & -- & -- \\
\hline 1034 & 8.0 & 3.00 & -- & -- & -- & -- & -- & -- & -- & -- \\
\hline 1038 & -- & -- & -- & -- & -- & -- & -- & -- & -- & -- \\
\hline 1039 & -- & -- & -- & -- & -- & -- & -- & -- & -- & -- \\
\hline 1051 & -- & -- & -- & -- & -- & -- & -- & -- & -- & -- \\
\hline 1052 & -- & -- & -- & -- & -- & -- & -- & -- & -- & -- \\
\hline 1053 & -- & -- & -- & -- & -- & -- & -- & -- & -- & -- \\
\hline 1054 & 70 & $<1$ & -- & -- & -- & -- & -- & -- & -- & -- \\
\hline
\end{tabular}


Appendix 1. Water Quality Data Used in This Report.-Continued

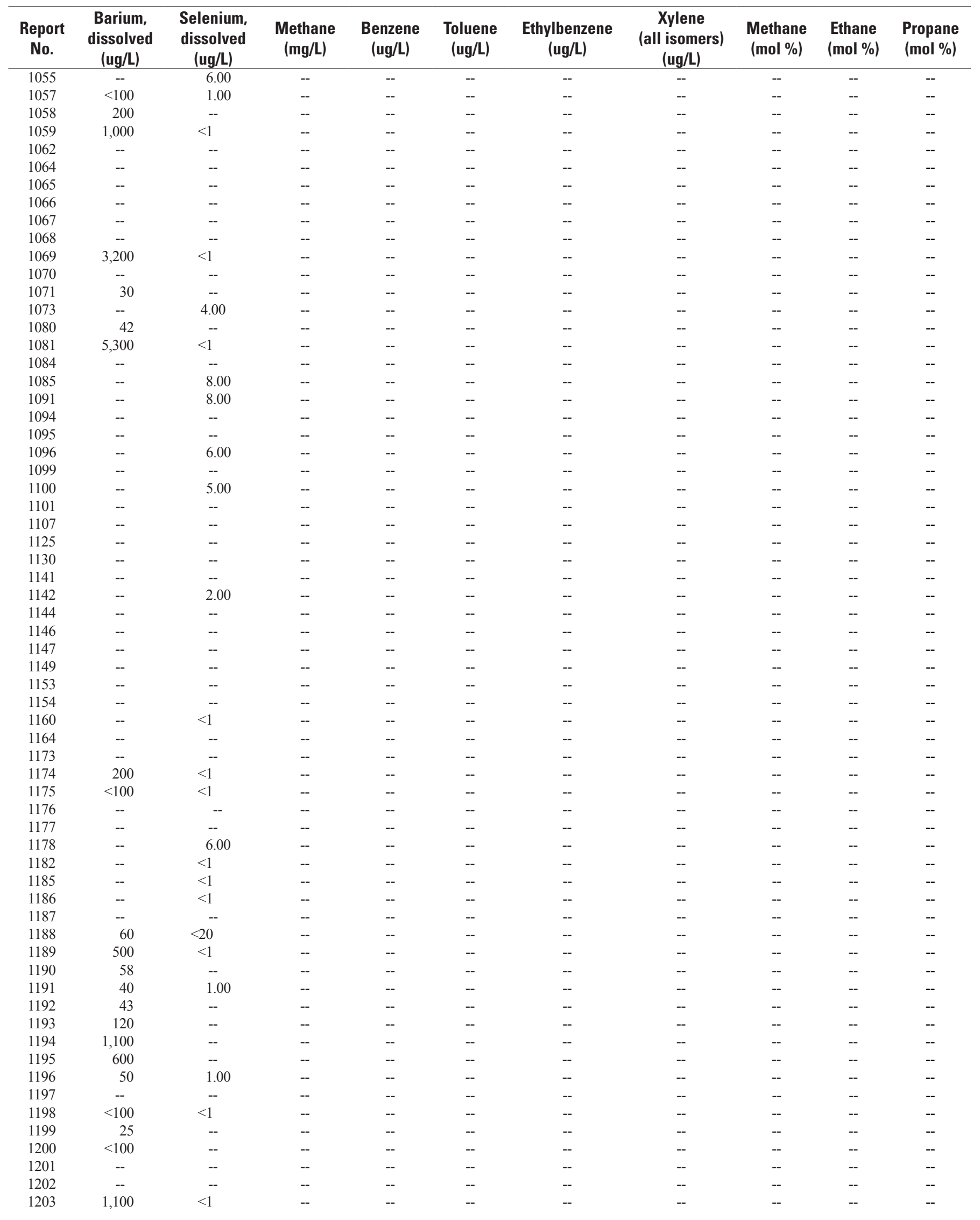


Appendix 1. Water Quality Data Used in This Report.-Continued

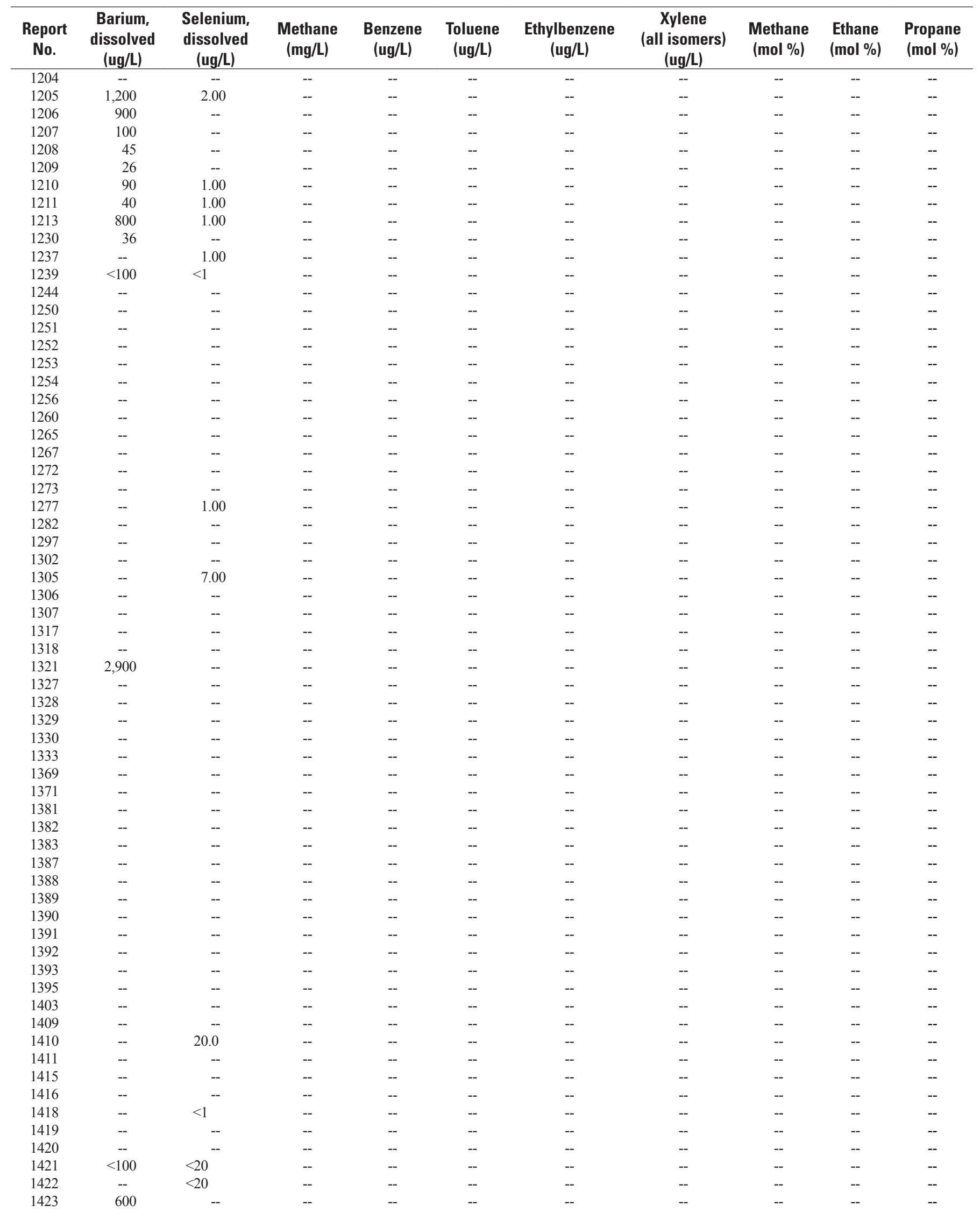


Appendix 1. Water Quality Data Used in This Report.-Continued

\begin{tabular}{|c|c|c|c|c|c|c|c|c|c|c|}
\hline $\begin{array}{c}\text { Report } \\
\text { No. }\end{array}$ & $\begin{array}{c}\text { Barium, } \\
\text { dissolved } \\
\text { (ug/L) }\end{array}$ & $\begin{array}{c}\text { Selenium, } \\
\text { dissolved } \\
\text { (ug/L) }\end{array}$ & $\begin{array}{l}\text { Methane } \\
\text { (mg/L) }\end{array}$ & $\begin{array}{c}\text { Benzene } \\
\text { (ug/L) }\end{array}$ & $\begin{array}{c}\text { Toluene } \\
\text { (ug/L) }\end{array}$ & $\begin{array}{c}\text { Ethylbenzene } \\
\text { (ug/L) }\end{array}$ & $\begin{array}{c}\text { Xylene } \\
\text { (all isomers) } \\
\text { (ug/L) }\end{array}$ & $\begin{array}{c}\text { Methane } \\
(\mathrm{mol} \%)\end{array}$ & $\begin{array}{l}\text { Ethane } \\
(\mathrm{mol} \%)\end{array}$ & $\begin{array}{r}\text { Propane } \\
\text { (mol \%) }\end{array}$ \\
\hline 1426 & $<100$ & $<1$ & -- & -- & -- & -- & -- & -- & -- & -- \\
\hline 1438 & -- & $<1$ & -- & -- & -- & -- & -- & -- & -- & -- \\
\hline 1443 & -- & 3.00 & -- & -- & -- & -- & -- & -- & -- & -- \\
\hline 1445 & -- & 4.00 & -- & -- & -- & -- & -- & -- & -- & -- \\
\hline 1448 & -- & 4.00 & -- & -- & -- & -- & -- & -- & -- & -- \\
\hline 1454 & 43 & -- & -- & -- & -- & -- & -- & -- & -- & -- \\
\hline 1461 & -- & -- & -- & -- & -- & -- & -- & -- & -- & -- \\
\hline 1463 & -- & -- & -- & -- & -- & -- & -- & -- & -- & -- \\
\hline 1464 & -- & -- & -- & -- & -- & -- & -- & -- & -- & -- \\
\hline 1465 & -- & -- & -- & -- & -- & -- & -- & -- & -- & -- \\
\hline 1466 & 700 & $<1$ & -- & -- & -- & -- & -- & -- & -- & -- \\
\hline 1476 & 41 & $<1$ & -- & 43.0 & $<3$ & $<3$ & $<3$ & -- & -- & -- \\
\hline 1477 & $<100$ & $<1$ & -- & -- & -- & -- & -- & -- & -- & -- \\
\hline 1478 & -- & -- & -- & -- & -- & -- & -- & -- & -- & -- \\
\hline 1480 & -- & -- & -- & -- & -- & -- & -- & -- & -- & -- \\
\hline 1482 & -- & -- & -- & -- & -- & -- & -- & -- & -- & -- \\
\hline 1484 & $<100$ & $<1$ & -- & -- & -- & -- & -- & -- & -- & -- \\
\hline 1486 & 300 & $<1$ & -- & -- & -- & -- & -- & -- & -- & -- \\
\hline 1487 & -- & $<1$ & -- & -- & -- & -- & -- & -- & -- & -- \\
\hline 1488 & $<100$ & $<1$ & -- & -- & -- & -- & -- & -- & -- & -- \\
\hline 1492 & -- & -- & -- & -- & -- & -- & -- & -- & -- & -- \\
\hline 1497 & -- & -- & -- & -- & -- & -- & -- & -- & -- & -- \\
\hline 1498 & -- & $<1$ & -- & -- & -- & -- & -- & -- & -- & -- \\
\hline 1499 & $<200$ & 1.00 & -- & -- & -- & -- & -- & -- & -- & -- \\
\hline 1515 & 649 & 1.22 & -- & -- & -- & -- & -- & -- & -- & -- \\
\hline 1516 & 4,841 & $<0.7$ & -- & -- & -- & -- & -- & -- & -- & -- \\
\hline 1517 & 1,786 & $<15$ & -- & -- & -- & -- & -- & -- & -- & -- \\
\hline 1518 & $<200$ & $<1$ & -- & -- & -- & -- & -- & -- & -- & -- \\
\hline 1519 & $<100$ & $<1$ & -- & -- & -- & -- & -- & -- & -- & -- \\
\hline 1523 & -- & 3.00 & -- & -- & -- & -- & -- & -- & -- & -- \\
\hline 1526 & -- & 7.00 & -- & -- & -- & -- & -- & -- & -- & -- \\
\hline 1530 & -- & $<1$ & -- & -- & -- & -- & -- & -- & -- & -- \\
\hline 1532 & -- & 9.00 & -- & -- & -- & -- & -- & -- & -- & -- \\
\hline 1535 & 15 & 2.04 & -- & -- & -- & -- & -- & -- & -- & -- \\
\hline 1536 & -- & 4.00 & -- & -- & -- & -- & -- & -- & -- & -- \\
\hline 1538 & 423 & $<0.7$ & -- & -- & -- & -- & -- & -- & -- & -- \\
\hline 1539 & 1,474 & $<20$ & -- & -- & -- & -- & -- & -- & -- & -- \\
\hline 1539 & 1,403 & $<20$ & -- & -- & -- & -- & -- & -- & -- & -- \\
\hline 1540 & 134 & 3.79 & -- & -- & -- & -- & -- & -- & -- & -- \\
\hline 1541 & 2,437 & $<2$ & -- & -- & -- & -- & -- & -- & -- & -- \\
\hline 1542 & 56 & $<5$ & -- & -- & -- & -- & -- & -- & -- & -- \\
\hline 1546 & 55 & $<1$ & -- & -- & -- & -- & -- & -- & -- & -- \\
\hline 1547 & 420 & $<1$ & -- & -- & -- & -- & -- & -- & -- & -- \\
\hline 1549 & -- & 5.00 & -- & -- & -- & -- & -- & -- & -- & -- \\
\hline 1552 & -- & 6.00 & -- & -- & -- & -- & -- & -- & -- & -- \\
\hline 1555 & -- & -- & -- & -- & -- & -- & -- & -- & -- & -- \\
\hline 1558 & -- & -- & -- & -- & -- & -- & -- & -- & -- & -- \\
\hline 1559 & -- & -- & -- & -- & -- & -- & -- & -- & -- & -- \\
\hline
\end{tabular}


Appendix 1. Water Quality Data Used in This Report.-Continued

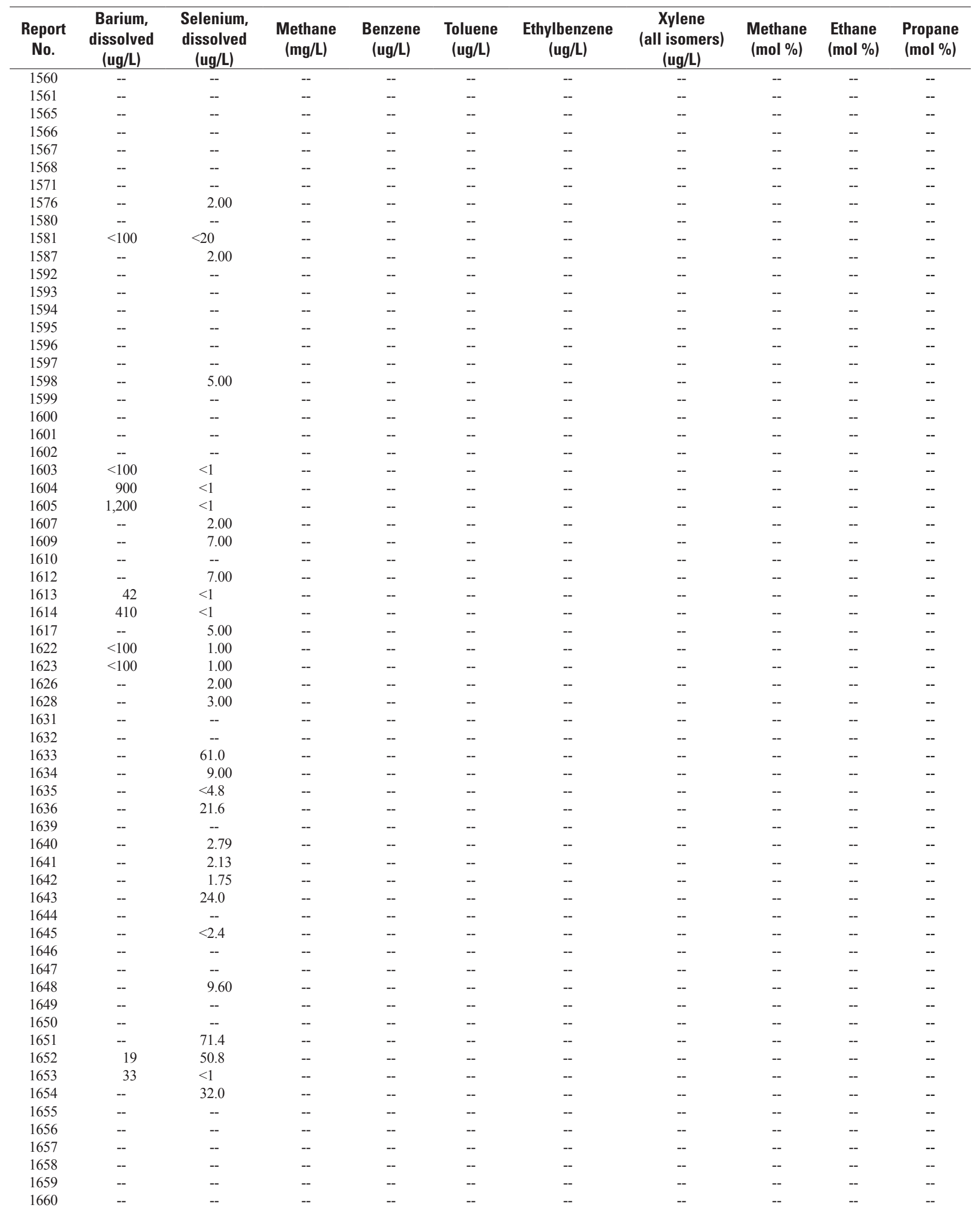


Appendix 1. Water Quality Data Used in This Report.-Continued

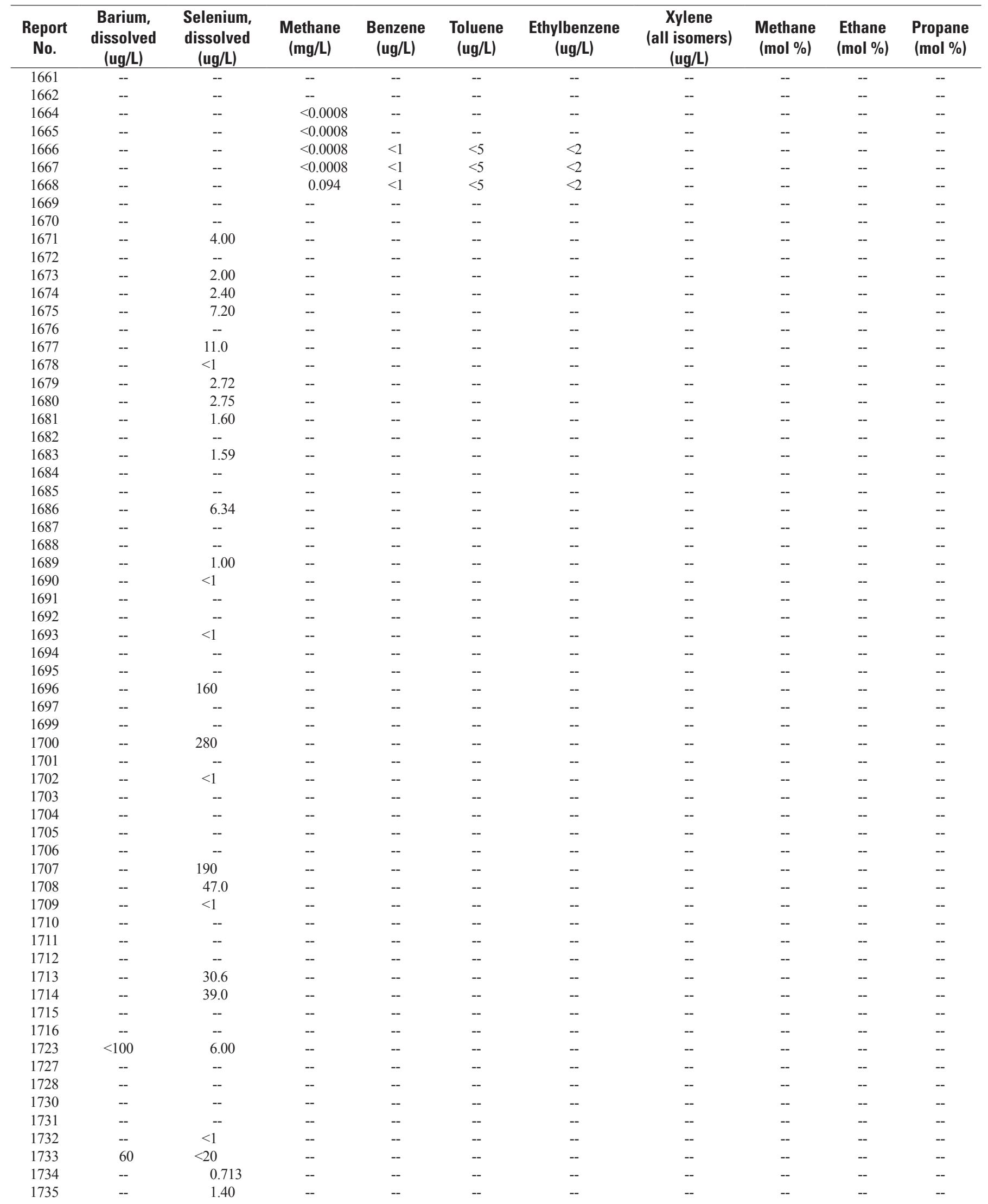


Appendix 1. Water Quality Data Used in This Report.-Continued

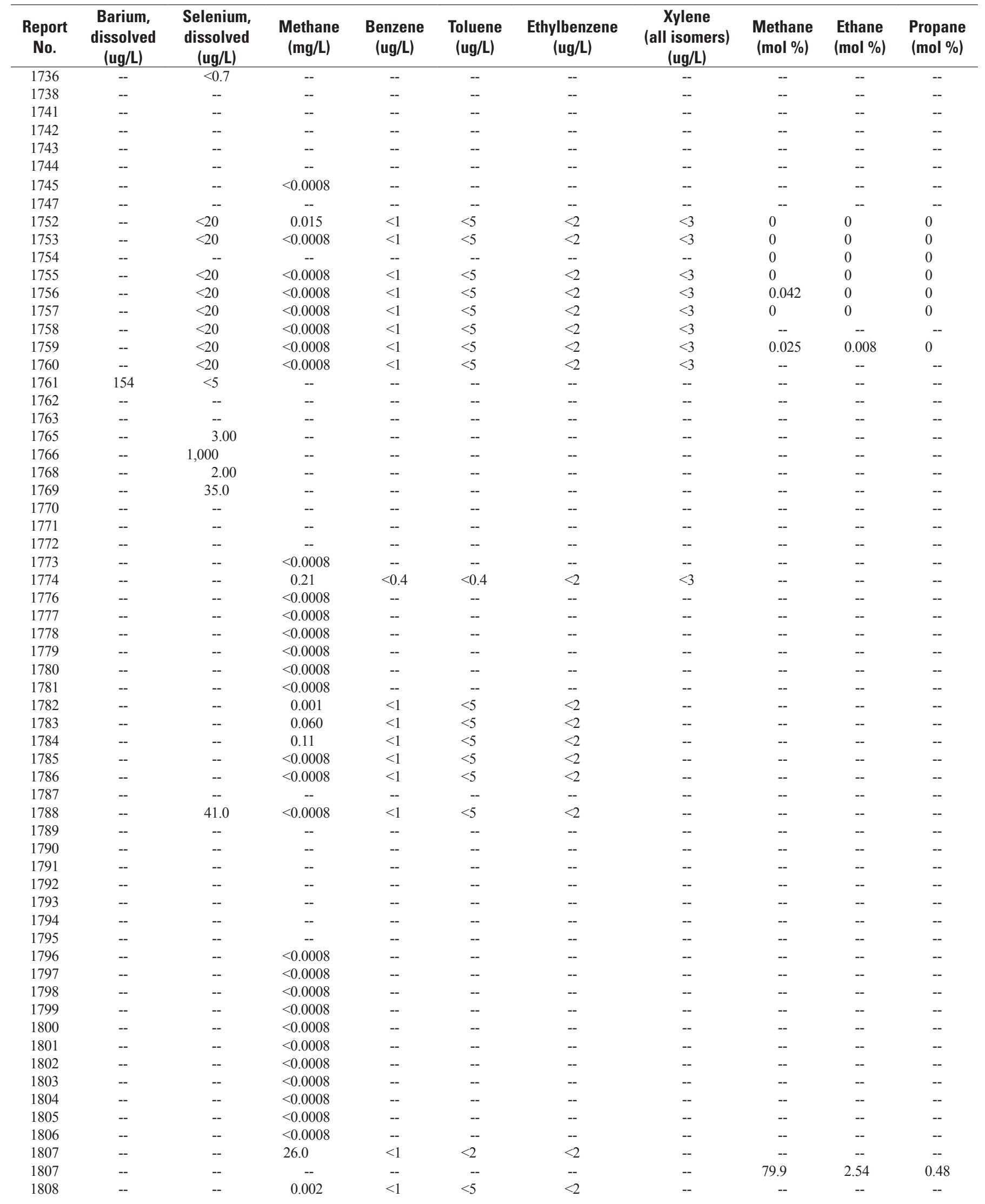


Appendix 1. Water Quality Data Used in This Report.-Continued

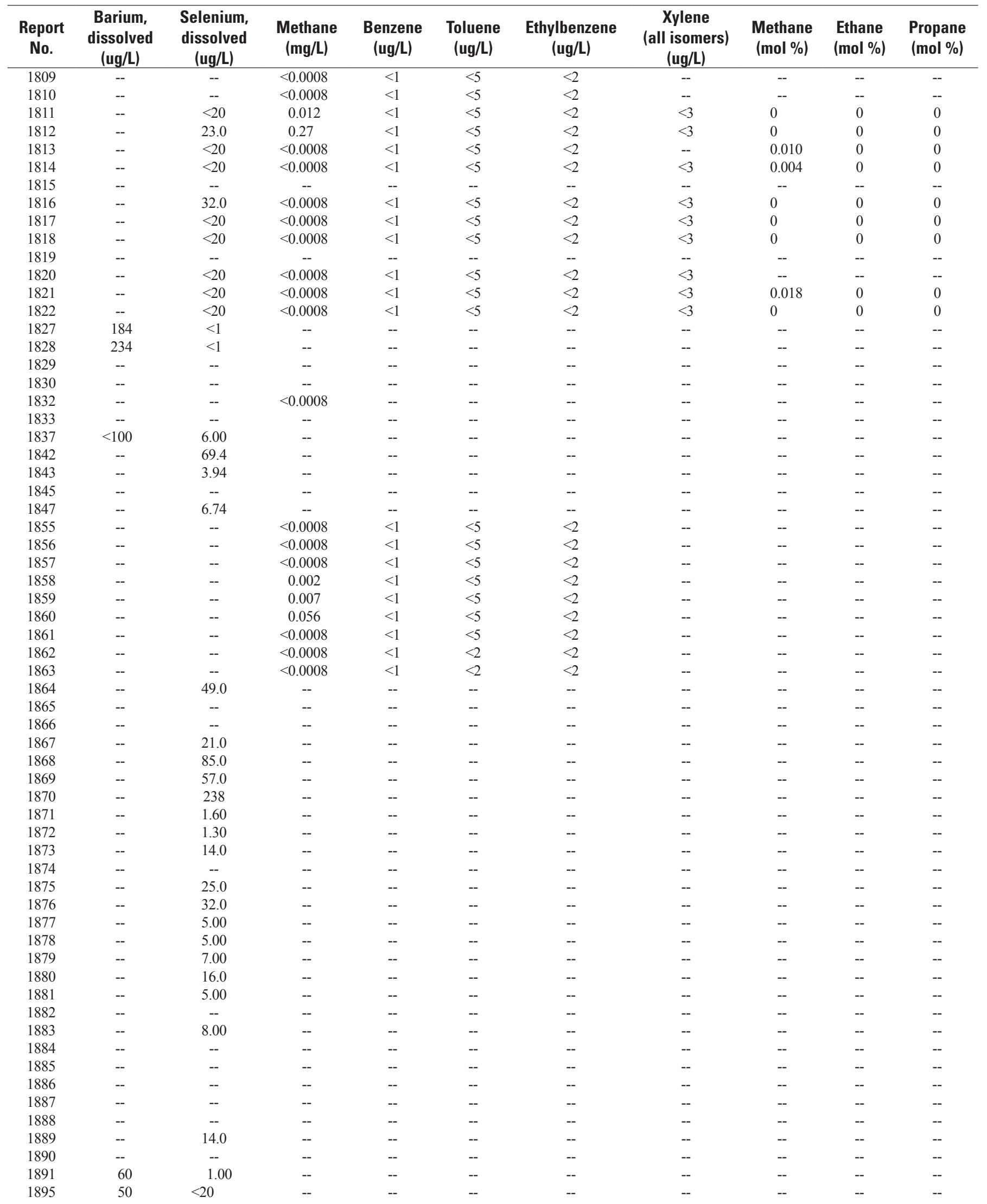


Appendix 1. Water Quality Data Used in This Report.-Continued

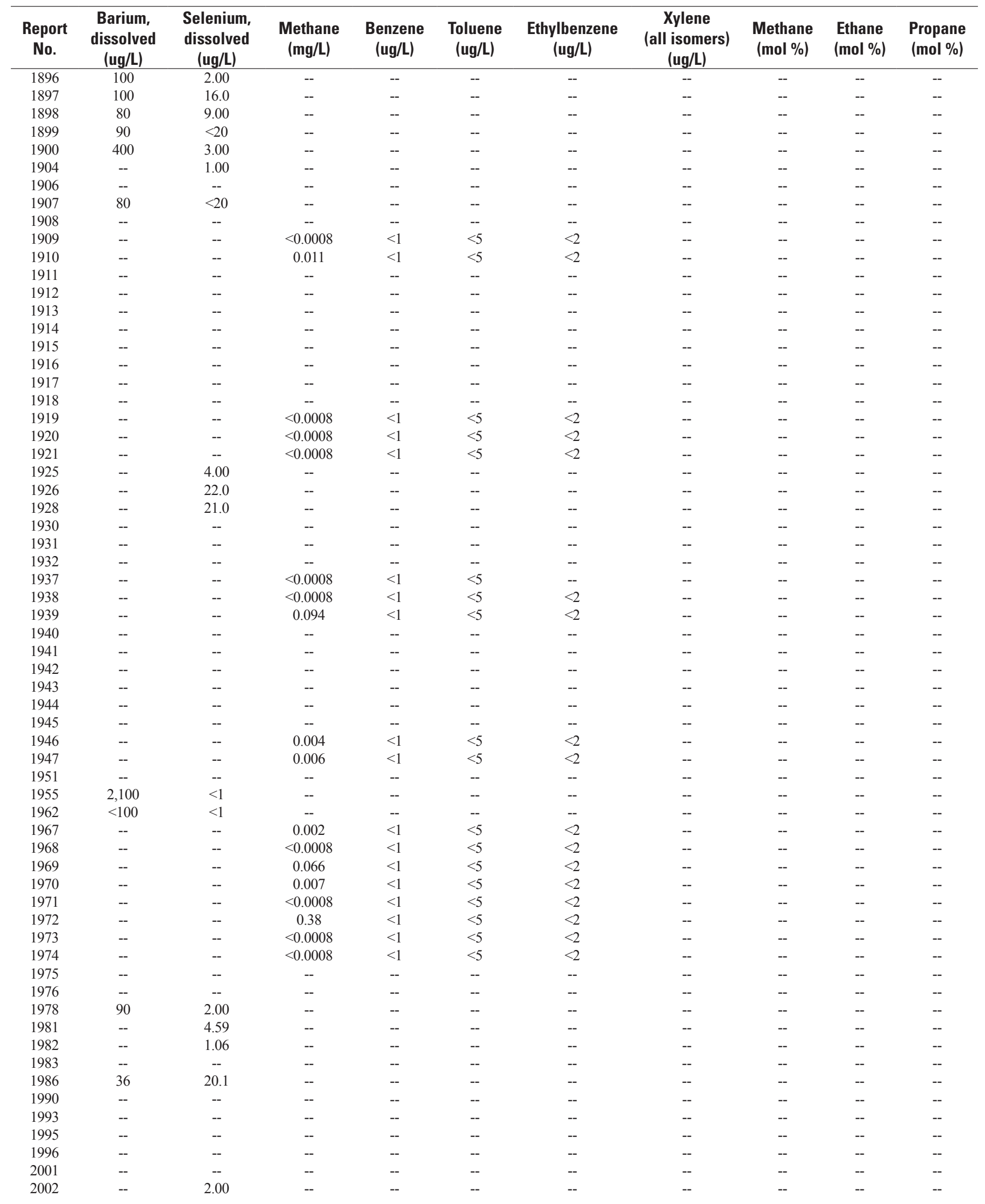


Appendix 1. Water Quality Data Used in This Report.-Continued

\begin{tabular}{|c|c|c|c|c|c|c|c|c|c|c|}
\hline $\begin{array}{l}\text { Report } \\
\text { No. }\end{array}$ & $\begin{array}{c}\text { Barium, } \\
\text { dissolved } \\
\text { (ug/L) }\end{array}$ & $\begin{array}{l}\text { Selenium, } \\
\text { dissolved } \\
\text { (ug/L) }\end{array}$ & $\begin{array}{l}\text { Methane } \\
\text { (mg/L) }\end{array}$ & $\begin{array}{c}\text { Benzene } \\
\text { (ug/L) }\end{array}$ & $\begin{array}{c}\text { Toluene } \\
\text { (ug/L) }\end{array}$ & $\begin{array}{l}\text { Ethylbenzene } \\
\text { (ug/L) }\end{array}$ & $\begin{array}{c}\text { Xylene } \\
\text { (all isomers) } \\
\text { (ug/L) }\end{array}$ & $\begin{array}{c}\text { Methane } \\
(\mathrm{mol} \%)\end{array}$ & $\begin{array}{l}\text { Ethane } \\
(\mathrm{mol} \%)\end{array}$ & $\begin{array}{r}\text { Propane } \\
\text { (mol \%) }\end{array}$ \\
\hline 2003 & -- & -- & -- & -- & -- & -- & -- & -- & -- & -- \\
\hline 2012 & -- & -- & -- & -- & -- & -- & -- & -- & -- & -- \\
\hline 2013 & -- & -- & -- & -- & -- & -- & -- & -- & -- & -- \\
\hline 2014 & -- & 31.0 & -- & -- & -- & -- & -- & -- & -- & -- \\
\hline 2015 & -- & -- & $<0.0008$ & $<1$ & $<2$ & $<2$ & -- & -- & -- & -- \\
\hline 2018 & -- & -- & -- & -- & -- & -- & -- & -- & -- & -- \\
\hline 2019 & -- & -- & -- & -- & -- & -- & -- & -- & -- & -- \\
\hline 2020 & -- & -- & -- & -- & -- & -- & -- & -- & -- & -- \\
\hline 2021 & -- & -- & -- & -- & -- & -- & -- & -- & -- & -- \\
\hline 2022 & -- & -- & -- & -- & -- & -- & -- & -- & -- & -- \\
\hline 2023 & -- & -- & -- & -- & -- & -- & -- & -- & -- & -- \\
\hline 2030 & -- & -- & -- & -- & -- & -- & -- & -- & -- & -- \\
\hline 2033 & -- & $<1$ & -- & -- & -- & -- & -- & -- & -- & -- \\
\hline 2034 & -- & -- & -- & -- & -- & -- & -- & -- & -- & -- \\
\hline 2035 & -- & -- & -- & -- & -- & -- & -- & -- & -- & -- \\
\hline 2037 & -- & -- & -- & -- & -- & -- & -- & -- & -- & -- \\
\hline 2040 & -- & -- & -- & -- & -- & -- & -- & -- & -- & -- \\
\hline 2041 & 20 & $<20$ & -- & -- & -- & -- & -- & -- & -- & -- \\
\hline 2044 & -- & -- & -- & -- & -- & -- & -- & -- & -- & -- \\
\hline 2046 & -- & $<1$ & -- & -- & -- & -- & -- & -- & -- & -- \\
\hline 2046 & -- & $<1$ & -- & -- & -- & -- & -- & -- & -- & -- \\
\hline 2049 & -- & -- & -- & -- & -- & -- & -- & -- & -- & -- \\
\hline 2050 & -- & -- & -- & -- & -- & -- & -- & -- & -- & -- \\
\hline 2051 & -- & -- & -- & -- & -- & -- & -- & -- & -- & -- \\
\hline 2065 & -- & -- & -- & -- & -- & -- & -- & -- & -- & -- \\
\hline 2066 & -- & -- & -- & -- & -- & -- & -- & -- & -- & -- \\
\hline 2067 & -- & -- & -- & -- & -- & -- & -- & -- & -- & -- \\
\hline 2068 & -- & -- & -- & -- & -- & -- & -- & -- & -- & -- \\
\hline 2069 & -- & -- & -- & -- & -- & -- & -- & -- & -- & -- \\
\hline 2070 & -- & -- & -- & -- & -- & -- & -- & -- & -- & -- \\
\hline 2071 & -- & -- & -- & -- & -- & -- & -- & -- & -- & -- \\
\hline 2072 & -- & -- & -- & -- & -- & -- & -- & -- & -- & -- \\
\hline 2076 & -- & 24.9 & -- & -- & -- & -- & -- & -- & -- & -- \\
\hline 2077 & -- & -- & -- & -- & -- & -- & -- & 66.1 & 0.47 & 0.013 \\
\hline 2078 & -- & -- & -- & -- & -- & -- & -- & -- & -- & -- \\
\hline 2079 & -- & -- & -- & -- & -- & -- & -- & -- & -- & -- \\
\hline 2080 & -- & -- & -- & -- & -- & -- & -- & -- & -- & -- \\
\hline 2082 & -- & -- & -- & -- & -- & -- & -- & -- & -- & -- \\
\hline 2083 & -- & -- & -- & -- & -- & -- & -- & -- & -- & -- \\
\hline 2084 & -- & -- & -- & -- & -- & -- & -- & -- & -- & -- \\
\hline 2085 & -- & $<1$ & -- & -- & -- & -- & -- & -- & -- & -- \\
\hline 2088 & -- & -- & -- & -- & -- & -- & -- & -- & -- & -- \\
\hline 2089 & -- & -- & -- & -- & -- & -- & -- & -- & -- & -- \\
\hline 2090 & -- & -- & -- & -- & -- & -- & -- & -- & -- & -- \\
\hline 2091 & -- & -- & -- & -- & -- & -- & -- & -- & -- & -- \\
\hline 2094 & -- & -- & -- & -- & -- & -- & -- & -- & -- & -- \\
\hline 2095 & -- & -- & -- & -- & -- & -- & -- & -- & -- & -- \\
\hline 2096 & -- & -- & -- & -- & -- & -- & -- & -- & -- & -- \\
\hline
\end{tabular}


Appendix 1. Water Quality Data Used in This Report.-Continued

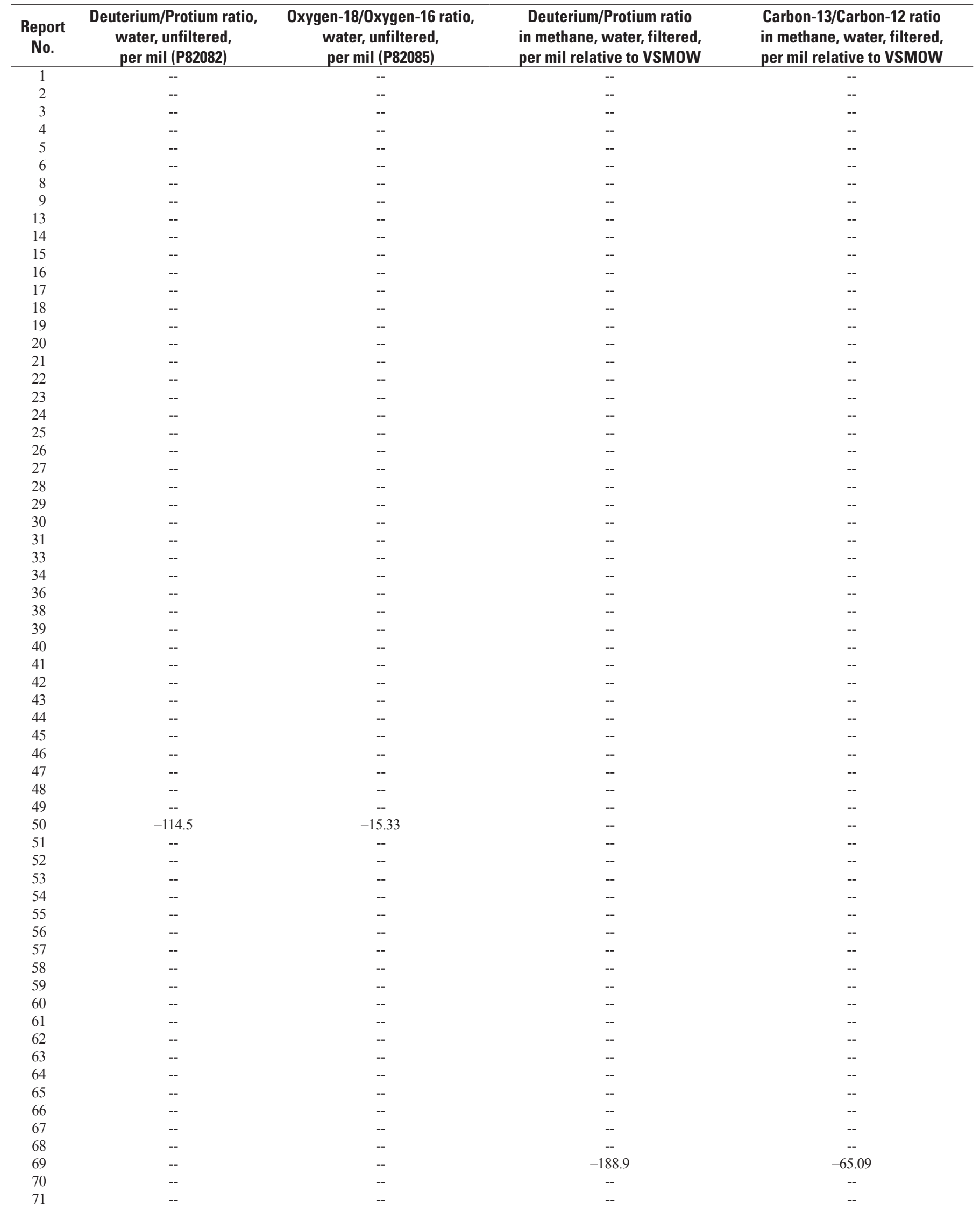


Appendix 1. Water Quality Data Used in This Report.-Continued

\begin{tabular}{|c|c|c|c|c|}
\hline $\begin{array}{c}\text { Report } \\
\text { No. }\end{array}$ & $\begin{array}{c}\text { Deuterium/Protium ratio, } \\
\text { water, unfiltered, } \\
\text { per mil (P82082) }\end{array}$ & $\begin{array}{c}\text { Oxygen-18/0xygen-16 ratio, } \\
\text { water, unfiltered, } \\
\text { per mil (P82085) }\end{array}$ & $\begin{array}{l}\text { Deuterium/Protium ratio } \\
\text { in methane, water, filtered, } \\
\text { per mil relative to VSMOW }\end{array}$ & $\begin{array}{l}\text { Carbon-13/Carbon-12 ratio } \\
\text { in methane, water, filtered, } \\
\text { per mil relative to VSMOW }\end{array}$ \\
\hline 72 & -- & -- & -- & -- \\
\hline 73 & -- & -- & -- & -- \\
\hline 74 & -- & -- & -- & -- \\
\hline 75 & -- & -- & -- & -- \\
\hline 76 & -- & -- & -- & -- \\
\hline 77 & -- & -- & -- & -- \\
\hline 78 & -- & -- & -- & -- \\
\hline 79 & -- & -- & -- & -- \\
\hline 80 & -- & -- & -- & -- \\
\hline 81 & -- & -- & -- & -- \\
\hline 82 & -- & -- & -- & -- \\
\hline 83 & -- & -- & -- & -- \\
\hline 84 & -- & -- & -- & -- \\
\hline 85 & -- & -- & -- & -- \\
\hline 86 & -- & -- & -- & -- \\
\hline 87 & -- & -- & -- & -- \\
\hline 88 & -- & -- & -- & -- \\
\hline 89 & -- & -- & -228.7 & -55.51 \\
\hline 90 & -- & -- & -- & -- \\
\hline 91 & -- & -- & -- & -- \\
\hline 92 & -- & -- & -- & -- \\
\hline 93 & -- & -- & -- & -- \\
\hline 94 & -- & -- & -- & -- \\
\hline 95 & -- & -- & -- & -- \\
\hline 96 & -- & -- & -- & -- \\
\hline 97 & -- & -- & -- & -- \\
\hline 98 & -- & -- & -- & -- \\
\hline 99 & -- & -- & -168 & -40.33 \\
\hline 100 & -- & -- & -185.8 & -39.9 \\
\hline 101 & -- & -- & -- & -- \\
\hline 102 & -- & -- & -189.3 & -41.3 \\
\hline 103 & -- & -- & -- & -- \\
\hline 104 & -- & -- & -142.4 & -38.57 \\
\hline 105 & -- & -- & -- & -- \\
\hline 106 & -- & -- & -185.1 & -39.88 \\
\hline 107 & -- & -- & -- & -- \\
\hline 108 & -- & -- & -- & -- \\
\hline 109 & -- & -- & -200.3 & -55.86 \\
\hline 110 & -- & -- & -213.9 & -56.5 \\
\hline 111 & -- & -- & -- & -- \\
\hline 112 & -- & -- & -- & -- \\
\hline 113 & -- & -- & -169.7 & -37.83 \\
\hline 114 & -- & -- & -190.5 & -44.14 \\
\hline 115 & -- & -- & -- & -- \\
\hline 116 & -- & -- & -- & -- \\
\hline 117 & -- & -- & -- & -- \\
\hline 118 & -- & -- & -- & -- \\
\hline 119 & -- & -- & -- & -- \\
\hline 120 & -- & -- & -- & -- \\
\hline 121 & -- & -- & -- & -- \\
\hline 122 & -- & -- & -- & -- \\
\hline 123 & -- & -- & -- & -- \\
\hline 124 & -- & -- & -- & -- \\
\hline 125 & -- & -- & -- & -- \\
\hline 126 & -- & -- & -- & -- \\
\hline 127 & -- & -- & -- & -- \\
\hline 128 & -- & -- & -- & -- \\
\hline 129 & -- & -- & -- & -- \\
\hline 130 & -- & -- & -- & -- \\
\hline 131 & -- & -- & -190.1 & -43.87 \\
\hline 132 & -- & -- & -- & -- \\
\hline 133 & -- & -- & -- & -- \\
\hline 134 & -- & -- & -- & -- \\
\hline
\end{tabular}


Appendix 1. Water Quality Data Used in This Report.-Continued

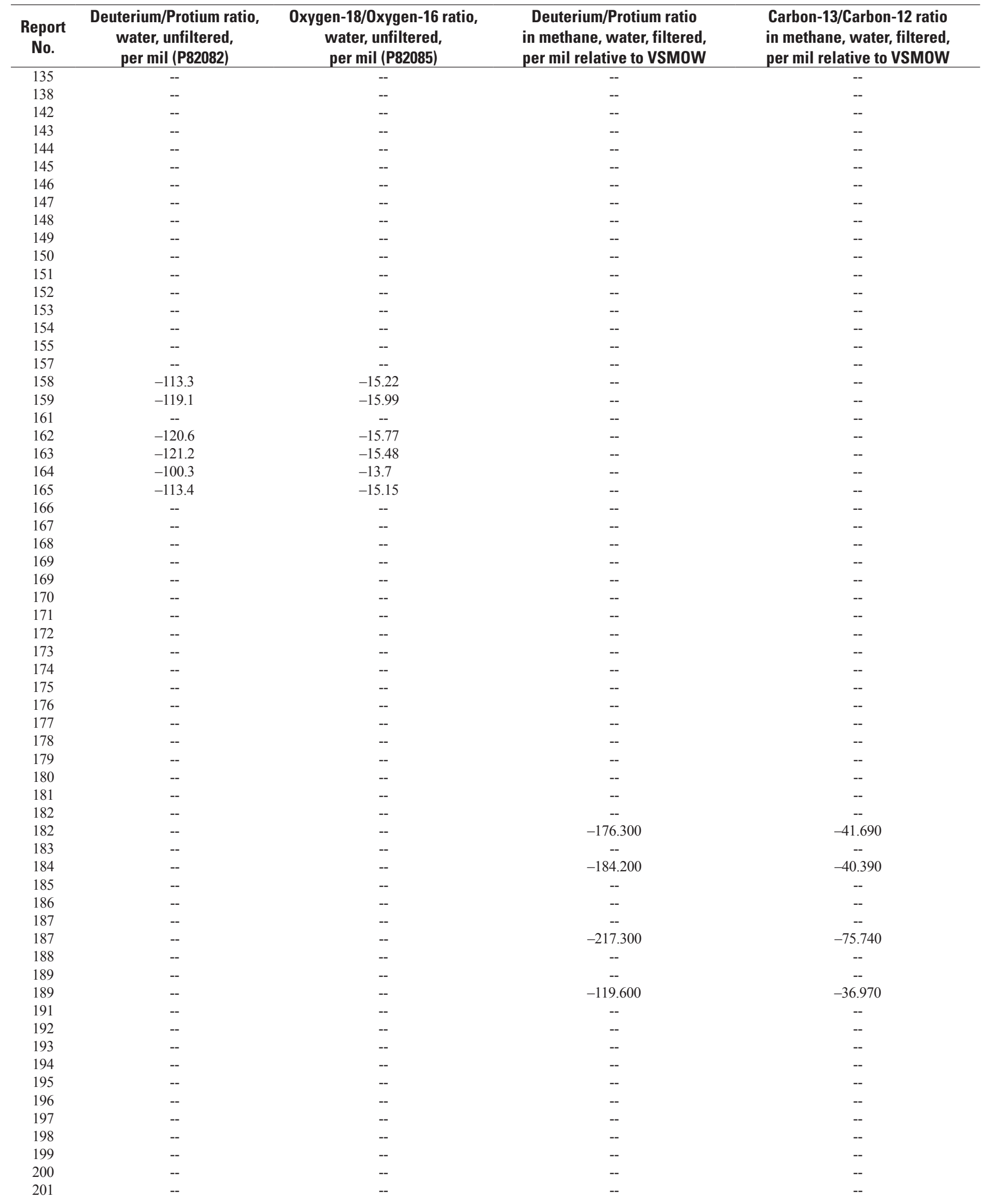


Appendix 1. Water Quality Data Used in This Report.-Continued

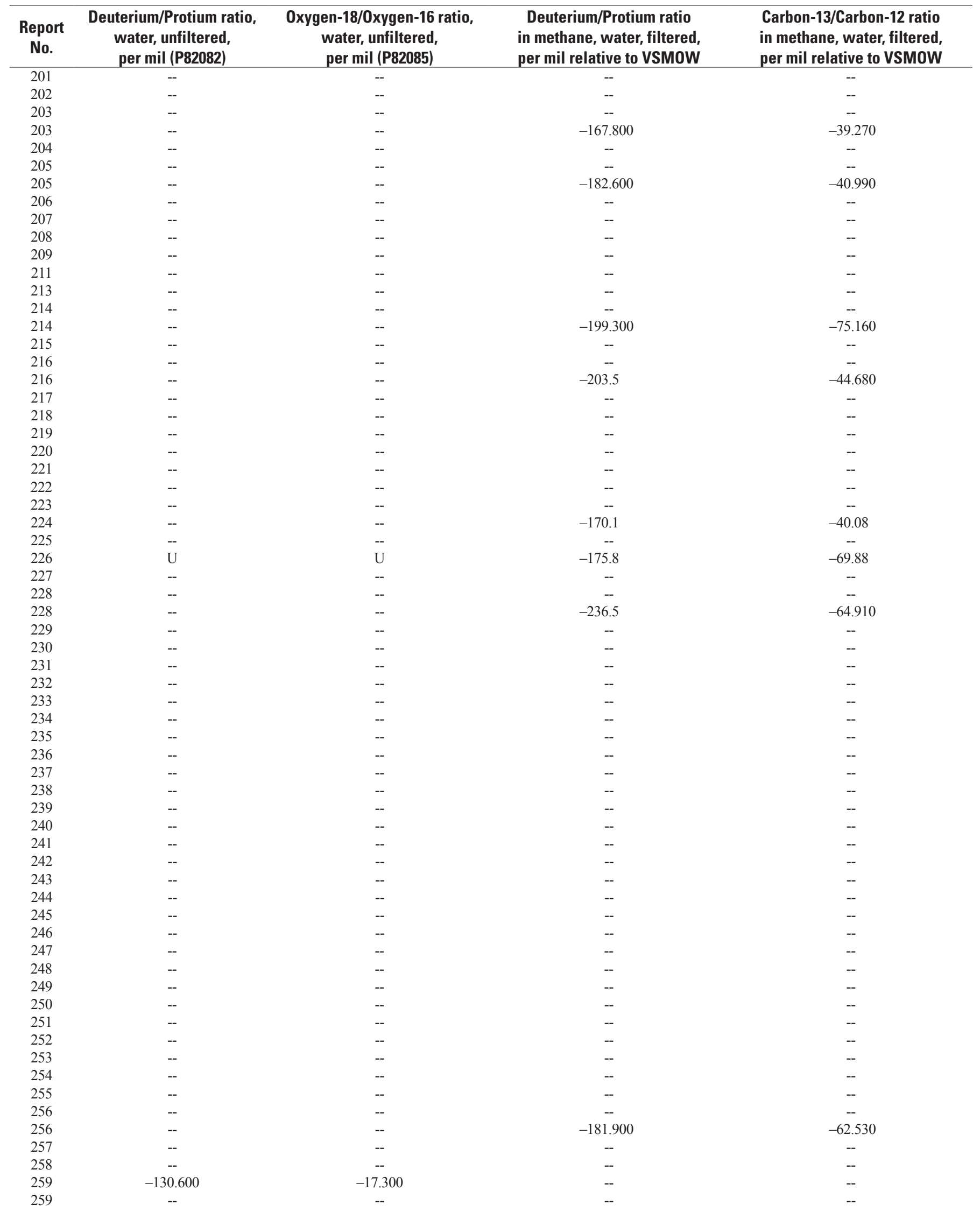


Appendix 1. Water Quality Data Used in This Report.-Continued

\begin{tabular}{|c|c|c|c|c|}
\hline $\begin{array}{c}\text { Report } \\
\text { No. }\end{array}$ & $\begin{array}{c}\text { Deuterium/Protium ratio, } \\
\text { water, unfiltered, } \\
\text { per mil (P82082) }\end{array}$ & $\begin{array}{c}\text { 0xygen-18/0xygen-16 ratio, } \\
\text { water, unfiltered, } \\
\text { per mil (P82085) }\end{array}$ & $\begin{array}{l}\text { Deuterium/Protium ratio } \\
\text { in methane, water, filtered, } \\
\text { per mil relative to VSMOW }\end{array}$ & $\begin{array}{l}\text { Carbon-13/Carbon-12 ratio } \\
\text { in methane, water, filtered, } \\
\text { per mil relative to VSMOW }\end{array}$ \\
\hline 260 & -- & -- & -- & -- \\
\hline 262 & -- & -- & -- & -- \\
\hline 263 & -- & -- & -- & -- \\
\hline 264 & -- & -- & -- & -- \\
\hline 264 & -- & -- & -- & -- \\
\hline 267 & -- & -- & -- & -- \\
\hline 268 & -- & -- & -- & -- \\
\hline 269 & -- & -- & -- & -- \\
\hline 270 & -- & -- & -- & -- \\
\hline 271 & -- & -- & -- & -- \\
\hline 272 & -- & -- & -- & -- \\
\hline 275 & -- & -- & -- & -- \\
\hline 276 & -- & -- & -161 & -36.45 \\
\hline 277 & -- & -- & -- & -- \\
\hline 278 & -- & -- & -- & -- \\
\hline 279 & -- & -- & -- & -- \\
\hline 280 & -- & -- & -98.1 & -50.88 \\
\hline 281 & -- & -- & -- & $\mathrm{U}$ \\
\hline 282 & -115.5 & -15.52 & -189 & -81.15 \\
\hline 283 & -- & -- & -- & -- \\
\hline 284 & -- & -- & -- & -- \\
\hline 285 & -- & -- & -- & -- \\
\hline 286 & -- & -- & -- & -- \\
\hline 287 & -- & -- & -- & -- \\
\hline 288 & -- & -- & -- & -- \\
\hline 289 & -- & -- & -- & -- \\
\hline 290 & -- & -- & -- & -- \\
\hline 291 & -- & -- & -- & -- \\
\hline 292 & -- & -- & -- & -- \\
\hline 293 & -- & -- & -- & -- \\
\hline 294 & -- & -- & -- & -- \\
\hline 295 & -- & -- & -- & -- \\
\hline 296 & -- & -- & -- & -- \\
\hline 297 & -- & -- & -- & -- \\
\hline 298 & -- & -- & -- & -- \\
\hline 299 & -- & -- & -- & -- \\
\hline 300 & -- & -- & -177.1 & -73.27 \\
\hline 301 & -- & -- & -187.9 & -74.7 \\
\hline 302 & -- & -- & -- & -- \\
\hline 303 & -- & -- & -- & -- \\
\hline 304 & -- & -- & -- & -- \\
\hline 305 & -- & -- & -- & -- \\
\hline 306 & -- & -- & -- & -- \\
\hline 307 & -- & -- & -- & -- \\
\hline 308 & -- & -- & -- & -- \\
\hline 309 & -- & -- & -- & -- \\
\hline 310 & -- & -- & -- & -- \\
\hline 311 & -- & -- & -188.4 & -53.68 \\
\hline 312 & -- & -- & - & -- \\
\hline 313 & -- & -- & -- & -- \\
\hline 314 & -- & -- & -- & -- \\
\hline 315 & -- & -- & -- & -- \\
\hline 316 & -- & -- & -223.2 & -58.41 \\
\hline 317 & -- & -- & -- & -- \\
\hline 318 & -- & -- & -- & -- \\
\hline 319 & -- & -- & -- & -- \\
\hline 320 & -- & -- & -- & -- \\
\hline 321 & -- & -- & -- & -- \\
\hline
\end{tabular}


Appendix 1. Water Quality Data Used in This Report.-Continued

\begin{tabular}{|c|c|c|c|c|}
\hline $\begin{array}{c}\text { Report } \\
\text { No. }\end{array}$ & $\begin{array}{c}\text { Deuterium/Protium ratio, } \\
\text { water, unfiltered, } \\
\text { per mil (P82082) }\end{array}$ & $\begin{array}{c}\text { Oxygen-18/0xygen-16 ratio, } \\
\text { water, unfiltered, } \\
\text { per mil (P82085) }\end{array}$ & $\begin{array}{l}\text { Deuterium/Protium ratio } \\
\text { in methane, water, filtered, } \\
\text { per mil relative to VSMOW }\end{array}$ & $\begin{array}{l}\text { Carbon-13/Carbon-12 ratio } \\
\text { in methane, water, filtered, } \\
\text { per mil relative to VSMOW }\end{array}$ \\
\hline 322 & -- & -- & -- & -- \\
\hline 323 & -- & -- & -- & -- \\
\hline 324 & -- & -- & -- & -- \\
\hline 325 & -- & -- & -132.4 & -39.24 \\
\hline 326 & -- & -- & -- & -- \\
\hline 327 & -- & -- & -- & -- \\
\hline 328 & -- & -- & -- & -- \\
\hline 329 & -- & -- & -- & -- \\
\hline 330 & -- & -- & -- & -- \\
\hline 331 & -- & -- & -- & -- \\
\hline 332 & -- & -- & -- & -- \\
\hline 333 & -- & -- & -- & -- \\
\hline 334 & -- & -- & -- & -- \\
\hline 335 & -- & -- & -- & -- \\
\hline 336 & -- & -- & -- & -- \\
\hline 337 & -- & -- & -- & -- \\
\hline 338 & -- & -- & -- & -- \\
\hline 339 & -- & -- & -- & -- \\
\hline 340 & -- & -- & -- & -- \\
\hline 341 & -- & -- & -- & -- \\
\hline 342 & -- & -- & -- & -- \\
\hline 343 & -- & -- & -- & -- \\
\hline 344 & -- & -- & -- & -- \\
\hline 345 & -- & -- & -- & -- \\
\hline 346 & -- & -- & -- & -- \\
\hline 347 & -- & -- & -- & -- \\
\hline 348 & -- & -- & -- & -- \\
\hline 349 & -- & -- & -- & -- \\
\hline 350 & -- & -- & -- & -- \\
\hline 351 & -- & -- & -- & -- \\
\hline 352 & -- & -- & -- & -- \\
\hline 353 & -- & -- & -- & -- \\
\hline 354 & -- & -- & -- & -- \\
\hline 355 & -- & -- & -- & -- \\
\hline 356 & -- & -- & -- & -- \\
\hline 357 & -- & -- & -- & -- \\
\hline 358 & -- & -- & -- & -- \\
\hline 359 & -- & -- & -186.8 & -46.28 \\
\hline 360 & -- & -- & -182.2 & -46.93 \\
\hline 361 & -- & -- & -75.26 & -- \\
\hline 362 & -- & -- & -- & -- \\
\hline 363 & -- & -- & -- & -- \\
\hline 364 & -- & -- & -199.3 & -75.16 \\
\hline 365 & -- & -- & -190.6 & -49.38 \\
\hline 366 & -- & -- & -- & -- \\
\hline 367 & -- & -- & -- & -- \\
\hline 368 & -- & -- & -- & -- \\
\hline 369 & -- & -- & -- & -- \\
\hline 370 & -- & -- & -- & -- \\
\hline 371 & -- & -- & -- & -- \\
\hline 372 & -- & -- & -- & -- \\
\hline 373 & -- & -- & -- & -- \\
\hline 374 & -- & -- & -- & -- \\
\hline 375 & -- & -- & -- & -- \\
\hline 376 & -- & -- & -54.09 & -- \\
\hline 377 & -- & -- & -- & -- \\
\hline 378 & -- & -- & -- & -- \\
\hline 379 & -- & -- & -- & -- \\
\hline 380 & -- & -- & -- & -- \\
\hline 381 & -- & -- & -- & -- \\
\hline 382 & -- & -- & -- & -- \\
\hline 383 & -- & -- & -- & -- \\
\hline 384 & -- & -- & -- & -- \\
\hline 385 & -- & -- & -- & -- \\
\hline
\end{tabular}


Appendix 1. Water Quality Data Used in This Report.-Continued

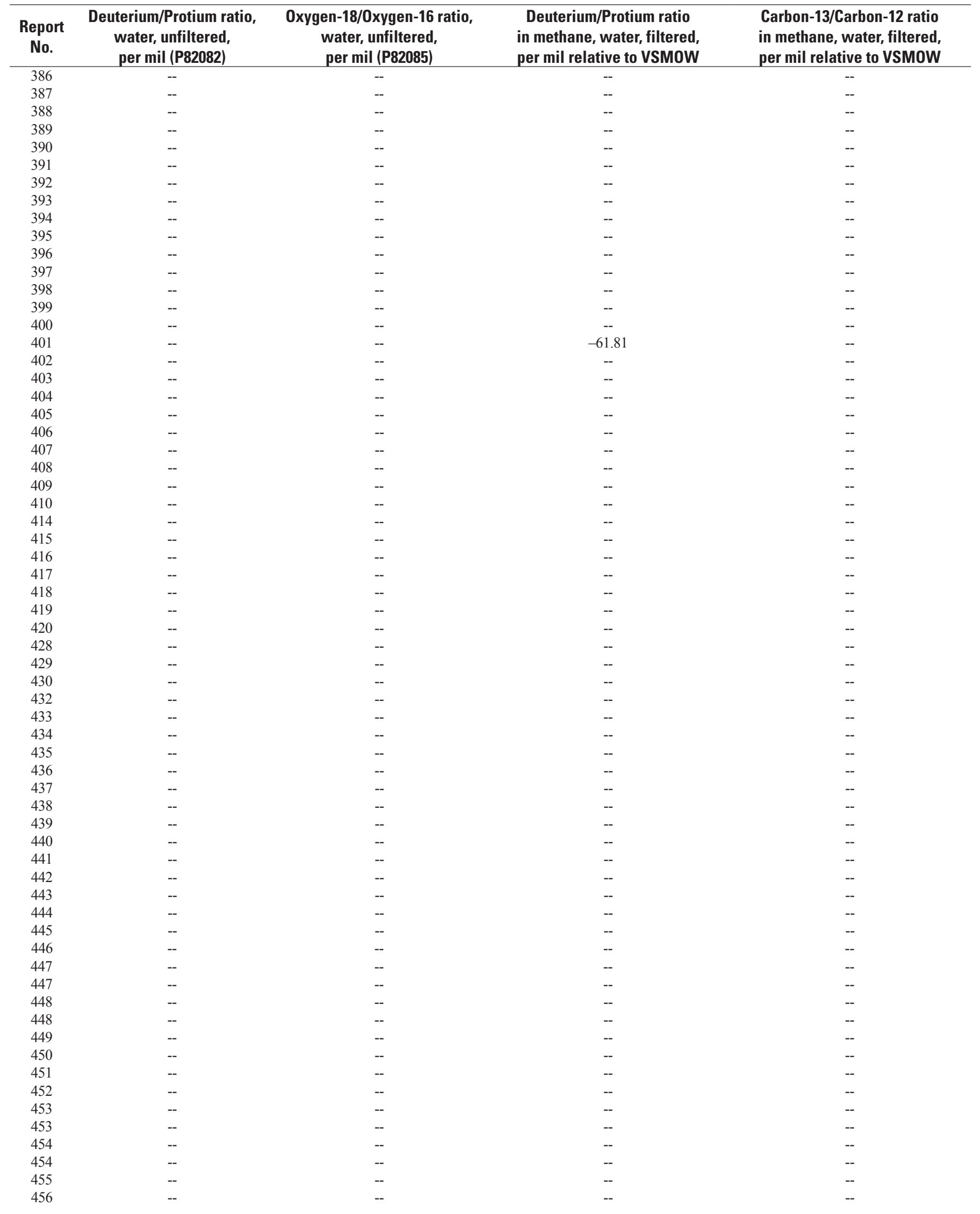


Appendix 1. Water Quality Data Used in This Report.-Continued

\begin{tabular}{|c|c|c|c|c|}
\hline $\begin{array}{c}\text { Report } \\
\text { No. }\end{array}$ & $\begin{array}{c}\text { Deuterium/Protium ratio, } \\
\text { water, unfiltered, } \\
\text { per mil (P82082) }\end{array}$ & $\begin{array}{c}\text { Oxygen-18/0xygen-16 ratio, } \\
\text { water, unfiltered, } \\
\text { per mil (P82085) }\end{array}$ & $\begin{array}{l}\text { Deuterium/Protium ratio } \\
\text { in methane, water, filtered, } \\
\text { per mil relative to VSMOW }\end{array}$ & $\begin{array}{l}\text { Carbon-13/Carbon-12 ratio } \\
\text { in methane, water, filtered, } \\
\text { per mil relative to VSMOW }\end{array}$ \\
\hline 457 & -- & -- & -- & -- \\
\hline 458 & -- & -- & -- & -- \\
\hline 459 & -- & -- & -- & -- \\
\hline 460 & -- & -- & -- & -- \\
\hline 461 & -- & -- & -- & -- \\
\hline 462 & -- & -- & -- & -- \\
\hline 463 & -- & -- & -- & -- \\
\hline 464 & -- & -- & -- & -- \\
\hline 465 & -48 & -3.9 & -- & -- \\
\hline 466 & -- & -- & -- & -- \\
\hline 468 & -- & -- & -- & -- \\
\hline 469 & -- & -- & -- & -- \\
\hline 470 & -- & -- & -- & -- \\
\hline 471 & -- & -- & -- & -- \\
\hline 472 & -- & -- & -- & -- \\
\hline 473 & -- & -- & -- & -- \\
\hline 474 & -- & -- & -- & -- \\
\hline 475 & -- & -- & -- & -- \\
\hline 476 & -- & -- & -- & -- \\
\hline 477 & -- & -- & -- & -- \\
\hline 478 & -- & -- & -- & -- \\
\hline 479 & -- & -- & -- & -- \\
\hline 480 & -- & -- & -- & -- \\
\hline 481 & -- & -- & -- & -- \\
\hline 482 & -- & -- & -- & -- \\
\hline 483 & -- & -- & -- & -- \\
\hline 484 & -- & -- & -- & -- \\
\hline 485 & -- & -- & -- & -- \\
\hline 486 & -- & -- & -- & -- \\
\hline 487 & -- & -- & -- & -- \\
\hline 488 & -- & -- & -- & -- \\
\hline 489 & -- & -- & -- & -- \\
\hline 490 & -- & -- & -- & -- \\
\hline 491 & -- & -- & -- & -- \\
\hline 492 & -- & -- & -- & -- \\
\hline 493 & -- & -- & -- & -- \\
\hline 494 & -- & -- & -- & -- \\
\hline 495 & -- & -- & -- & -- \\
\hline 496 & -- & -- & -- & -- \\
\hline 497 & -- & -- & -- & -- \\
\hline 498 & -- & -- & -- & -- \\
\hline 499 & -- & -- & -- & -- \\
\hline 500 & -- & -- & -- & -- \\
\hline 501 & -- & -- & -- & -- \\
\hline 502 & -- & -- & -- & -- \\
\hline 503 & -- & -- & -- & -- \\
\hline 504 & -- & -- & -- & -- \\
\hline 505 & -- & -- & -- & -- \\
\hline 506 & -- & -- & -- & -- \\
\hline 507 & -- & -- & -- & -- \\
\hline 508 & -- & -- & -- & -- \\
\hline 509 & -- & -- & -- & -- \\
\hline 510 & -- & -- & -- & -- \\
\hline 511 & -- & -- & -- & -- \\
\hline 512 & -- & -- & -- & -- \\
\hline 513 & $\mathrm{U}$ & $\mathrm{U}$ & $\mathrm{U}$ & $\mathrm{U}$ \\
\hline 514 & -- & - & - & - \\
\hline 515 & -- & -- & -- & -- \\
\hline 516 & -- & -- & -- & -- \\
\hline 517 & -- & -- & -- & -- \\
\hline 518 & -- & -- & -- & -- \\
\hline 519 & -- & -- & -- & -- \\
\hline 520 & -- & -- & -- & -- \\
\hline 521 & -- & -- & -- & -- \\
\hline
\end{tabular}


Appendix 1. Water Quality Data Used in This Report.-Continued

\begin{tabular}{|c|c|c|c|c|}
\hline $\begin{array}{c}\text { Report } \\
\text { No. }\end{array}$ & $\begin{array}{c}\text { Deuterium/Protium ratio, } \\
\text { water, unfiltered, } \\
\text { per mil (P82082) }\end{array}$ & $\begin{array}{c}\text { 0xygen-18/0xygen-16 ratio, } \\
\text { water, unfiltered, } \\
\text { per mil (P82085) } \\
\end{array}$ & $\begin{array}{l}\text { Deuterium/Protium ratio } \\
\text { in methane, water, filtered, } \\
\text { per mil relative to VSMOW }\end{array}$ & $\begin{array}{l}\text { Carbon-13/Carbon-12 ratio } \\
\text { in methane, water, filtered, } \\
\text { per mil relative to VSMOW }\end{array}$ \\
\hline 522 & -- & $-\operatorname{lol}^{-}$ & -- & -- \\
\hline 524 & -- & -- & -- & -- \\
\hline 525 & -- & -- & -- & -- \\
\hline 526 & -- & -- & -- & -- \\
\hline 527 & -- & -- & -- & -- \\
\hline 530 & -- & -- & -43.38 & -- \\
\hline 531 & -- & -- & -- & -- \\
\hline 532 & -- & -- & -- & -- \\
\hline 533 & -- & -- & -- & -- \\
\hline 534 & -- & -- & -- & -- \\
\hline 535 & -- & -- & -- & -- \\
\hline 540 & -- & -- & -- & -- \\
\hline 541 & -- & -- & -- & -- \\
\hline 542 & -- & -- & -- & -- \\
\hline 543 & -- & -- & -- & -- \\
\hline 544 & -- & -- & -- & -- \\
\hline 558 & -- & -- & -- & -- \\
\hline 559 & -- & -- & -- & -- \\
\hline 560 & -- & -- & -- & -- \\
\hline 561 & -- & -- & -- & -- \\
\hline 562 & -- & -- & -- & -- \\
\hline 563 & -- & -- & -- & -- \\
\hline 564 & -- & -- & -- & -- \\
\hline 565 & -- & -- & -- & -- \\
\hline 577 & -- & -- & -- & -- \\
\hline 578 & -- & -- & -- & -- \\
\hline 579 & -- & -- & -- & -- \\
\hline 580 & -- & -- & -- & -- \\
\hline 581 & -- & -- & -- & -- \\
\hline 582 & -- & -- & -- & -- \\
\hline 583 & -- & -- & -- & -- \\
\hline 584 & -- & -- & -- & -- \\
\hline 585 & -- & -- & -- & -- \\
\hline 586 & -- & -- & -- & -- \\
\hline 587 & -- & -- & -- & -- \\
\hline 588 & -- & -- & -- & -- \\
\hline 589 & -- & -- & -- & -- \\
\hline 590 & -- & -- & -- & -- \\
\hline 591 & -- & -- & -- & -- \\
\hline 592 & -- & -- & -- & -- \\
\hline 593 & -- & -- & -- & -- \\
\hline 594 & -- & -- & -- & -- \\
\hline 595 & -- & -- & -- & -- \\
\hline 596 & -- & -- & -- & -- \\
\hline 597 & -- & -- & -- & -- \\
\hline 598 & -- & -- & -- & -- \\
\hline 599 & -- & -- & -- & -- \\
\hline 600 & -- & -- & -- & -- \\
\hline
\end{tabular}


Appendix 1. Water Quality Data Used in This Report.-Continued

\begin{tabular}{|c|c|c|c|c|}
\hline $\begin{array}{c}\text { Report } \\
\text { No. }\end{array}$ & $\begin{array}{c}\text { Deuterium/Protium ratio, } \\
\text { water, unfiltered, } \\
\text { per mil (P82082) }\end{array}$ & $\begin{array}{c}\text { Oxygen-18/0xygen-16 ratio, } \\
\text { water, unfiltered, } \\
\text { per mil (P82085) }\end{array}$ & $\begin{array}{l}\text { Deuterium/Protium ratio } \\
\text { in methane, water, filtered, } \\
\text { per mil relative to VSMOW }\end{array}$ & $\begin{array}{l}\text { Carbon-13/Carbon-12 ratio } \\
\text { in methane, water, filtered, } \\
\text { per mil relative to VSMOW }\end{array}$ \\
\hline 601 & -- & -- & -- & -- \\
\hline 602 & -- & -- & -- & -- \\
\hline 603 & -- & -- & -- & -- \\
\hline 604 & -- & -- & -- & -- \\
\hline 605 & $\mathrm{U}$ & U & U & U \\
\hline 606 & $\mathrm{U}$ & U & U & U \\
\hline 607 & -- & -- & -- & -- \\
\hline 608 & -- & -- & -- & -- \\
\hline 609 & -- & -- & -- & -- \\
\hline 610 & -- & -- & -- & -- \\
\hline 611 & -- & -- & -- & -- \\
\hline 612 & -- & -- & -- & -- \\
\hline 613 & -- & -- & -- & -- \\
\hline 614 & -- & -- & -- & -- \\
\hline 615 & -- & -- & -- & -- \\
\hline 616 & -- & -- & -- & -- \\
\hline 617 & -- & -- & -- & -- \\
\hline 623 & -118 & -15.44 & -- & -- \\
\hline 624 & -120.6 & -15.75 & -- & -- \\
\hline 625 & -- & -- & -- & -- \\
\hline 626 & -- & -- & -- & -- \\
\hline 627 & -115.7 & -15.18 & -- & -- \\
\hline 628 & -- & -- & -- & -- \\
\hline 629 & -- & -- & -- & -- \\
\hline 630 & -- & -- & -- & -- \\
\hline 631 & -- & -- & -- & -- \\
\hline 632 & -- & -- & -- & -- \\
\hline 633 & -- & -- & -- & -- \\
\hline 634 & -- & -- & -- & -- \\
\hline 635 & -- & -- & -- & -- \\
\hline 636 & -- & -- & -- & -- \\
\hline 637 & -- & -- & -- & -- \\
\hline 638 & -- & -- & -- & -- \\
\hline 639 & -- & -- & -- & -- \\
\hline 640 & -- & -- & -- & -- \\
\hline 641 & -- & -- & -- & -- \\
\hline 642 & -- & -- & -- & -- \\
\hline 643 & -- & -- & -- & -- \\
\hline 644 & -- & -- & -- & -- \\
\hline 645 & -- & -- & -- & -- \\
\hline 646 & -- & -- & -- & -- \\
\hline 647 & -- & -- & -- & -- \\
\hline 649 & -- & -- & -- & -- \\
\hline 650 & -- & -- & -- & -- \\
\hline 651 & -- & -- & -- & -- \\
\hline 652 & -- & --- & -- & -- \\
\hline 653 & -- & -- & -- & -- \\
\hline 654 & -- & -- & -- & -- \\
\hline 655 & -- & -- & -- & -- \\
\hline 656 & -- & -- & -- & -- \\
\hline 657 & -- & -- & -- & - \\
\hline 658 & -- & -- & -- & -- \\
\hline 660 & -- & -- & -- & -- \\
\hline 660 & -- & -- & -- & -- \\
\hline 661 & -- & -- & -- & -- \\
\hline 662 & -- & -- & -- & -- \\
\hline 663 & -- & -- & -- & -- \\
\hline 663 & -- & -- & -- & -- \\
\hline 664 & -- & -- & -- & -- \\
\hline 665 & -- & -- & -- & -- \\
\hline 666 & -- & -- & -- & -- \\
\hline 667 & -- & -- & -- & -- \\
\hline 668 & -- & -- & -- & -- \\
\hline 669 & -- & -- & -- & -- \\
\hline
\end{tabular}


Appendix 1. Water Quality Data Used in This Report.-Continued

\begin{tabular}{|c|c|c|c|c|}
\hline $\begin{array}{c}\text { Report } \\
\text { No. }\end{array}$ & $\begin{array}{c}\text { Deuterium/Protium ratio, } \\
\text { water, unfiltered, } \\
\text { per mil (P82082) }\end{array}$ & $\begin{array}{c}\text { 0xygen-18/0xygen-16 ratio, } \\
\text { water, unfiltered, } \\
\text { per mil (P82085) }\end{array}$ & $\begin{array}{l}\text { Deuterium/Protium ratio } \\
\text { in methane, water, filtered, } \\
\text { per mil relative to VSMOW }\end{array}$ & $\begin{array}{l}\text { Carbon-13/Carbon-12 ratio } \\
\text { in methane, water, filtered, } \\
\text { per mil relative to VSMOW }\end{array}$ \\
\hline 670 & -- & -- & -- & -- \\
\hline 672 & -- & -- & -- & -- \\
\hline 673 & -- & -- & -- & -- \\
\hline 674 & -- & -- & -- & -- \\
\hline 675 & -- & -- & -- & -- \\
\hline 678 & -- & -- & -- & -- \\
\hline 679 & -- & -- & -- & -- \\
\hline 680 & -- & -- & -- & -- \\
\hline 681 & - & -- & -- & -- \\
\hline 682 & -- & -- & -- & -- \\
\hline 683 & -- & -- & -- & -- \\
\hline 688 & -- & -- & -- & -- \\
\hline 689 & -- & -- & -- & -- \\
\hline 690 & -- & -- & -- & -- \\
\hline 691 & -- & -- & -- & -- \\
\hline 692 & -- & -- & -- & -- \\
\hline 693 & -- & -- & -- & -- \\
\hline 694 & -- & -- & -- & -- \\
\hline 695 & -- & -- & -- & -- \\
\hline 696 & -- & -- & -- & -- \\
\hline 699 & -- & -- & -- & -- \\
\hline 704 & -- & -- & -- & --- \\
\hline 706 & -- & -- & -- & -- \\
\hline 707 & -- & -- & -- & -- \\
\hline 708 & -- & -- & -- & -- \\
\hline 718 & -- & -- & -- & -- \\
\hline 719 & -- & -- & -- & -- \\
\hline 720 & -- & -- & -- & -- \\
\hline 721 & -- & -- & -- & -- \\
\hline 722 & -- & -- & -- & -- \\
\hline 723 & -- & -- & -- & -- \\
\hline 724 & -- & -- & -- & -- \\
\hline 725 & -- & -- & -- & -- \\
\hline 726 & -- & -- & -- & -- \\
\hline 727 & -- & -- & -- & -- \\
\hline 728 & -- & -- & -- & -- \\
\hline 729 & -- & -- & -- & -- \\
\hline 730 & -- & -- & -- & -- \\
\hline 731 & -- & -- & -- & -- \\
\hline 732 & -- & -- & -- & -- \\
\hline 733 & -- & -- & -- & -- \\
\hline 734 & -- & -- & -- & -- \\
\hline 735 & -- & -- & -- & -- \\
\hline 736 & -- & -- & -- & -- \\
\hline 737 & -- & -- & -- & -- \\
\hline 738 & -- & -- & -- & -- \\
\hline 739 & -- & -- & -- & -- \\
\hline 740 & -- & -- & -- & -- \\
\hline 741 & -- & -- & -- & -- \\
\hline
\end{tabular}


Appendix 1. Water Quality Data Used in This Report.-Continued

\begin{tabular}{|c|c|c|c|c|}
\hline $\begin{array}{c}\text { Report } \\
\text { No. }\end{array}$ & $\begin{array}{c}\text { Deuterium/Protium ratio, } \\
\text { water, unfiltered, } \\
\text { per mil (P82082) }\end{array}$ & $\begin{array}{c}\text { Oxygen-18/0xygen-16 ratio, } \\
\text { water, unfiltered, } \\
\text { per mil (P82085) }\end{array}$ & $\begin{array}{l}\text { Deuterium/Protium ratio } \\
\text { in methane, water, filtered, } \\
\text { per mil relative to VSMOW }\end{array}$ & $\begin{array}{l}\text { Carbon-13/Carbon-12 ratio } \\
\text { in methane, water, filtered } \\
\text { per mil relative to VSMOW }\end{array}$ \\
\hline 742 & -- & -- & -- & -- \\
\hline 743 & -- & -- & -- & -- \\
\hline 744 & -- & -- & -- & -- \\
\hline 745 & -- & -- & -- & -- \\
\hline 746 & -- & -- & -- & -- \\
\hline 747 & -- & -- & -- & -- \\
\hline 748 & -- & -- & -- & -- \\
\hline 749 & -- & -- & -- & -- \\
\hline 750 & -- & -- & -- & -- \\
\hline 751 & -- & -- & -- & -- \\
\hline 752 & -- & -- & -- & -- \\
\hline 753 & -- & -- & -- & -- \\
\hline 754 & -- & -- & -- & -- \\
\hline 755 & -- & -- & -- & -- \\
\hline 756 & -- & -- & -- & -- \\
\hline 757 & -- & -- & -- & -- \\
\hline 758 & -- & -- & -- & -- \\
\hline 759 & -- & -- & -- & -- \\
\hline 760 & -- & -- & -- & -- \\
\hline 761 & -- & -- & -- & -- \\
\hline 762 & -- & -- & -- & -- \\
\hline 763 & -- & -- & -- & -- \\
\hline 764 & -- & -- & -- & -- \\
\hline 765 & -- & -- & -- & -- \\
\hline 766 & -- & -- & -- & -- \\
\hline 767 & -- & -- & -- & -- \\
\hline 768 & -- & -- & -- & -- \\
\hline 769 & -- & -- & -- & -- \\
\hline 770 & -- & -- & -- & -- \\
\hline 771 & -- & -- & -- & -- \\
\hline 772 & -- & -- & -- & -- \\
\hline 773 & -- & -- & -- & -- \\
\hline 774 & -- & -- & -- & -- \\
\hline 775 & -- & -- & -- & -- \\
\hline 776 & -- & -- & -- & -- \\
\hline 777 & -- & -- & -- & -- \\
\hline 778 & -- & -- & -- & -- \\
\hline 779 & -- & -- & -- & -- \\
\hline 780 & -- & -- & -- & -- \\
\hline 781 & -- & -- & -- & -- \\
\hline 782 & -- & -- & -- & -- \\
\hline 783 & -- & -- & -- & -- \\
\hline 784 & -- & -- & -- & -- \\
\hline 785 & -- & -- & -- & -- \\
\hline 786 & -- & -- & -- & -- \\
\hline 787 & -- & -- & -- & -- \\
\hline 788 & -- & -- & -- & -- \\
\hline 789 & -- & -- & -- & -- \\
\hline 790 & -- & -- & -- & -- \\
\hline 791 & -- & -- & -- & -- \\
\hline 792 & -- & -- & -- & - \\
\hline 793 & -- & -- & -- & -- \\
\hline 794 & -- & -- & -- & -- \\
\hline 795 & -- & -- & -- & -- \\
\hline 796 & -- & -- & -- & -- \\
\hline 797 & -- & -- & -- & -- \\
\hline 798 & -- & -- & -- & -- \\
\hline 799 & -- & -- & -- & -- \\
\hline 800 & -- & -- & -- & -- \\
\hline 801 & -- & -- & -- & -- \\
\hline 802 & -- & -- & -- & -- \\
\hline 803 & -- & -- & -- & -- \\
\hline 804 & -112.5 & -15.02 & -- & -- \\
\hline 805 & -115 & -14.98 & -- & -- \\
\hline
\end{tabular}


Appendix 1. Water Quality Data Used in This Report.-Continued

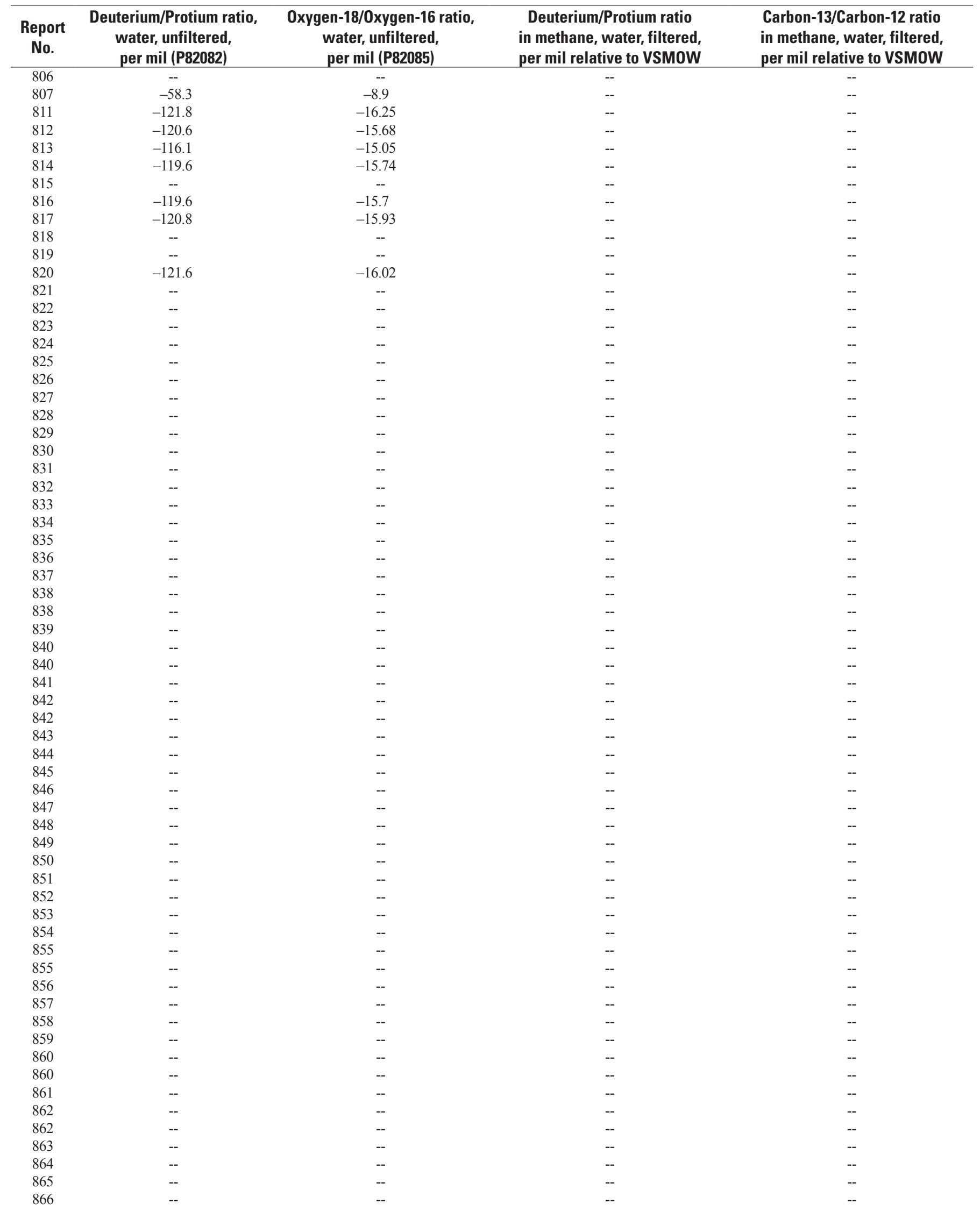


Appendix 1. Water Quality Data Used in This Report.-Continued

\begin{tabular}{|c|c|c|c|c|}
\hline $\begin{array}{c}\text { Report } \\
\text { No. }\end{array}$ & $\begin{array}{l}\text { Deuterium/Protium ratio, } \\
\text { water, unfiltered, } \\
\text { per mil (P82082) }\end{array}$ & $\begin{array}{l}\text { Oxygen-18/0xygen-16 ratio, } \\
\text { water, unfiltered, } \\
\text { per mil (P82085) }\end{array}$ & $\begin{array}{l}\text { Deuterium/Protium ratio } \\
\text { in methane, water, filtered, } \\
\text { per mil relative to VSMOWW }\end{array}$ & $\begin{array}{l}\text { Carbon-13/Carbon-12 ratio } \\
\text { in methane, water, filtered, } \\
\text { per mil relative to VSMOW }\end{array}$ \\
\hline 866 & -- & -- & -- & -- \\
\hline 867 & -- & -- & -- & -- \\
\hline 868 & -- & -- & -- & -- \\
\hline 869 & -- & -- & -- & -- \\
\hline 870 & -- & -- & -- & -- \\
\hline 871 & -- & -- & -- & -- \\
\hline 872 & -- & -- & -- & -- \\
\hline 873 & -- & -- & -- & -- \\
\hline 874 & -- & -- & -- & -- \\
\hline 875 & -- & -- & -- & -- \\
\hline 876 & -- & -- & -- & -- \\
\hline 877 & -- & -- & -- & -- \\
\hline 878 & -- & -- & -- & -- \\
\hline 879 & -- & -- & -- & -- \\
\hline 880 & -- & -- & -- & -- \\
\hline 881 & -- & -- & -- & -- \\
\hline 882 & -- & -- & -- & -- \\
\hline 883 & -- & -- & -- & -- \\
\hline 884 & -- & -- & -- & -- \\
\hline 885 & -- & -- & -- & -- \\
\hline 886 & -- & -- & -- & -- \\
\hline 887 & -- & -- & -- & -- \\
\hline 888 & -- & -- & -- & -- \\
\hline 889 & -- & -- & -- & -- \\
\hline 890 & -- & -- & -- & -- \\
\hline 891 & -- & -- & -- & -- \\
\hline 892 & -- & -- & -- & -- \\
\hline 893 & -- & -- & -- & -- \\
\hline 894 & -- & -- & -- & -- \\
\hline 895 & -- & -- & -- & -- \\
\hline 896 & -- & -- & -- & -- \\
\hline 897 & -- & -- & -- & -- \\
\hline 898 & -- & -- & -- & -- \\
\hline 898 & -- & -- & -- & -- \\
\hline 899 & -- & -- & -- & -- \\
\hline 899 & -- & -- & -- & -- \\
\hline 900 & -- & -- & -- & -- \\
\hline 901 & -- & -- & -- & -- \\
\hline 902 & -- & -- & -- & -- \\
\hline 903 & -- & -- & -- & -- \\
\hline 904 & -- & -- & -- & -- \\
\hline 905 & -- & -- & -- & -- \\
\hline 905 & -- & -- & -- & -- \\
\hline 906 & -- & -- & -- & -- \\
\hline 907 & -- & -- & -- & -- \\
\hline 907 & -- & -- & -- & -- \\
\hline 908 & -- & -- & -- & -- \\
\hline 908 & -- & -- & -- & -- \\
\hline 909 & -- & -- & -- & -- \\
\hline 910 & -- & -- & -- & -- \\
\hline 911 & -- & -- & -- & -- \\
\hline 912 & -- & -- & -- & -- \\
\hline 913 & -- & -- & -- & -- \\
\hline 913 & -- & -- & -- & -- \\
\hline 915 & -- & -- & -- & -- \\
\hline 916 & -- & -- & -- & -- \\
\hline 916 & -- & -- & -- & -- \\
\hline 917 & -- & -- & -- & -- \\
\hline 918 & -- & -- & -- & -- \\
\hline 918 & -- & -- & -- & -- \\
\hline 919 & -- & -- & -- & -- \\
\hline 920 & -- & -- & -- & -- \\
\hline 921 & -- & -- & -- & -- \\
\hline 922 & -- & -- & -- & -- \\
\hline
\end{tabular}


Appendix 1. Water Quality Data Used in This Report.-Continued

\begin{tabular}{|c|c|c|c|c|}
\hline $\begin{array}{c}\text { Report } \\
\text { No. }\end{array}$ & $\begin{array}{c}\text { Deuterium/Protium ratio, } \\
\text { water, unfiltered, } \\
\text { per mil (P82082) }\end{array}$ & $\begin{array}{c}\text { 0xygen-18/0xygen-16 ratio, } \\
\text { water, unfiltered, } \\
\text { per mil (P82085) }\end{array}$ & $\begin{array}{l}\text { Deuterium/Protium ratio } \\
\text { in methane, water, filtered, } \\
\text { per mil relative to VSMOW }\end{array}$ & $\begin{array}{l}\text { Carbon-13/Carbon-12 ratio } \\
\text { in methane, water, filtered, } \\
\text { per mil relative to VSMOW }\end{array}$ \\
\hline 923 & -- & -- & -- & -- \\
\hline 924 & -- & -- & -- & -- \\
\hline 925 & -- & -- & -- & -- \\
\hline 925 & -- & -- & -- & -- \\
\hline 926 & -- & -- & -- & -- \\
\hline 928 & -- & -- & -- & -- \\
\hline 930 & -- & -- & -- & -- \\
\hline 931 & -- & -- & -- & -- \\
\hline 932 & -- & -- & -- & -- \\
\hline 933 & -- & -- & -- & -- \\
\hline 934 & -- & -- & -- & -- \\
\hline 937 & -- & -- & -- & -- \\
\hline 938 & -- & -- & -- & -- \\
\hline 939 & -- & -- & -- & -- \\
\hline 940 & -- & -- & -- & -- \\
\hline 941 & -- & -- & -- & -- \\
\hline 942 & -- & -- & -- & -- \\
\hline 943 & -- & -- & -- & -- \\
\hline 944 & -- & -- & -- & -- \\
\hline 945 & -- & -- & -- & -- \\
\hline 946 & -- & -- & -- & -- \\
\hline 947 & -- & -- & -- & -- \\
\hline 948 & -- & -- & -- & -- \\
\hline 949 & -- & -- & -- & -- \\
\hline 950 & -- & -- & -- & -- \\
\hline 951 & -- & -- & -- & -- \\
\hline 952 & -- & -- & -- & -- \\
\hline 953 & -- & -- & -- & -- \\
\hline 954 & -- & -- & -- & -- \\
\hline 955 & -- & -- & -- & -- \\
\hline 956 & -- & -- & -- & -- \\
\hline 957 & -- & -- & -- & -- \\
\hline 958 & -- & -- & -- & -- \\
\hline 959 & -- & -- & -- & -- \\
\hline 960 & -- & -- & -- & -- \\
\hline 961 & -- & -- & -- & -- \\
\hline 962 & -- & -- & -- & -- \\
\hline 963 & -- & -- & -- & -- \\
\hline 964 & -- & -- & -- & -- \\
\hline 965 & -- & -- & -- & -- \\
\hline 966 & -- & -- & -- & -- \\
\hline 967 & -- & -- & -- & -- \\
\hline 968 & -- & -- & -- & -- \\
\hline 969 & -- & -- & -- & -- \\
\hline 970 & -- & -- & -- & -- \\
\hline 971 & -- & -- & -- & -- \\
\hline 972 & -- & -- & -- & -- \\
\hline 973 & -- & -- & -- & -- \\
\hline 974 & -- & -- & -- & -- \\
\hline 975 & -- & -- & -- & -- \\
\hline 976 & -- & -- & -- & -- \\
\hline 977 & -- & -- & -- & -- \\
\hline 978 & -- & -- & -- & -- \\
\hline 979 & -- & -- & -- & -- \\
\hline 980 & -- & -- & -- & -- \\
\hline 981 & -- & -- & -- & -- \\
\hline 982 & -- & -- & -- & -- \\
\hline 983 & -- & -- & -- & -- \\
\hline
\end{tabular}


Appendix 1. Water Quality Data Used in This Report.-Continued

\begin{tabular}{|c|c|c|c|c|}
\hline $\begin{array}{c}\text { Report } \\
\text { No. }\end{array}$ & $\begin{array}{l}\text { Deuterium/Protium ratio, } \\
\text { water, unfiltered, } \\
\text { per mil (P82082) }\end{array}$ & $\begin{array}{l}\text { Oxygen-18/0xygen-16 ratio, } \\
\text { water, unfiltered, } \\
\text { per mil (P82085) }\end{array}$ & $\begin{array}{l}\text { Deuterium/Protium ratio } \\
\text { in methane, water, filtered, } \\
\text { per mil relative to VSMOWW }\end{array}$ & $\begin{array}{l}\text { Carbon-13/Carbon-12 ratio } \\
\text { in methane, water, filtered, } \\
\text { per mil relative to VSMOW }\end{array}$ \\
\hline 984 & -- & -- & - & -- \\
\hline 985 & -- & -- & -- & -- \\
\hline 986 & -- & -- & -- & -- \\
\hline 987 & -- & -- & -- & -- \\
\hline 988 & -- & -- & -- & -- \\
\hline 989 & -- & -- & -- & -- \\
\hline 990 & -- & -- & -- & -- \\
\hline 991 & -- & -- & -- & -- \\
\hline 992 & -- & -- & -- & -- \\
\hline 993 & -- & -- & -- & -- \\
\hline 994 & -- & -- & -- & -- \\
\hline 995 & -- & -- & -- & -- \\
\hline 996 & -- & -- & -- & -- \\
\hline 997 & -- & -- & -- & -- \\
\hline 998 & -- & -- & -- & -- \\
\hline 999 & -- & -- & -- & -- \\
\hline 1000 & -- & -- & -- & -- \\
\hline 1001 & -- & -- & -- & -- \\
\hline 1002 & -- & -- & -- & -- \\
\hline 1003 & -- & -- & -- & -- \\
\hline 1004 & -- & -- & -- & -- \\
\hline 1005 & -- & -- & -- & -- \\
\hline 1006 & -- & - & -- & -- \\
\hline 1007 & -- & -- & -- & -- \\
\hline 1008 & -- & -- & -- & -- \\
\hline 1009 & -- & -- & -- & -- \\
\hline 1010 & -- & -- & -- & -- \\
\hline 1011 & -- & -- & -- & -- \\
\hline 1012 & -- & -- & -- & -- \\
\hline 1013 & -- & -- & -- & -- \\
\hline 1014 & -- & -- & -- & -- \\
\hline 1015 & -- & -- & -- & -- \\
\hline 1016 & -- & -- & -- & -- \\
\hline 1017 & -- & -- & -- & -- \\
\hline 1018 & -- & -- & -- & -- \\
\hline 1019 & -- & -- & -- & -- \\
\hline 1020 & -- & -- & -- & -- \\
\hline 1021 & -- & -- & -- & -- \\
\hline 1022 & -- & -- & -- & -- \\
\hline 1023 & -- & -- & -- & -- \\
\hline 1024 & -- & -- & -- & -- \\
\hline 1025 & -- & -- & -- & -- \\
\hline 1026 & -- & -- & -- & -- \\
\hline 1027 & -- & -- & -- & -- \\
\hline 1028 & -- & -- & -- & -- \\
\hline 1029 & -- & -- & -- & -- \\
\hline 1030 & -- & -- & -- & -- \\
\hline 1031 & -- & -- & -- & -- \\
\hline 1032 & -- & -- & -- & -- \\
\hline 1033 & -- & -- & -- & -- \\
\hline 1034 & -- & -- & -- & -- \\
\hline 1038 & -- & -- & -- & -- \\
\hline 1039 & -- & -- & -- & -- \\
\hline 1051 & -- & -- & -- & -- \\
\hline 1052 & -- & -- & -- & -- \\
\hline 1053 & -- & -- & -- & -- \\
\hline 1054 & -- & -- & -- & -- \\
\hline 1055 & -- & -- & -- & -- \\
\hline 1057 & -- & -- & -- & -- \\
\hline 1058 & -- & -- & -- & -- \\
\hline 1059 & -- & -- & -- & -- \\
\hline 1062 & -- & -- & -- & -- \\
\hline 1064 & -- & -- & -- & -- \\
\hline
\end{tabular}


Appendix 1. Water Quality Data Used in This Report.-Continued

\begin{tabular}{|c|c|c|c|c|}
\hline $\begin{array}{c}\text { Report } \\
\text { No. }\end{array}$ & $\begin{array}{c}\text { Deuterium/Protium ratio, } \\
\text { water, unfiltered, } \\
\text { per mil (P82082) }\end{array}$ & $\begin{array}{c}\text { 0xygen-18/0xygen-16 ratio, } \\
\text { water, unfiltered, } \\
\text { per mil (P82085) }\end{array}$ & $\begin{array}{l}\text { Deuterium/Protium ratio } \\
\text { in methane, water, filtered, } \\
\text { per mil relative to VSMOW }\end{array}$ & $\begin{array}{l}\text { Carbon-13/Carbon-12 ratio } \\
\text { in methane, water, filtered, } \\
\text { per mil relative to VSMOW }\end{array}$ \\
\hline 1065 & -- & -- & -- & -- \\
\hline 1067 & -- & -- & -- & -- \\
\hline 1068 & -- & -- & -- & -- \\
\hline 1069 & -- & -- & -- & -- \\
\hline 1070 & -- & -- & -- & -- \\
\hline 1080 & -140 & -17.7 & -- & -- \\
\hline 1081 & -- & -- & -- & -- \\
\hline 1084 & -- & -- & -- & -- \\
\hline 1085 & -- & -- & -- & -- \\
\hline 1091 & -- & -- & -- & -- \\
\hline 1094 & -- & -- & -- & -- \\
\hline 1099 & -- & -- & -- & -- \\
\hline 1100 & -- & -- & -- & -- \\
\hline 1101 & -- & -- & -- & -- \\
\hline 1107 & -- & -- & -- & -- \\
\hline 1125 & -- & -- & -- & -- \\
\hline 1130 & -- & -- & -- & -- \\
\hline 1141 & -- & -- & -- & -- \\
\hline 1142 & -- & -- & -- & -- \\
\hline 1144 & -- & -- & -- & -- \\
\hline 1146 & -- & -- & -- & -- \\
\hline 1147 & -- & -- & -- & -- \\
\hline 1149 & -- & -- & -- & -- \\
\hline 1153 & -- & -- & -- & -- \\
\hline 1154 & -- & -- & -- & -- \\
\hline 1160 & -- & -- & -- & -- \\
\hline 1164 & -- & -- & -- & -- \\
\hline 1173 & -- & -- & -- & -- \\
\hline 1174 & -- & -- & -- & -- \\
\hline 1175 & -- & -- & -- & -- \\
\hline 1176 & -- & -- & -- & -- \\
\hline 1177 & -- & -- & -- & -- \\
\hline 1178 & -- & -- & -- & -- \\
\hline 1182 & -- & -- & -- & -- \\
\hline 1185 & -- & -- & -- & -- \\
\hline 1186 & -- & -- & -- & -- \\
\hline 1187 & -- & -- & -- & -- \\
\hline 1188 & -- & -- & -- & -- \\
\hline 1189 & -- & -- & -- & -- \\
\hline 1190 & -- & -- & -- & -- \\
\hline 1191 & -- & -- & -- & -- \\
\hline 1192 & -- & -- & -- & -- \\
\hline 1193 & -- & -- & -- & -- \\
\hline 1194 & -- & -- & -- & -- \\
\hline 1195 & -- & -- & -- & -- \\
\hline 1196 & -- & -- & -- & -- \\
\hline 1197 & -- & -- & -- & -- \\
\hline 1198 & -- & -- & -- & -- \\
\hline 1199 & -- & -- & -- & -- \\
\hline 1200 & -- & -- & -- & -- \\
\hline 1201 & -- & -- & -- & -- \\
\hline 1202 & -- & -- & -- & -- \\
\hline 1203 & -- & -- & -- & -- \\
\hline 1204 & -- & -- & -- & -- \\
\hline 1205 & -- & -- & -- & -- \\
\hline 1206 & -- & -- & -- & -- \\
\hline 1207 & -- & -- & -- & -- \\
\hline 1208 & -- & -- & -- & -- \\
\hline
\end{tabular}


Appendix 1. Water Quality Data Used in This Report.-Continued

\begin{tabular}{|c|c|c|c|c|}
\hline $\begin{array}{c}\text { Report } \\
\text { No. }\end{array}$ & $\begin{array}{l}\text { Deuterium/Protium ratio, } \\
\text { water, unfiltered, } \\
\text { per mil (P82082) }\end{array}$ & $\begin{array}{l}\text { Oxygen-18/0xygen-16 ratio, } \\
\text { water, unfiltered, } \\
\text { per mil (P82085) }\end{array}$ & $\begin{array}{l}\text { Deuterium/Protium ratio } \\
\text { in methane, water, filtered, } \\
\text { per mil relative to VSMOWW }\end{array}$ & $\begin{array}{l}\text { Carbon-13/Carbon-12 ratio } \\
\text { in methane, water, filtered, } \\
\text { per mil relative to VSMOW }\end{array}$ \\
\hline 1209 & -- & -- & -- & -- \\
\hline 1210 & -- & -- & -- & -- \\
\hline 1211 & -- & -- & -- & -- \\
\hline 1213 & -- & -- & -- & -- \\
\hline 1230 & -- & -- & -- & -- \\
\hline 1237 & -- & -- & -- & -- \\
\hline 1239 & -- & -- & -- & -- \\
\hline 1244 & -- & -- & -- & -- \\
\hline 1250 & -- & -- & -- & -- \\
\hline 1251 & -- & -- & -- & -- \\
\hline 1252 & -- & -- & -- & -- \\
\hline 1253 & -- & -- & -- & -- \\
\hline 1254 & -- & -- & -- & -- \\
\hline 1256 & -- & -- & -- & -- \\
\hline 1260 & -- & -- & -- & -- \\
\hline 1265 & -- & -- & -- & -- \\
\hline 1267 & -- & -- & -- & -- \\
\hline 1272 & -- & -- & -- & -- \\
\hline 1273 & -- & -- & -- & -- \\
\hline 1277 & -- & -- & -- & -- \\
\hline 1282 & -- & -- & -- & -- \\
\hline 1297 & -- & -- & -- & -- \\
\hline 1302 & -- & -- & -- & -- \\
\hline 1305 & -- & -- & -- & -- \\
\hline 1306 & -- & -- & -- & -- \\
\hline 1307 & -- & -- & -- & -- \\
\hline 1317 & -- & -- & -- & -- \\
\hline 1318 & -- & -- & -- & -- \\
\hline 1321 & -- & -- & -- & -- \\
\hline 1327 & -- & -- & -- & -- \\
\hline 1328 & -- & -- & -- & -- \\
\hline 1329 & -- & .- & -- & -- \\
\hline 1330 & -- & -- & -- & -- \\
\hline 1333 & -- & -- & -- & -- \\
\hline 1369 & -- & -- & -- & -- \\
\hline 1371 & -- & -- & -- & -- \\
\hline 1381 & -- & .- & -- & -- \\
\hline 1382 & -- & -- & -- & -- \\
\hline 1383 & -- & -- & -- & -- \\
\hline 1387 & -- & -- & -- & -- \\
\hline 1388 & -- & -- & -- & -- \\
\hline 1389 & -- & -- & -- & -- \\
\hline 1390 & -- & -- & -- & -- \\
\hline 1391 & -- & -- & -- & -- \\
\hline 1392 & -- & -- & -- & -- \\
\hline 1393 & -- & -- & -- & -- \\
\hline 1395 & -- & -- & -- & -- \\
\hline 1403 & -- & -- & -- & -- \\
\hline 1409 & -- & -- & -- & -- \\
\hline 1410 & -- & -- & -- & -- \\
\hline 1411 & -- & -- & -- & -- \\
\hline 1415 & -- & -- & -- & -- \\
\hline 1416 & -- & -- & -- & -- \\
\hline 1418 & -- & -- & -- & -- \\
\hline 1419 & -- & -- & -- & -- \\
\hline 1420 & -- & -- & -- & -- \\
\hline 1421 & -- & -- & -- & -- \\
\hline 1422 & -- & .- & -- & -- \\
\hline 1423 & -136 & -17.8 & -- & -- \\
\hline 1426 & -- & -- & -- & -- \\
\hline 1433 & -- & -- & -- & -- \\
\hline 1438 & -- & -- & -- & -- \\
\hline 1443 & -- & -- & -- & -- \\
\hline
\end{tabular}


Appendix 1. Water Quality Data Used in This Report.-Continued

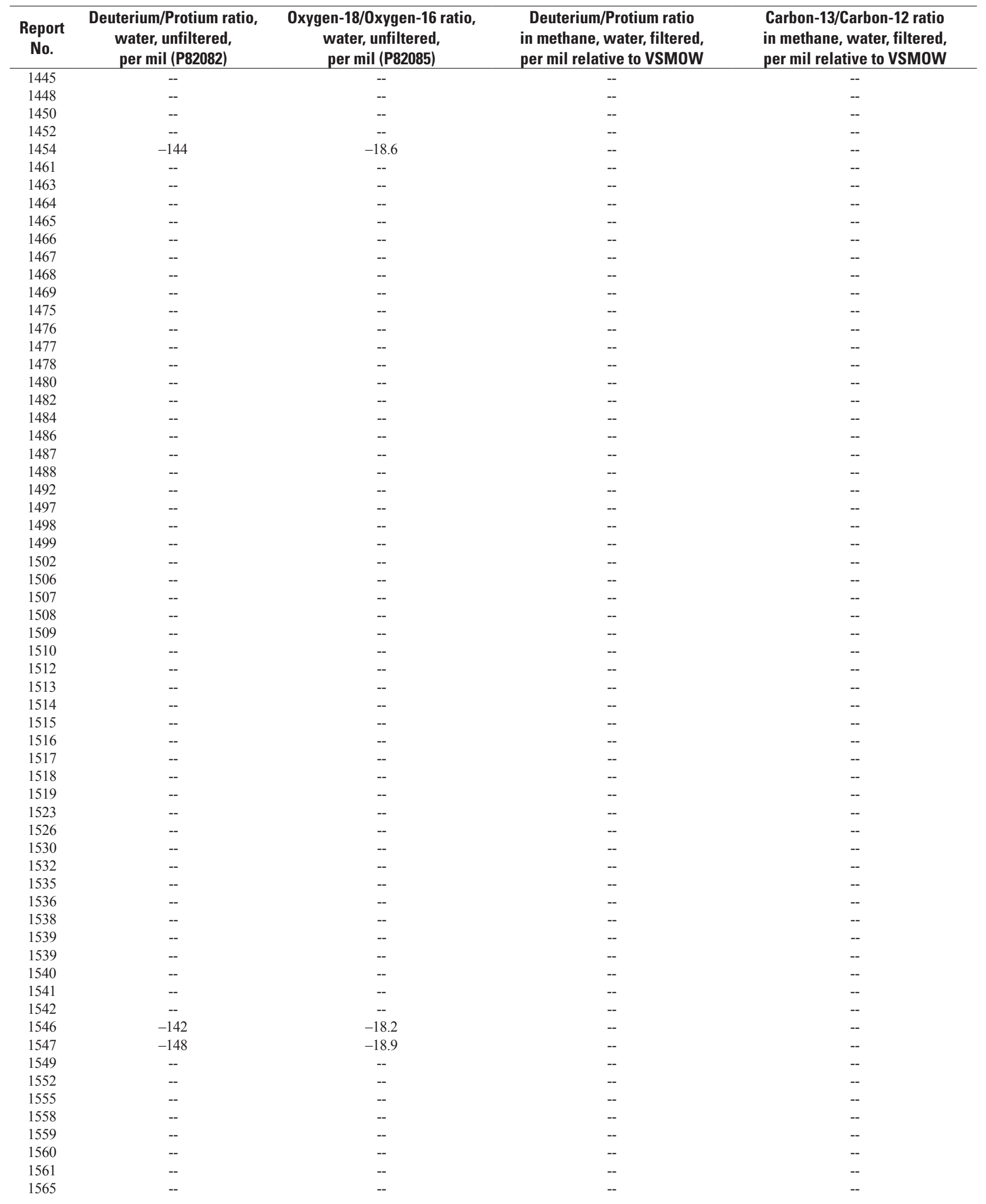


Appendix 1. Water Quality Data Used in This Report.-Continued

\begin{tabular}{|c|c|c|c|c|}
\hline $\begin{array}{c}\text { Report } \\
\text { No. }\end{array}$ & $\begin{array}{c}\text { Deuterium/Protium ratio, } \\
\text { water, unfiltered, } \\
\text { per mil (P82082) }\end{array}$ & $\begin{array}{c}\text { Oxygen-18/0xygen-16 ratio, } \\
\text { water, unfiltered, } \\
\text { per mil (P82085) }\end{array}$ & $\begin{array}{l}\text { Deuterium/Protium ratio } \\
\text { in methane, water, filtered, } \\
\text { per mil relative to VSMOW }\end{array}$ & $\begin{array}{l}\text { Carbon-13/Carbon-12 ratio } \\
\text { in methane, water, filtered, } \\
\text { per mil relative to VSMOW }\end{array}$ \\
\hline 1566 & -- & -- & -- & -- \\
\hline 1567 & -- & -- & -- & -- \\
\hline 1568 & -- & -- & -- & -- \\
\hline 1571 & -- & -- & -- & -- \\
\hline 1576 & -- & -- & -- & -- \\
\hline 1580 & -- & -- & -- & -- \\
\hline 1581 & -- & -- & -- & -- \\
\hline 1587 & -- & -- & -- & -- \\
\hline 1592 & -- & -- & -- & -- \\
\hline 1593 & -- & -- & -- & -- \\
\hline 1594 & -- & -- & -- & -- \\
\hline 1595 & -- & -- & -- & -- \\
\hline 1596 & -- & -- & -- & -- \\
\hline 1597 & -- & -- & -- & -- \\
\hline 1598 & -- & -- & -- & -- \\
\hline 1599 & -- & -- & -- & -- \\
\hline 1600 & -- & -- & -- & -- \\
\hline 1601 & -- & -- & -- & -- \\
\hline 1602 & -- & -- & -- & -- \\
\hline 1603 & -- & -- & -- & -- \\
\hline 1604 & -- & -- & -- & -- \\
\hline 1605 & -- & -- & -- & -- \\
\hline 1607 & -- & -- & -- & -- \\
\hline 1609 & -- & -- & -- & -- \\
\hline 1610 & -- & -- & -- & -- \\
\hline 1612 & -- & -- & -- & -- \\
\hline 1613 & -139 & -17.5 & -- & -- \\
\hline 1614 & -150 & -19.2 & -- & -- \\
\hline 1617 & -- & -- & -- & -- \\
\hline 1622 & -- & -- & -- & -- \\
\hline 1623 & -- & -- & -- & -- \\
\hline 1626 & -- & -- & -- & -- \\
\hline 1628 & -- & -- & -- & -- \\
\hline 1631 & -- & -- & -- & -- \\
\hline 1632 & -- & -- & -- & -- \\
\hline 1633 & -- & -- & -- & -- \\
\hline 1634 & -- & -- & -- & -- \\
\hline 1635 & -- & -- & -- & -- \\
\hline 1636 & -- & -- & -- & -- \\
\hline 1639 & -- & -- & -- & -- \\
\hline 1640 & -- & -- & -- & -- \\
\hline 1641 & -- & -- & -- & -- \\
\hline 1642 & -- & -- & -- & -- \\
\hline 1643 & -- & -- & -- & -- \\
\hline 1644 & -- & -- & -- & -- \\
\hline 1645 & -- & -- & -- & -- \\
\hline 1646 & -- & -- & -- & -- \\
\hline 1647 & -- & -- & -- & -- \\
\hline 1648 & -- & -- & -- & -- \\
\hline 1649 & -- & -- & -- & -- \\
\hline 1650 & -- & -- & -- & -- \\
\hline 1651 & -- & -- & -- & -- \\
\hline 1652 & -- & -- & -- & -- \\
\hline 1653 & -- & -- & -- & -- \\
\hline 1654 & -- & -- & -- & -- \\
\hline 1655 & -- & -- & -- & -- \\
\hline 1656 & -- & -- & -- & -- \\
\hline 1657 & -- & -- & -- & -- \\
\hline 1658 & -- & -- & -- & -- \\
\hline 1659 & -- & -- & -- & -- \\
\hline 1660 & -- & -- & -- & -- \\
\hline 1661 & -- & -- & -- & -- \\
\hline 1662 & -- & -- & -- & -- \\
\hline 1664 & -- & -- & -- & -- \\
\hline
\end{tabular}


Appendix 1. Water Quality Data Used in This Report.-Continued

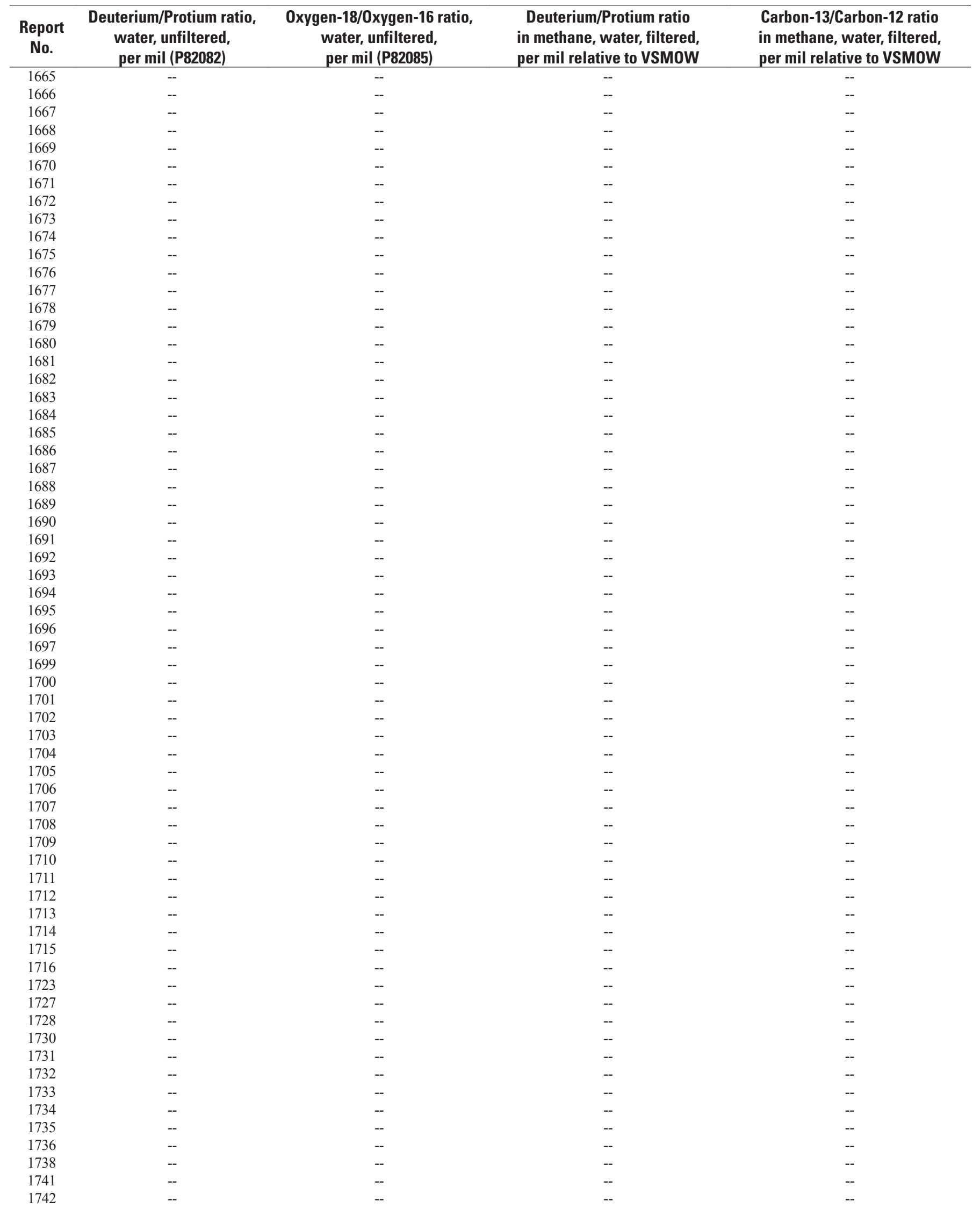


Appendix 1. Water Quality Data Used in This Report.-Continued

\begin{tabular}{|c|c|c|c|c|}
\hline $\begin{array}{c}\text { Report } \\
\text { No. }\end{array}$ & $\begin{array}{c}\text { Deuterium/Protium ratio, } \\
\text { water, unfiltered, } \\
\text { per mil (P82082) }\end{array}$ & $\begin{array}{c}\text { Oxygen-18/0xygen-16 ratio, } \\
\text { water, unfiltered, } \\
\text { per mil (P82085) }\end{array}$ & $\begin{array}{l}\text { Deuterium/Protium ratio } \\
\text { in methane, water, filtered, } \\
\text { per mil relative to VSMOW }\end{array}$ & $\begin{array}{l}\text { Carbon-13/Carbon-12 ratio } \\
\text { in methane, water, filtered, } \\
\text { per mil relative to VSMOW }\end{array}$ \\
\hline 1743 & -- & $-\operatorname{lol}^{-}$ & -- & -- \\
\hline 1745 & -- & -- & -- & -- \\
\hline 1747 & -- & -- & -- & -- \\
\hline 1752 & $\mathrm{U}$ & $\mathrm{U}$ & $\mathrm{U}$ & $\mathrm{U}$ \\
\hline 1753 & -- & -- & -- & -- \\
\hline 1756 & -- & -- & -- & -- \\
\hline 1757 & -- & -- & -- & -- \\
\hline 1758 & -- & -- & -- & -- \\
\hline 1759 & -- & -- & -- & -- \\
\hline 1760 & -- & -- & -- & -- \\
\hline 1761 & -- & -- & -- & -- \\
\hline 1768 & -124 & -16.4 & -- & -- \\
\hline 1769 & -121 & -16.25 & -- & -- \\
\hline 1770 & -- & -- & -- & -- \\
\hline 1771 & -- & -- & -- & -- \\
\hline 1772 & -- & -- & -- & -- \\
\hline 1773 & -- & -- & -- & -- \\
\hline 1774 & -- & -- & -- & -- \\
\hline 1776 & -- & -- & -- & -- \\
\hline 1777 & -- & -- & -- & -- \\
\hline 1778 & -- & -- & -- & -- \\
\hline 1779 & -- & -- & -- & -- \\
\hline 1780 & -- & -- & -- & -- \\
\hline 1781 & -- & -- & -- & -- \\
\hline 1791 & -- & -- & -- & -- \\
\hline 1792 & -- & -- & -- & -- \\
\hline 1793 & -- & -- & -- & -- \\
\hline 1794 & -- & -- & -- & -- \\
\hline 1795 & -- & -- & -- & -- \\
\hline 1796 & -- & -- & -- & -- \\
\hline 1797 & -- & -- & -- & -- \\
\hline 1798 & -- & -- & -- & -- \\
\hline 1799 & -- & -- & -- & -- \\
\hline 1800 & -- & -- & -- & -- \\
\hline 1801 & -- & -- & -- & -- \\
\hline 1802 & -- & -- & -- & -- \\
\hline 1803 & -- & -- & -- & -- \\
\hline 1804 & -- & -- & -- & -- \\
\hline 1805 & -- & -- & -- & -- \\
\hline 1806 & -- & -- & -- & -- \\
\hline 1807 & -- & -- & -- & -- \\
\hline 1807 & -- & -- & -- & -40.310 \\
\hline 1808 & -- & -- & -- & -- \\
\hline 1809 & -- & -- & -- & -- \\
\hline 1810 & -- & -- & -- & -- \\
\hline 1811 & $\mathrm{U}$ & $\mathrm{U}$ & $\mathrm{U}$ & $\mathrm{U}$ \\
\hline 1812 & $\mathrm{U}$ & $\mathrm{U}$ & $\mathrm{U}$ & $\mathrm{U}$ \\
\hline 1813 & -- & -- & -- & -- \\
\hline
\end{tabular}


Appendix 1. Water Quality Data Used in This Report.-Continued

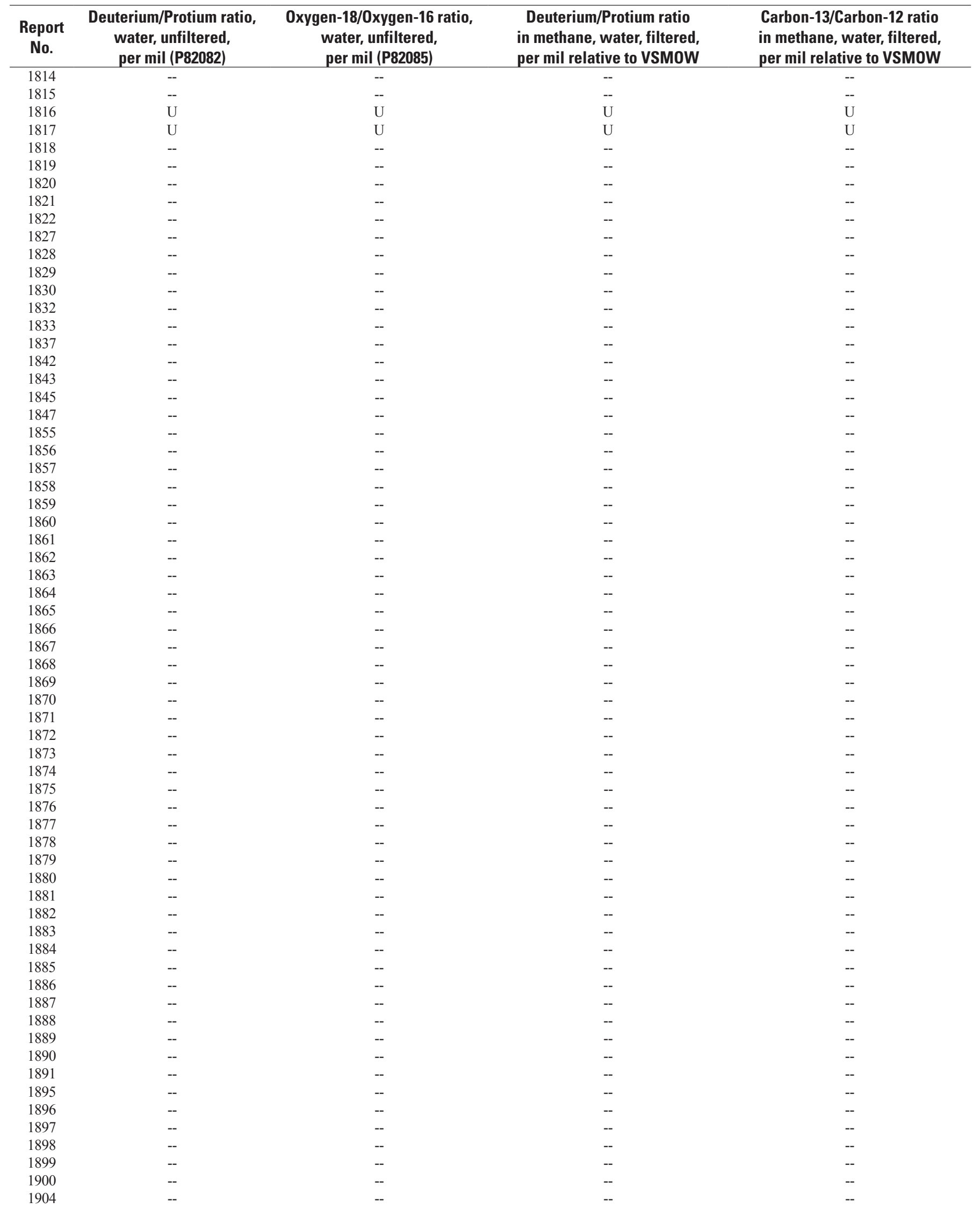


Appendix 1. Water Quality Data Used in This Report.-Continued

\begin{tabular}{|c|c|c|c|c|}
\hline $\begin{array}{c}\text { Report } \\
\text { No. }\end{array}$ & $\begin{array}{l}\text { Deuterium/Protium ratio, } \\
\text { water, unfiltered, } \\
\text { per mil (P82082) }\end{array}$ & $\begin{array}{l}\text { Oxygen-18/0xygen-16 ratio, } \\
\text { water, unfiltered, } \\
\text { per mil (P82085) }\end{array}$ & $\begin{array}{l}\text { Deuterium/Protium ratio } \\
\text { in methane, water, filtered, } \\
\text { per mil relative to VSMOW }\end{array}$ & $\begin{array}{l}\text { Carbon-13/Carbon-12 ratio } \\
\text { in methane, water, filtered, } \\
\text { per mil relative to VSMOW }\end{array}$ \\
\hline 1906 & -- & - & -- & - \\
\hline 1907 & -- & -- & -- & -- \\
\hline 1908 & -- & -- & -- & -- \\
\hline 1909 & -- & -- & -- & -- \\
\hline 1910 & -- & -- & -- & -- \\
\hline 1911 & -- & -- & -- & -- \\
\hline 1912 & -- & -- & -- & -- \\
\hline 1913 & -- & -- & -- & -- \\
\hline 1914 & -- & -- & -- & -- \\
\hline 1915 & -- & -- & -- & -- \\
\hline 1916 & -- & -- & -- & -- \\
\hline 1917 & -- & -- & -- & -- \\
\hline 1918 & -- & -- & -- & -- \\
\hline 1919 & -- & -- & -- & -- \\
\hline 1920 & -- & -- & -- & -- \\
\hline 1921 & -- & -- & -- & -- \\
\hline 1925 & -117 & -15.75 & -- & -- \\
\hline 1926 & -116 & -15.5 & -- & -- \\
\hline 1928 & -- & -- & -- & -- \\
\hline 1930 & -- & -- & -- & -- \\
\hline 1931 & -- & -- & -- & -- \\
\hline 1932 & -- & -- & -- & -- \\
\hline 1937 & -- & -- & -- & -- \\
\hline 1938 & -- & -- & -- & -- \\
\hline 1939 & -- & -- & -- & -- \\
\hline 1940 & -- & -- & -- & -- \\
\hline 1941 & -- & -- & -- & -- \\
\hline 1942 & -- & -- & -- & -- \\
\hline 1943 & -- & -- & -- & -- \\
\hline 1944 & -- & -- & -- & -- \\
\hline 1945 & -- & -- & -- & -- \\
\hline 1946 & -- & -- & -- & -- \\
\hline 1947 & -- & -- & -- & -- \\
\hline 1951 & -- & -- & -- & -- \\
\hline 1955 & -- & -- & -- & -- \\
\hline 1962 & -- & -- & -- & -- \\
\hline 1967 & -- & -- & -- & -- \\
\hline 1968 & -- & -- & -- & --. \\
\hline 1969 & -- & -- & -- & -- \\
\hline 1970 & -- & -- & -- & -- \\
\hline 1971 & -- & -- & -- & -- \\
\hline 1972 & -- & -- & -- & -- \\
\hline 1973 & -- & -- & -- & -- \\
\hline 1974 & -- & -- & -- & -- \\
\hline 1975 & -- & -- & -- & -- \\
\hline 1976 & -- & -- & -- & -- \\
\hline 1978 & -- & -- & -- & -- \\
\hline 1981 & -- & -- & -- & -- \\
\hline 1982 & -- & -- & -- & -- \\
\hline 1983 & -- & -- & -- & -- \\
\hline 1986 & -- & -- & -- & -- \\
\hline 1990 & -- & -- & -- & -- \\
\hline 1993 & -- & -- & -- & -- \\
\hline 1995 & -- & -- & -- & -- \\
\hline 1996 & -- & -- & -- & -- \\
\hline 2001 & -- & -- & -- & -- \\
\hline 2002 & -- & -- & -- & -- \\
\hline 2003 & -- & -- & -- & -- \\
\hline 2011 & -- & -- & -- & -- \\
\hline 2012 & -- & -- & -- & -- \\
\hline
\end{tabular}


Appendix 1. Water Quality Data Used in This Report.-Continued

\begin{tabular}{|c|c|c|c|c|}
\hline $\begin{array}{c}\text { Report } \\
\text { No. }\end{array}$ & $\begin{array}{c}\text { Deuterium/Protium ratio, } \\
\text { water, unfiltered, } \\
\text { per mil (P82082) }\end{array}$ & $\begin{array}{c}\text { Oxygen-18/0xygen-16 ratio, } \\
\text { water, unfiltered, } \\
\text { per mil (P82085) }\end{array}$ & $\begin{array}{l}\text { Deuterium/Protium ratio } \\
\text { in methane, water, filtered, } \\
\text { per mil relative to VSMOWW }\end{array}$ & $\begin{array}{l}\text { Carbon-13/Carbon-12 ratio } \\
\text { in methane, water, filtered, } \\
\text { per mil relative to VSMOW }\end{array}$ \\
\hline 2013 & -- & -- & -- & -- \\
\hline 2014 & -- & -- & -- & -- \\
\hline 2015 & -- & -- & -- & -- \\
\hline 2016 & -- & -- & -- & -- \\
\hline 2017 & -- & -- & -- & -- \\
\hline 2018 & -- & -- & -- & -- \\
\hline 2019 & -- & -- & -- & -- \\
\hline 2020 & -- & -- & -- & -- \\
\hline 2021 & -- & -- & -- & -- \\
\hline 2022 & -- & -- & -- & -- \\
\hline 2023 & -- & -- & -- & -- \\
\hline 2024 & -- & -- & -- & -- \\
\hline 2025 & -- & -- & -- & -- \\
\hline 2027 & -- & -- & -- & -- \\
\hline 2029 & -- & -- & -- & -- \\
\hline 2030 & -- & -- & -- & -- \\
\hline 2033 & -- & -- & -- & -- \\
\hline 2034 & -- & -- & -- & -- \\
\hline 2035 & -- & -- & -- & -- \\
\hline 2037 & -- & -- & -- & -- \\
\hline 2040 & -- & -- & -- & -- \\
\hline 2041 & -- & -- & -- & -- \\
\hline 2044 & -- & -- & -- & -- \\
\hline 2046 & -119 & -16.05 & -- & -- \\
\hline 2046 & -- & -- & -- & -- \\
\hline 2049 & -- & -- & -- & -- \\
\hline 2050 & -- & -- & -- & -- \\
\hline 2051 & -- & -- & -- & -- \\
\hline 2052 & -- & -- & -- & -- \\
\hline 2053 & -- & -- & -- & -- \\
\hline 2054 & -- & -- & -- & -- \\
\hline 2055 & -- & -- & -- & -- \\
\hline 2056 & -- & -- & -- & -- \\
\hline 2058 & -- & -- & -- & -- \\
\hline 2061 & -117 & -15.65 & -- & -- \\
\hline 2063 & -- & -- & -- & -- \\
\hline 2064 & -- & -- & -- & -- \\
\hline 2065 & -- & -- & -- & -- \\
\hline 2066 & -- & -- & -- & -- \\
\hline 2067 & -- & -- & -- & -- \\
\hline 2068 & -- & -- & -- & -- \\
\hline 2069 & -- & -- & -- & -- \\
\hline 2070 & -- & -- & -- & -- \\
\hline 2071 & -- & -- & -- & -- \\
\hline 2072 & -- & -- & -- & -- \\
\hline 2076 & -- & -- & -- & -- \\
\hline 2077 & -- & -- & -246.900 & -45.170 \\
\hline 2078 & -- & -- & -- & -- \\
\hline 2079 & -- & -- & -- & -- \\
\hline 2080 & -- & -- & -- & -- \\
\hline 2082 & -- & -- & -- & -- \\
\hline 2083 & -- & -- & -- & -- \\
\hline 2084 & -- & -- & -- & -- \\
\hline 2085 & -- & -- & -- & -- \\
\hline 2088 & -- & -- & -- & -- \\
\hline 2089 & -- & -- & -- & -- \\
\hline 2090 & -- & -- & -- & -- \\
\hline 2091 & -- & -- & -- & -- \\
\hline 2094 & -- & -- & -- & -- \\
\hline 2095 & -- & -- & -- & -- \\
\hline 2096 & -- & -- & -- & -- \\
\hline
\end{tabular}


㺃 